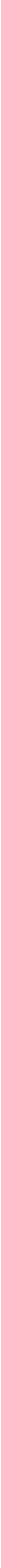




\section{NAVAL OCEAN SYSTEMS CENTER}

E. G. SCHWEIZER, CAPT, USN

R. M. HILLYER

Commander

Technical Director

\section{ADMINISTRATIVE INFORMATION}

This reissue of Naval Electronics Laboratory Center (NELC) Technical Notes 2031, 2371, and 2569 was funded by the Office of Naval Technology. Arlington, VA 22217. The authors are currently members of the Marine Sciences and Technology Department, Naval Ocean Systems Center, San Diego, CA 92152-5000.

Released by

H.V. Hitney, Head

Tropospheric Branch
Under authority of

J.H. Richter, Head

Ocean and Atmospheric

Sciences Division 
UNCLASSIFIED

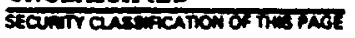

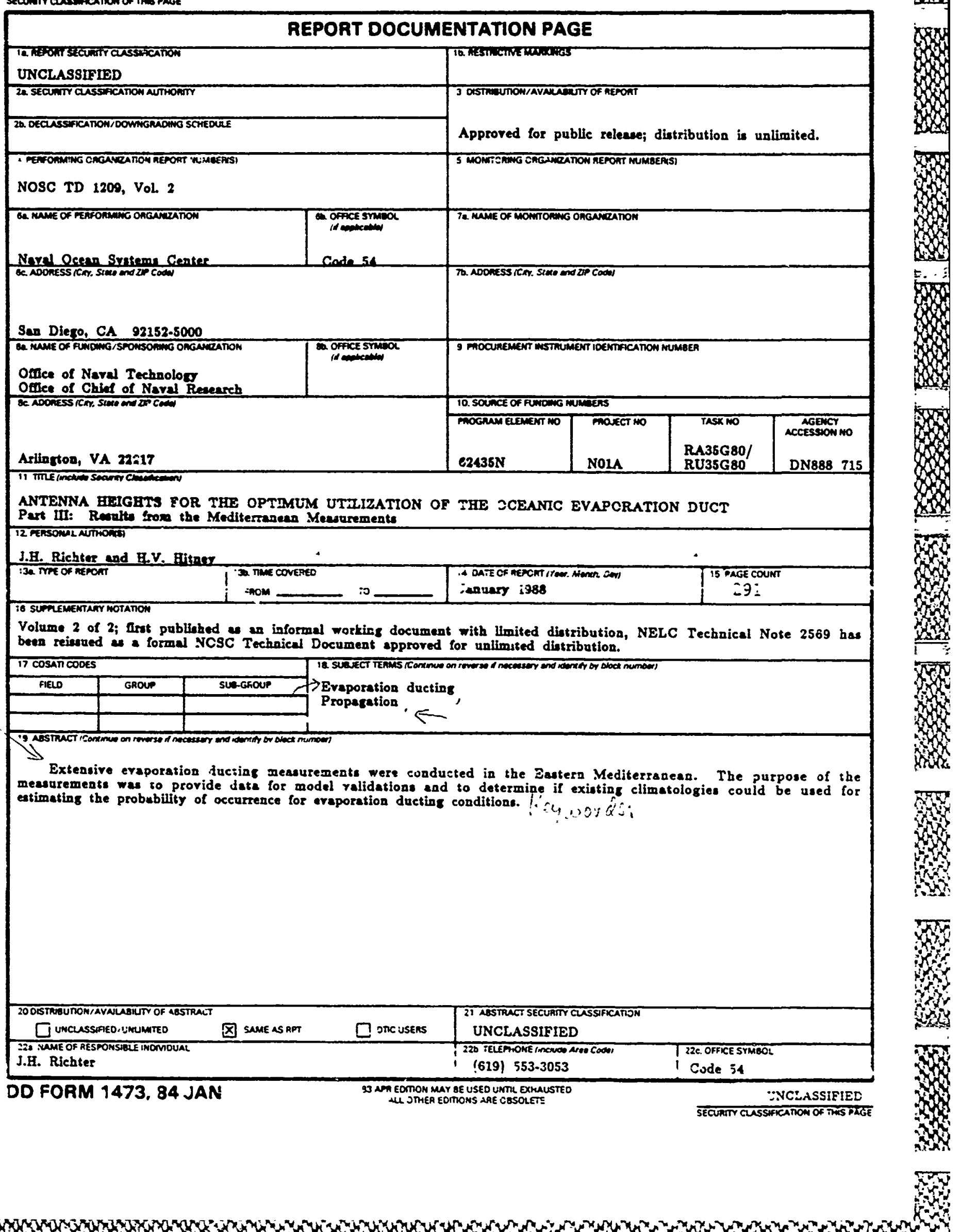





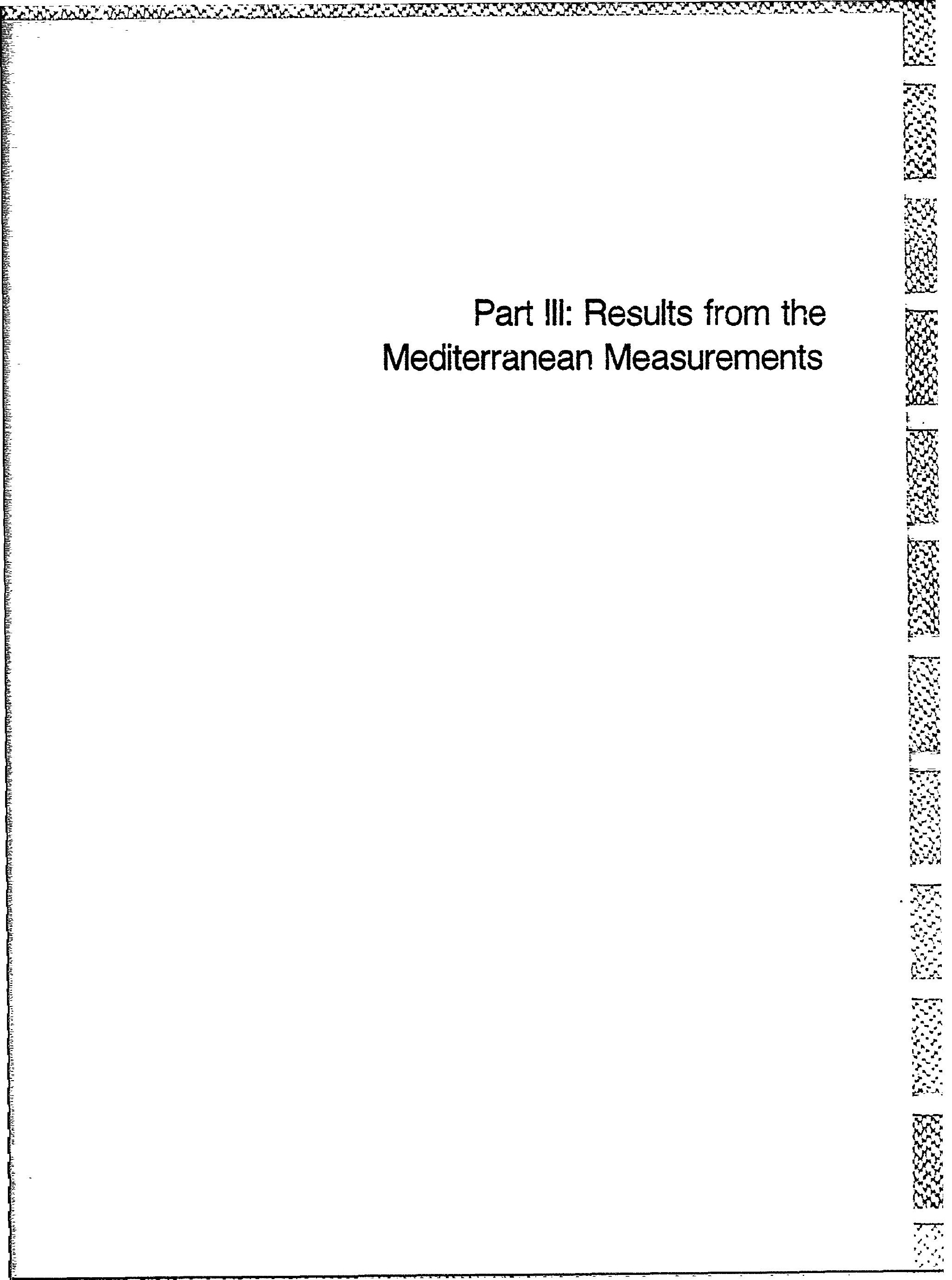


EUNTENTS

\begin{tabular}{llr} 
& & PAGE \\
I. & BACKGROUND & 1 \\
II. & OBJECTIVES & 3 \\
III. APPROACH & 4 \\
IV. & RESULTS & 5 \\
& A. Propagation Measurements & 6 \\
& B. Meteorological Comparisons & 6 \\
V. & CONCLUSIONS & 15 \\
VI. RECOMMENDATIONS & 21 \\
VII. REFERENCES & 23 \\
VIII. FIGURES & 24 \\
IX. TABLES & APPENDICES \\
X. & A. Detection Range Calculations & 25 \\
XI. RCKNOWIEDGEMENT & 200 \\
& B. & 283 \\
\hline
\end{tabular}


SUMMARY

Radio propagation measurements in the $1-40 \mathrm{GHz}$ frequency range were performed during 1972 in the Eastern Mediterranean. A propagation link between the islands of Mkonos and Naxos in the Aegean Sea was operated during four measurement perlods, each lasting approximately two weeks. The receiver terminal was equipped with vertically spaced antennas in order to obtain information on optimum shipboard antenna heights. The measurements showed that evaporation ducting is an important phenomenon, in particular for frequencies above S-band. For example, signal enhancements from evaporation ducting were measured $99 \%$ of the time for $X-b$ and frequencles. It was determined that the evaporation duct strongly affects propagation for all shipboard antema heights. Under conditions of strong ducting, 10w sited antennas (e.g. 15 feet above mean sea level) may receive higher signals than more conventional antema heights (e.g. $60-70$ feet above ms 1 ). For all measurements in the Mediterranean the low sited 8 -band antenna received equal or higher signals than the high antenna $47.4 \pi$ of the time. During $20 \%$ of the time signals received at the low sited $\mathrm{X}$-band antenna exceeded those received on the high sited antenna by $10 \mathrm{~dB}$. From the measurements one may conclude that the optimum location for an antema is high on the ship. When economics justify two antemas, an advantage can be obtained with both a high and low antenna.

The evaporation ducting effect sppears to have a broad maximum in the $\mathrm{X}$ - to Ku-band frequency range. Atmospheric absorption and sea surface roughness apparently counteract the effectiveness of the duct expected at higher frequencies. 
Simple meteorological measurements were found to be quite sufficient to describe ducting conditions. Horizontal homogeneity of the duct was found to be $800 d$ for the propagation path used in this investigation. Ducting effects deduced from long term meteorological averages compared well with the actual measurements, permitting estimates of ducting conditions to be made for any oceanic area for which such statistical meteorological data are avaflable. 


\section{BACKGRCUND}

Parts I and II of this series of reports (references 1 and 2) described microwave propagation measurements in the oceanic evaporation duct performed off the California and Florida coast. Ducting conditions were found to be signtficantly different in the two areas. At $X$-band antenna reversals (i.e. an antenna $64^{\prime}$ above mean sea level received less signal strength than an antenna at $16^{\circ}$ above mean sea level) occurred less than $10 \%$ of the time In Californla and $60 \%$ of the time in Florida. The apparent desendency of ducting conditions on geographic locations makes it important for Naval operations to be able to estimate occurrence and effects of ducting in various oceanic areas. As it is impractical to perform long term measurements in all oceanic areas of interest, one has to rely on available meteorological statistics. Available statistical meteorological data (from sources like the National Weather Records Center in Ashville, North Carolina) were not gathered with the accuracy one would like to describe evaporation duct parameters. However, one can hope that in the absence of any constant bias in the data the averages of long term statistical data are of sufficient quality to make rellable judgements of evaporation ducting conditions. In order to check tiis assumption, it was decided to perform extensive measurements during one year in a strategically important area which encounters a wide range of ducting conditions. The Mediterranean meets these conditions and two islands in the Aegean Sea were selected for the measurements. In order to encounter the full range of seasonal variacions, measurements were performed in four different seasons during 1972. 


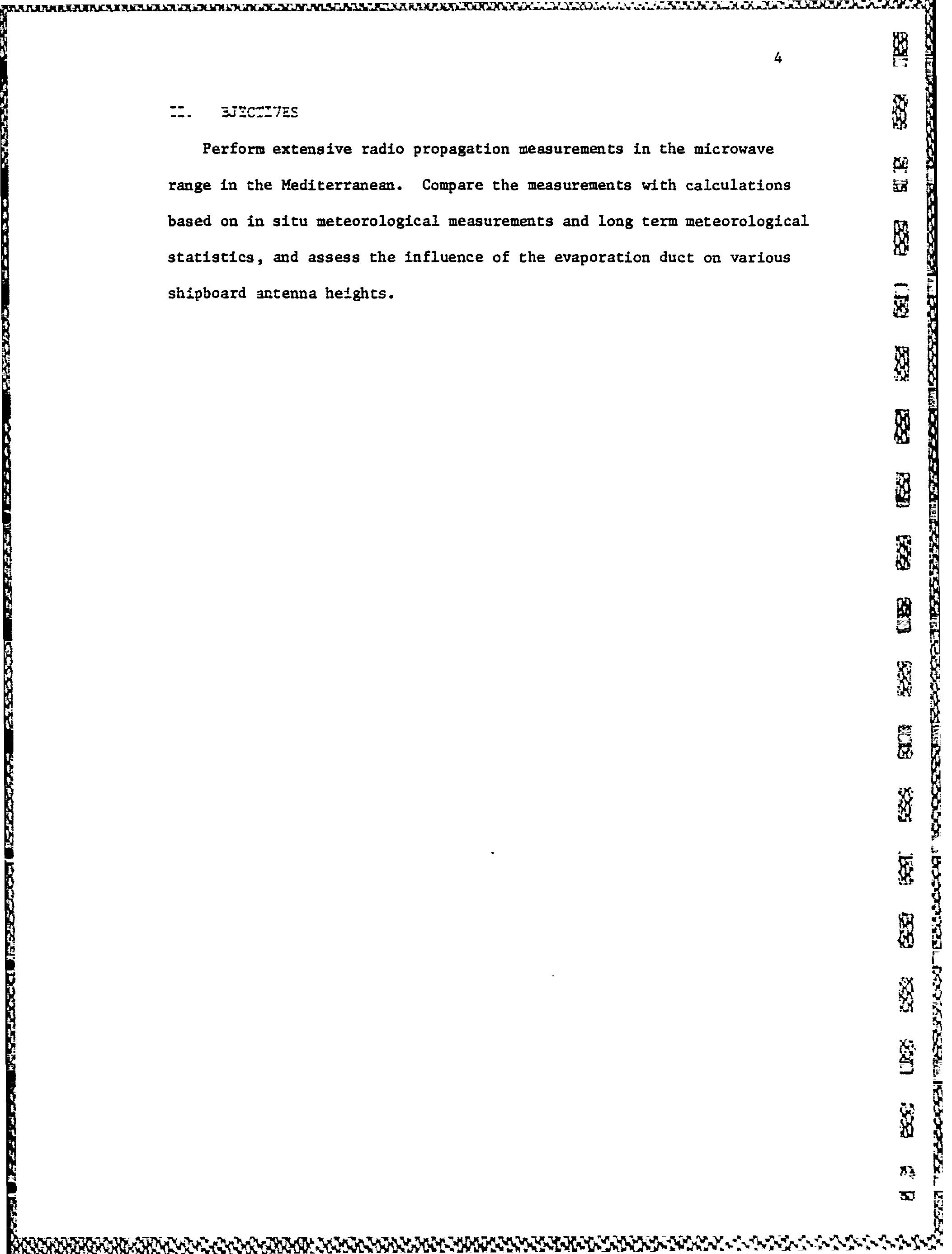




\section{APPROACH}

Following the approach described in reference 1, a propagation link was established between the islands of Nykonos and Naxos in the Aegean Sea. The transmitter was placed on the island of Naxos. The geographical location of the propagation path is shown in figure 1. The UTM coordinates (zone 35) for the transmitter and receiver sites were $4108.35 ; 355.70$ and 4143. 32 ; 351.65 respectively. The links were operated during four different perfods each of which lasted for two weeks. Table 1 lists frequencies and duration of the various measurement periods. The block diagrams for transmitters and recelvers are given in references $i-3$. Table 2 compiles the profagation link characteristlcs for all five frequencies used during the Mediterranean measurements. Three vertically spaced antennas were used for L-, S-, $\mathrm{X}-$, and $\mathrm{Ku}-\mathrm{b}$ and and two for $\mathrm{Ka-band.}$ Figure 2 shows the receiving terminal at 0 rnos Beach, Mykonos. The equipment was housed in the trailer to. the left of the mast. Figure 3 snows some of the receiving equipment inside the trailer. The upper row of receivers and recorders was for L-, S-, $X-$, and Ku-band while the equipment of the lower shelf belonged to the Ka-band receiving system described in reference 3 . Figure 4 shows the eransmitting antennas at vaxos. The $3^{\prime}$ antenna to the right radiated at $L_{-}, \mathrm{S}-$, and $\mathrm{X}$-band frequencies, the $1.5^{\prime}$ center antenna at $\mathrm{Ku}-$ band and the $3^{\prime}$ antenna to the left at Ka-band frequencies. Commercial power was used during all measurement periods at Naxos and during the sumer and jall periods at Mykonos. In the winter and spring periods, diesel generators were used at Mykonos. 
IV. RESULTS

A. Propagation Measurements

$\Delta$

Figures 5-134 and tables 3-62 show the results of the measurements

in graphical and tabular form. The time indicated on the figures is eastern European standard t1me. Groupling was done by measurement period and frequency. Figures 5-11 sumarize the L-band measurements for the vinter period. The upper part of figure 5 gives the path $108 s$ as a function of time for the high L-band antenna. The upper parts of figures 6 and 7 show path loss for the middle and the low antennas. Two dashed reference lines in this presentation represent free space path loss and path loss due to diffraction assuming a standard atmosphere. The L-band path loss values for all three antenna helghts stay close to the diffraction field value which means that the evaporation duct helghts encountered have little influence on L-band frequencies. The lower part of figures $5-7$ shows the path loss difference For different anteñna configurations. Positive differences mean higher signals on the higher antmnas and negative vaiues higher signals on the lower antennas. In all three cases (high-low, midlow, high mid) the higher antenna received higher signals than the lower antenna. This is what one would expect if evaporation ducting is not a significant factor influencing propagation conditions. Fading for the three antenna heights is plotted in figure 8 . Fading is here defined as the maximum deviation from the mean signal during a five minute period. Rapid fluctuations wer suppressed oy a four secona ime constant in the receiver. In figure 8 , fading increases with decreasing antenna height or, in othe: words, higher fluctuations were observed for the weaker signals. Except for path loss, the information graphically displayed in 
figures $5-8$ is presented in tabular form in table 3 . The upper portion of this table gives the percentage of time the difference between an antenna pair exceeds a certain value in $\mathrm{dB}$. As an example, signals received on the high antenna during the February measurement period exceeded those recelved on tine low antenna by more than $15 \mathrm{~dB}$ during $54.5 \%$ of the time. The lower portion of table 3 lists for the three antennas the percentage of time certain fading values in $d B$ were exceeded. As an example, the fading observed on the high antenna exceeded $3 \mathrm{~dB}$ during $0.6 \%$ of the time. Figures 9-11 present the statistical information in graphic form. Figure 9 shows the percentage of occurrence of path loss values in $5 \mathrm{~dB}$ intervals. The distribution shifts toward higher path $108 s$ values with decreasing antenna height. Fading distributions in $0.5 \mathrm{~dB}$ intervals are displayed in figure 10. The numerical values of figures 9 and 10 are listed in table 4. F1gure 11 shows the frequency distribution of path loss difference besween antennas plotted for $2 \mathrm{AB}$ intervals. The path loss differences are aiways positive and are highest for maximum antenna separarion. Tabie $j$ contains the corresponding numertcal values.

Figures $12-18$ and tables $6-8$ contain the S-band propagation measurements for the winter period. Path loss values as shown in the upper portions of the figures are consistently above diffraction field values which means that S-band frequencles were apparently influenced by ducting conditions. However, the path loss differences between antennas are dimost always positive, i.a. received signal strength increased $\forall i t h$ antenna height. One may conclude that ducting conditions were not strong enough to cause trapping of $10 \mathrm{~cm}$ waves. Fading in figure 15 follows the 
same zend obseryed at L-band frequencies, is incraases izit fecreas=ng

antenna height (or decreasing signals).

While L-band frequencies were apparently not affected by ducting

conditions, and S-band frequencies only moderately so, there is a strong influence on propagation of $X-b$ and frequencies as evidenced in figures 19-28 and tables 9-12. The upper portions of figures 19-21 show that observed path loss values approach and even become less than the free space path loss value. Also, the differences between path loss values measured at the three different antenna helghts frequently assume negative values, 1.e. higher signals are observed on antennas closer to the surface. The statistical comparison of antenna performance in table 9 shows that during 49.3\% of the t1me the high antenna observed higher signals than the low antenna. In other words, for the ducting conditions encountered during this meaurement period, none of the three antenna heights showed a distince advantage over the other. Also, the fading (figure 22 and table 9) is quite similar For all antennas. Figures $23-25$ show the frequency distributions of path loss, fading, and path loss differences. The spread of path loss difference values is largest for the maximum antenna separation (high-low) and smallest fur the antennas which are closest to each other (mid-low). This spread is both an indlcation of stronger ducting effects on lower antennas and spatial decorrelation between antennas.

The formar for presenting the data shown so far has been carefully W

ehosen to provide a systems designer with the maximum information on systems performance under varying ducting conditions and the relative merits of antennas at different heights. Path loss was chosen as the primary 
parameter in this format. While path loss represents an excellent quantity independent of specific syetems parameters, it was realized that this very independence might limit its usefulness under specific operational circumstances. Therefore, it was attempted to translate propagation conditions into an operationally important parametez for judging radar performance. The parameter chosen was detection range. Surveillance radar performance is often described by the range a target with a specified radar cross section (e.g. one square meter) can be detected under free space propagation conditions. All of the calculations presented here assume a free space detection range of 200 nautical miles at $9.6 \mathrm{GHz}$ and all detection range figures thus have units of nautical miles. The procedure for the detection range calculations is described in Appendix A. Figure 26 shows the Erequency distribution of detection range calculated for the propagation conditions encountered during the winter measurement period. In the absence of any ducting the detection ranges vouid have jeen 16,14 , and 12 nautical miles for the higin, middle, and low antenna respectively. Figure 26 illustrates the fact that detection ranges due to ducting are significantly extended a large percentage of the time. It also shows that extreme extensions of detection ranges are obtained for the lowest antenna under conditions of strong ducting. The nean detection canges were $28 \mathrm{n}$ miles for the high and the middle antenna and $31 \mathrm{n}$ miles for the low antenna. These mean decection ranges represent range extensions comfared to no jucring of $753,100 z$, and $158 \%$ Eor tise high, midàle, anc jow antenna respectively. Figure 27 shows the cumulative distribution of detection range. In analogy to the path loss difference presentations of figure 25, detection zange differences are plotted in figure 28. The 
abscissa in this presentation is in nautical miles and the graphs show the percentage of time for which one antenna exceeds the detection range of another antenna by a certain value. Positive values mean longer detection ranges for the higher antenna and negative values longer detection ranges for the lowe: antenna.

The spring measurements were conducted during 18 April to 1 May 1972. Power and equipment fallures caused the loss of some data during the pertod. The path $108 s$ curves for the three L-band antennas in figures 29-31 show more variation compared to the winter period. In particular, during the second half of the spring period the signals vary between free space and diffraction values which is an indication of highly variable atmospherlc refractive conditions. The higher variability is also reflected in the fading of f1gure 32 . S-band frequencies are even more affected as seen from the path loss curves for the three antenna heights in figures 36-39. Tree space values 3re exceeded for over $20 \%$ of the time for the high antenna, $14 \%$ for the midde antenna and $11 \%$ for the low antenna. Signal reversals between high and low antenna occurred during 4.4\% of the time (table 16). Also for S-band frequencies the fading was somewhat higher than during the winter period. The path loss values measured during the spring period for $X$-band frequencies exceed free space values $70 \%$ of the time at the high antenna and $57 \%$ of the time at the middle and low antennas (figures 43-45). However these low path loss (or ilgi signal; vaiues are accompanied by strong fading (figure 46). The low antenna recelved higher signals than the high antenna $27.9 \%$ of the time. It is interesting to note that even though atmospheric refractive sondirion caused higher signals compazed to the vinter period, 
antenna reversals occurred less frequently in the spring period than in winter. A qualitative explanation will be given later in discussion of meteorological measurements. Path loss in terms of detection range is . shown in figures 50 and 51 . The interval of 190-200 nautical miles in figure 50 includes all detection ranges exceeding 200 nautical miles. The mean detection ranges calculated from the presentation in figure 50 for the three antenna helghts are $113 \mathrm{n} \mathrm{ml}$. (high), $94 \mathrm{n}$ mi (mid), $98 \mathrm{n}$ mi (1ow).

The summer measurement period from 31 July - 14 August 1972 also encountered strong ducting conditions. L-band signals again varied between diffraction and free space values (figures 53-55) and exhibited deep fading during periods of high signal enhancement. During $1 \%$ of the time did the signal received on the low antenna equal or exceed that recelved on the high antenna (table 23). S-band signals (figures 60-62) during this period were consistently enhanced and exceeded the Eree space path loss value a significant portion of the time. The low antenna received equal or higher signals than the high antenna during $8.6 \%$ of the time (table 26). The most dramatic effect of the ducting conditions durIng the summer measurement period was experienced for $\mathrm{x}$-band frequencies. Signals on the lower antenna fell below free space values only $7 \%$ of the time which is an indication of unusually persistent ducting conditions. Signals received on the lower antenna equaled or exceeded those received on the higier antenna $93 \%$ of the time. Figure 70 shows a jecrease $0 \dot{i}$ fading with antenna height which may be interpreted as a result of more complete trapping of energy for the lower antenna. The higher signals on the low antenna are of course reflected in ionger detection ranges of 

ducting. While ducting should increase with increasing frequency, absorption and roughness of the boundarles (noteably sea surface roughness) became more important for higher frequencies. Therefore, not all of the signal enhancements expected from ducting will be realized. Estimates of signai iosses due to atmospieric absorption and sea surface roughness are given in reference 4. The path loss values for Ka-band shown in figures 115 and 116 (only two antenna heights at 8.6 and $3.6 \mathrm{~m}$ above msl were used) do not reach or exceed free space values as it was observed for $X$ - and Ru-band frequencies. Yontheless, the received signal levels were consistently high and most of the time within 30-65 $\mathrm{dB}$ above the value one would expect without ducting. Figure 115 shows path loss as a function of time for the recelving mtenna located at $8.6 \mathrm{~m}$ above msl. The received signal levels are consistenty above the diffraction Jalues, most of the time $30-4 j \mathrm{~dB}$, but not quite as hizh as tine signals on the zower antenna. A comparison of the gigna1 ieveis of the to re:tivally spaced leceiting antennas shows that the iower antenna receives higher signals a larger percentage of the tige. The difference in the path loss values is plotted in figure 115 . The zero level on the ordinate represents equal power on both antennas. Positive valises indicate nigher signals on the higher antenna and negative values higher signals on the lower antenna. The information in figure 115 is also expressed in table 49 which gives the percentage of time the path loss difference received on the tro antenuas exceeds a certain vaiue. For exampie, the nigher antenna received signals at least $10 \mathrm{~dB}$ larger than the lower antenna $1.8 \%$ of the time; likewise, the lower antenna exceeded the signal levels received on the higher antenna $65.7 \%$ of the time. Figure 117 shows the fading joserved on both receiving antennas. Fading is here defined as maximum peak 

antennas are averaged in figures 121 and 122 . Tne curresponding numericai values are listed in tables 52 and 53. The S-band datz are averaged in figures 123-125 and tables 54 and 55. Table 56 shows the percentage of time path loss differences between high and low X-band antennas exceed certain $\mathrm{dB}$ values. For example, for all seasons combined, signals recelved on the high antenna exceeded those rectsived on the low antenna during $52.6 \%$ of the time. During $20 \%$ of the time the signal received at the low $\mathrm{X}$-band antenna exceeded that received at the high antenna by $10 \mathrm{~dB}$. The frequency distributions of path loss for X-band in figure 126 show again the wider spread for the low antenna. The detection ranges for the hypothetical $200 \mathrm{n}$ ml free space detection range radar are shown in figures 129 and 130. The wean detection ranges calculated from this presentation are 60,61 , and $79 \mathrm{n}$ mi for the high, middle, and low antenna respectiveiy. 81gures 132-134 show the Ku-band data averaged for the surme: and Eali season.

\section{B. Meteorological Comparisons}

Three aspects were pursued in the meteoroiogical phase of the program. First, the complex processes in the oceanic boundary layer demand sophisticated measurements for their detailed descruption; however, only simple meteorological measurements can be performed under operational conditions and only simple meteorological measuremerts are routinely performed by meteorological oficices around the world. Thereiore, the assessment of evaporation jucting effects on :adio propagation beccmes practical on:-: if simple measurements can be used to estimate ducting phenomena. For this reason, it was decided to use routinely obtained data from a meteorologtcal station of the Greek Neather jervice on liaxos. The data consisted 
of surface temperature and humidty neasuraments :akan jyery inzee .scus and water temperatures measured twice daily. F1gures 135-146 show the air-sea temperature difference, air temperature, relative humidity, wind speed, and duct height calculated from these measurements for the four measurement periods. Duct helght $\delta$ was calculated according to the following formulas (log-linear profile):

$$
\begin{aligned}
& \delta=-\left[\frac{0.0013\left(\ln \frac{z_{1}}{z_{0}}+\frac{z_{1}}{L^{\varphi}}\right)}{\phi_{A}-\phi_{S}}+\frac{\alpha}{L^{\prime}}\right]^{-1}=m \\
& { }_{A}=\frac{77.6}{T_{A}}\left[1000+\frac{4810}{T_{A}} e\right] \\
& \phi_{\mathrm{S}}=\frac{77.6}{\mathrm{~T}_{\mathrm{SW}}}\left[1000+\frac{4810}{\mathrm{~T}_{\mathrm{SW}}} \mathrm{e}_{\mathrm{SW}}\right] \\
& L^{\prime}=\frac{\frac{(u \cdot 51.4444)^{2}}{980\left(T_{A}-T_{S W}\right) / T_{S W}}-\approx z_{1}}{\ln \left(z_{1} / z_{0}\right)} \mathrm{cm} \\
& z_{1}=500 \mathrm{~cm} \\
& z_{0}=0.0015 \mathrm{~cm} \\
& x=2.0 \\
& u=\text { wind speed in knots }
\end{aligned}
$$




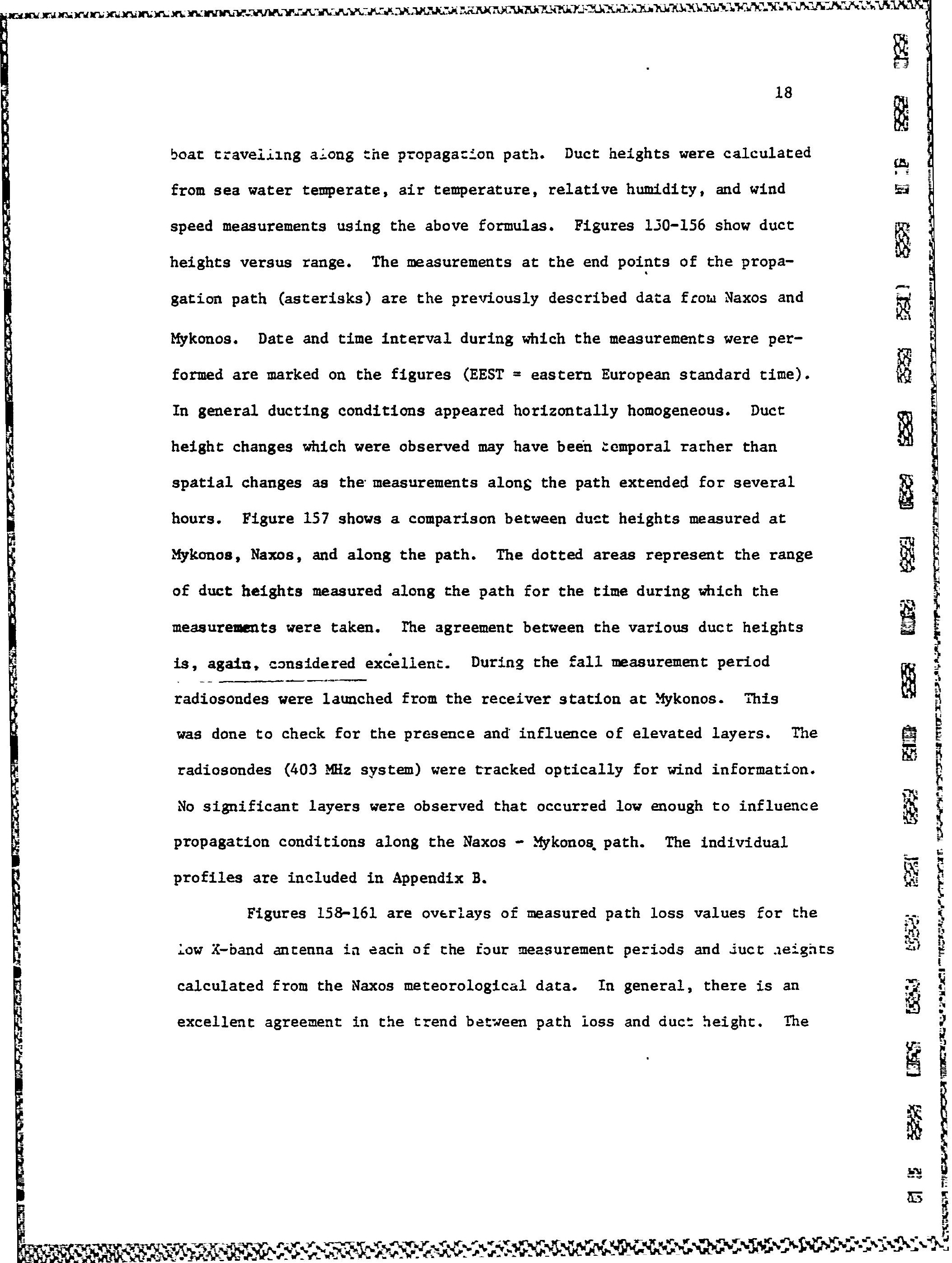


agreement is particularly good for the sinter and fall periods. The agreement in the trend between duct heights and path loss might be improved by using different models to calculate duct height for thermally stable and unstable conditions. Those models are described in reference 5. Figure 162 shows the measured path loss for tine low X-band antenna during the fall period and path loss values calculated from the Naxos duct height (indicated by circles). The relationship between duct height and path loss for the geometry under consideration shown in figure 163, has been calculated with NELC's full wave solution wave guide computer program which will be described in a report which is presently under preparation. The general agreement between calculated and measured path loss values in figure 162 is very good if one considers the shortcomings of the meteorological measurements. The correlation coeficient between salsulated values ard corresponding measured values was claculated to be 0.63 . If one elimiaates in this comparizon duct heights associated with riad speeds 0 is less than 5 knots, the correlation coefficient increases to 0.71 . (The elimination of duct heights for wind speeds less than 5 knots may be justified on the basis that wind measurements in this range are quite unreliable but may have a strong influence on atmospheric stability and duct helght). Boti correlation coefficients 0.63 and 0.71 are signticant at the $99 \%$ level. This means there is only a $1 \%$ probability that the data are actualiy uncorrelated. Tne lay conclude that the compariscn jetieen the netecroiog:cal and radio data shows that simple in situ meteorological measurements are quite adequate to estimate propagation conditions. 
The third aspect of the meteorological phase of this program was to check the usefulness of avallable long-term meteorological statistics to predict radio propagation conditions. Only if this can be done, can successfull predictions of ducting conditions be made without actually performing extensive measurements. Figure 164 is the irequency distribution of duct height based on five years of meteorological averages and the duct height distributions based on the Naxos meteorological measurements taken during the four measurement perfods in 1972. The agreement between the two distributions is considered quite good. These duct height distributions may also be used to estimate antenna reversals, i.e., the percentage of time the low antenna receives equal or higher signals than the high antenna. From a famlly of height-gain functions generated with the previously mentioned wavegutde program it was concluded that reversals would be expected for duc: heights between 10 and $30 \mathrm{~m}$. Table 62 shows that for individual seasons caiculated and measured ralues may differ appreciably. Jne reaily wouid not expeci a specific two week period to be identical with a five year seasonal average. However, all measured data averaged (thereby forming a larger sample size) compare guite favorably with the average for the entire five year period. Based on these and similar previous comparisons for the California off-shore area (reference 1), one may conclude that long term meteorological statistics are quite useful in estimating average ducring snditions for oceanic arees. 


\section{v. CONCLUSIONS}

Extensive radio propagation measurements in the Mediterranean have shown that evaporation ducting is an important phenomenon in particular for frequencles above S-band. For example, signal enhancements from evaporation ducting have been measured $99 \%$ of the time for $\mathrm{X}$-band frequencies. It was determined that the evaporation duct strongly affects all shipboard antenna heights. Under conditions of strong ducting, low sited antennas (e.g. 15 feet above mean sea level) may receive higher signals than more conventional antenna helghts (e.8. 60-70 feet above msl). For all measurements in the Mediterranean the low sited X-band antenna received equa.i or higher signals than the high antenna $47.4 \%$ of the time. Durdng 207 of the time signals received at the low sited $x$-band antenna exceeded those received on the high sited antenna by $10 \mathrm{~dB}$. From the measurements one may conclude that the optimum location for an antenna is nigi on the ship. When economics justify two antennas, an advantage can be obtained with both a high and low antenna.

The evaporation ducting effect appears to have a broad maximum in the X- to Ku-band frequency range. Atmospheric absorption and sea surface roughness apparently counteract the effectiveness of the duct expected at higher frequencies.

Simple meteorological measurements were found to be quite sufficient to descrioe jucting conditions. Horizontal homogeneity of the duce vas found to be good for the propagation path used in this investigation. Ducting effects deduced from long term meteorologi=al averages compared well 
with the average of all measurements for the year permitting estimates of average ducting conditions to be made for any oceanic area for which such statistical meteorological data are available. 
VI. RECOMENDATIONS

The path loss information obtained from the propagation measurements had been translated in this report into detection range information for $\mathrm{x}$-band. Hypothetical radar parameters were assumed for this purpose. Target height versus detection range plots for various ground based duct heights can be generated for actual radars (reference 6). Those curves could be easily used under operational conditions in connection with simple meteorological measurements performed on board ship and in connection with duct height distributions calculated from long term meteorological averages. It is, therefore, recommended to apply this technique to a suitable surface survelllance radar (e.8. SPS - 55) and conduct an evaluation under opezational conditions. 


\section{REFERENCES}

1. Richter, J. H. and H. V. Hitney, "Antenna heights for optimum utilization of the oceanic surface evaporation duct", Part I: Results from the Pacific measurements, NELC TN 2031, 4 May 1972.

2. Richter, J. H. and H. V. Hitney, "Antenna heights for optimum utilization of the oceanic surface evaporation duct", Part II: Results from the Key West measurements, NELC TN 2371, 10 May 1973.

3. Richter, J. H., H. V. Hitney, K. D. Anderson, and M. L. Phares, "Propagation measurements of $37 \mathrm{GHz}$ in the oceanic surface evaporation duct", NELC TN 2422, 3 July 1973.

4. Hitney, H. V., "Propagation Calculations of $37 \mathrm{GHz}$ in the oceanic evaporation duct", NELC TN 2421, 3 July 1973.

5. Jeske, H., "The state of radar-range prediction over sea", AGARD Conference Proceedings No. 70, Part II, p. 50.1 - 50.10, Februar: 1971.

6. Hitney, H. V., "The influence of the oceanic surface evaporation duct on detection range for a $3.3 \mathrm{GHz}$ radar", NELC TN 2322, 15 March 1973. 
VIII. FIGURES

PAGE

1. Geographic location of propagation path 36

2. Recelving terminal at Ornos Beach, Mykonos 37

3. Receiving equipment 38

4. Transmitting antennas at Naxos 39

WINTER PERIOD (8-22 FEBRUARY 1972)

5. Path loss for high L-band antenna and path loss difference high-low antenna

6. Path loss for middle L-band antenna and path loss difference mid-low antenna

7. Path loss for low L-band antenna and path loss difference high-mid antenna

8. Rading L-band

9. Frequency distributions of path loss for L-band

10. Frequency distributions of Fading L-band

1.1. Frequency distributions of path loss differences between antennas for $L-$-band

12. Path loss for high S-band antenna and path loss difference high-low antenna

13. Path loss for middle S-band antenna and path loss difference mid-low antenna

14. Path loss for low S-band antenna and path loss difference high-mid antenna 
15. Fading S-band

16. Frequency distributions of path loss for s-band

17. Frequency distributions of fading for s-band

18. Frequency distributions of path loss differences between antennas for S-band

19. Path loss for high X-band antenna and path loss difference high-10w antenna

20. Path loss middle $X$-band antenna and path loss difference mid-low antenna

21. Path loss low X-band antenna and path loss difference highmid antenna

22. Fading X-band

23. Frequency distribution of path loss for $\mathrm{X}$-band

24. Frequency distribution of fading $x$-band

25. Frequency distribution of differences between antennas for $\mathrm{X}$-band

26. Frequency distribution of detection range for $\mathrm{x}$-band

27. Cumulative distribution of detection range for $\mathrm{X}$-band

28. Frequency distribution of detection range differences between antennas for $X$-band

SPRING PERIOD (18-28 APRIL 1972)

29. Path loss for high L-band antenna and path loss difference high-low antenna 
30. Path loss for middle L-band antenna and path loss difference mid-low antenna

31. Path loss for low L-band antenna and path loss difference high-mid antenna

32. Fading L-band

33. Frequency distributions of path loss for L-band

34. Frequency distributions of fading L-band

35. Frequency distributions of path loss differences between antennas for L-band

36. Path loss for high S-band antenna and path loss difference h1gh-10w antenna

37. Path loss for middle S-band antenna and path loss difference mid-Iow antenna

38. Path loss for low S-band antenna and path loss difference high-mid antenna

39. Fading S-band

40. Frequency distributions of path loss for S-band

41. Frequency distributions of fading for S-band

42. Frequency distributions of path loss differences between antennas for s-band

43. Path loss for high $\mathrm{X}$-band antenna and path loss difference high-low antenna

44. Path loss middle $X$-band antenna and path loss difference mid-low antenna 
45. Path loss low $\mathrm{X}$-band antenna and path loss difference nigh80 mid antenna

46. Fading $X$-band

48. Frequency d1stribution of fading $X$-band

49. Frequency distribution of differences between antennas for X-band

50. Frequency distribution of detection range for $x$-band 85

51. Cumulative discribution of detection range for $\mathrm{X}$-band

52. Frequency distribution of detection range differences between antennas for $\mathrm{X}$-band

SUMER PERIOD (31 JULY - 14 AUGUST 1972)

53. Path loss for high L-band antenna and path loss difference high-1ow antenna

54. Path loss for middle L-band antenna and path loss difference mid-low antenna

55. Path loss for low L-band antenna and path loss difference high-mid antenna

56. Fading L-band

57. Frequency distributions of path loss for L-band

58. Erequency distributions of fading I-band

59. Frequency distributions of path loss differences between antennas' for L-band 
60. Path loss for high S-band antenna and path loss difference high-low antenna

61. Path loss for middle S-band antenna and path loss difference mid-1ow antenna

62. Path loss for low S-band antenna and path loss difference high-mid antenna

63. Fading S-band

64. Frequency distributions of path loss for S-band

65. Frequency distributions of fading for s-band

66. Frequency distributions of path loss differences between antennas for s-band

67. Path loss for high $X$-band antenna and path loss difference high-low antenna

68. Path loss middle $\mathrm{X}$-band antenna and path loss difference. mid-low antenna

69. Path loss low X-band antenna and path loss difference highmid antenna

70. Fading $\mathrm{X}$-band

71. Frequency distribution of path loss for $\mathrm{X}$-band

72. Frequency distribution of fading $x$-band

73. Frequency distribution of differences between antennas for $\mathrm{x}$-band

74. Frequency distribution of detection range for X-band 109

75. Cumulative distribution of detection range for $\mathrm{X}$-band 
76. Frequency distribution of detection range differences between antennas for $\mathrm{X}$-band

77. Path loss for high Ku-band antenna and path loss difference high-low antenna

78. Path loss for mid Ku-band antenna and path loss difíerence midlow antenna

79. Path loss for low Ku-band antenna and path loss difference high-mid antenna

80. Fading Ku-band

81. Frequency distributions of path loss for Ku-band

82. Frequency distributions of fading for Ku-band

83. Frequency distributions of path loss differences between antennas for Ku-band

FALL PERIOD (7-21 NOVEMBER 1972)

84. Path loss for high $L$-band antenna and path loss difference high-low antenna

85. Path loss for middle L-band antenna and path loss diEEerence mid-1ow antenna

86. Path loss for low L-band antenna and path loss difference high-mid antenna

87. Fading $L-b$ and

88. Frequency distributions of path loss for L-band

89. Frequency distributions of fading $L-b$ and 
90. Frequency distributions of path loss differences between antennas for $\mathrm{L}-\mathrm{b}$ and

91. Path loss for high S-band antenna and path loss difference righ-low antenna

92. Path loss for middle S-band antenna and path loss difference mid-low antenna

93. Path loss for low S-band antenna and path loss difference high-mid antenna

94. Fading S-band

95. Frequency distributions of path loss for s-band

96. Frequency distributions of fading for s-band

97. Frequency distributions of path loss differences between antennas for $S-b a n d$

98. Path loss for high $\mathrm{x}$-band antenna and path loss difference high-low antenna

99. Path loss middle $\mathrm{X}$-band antenna and path loss difference mid-1ow antenna

100. Path loss low $\mathrm{X}$-band antenna and path loss difference highmid antenna

101. Fading X-band

102. Frequency distribution of path loss for $\mathrm{x}$-band

103. Frequency jistribution of Eading $z$-band

104. Frequency distribution of differences between antennas for $\mathrm{X}$-band 
ALL SEASONS COMBINED

120. Frequency distributions of path loss for L-band

121. Frequency distributions of fading L-band

122. Frequenc; distributions of path Ioss differences betideen. antennas for L-band

123. Frequency distributions of path loss for s-band

124. Frequency distributions of fading for S-band

125. Frequency distributions of path loss differences between antennas for $S-b a n d$

126. Frequency distribution of path loss for $\mathrm{X}$-band

127. Frequency distribution of fading $x$-band

128. Frequency distribution of differences between antennas for $x$-band

129. Frequency jistribution of ieraction range for $x$-band

130. Cumulative distribution of detaction range for $\mathrm{x}$-band

131. Frequency distribution of detection range difierences between antennas for $\mathrm{X}$-band

132. Frequency distributions of path loss for Ku-band

133. Frequency distributions of fading for Ku-band

134. Frequency distributions of path loss differences betiveen antennas For ku-band 

153. Duct height measurements along the propagation path

154. Duct helght measurements along the propagation path

155. Duct height measurements along the propagation path

156. Duct height measurements along the propagation path

157. Duct heights calculated from meteorological measurements at Naxos and at Nykonos (crosses) and along the propagation path (shaded areas)

158. Path 108 sor 1 ow $X-b a n d$ antenna and duct helght, winter pertod

159. Path $108 s$ for low $X$-band antenna and duct height, spring period

160. Path loss for low $\mathrm{X}$-band antenna and duct height, summer perlod

161. Path loss for low $\mathrm{X}$-jand antenna and duct height, Eall period

162. Measured path loss for low X-band antenna during fall period (solid line) and calculated path loss from Naxos duct heights (circles)

163. Duct height-path loss relationship for low $\mathrm{X}$-band antenna

164. Duct height distribution from Naxos measurements and from five year meteorological averages for the area of tine propagation path 


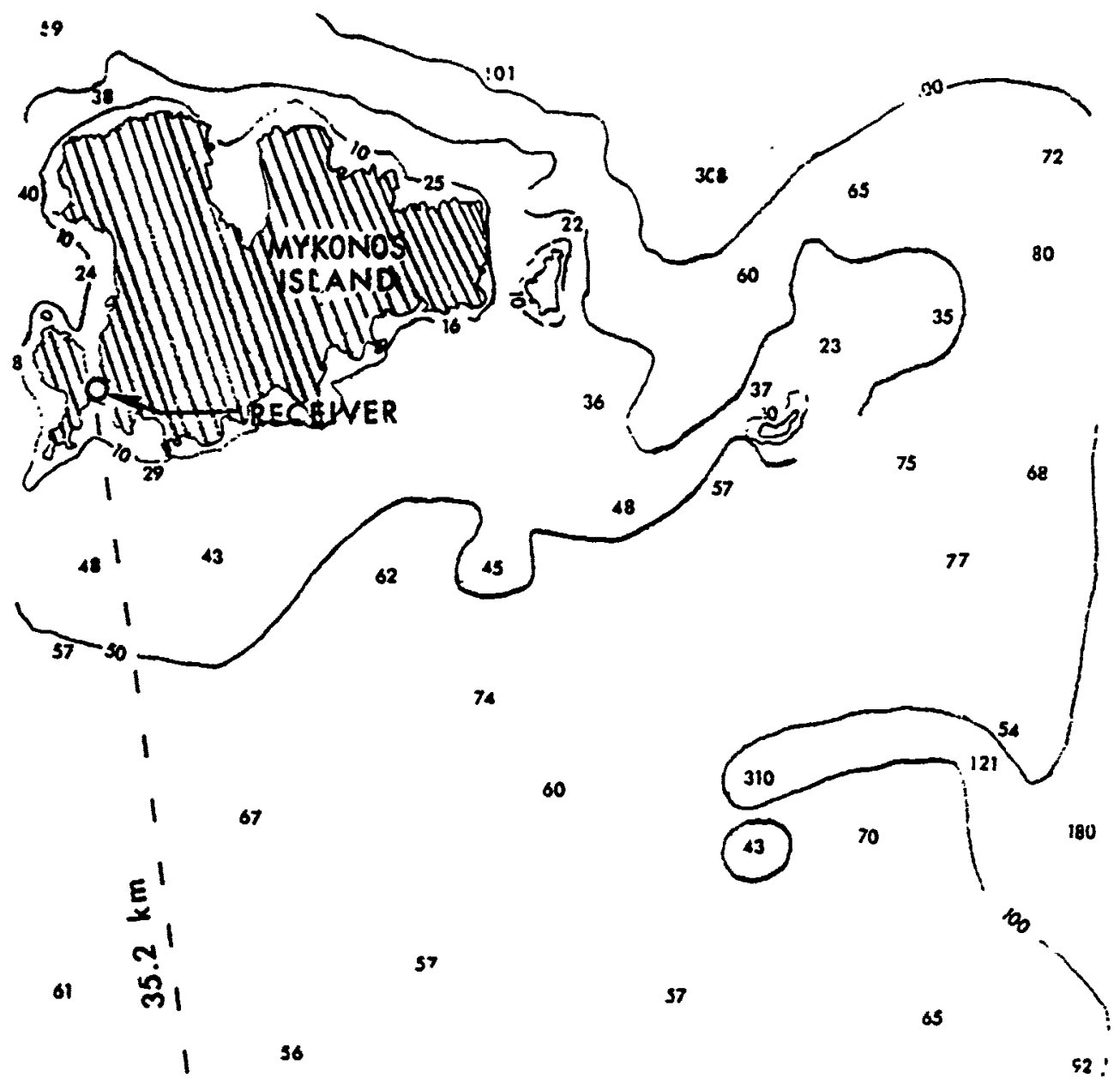

8

6

6

8

D

W

i

$\$$

基

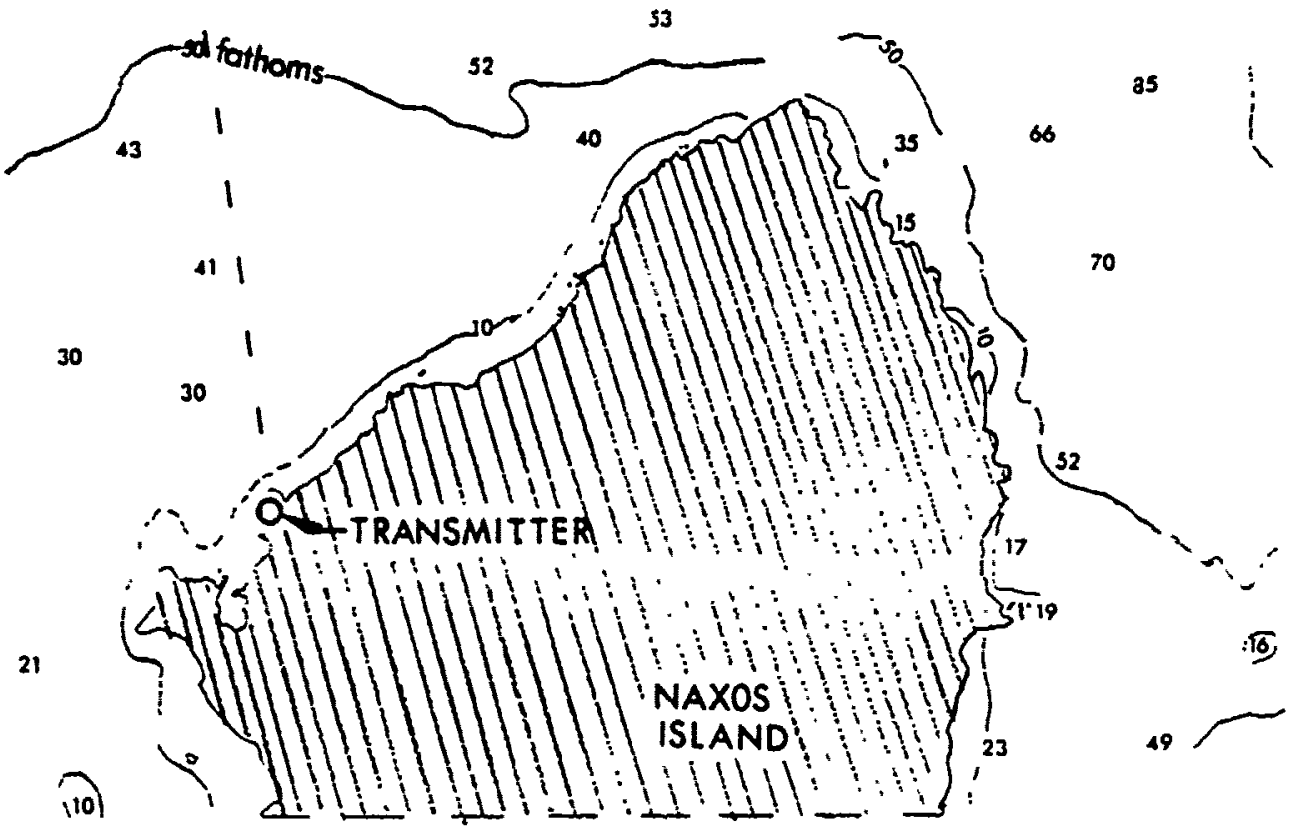

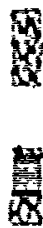

․․

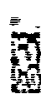

$y$

8

믈

Fizure 1. Geograghic locarzon of propagarion path 


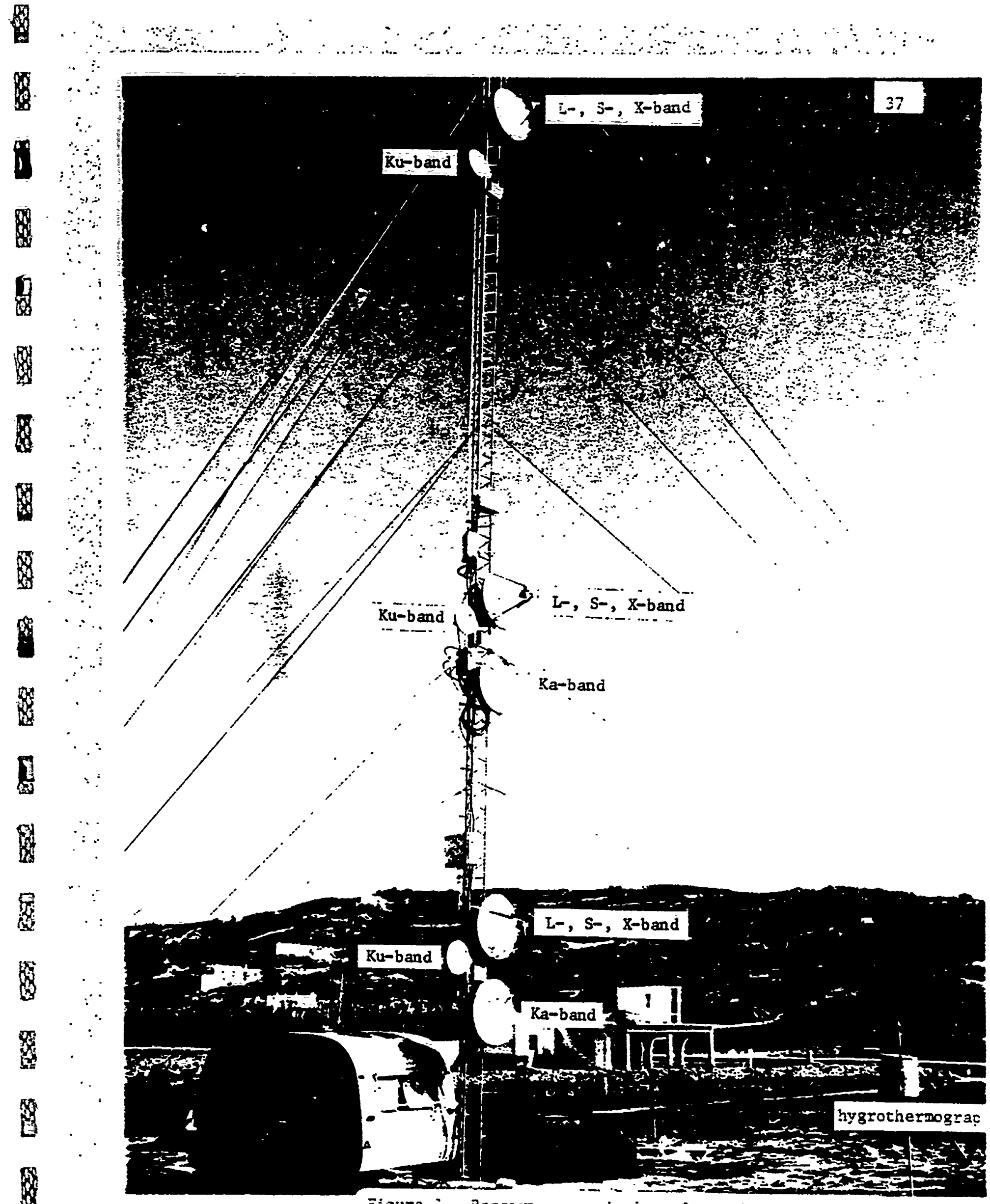

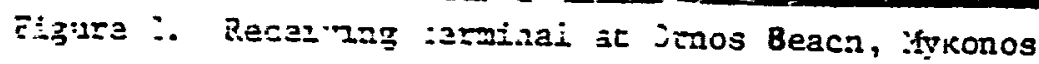




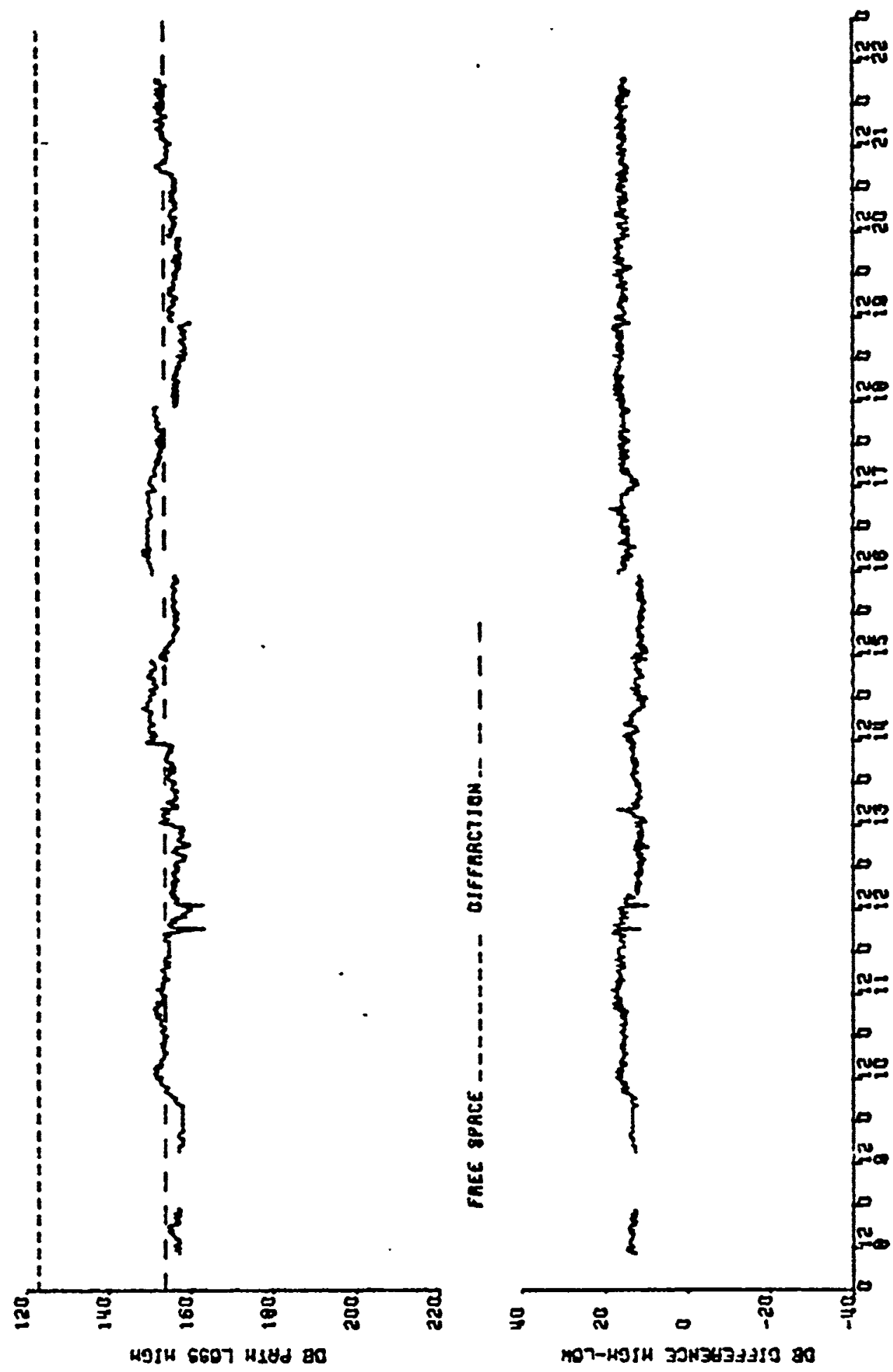

:

8

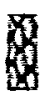

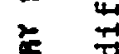

总

9

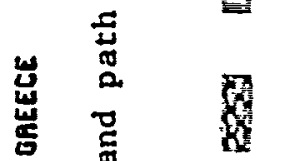

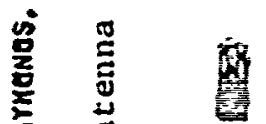

元

总

要

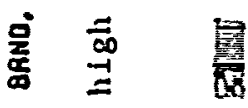

w

ज

8

$\stackrel{\leftrightarrow}{\stackrel{7}{7}}$

8

8

8 

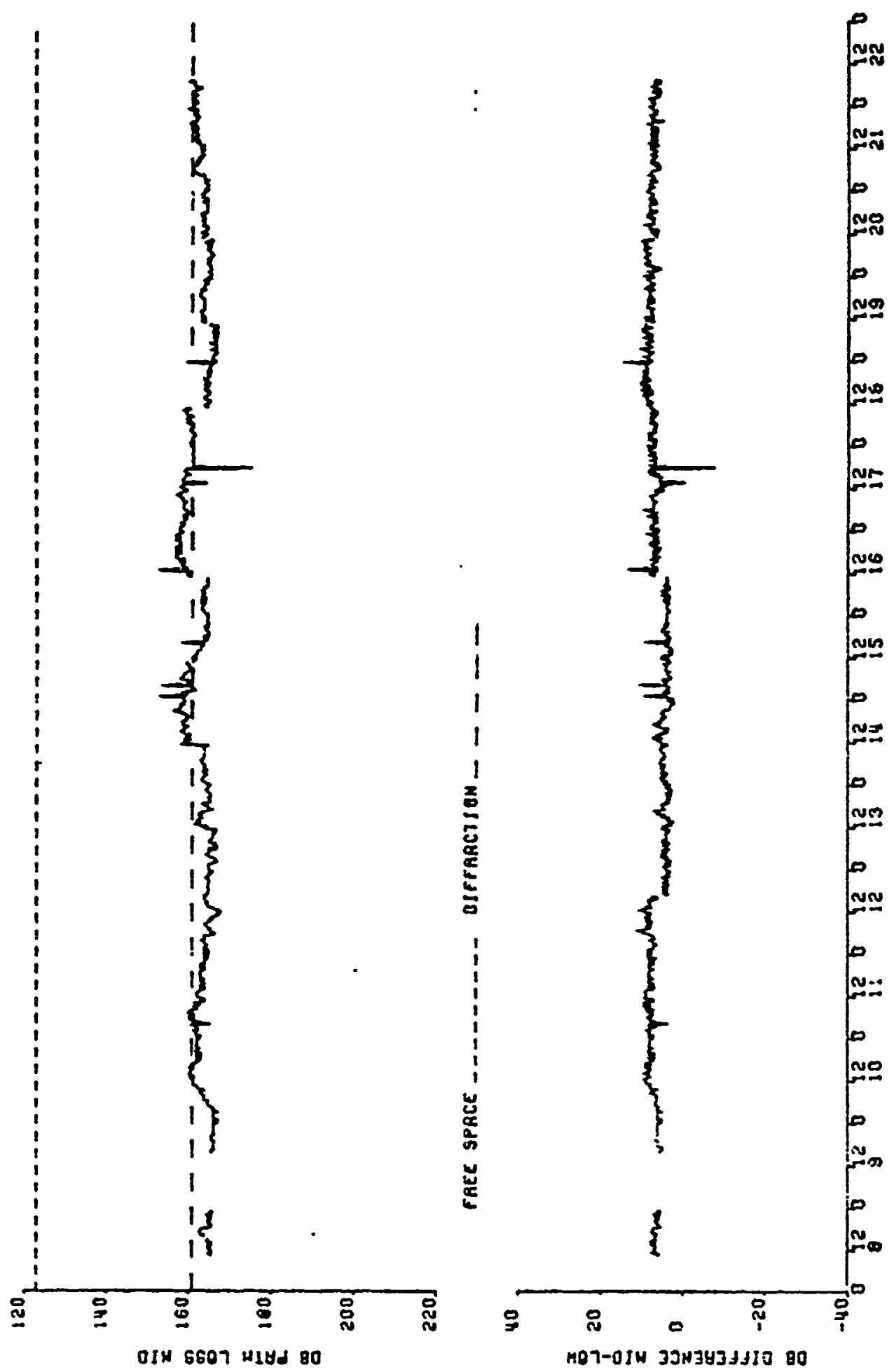

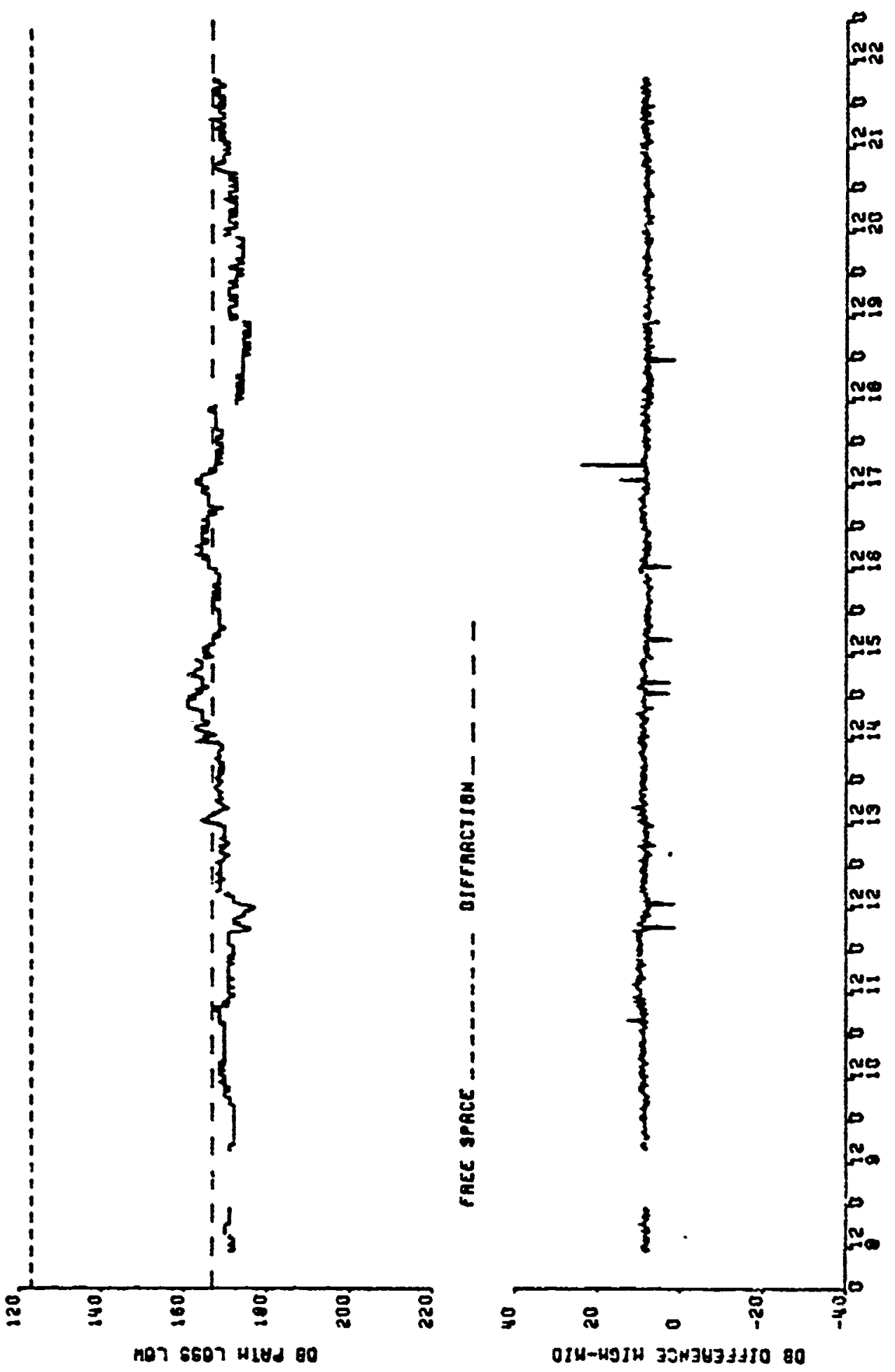

1

2

8

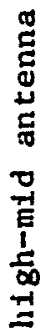

(

祭

获

空

80

$\sqrt{x}$

疋

등

ำ

新

8

ญี

总

芒

ఎ

a

8

驾

d

$\stackrel{5}{=}$

$\therefore \quad$

3

跑

W

se

$\times$ 
8
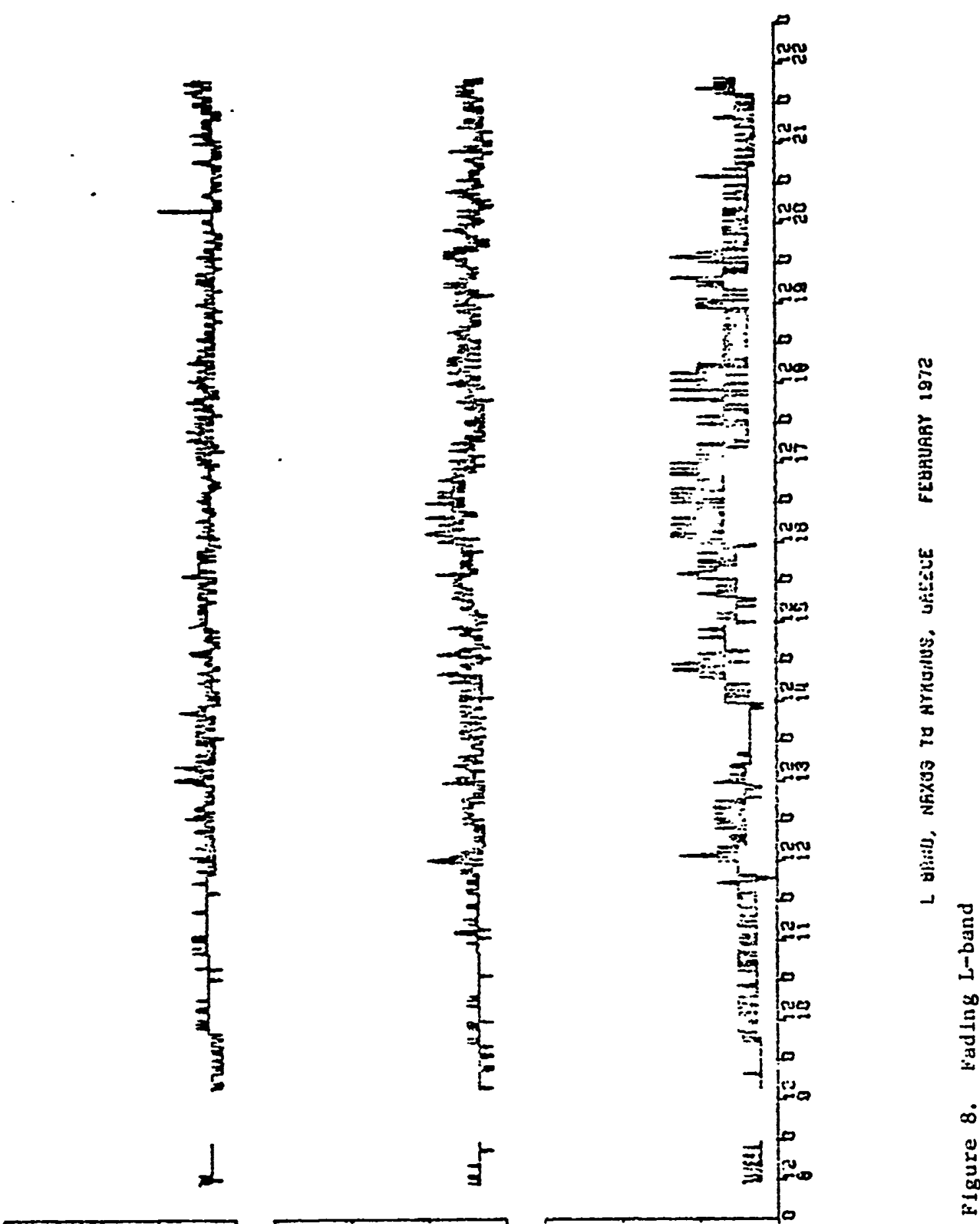

$\sqrt{2}$

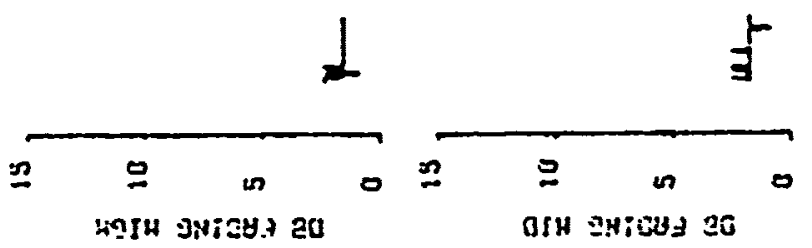

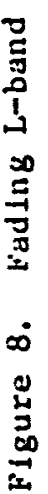




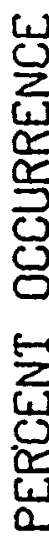
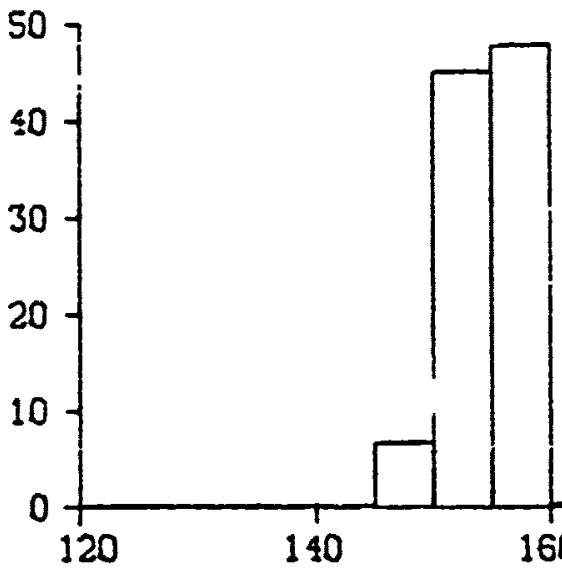

HIGH ANTENNA

DB PATH LOSS

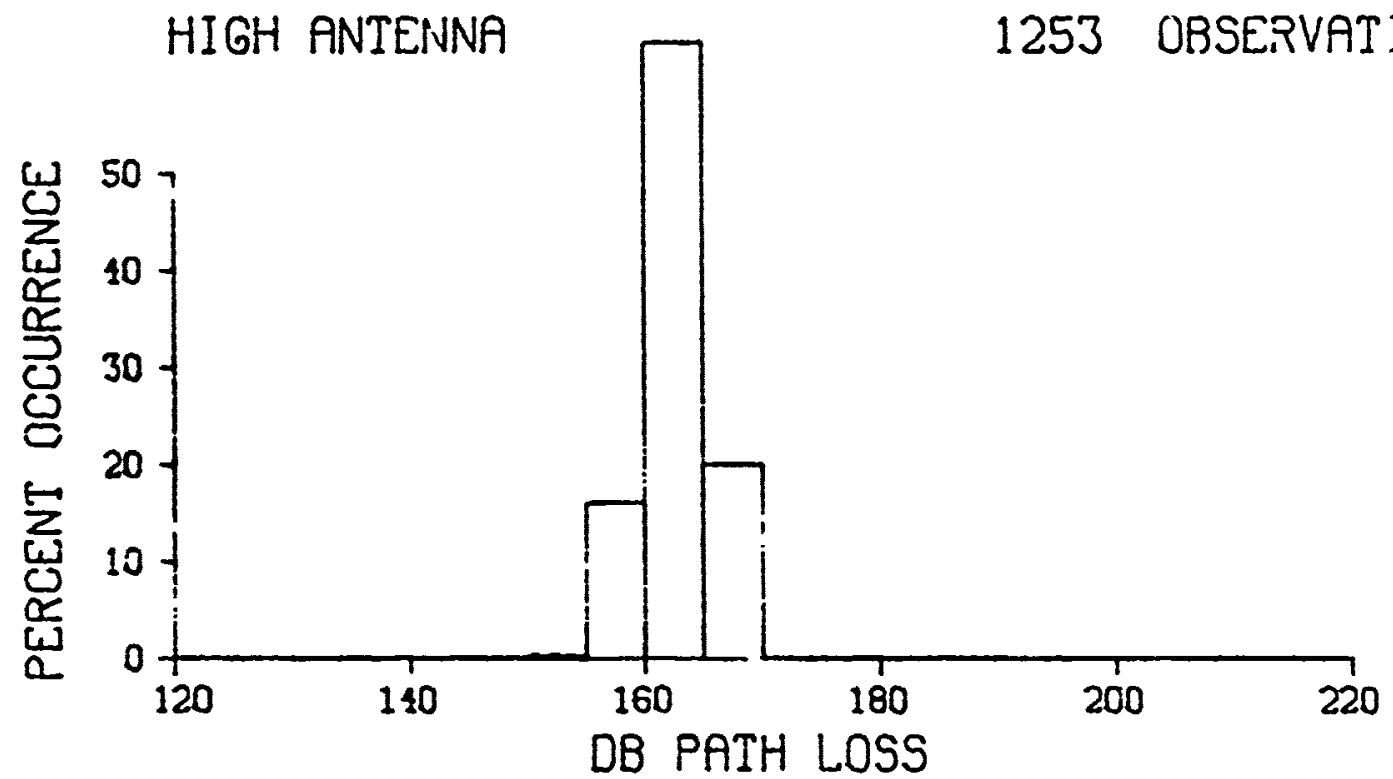

MID ANIENNA

1253 OBSERVATIONS

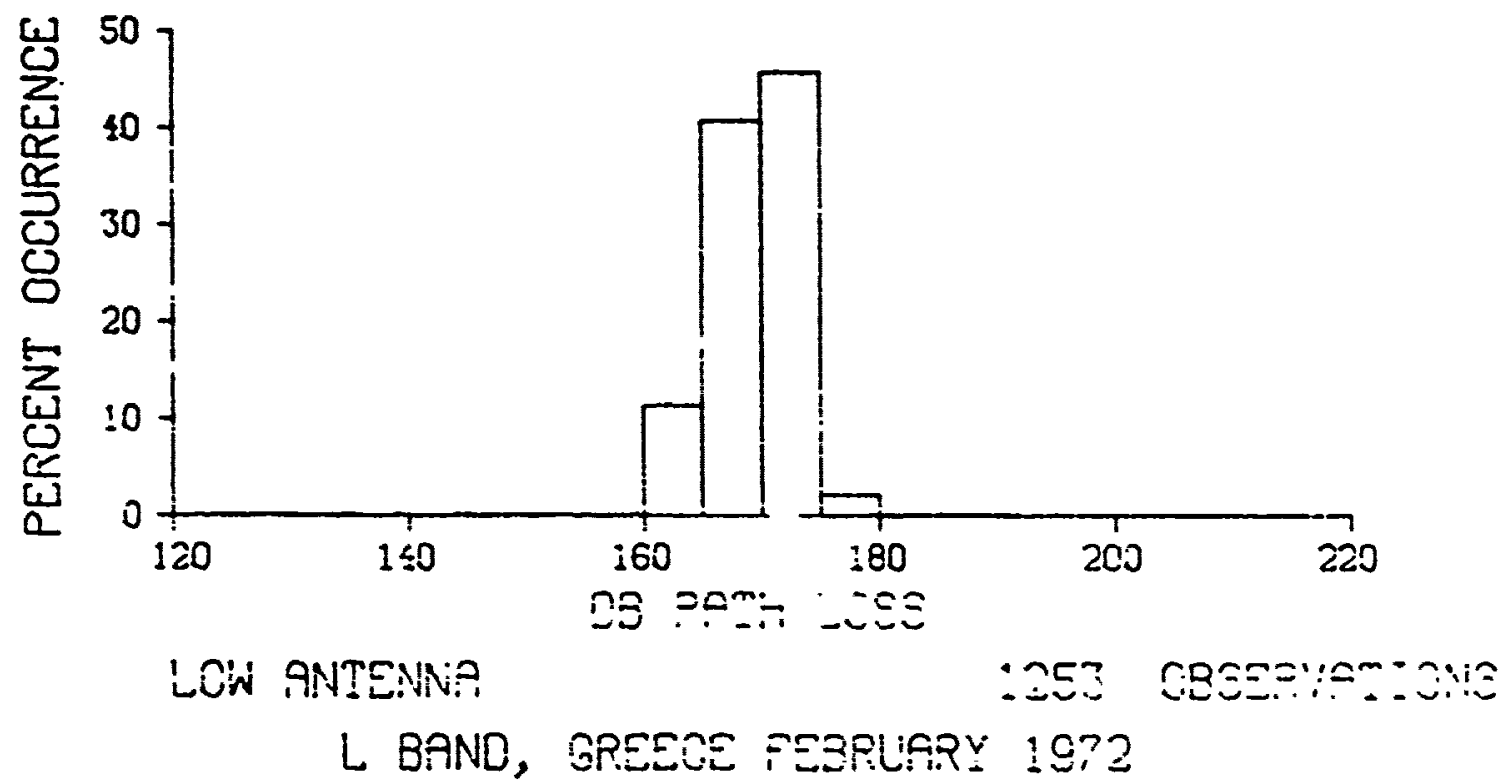

$\underset{3}{a}$ 


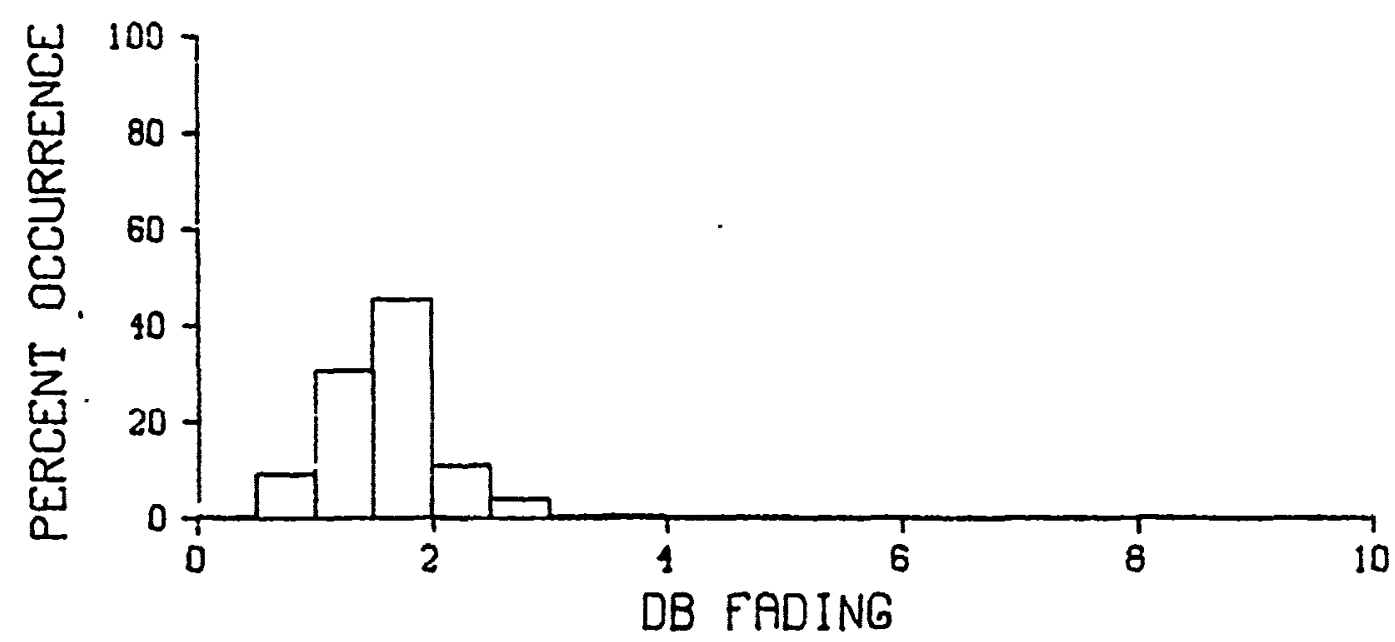

HIGH ANTENNA

1253 OBSERVATIONS 葡

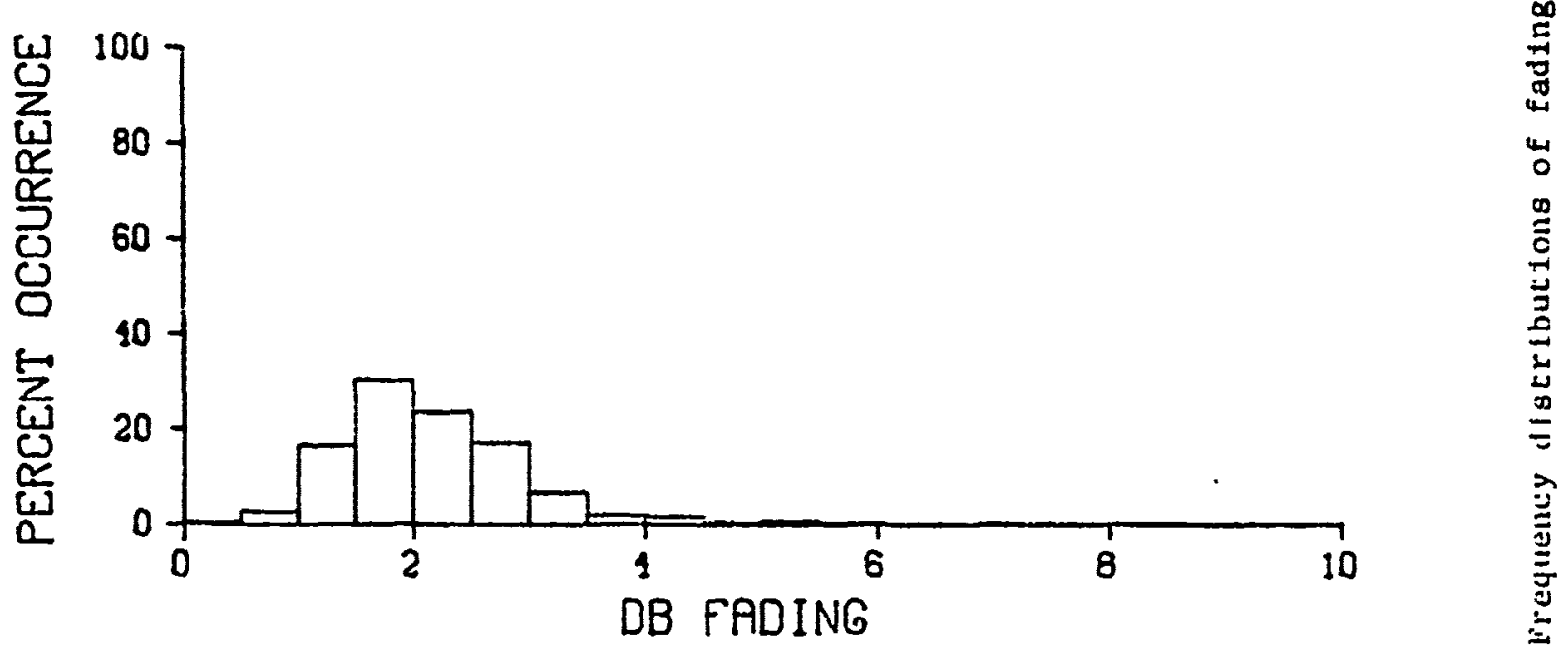

MID ANTENNA

1253 OBSERVATIONS

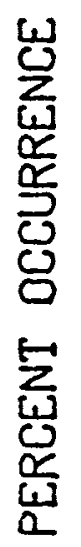

100
80
$60-$
$10-$
20
0

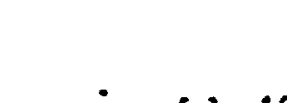




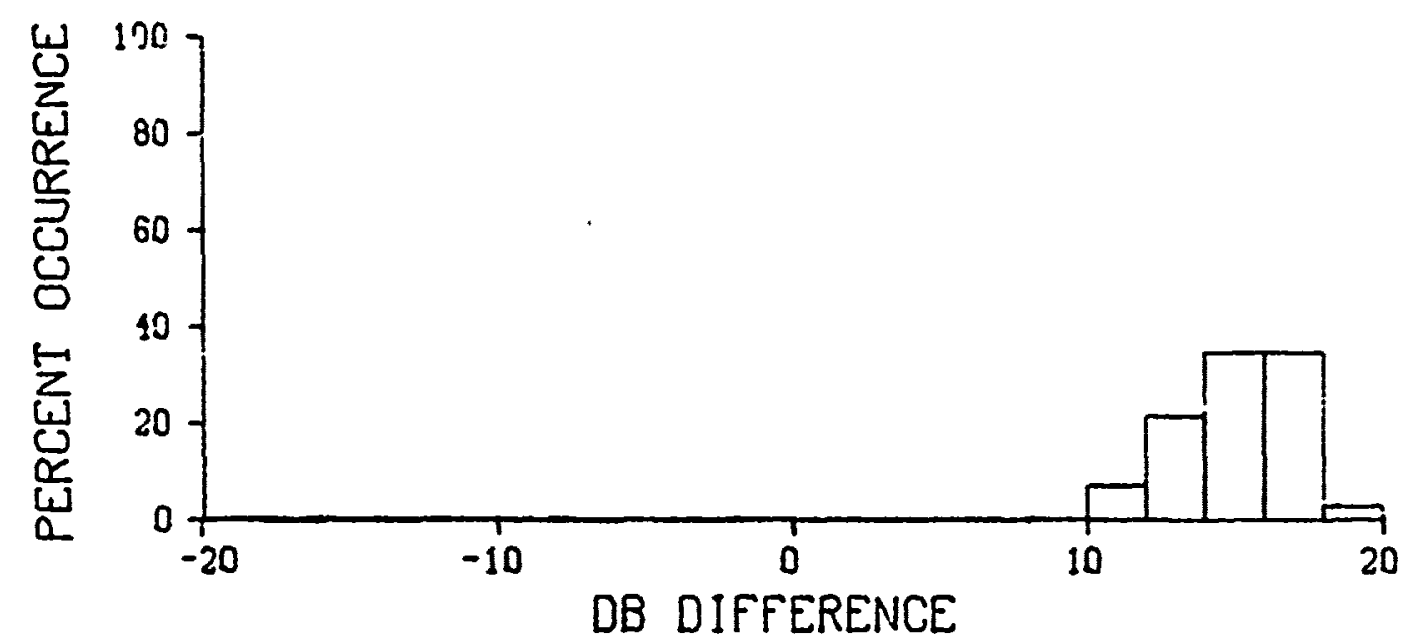

HIGH-LOW

1253 OBSERVATIONS

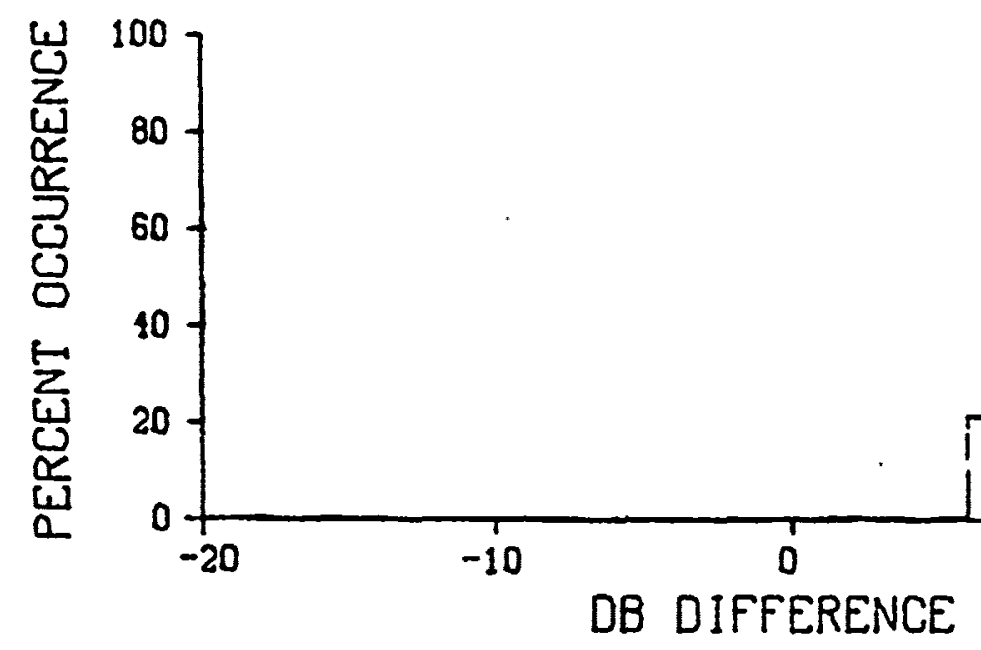

HIGH-MID
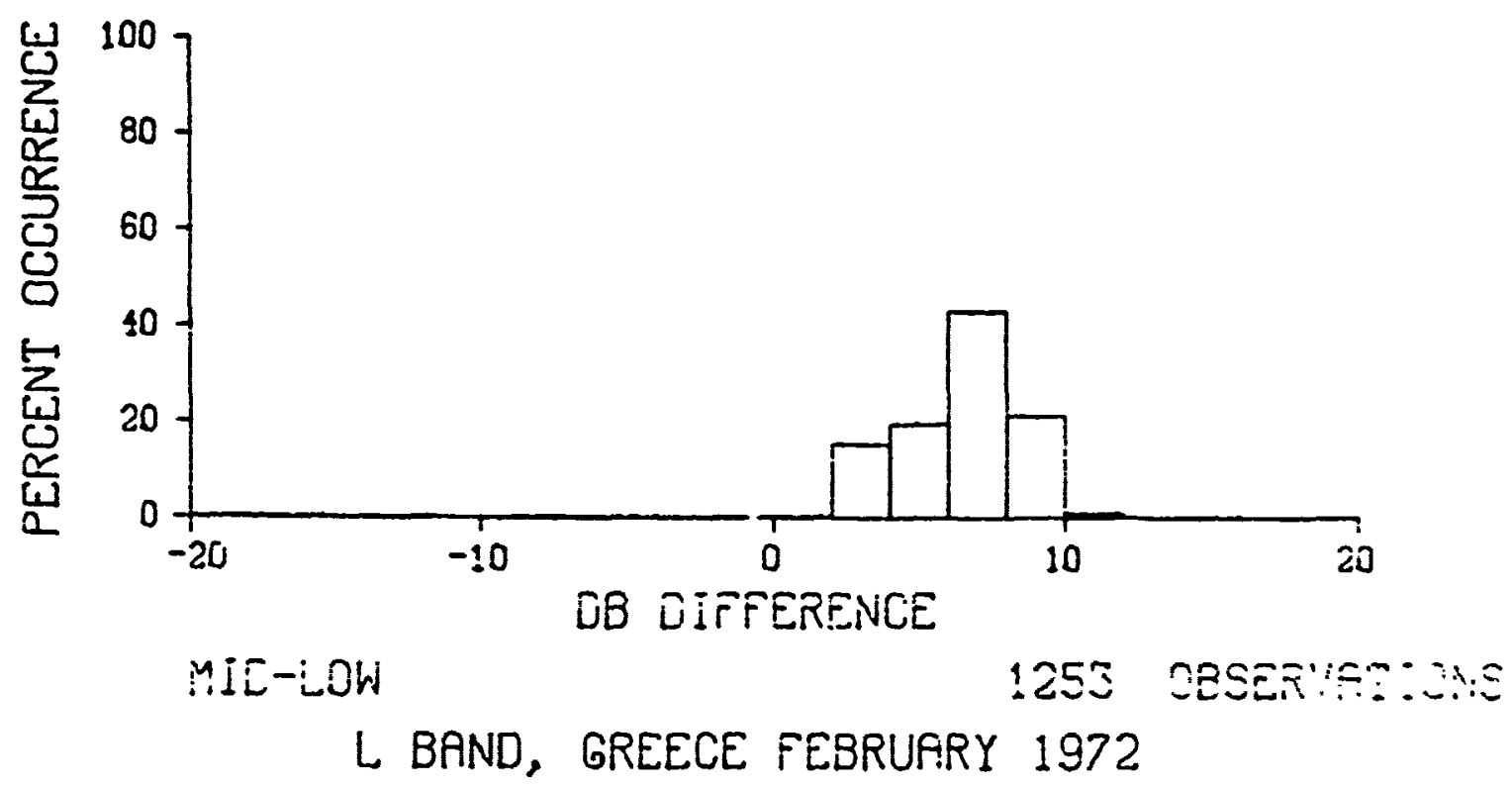

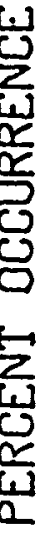




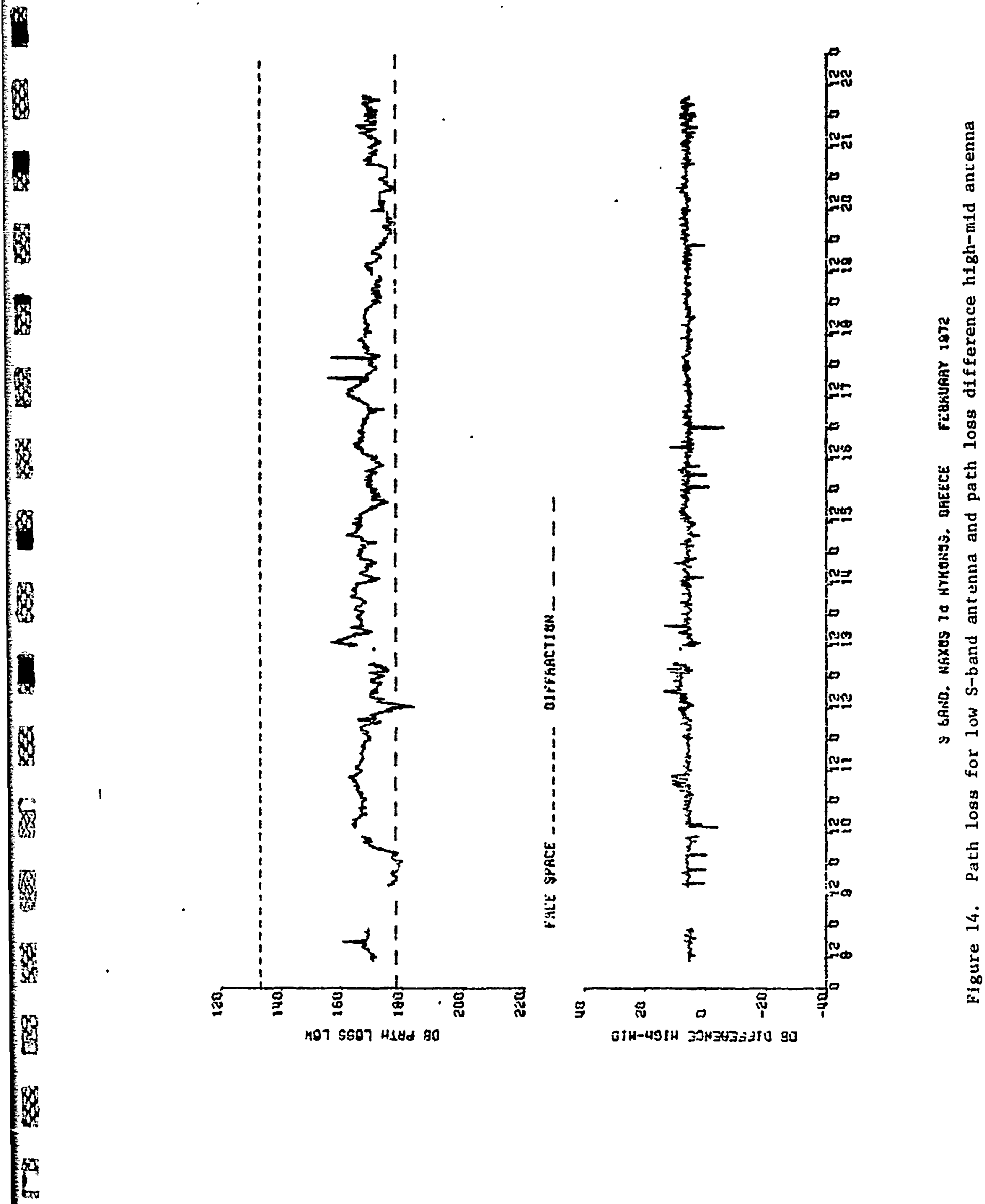

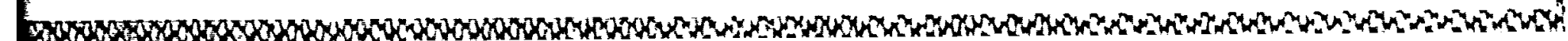




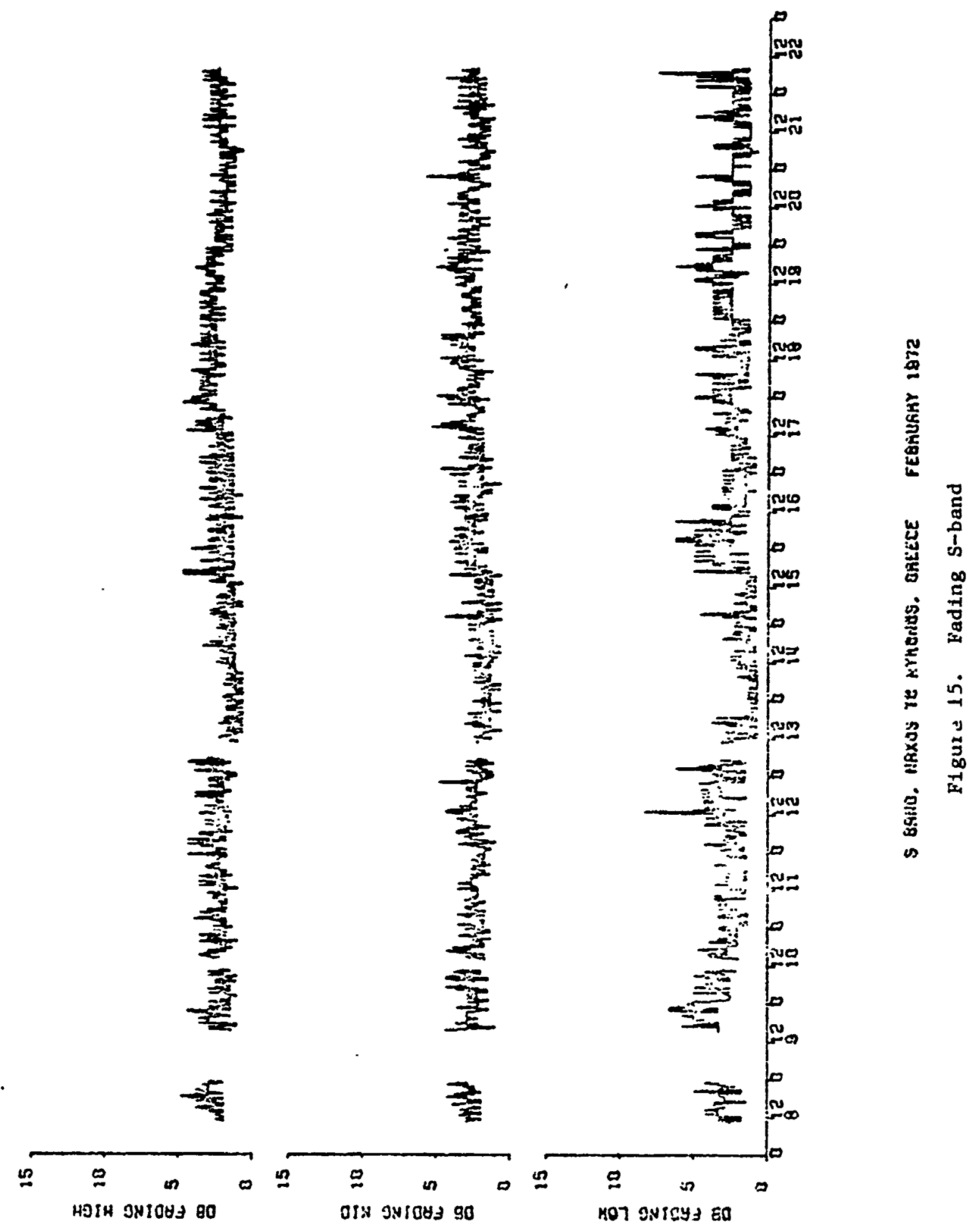

8

a

$=$

0

Q

8

3

8

8

8

承

8

8

5

x

绕

8

n

$x$ 


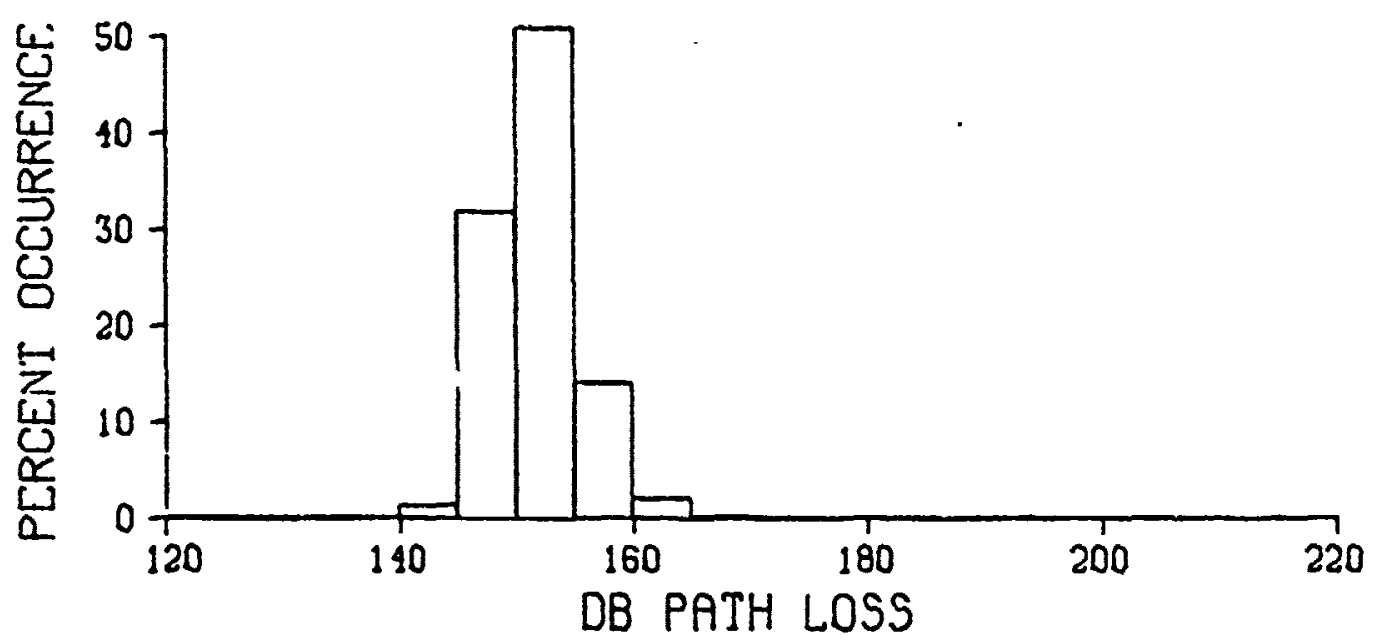

HIGH ANTENNA

1216 OESERVATIONS

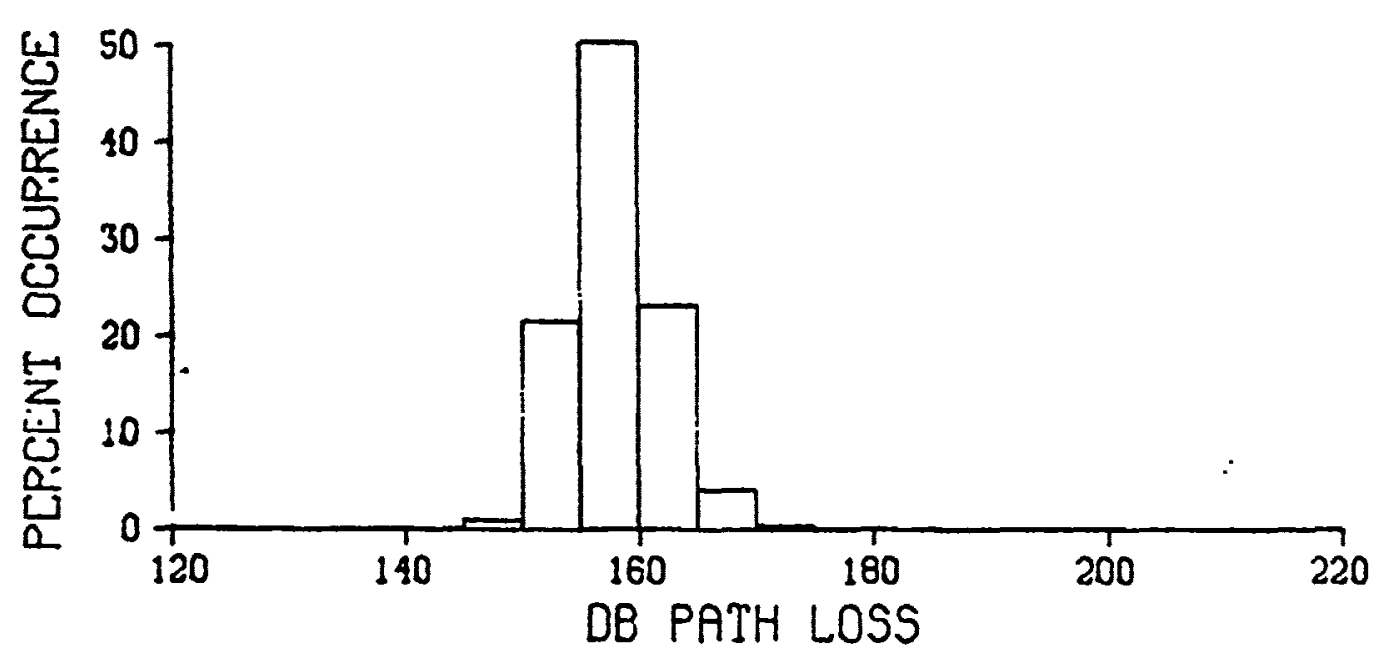

MID ANTENNA

1216 OBSERVATIONS

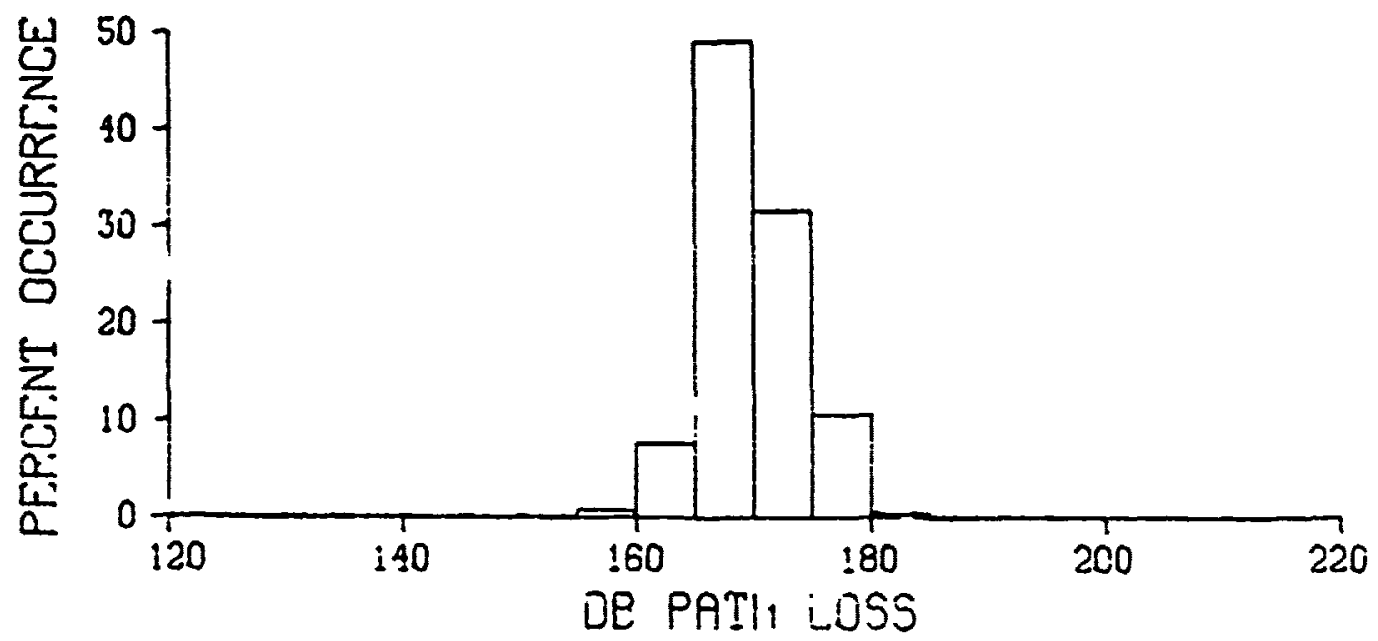

W

8

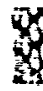

8

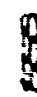

LOH RNTENATH

:213 OESFRYAT:DNS

$S$ BAND, GP.EF.CE FEBRURRY 1972

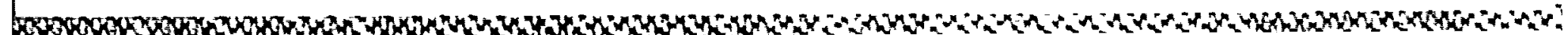



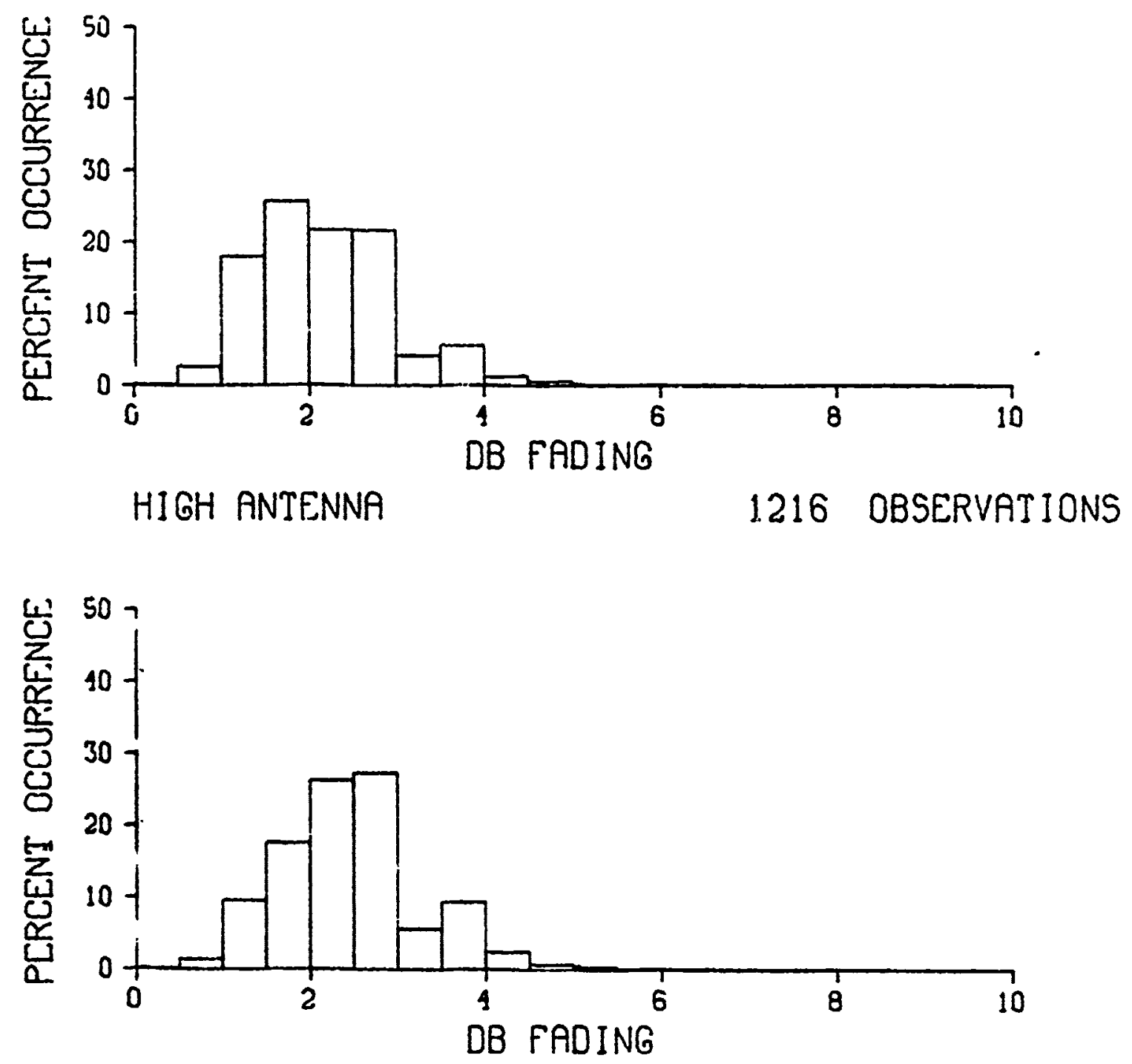

1216 OBSERVATIONS

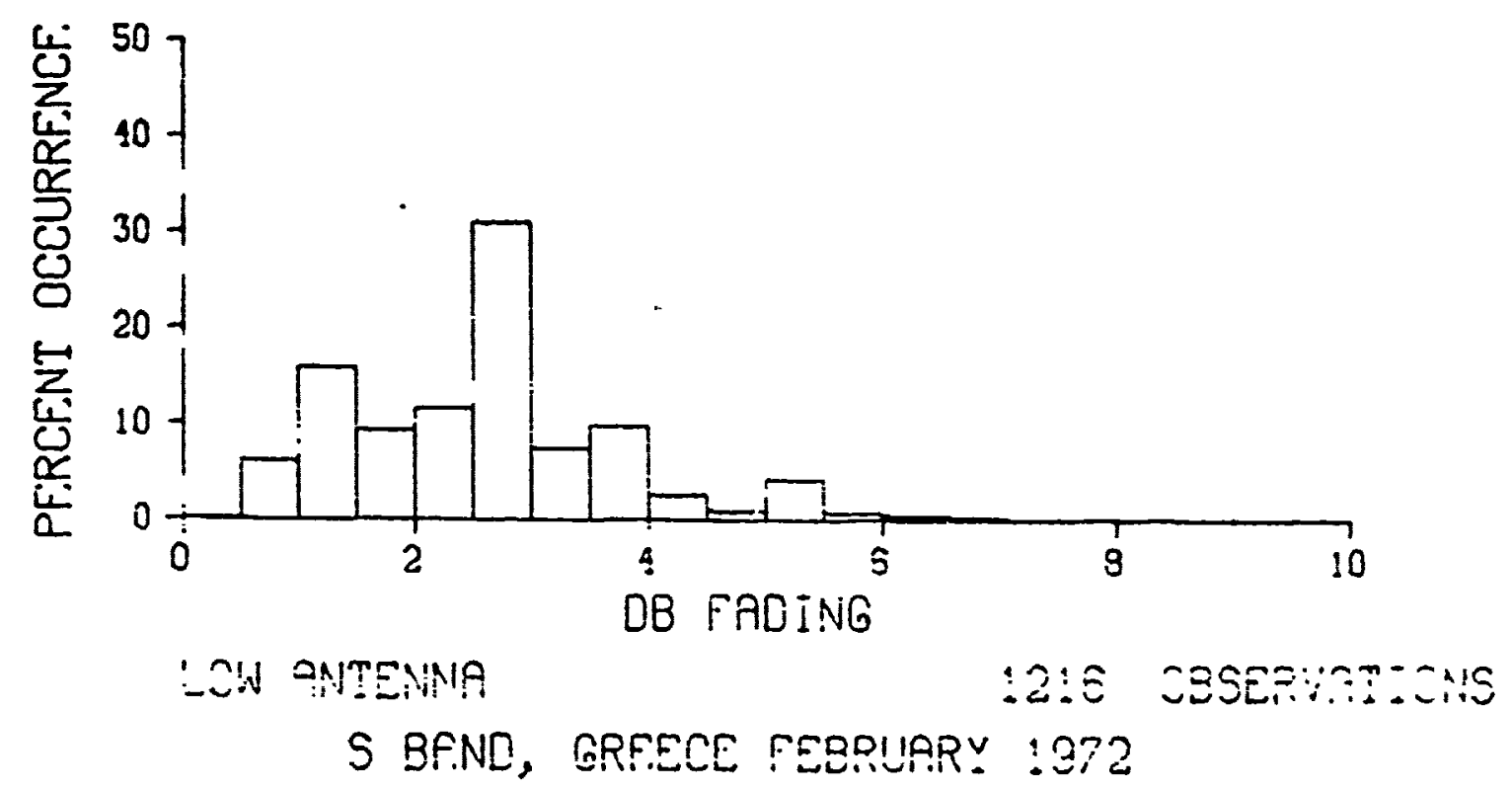

I 
6

8

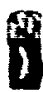

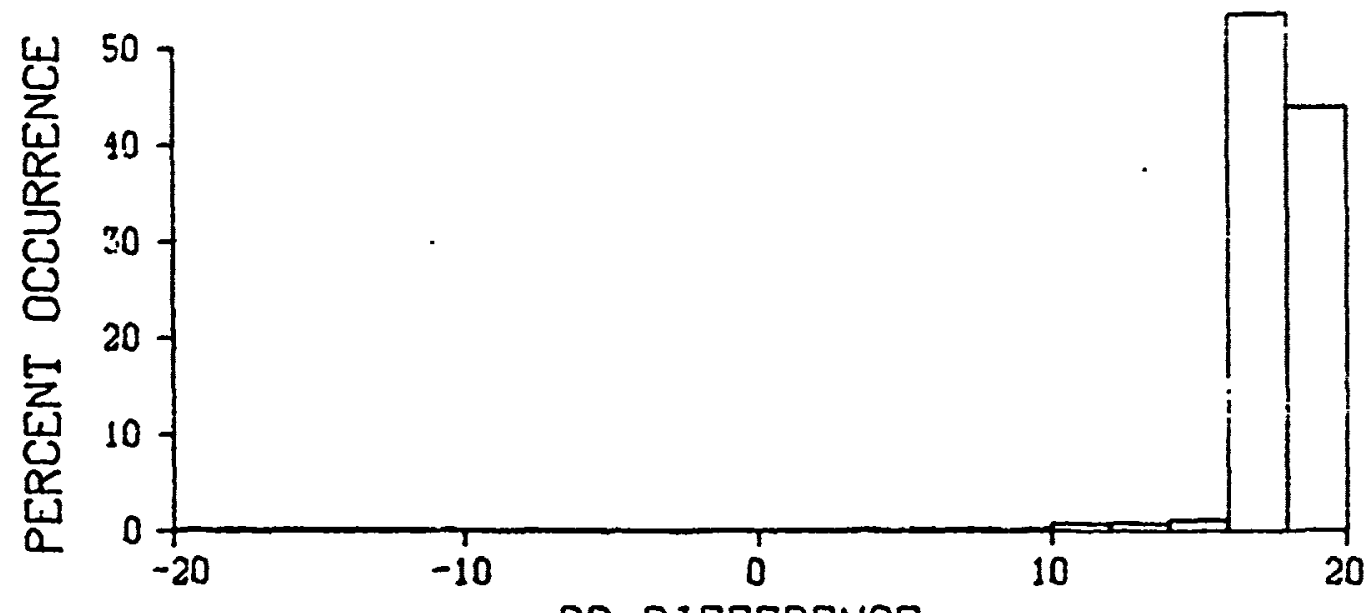

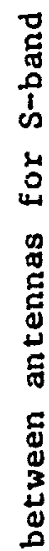

8
8
8
8

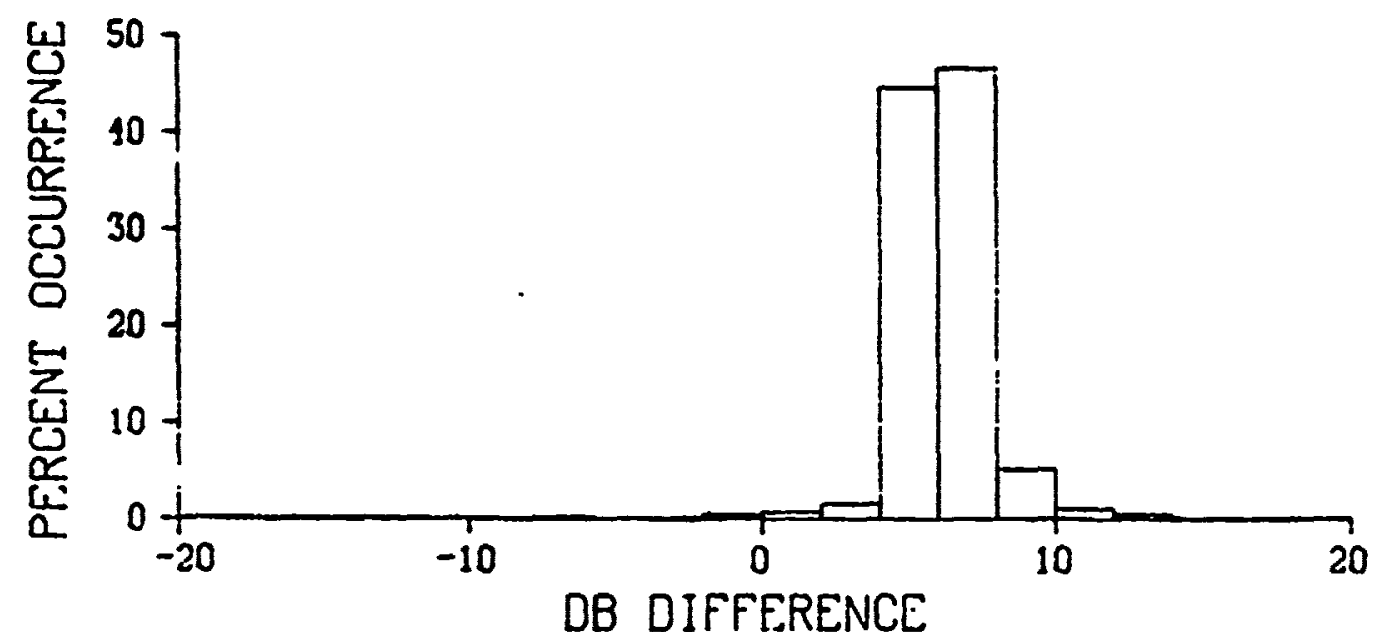

HIGH-MID

1216 OBSFRVATIONS
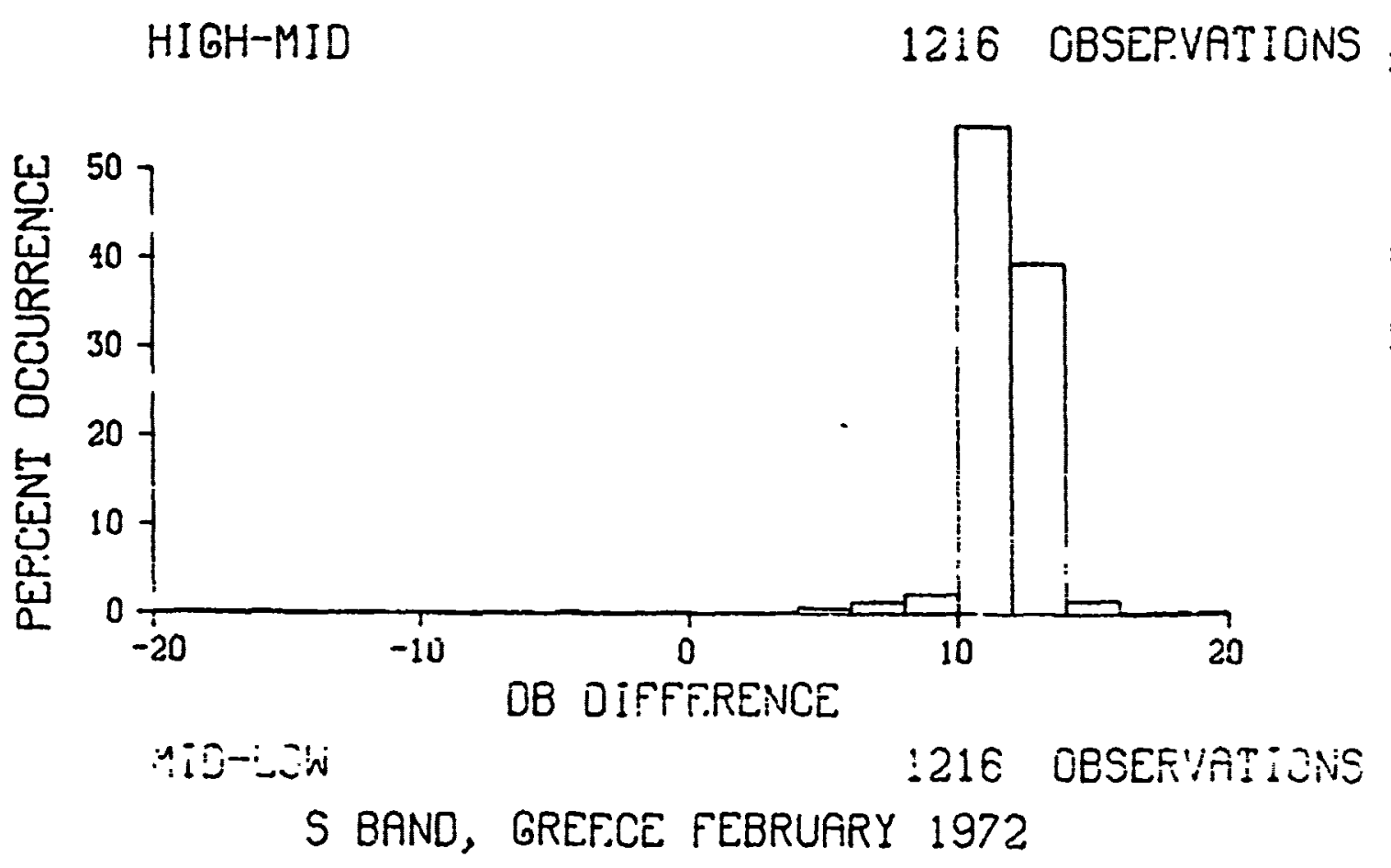

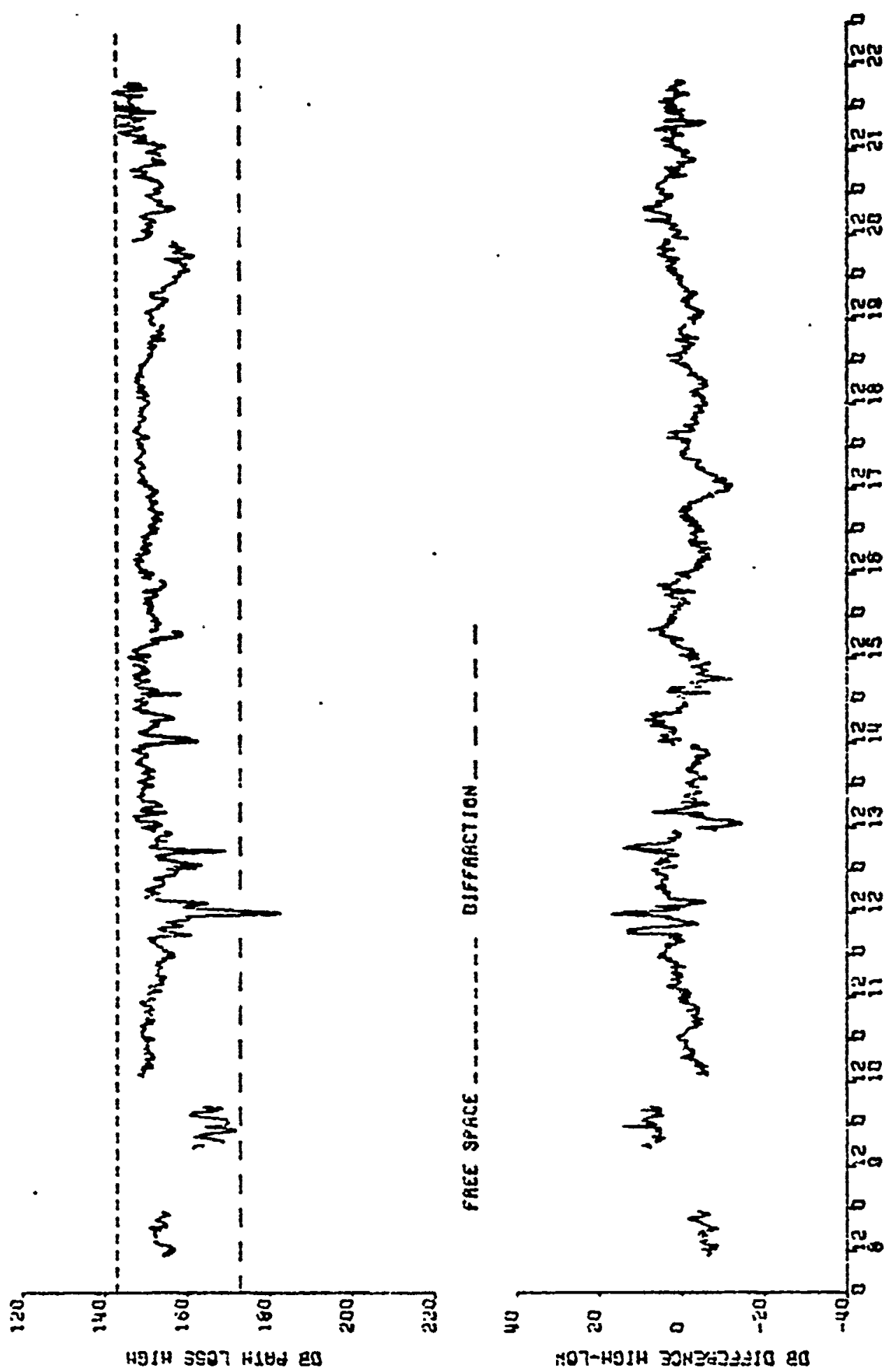

琣

did

$\widetilde{3}$

$\mathbb{8}$

W

8

13

8

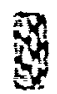

畨

8

I:
0
0

专

8

$\stackrel{2}{2}$

8

$x$

0

8

$\underline{9}$

s. 

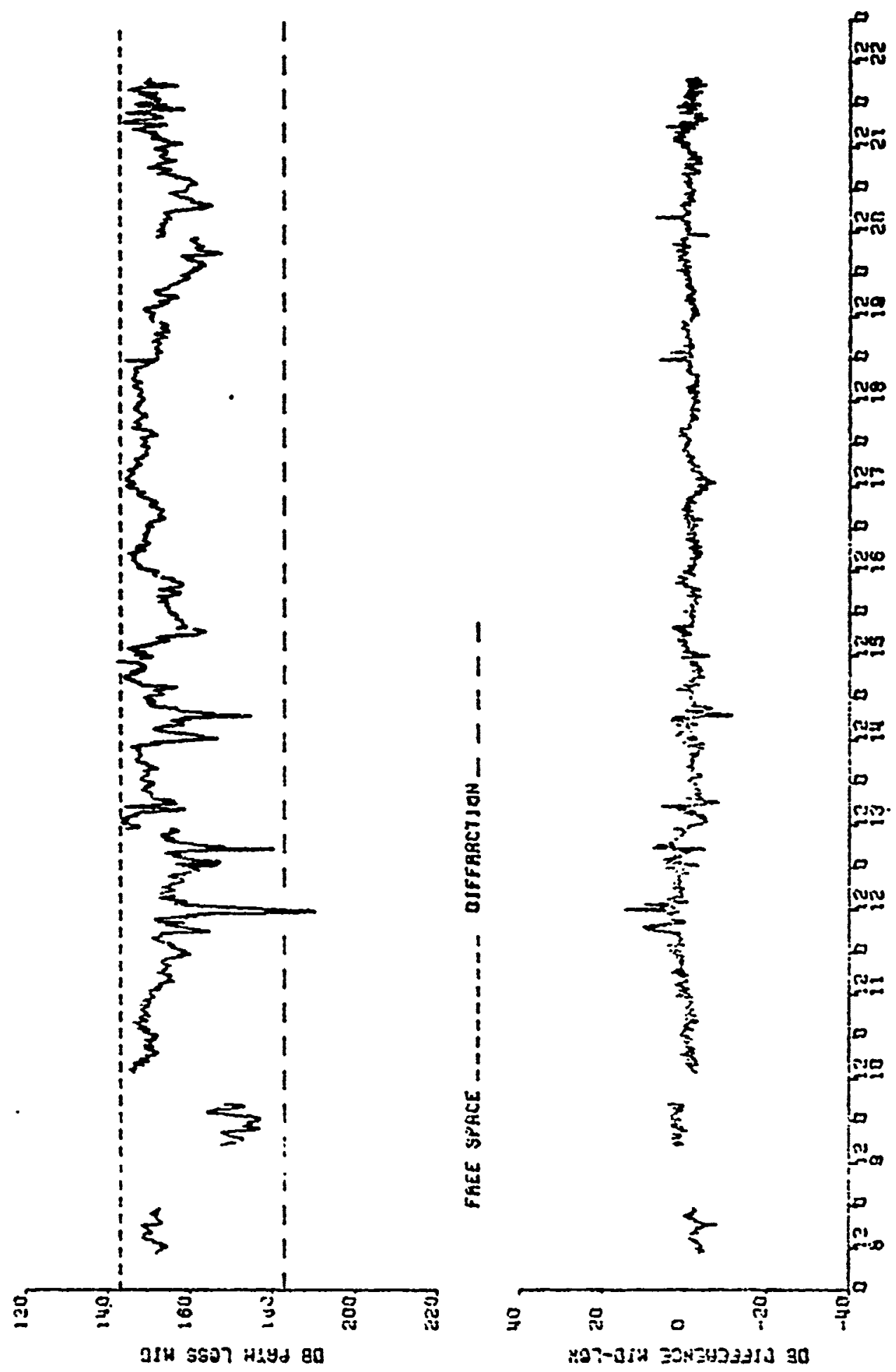

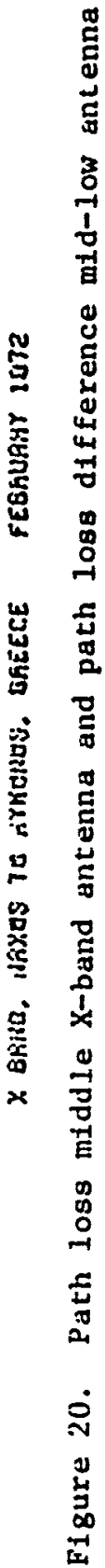

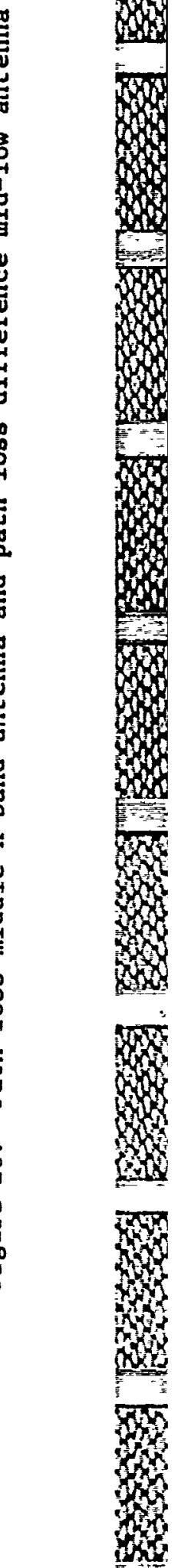



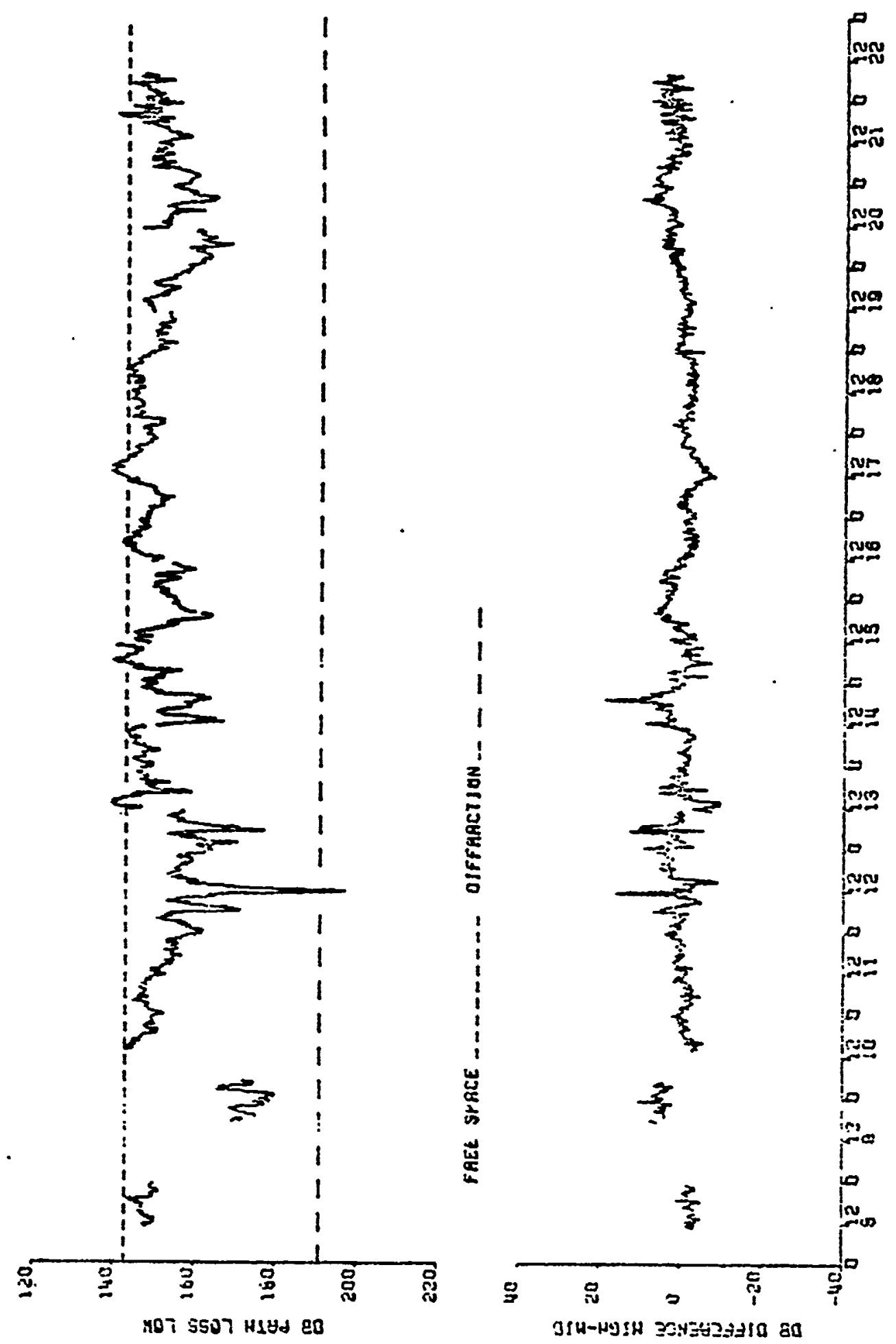

8

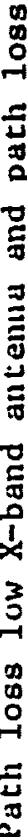

武

$B$

告

s

g

望

泼

总

甄

8

影

$\dot{\sim}$

9

to 


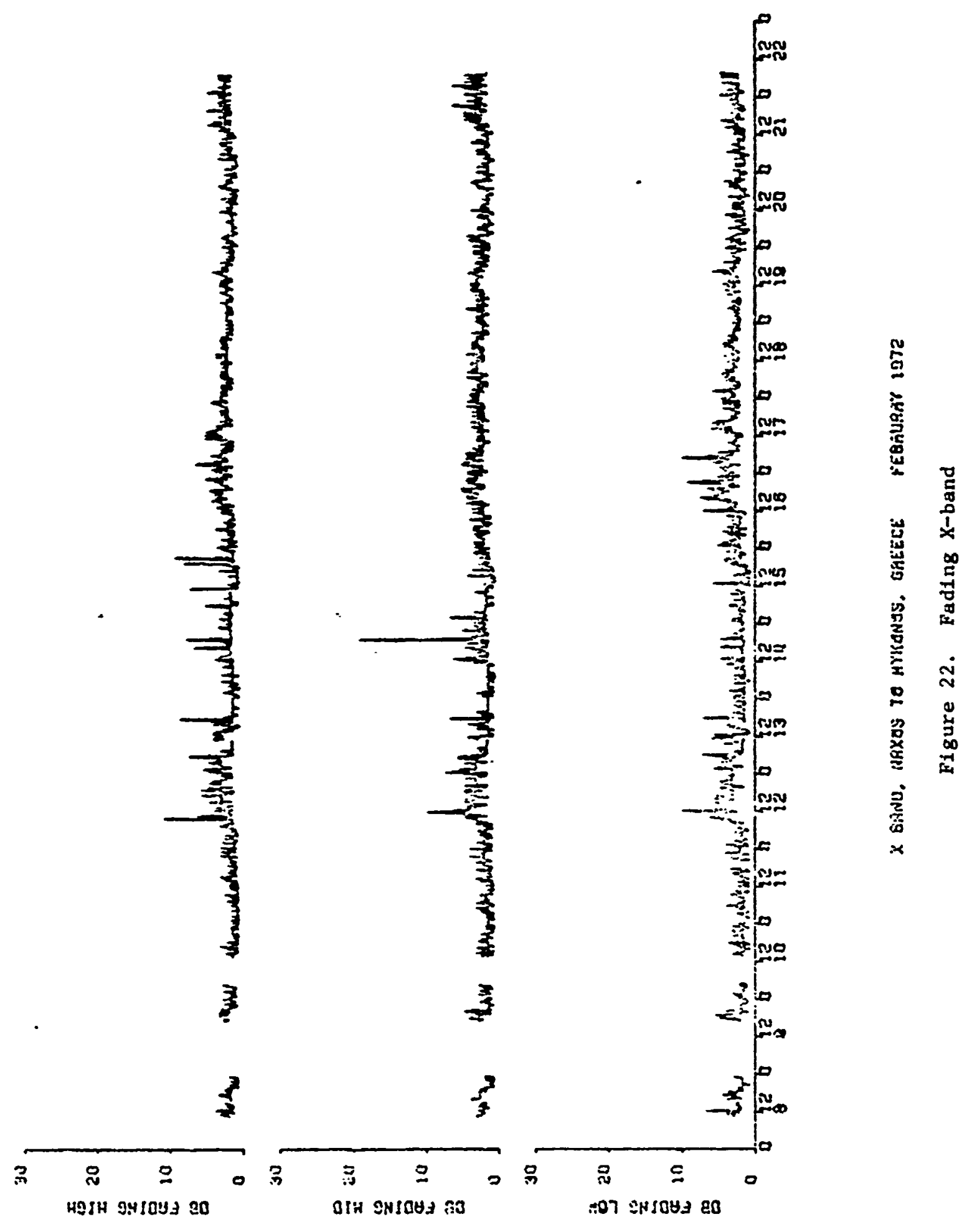



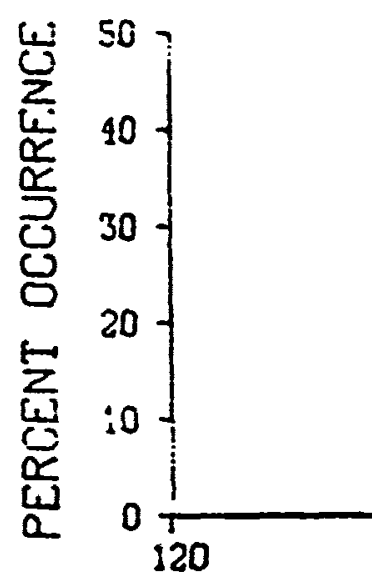

HIGH ANTENNA

1202 OBSERVATIONS

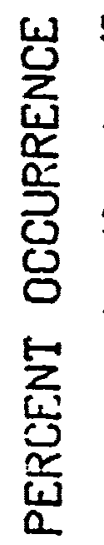

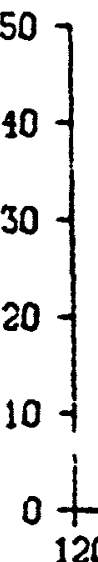

MID ANTENNA

1202 OBSERVATIONS

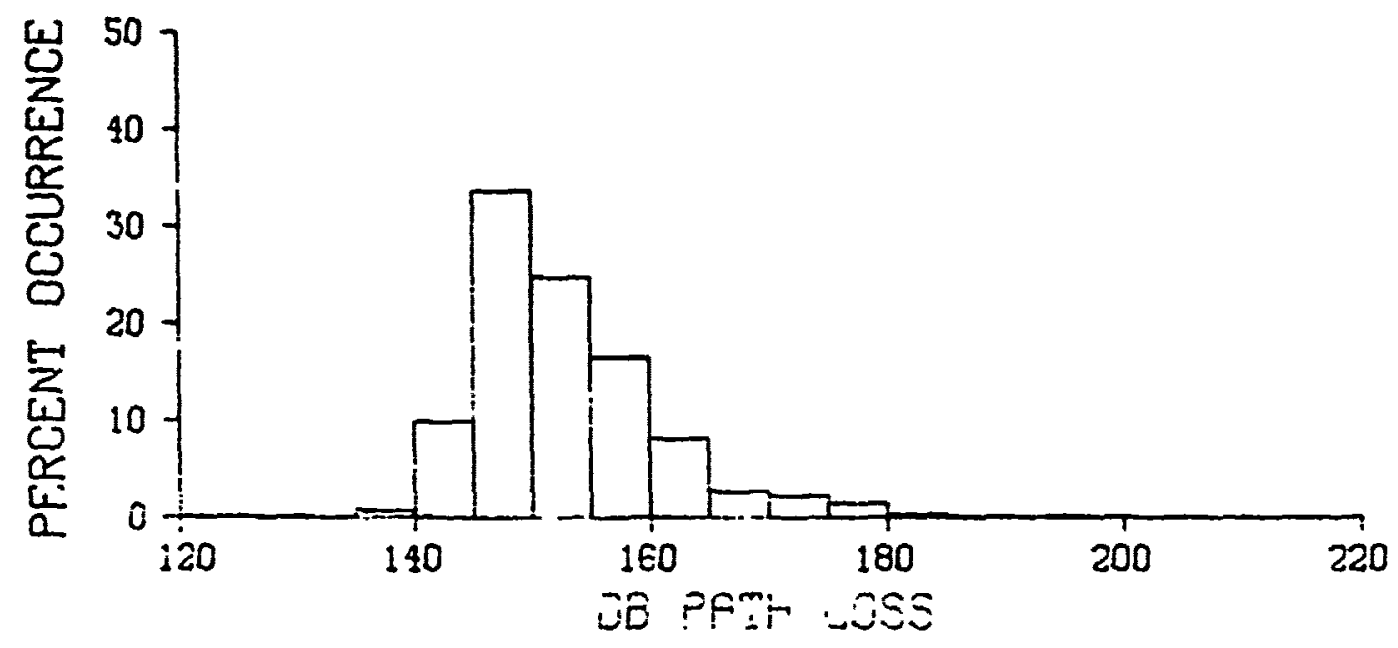

LOW ONEEINIS

1292 CSSE:*:-2:2:

$X$ BAND, OREECE FEBRLIRRY :972 


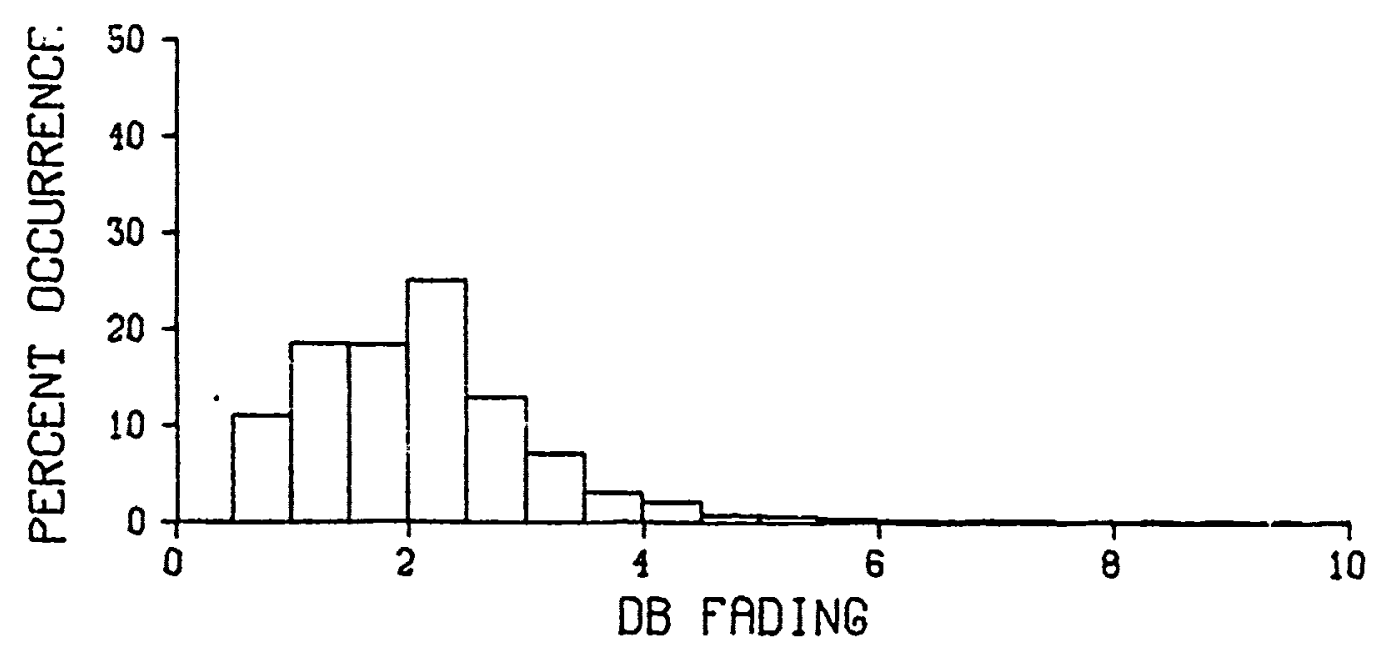

HIGH ANTENNA

1202 OBSERVATIONS

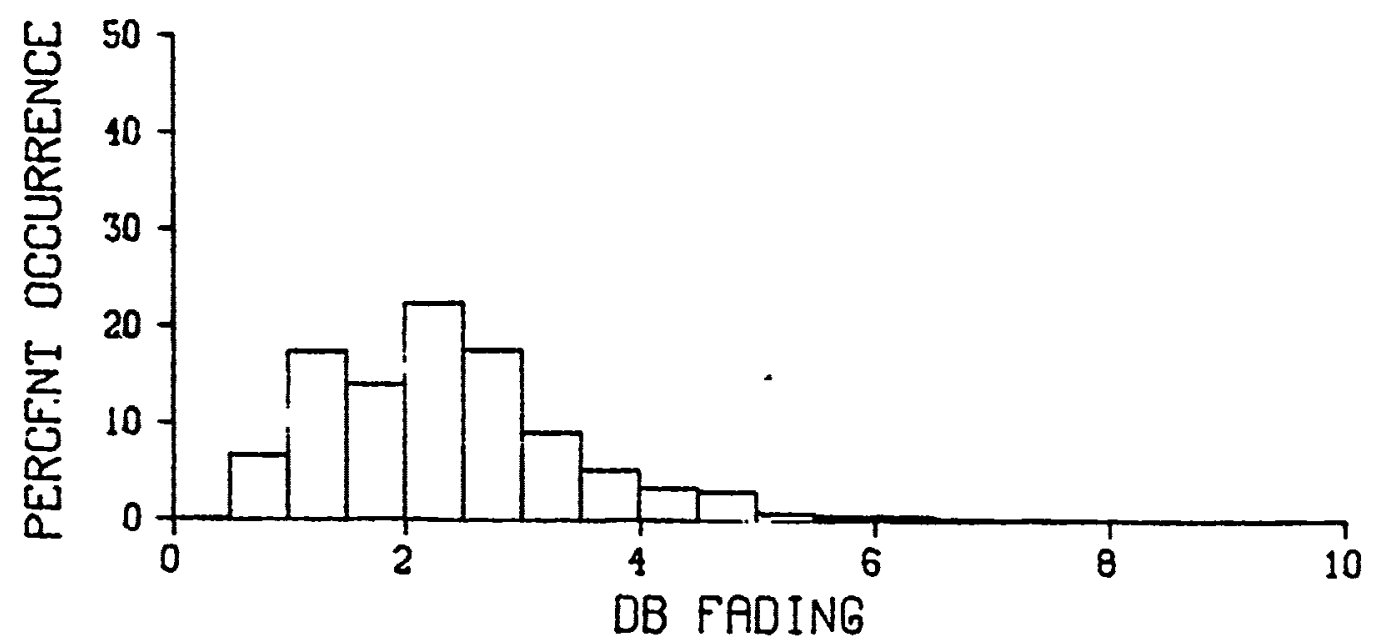

MID ANTENNA

1202 OBSERVATIONS

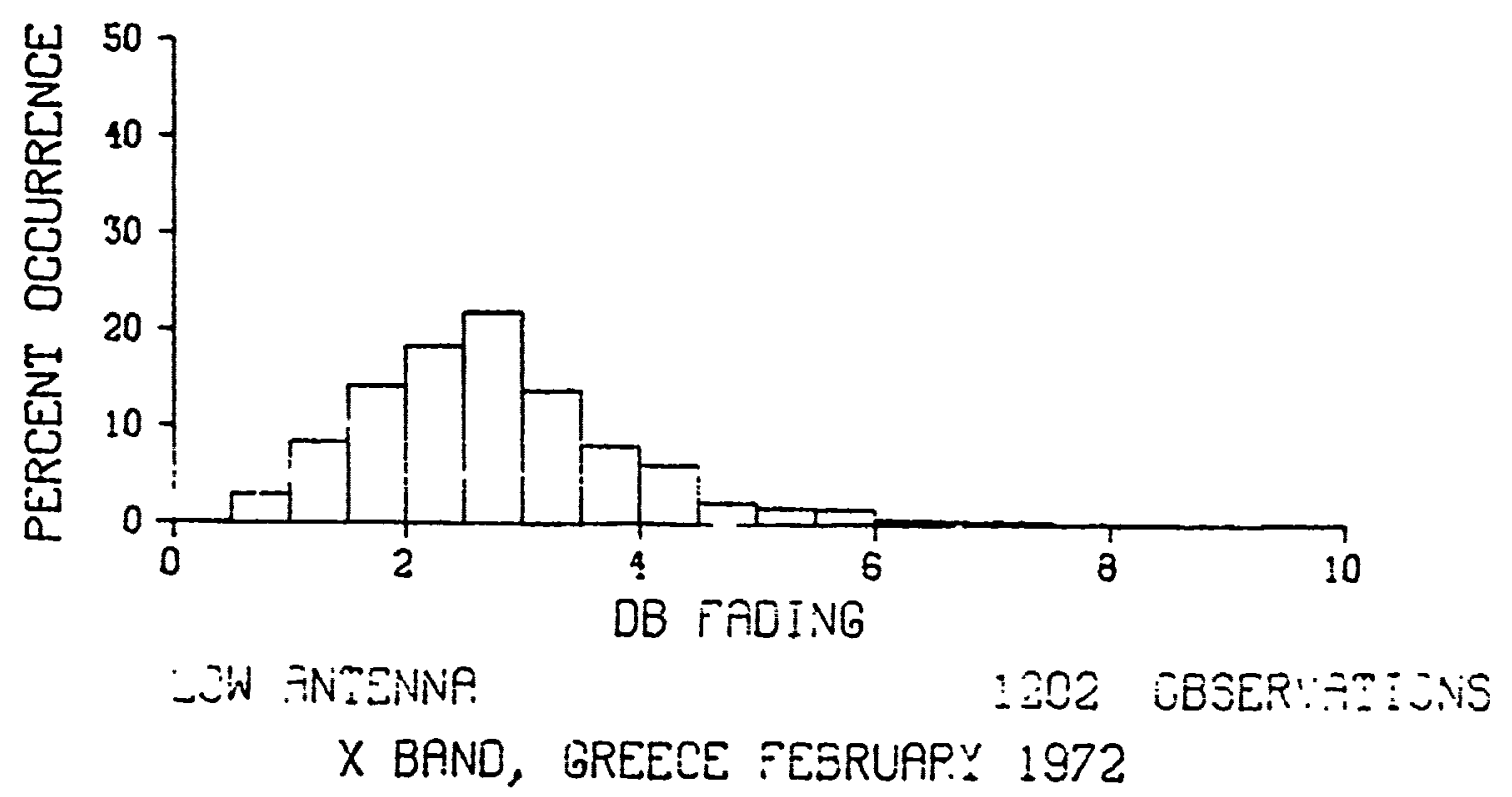




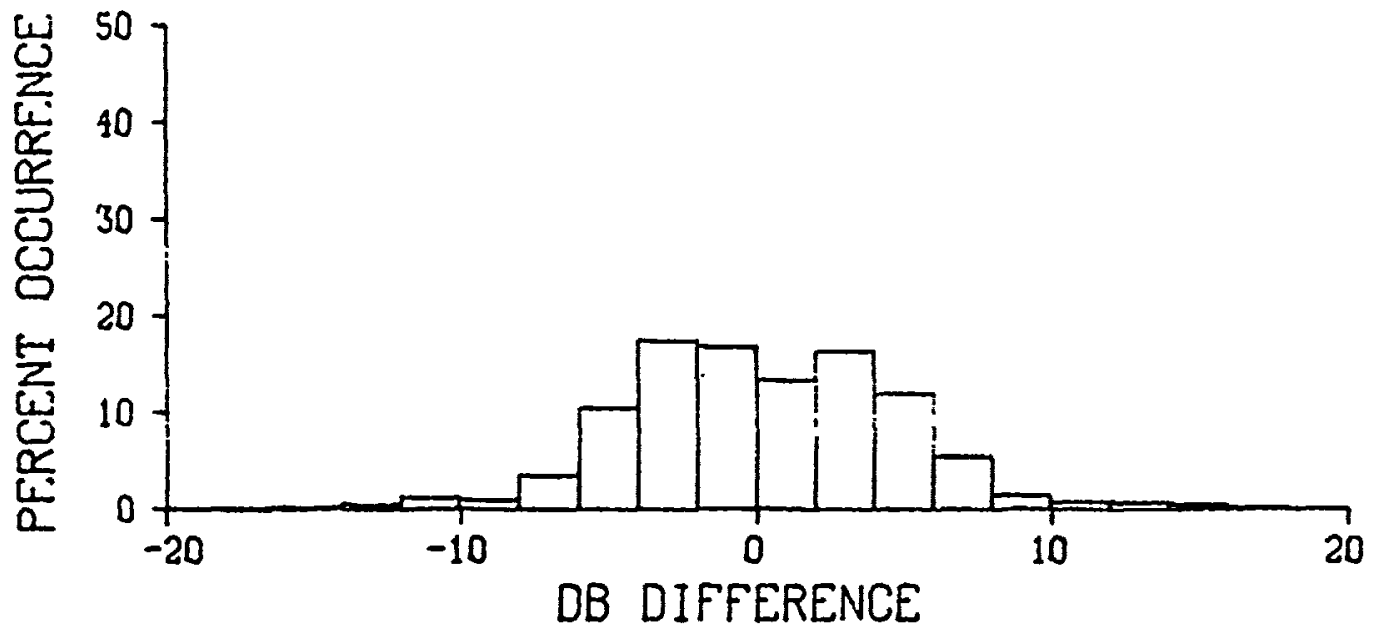

(⿻日木

ㅍiv

a

Xิ

HIGH-LOW

1202 OBSERVATIONS

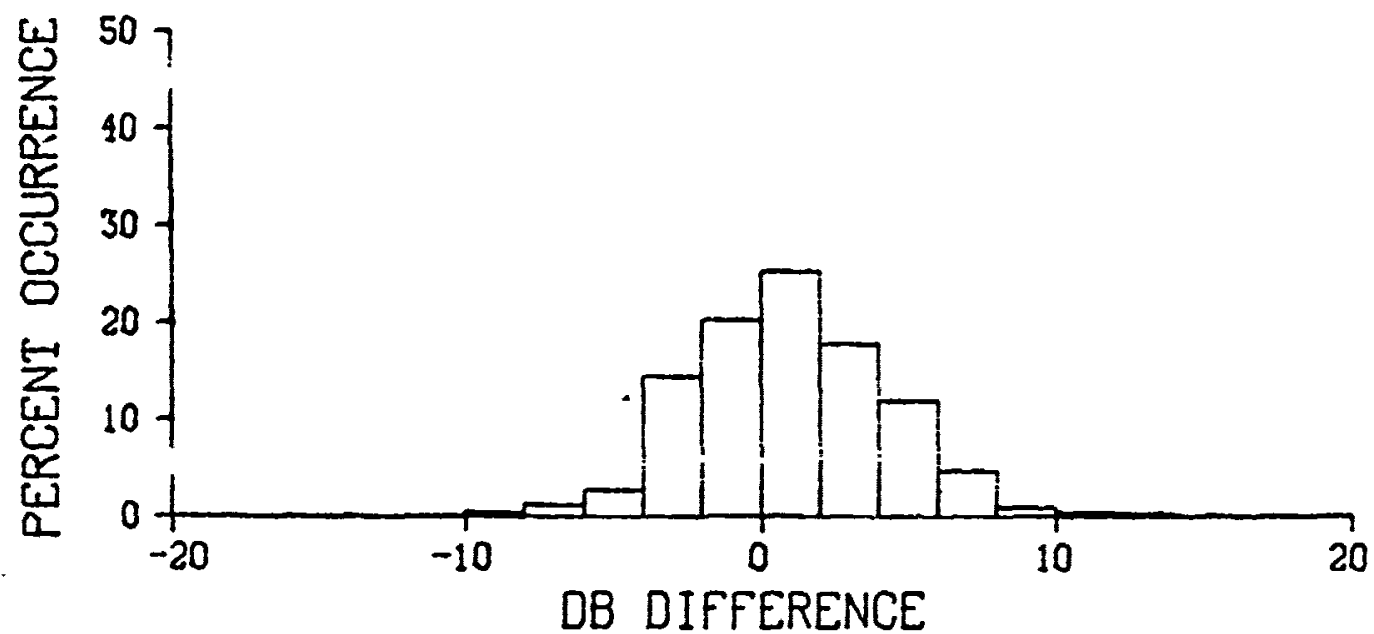

HIGH-MID

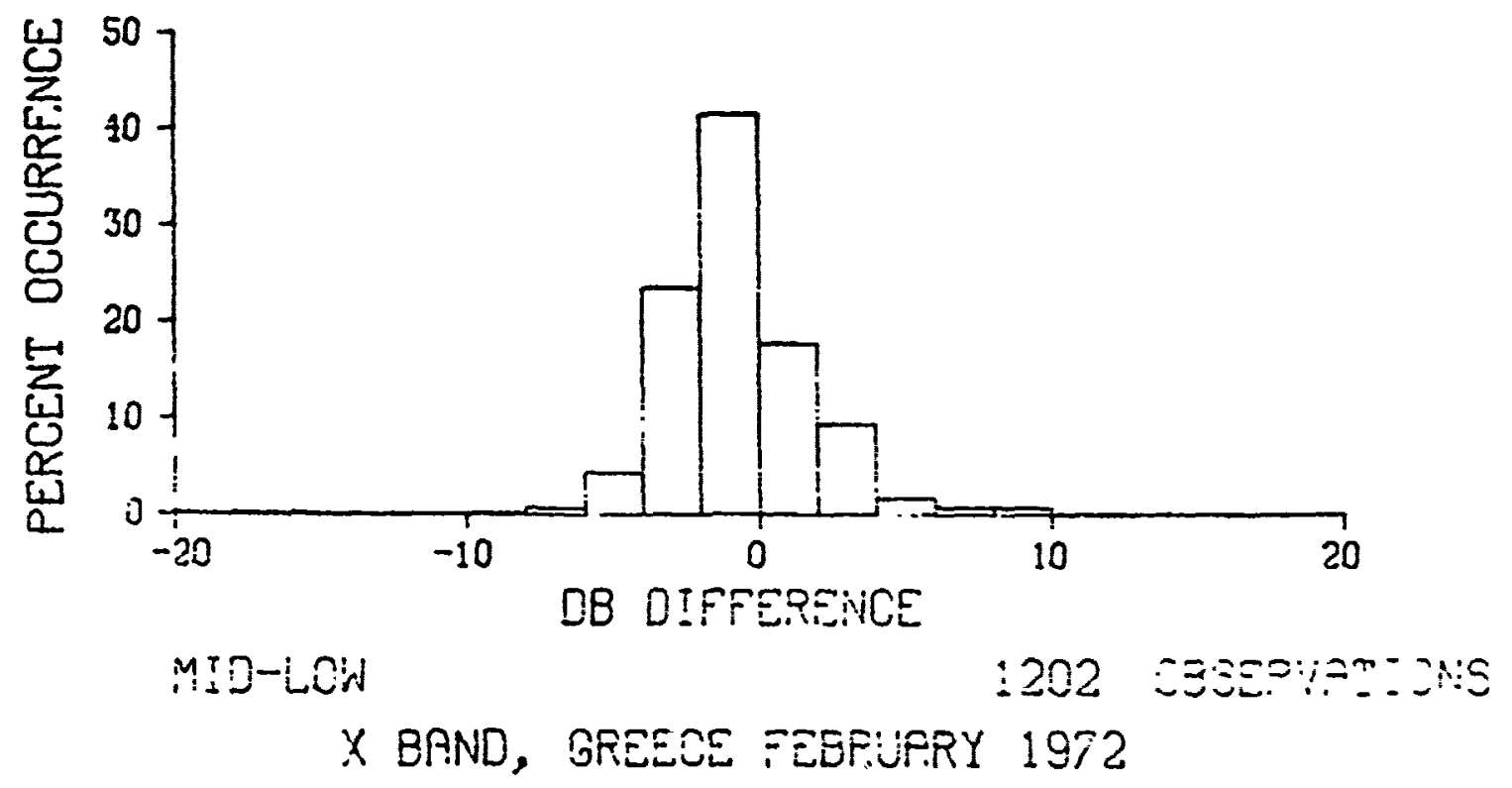



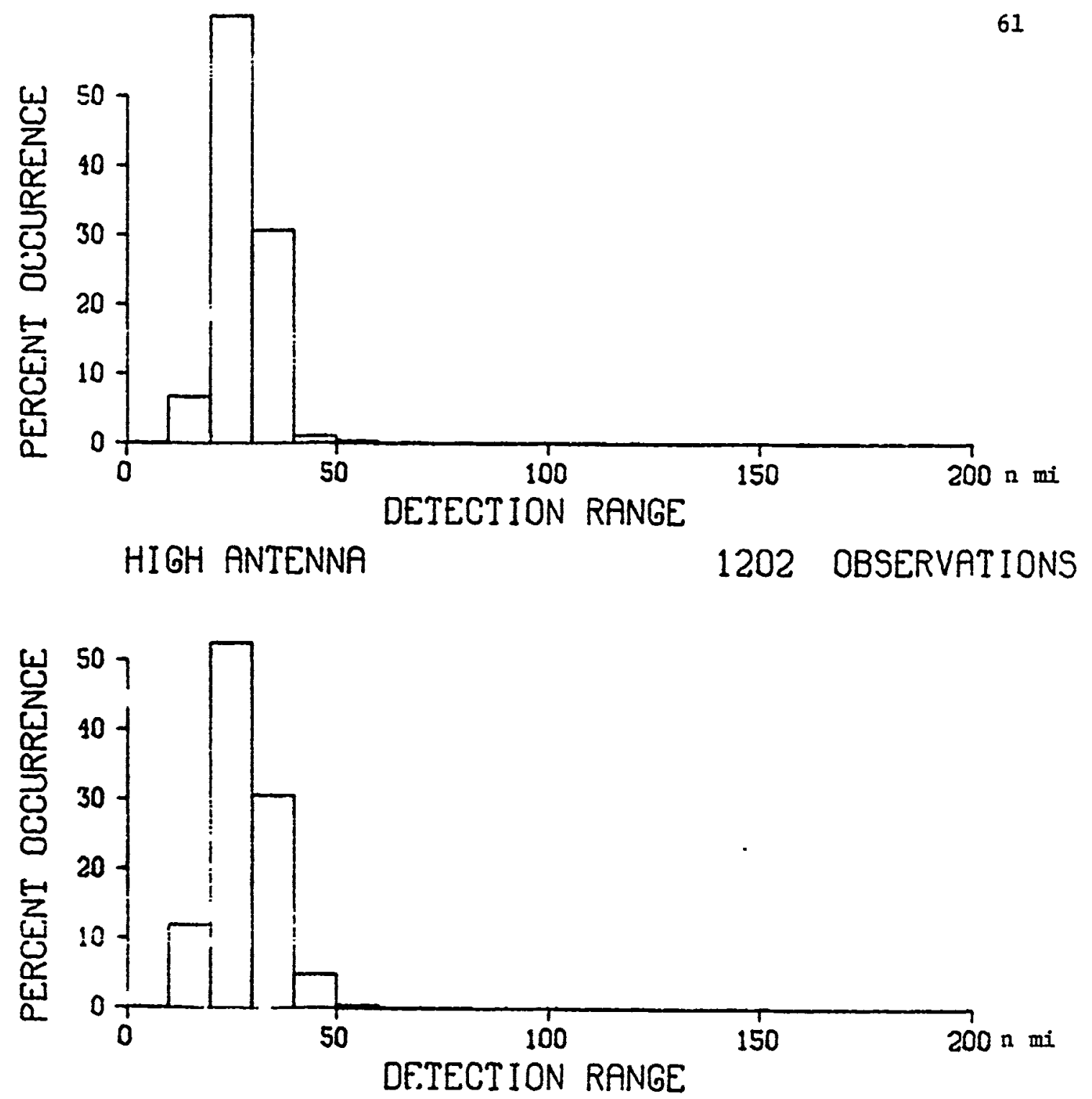

MID ANTENNA 1202 OBSERVATIONS

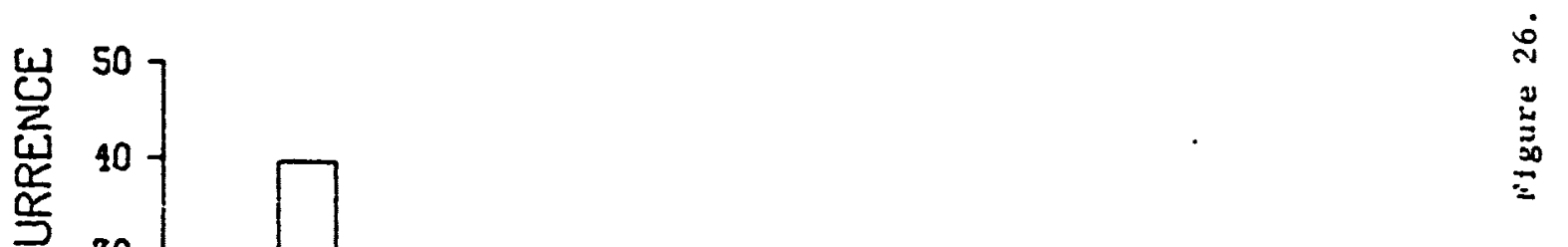


是

100
80
60
40
20
0

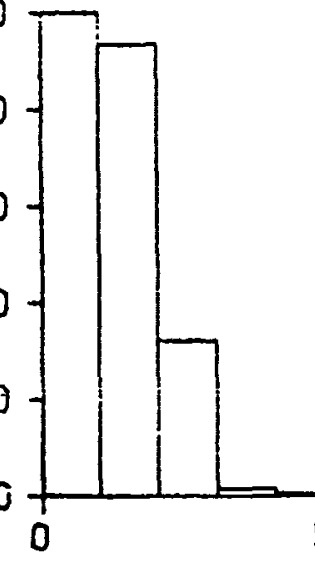

50

DETECTION RANGE

HIGH ANTENNA

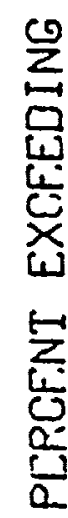

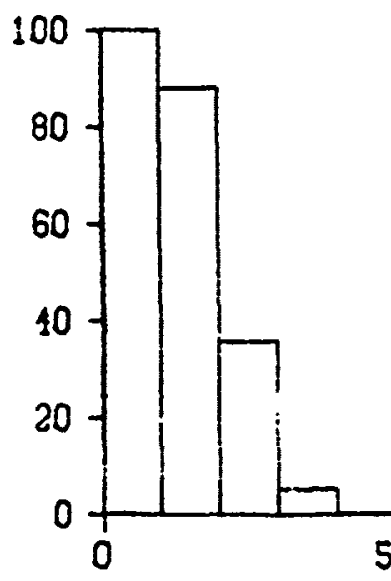

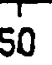

MID ANTENNA

\section{DETECTION RANGE}

150

1202 OESERVATIONS

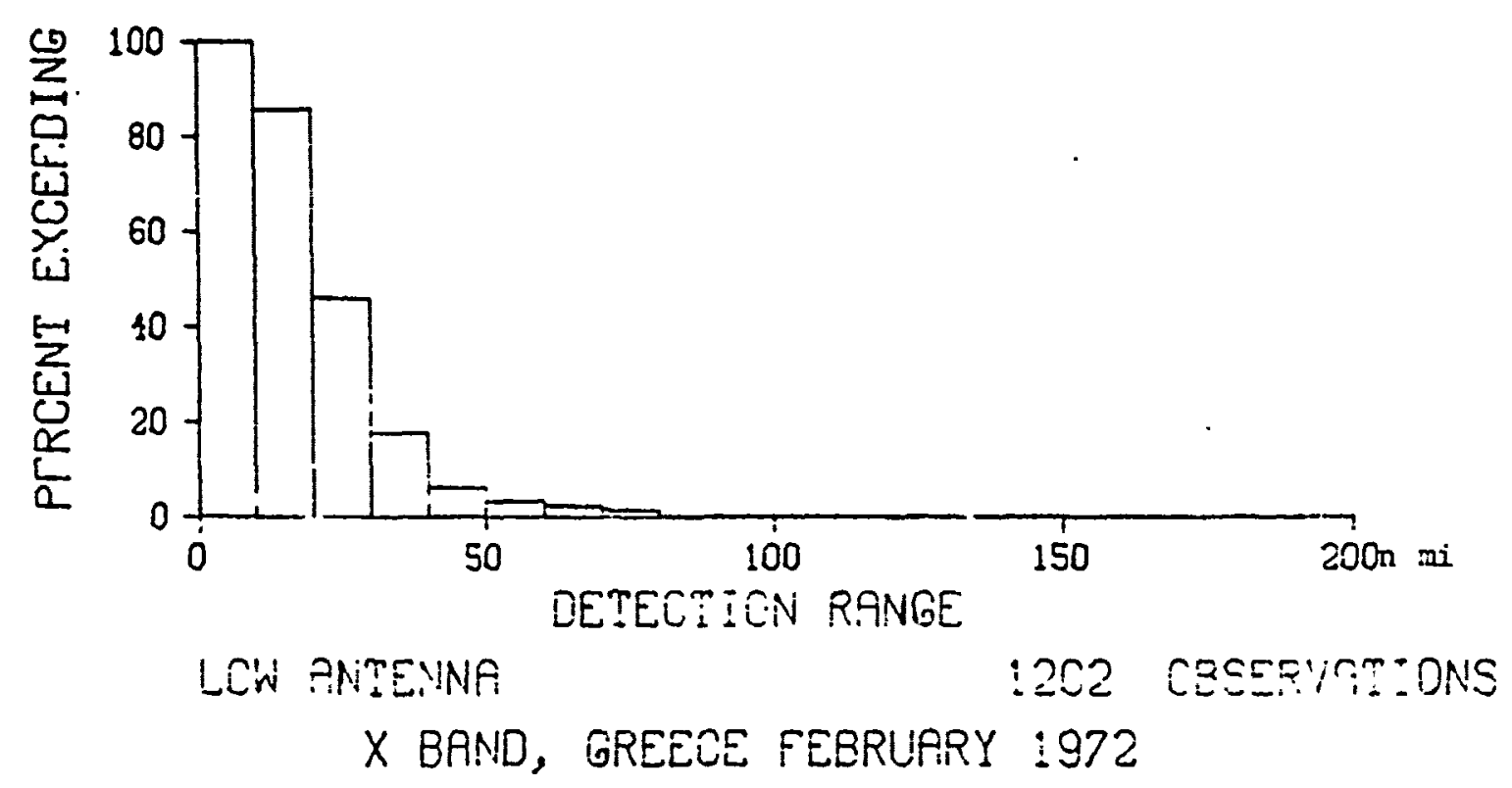




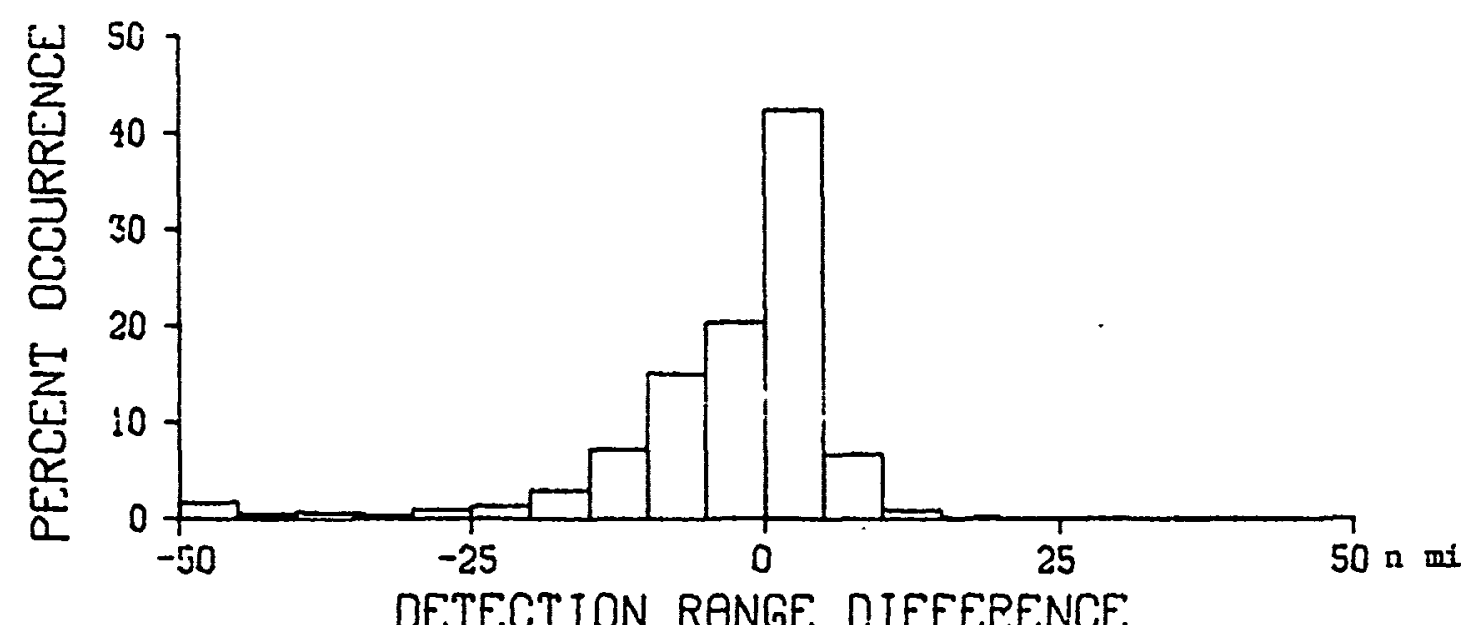
HIGH-LOW
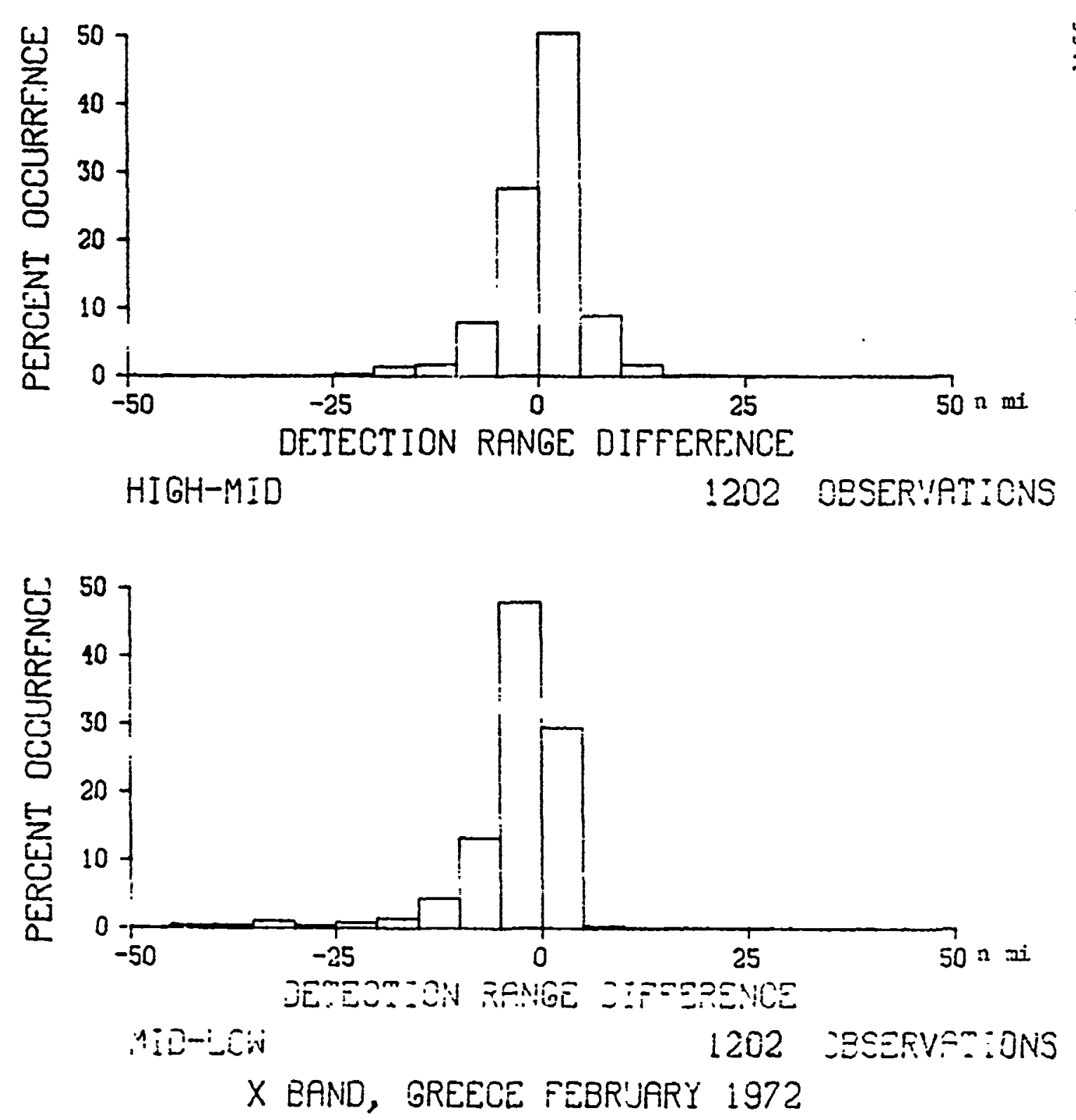

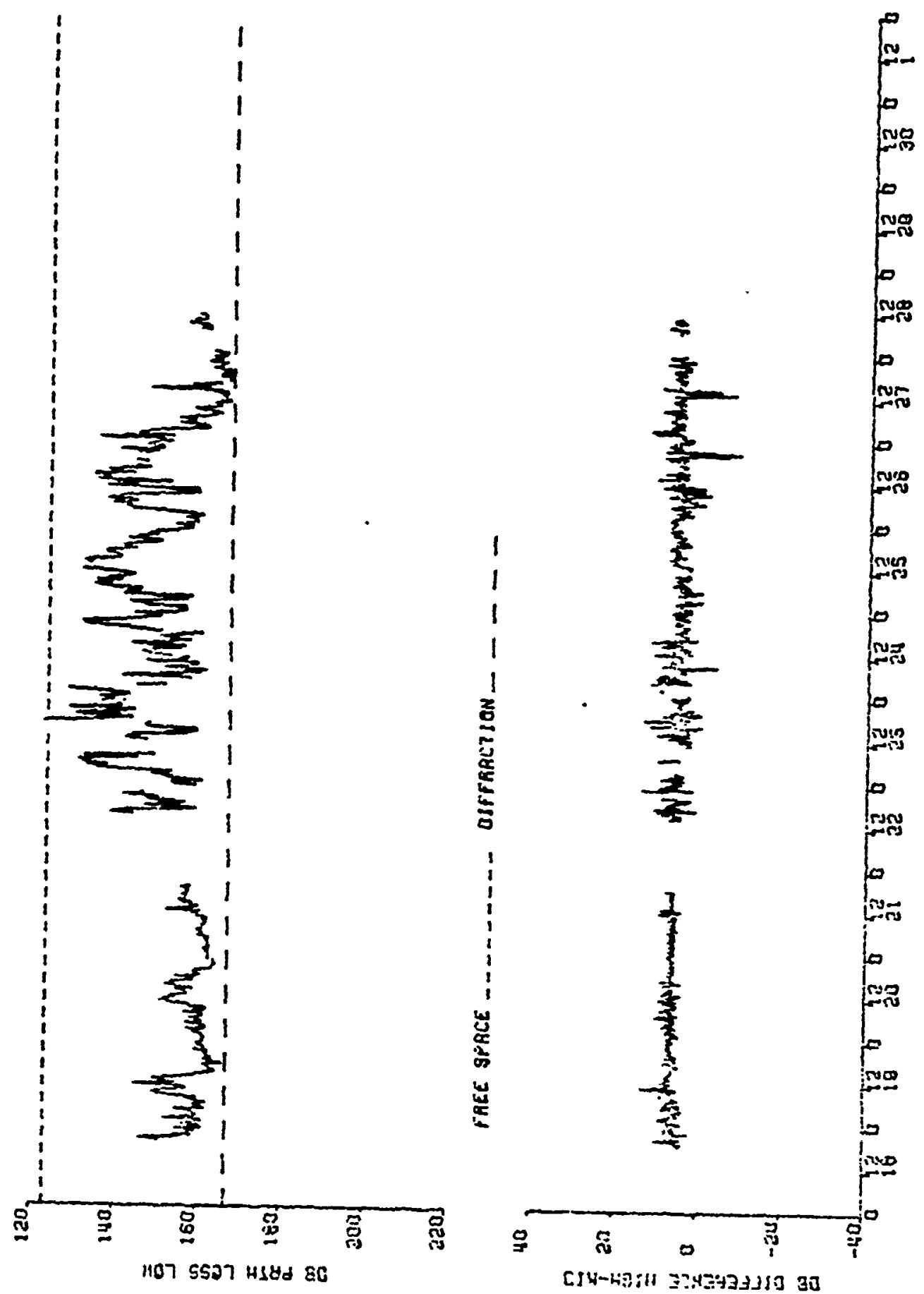

ח

.

垔

兽

R

武

8

吾

ถ

oun

를

马

產

क

韋

㝢

1

䓛

的

品

吾

X

$\dot{m}$

X

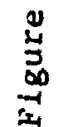

8

$x$ 


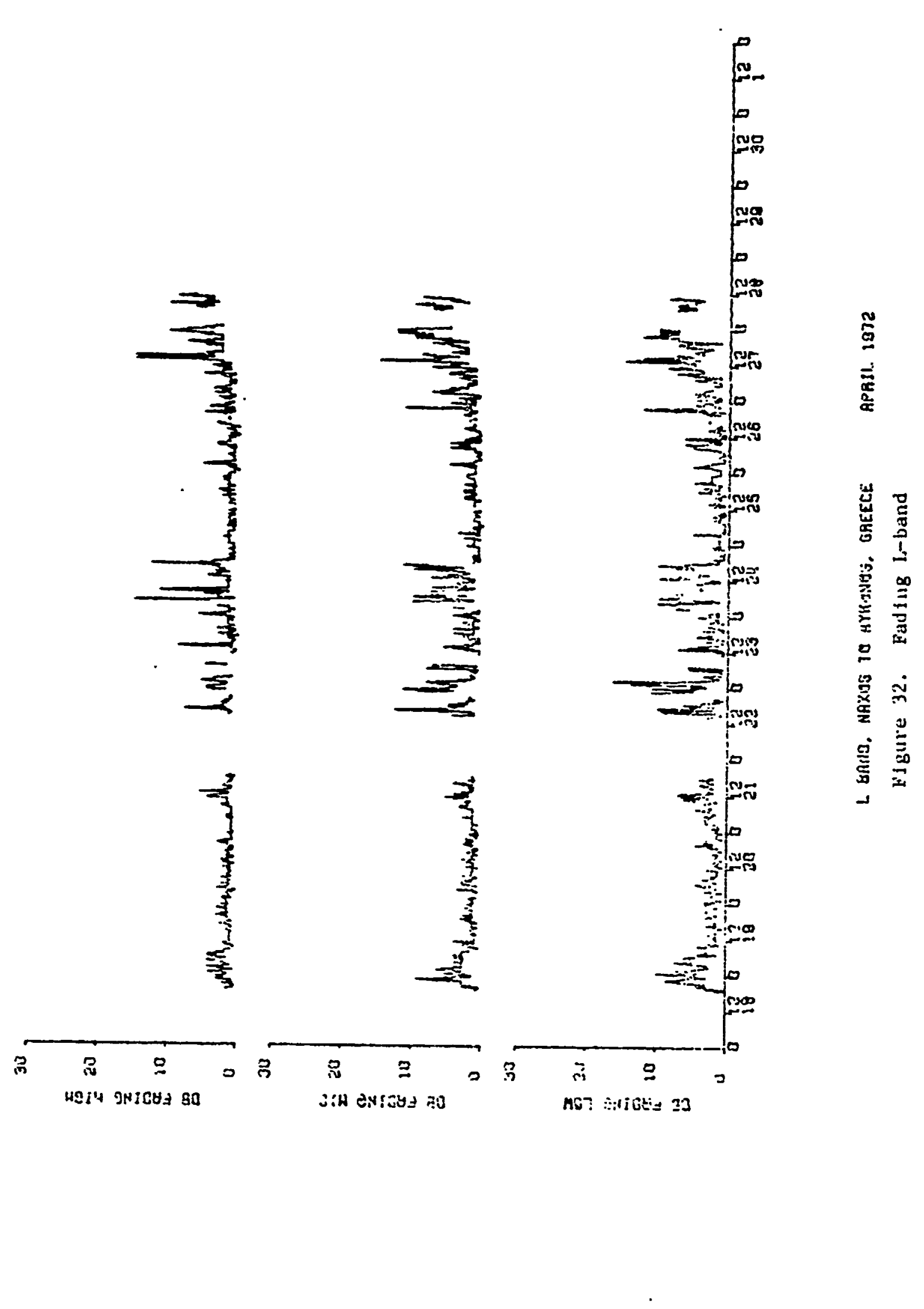




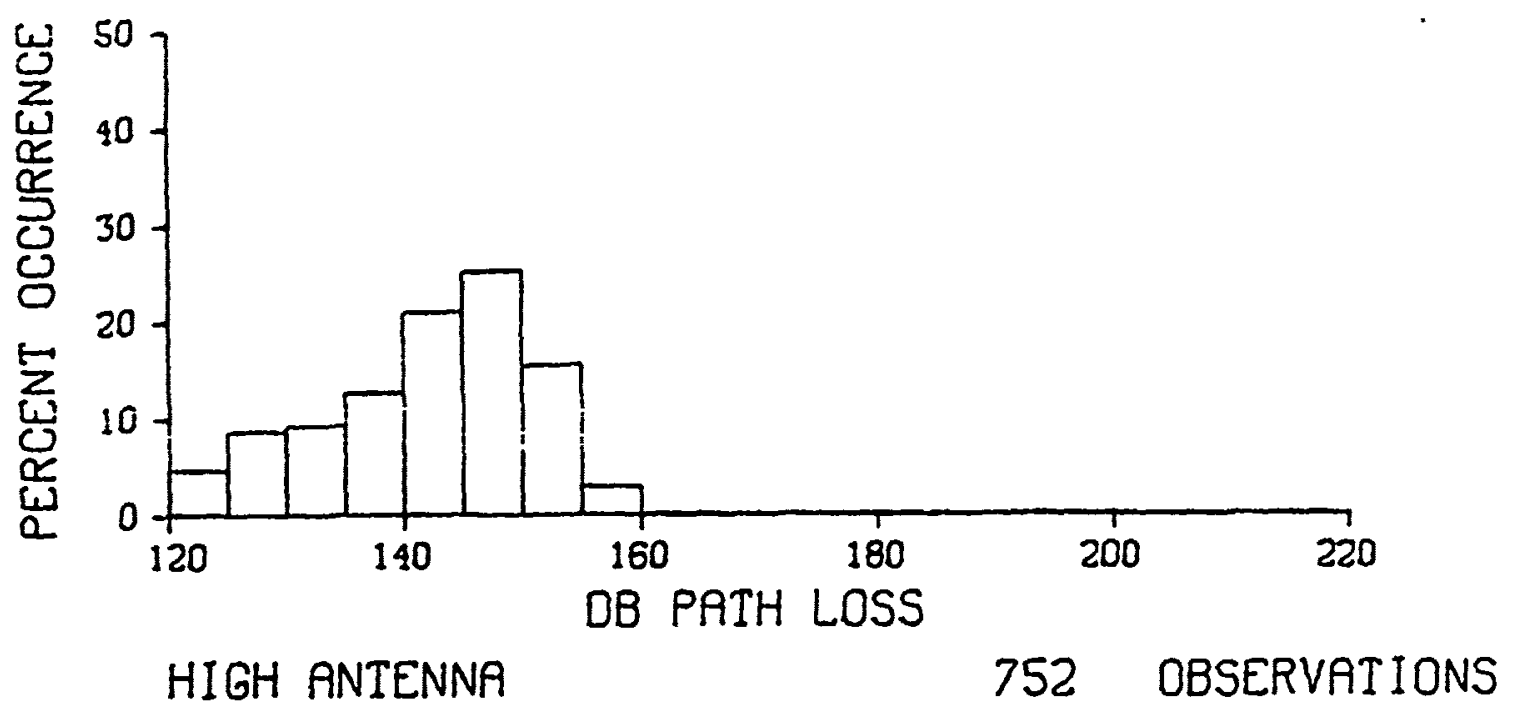

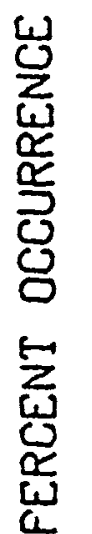

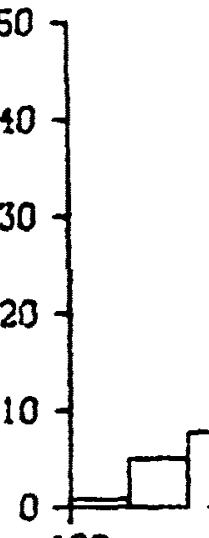
120
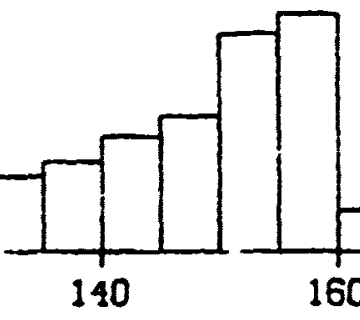

DB PATH LOSS

MID ANTENNA

758 OBSERVATIONS

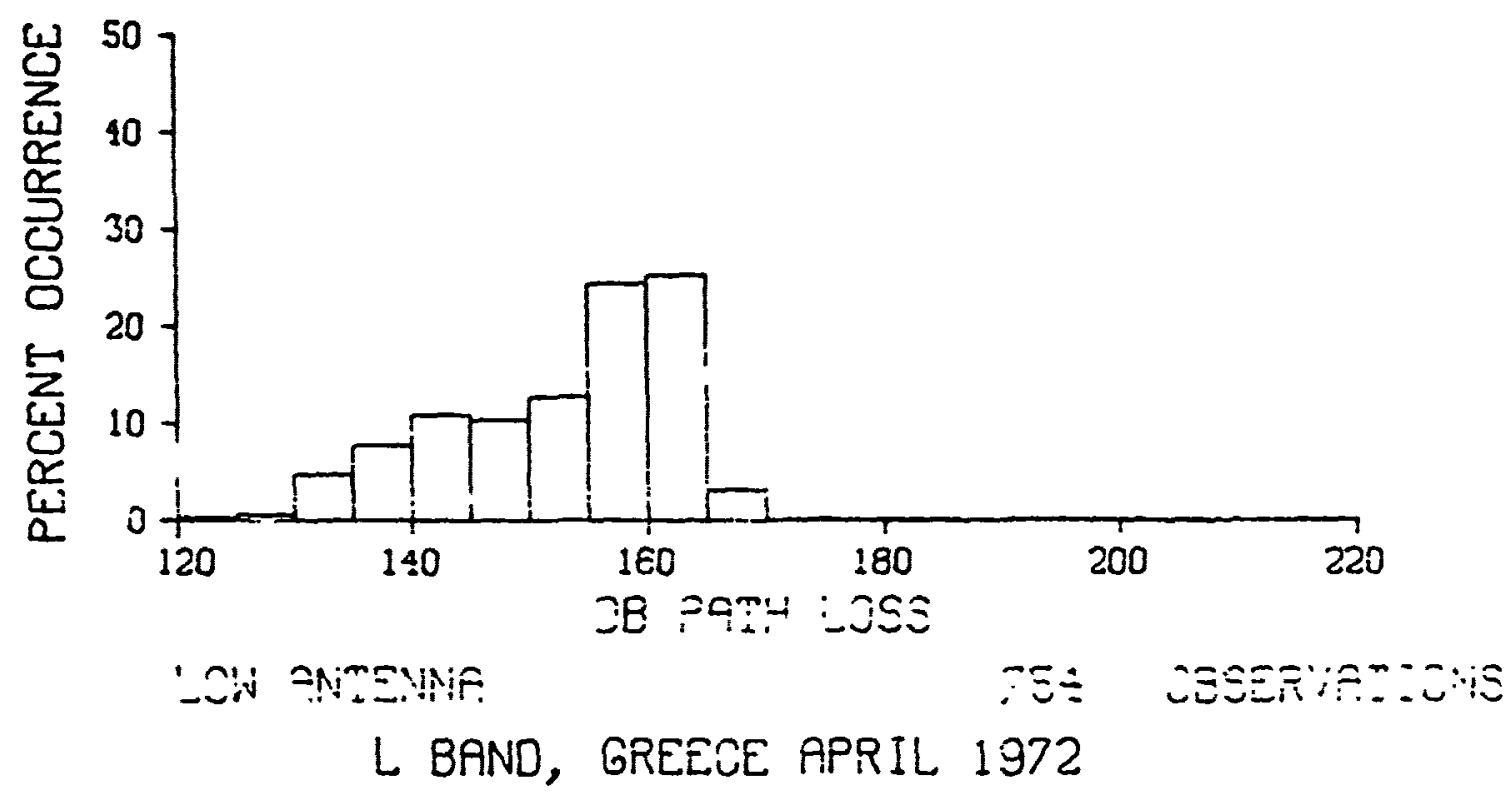

$\underline{H}$

난

8

$\overline{3}$

W

8

8

bi

9

8

5

8

th

9

a

8

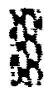

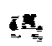
3 


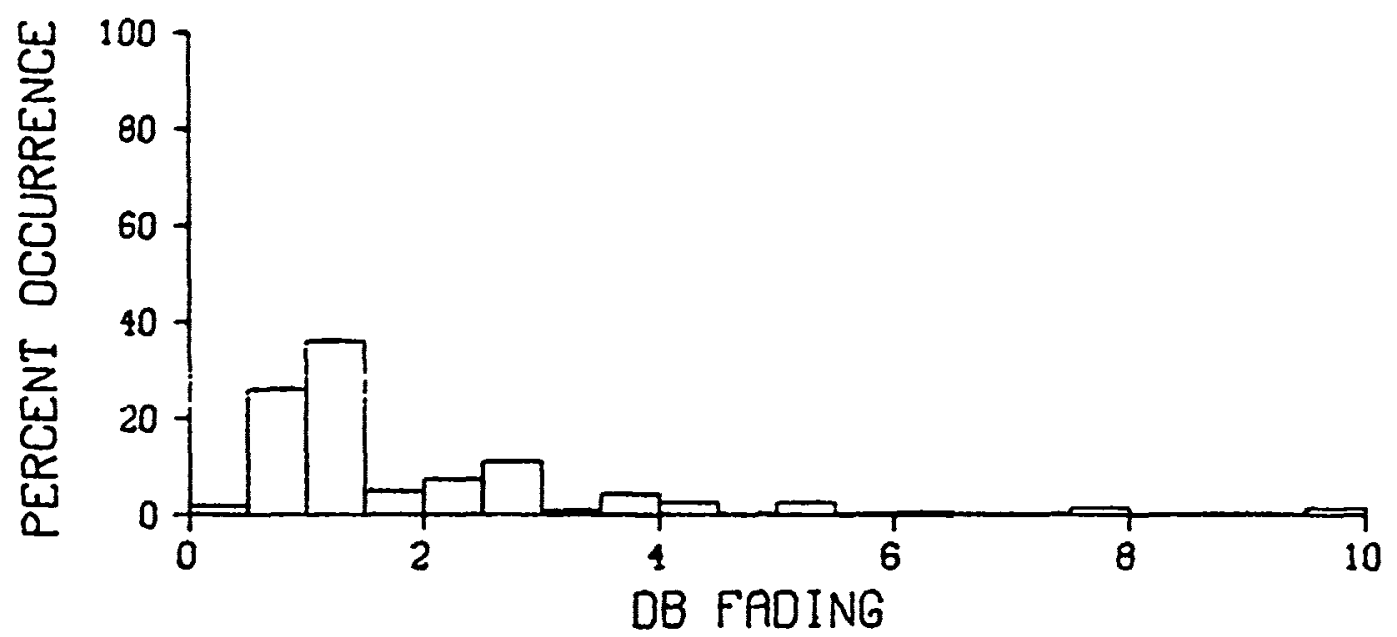

HIGH ANTENNA

752 OBSERVATIONS

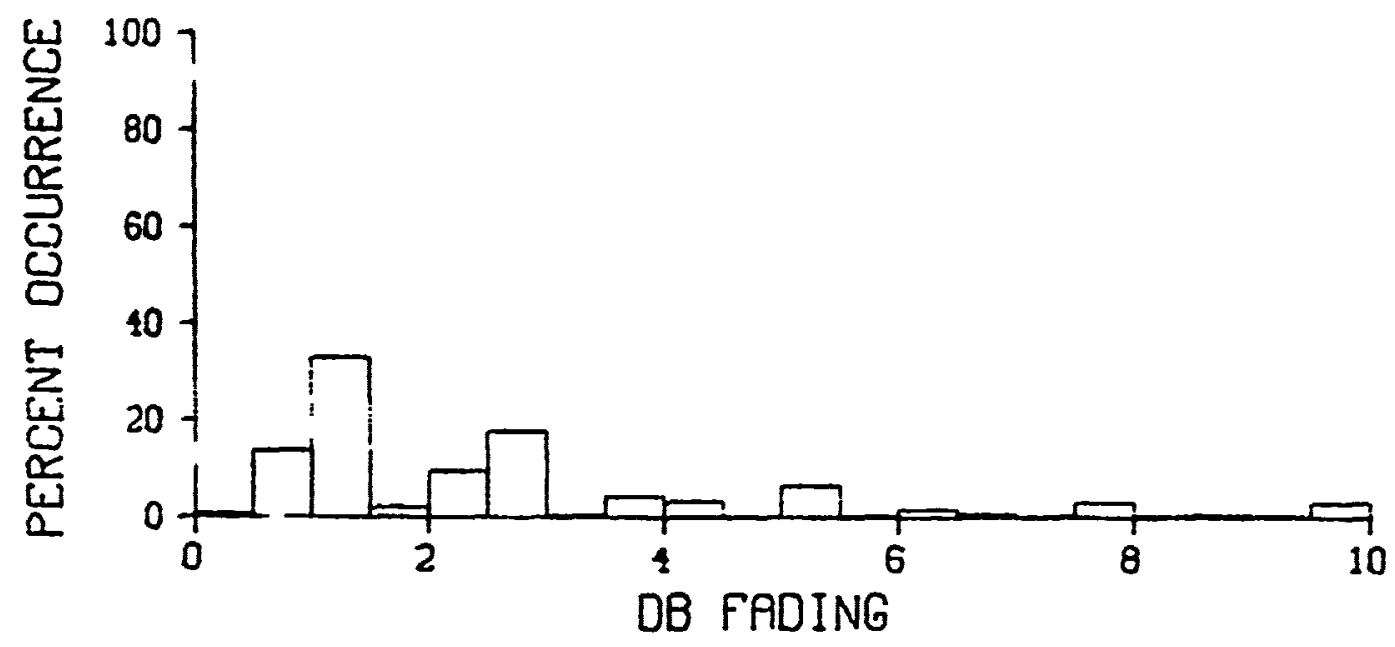

MID ANTENNA

758 OBSERVATIONS

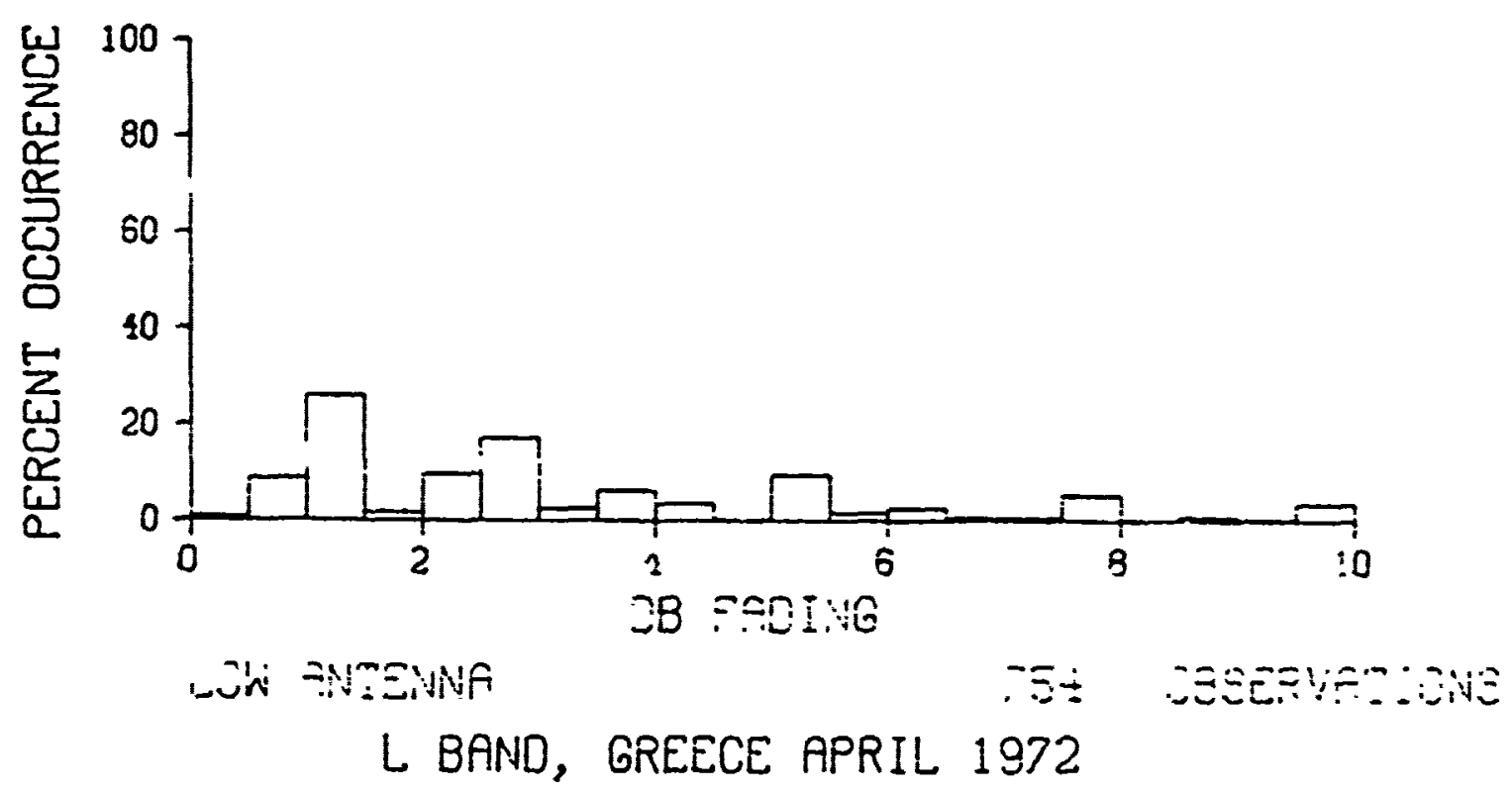



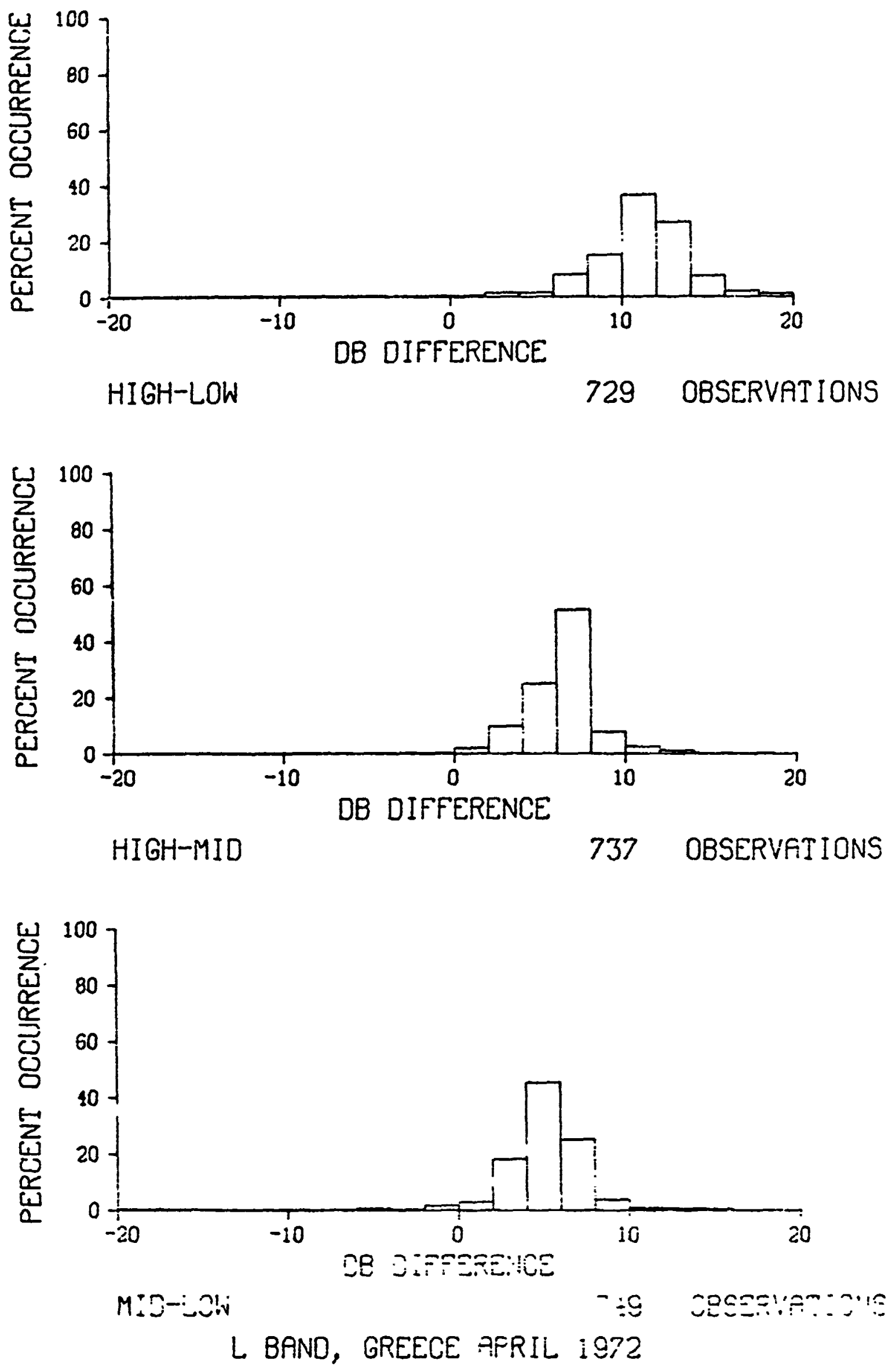

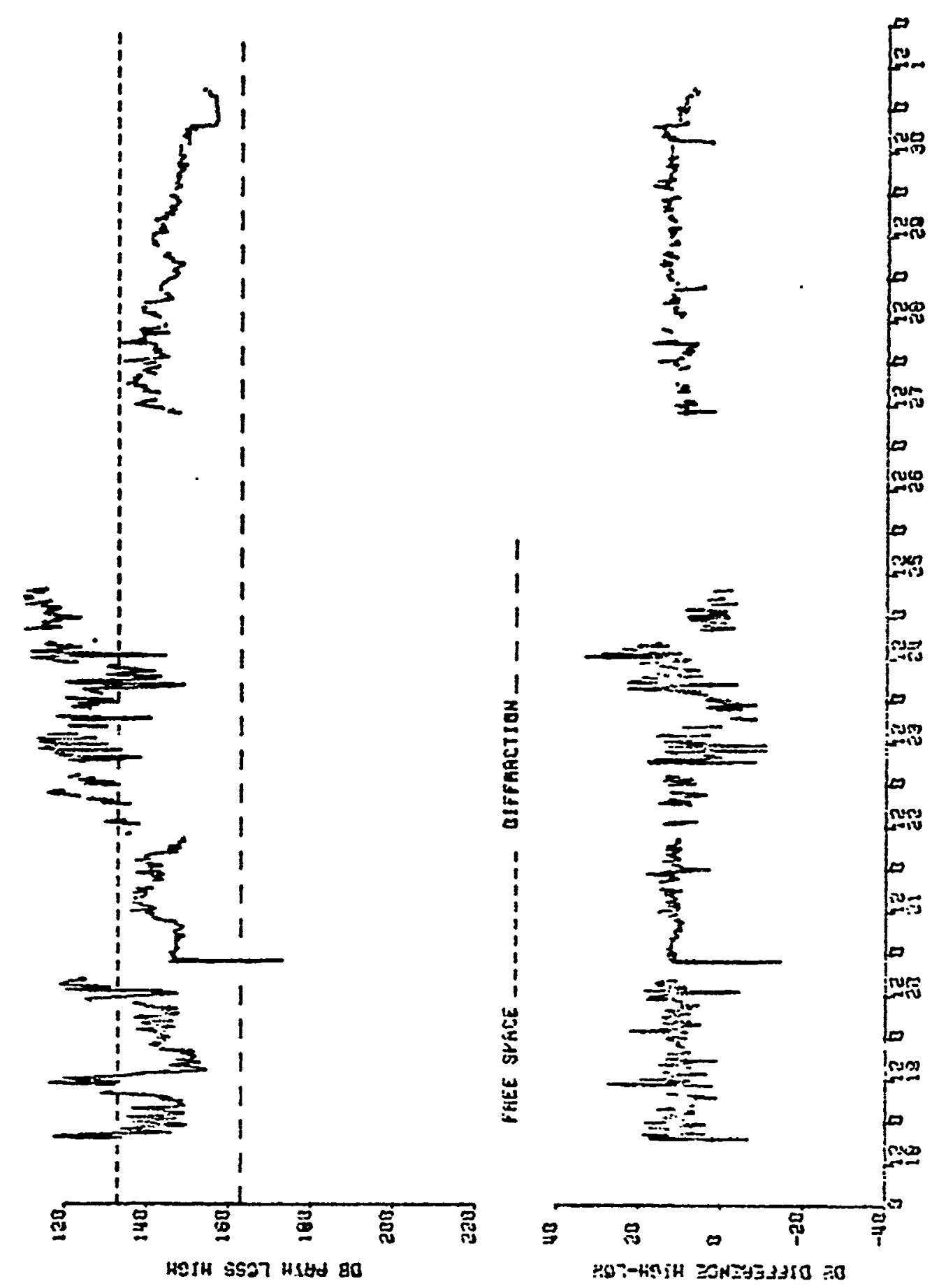

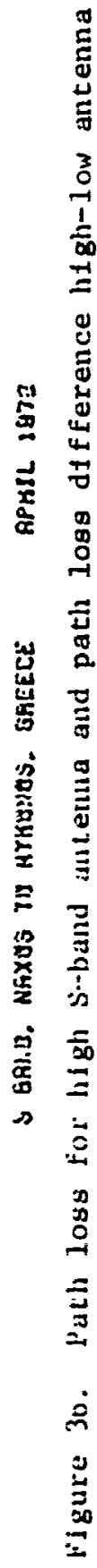



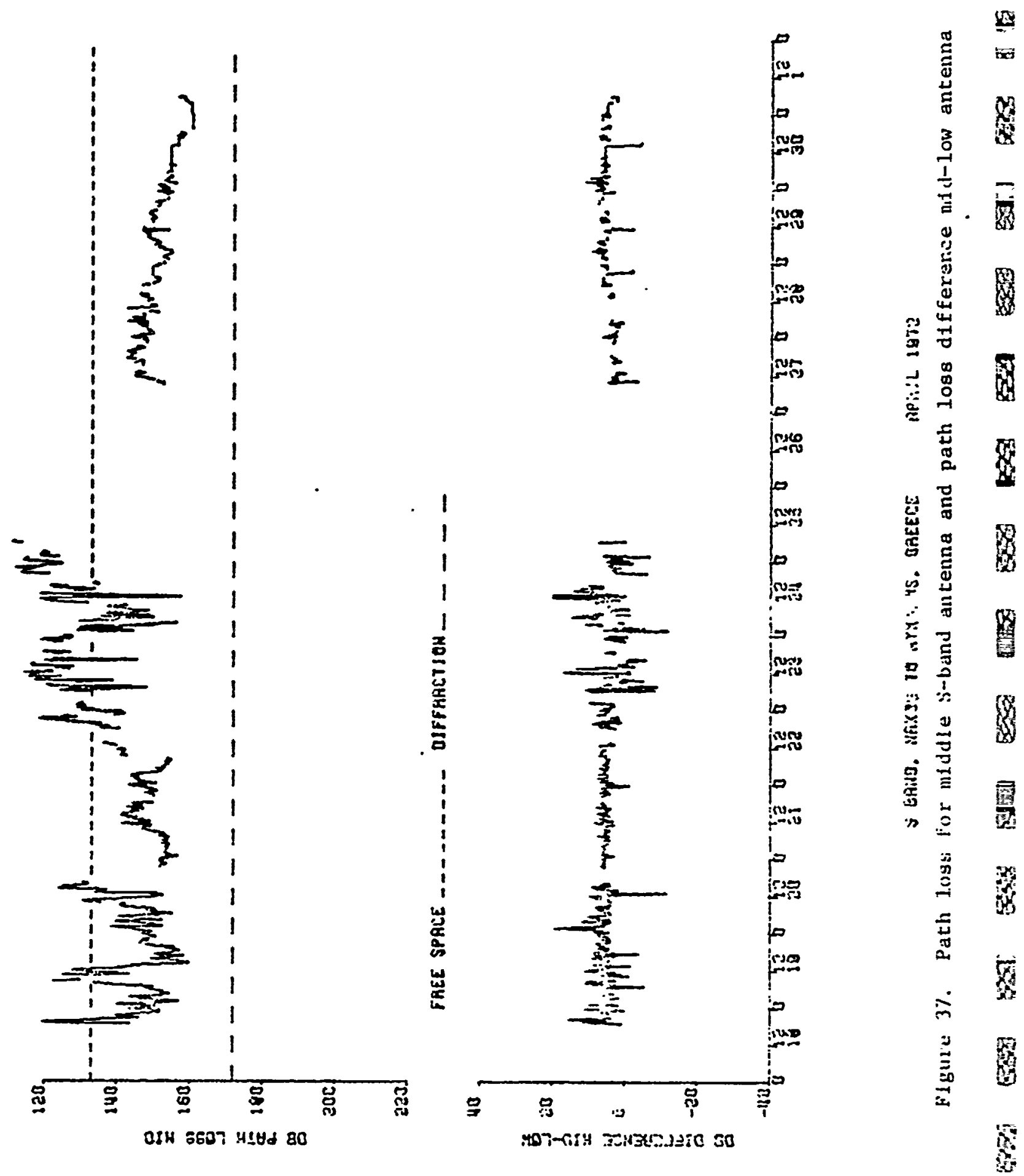

8

bo

$\mathbf{M}$

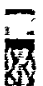

9

8

8

8

8



翌

w

8

$B$

8



$N$ 

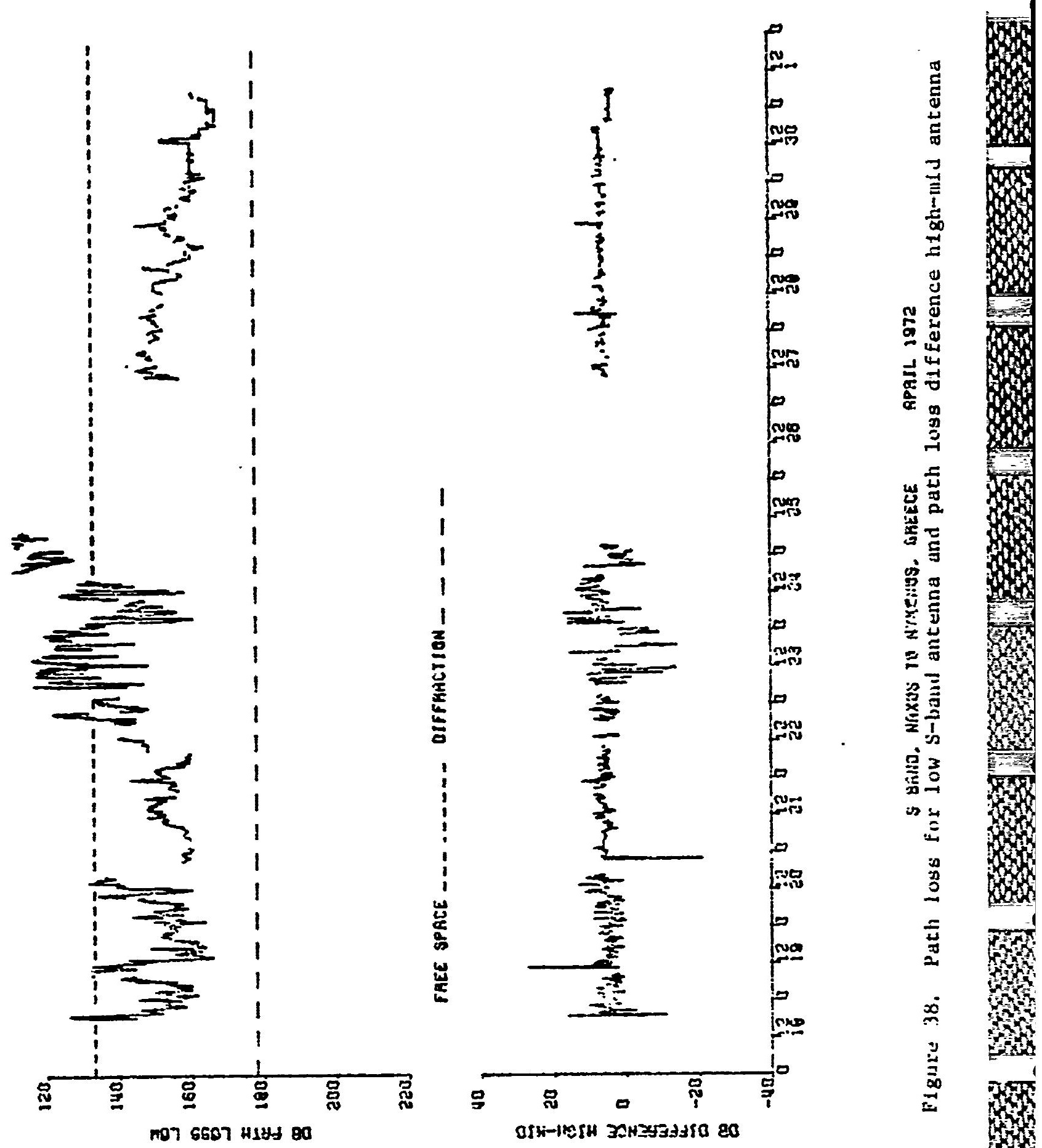

MN

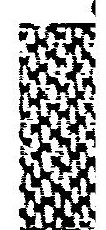





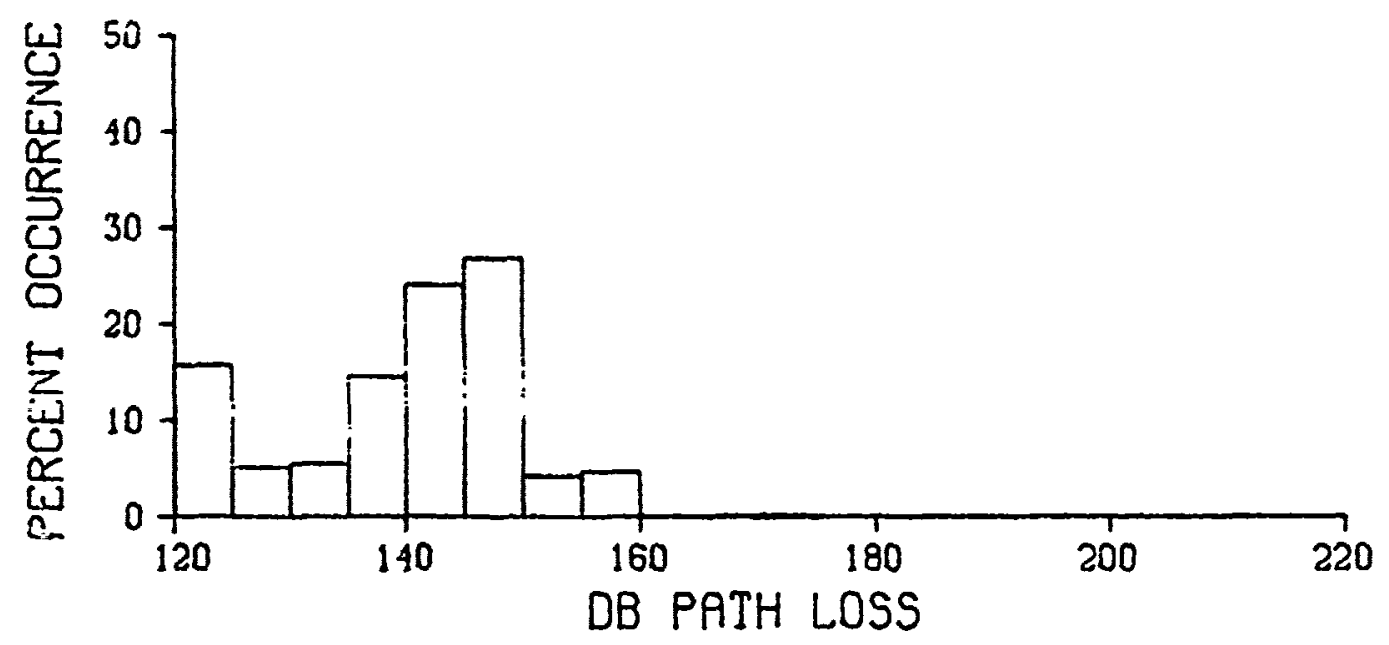

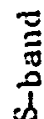

HIGH ANTENNA

786 OBSERVATIONS

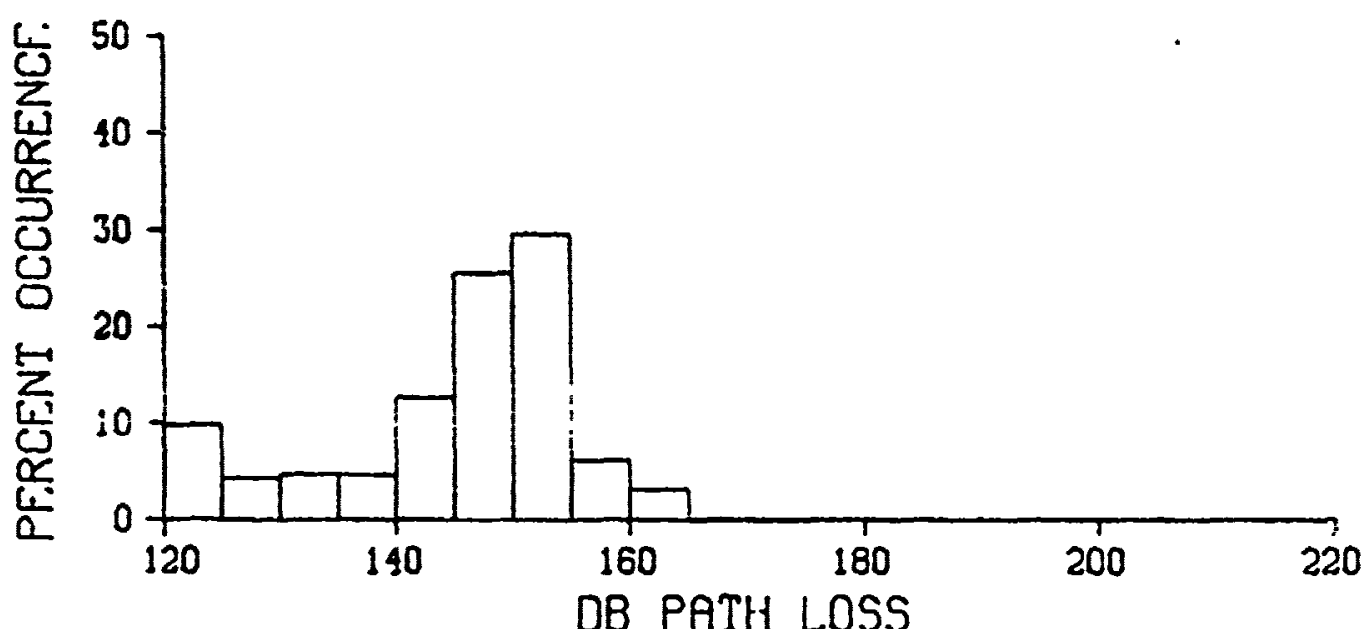

MID ANTENNA

77a GBSERVATITUIVS

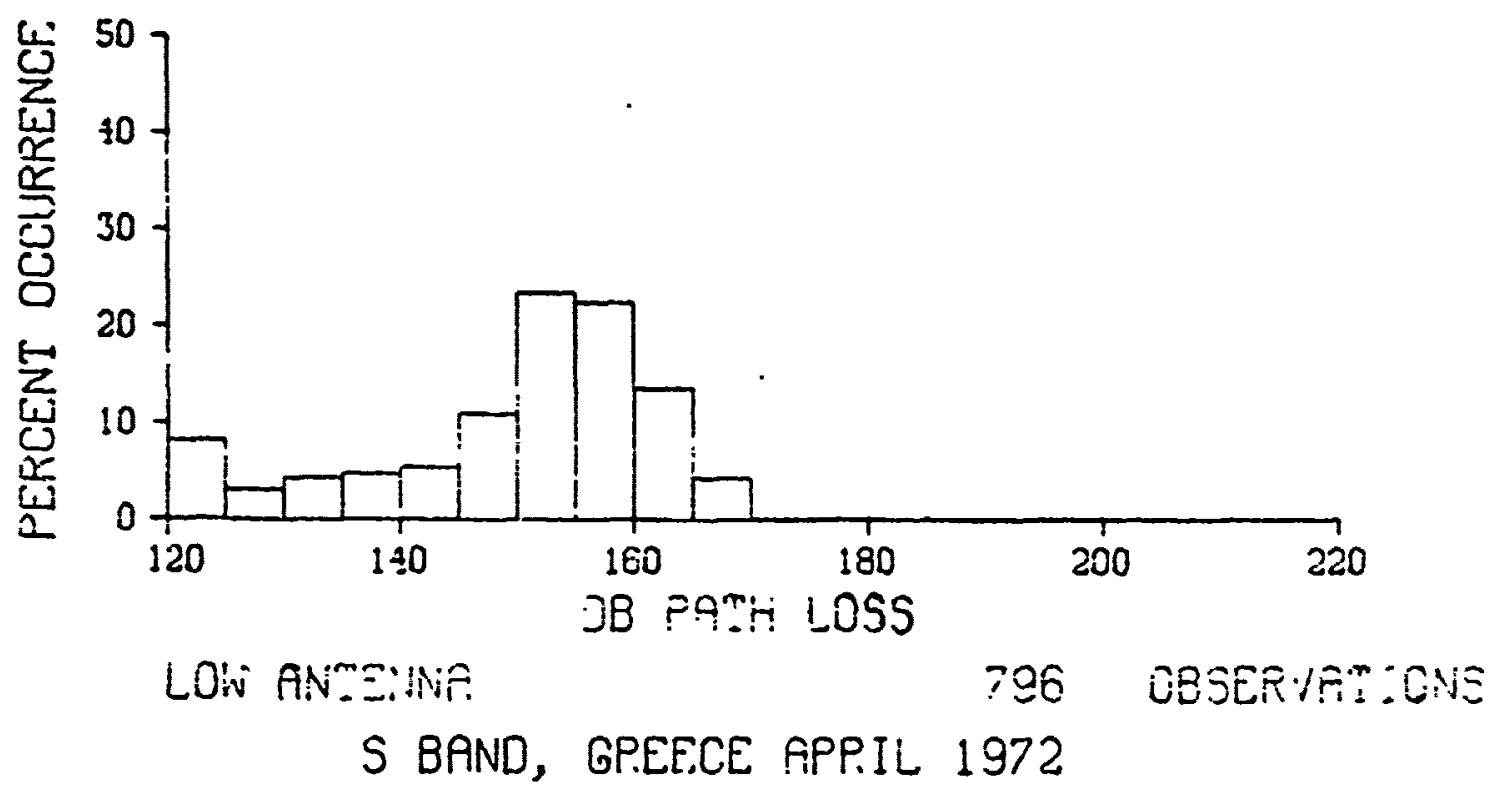




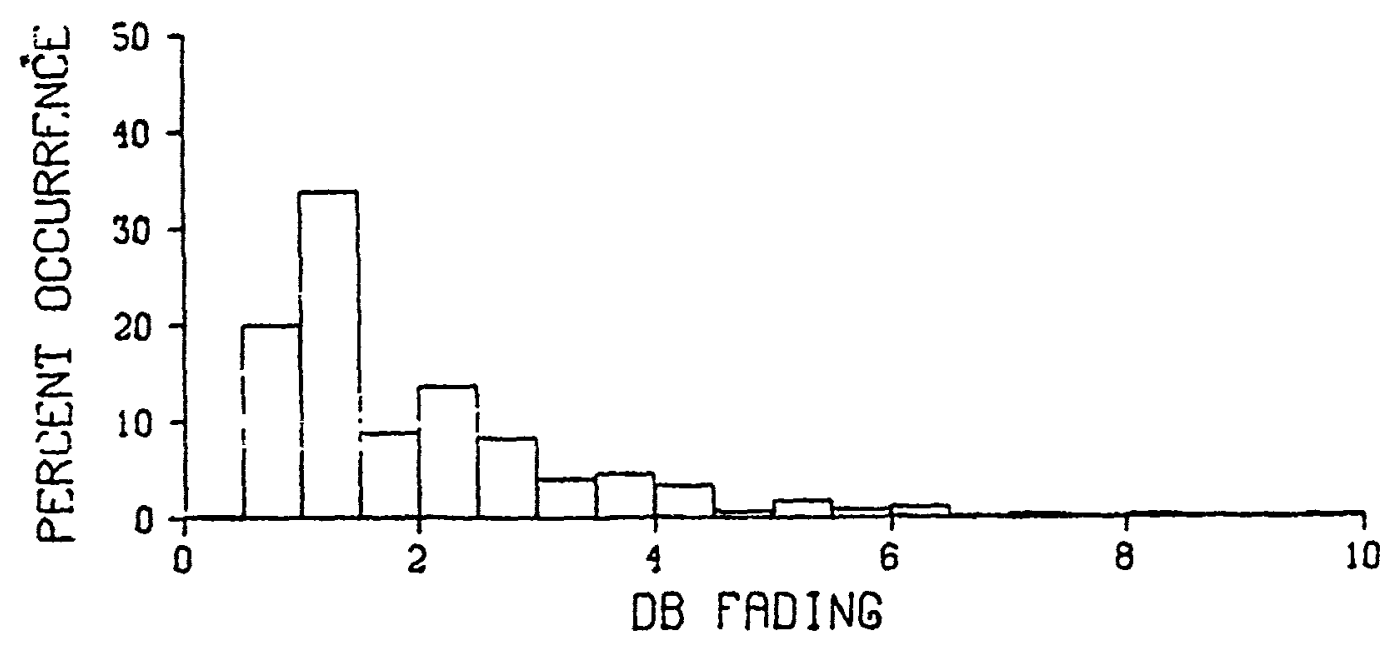

HIGH ANTENNA

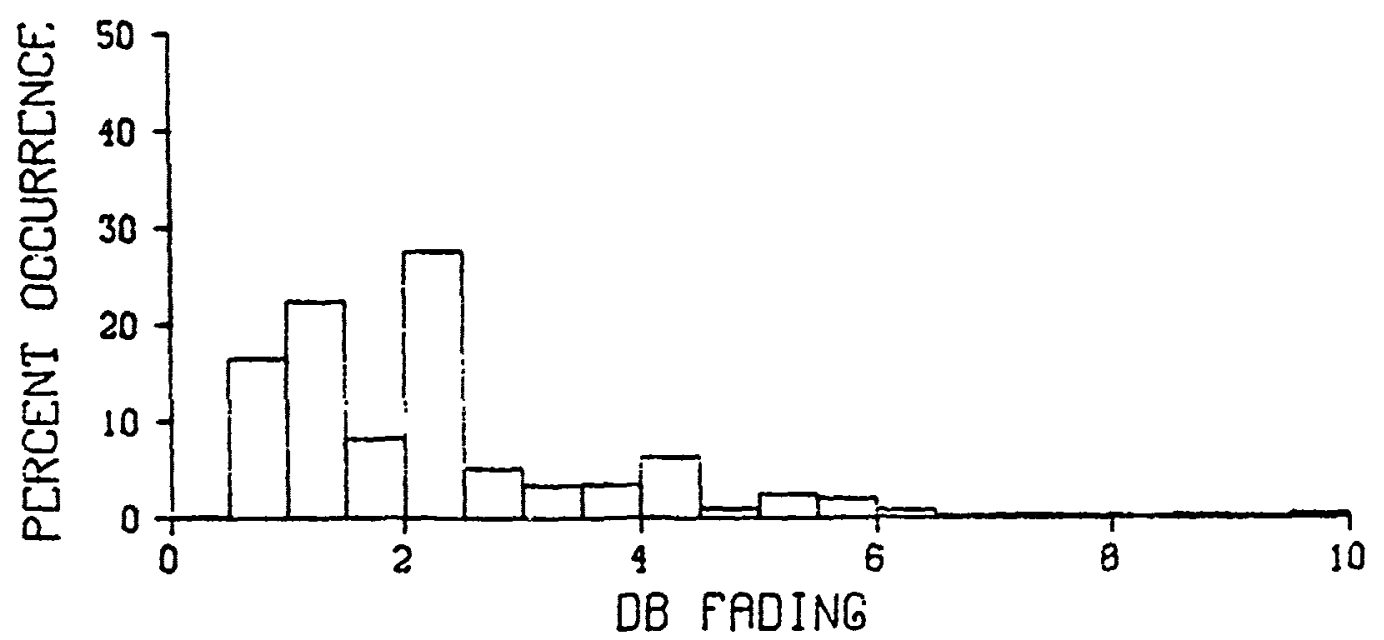

MID ANTENNR
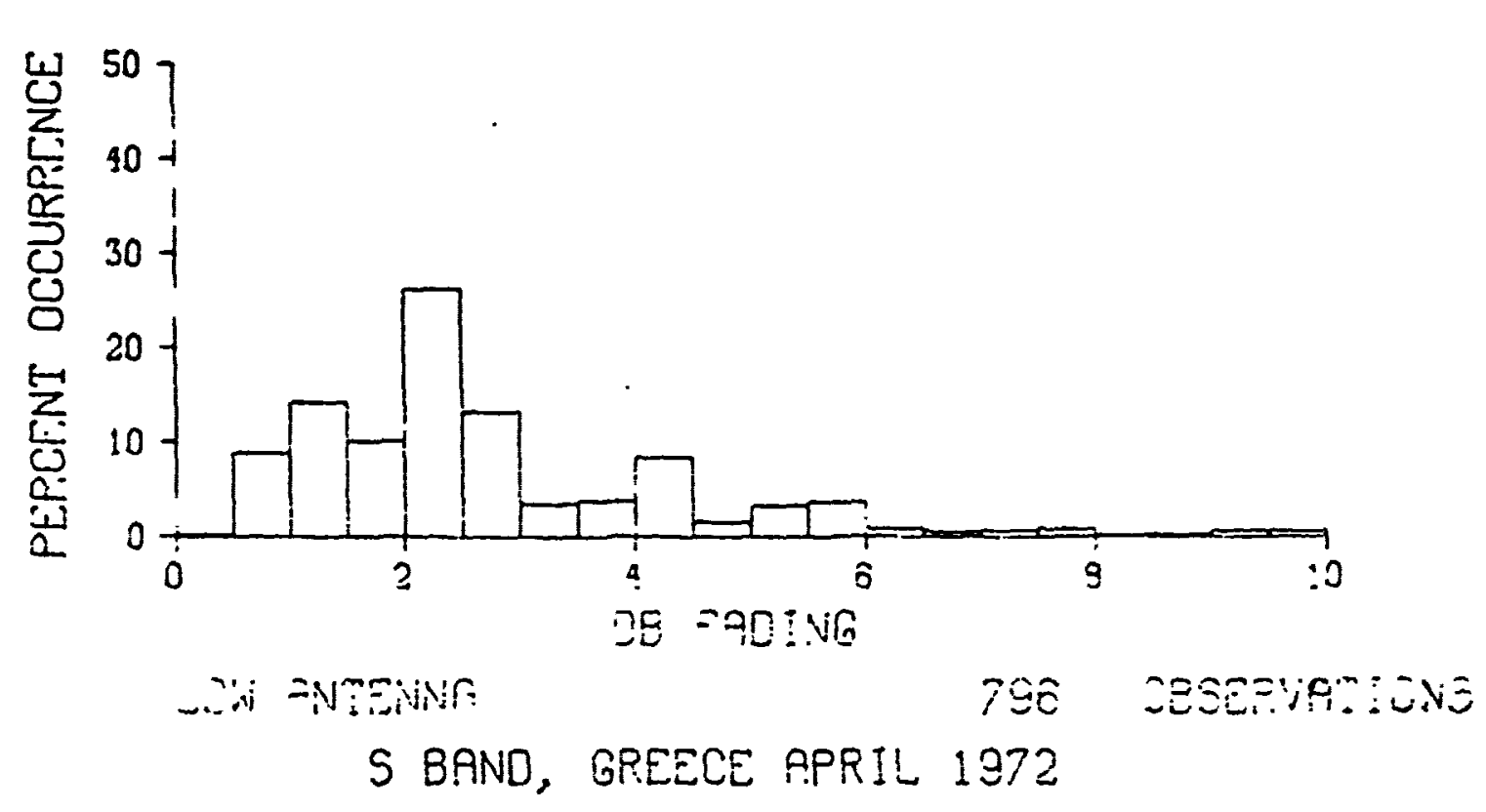


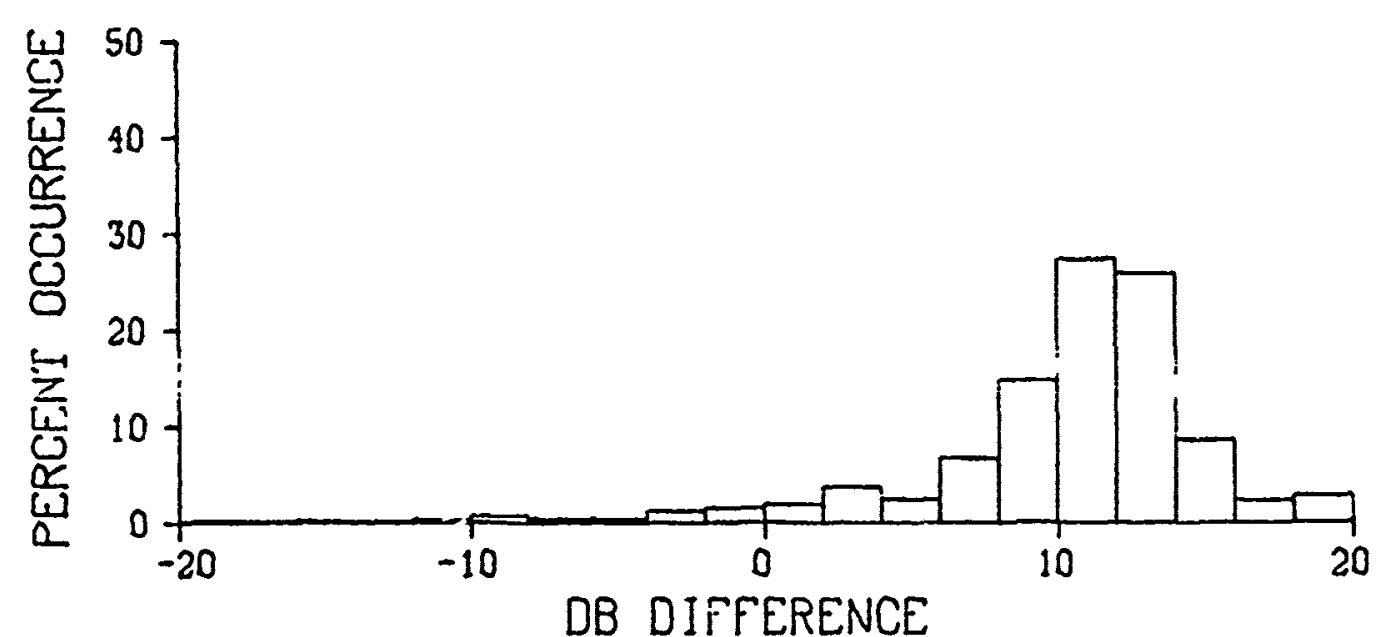

HIGH-LOW

706 OBSERVATIONS

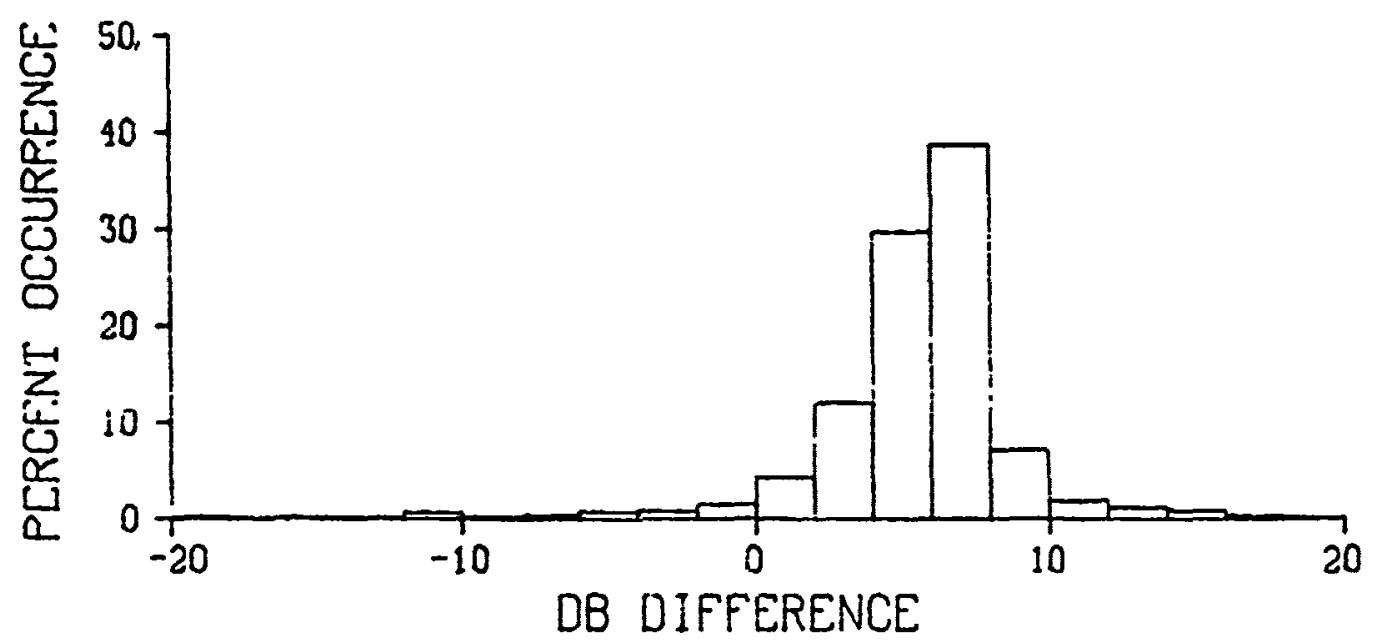

HIGH-MID

691 OBSEPVYTIIONS

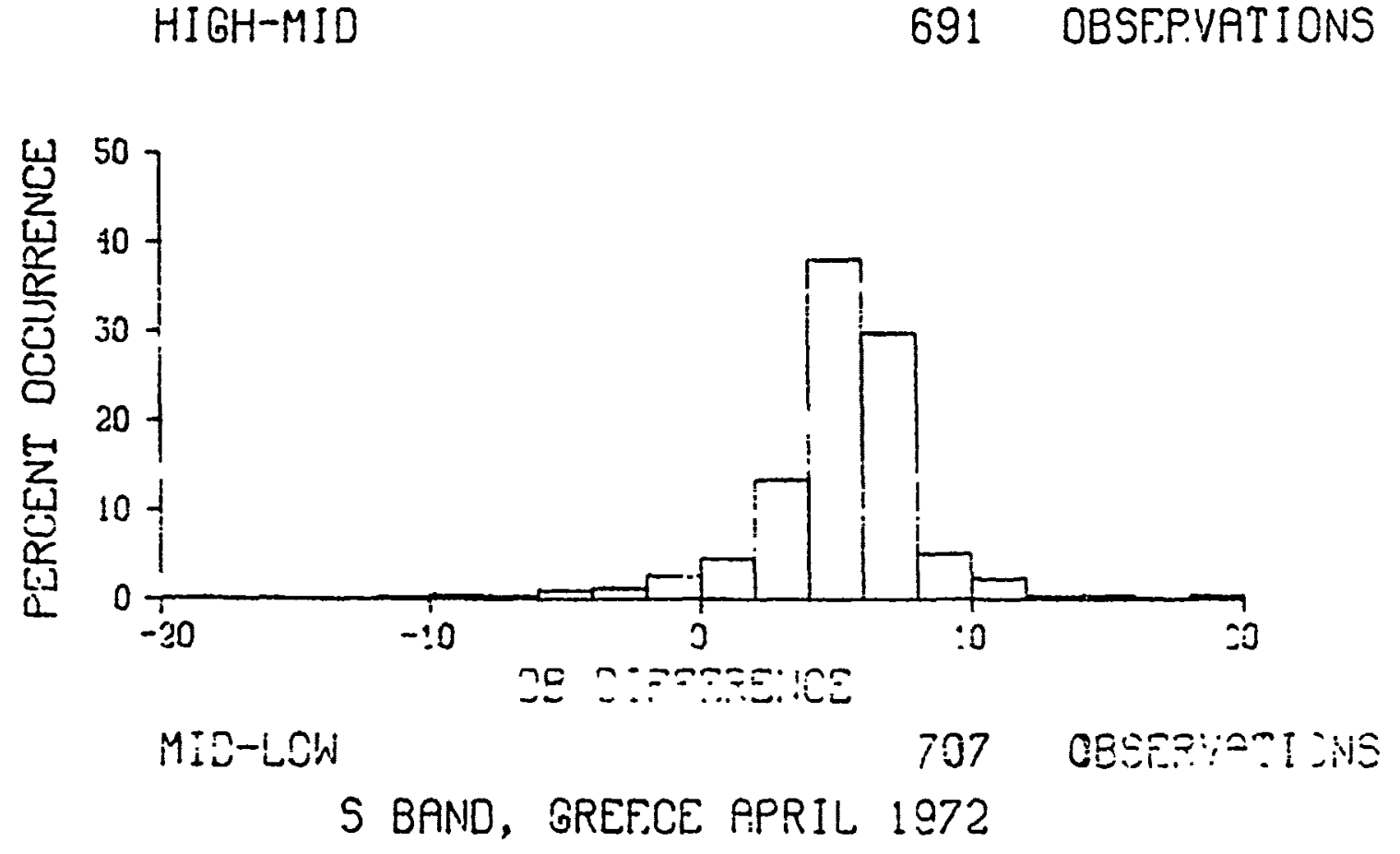



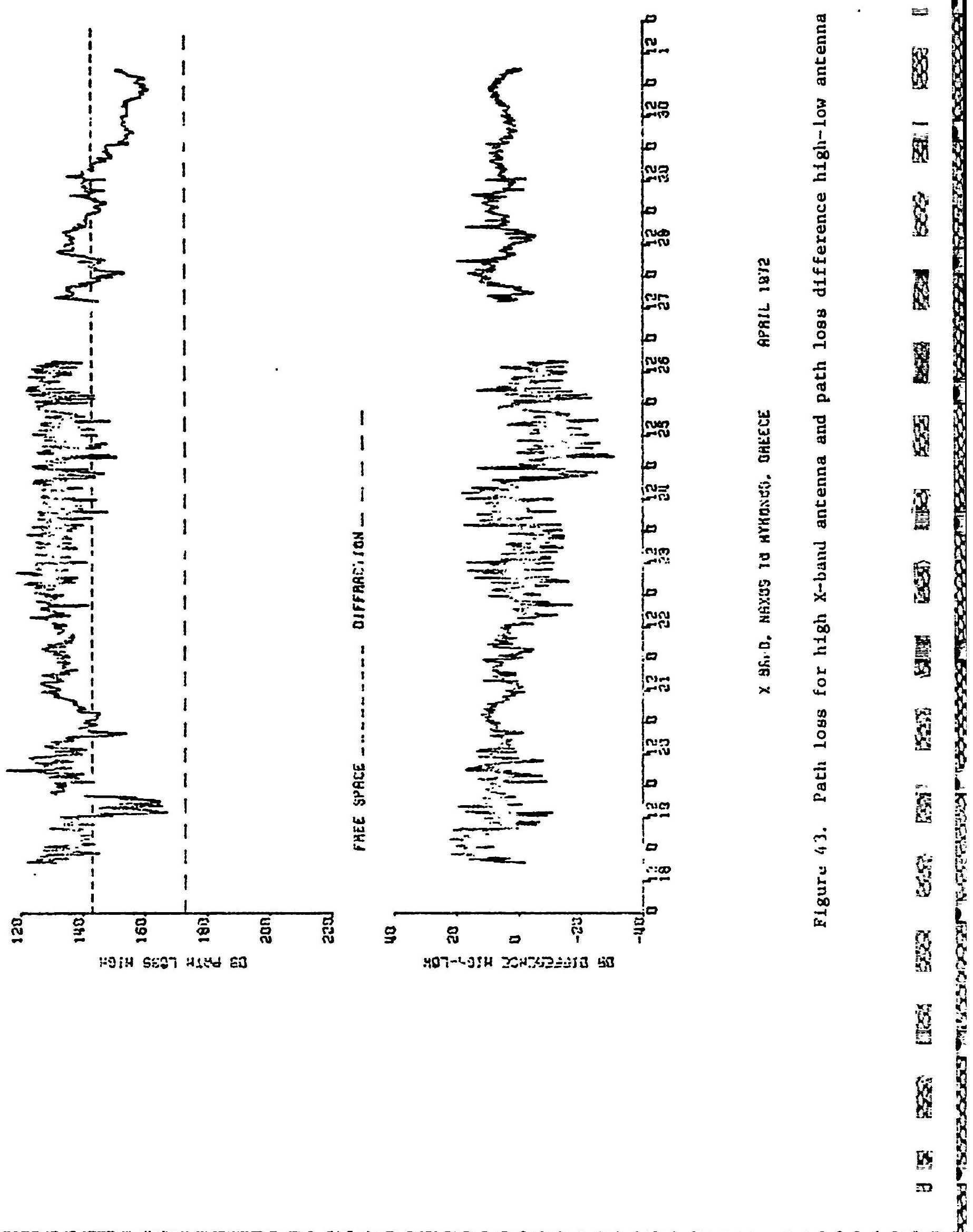
8

3

a

B

80

8

9

8

?
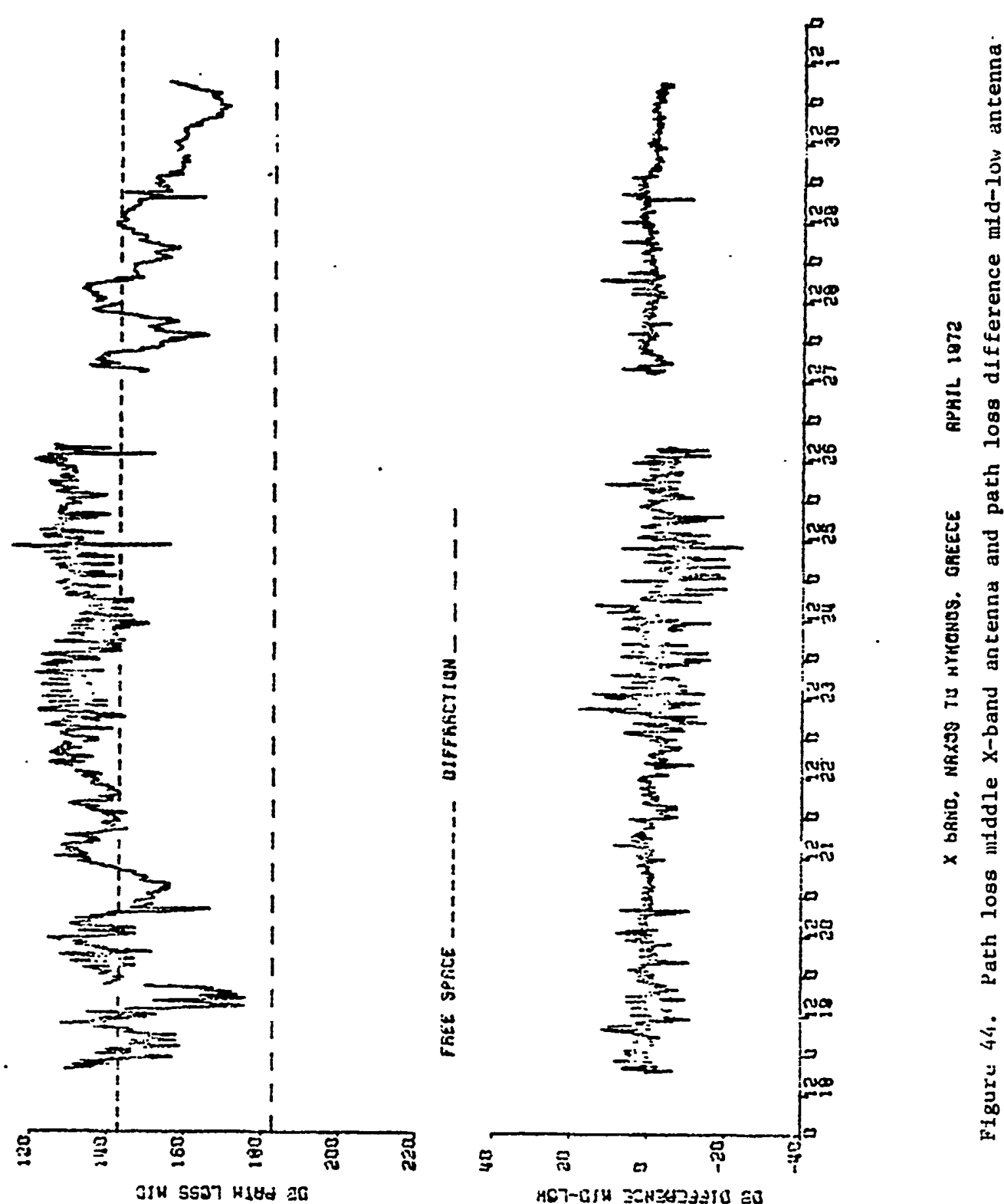

$\mathbb{B}$

8

st

3

疍

嘼

8

3

3

3

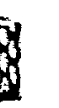

9

?

8

8

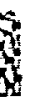

งสน Sรมา K1

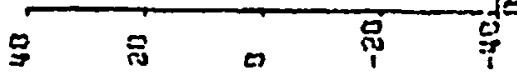



8

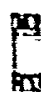



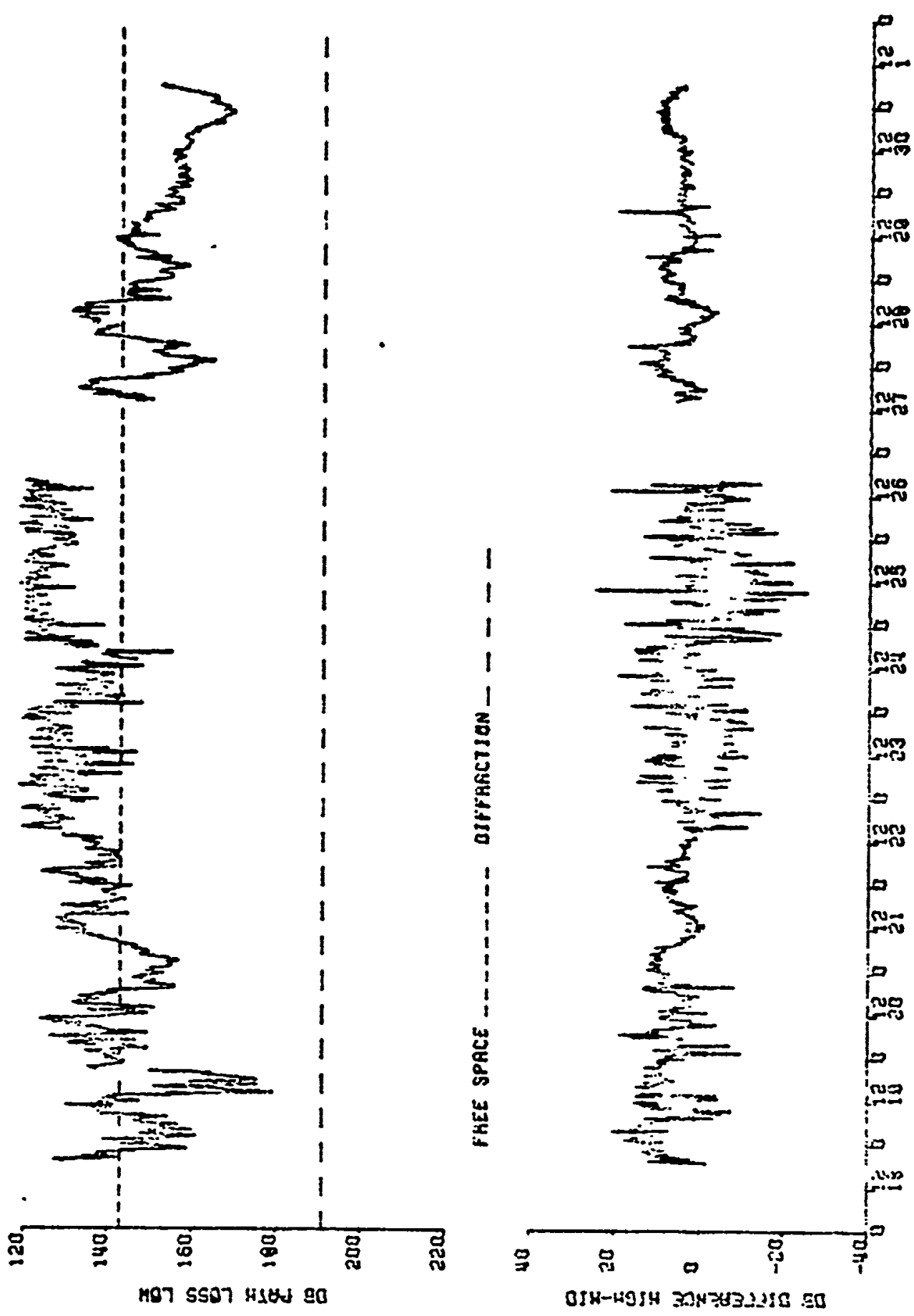

DI 


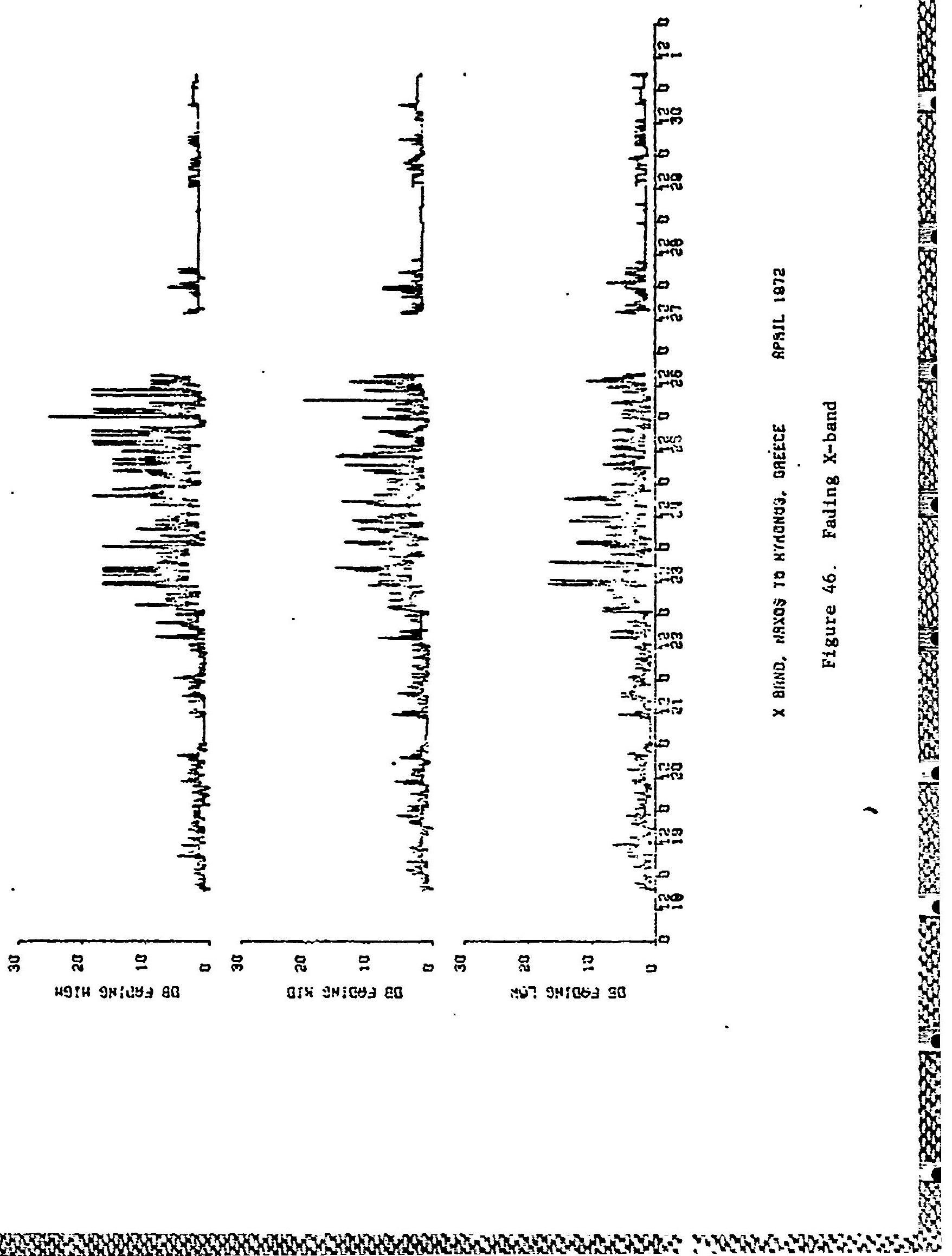


8

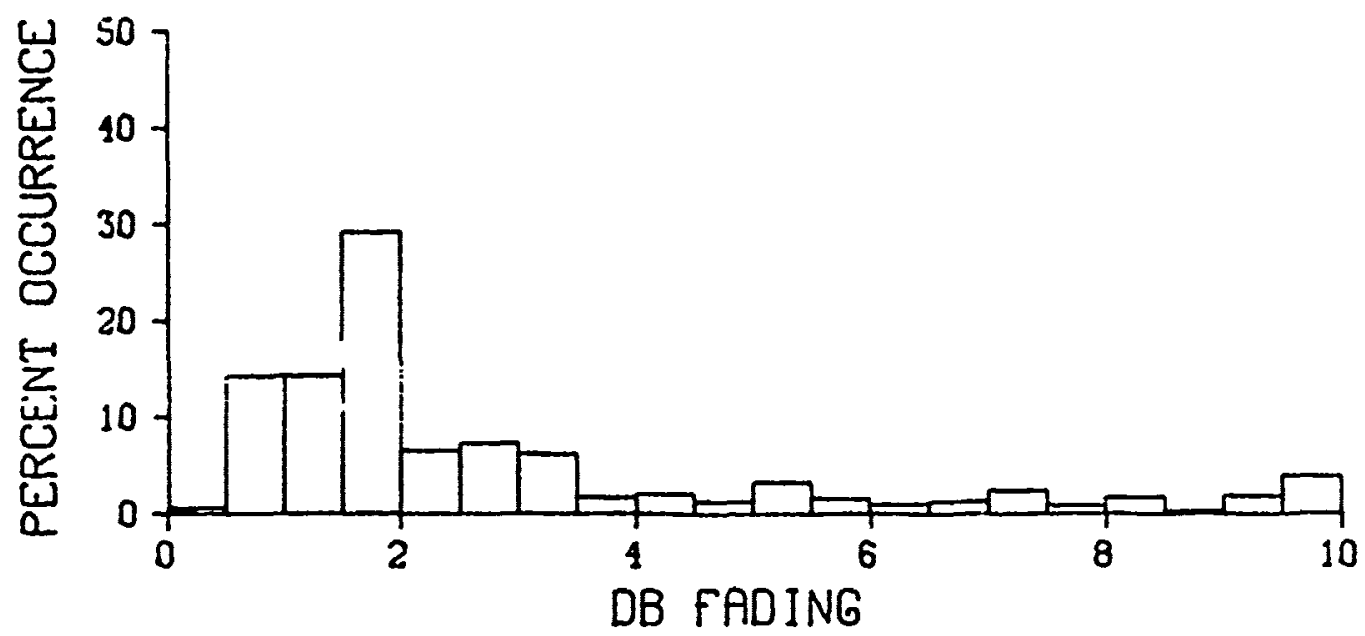

HIGH ANTENNA

1069 OBSERVATIONS

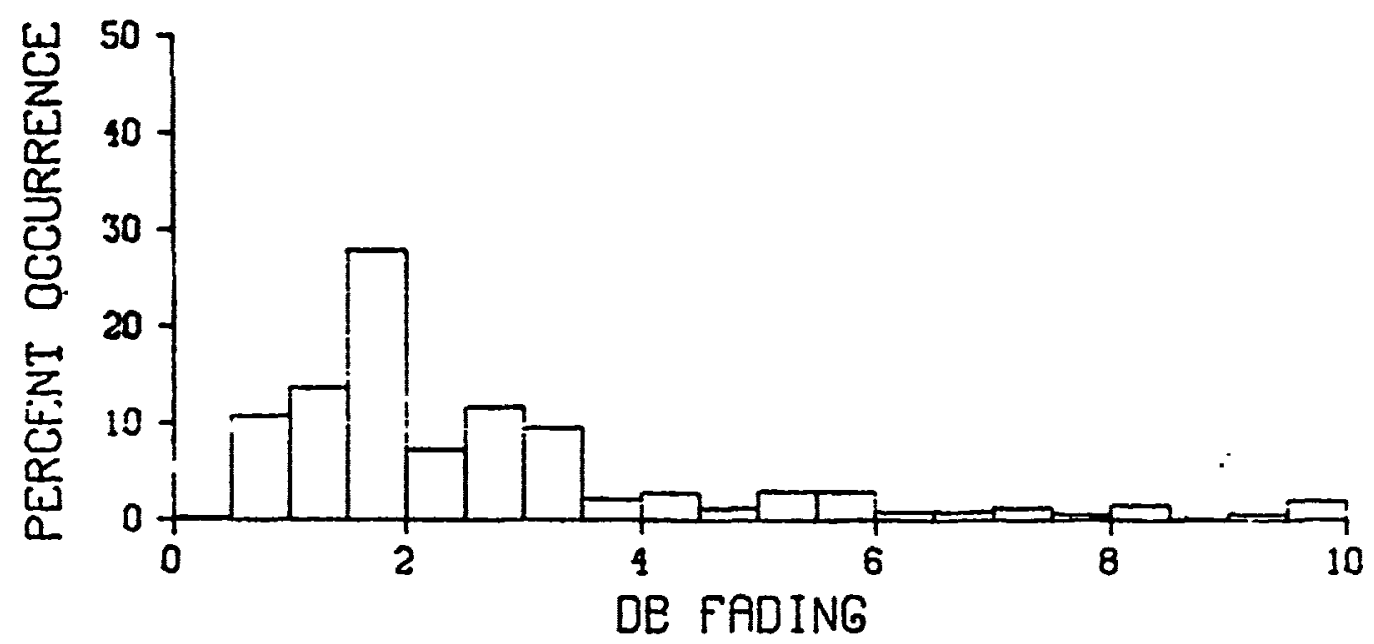

MID ANTENNF

¿OT1 OBSERVVTIONS

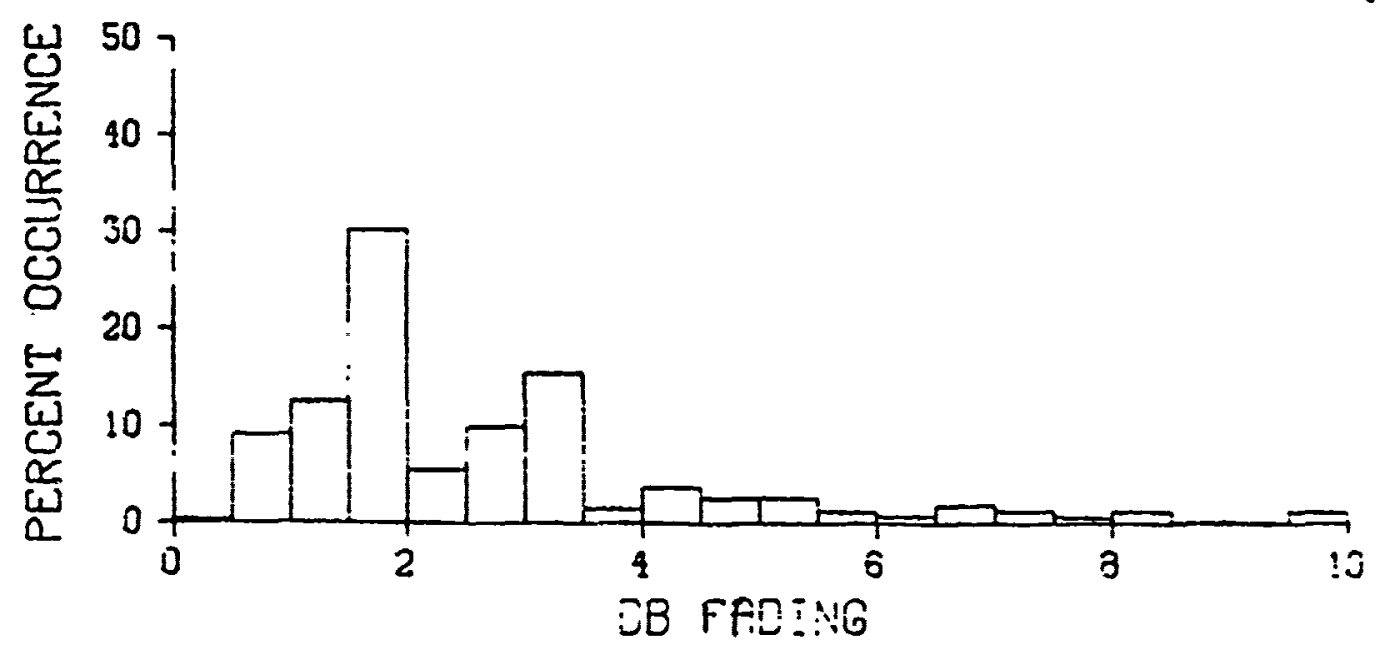

- Uื

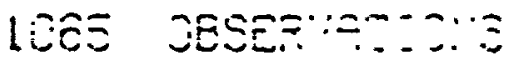

$X$ BAND, GREECE APRIL 1972 

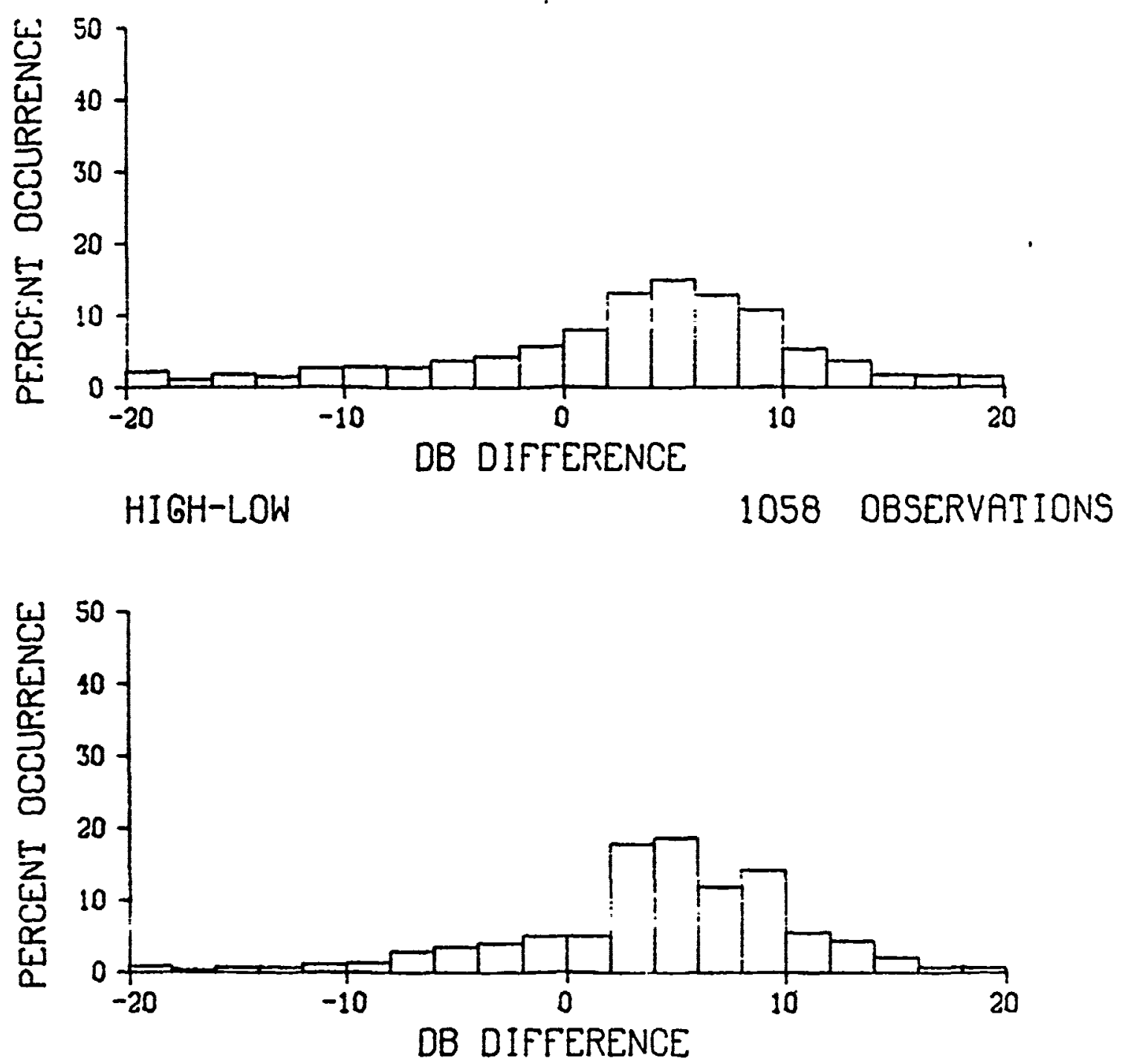

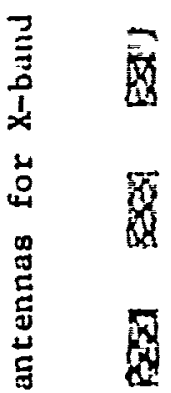

HIGH-MID

1065 OBSERVATIONS

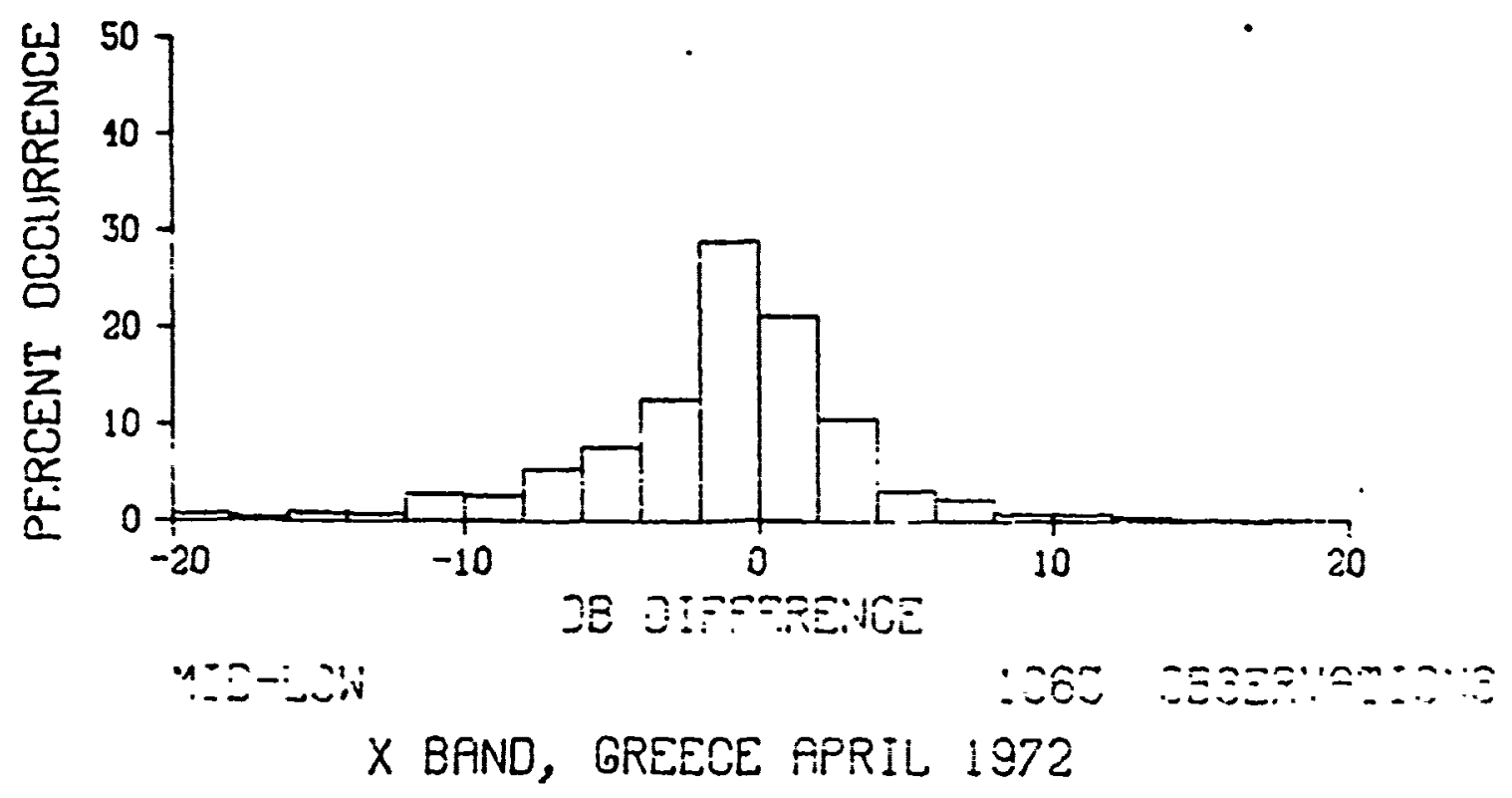

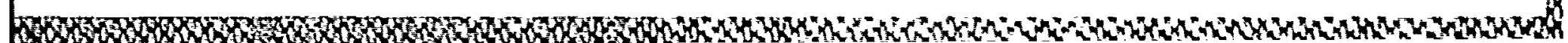



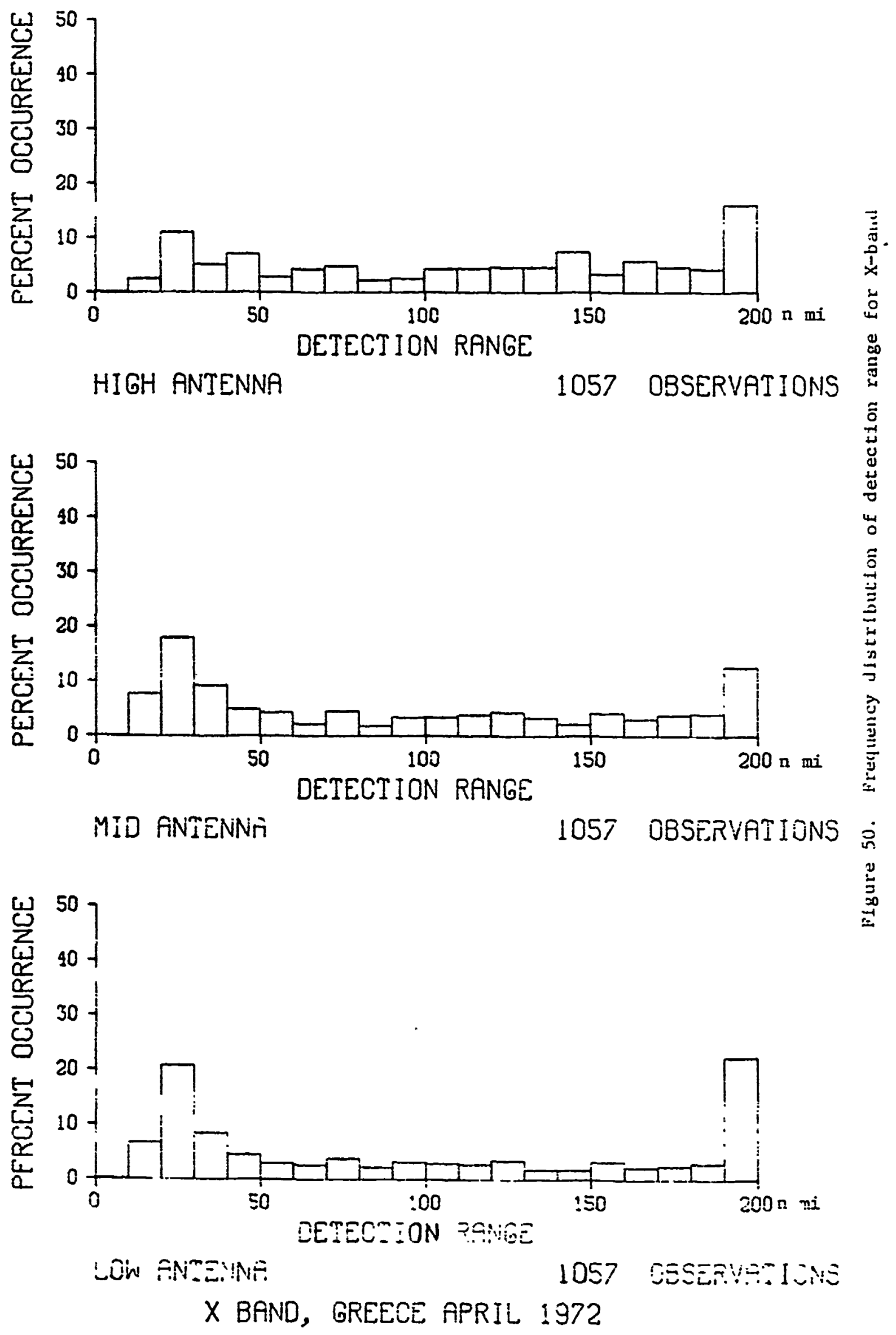

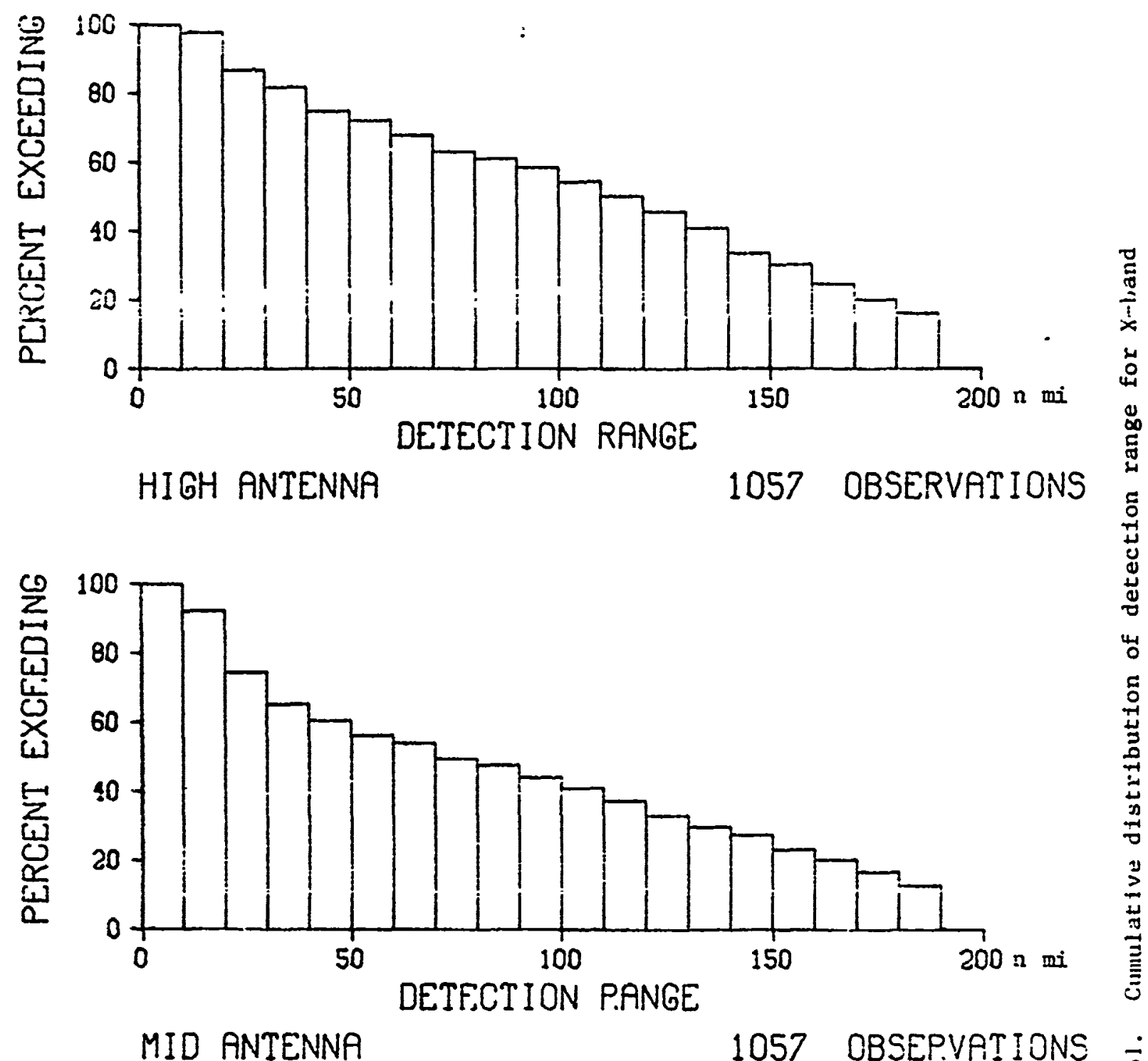

1057 OBSEPVATIIONS $\dot{\bar{r}}$

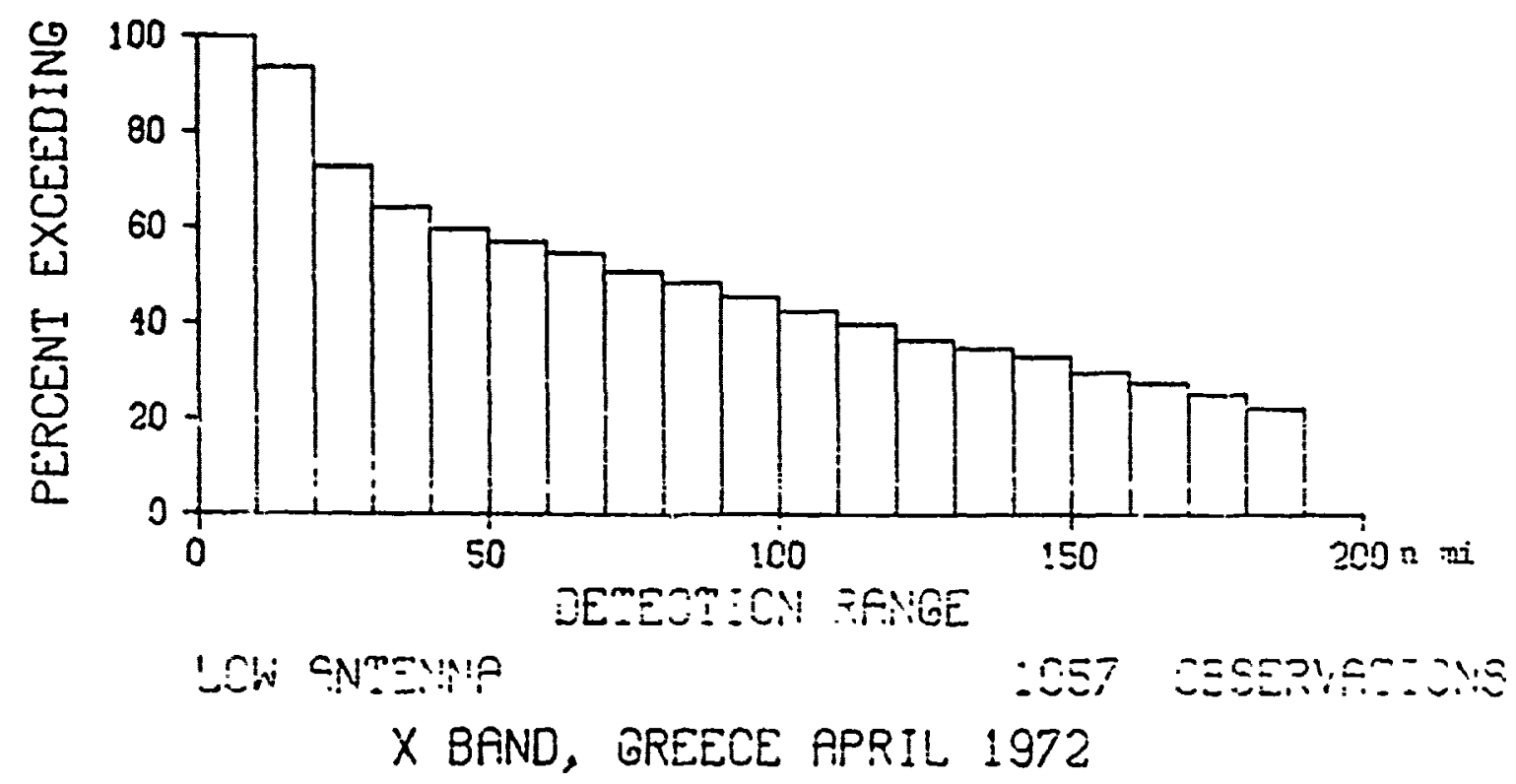



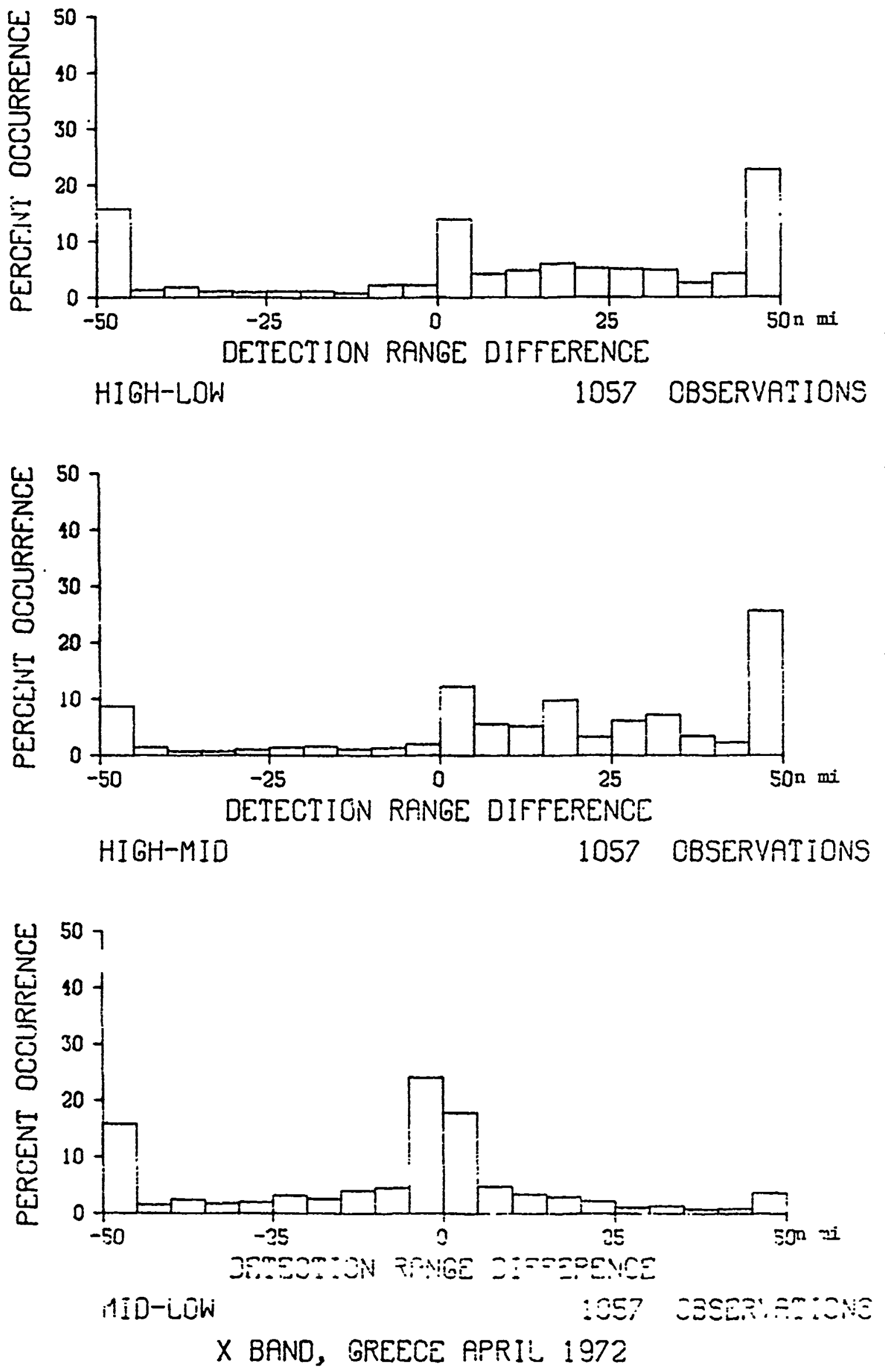

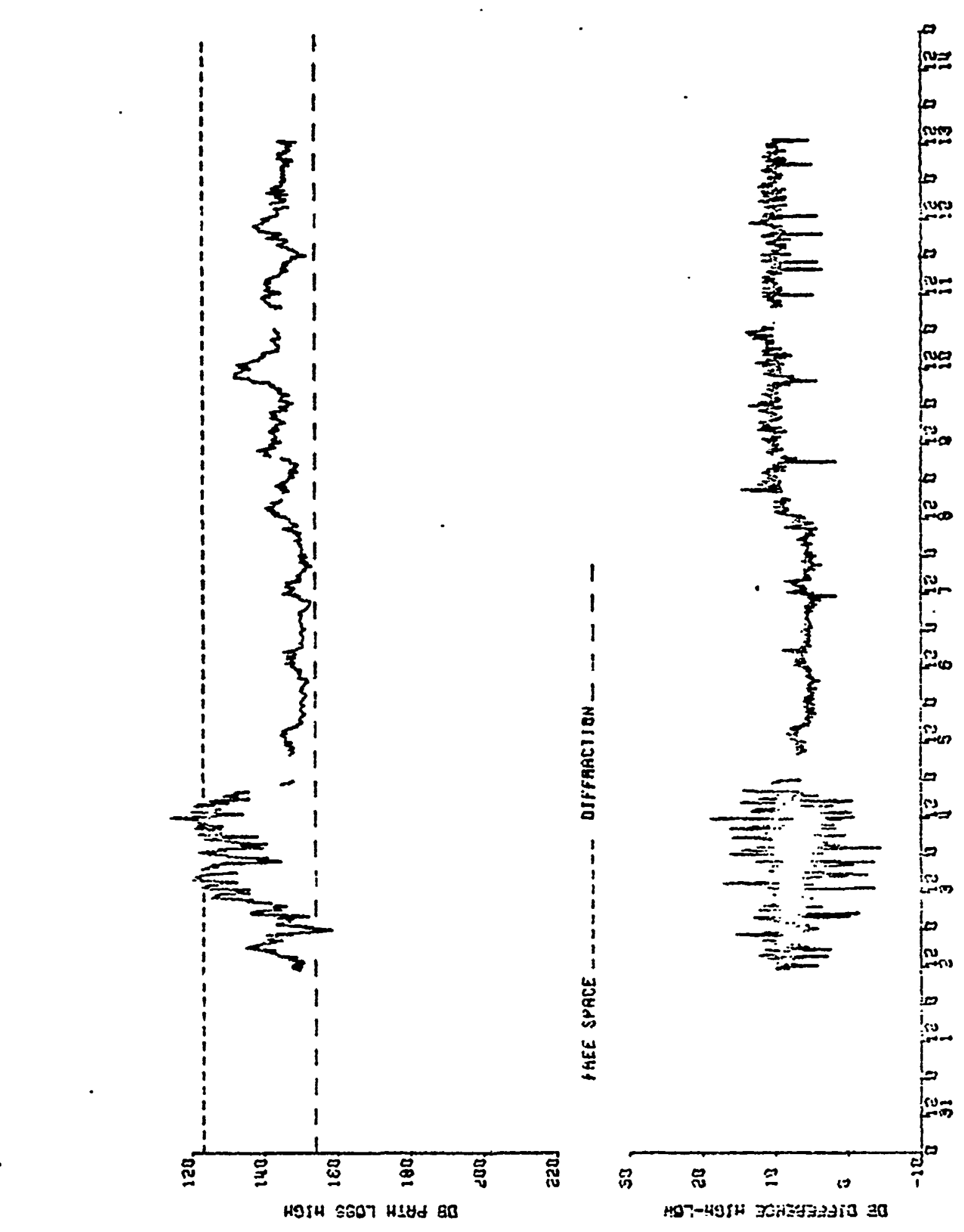

8

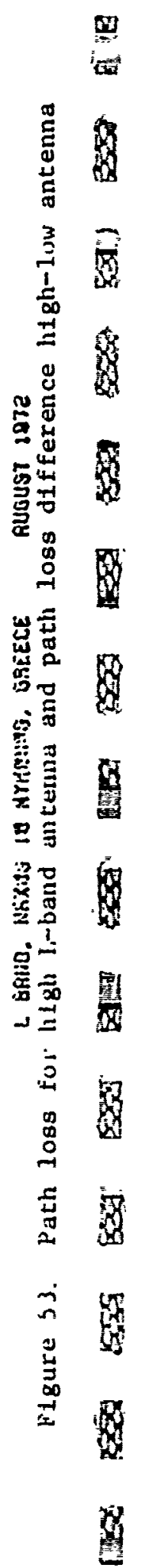

i

0

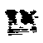

n

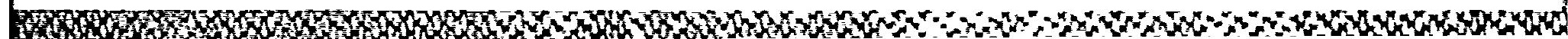



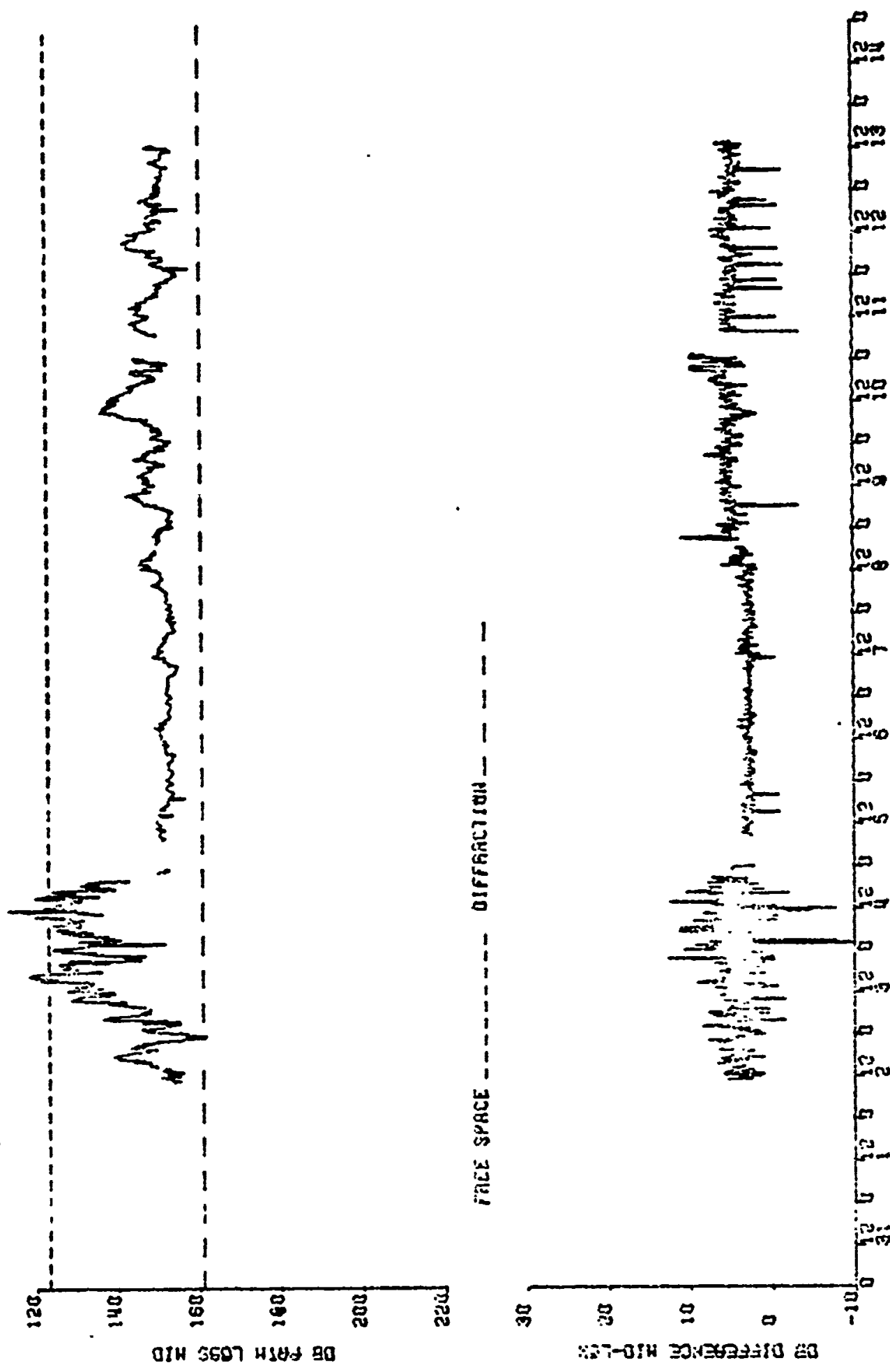

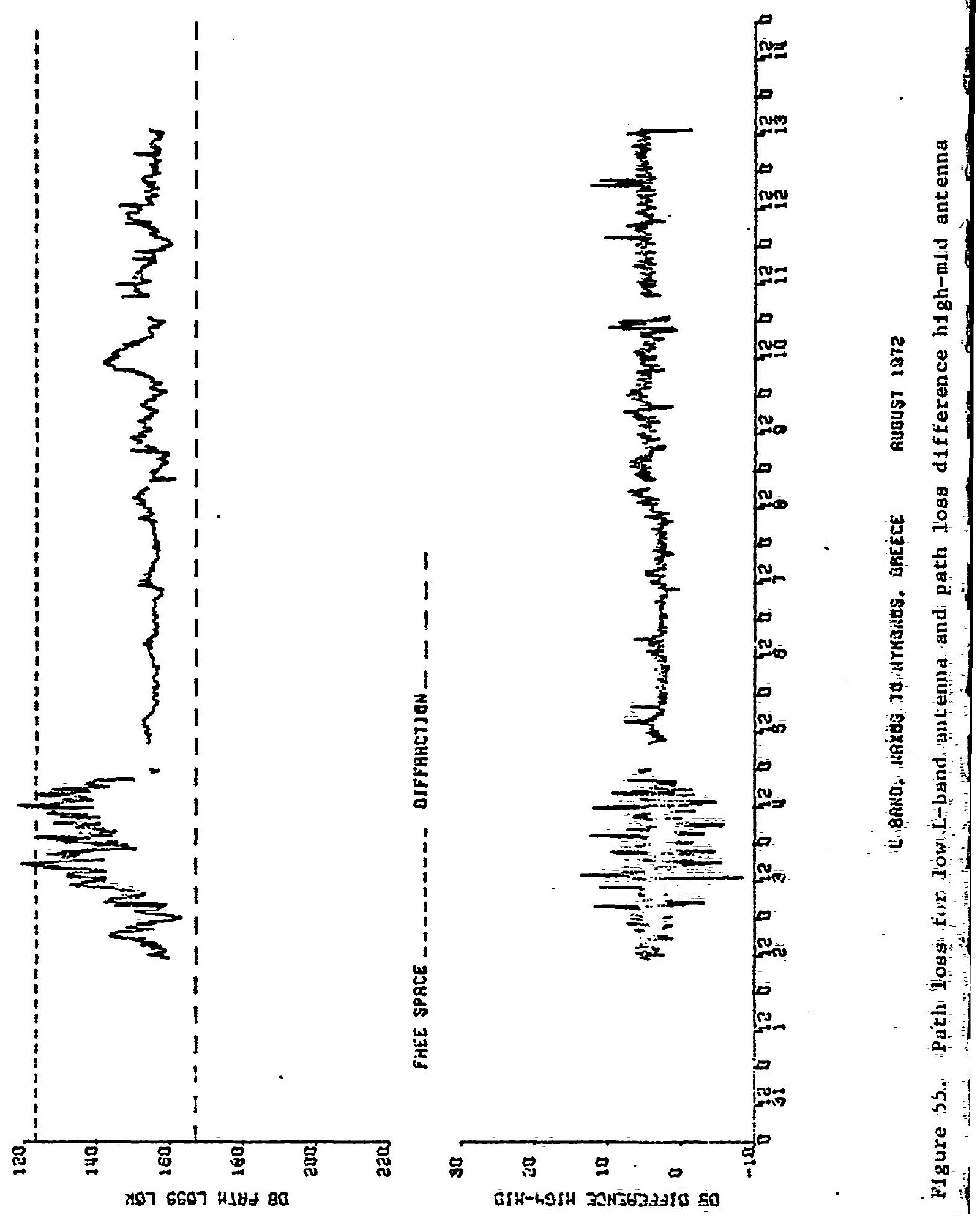


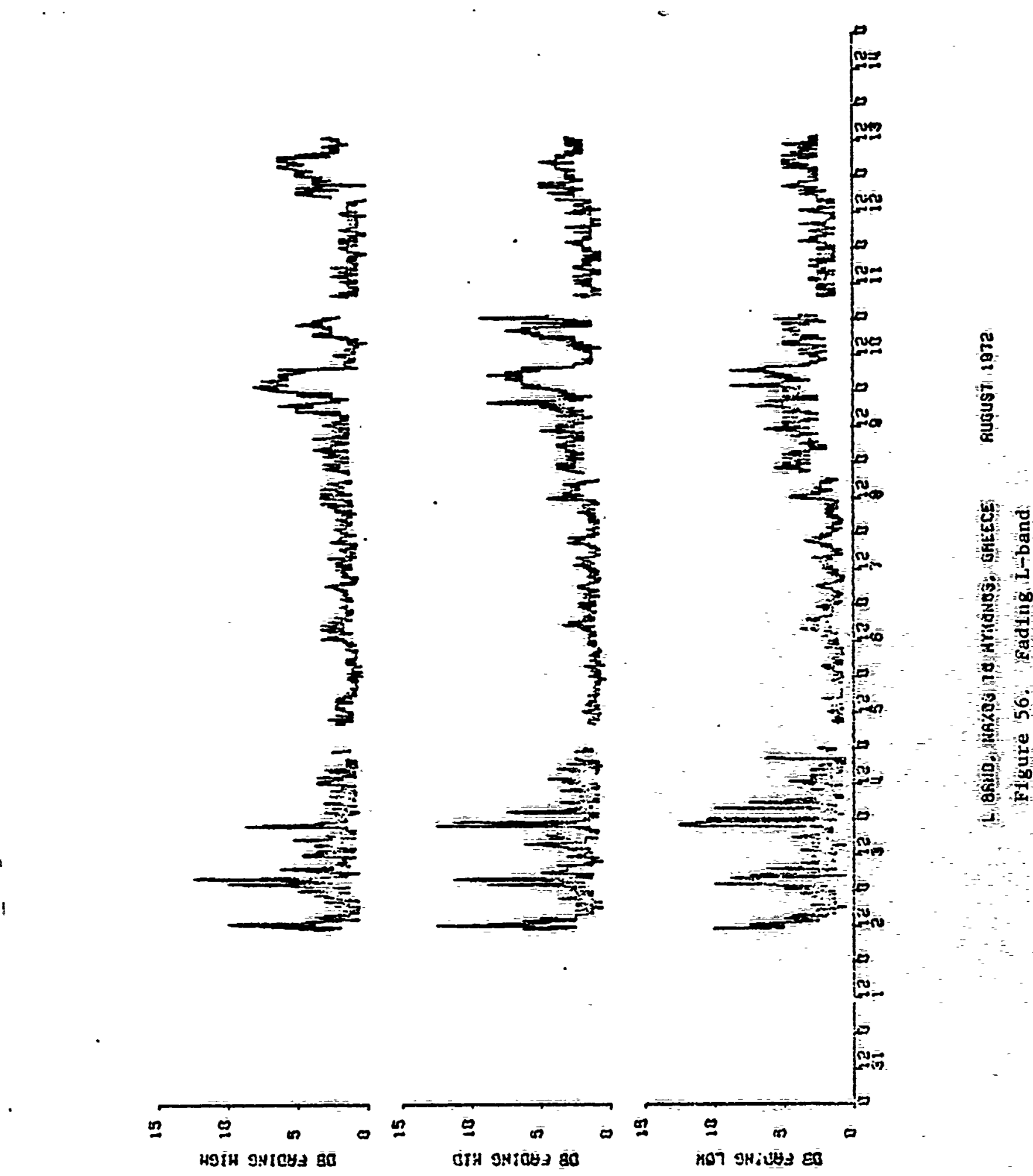



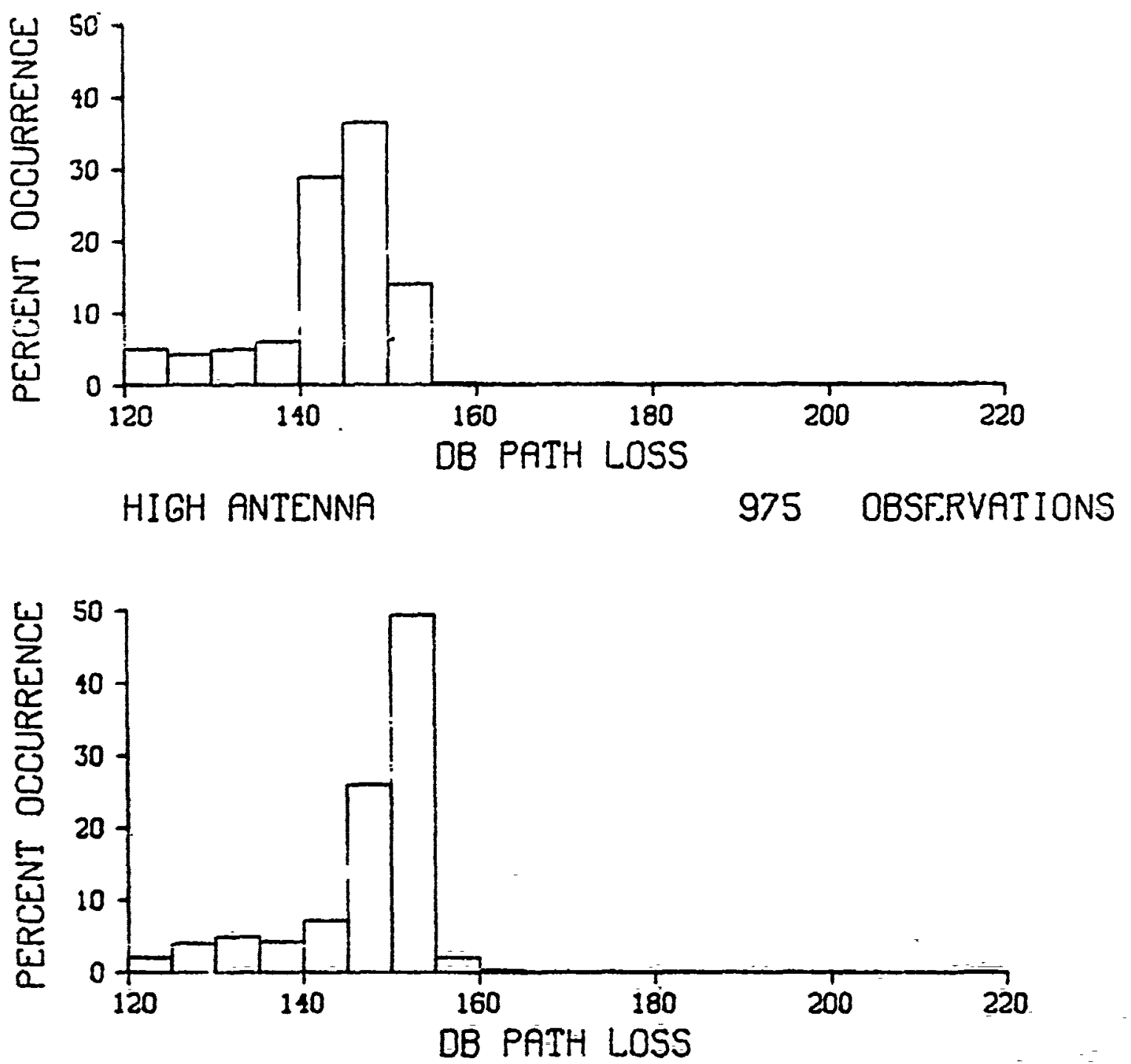

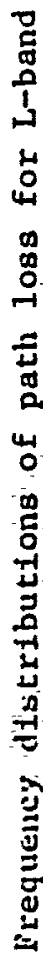

MID ANTENNA

975 OBSERVATIONS iิ

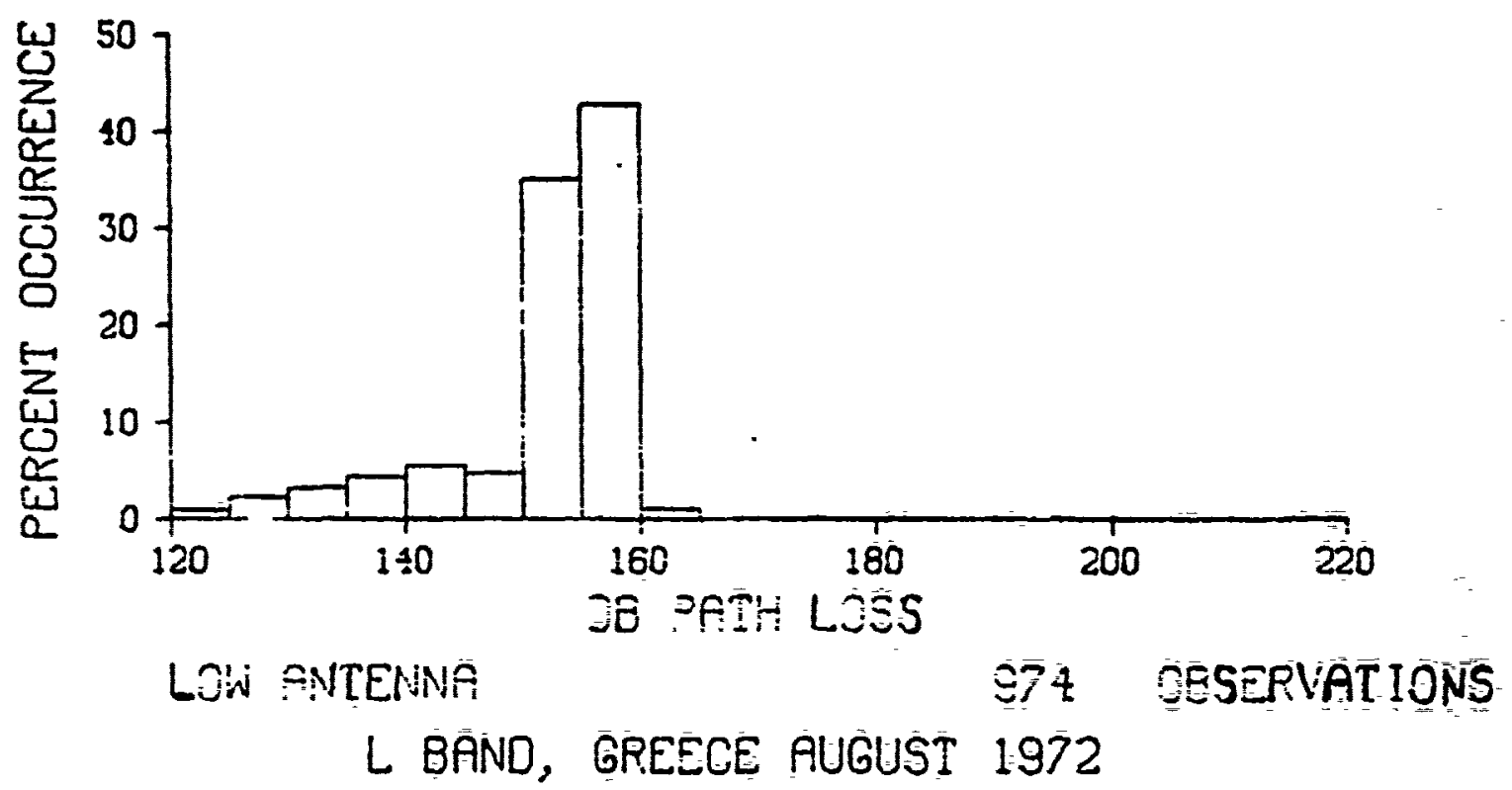




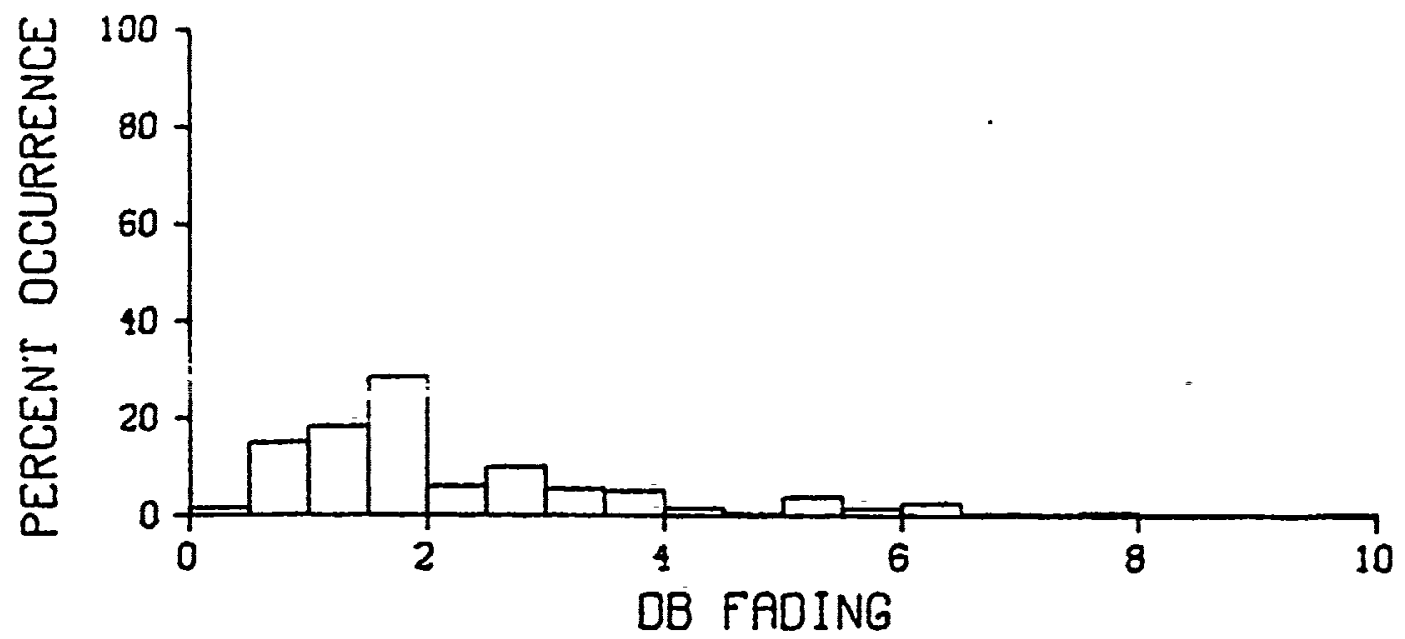

HIGH ANTENNA

975 OBSERVATIONS
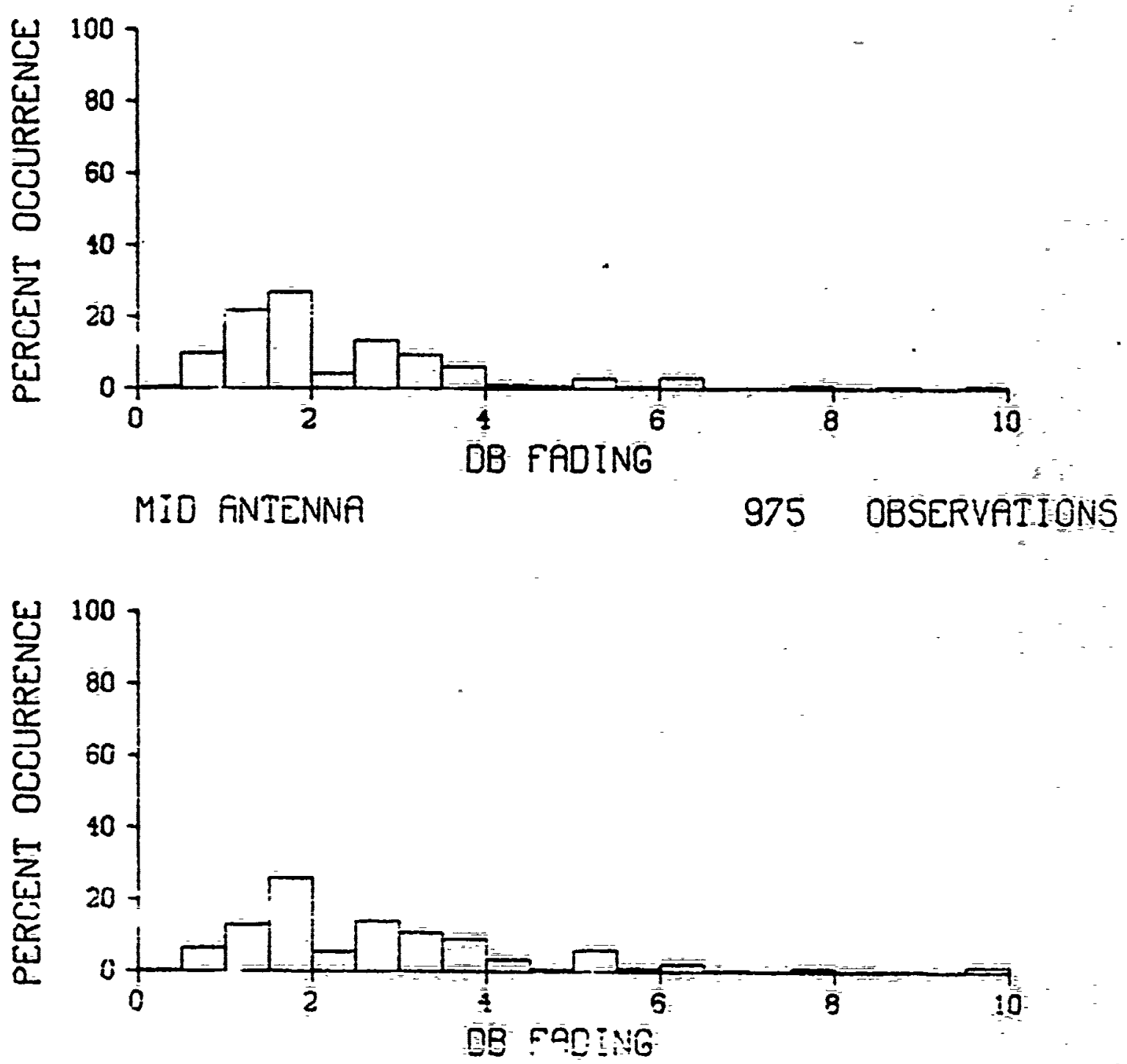

IIN BNTENAF

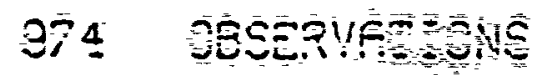

L BAND, GREECE AUGUST 1972 


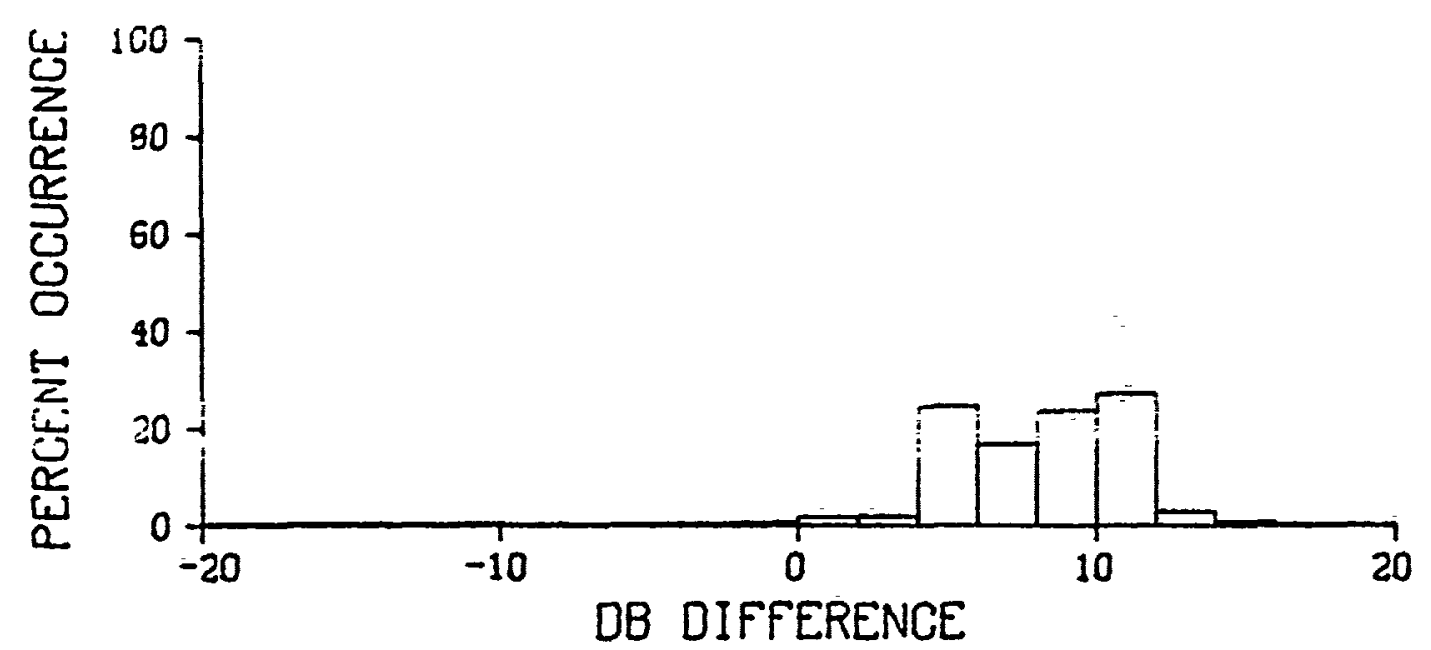

HIGH-LOW

968 OBSERVATIONS

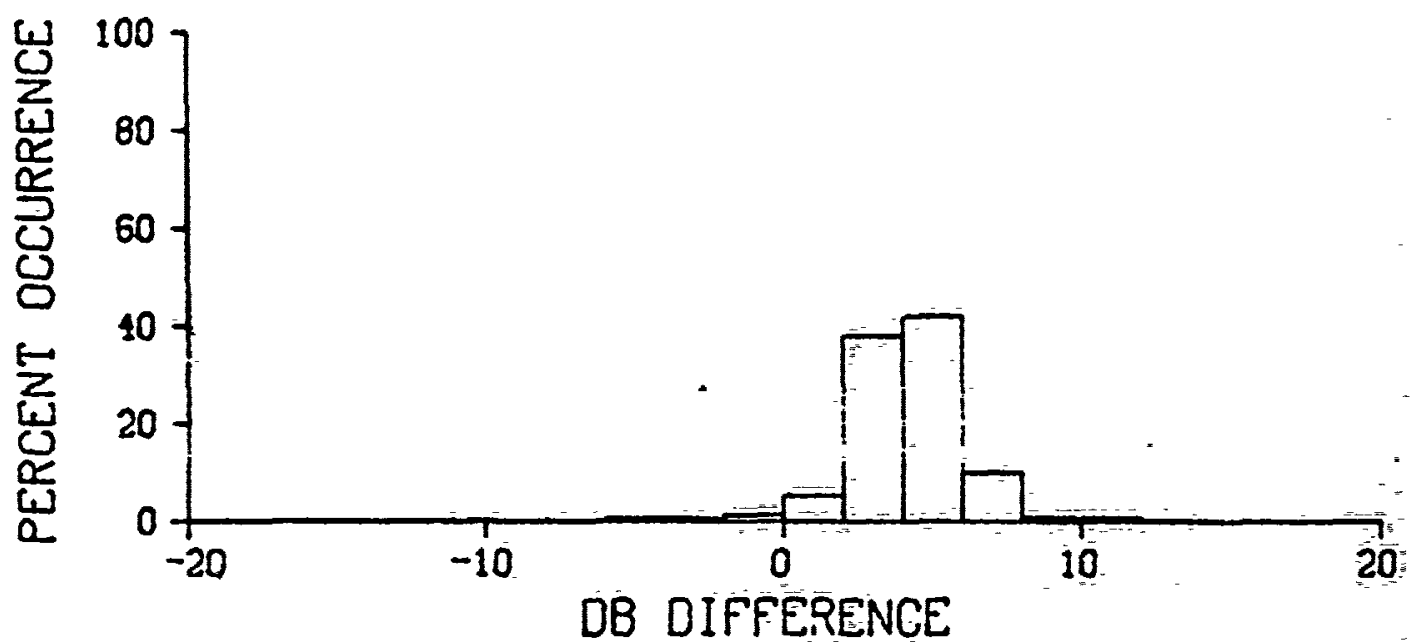

HIGH-MID

970 OBSERVATIONS

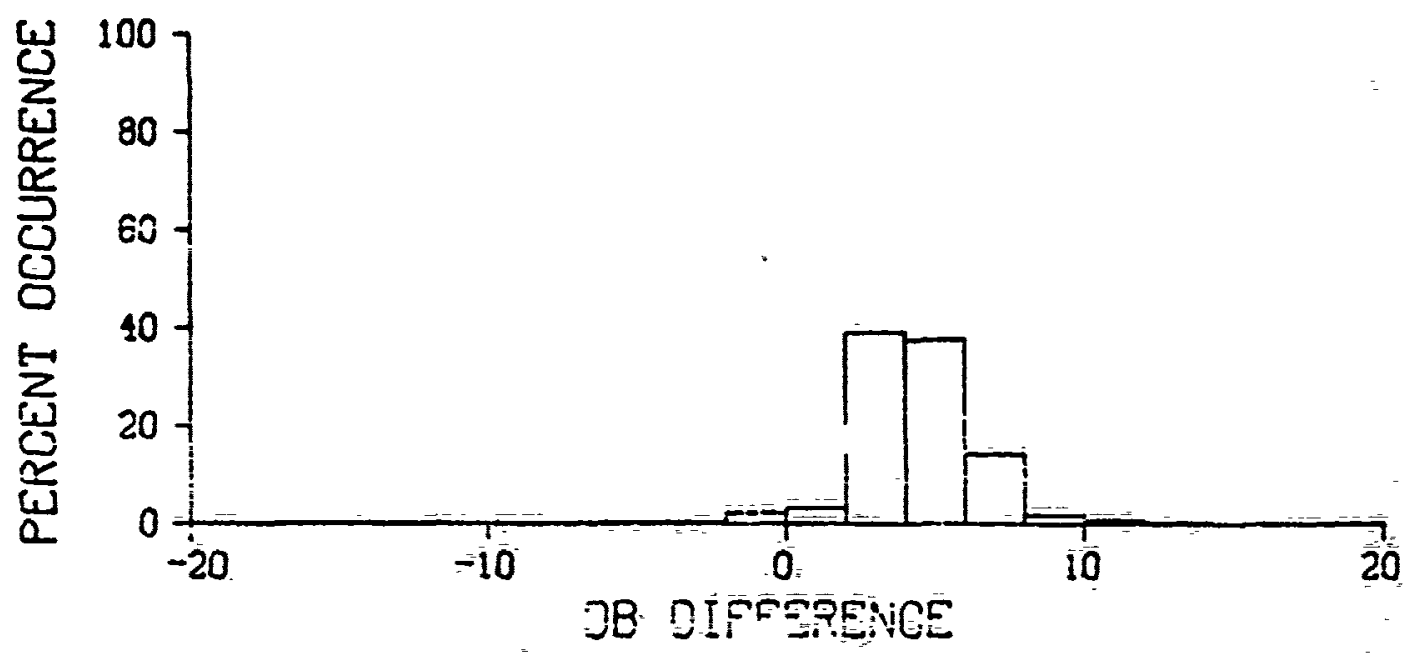

Aㄴ.

छ:

L BANG, GREECE ALGGUST 1972 

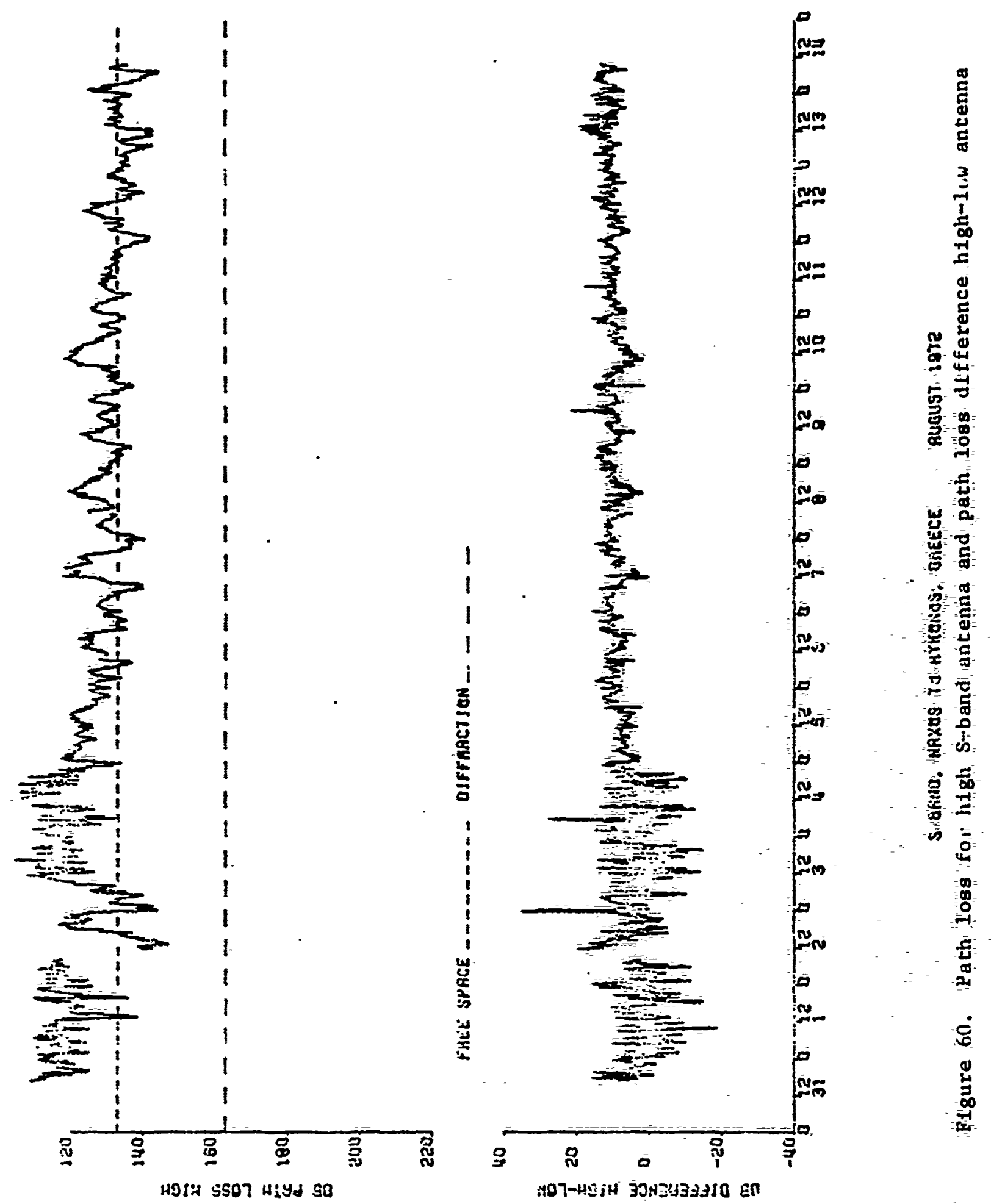

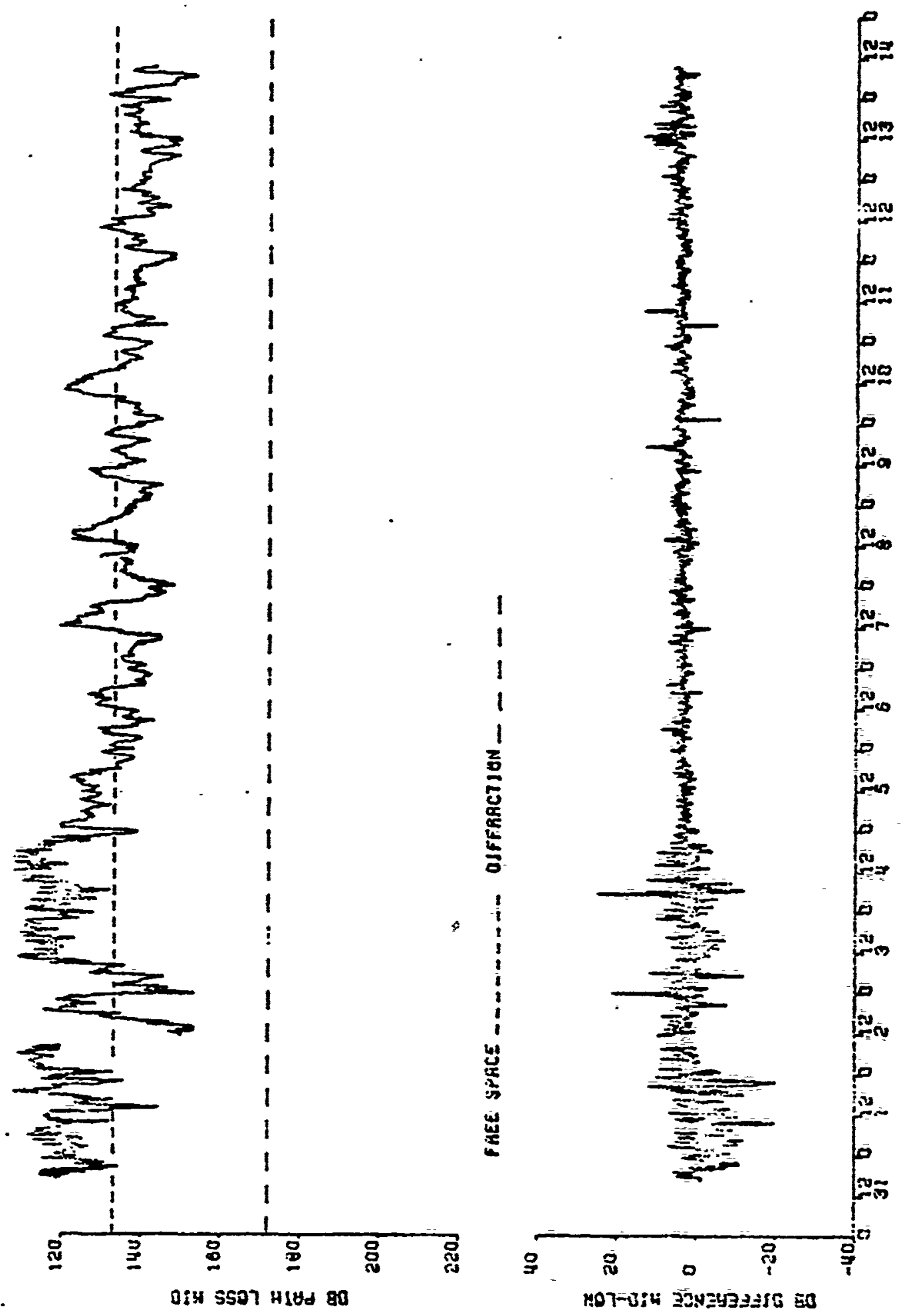



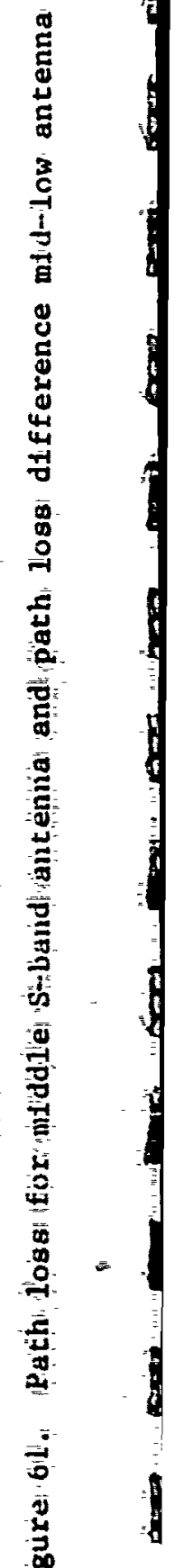




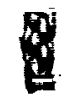

政

8

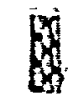

4
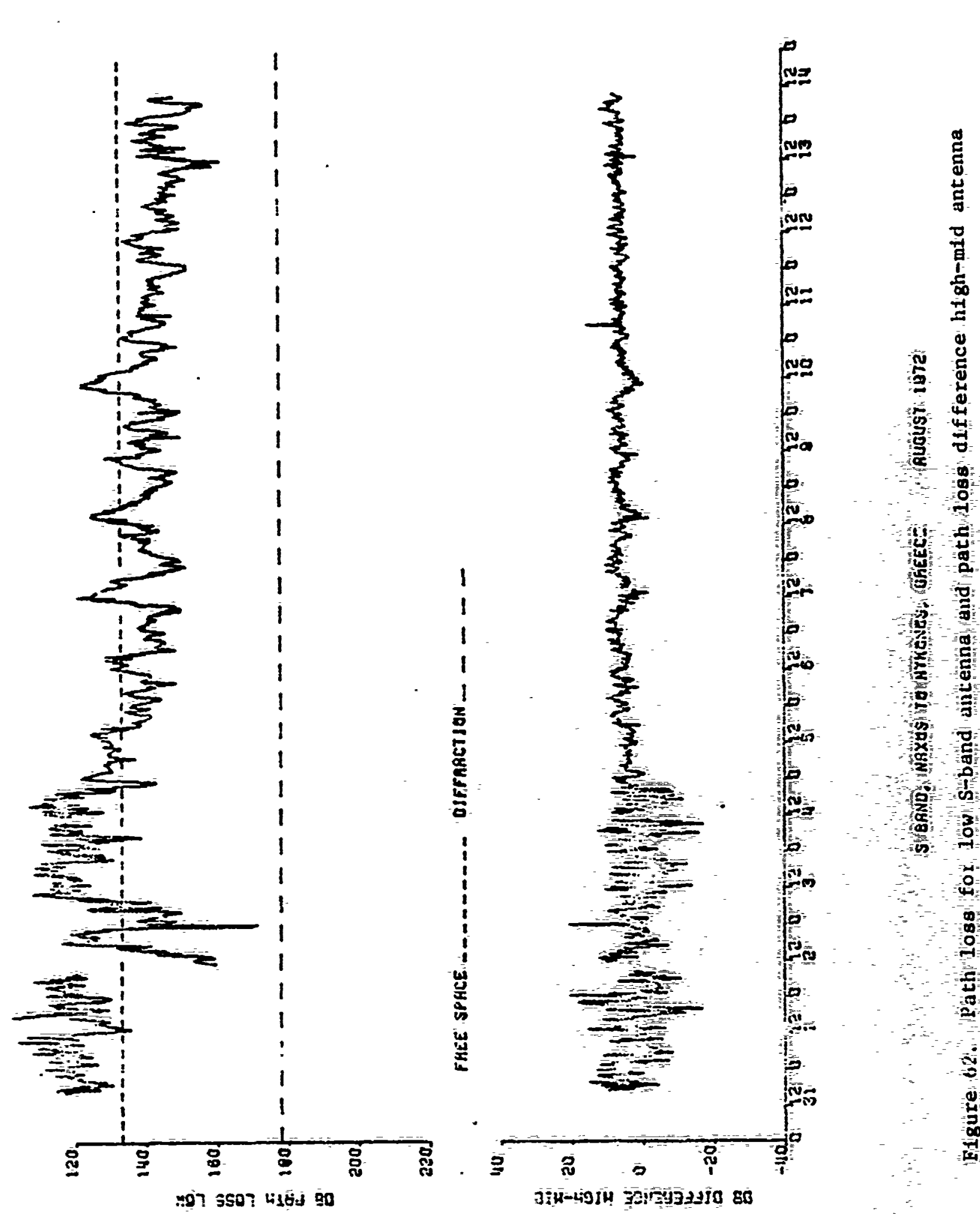

苞

ja



4

19

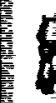

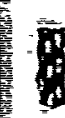

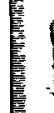

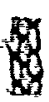

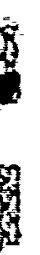

E 

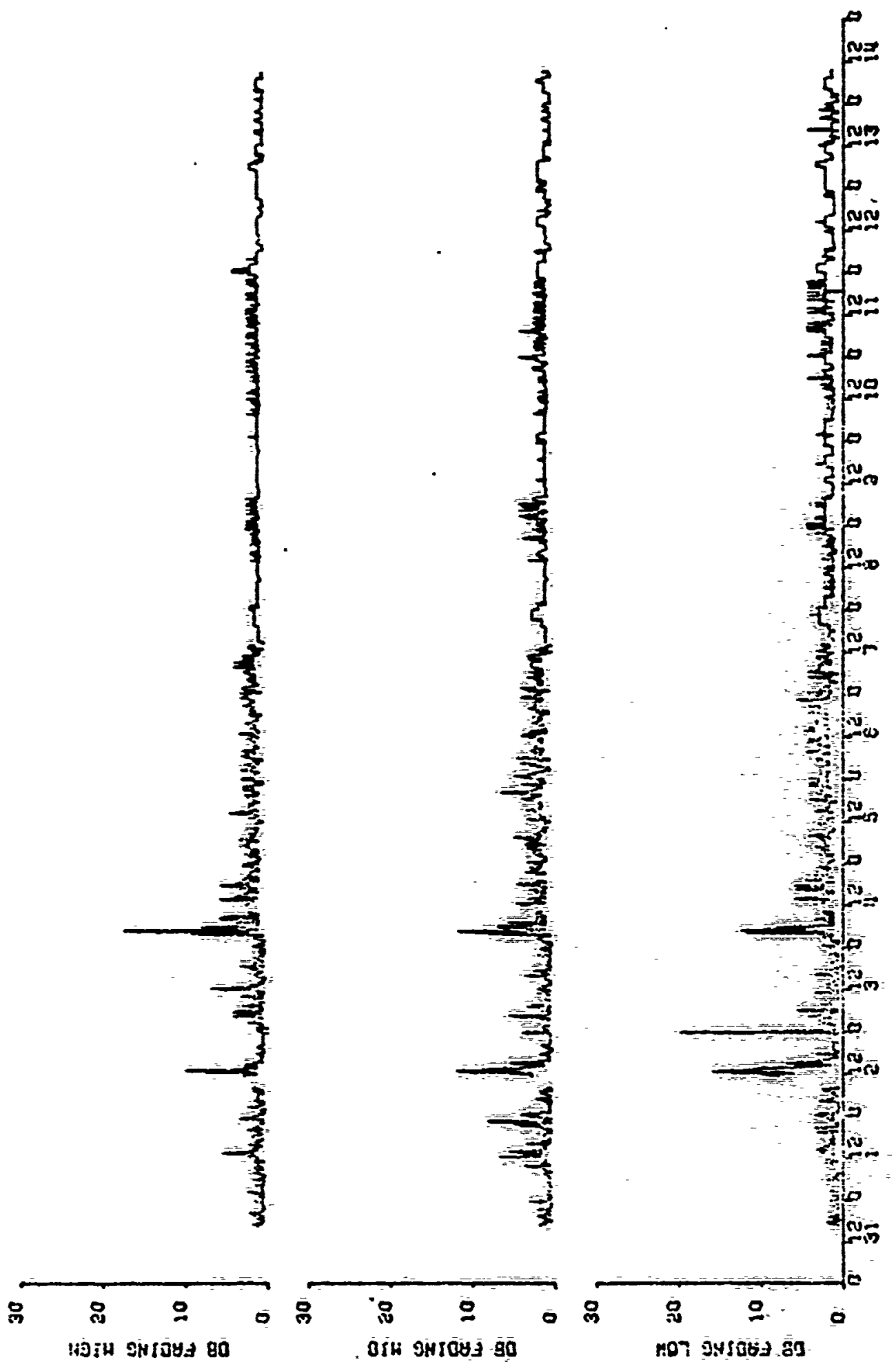

D

㕵

8

4

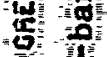

期 


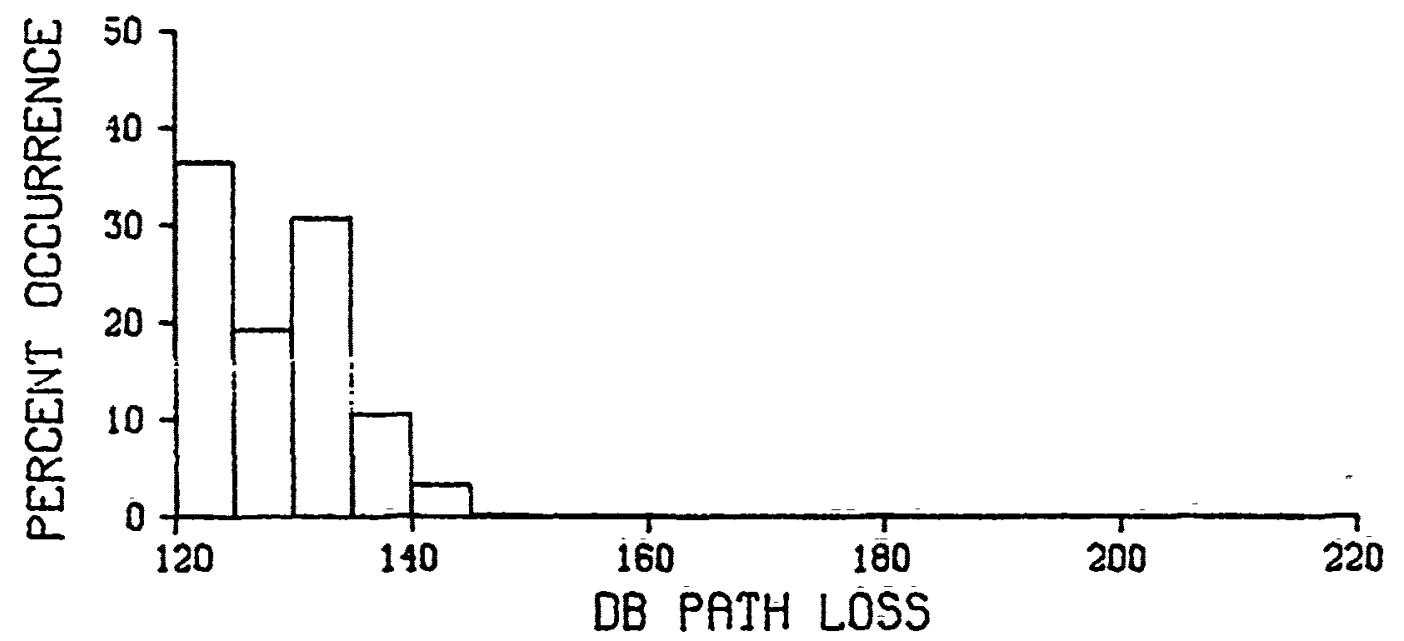

HIGH ANTENNA

1279 OBSERVATIONS

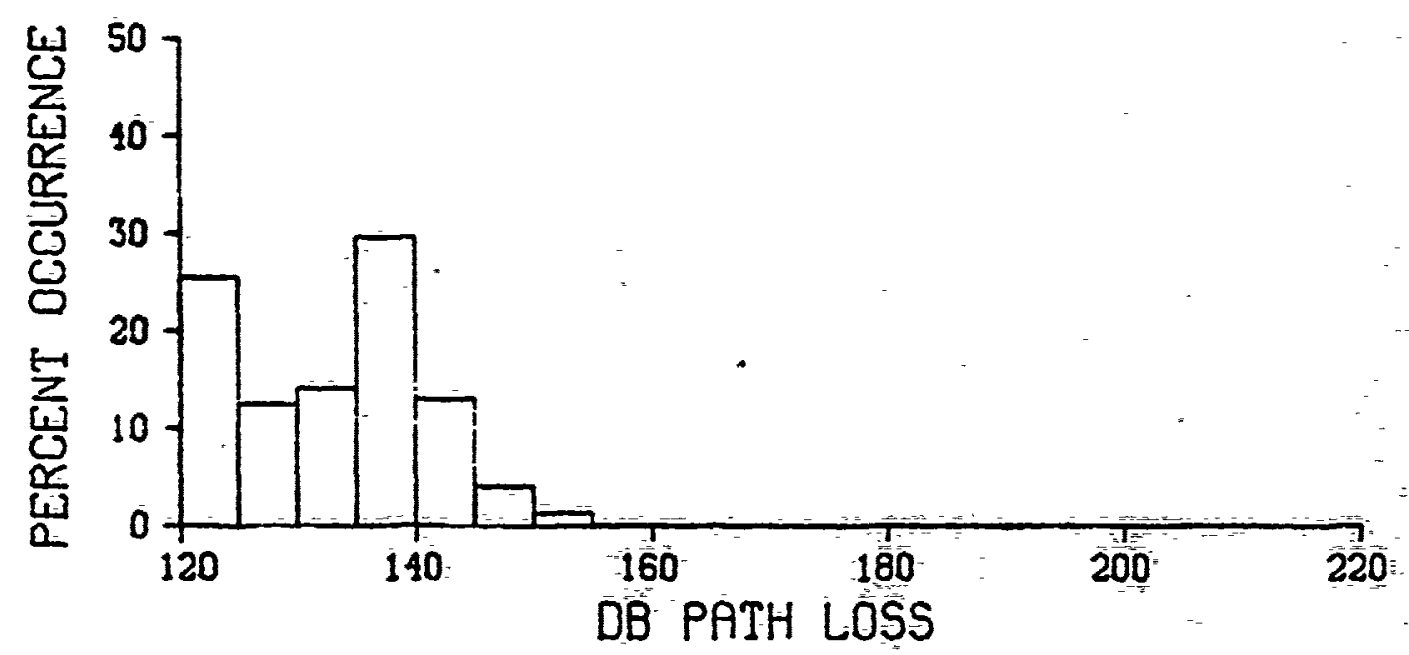

की

MID ANTENNA

1281 OBESRVATUTHOS

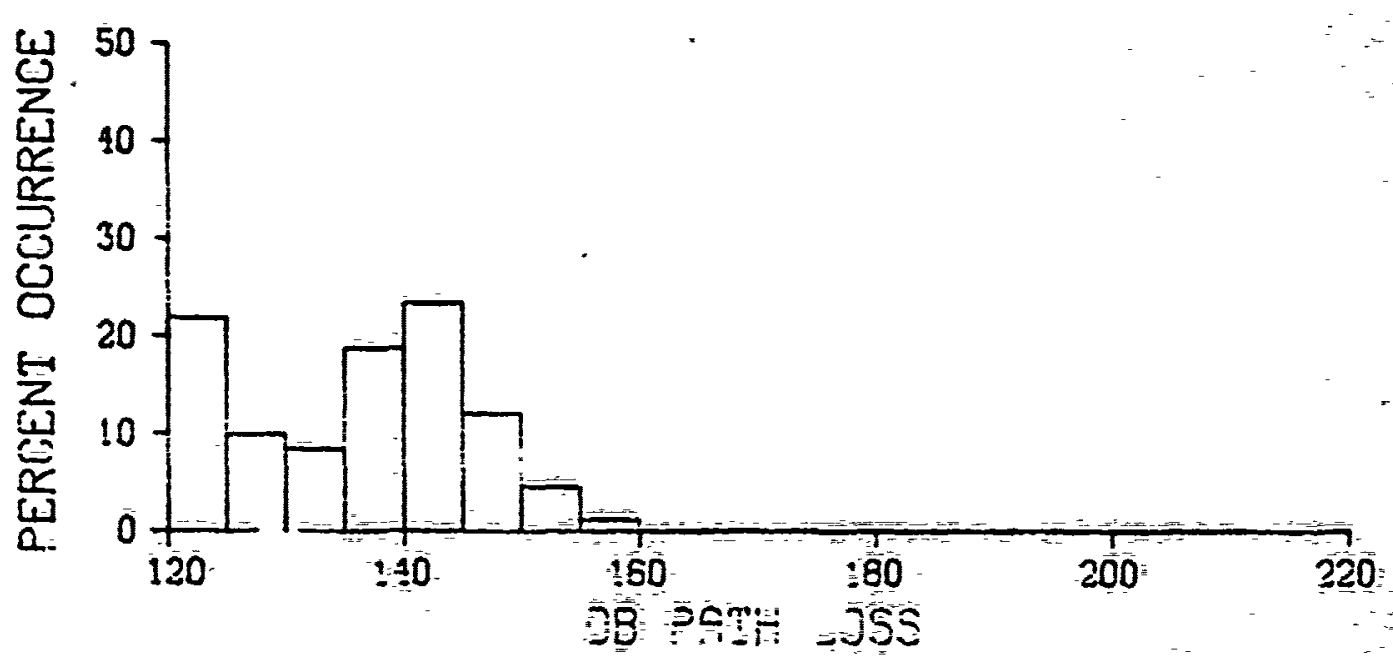

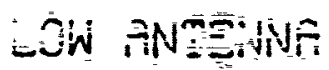

129: CBSERYATHA S BANE, GREECE FUGUST 1972 


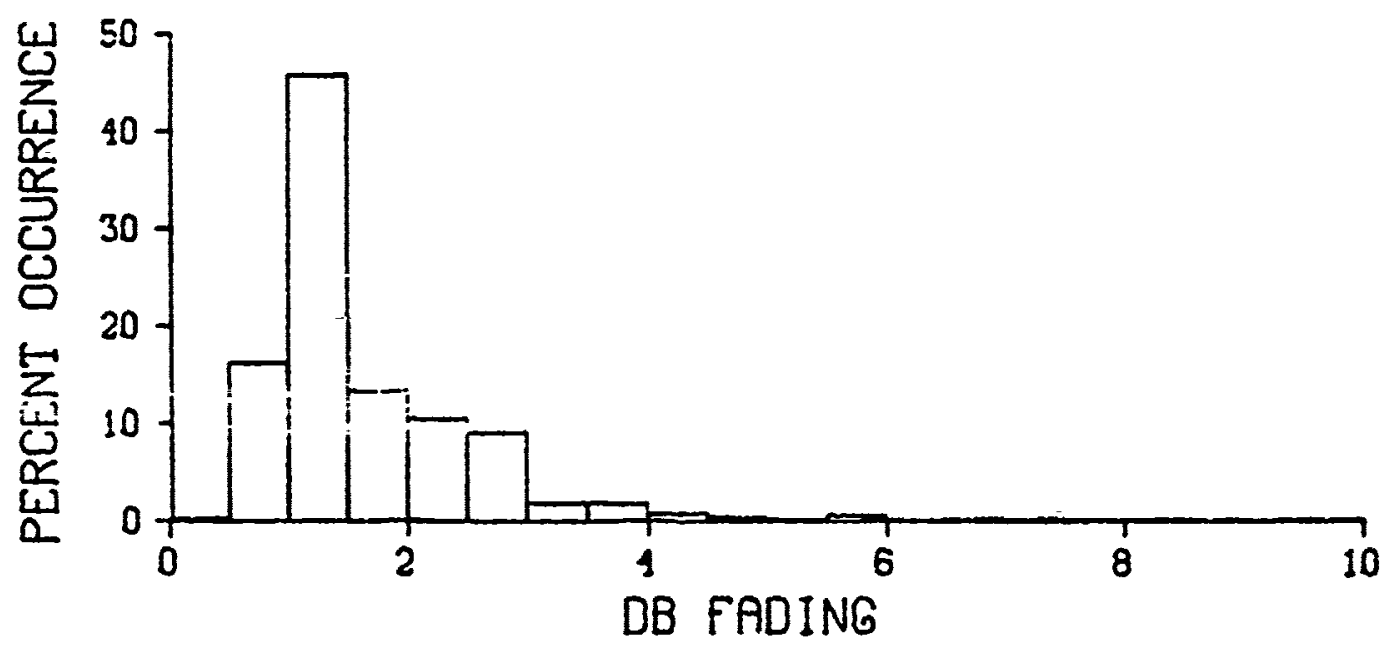

㻤 HIGH ANTENNA 1279 OBSERVATIONS

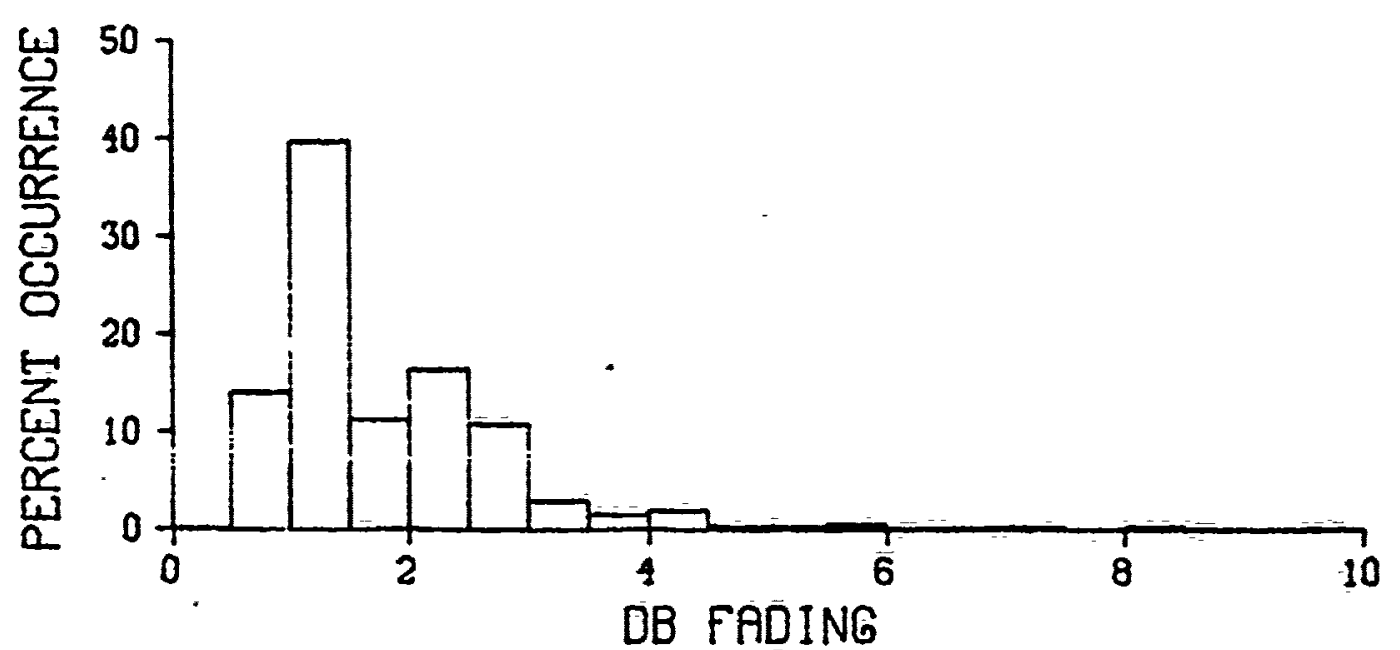
so

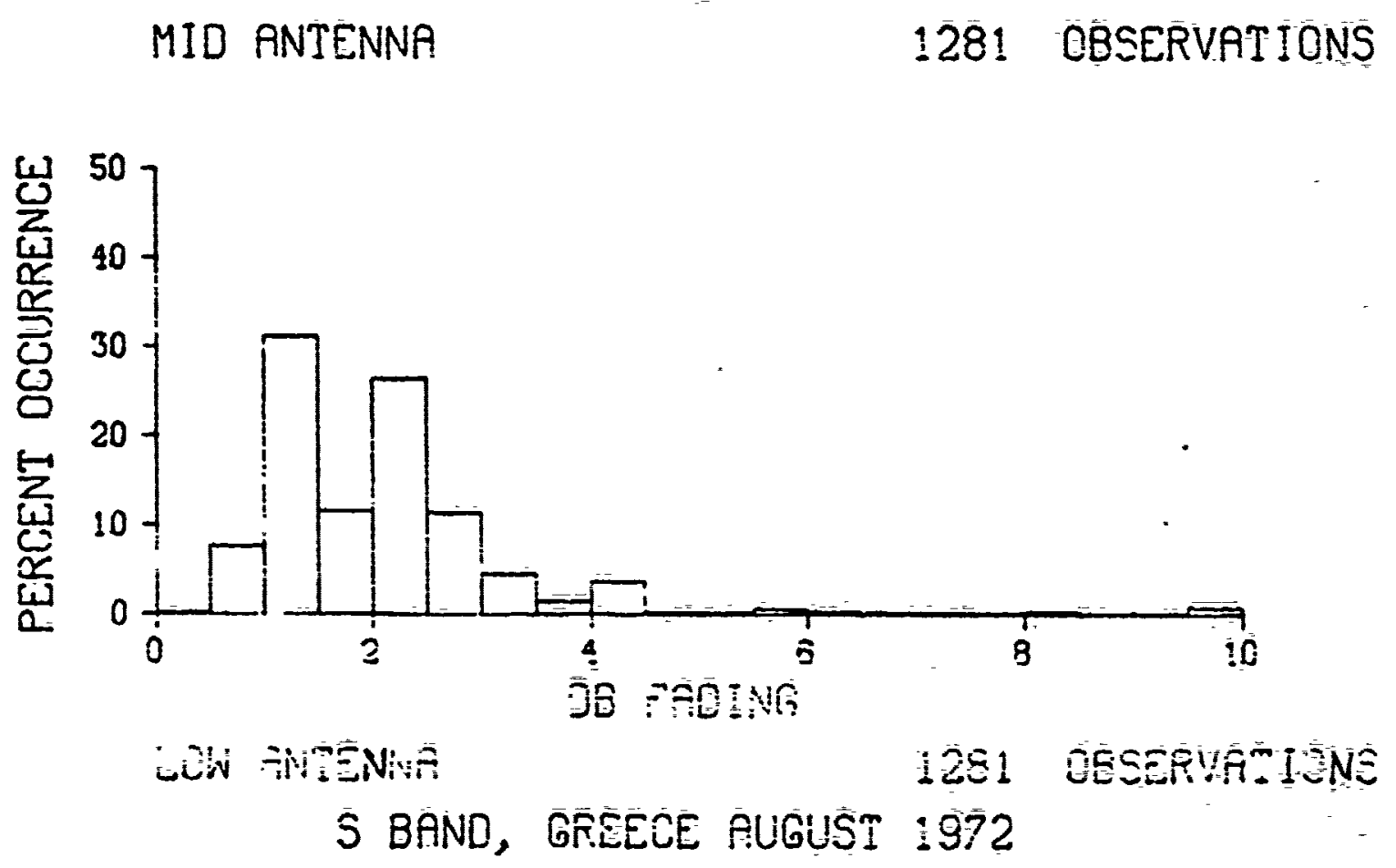



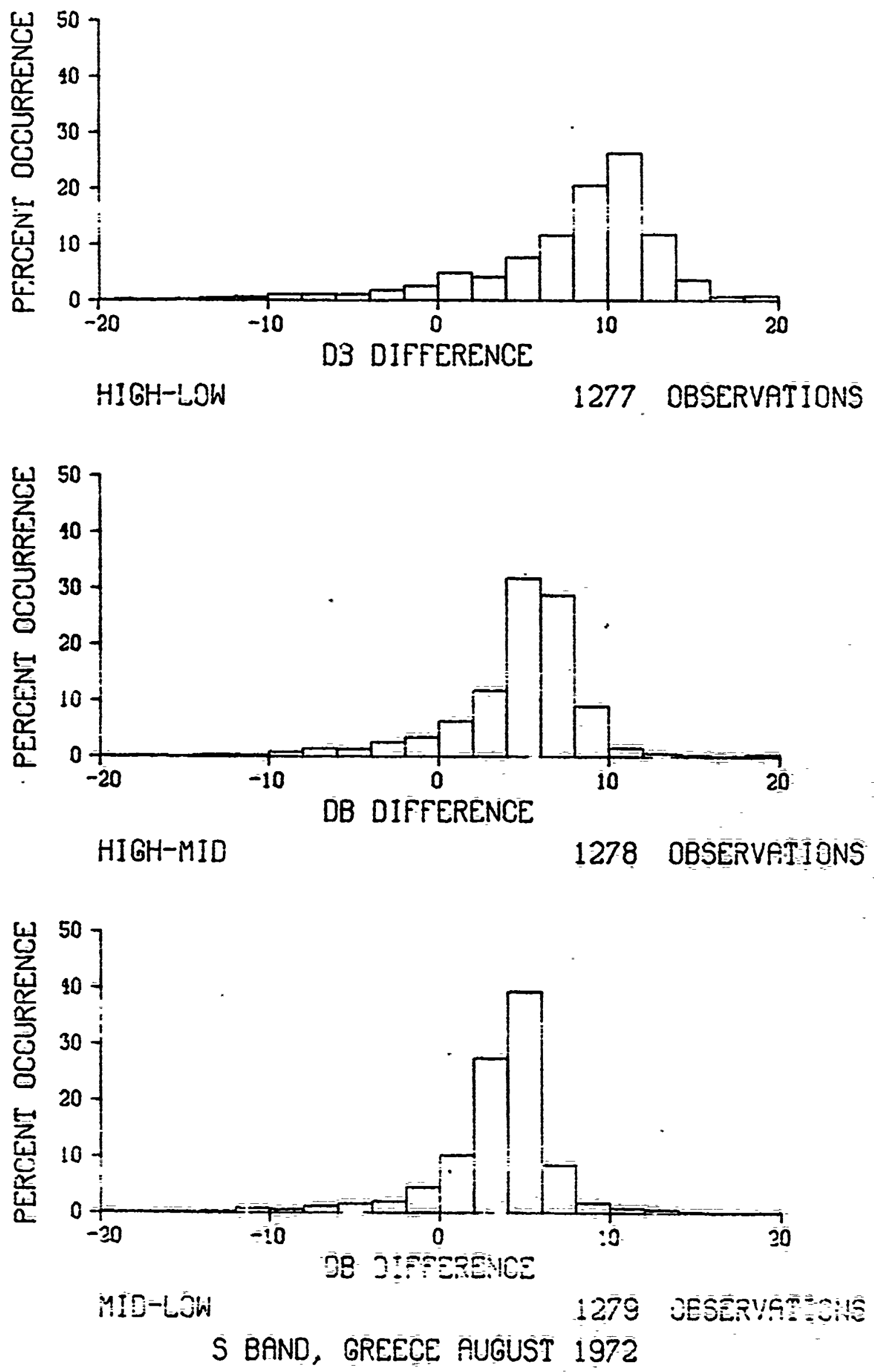

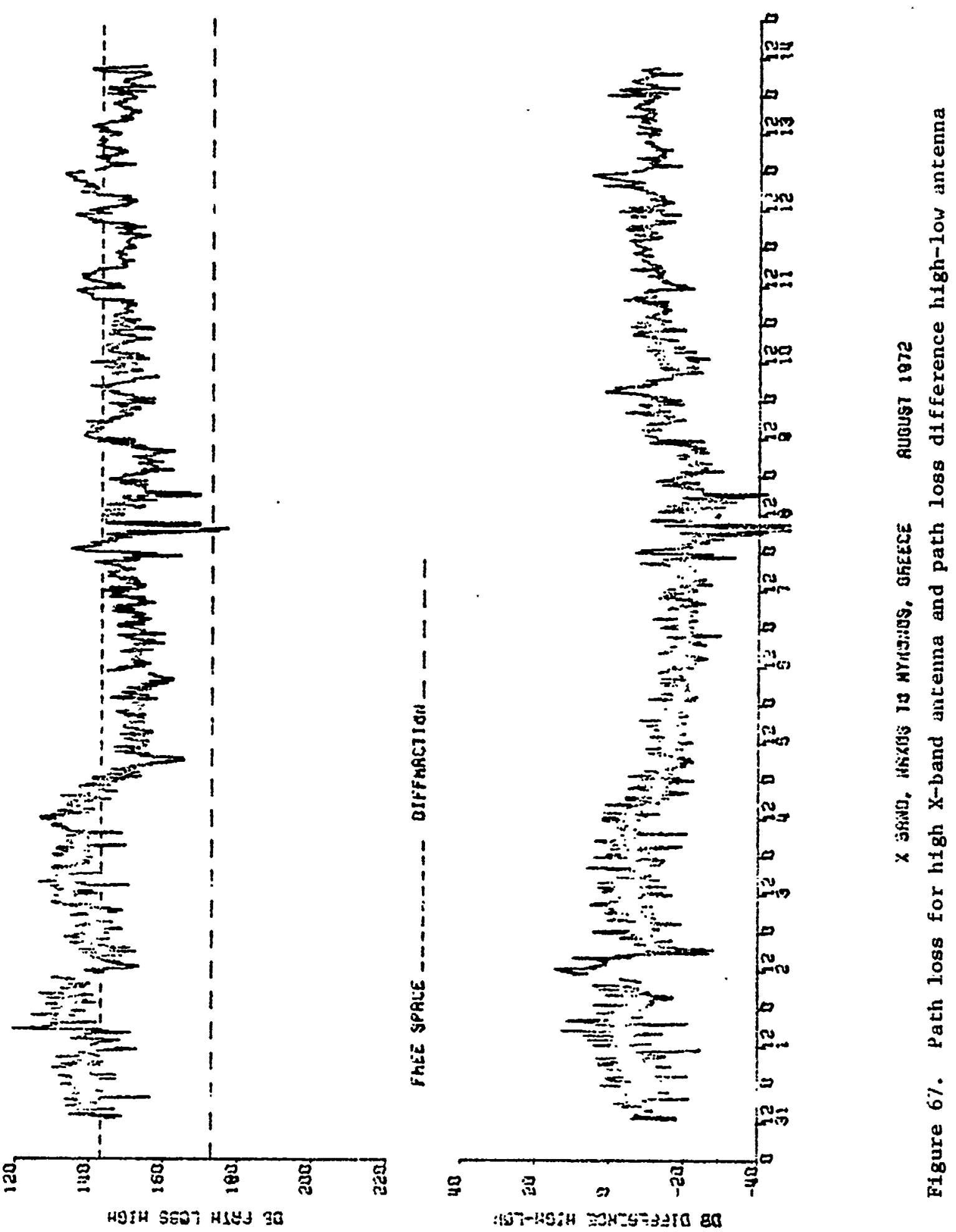
$F^{\prime}$

$\alpha$

$E$
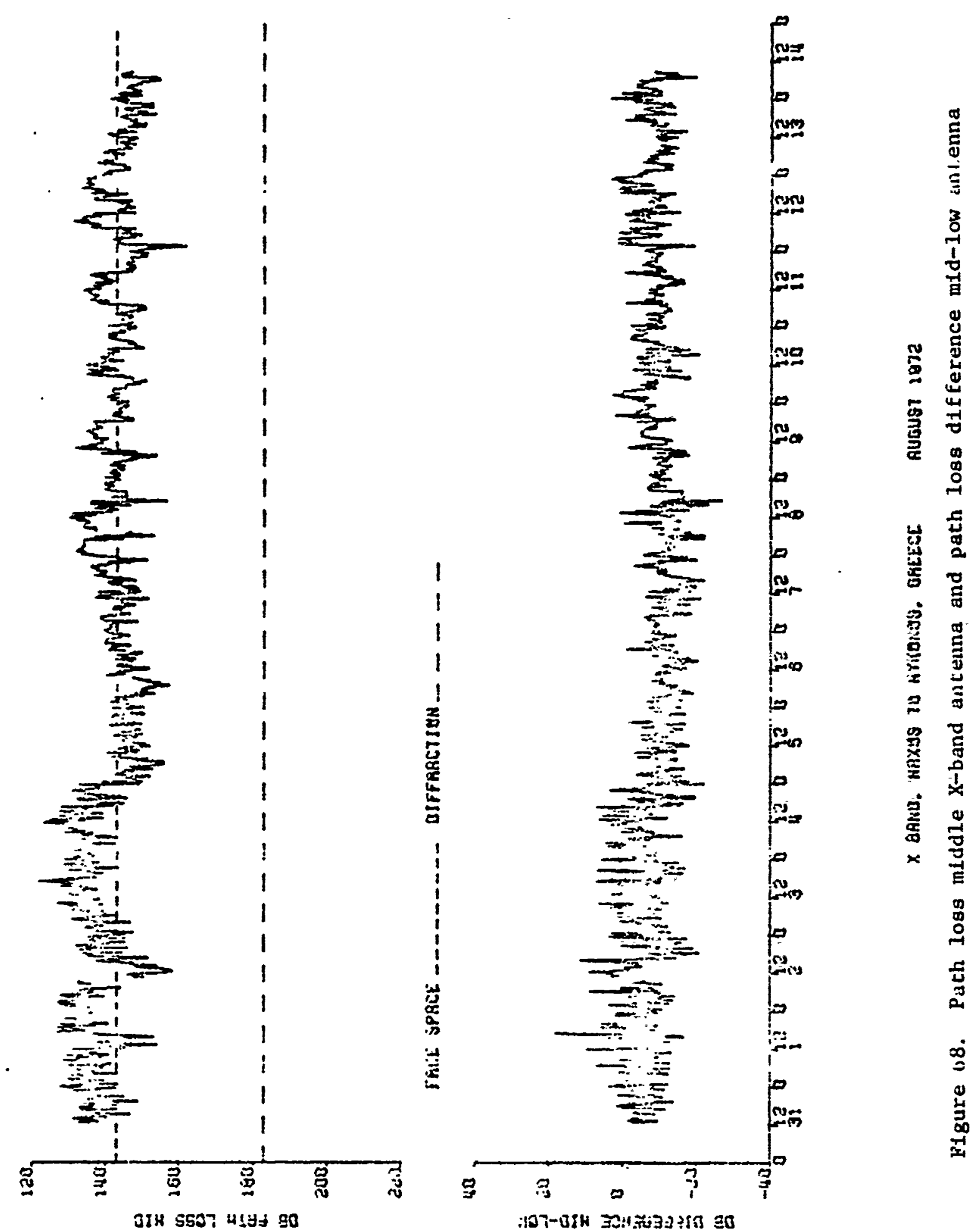

:107-814 ב2.4. 

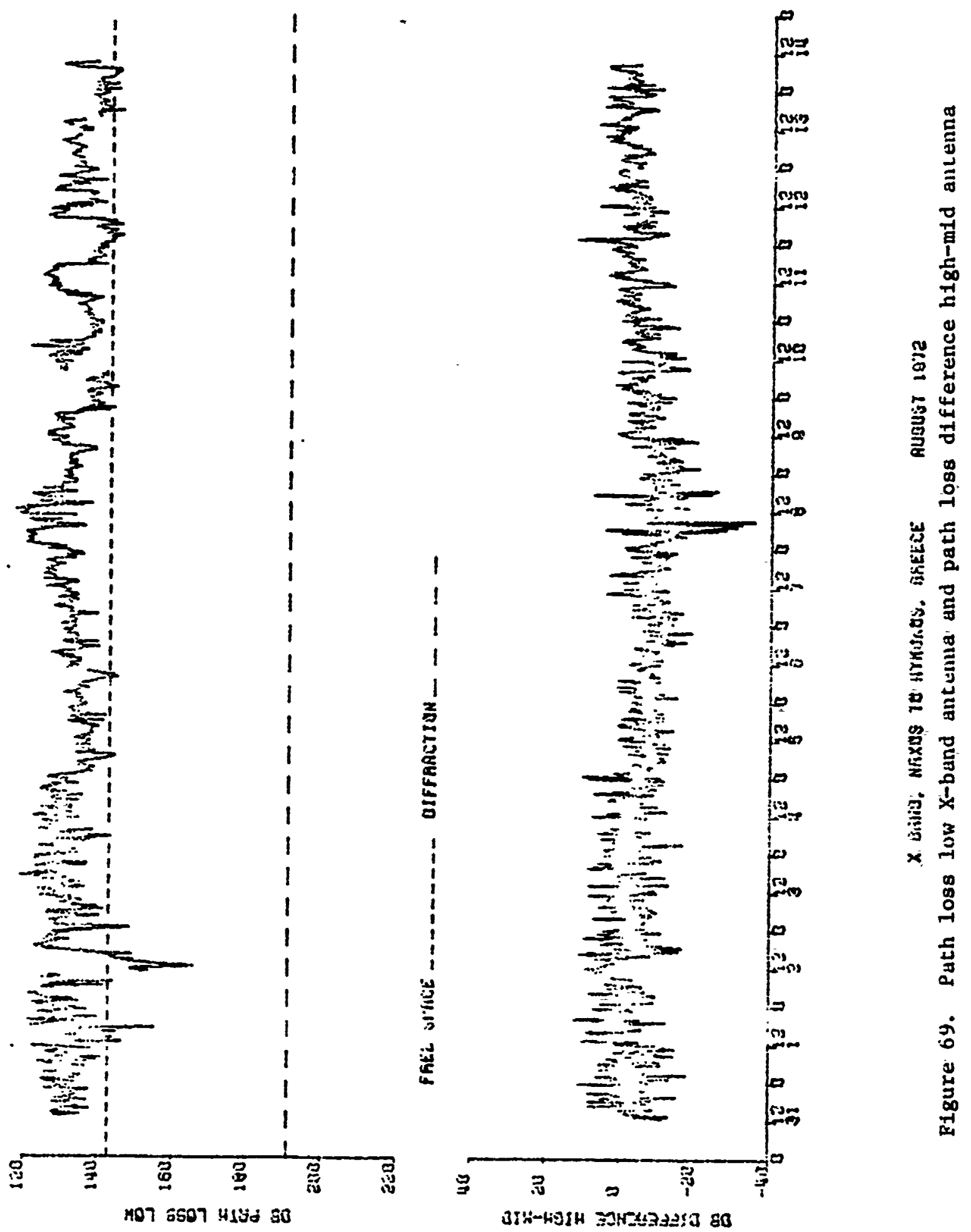


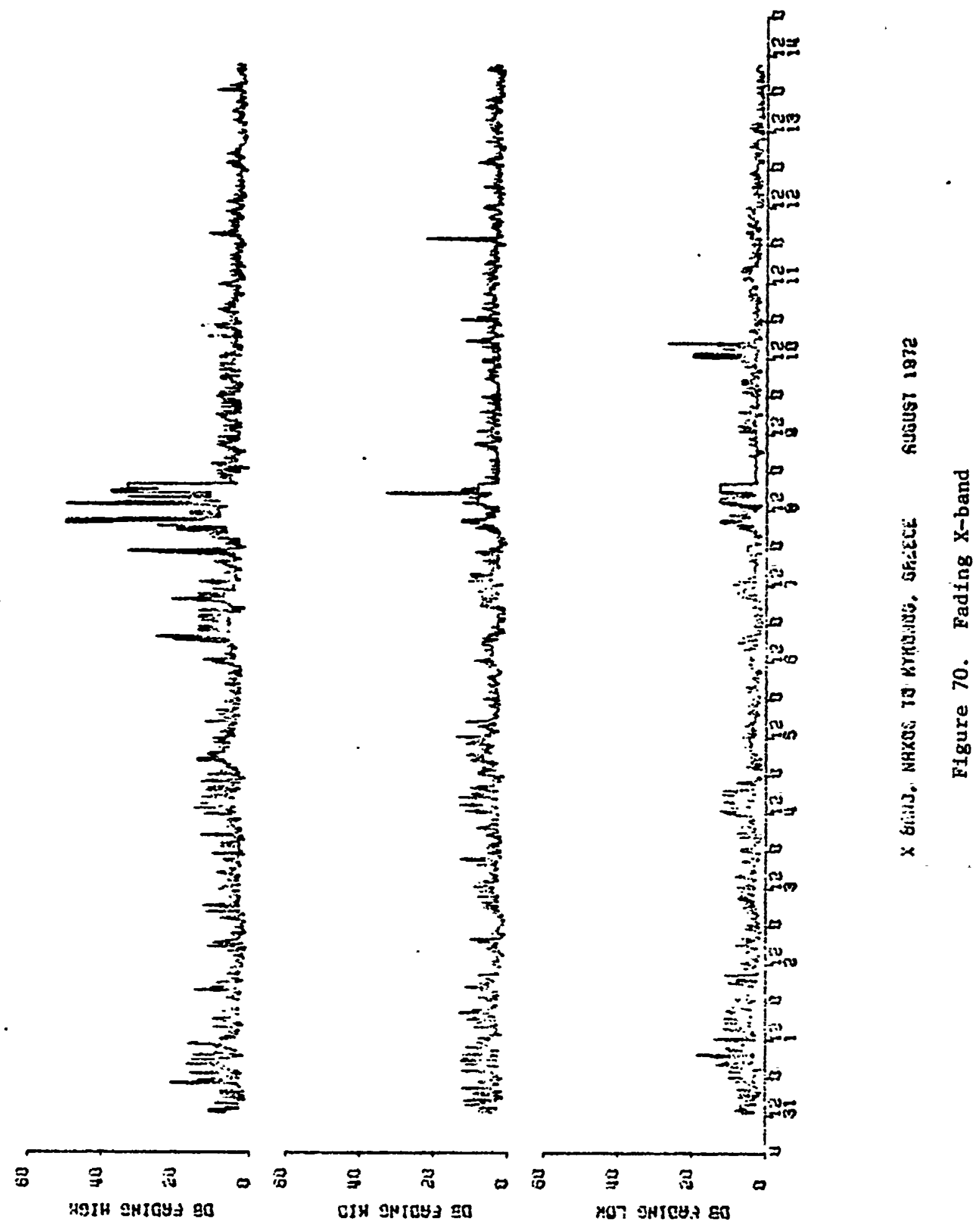




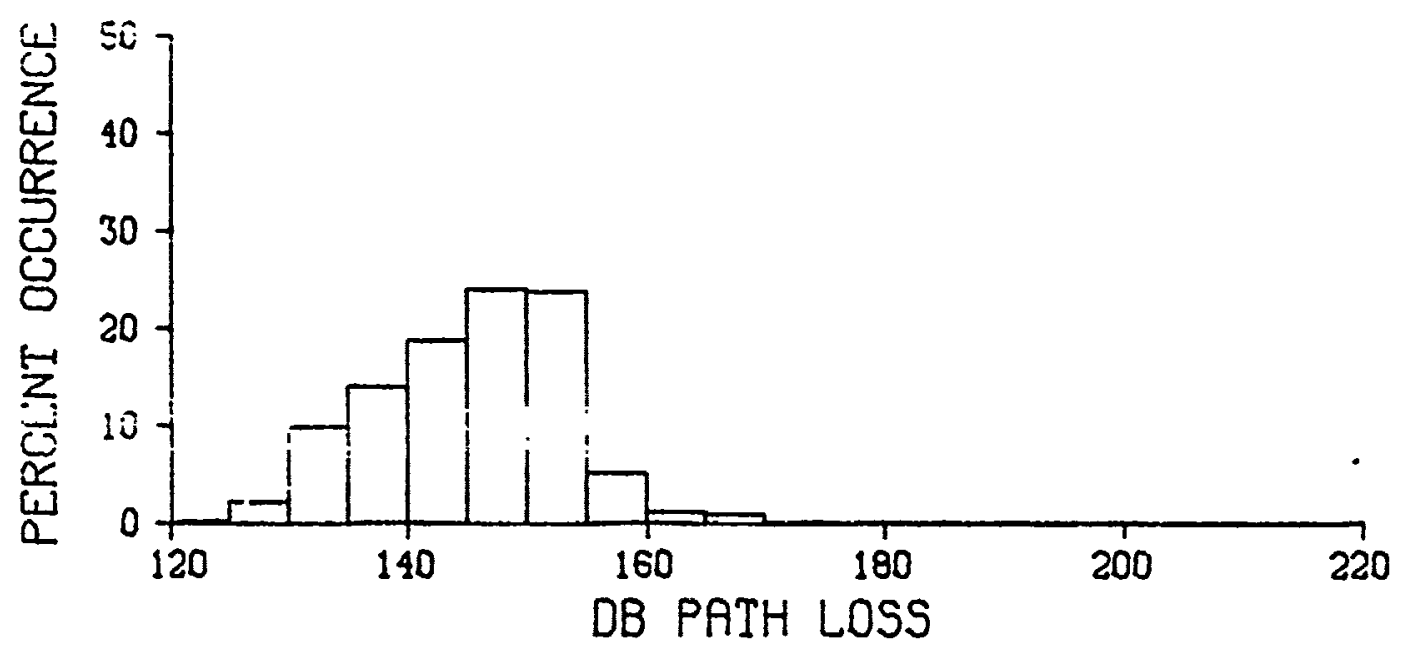

8

HIGH RNTENNA

1292 OESERVATIONS
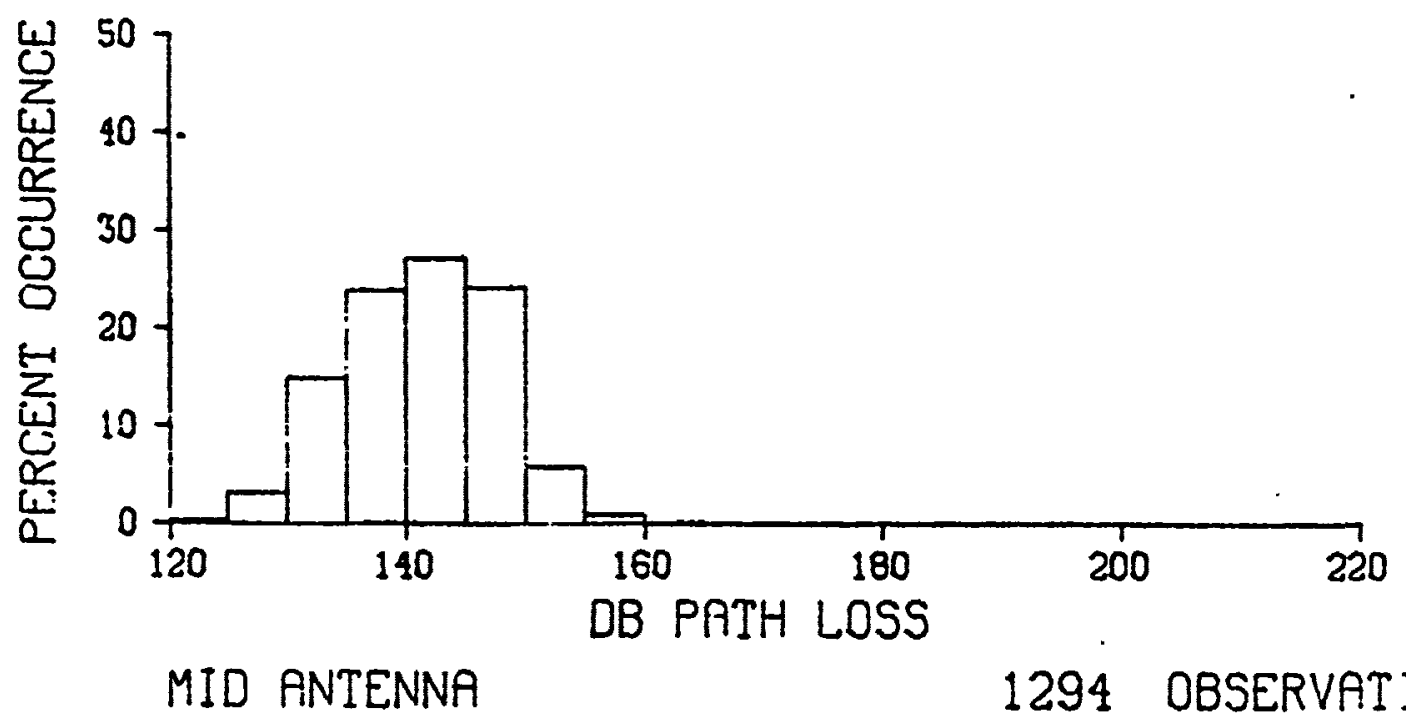

1294 OBSERVATIONS

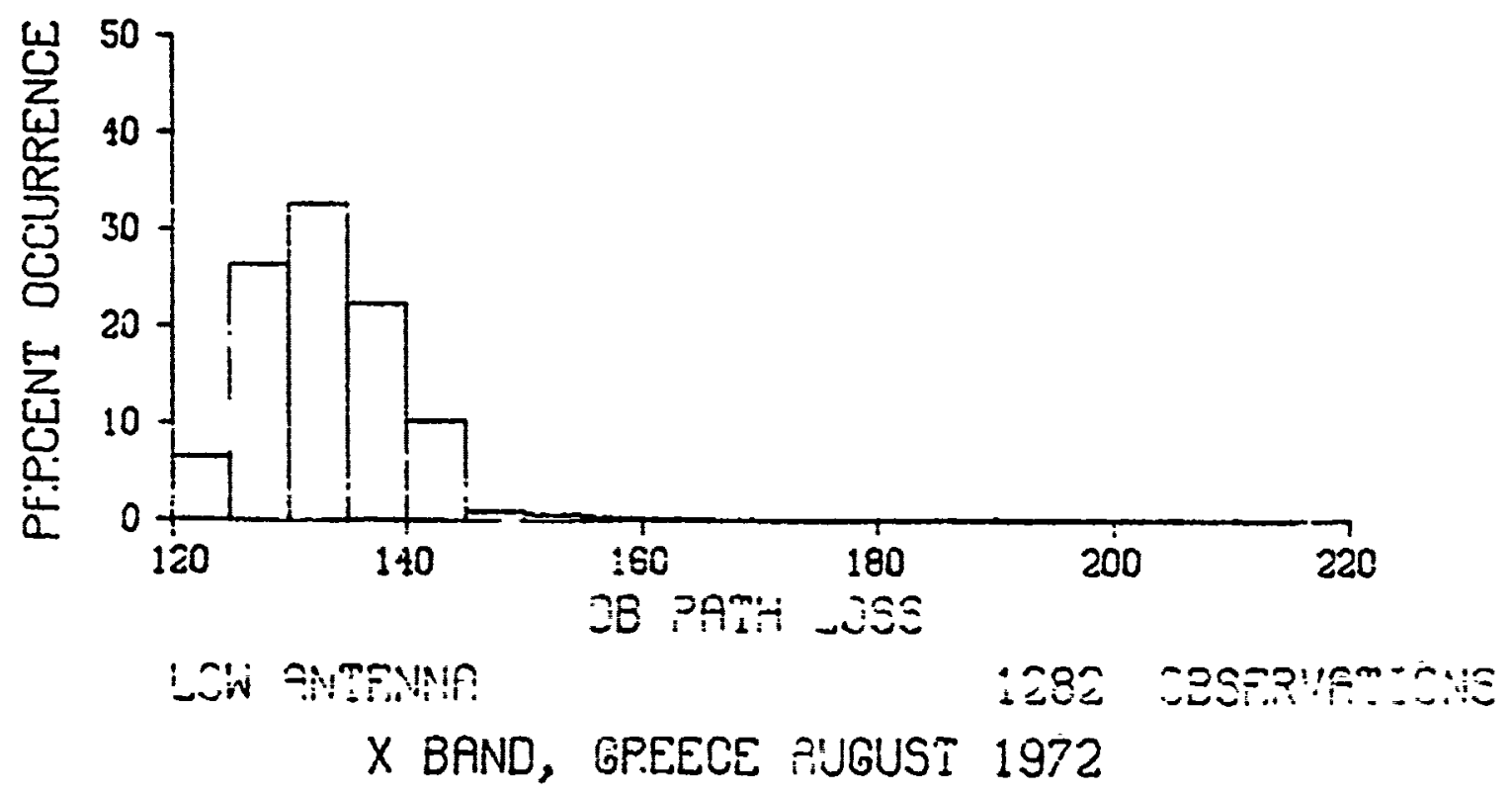




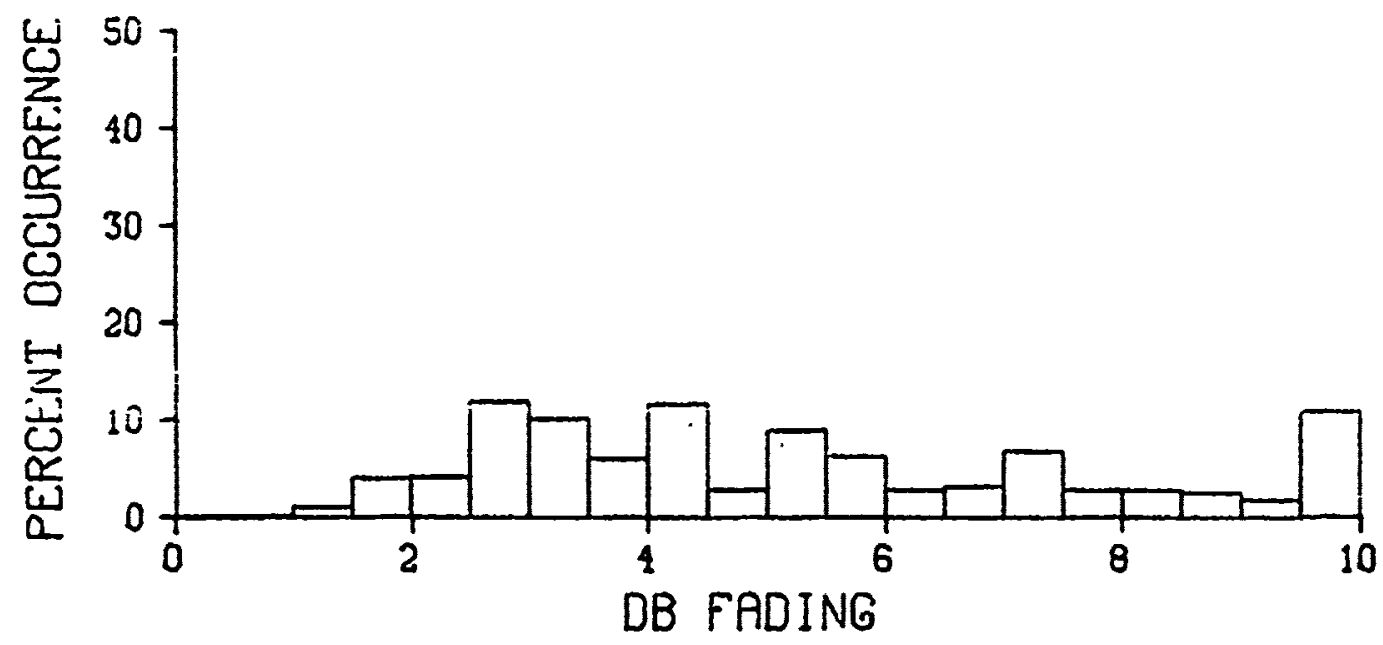

HIGH ANTENNA

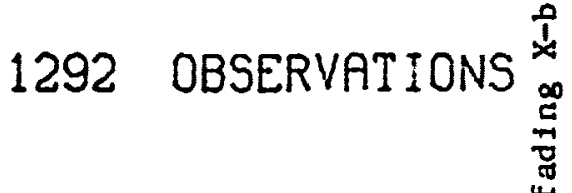

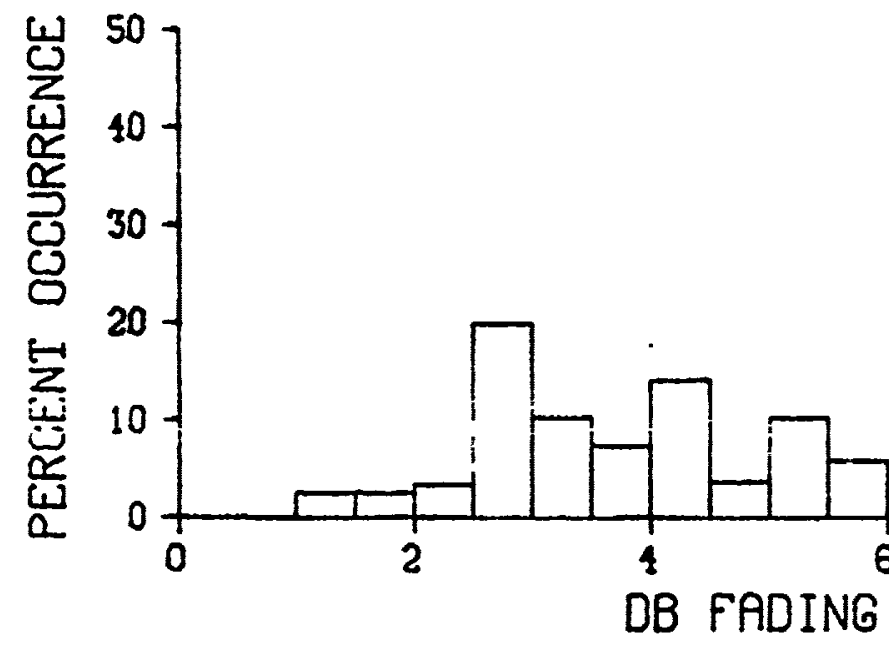

MID ANTENNA 1294 OBSERVATIONS 芦

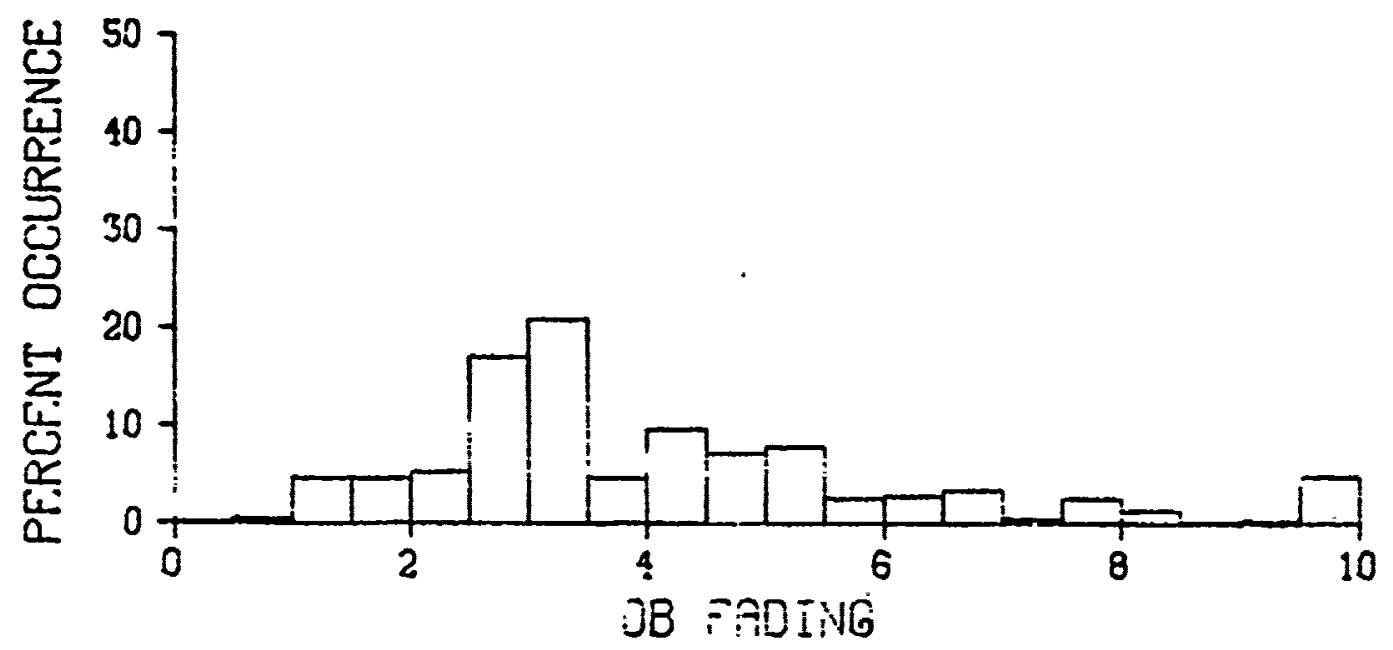

- Th GN:EVWA

1282 OBSERIFM:ONS

$X$ BAND, GREECE AUGUST 1972 


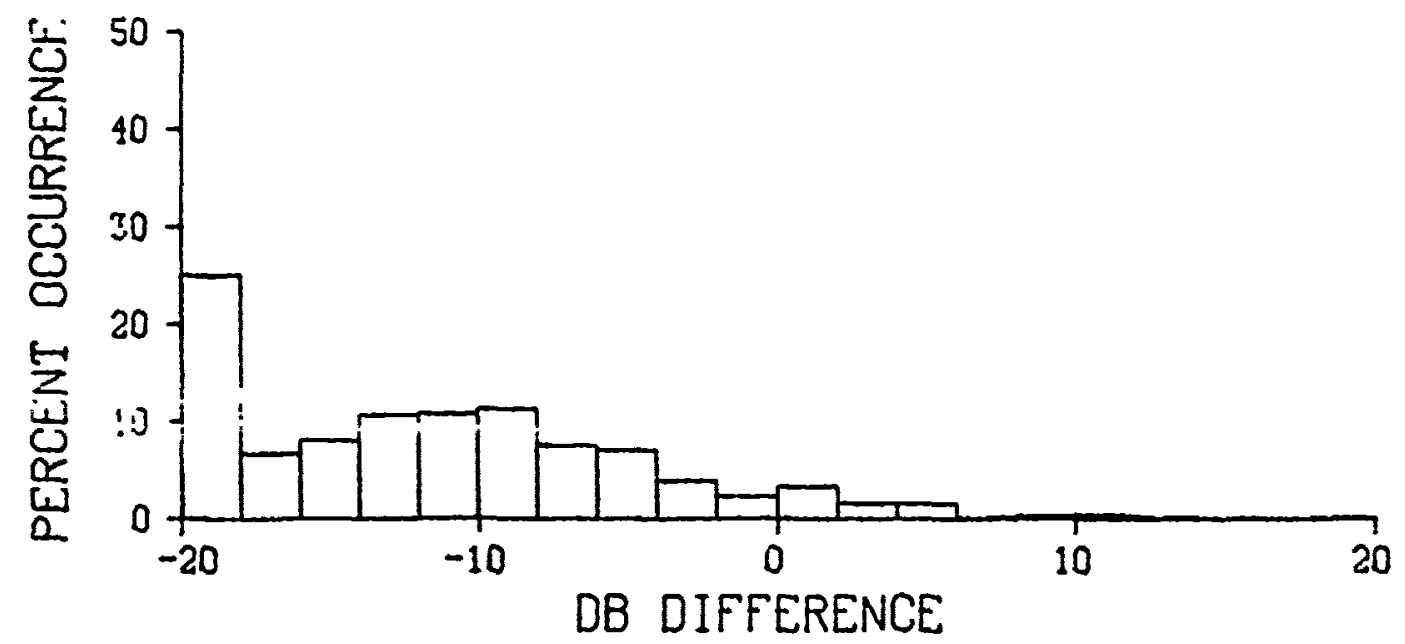

HIGH-LOW

1278 OBSERVATIONS

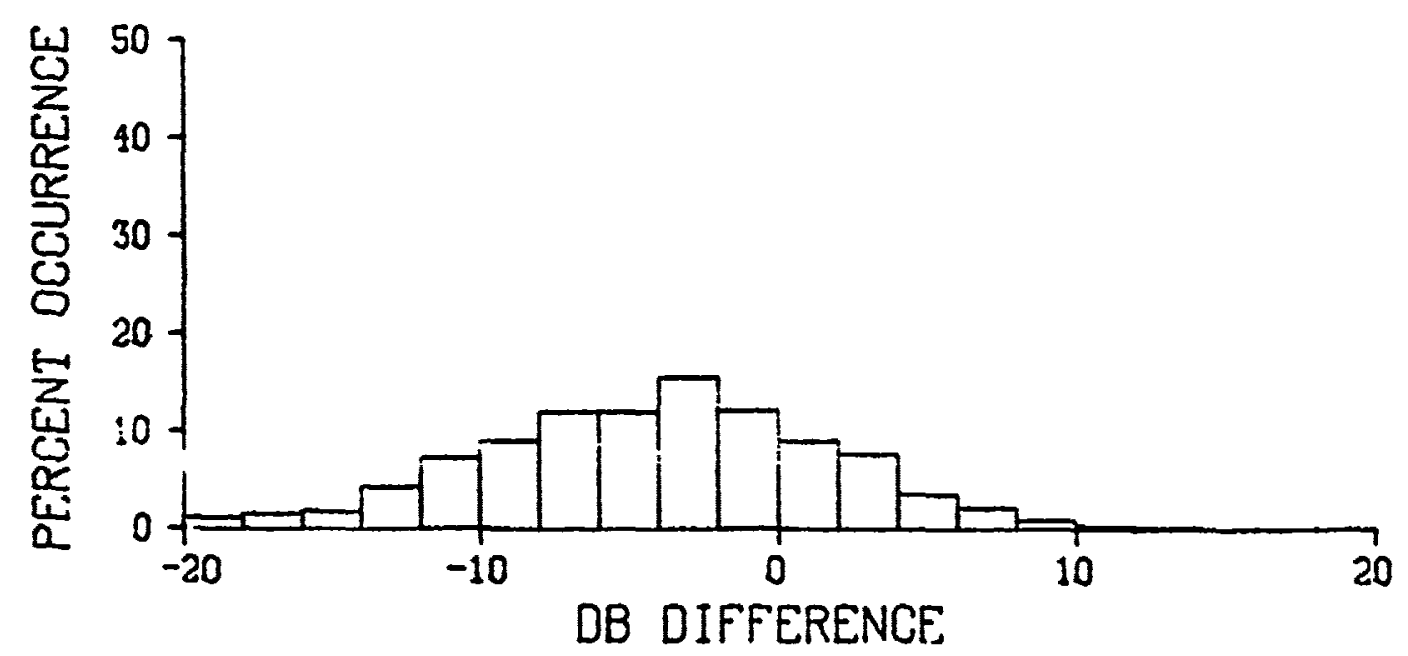

HIGH-MID

1289 OBSERVATIONS
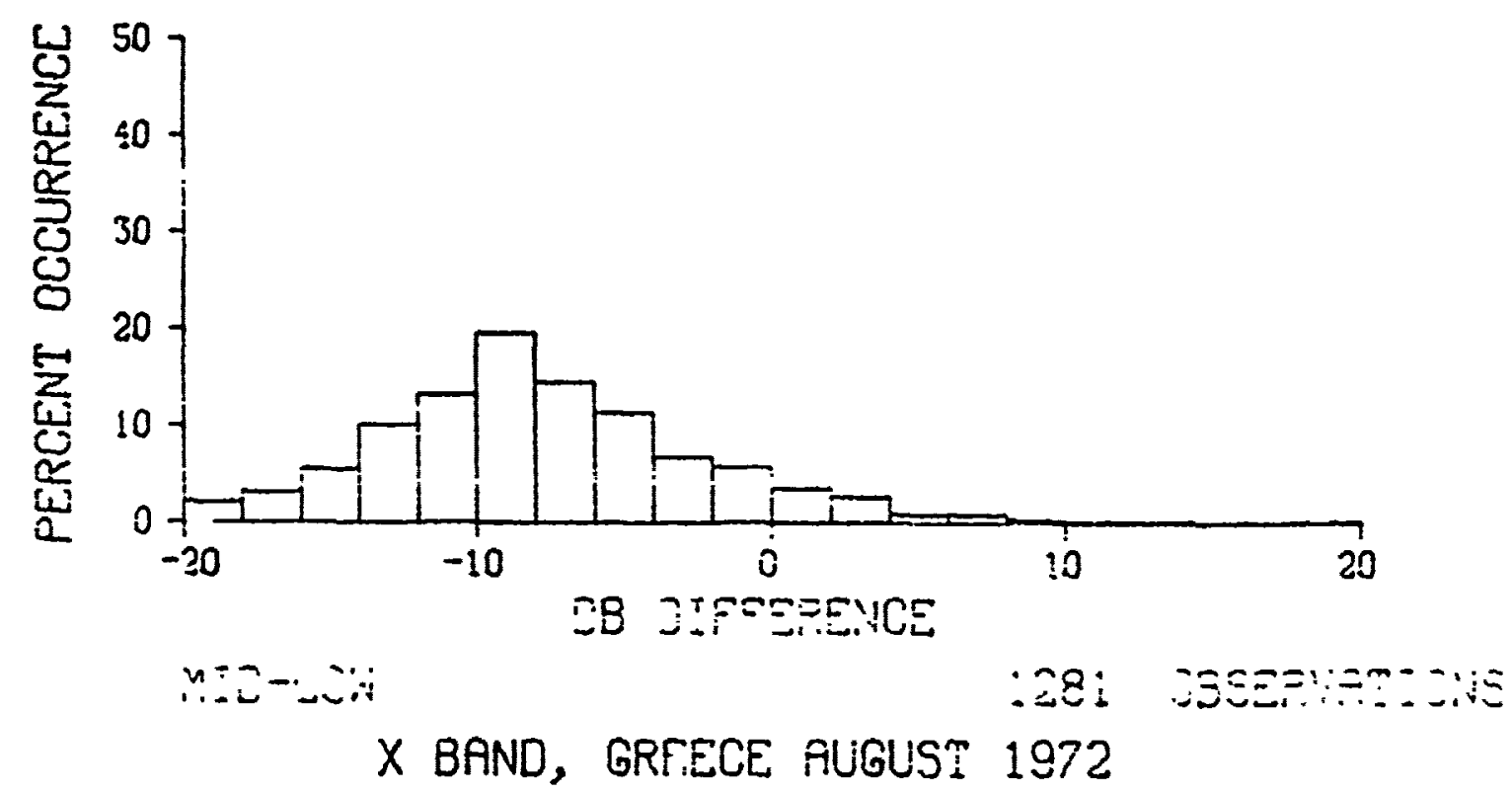


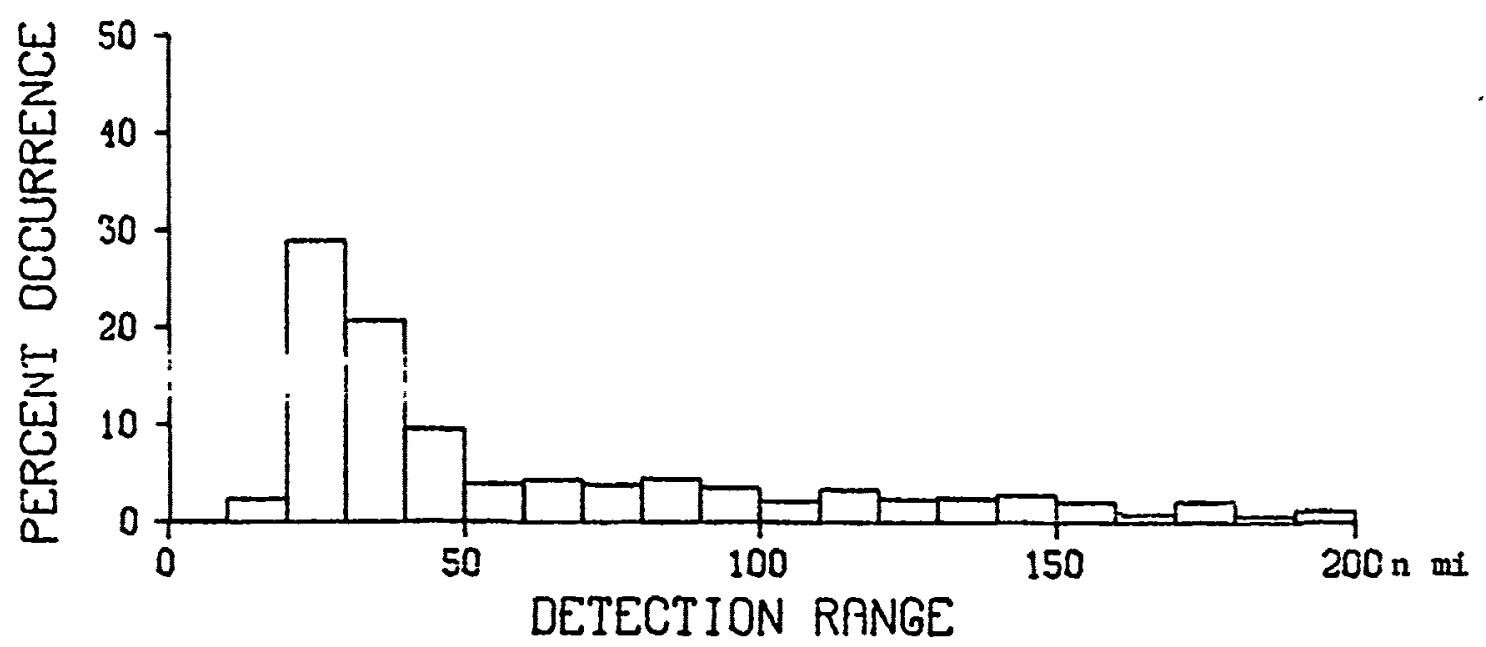

HIGH ANTENNP

1277 OBSERVIFTIONS

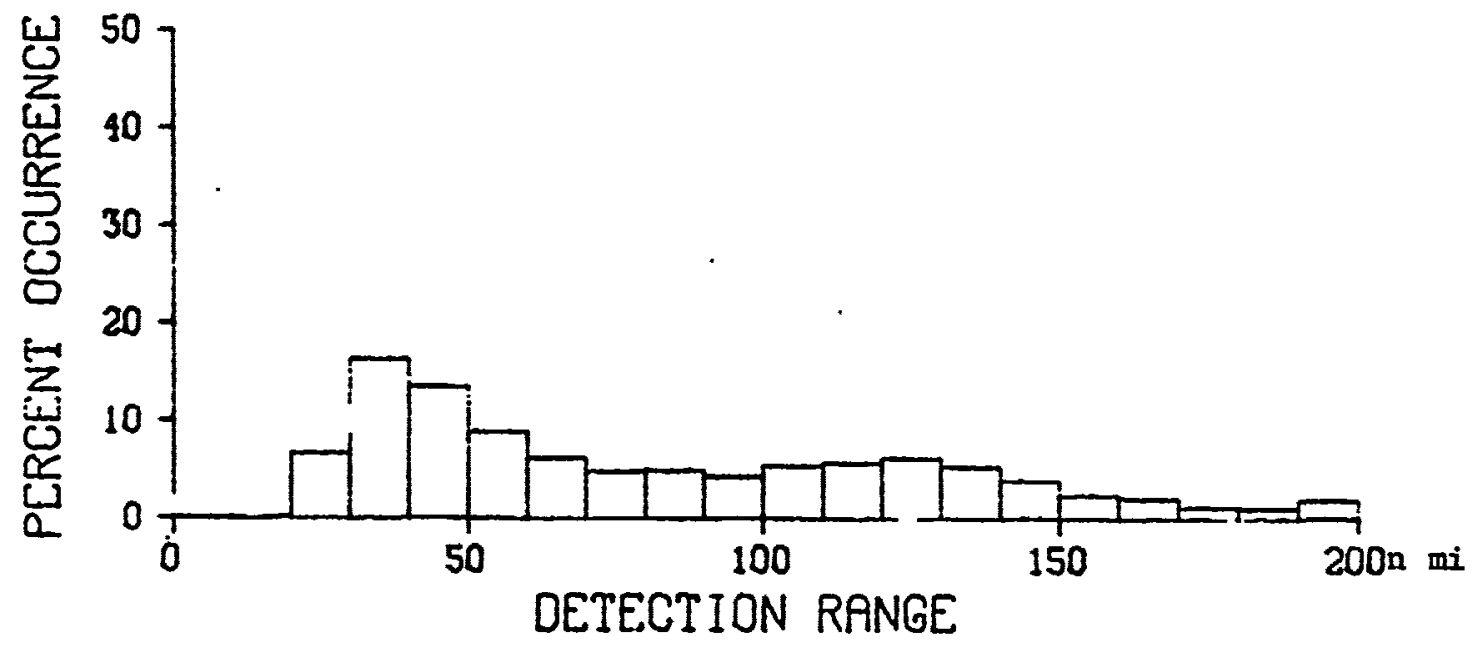

MID ANTENNA

1277 OBSERVATIONS

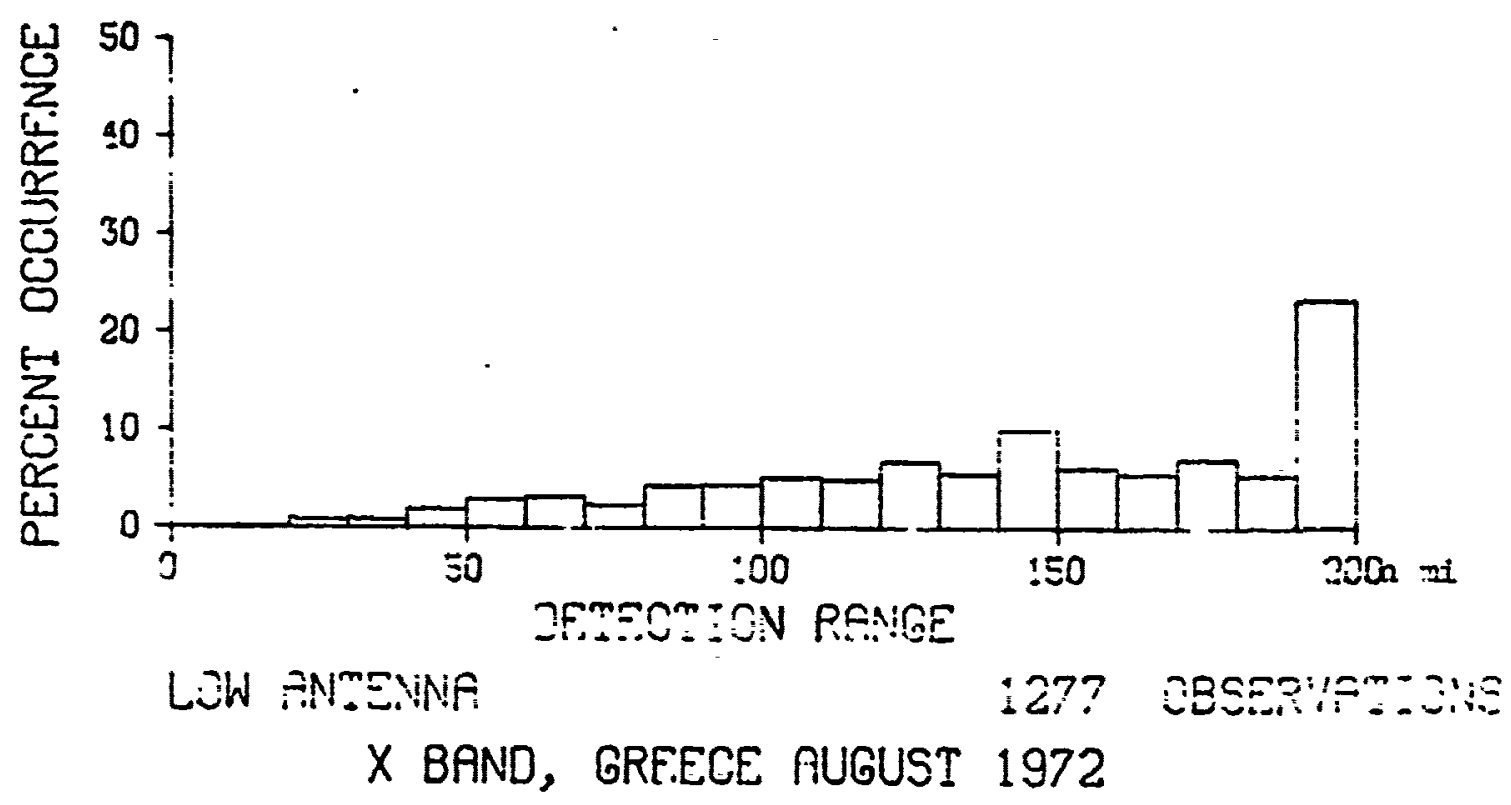




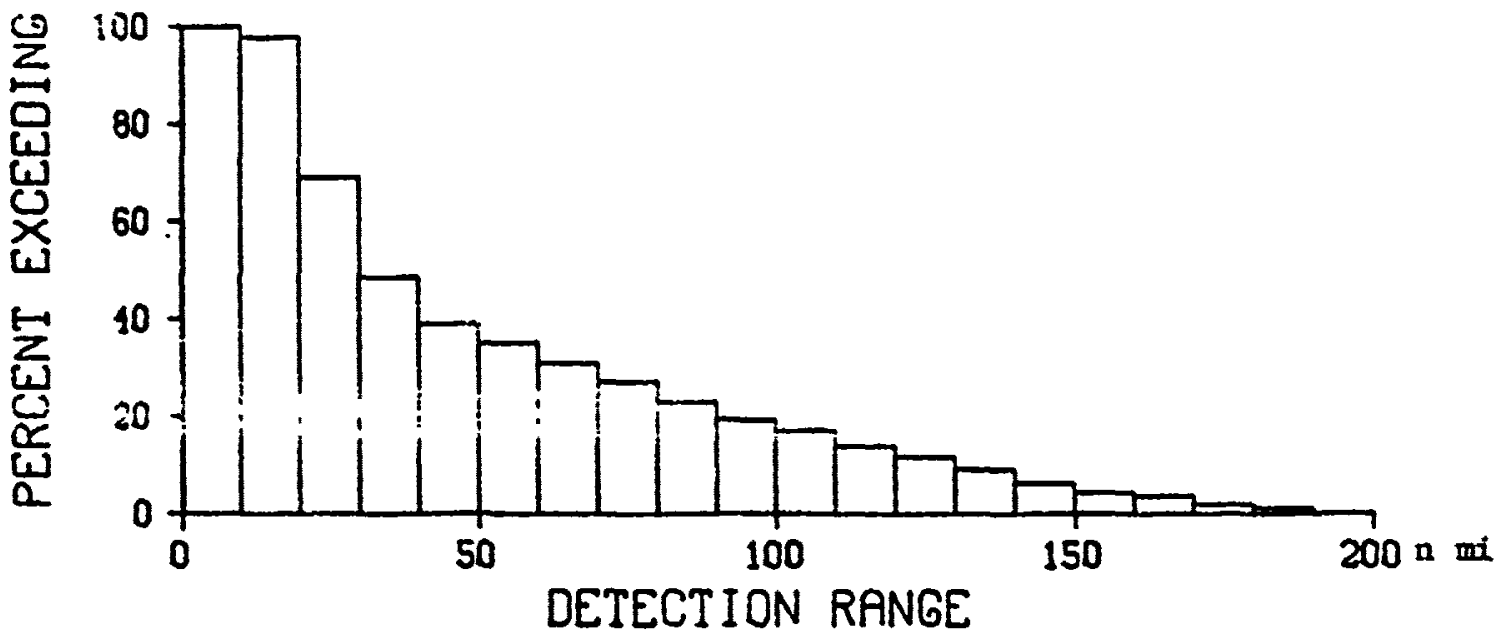

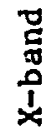
HIGH ANTENNA

1277 OBSERVATIONS

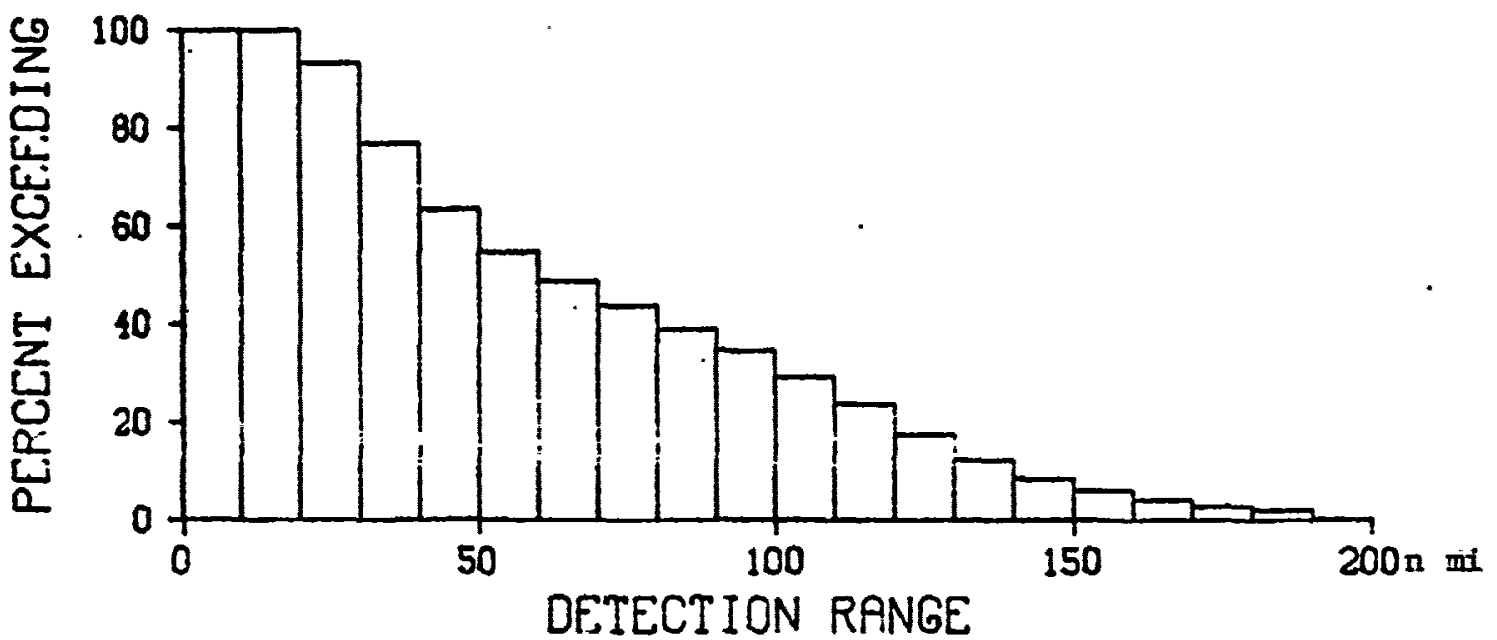

MID ANTENNA

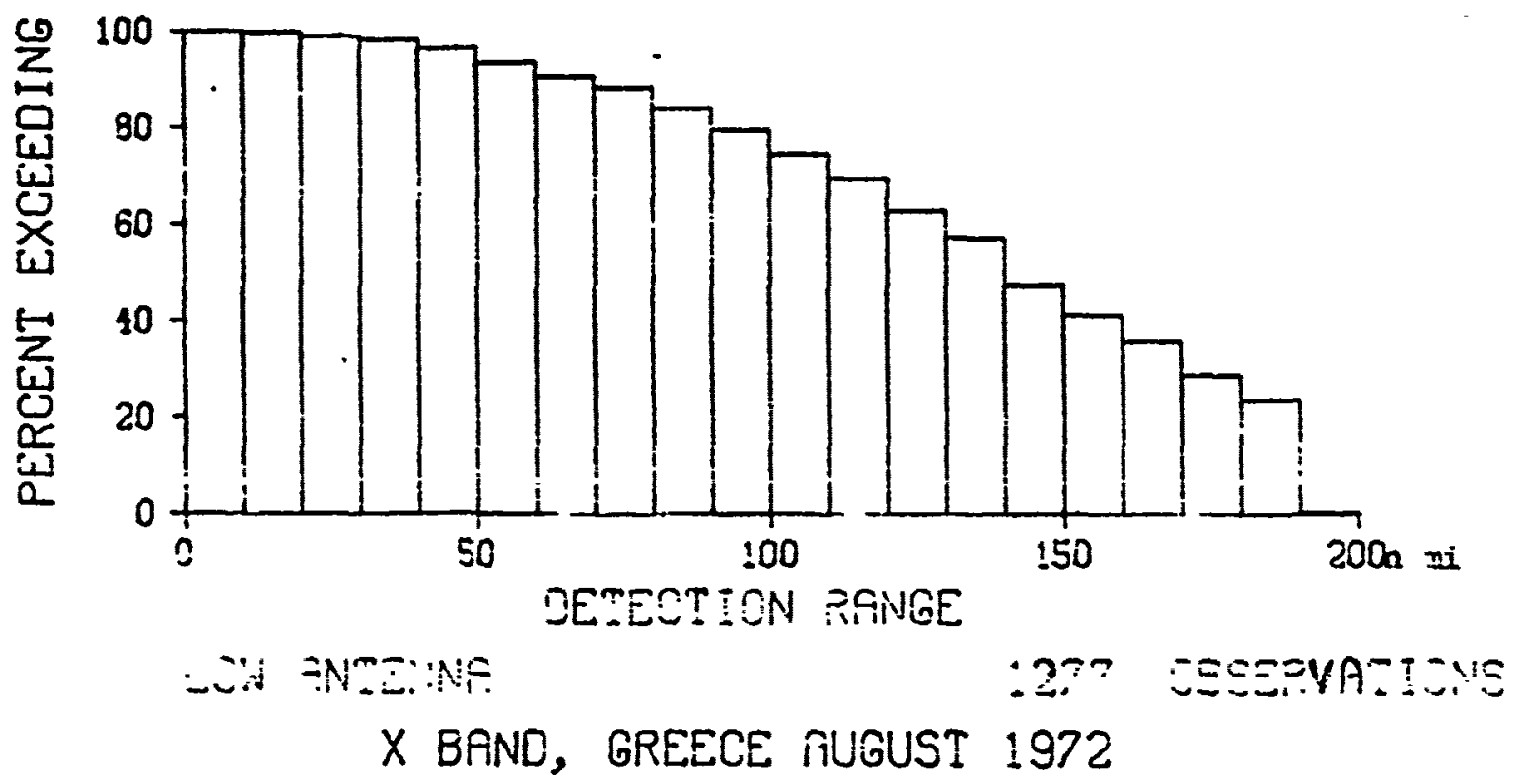

$\stackrel{0}{2}$ 

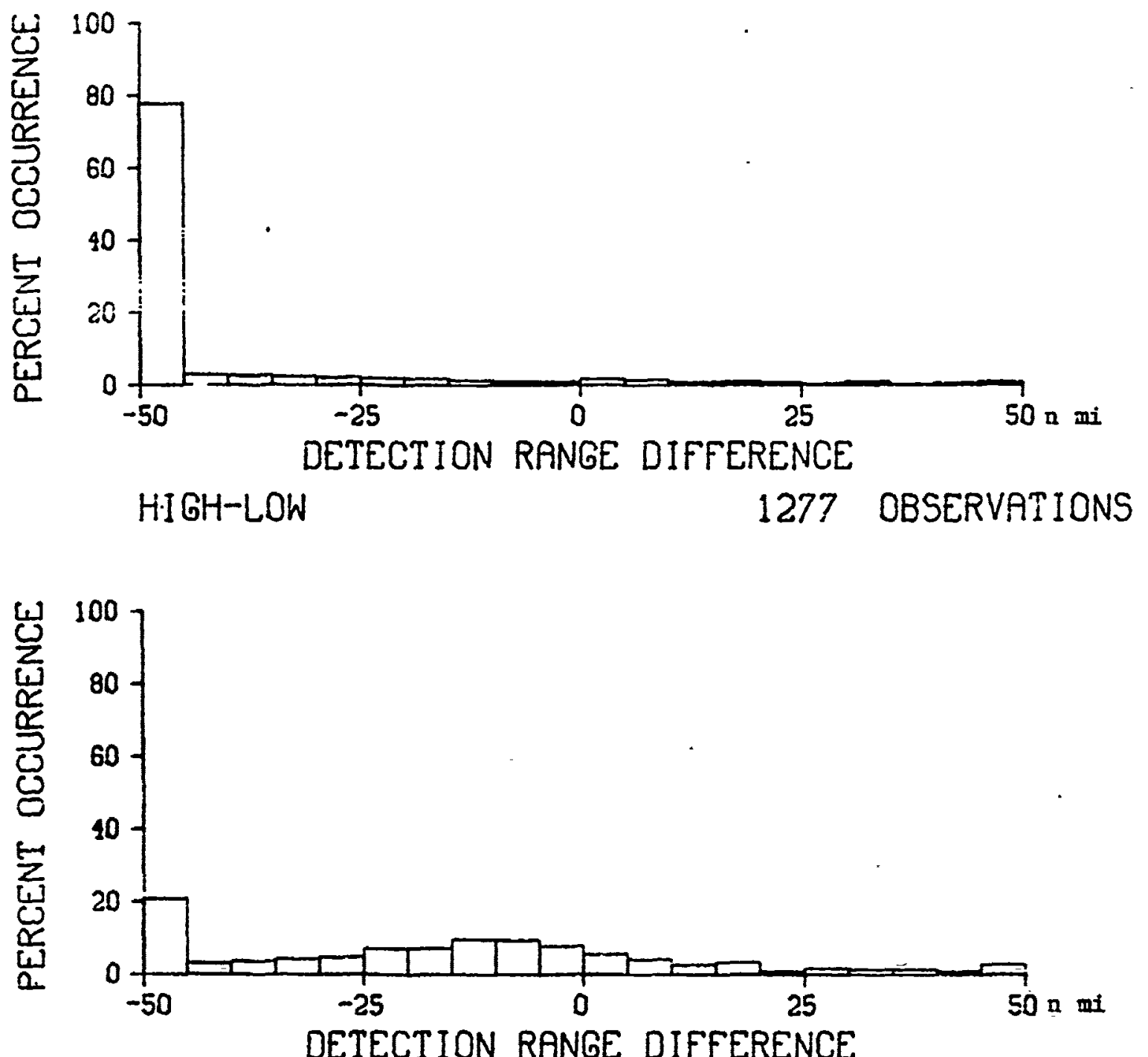

HIGH-MID

1277 CBSEPVATIONS

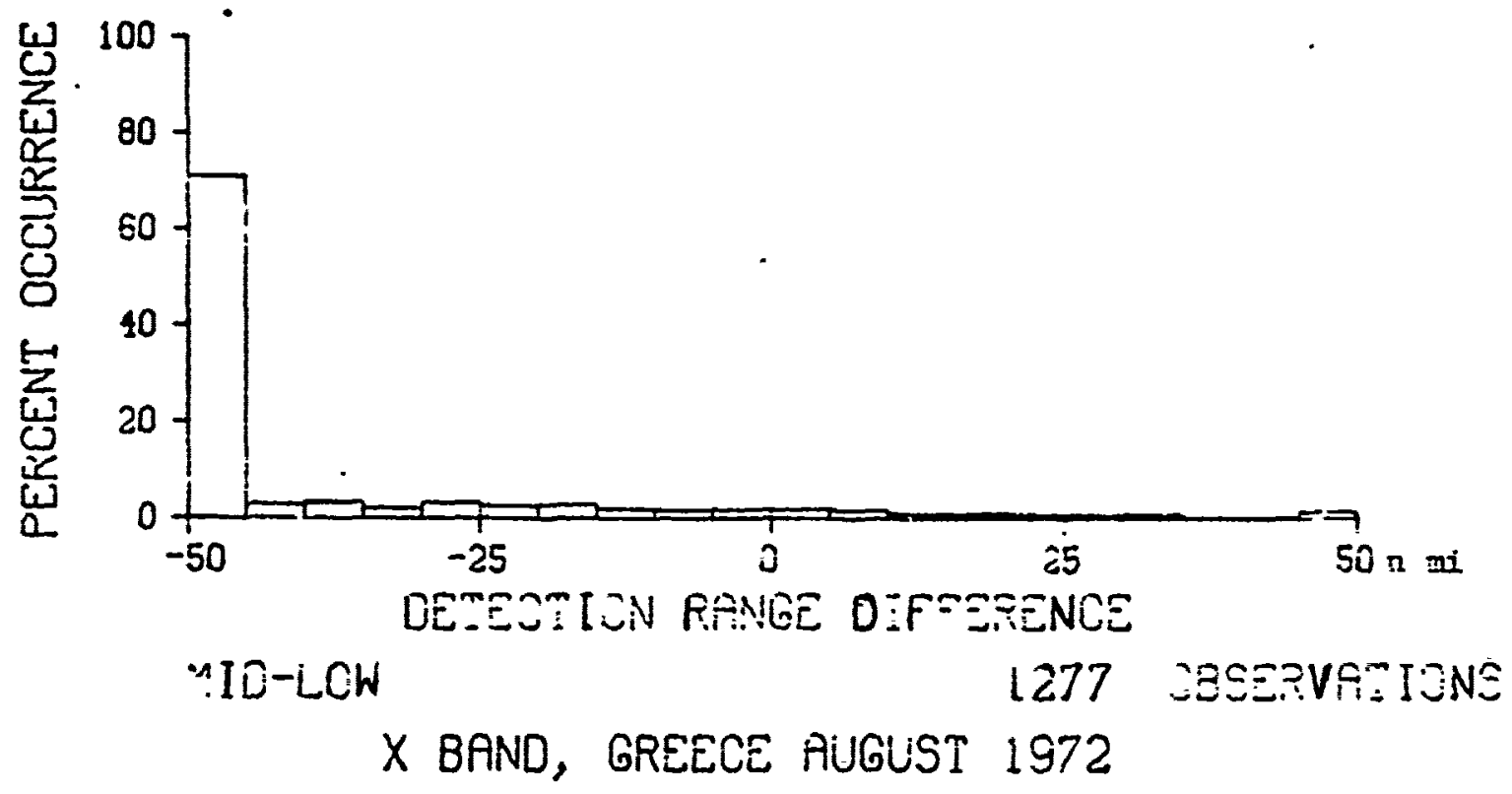



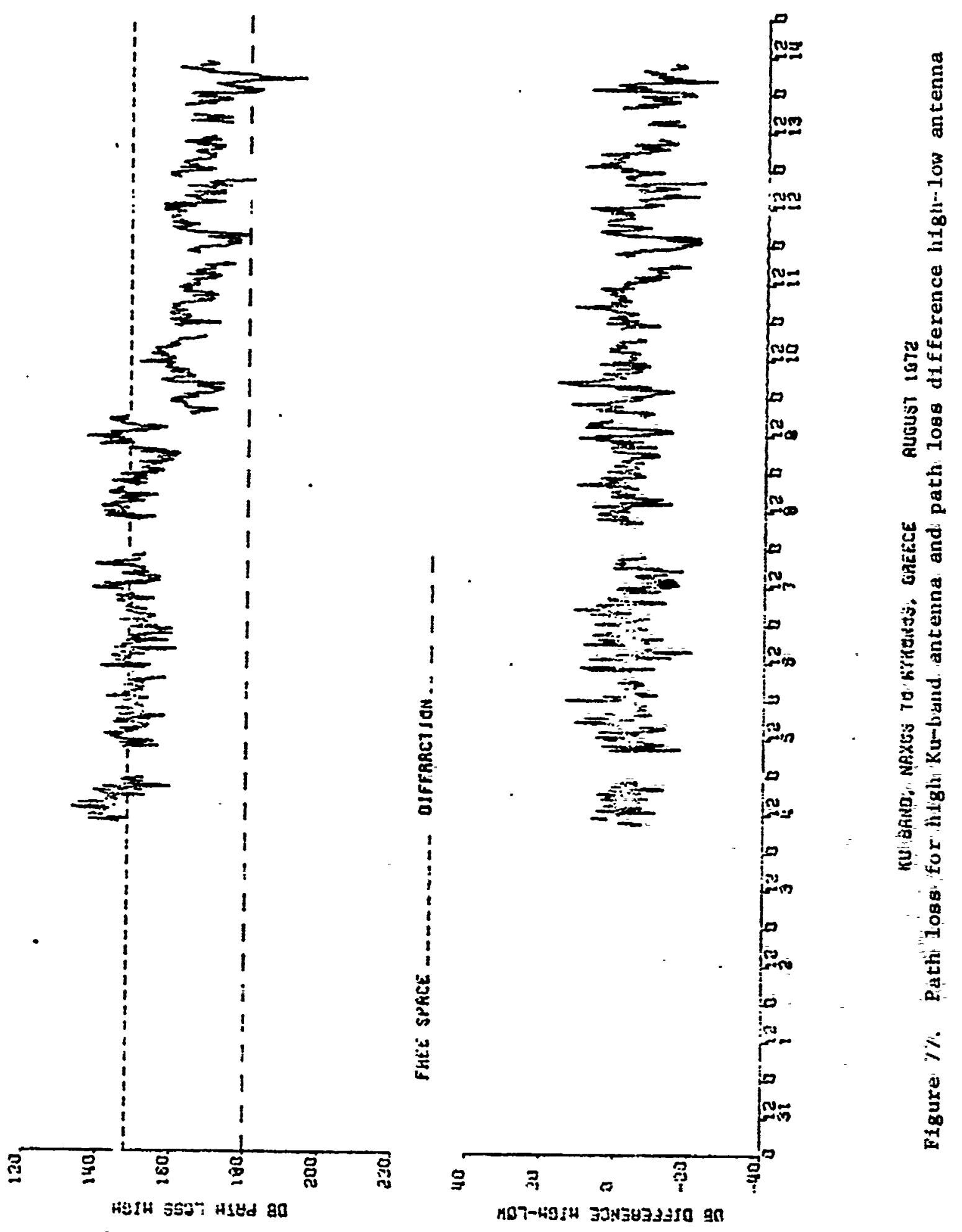


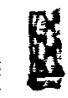

8

1

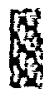
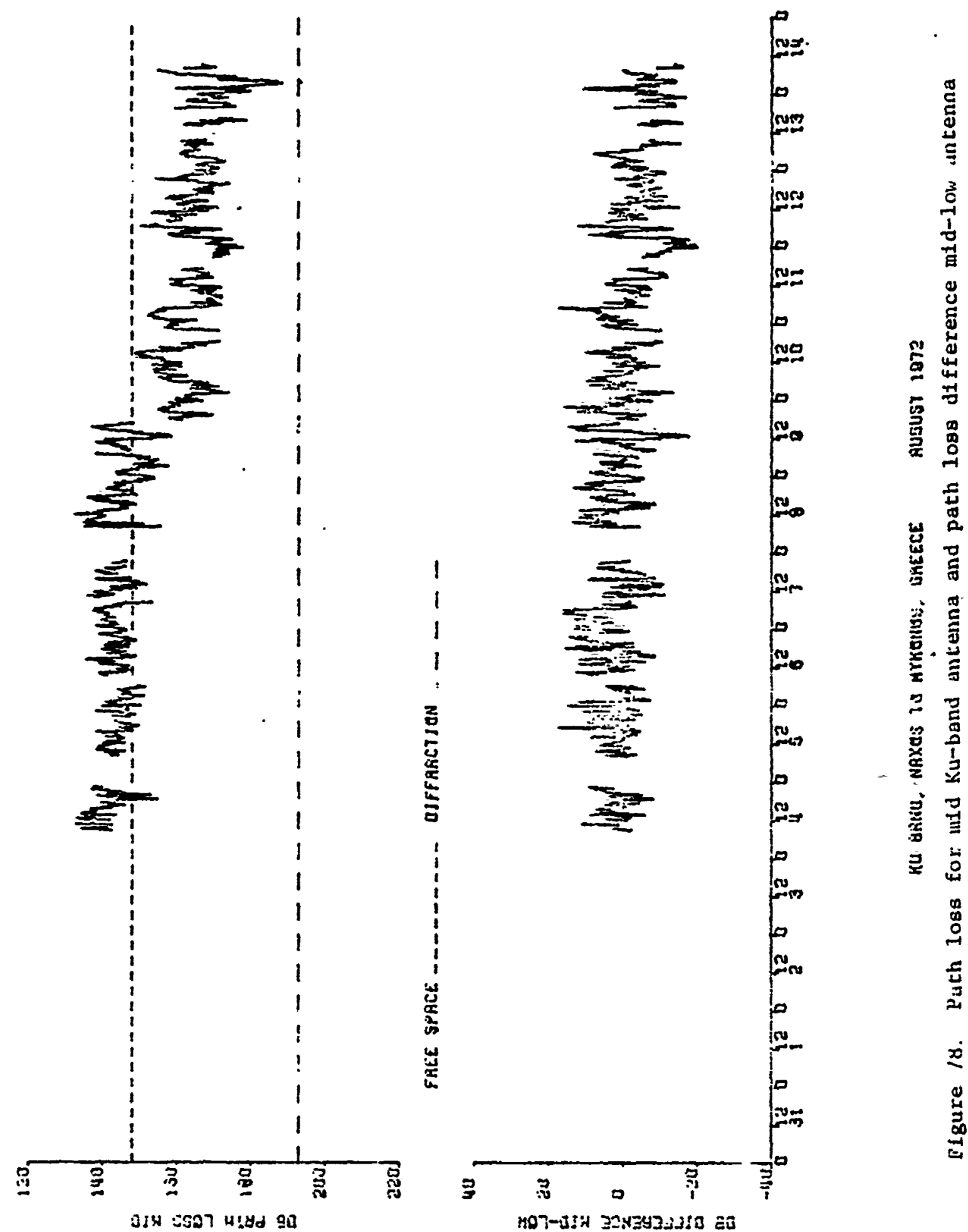

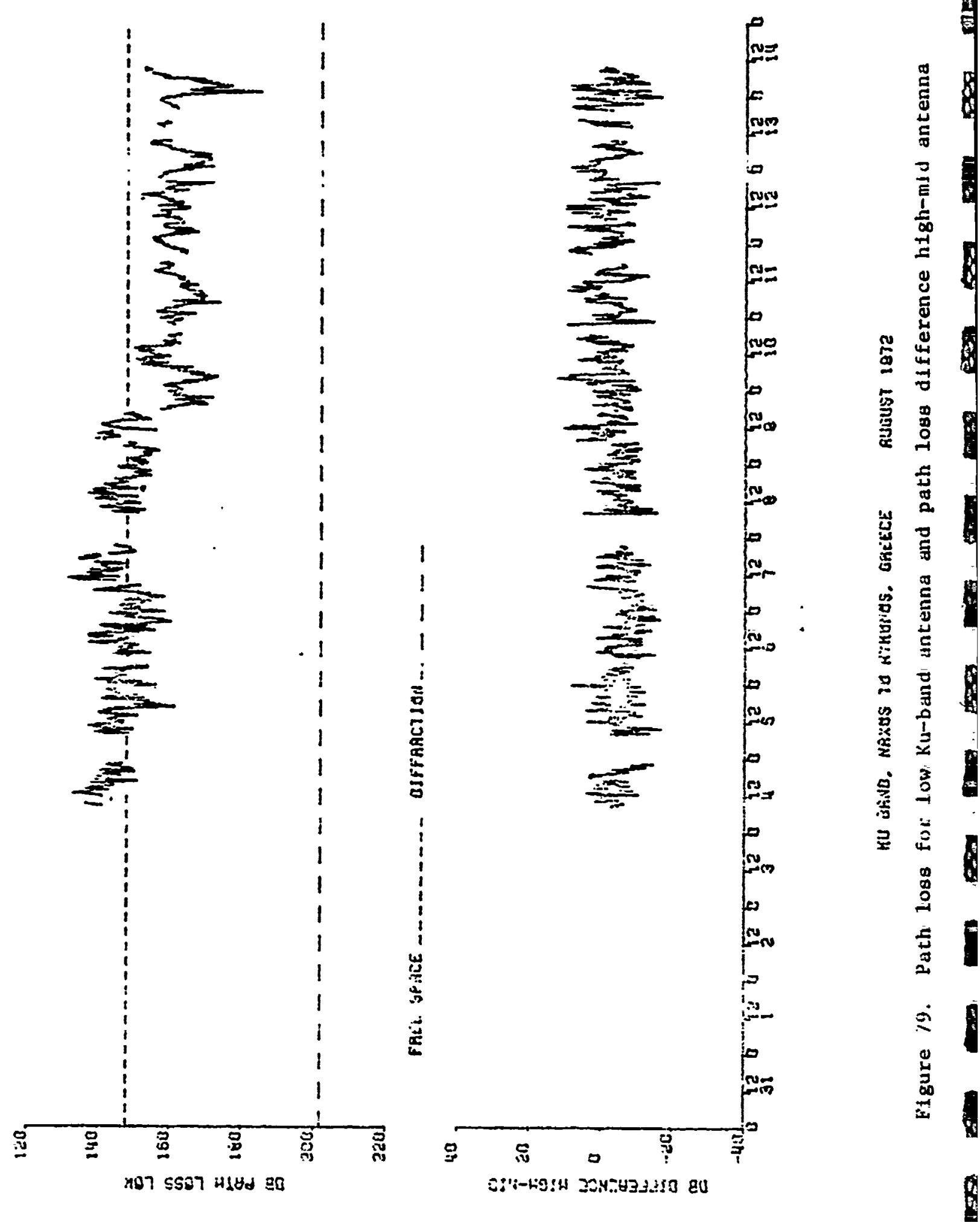
8

8

8

6
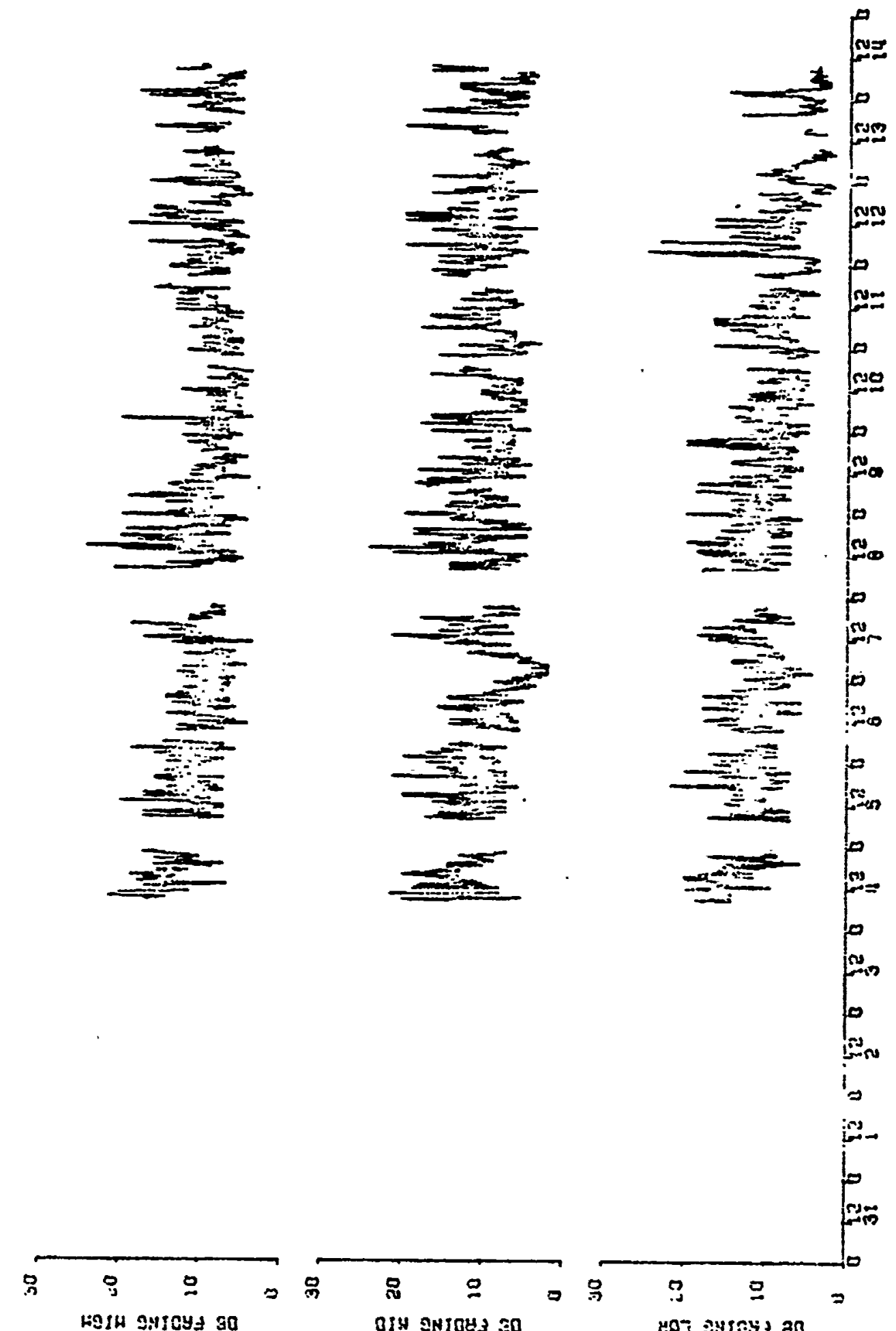

65

8

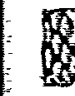

tis

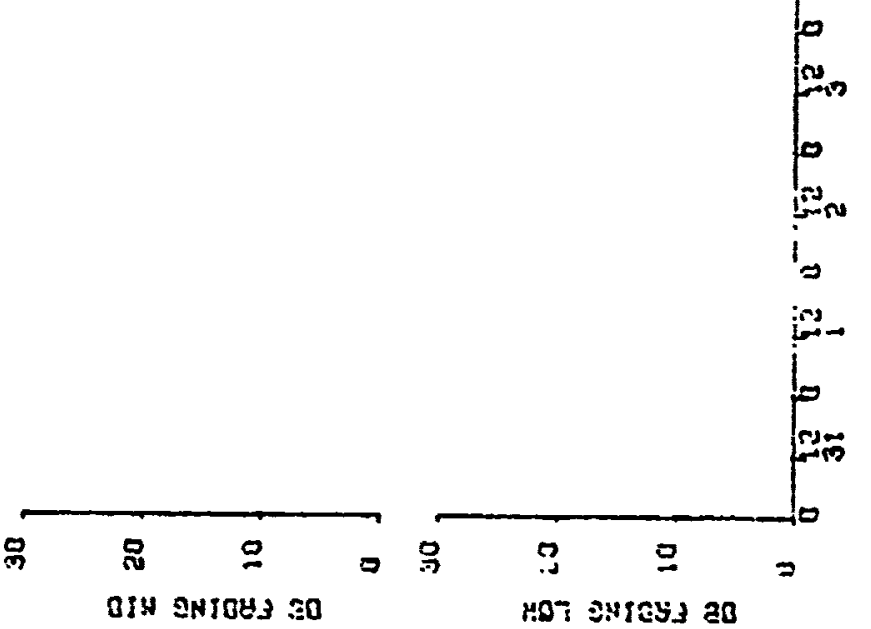

8

8 


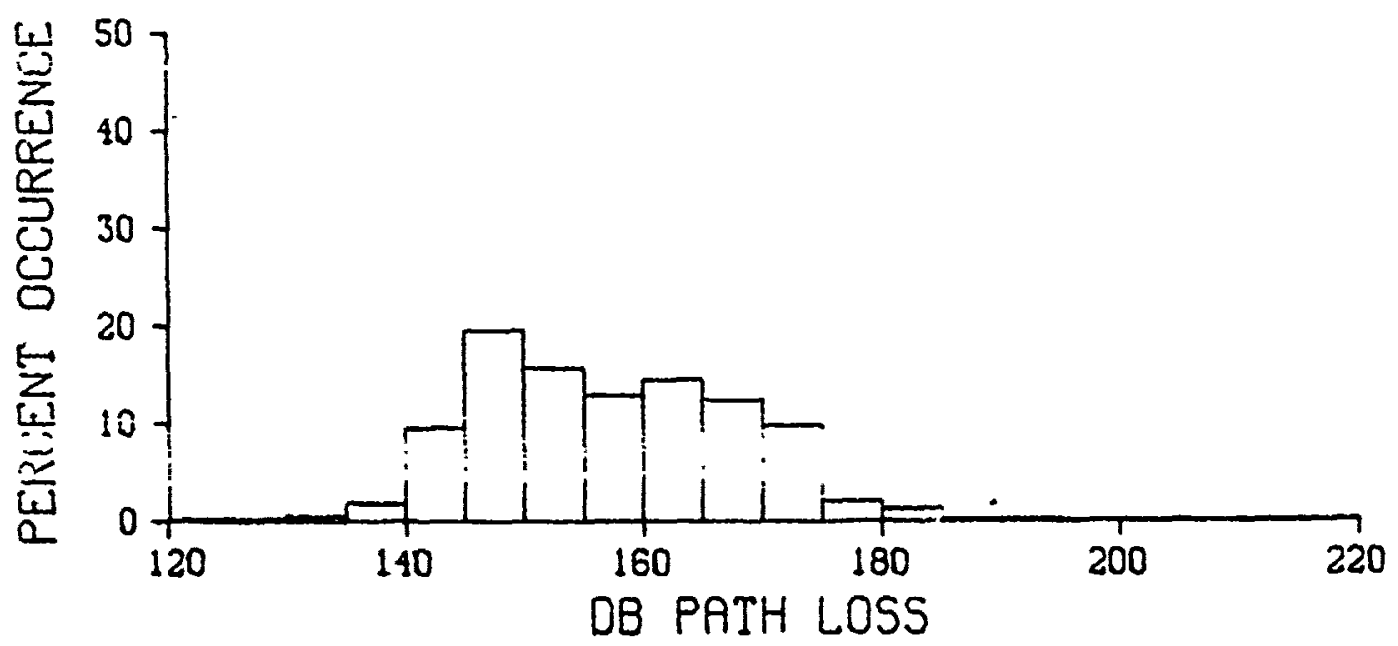

HIGH ANTENNA

777 OBSERVATIONS

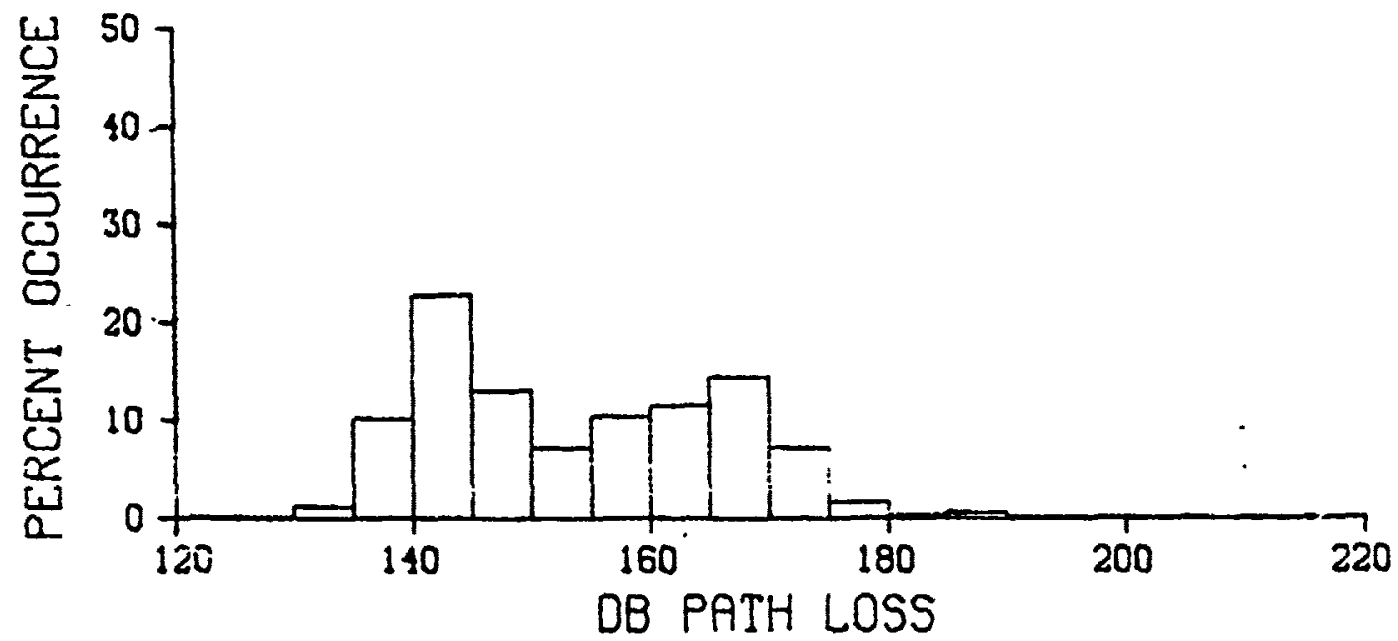

MID ANTENNA

772 OBSERVATIONS

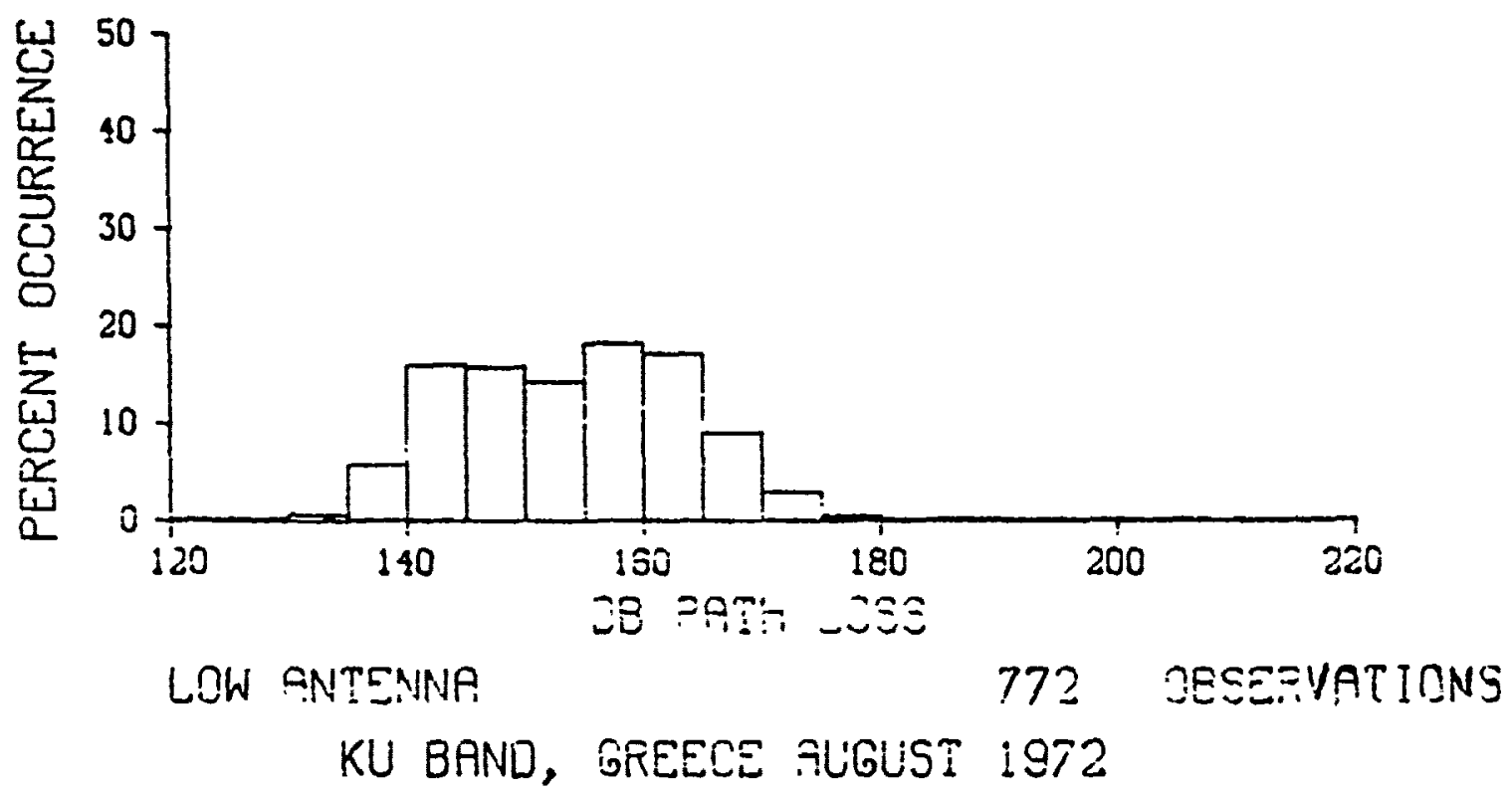




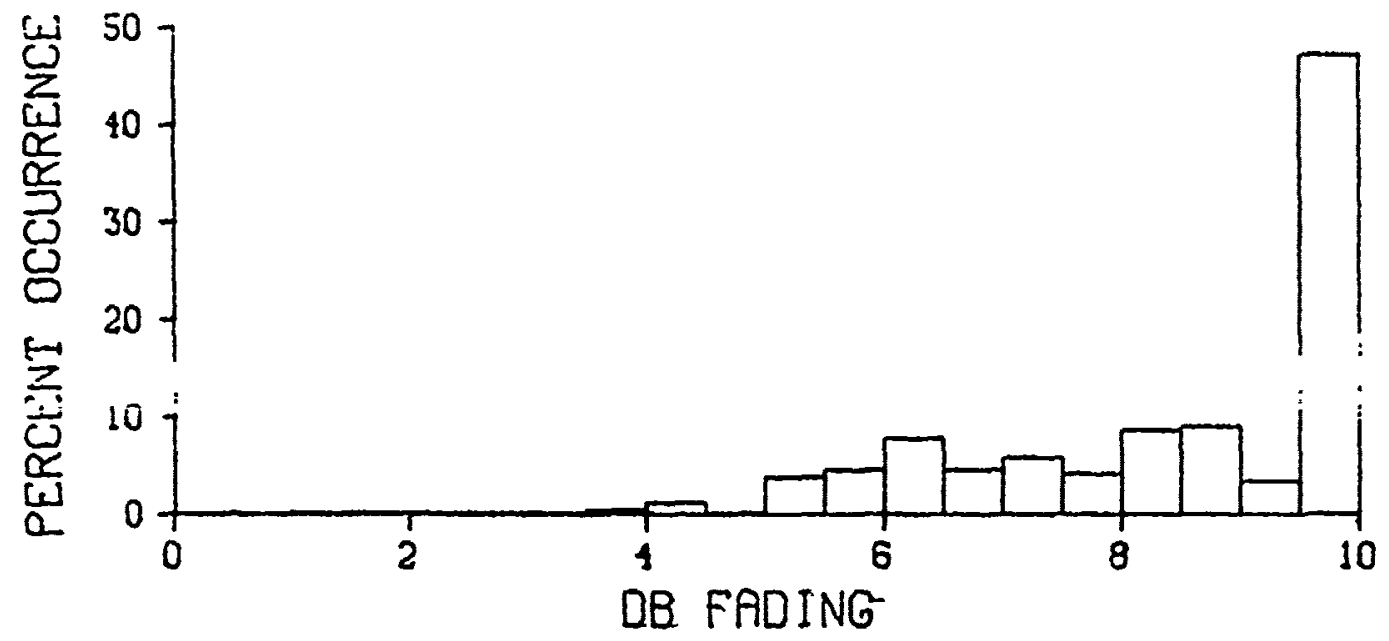

章

6

HIGF ANTENNA

777 OBSERVATIONS

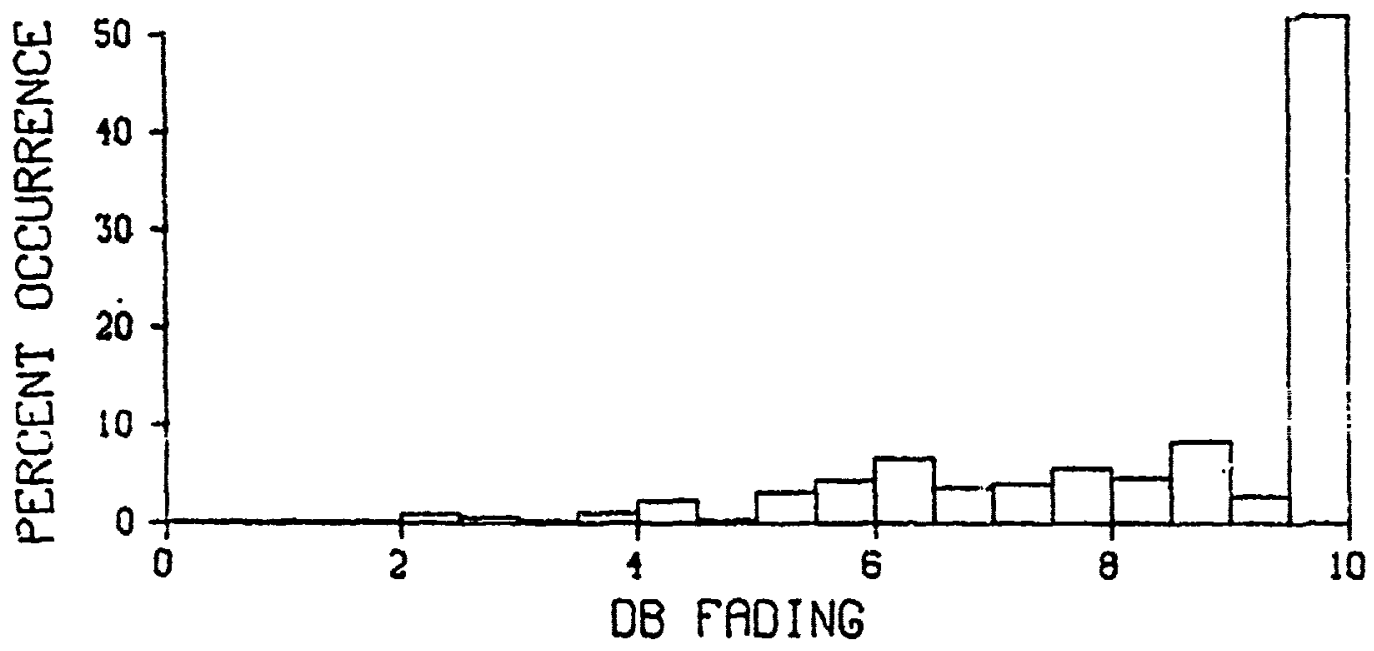

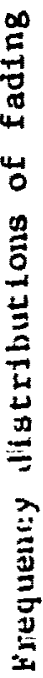

8

MID ANTENNA

772 OBSERVATIONS

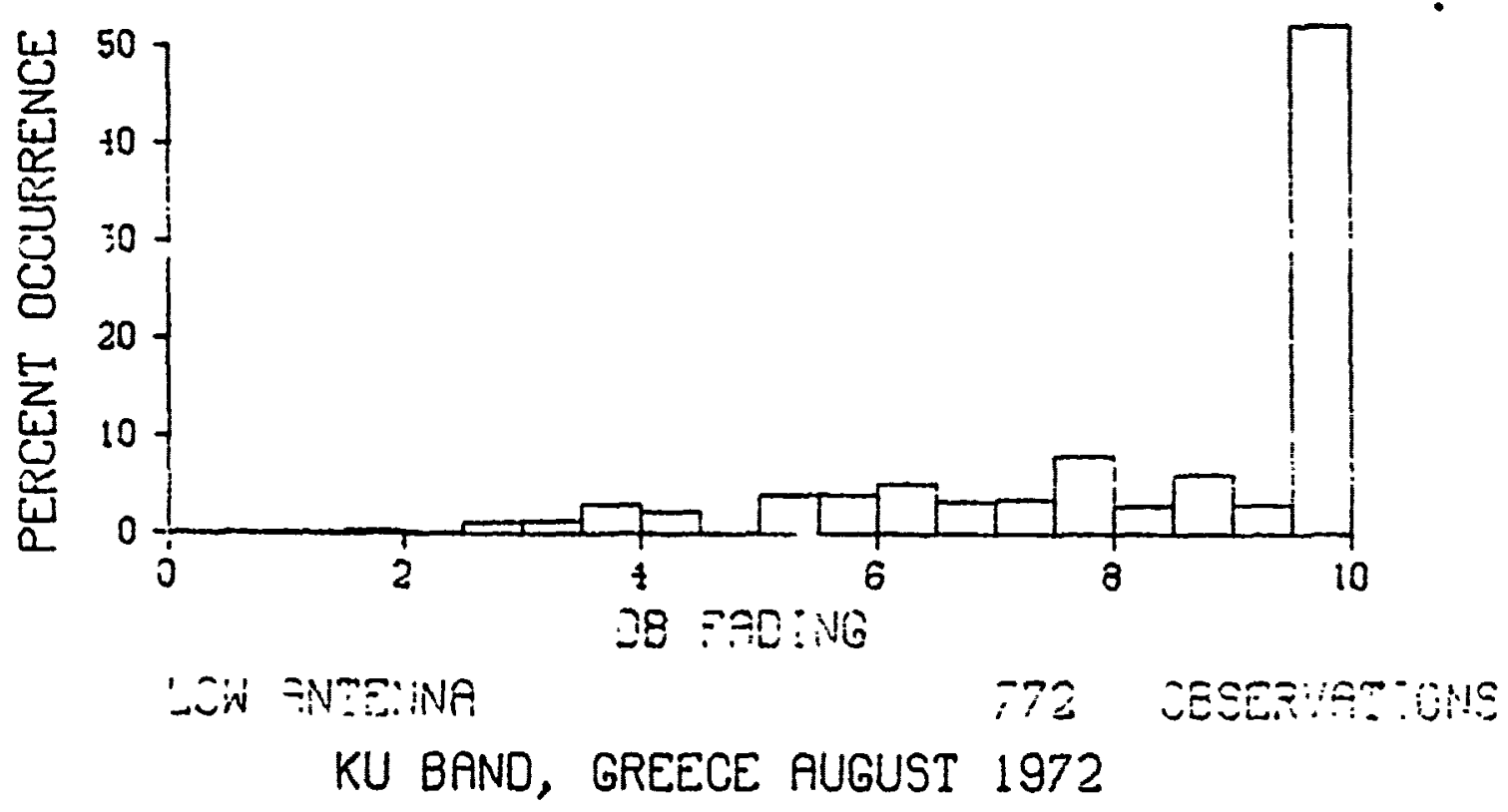



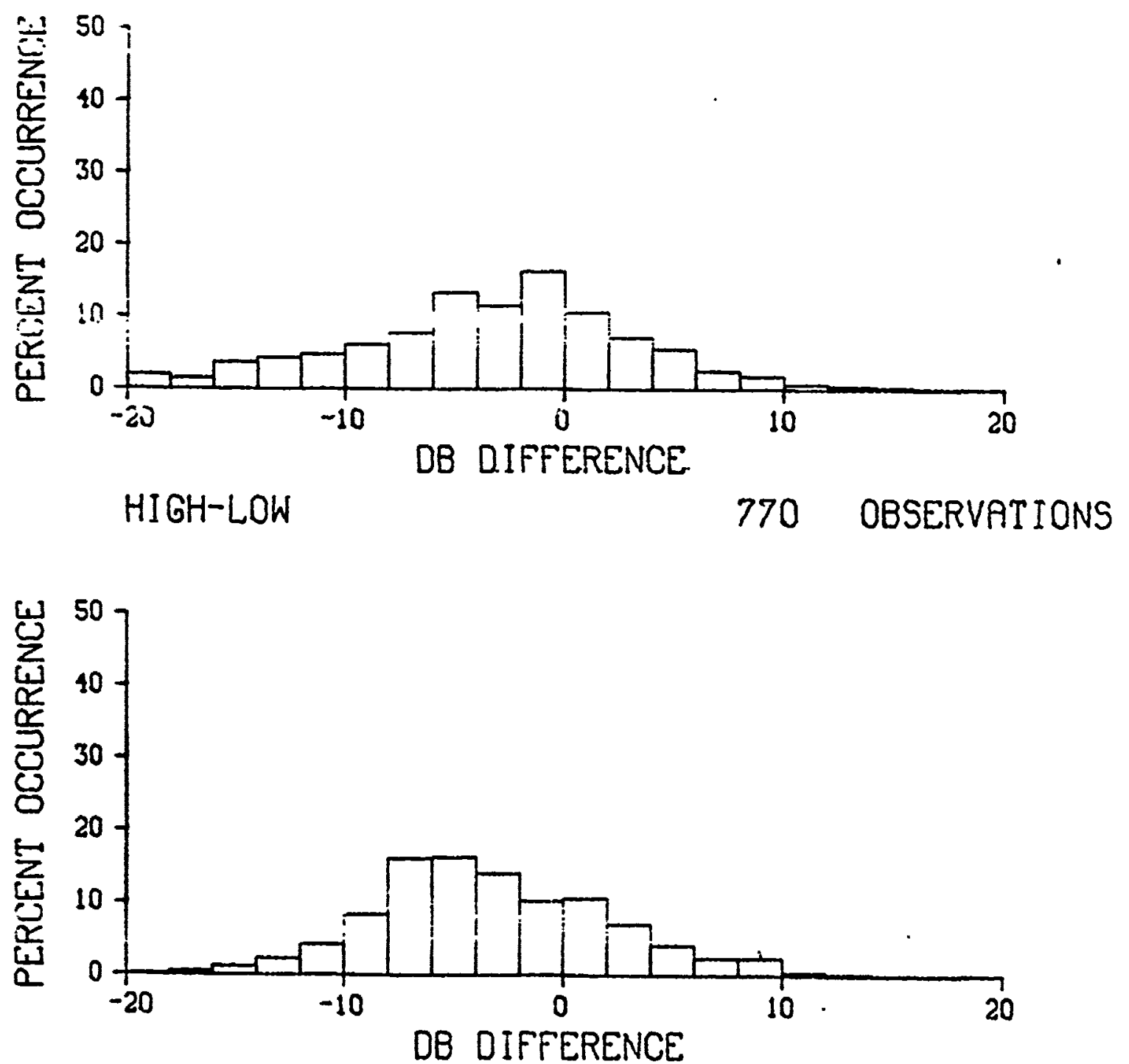

HIGH-MID

771 OBSERVATIONS

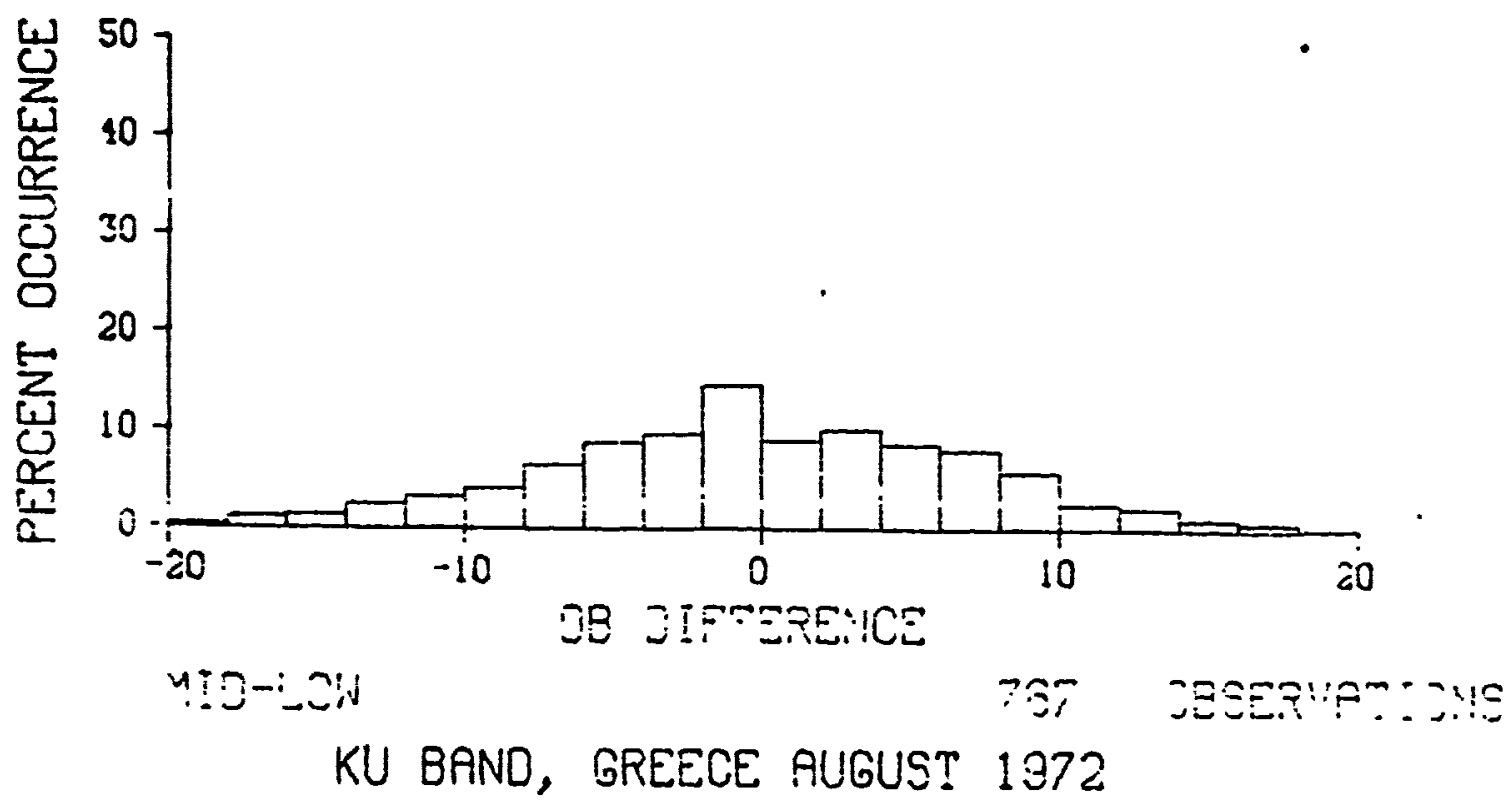



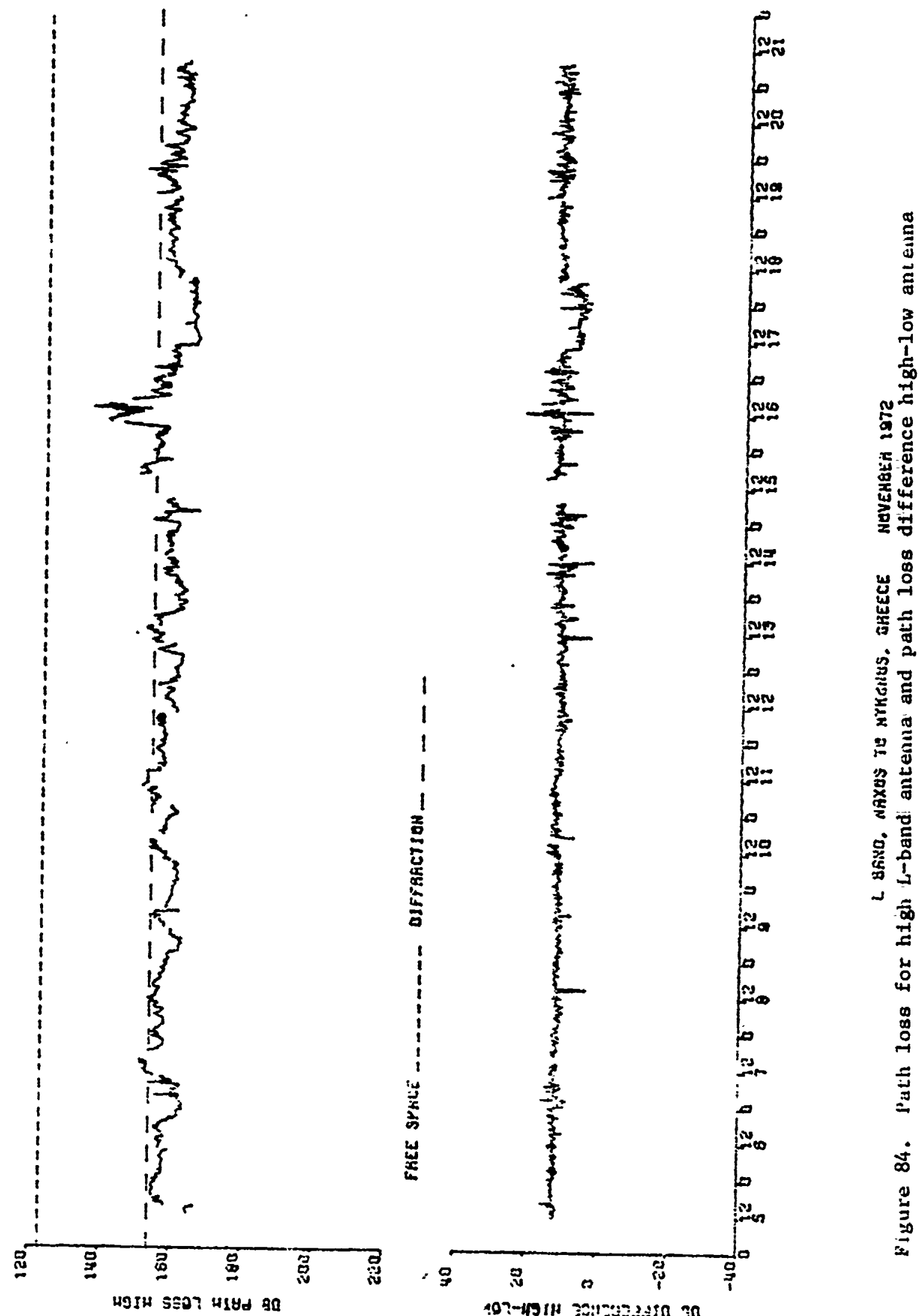

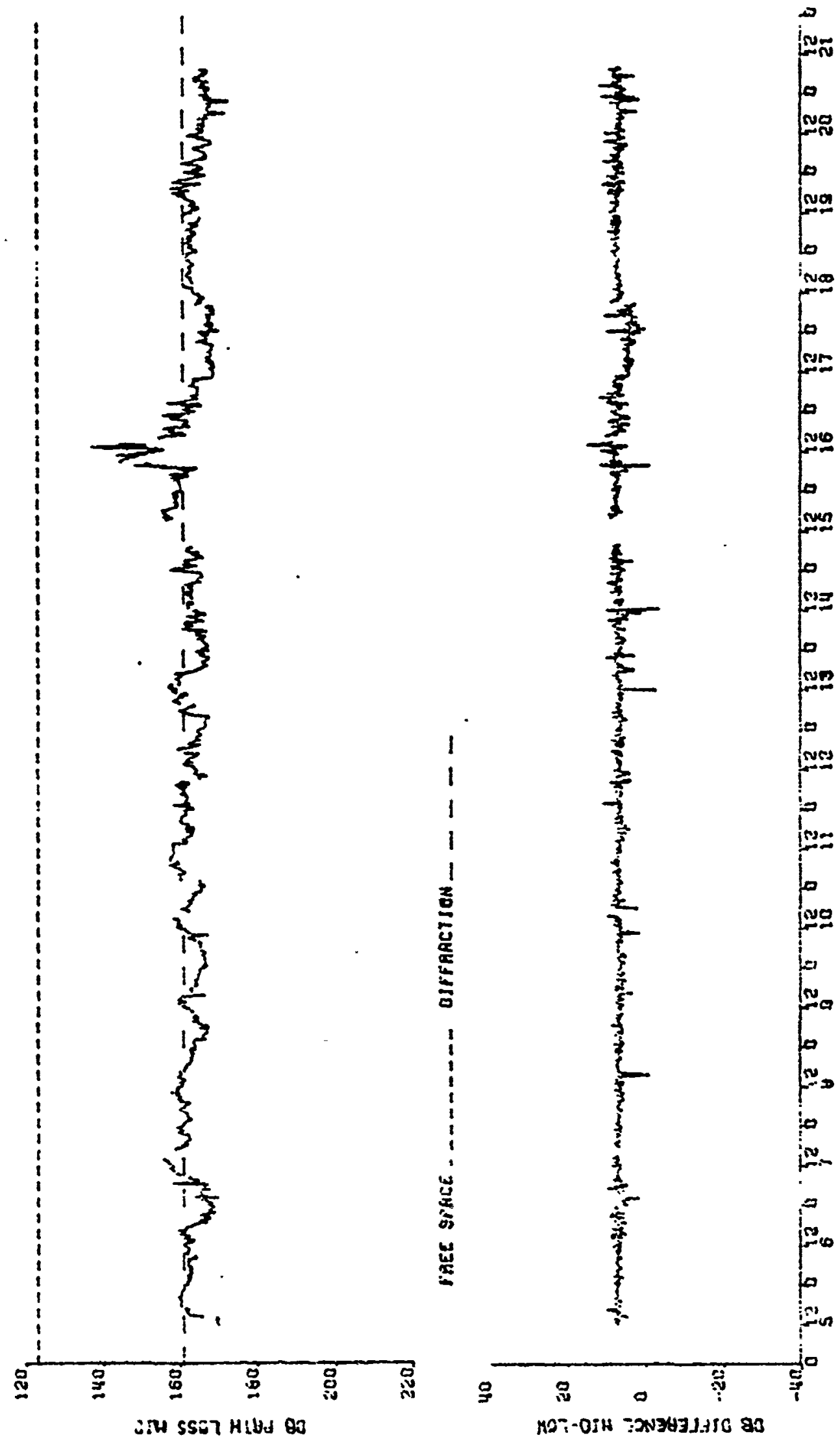

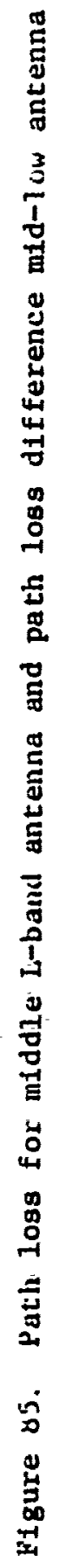




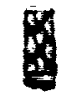

8

1

$x$
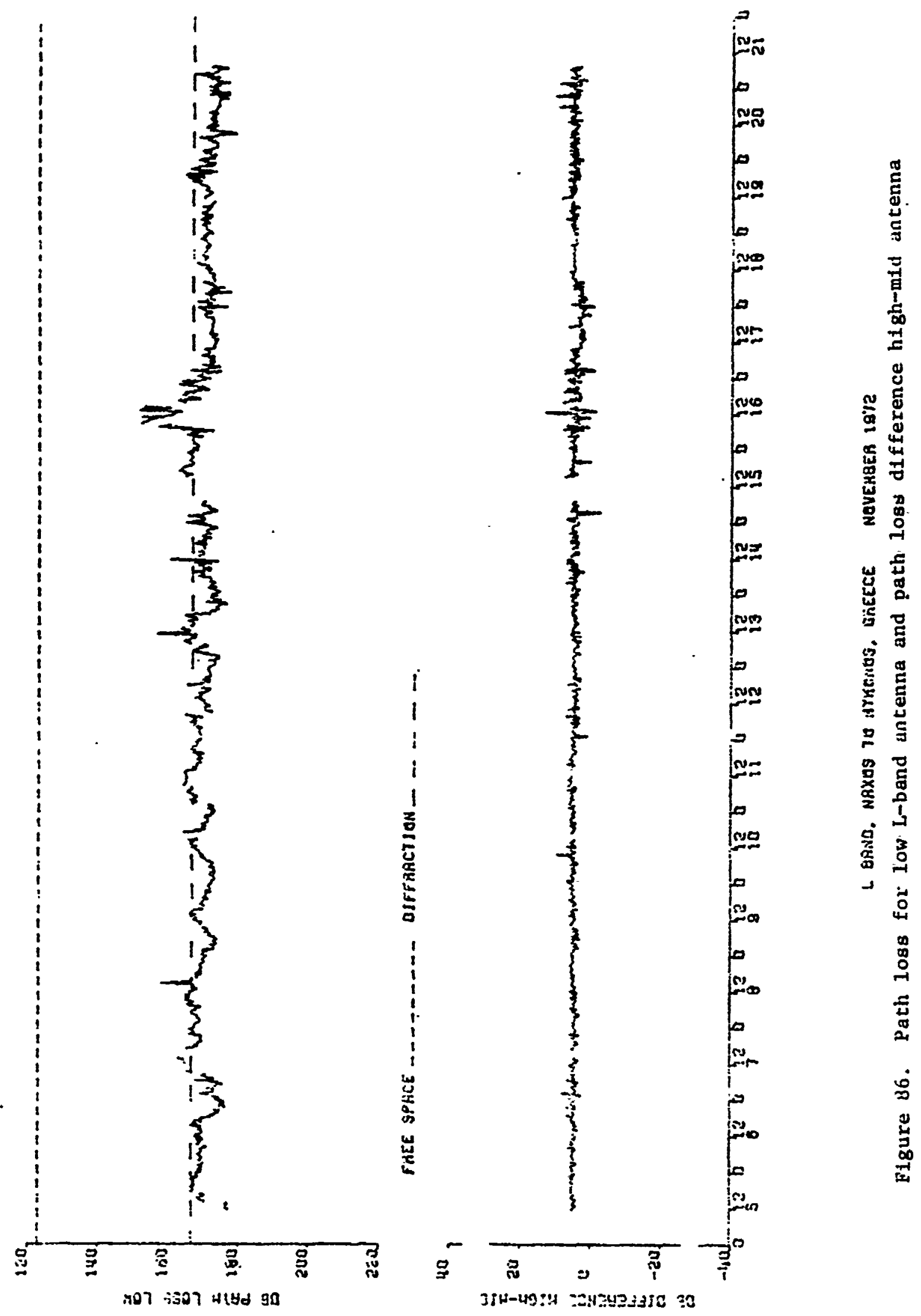

19

b 


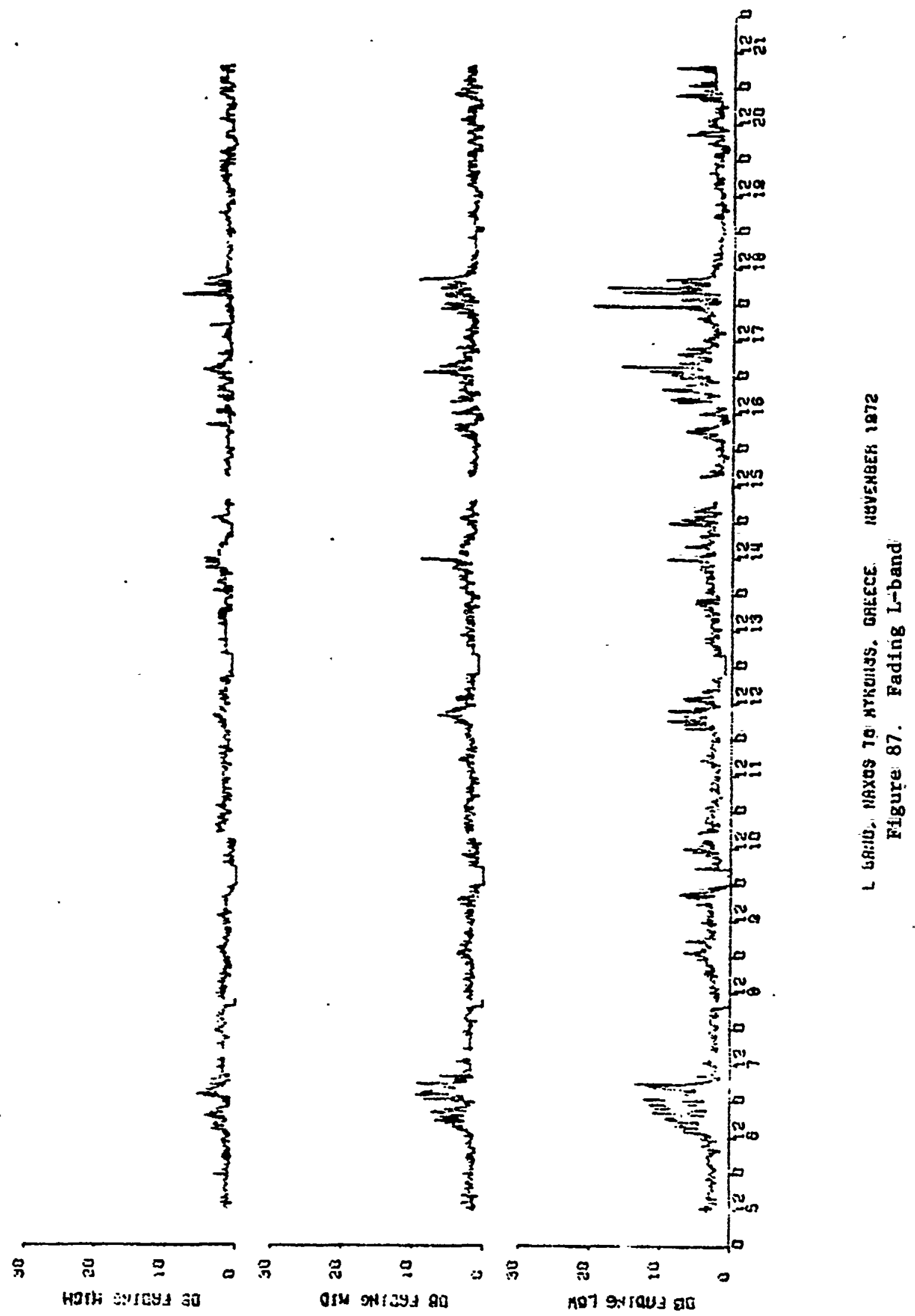




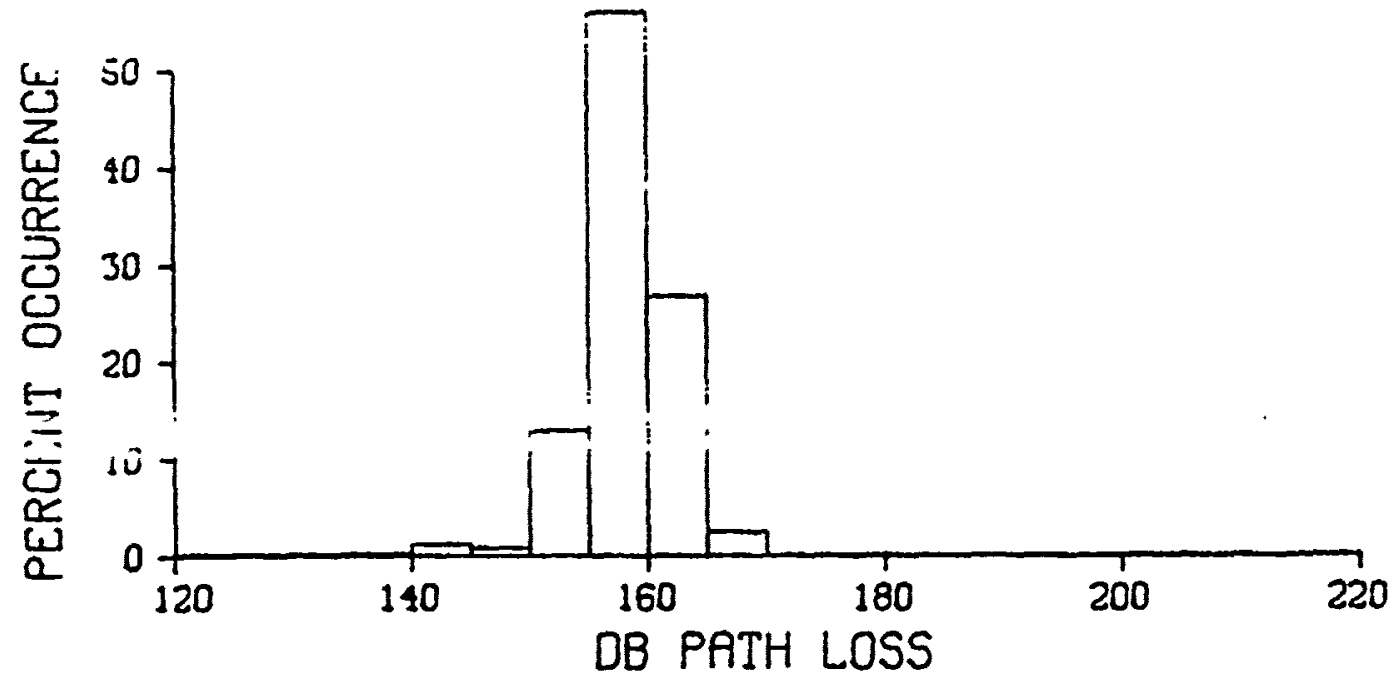

㽞 HIGH RNTENNA 1405 OBSERVATIONS 总
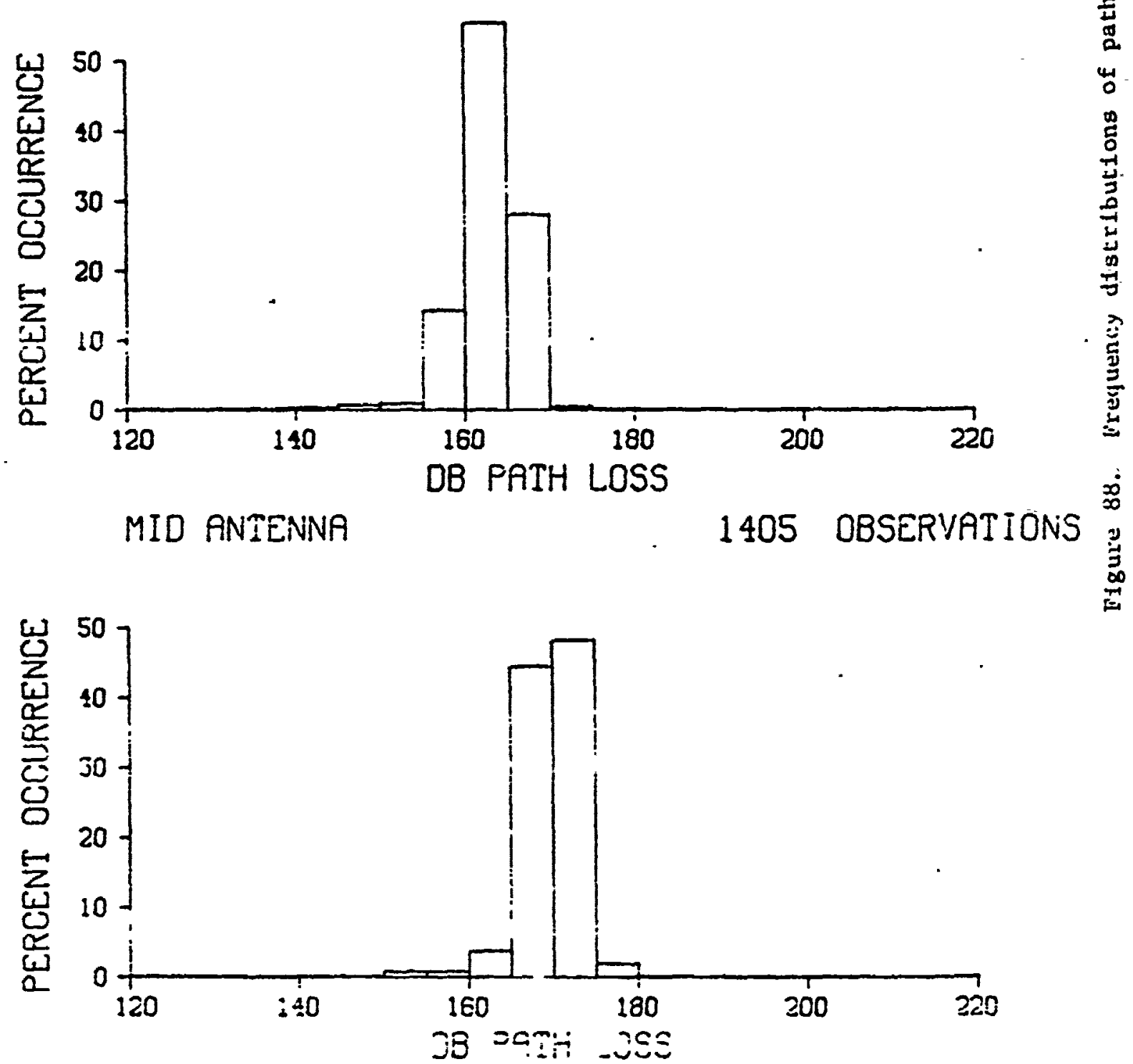

LCW PNOENHî

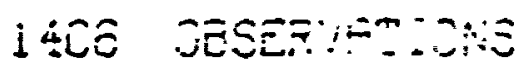

$\triangle$ BFND, GREECE NOVEMEER 1972 

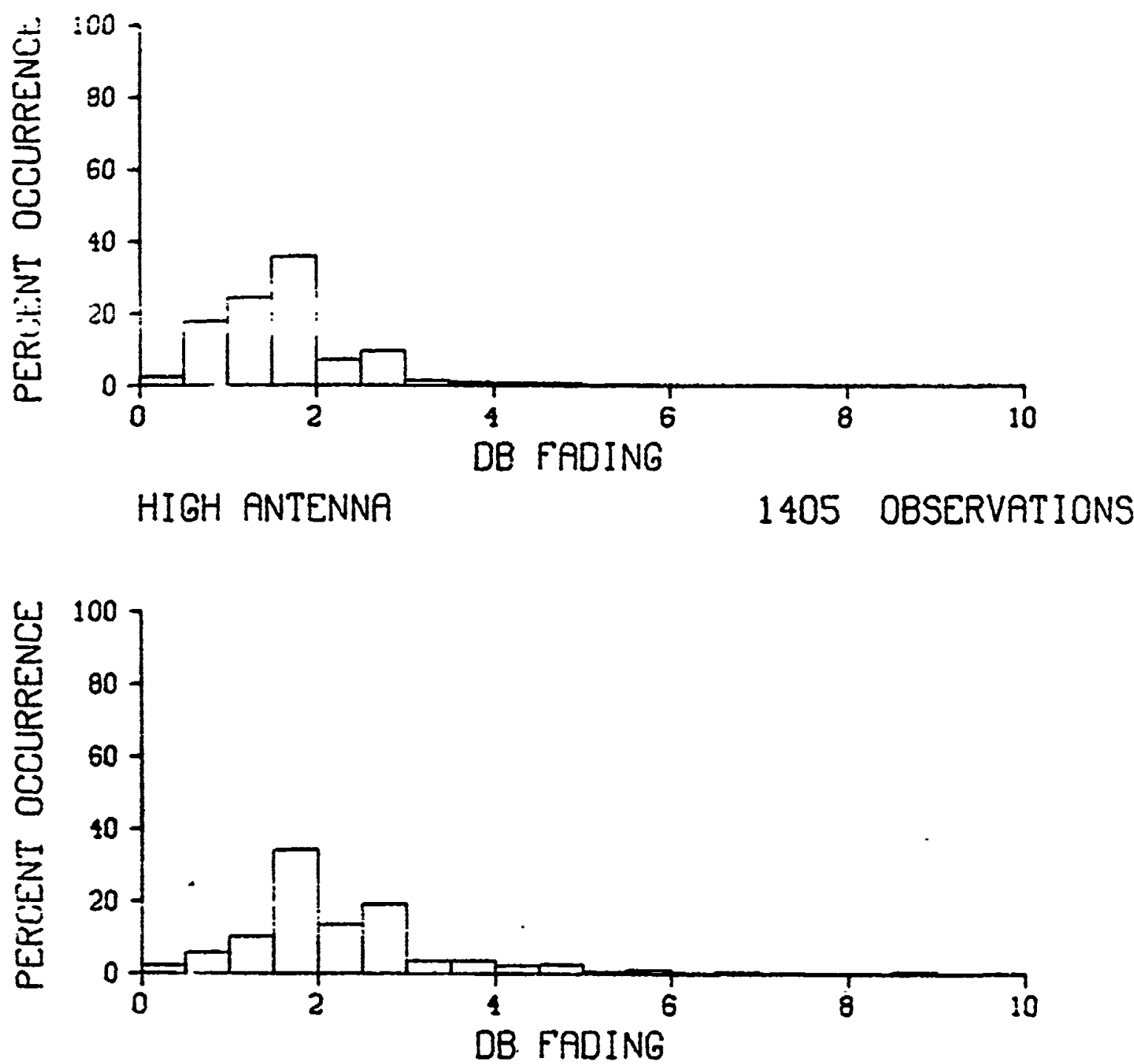

:

MID ANTENNA

1405 OBSERVATIONS

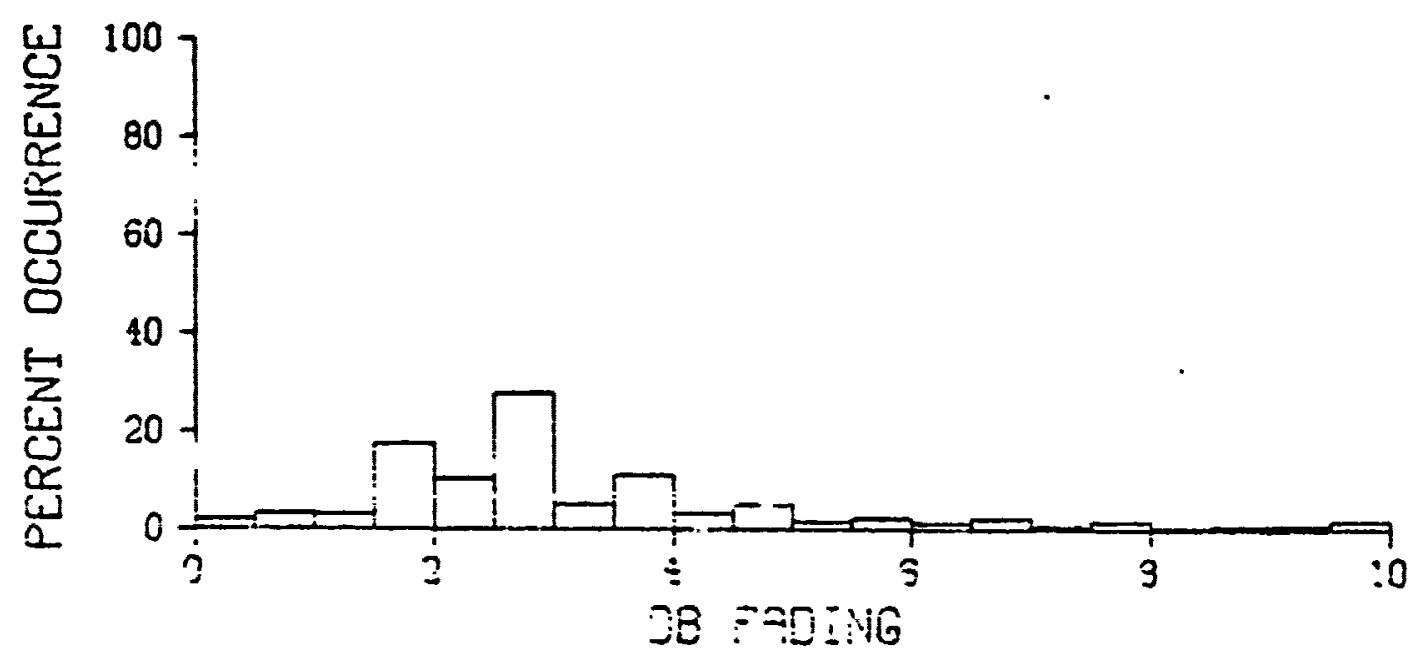

- 20 ing

บำ

$L$ BAND, GREECE NGVEMBER 1972 

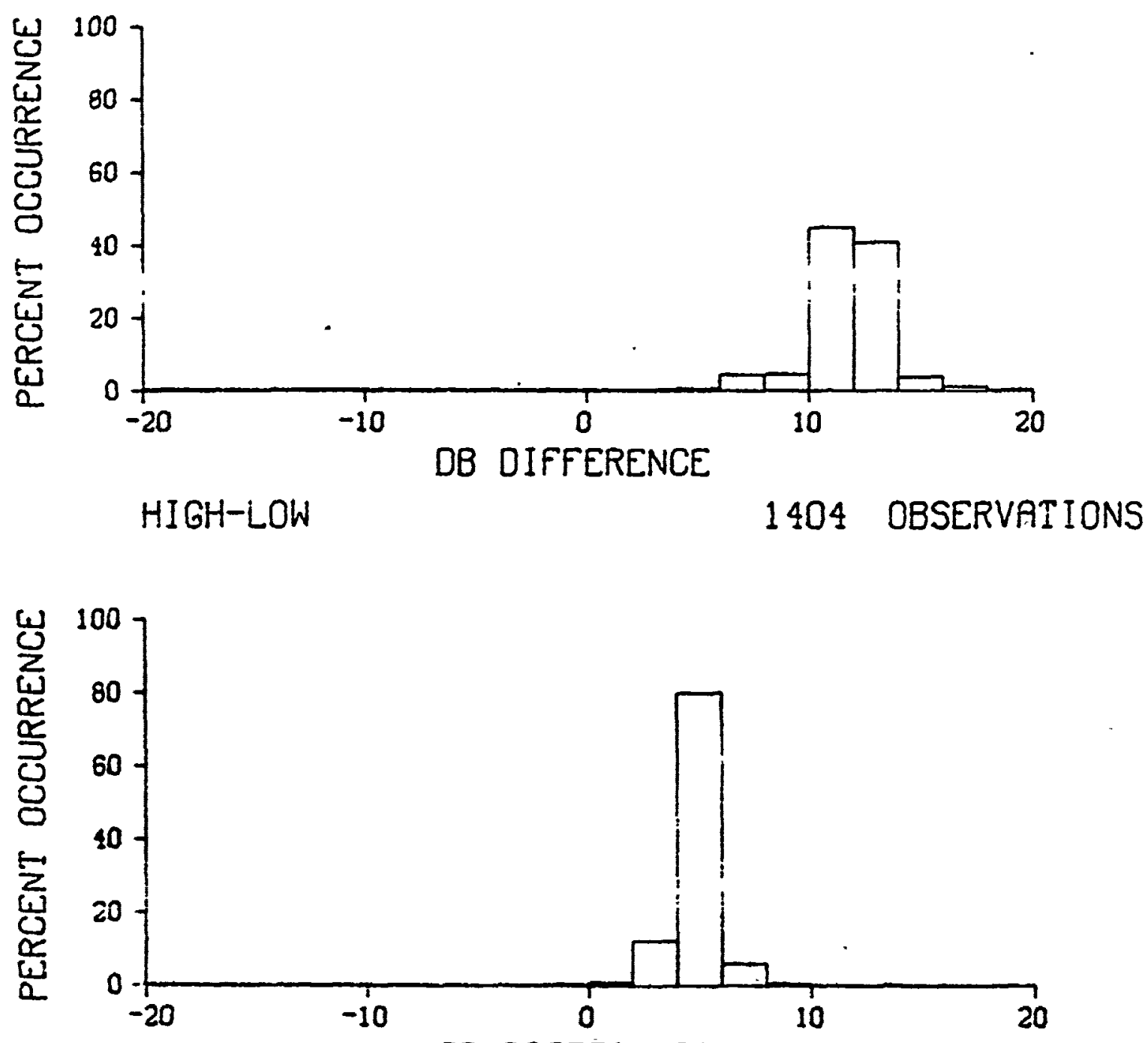

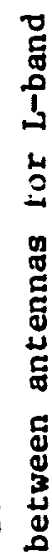

8

8

DB DIFFERENCE

HIGH-MID

1403 OBSERVATIONS $\stackrel{5}{\frac{1}{7}}$

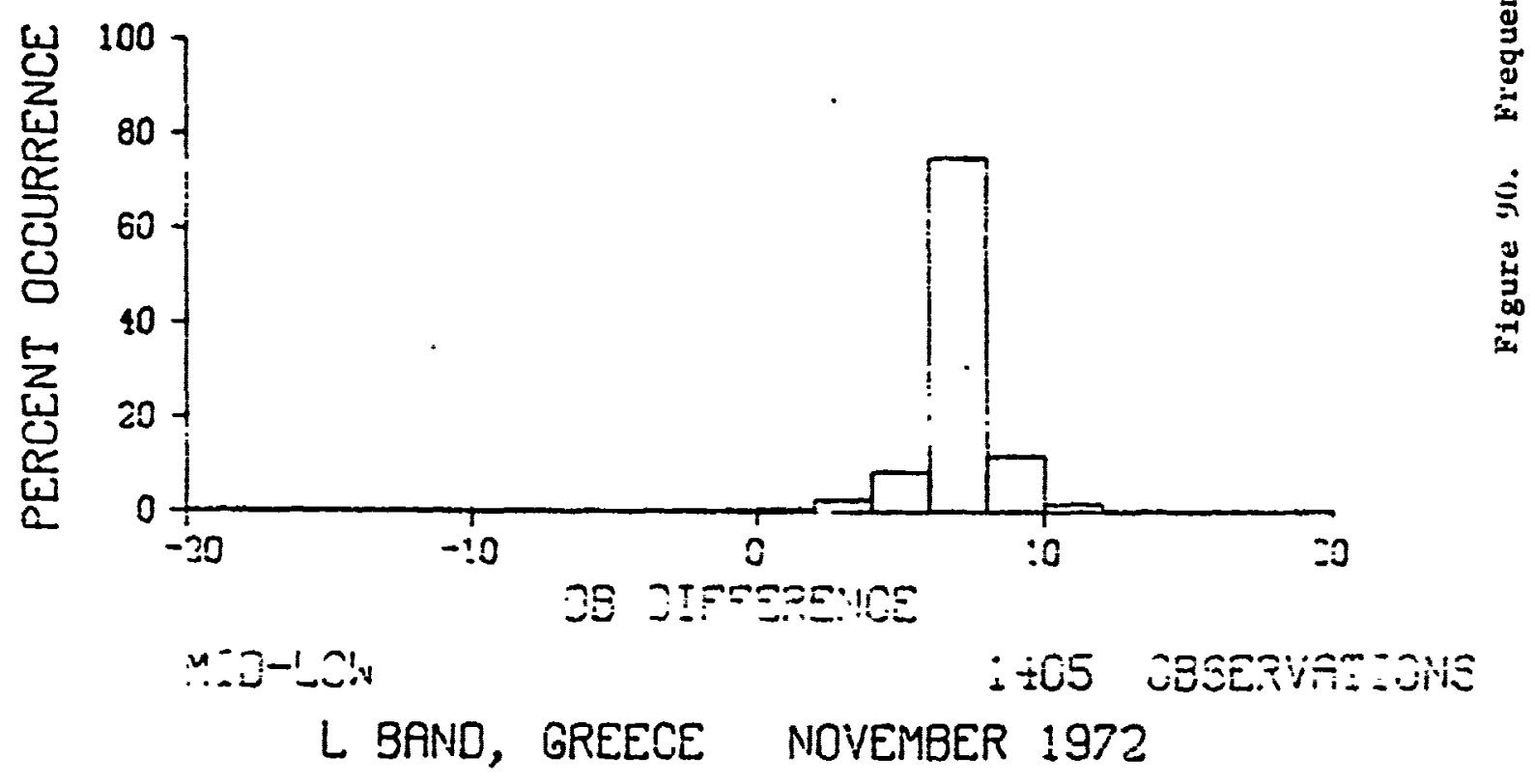



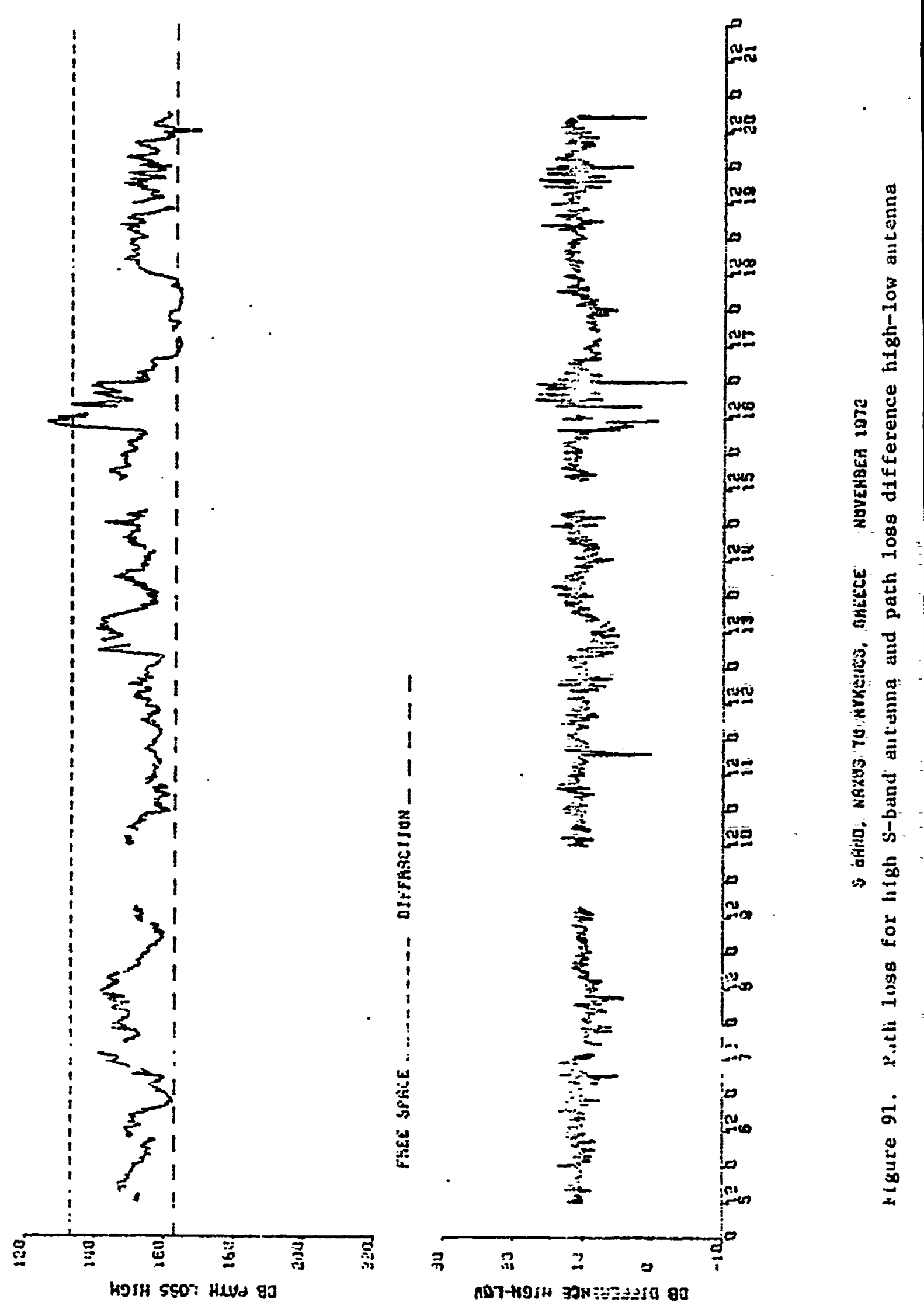
a

8

8
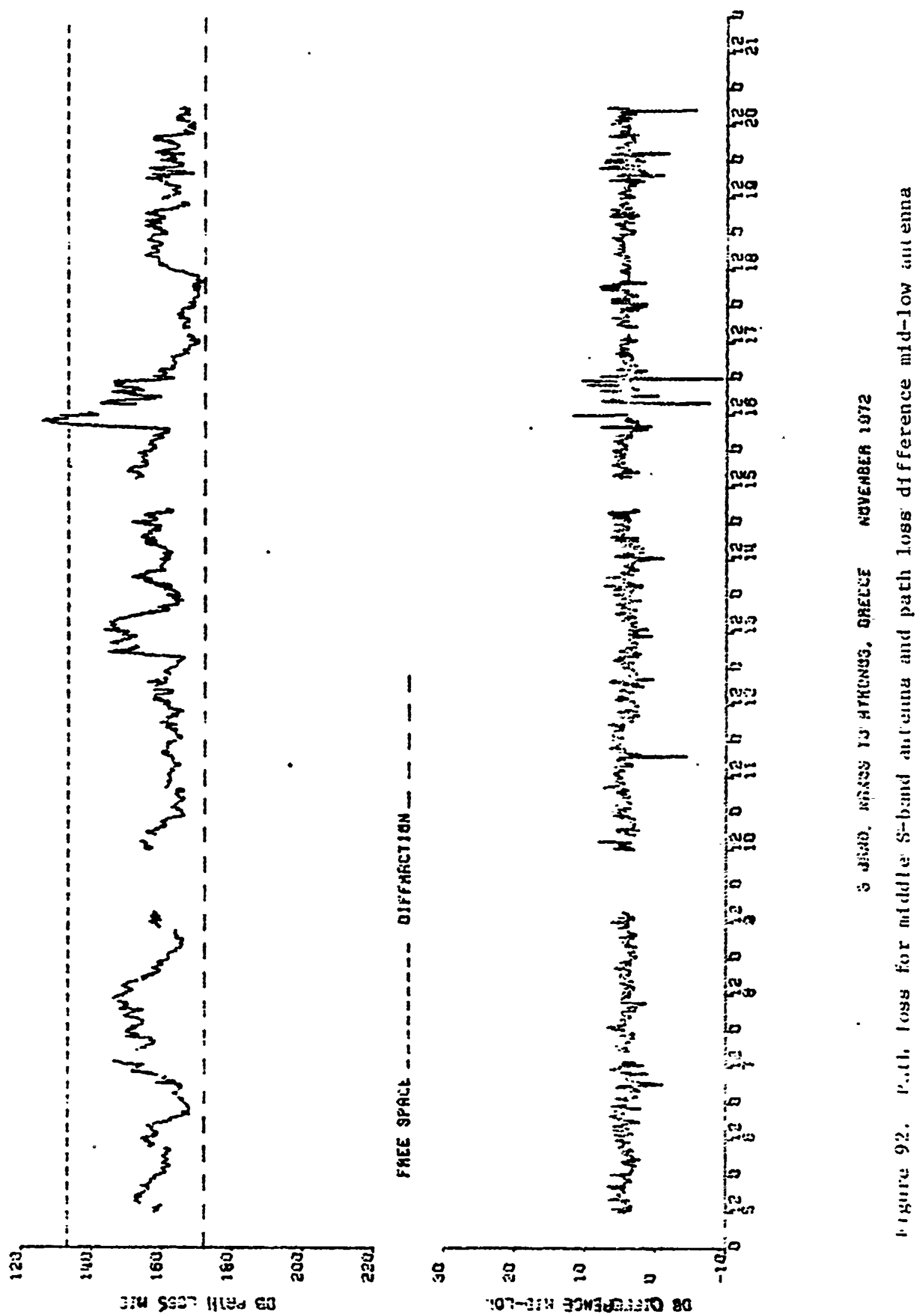

9

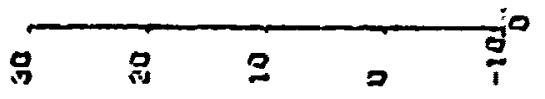

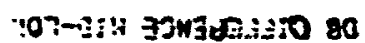

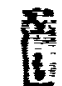



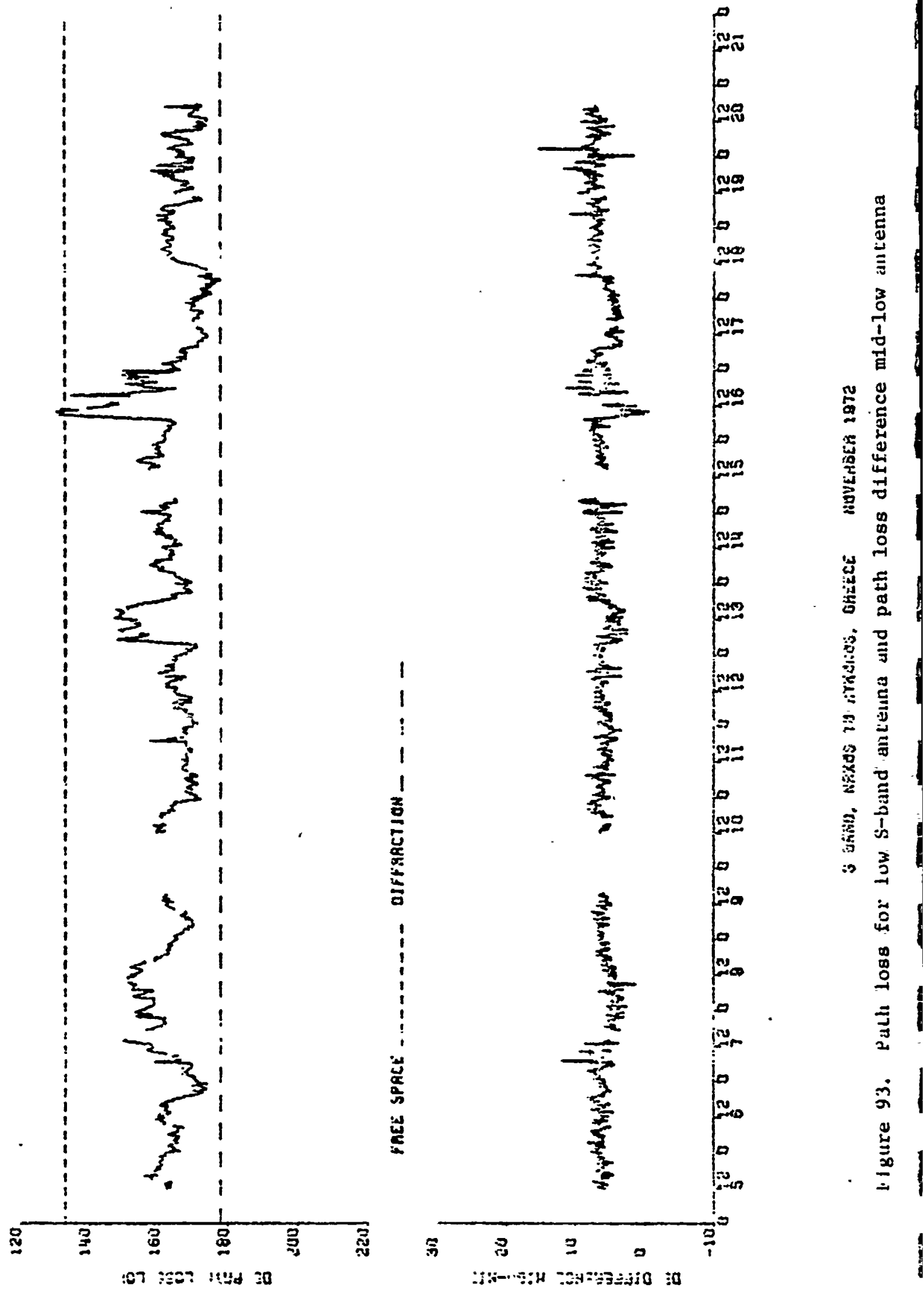


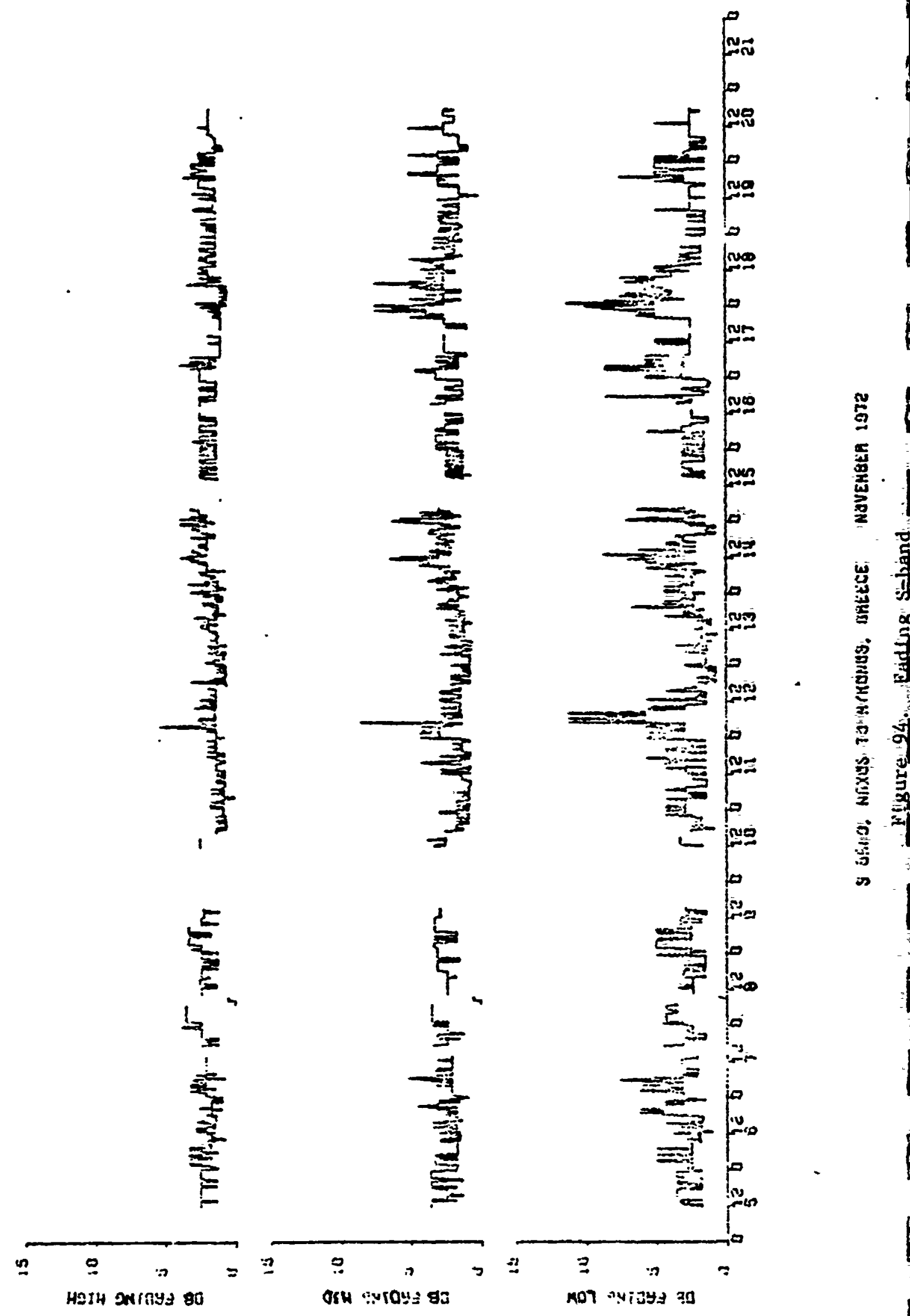


8

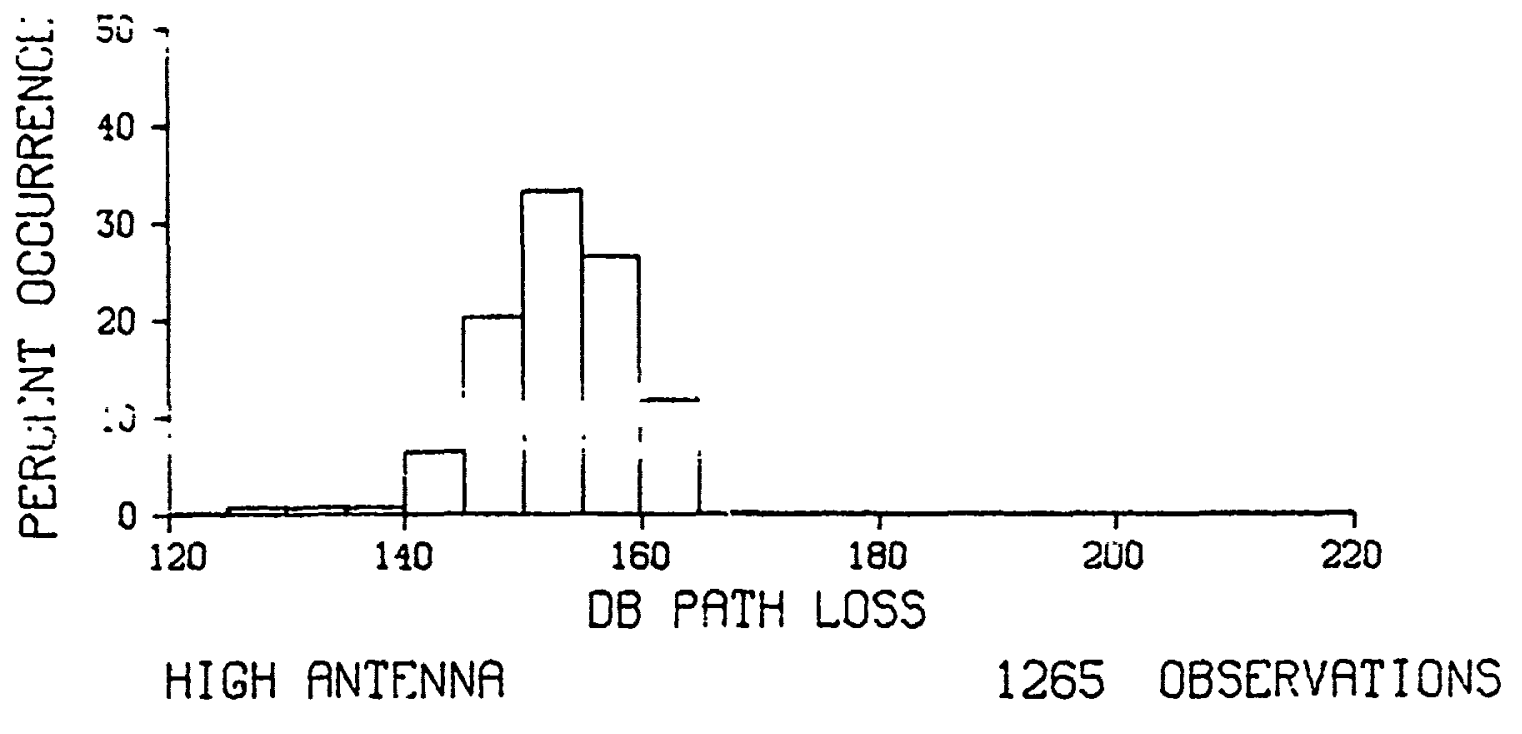

录

8

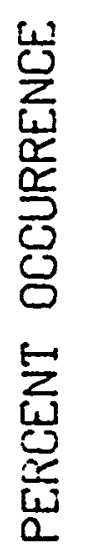

$\left.\begin{array}{l}50 \\ 40 \\ 30 \\ 20 \\ 10\end{array}\right]$

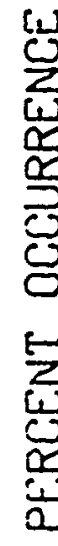

MID ANTENNA

1257 OBSERVATIONS

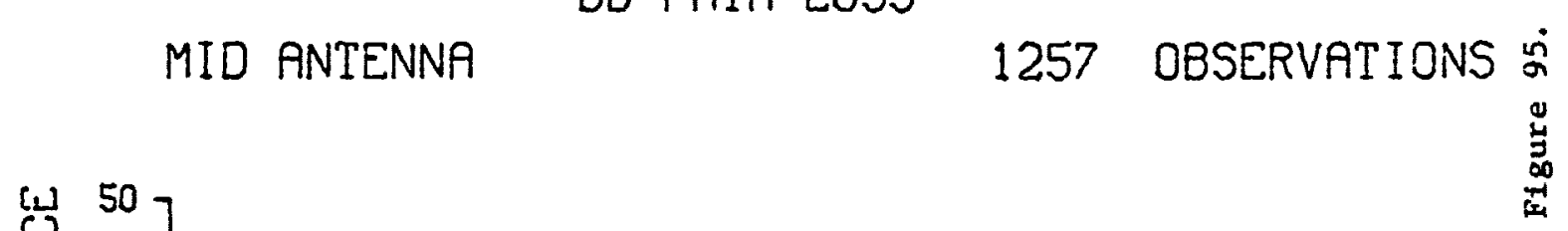

5

8

$40-$

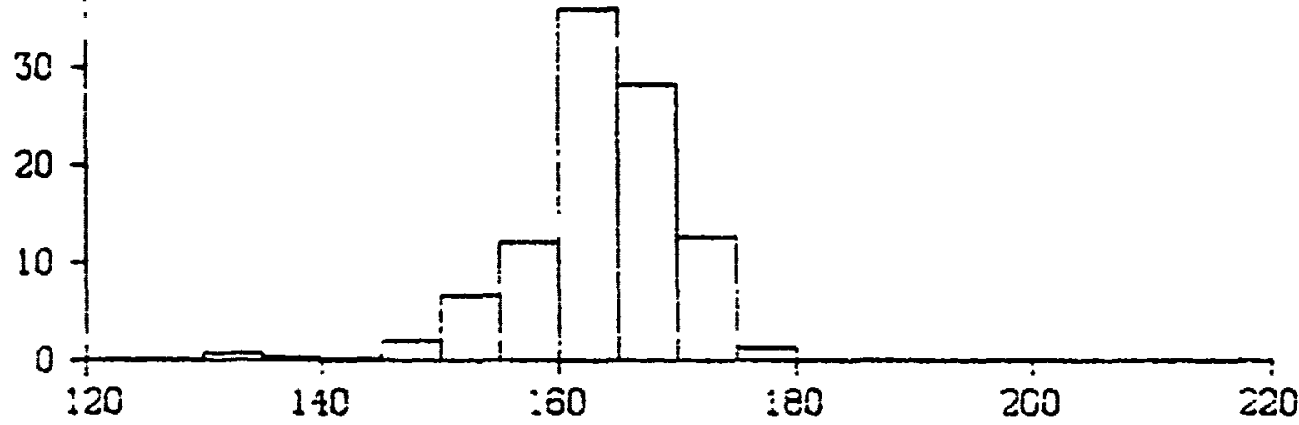

IE =9:-

LAH GNMEINA

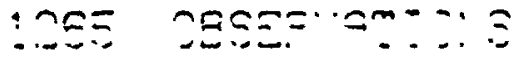

$S$ BAND, GREECE NOVEMEER 1972

2 


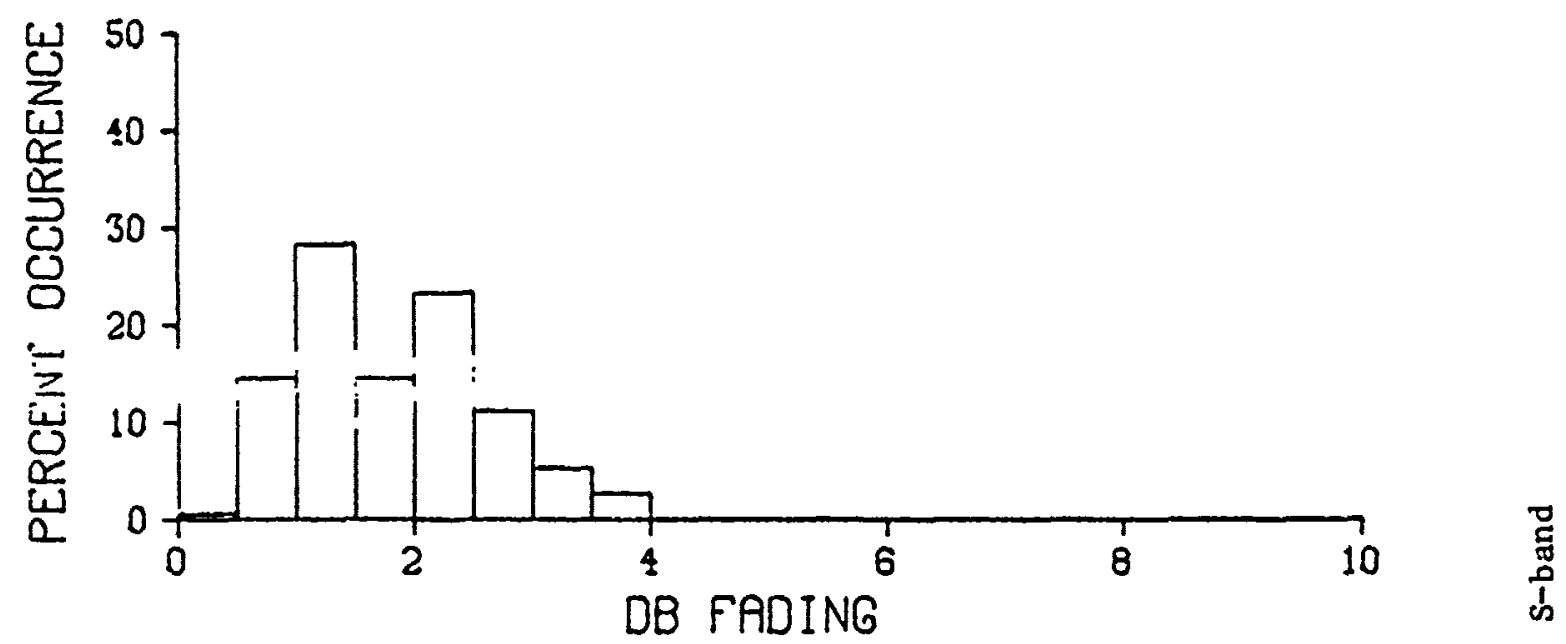
HIGH ANTENNA 1265 OESERVATIONS 芩

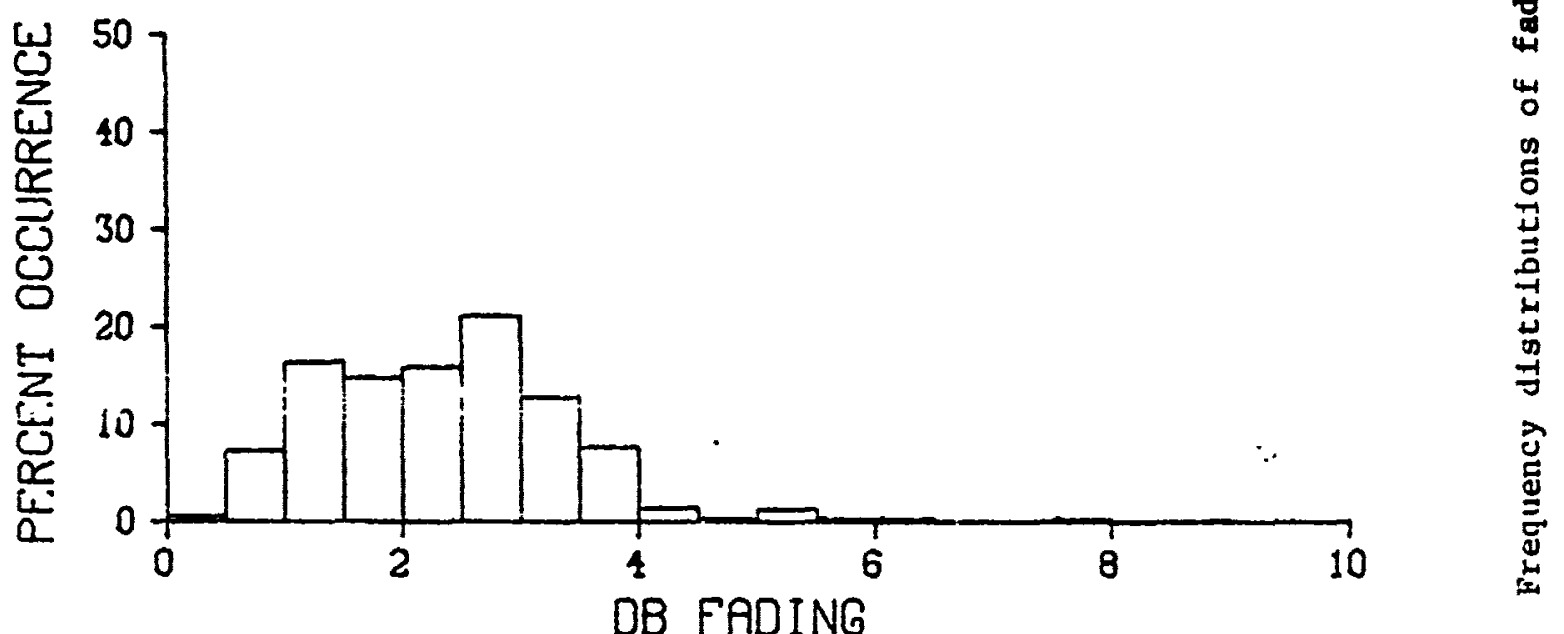
MID ANTENNA 1257 OBSERVATIONS

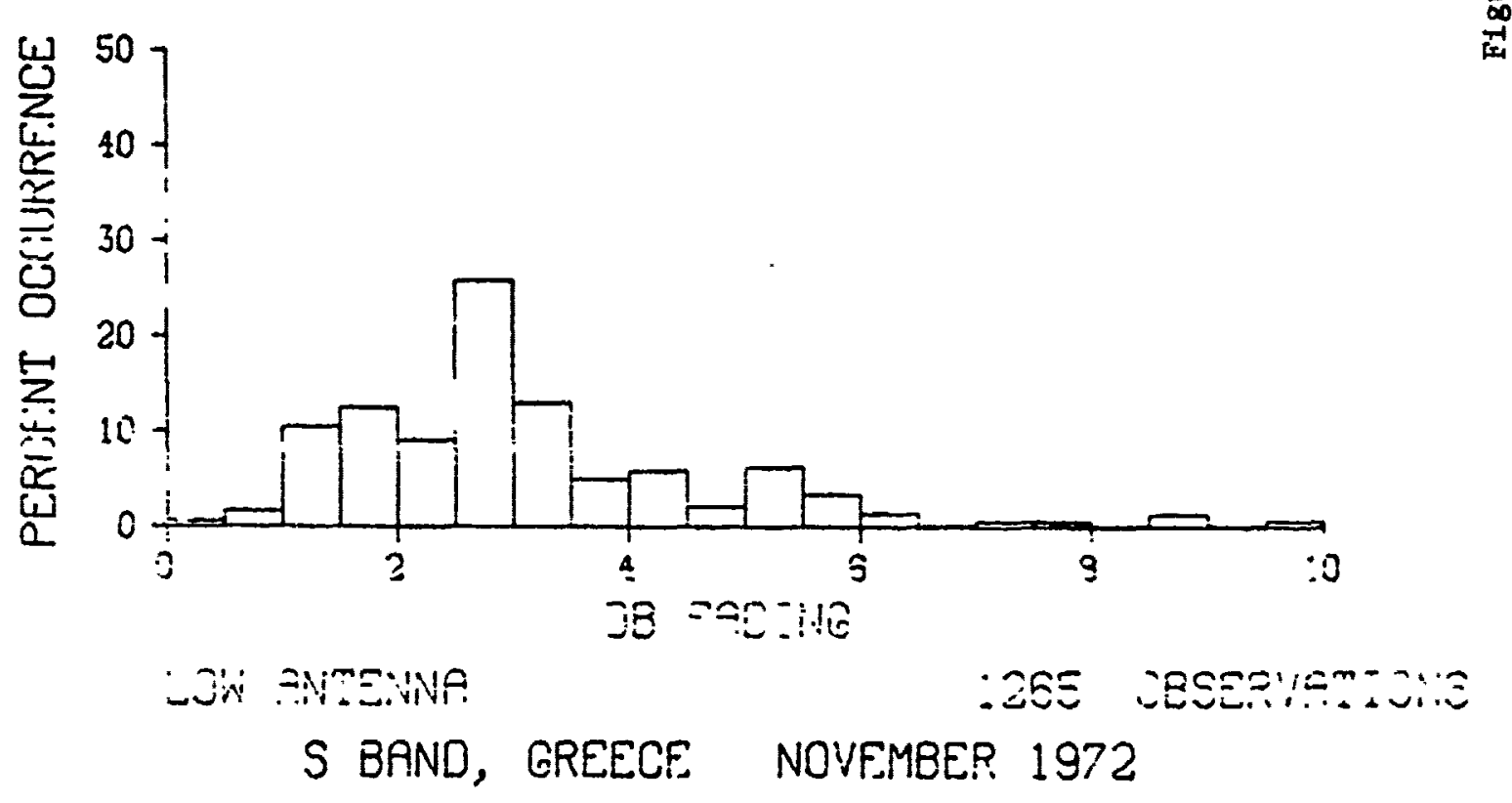



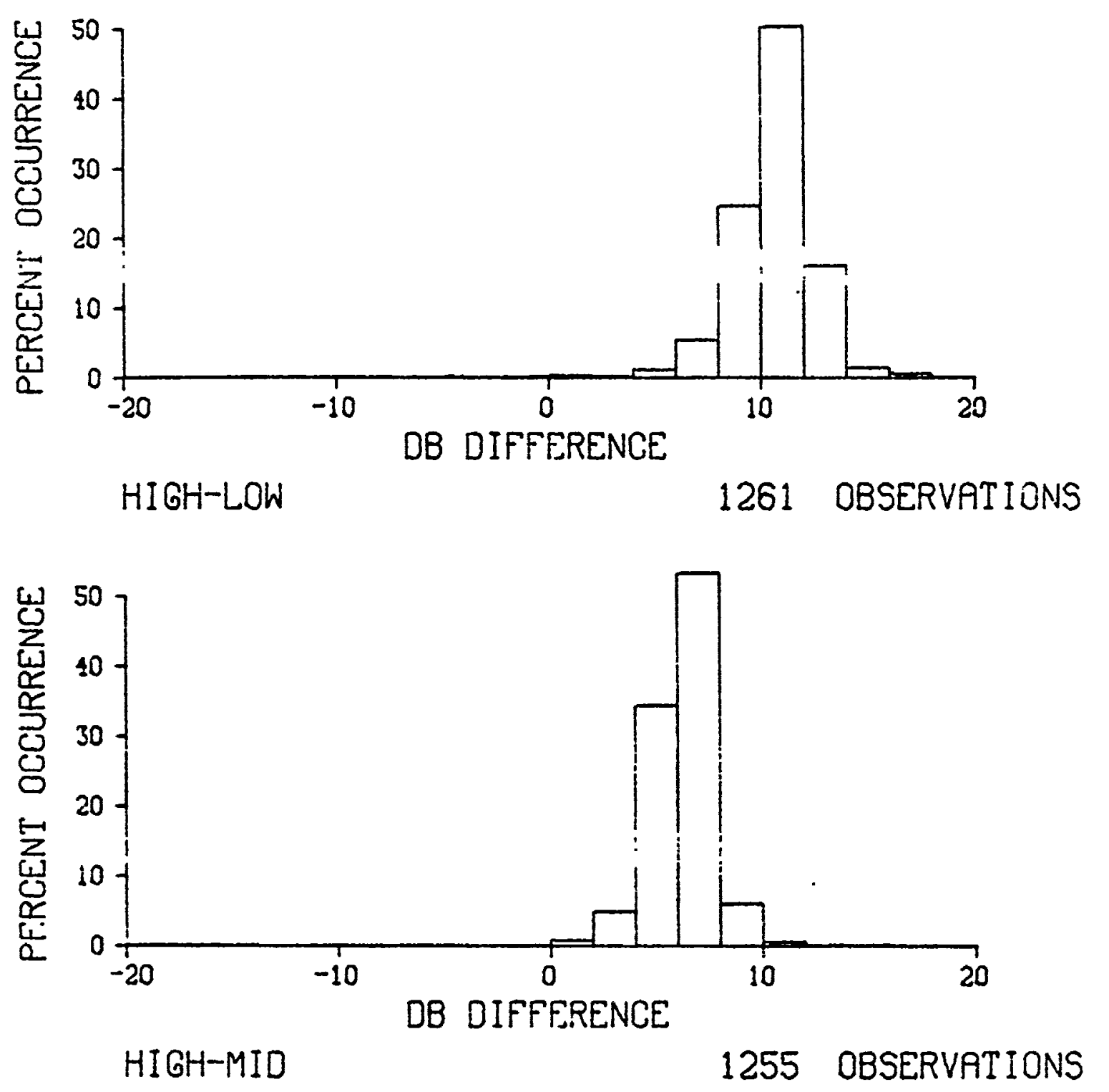

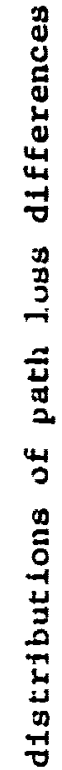

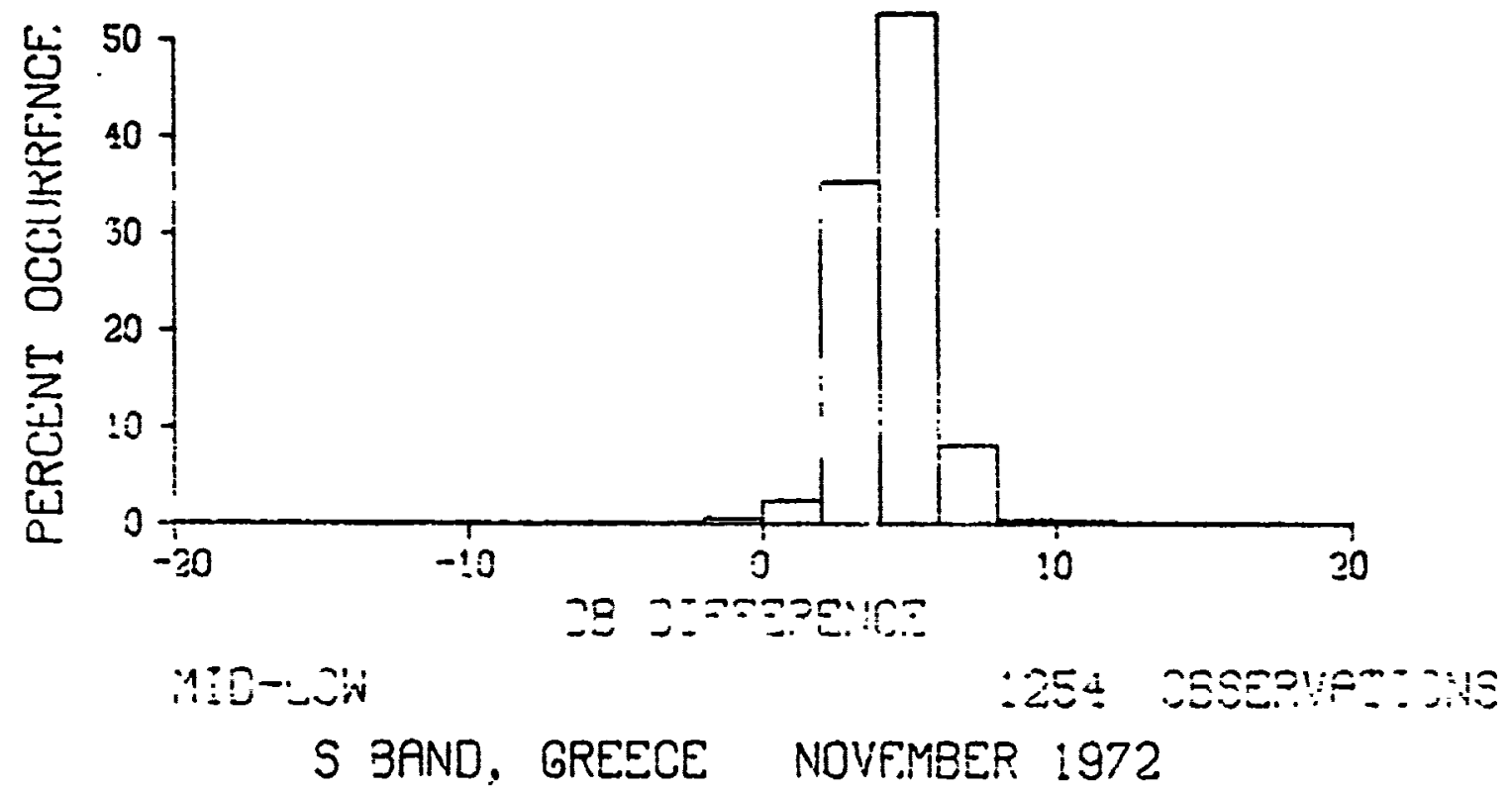

仓े 
8

8

8

8

w

8
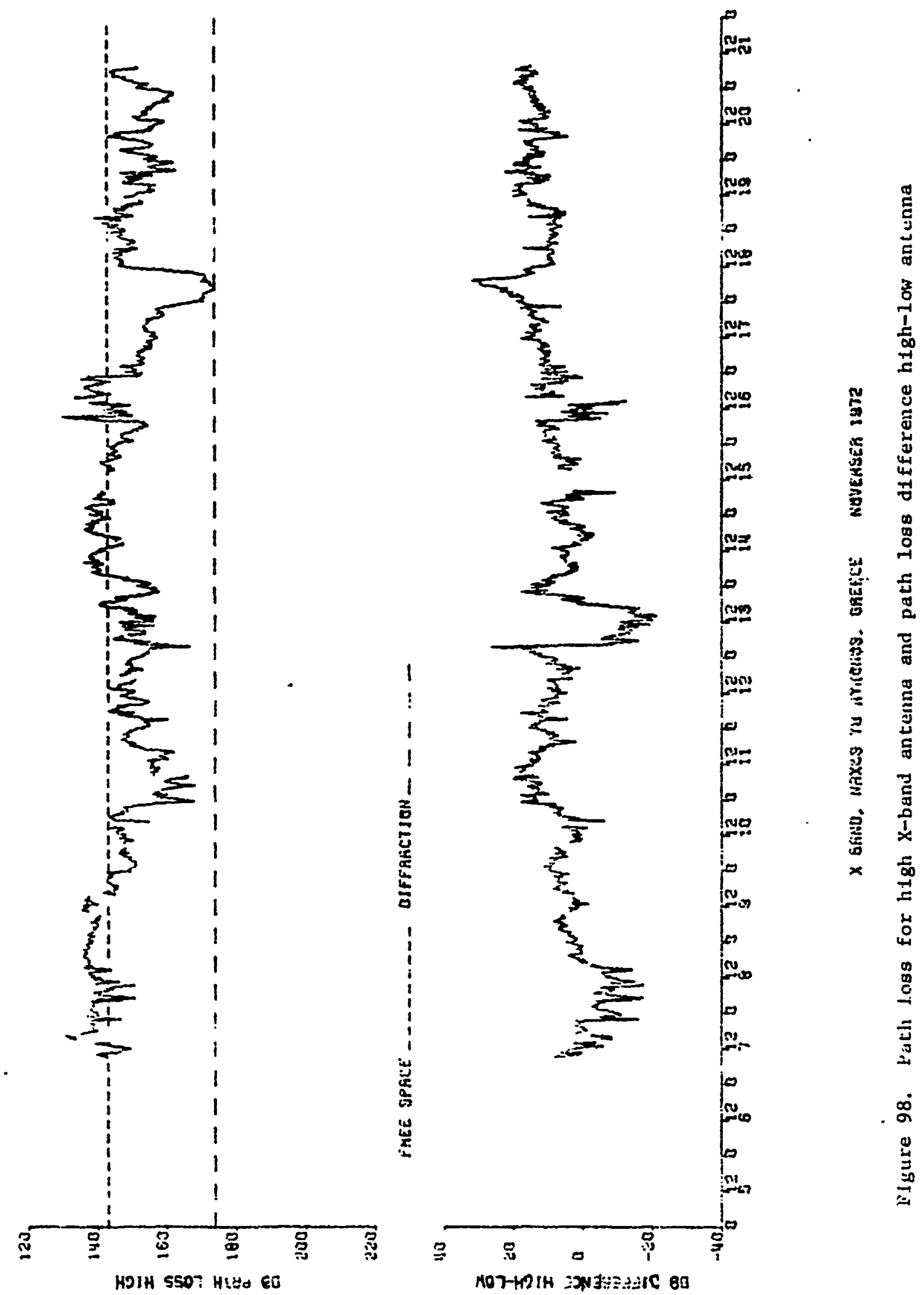

8

HOIH 5507 HIHA EO

8 

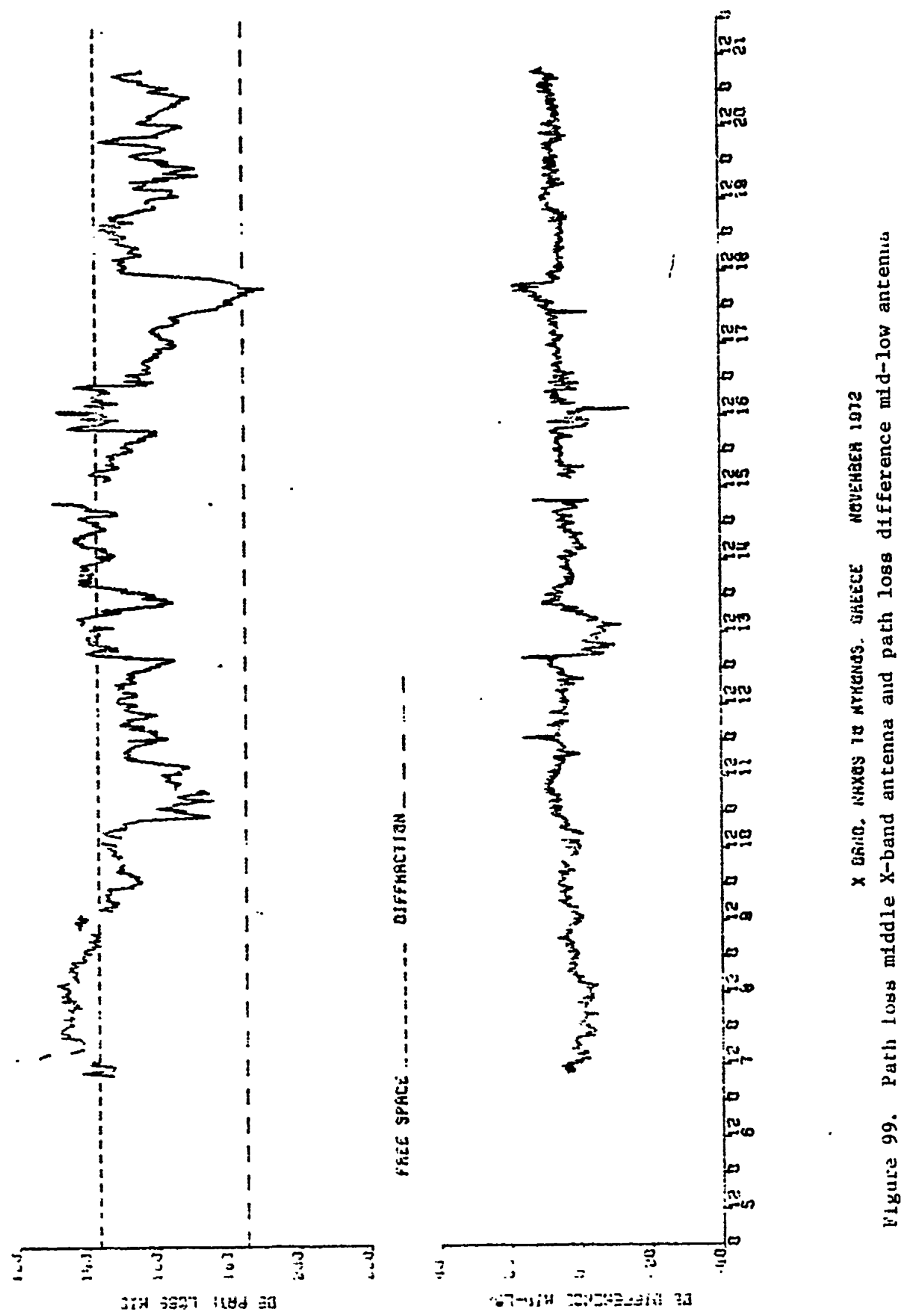
8

B

?
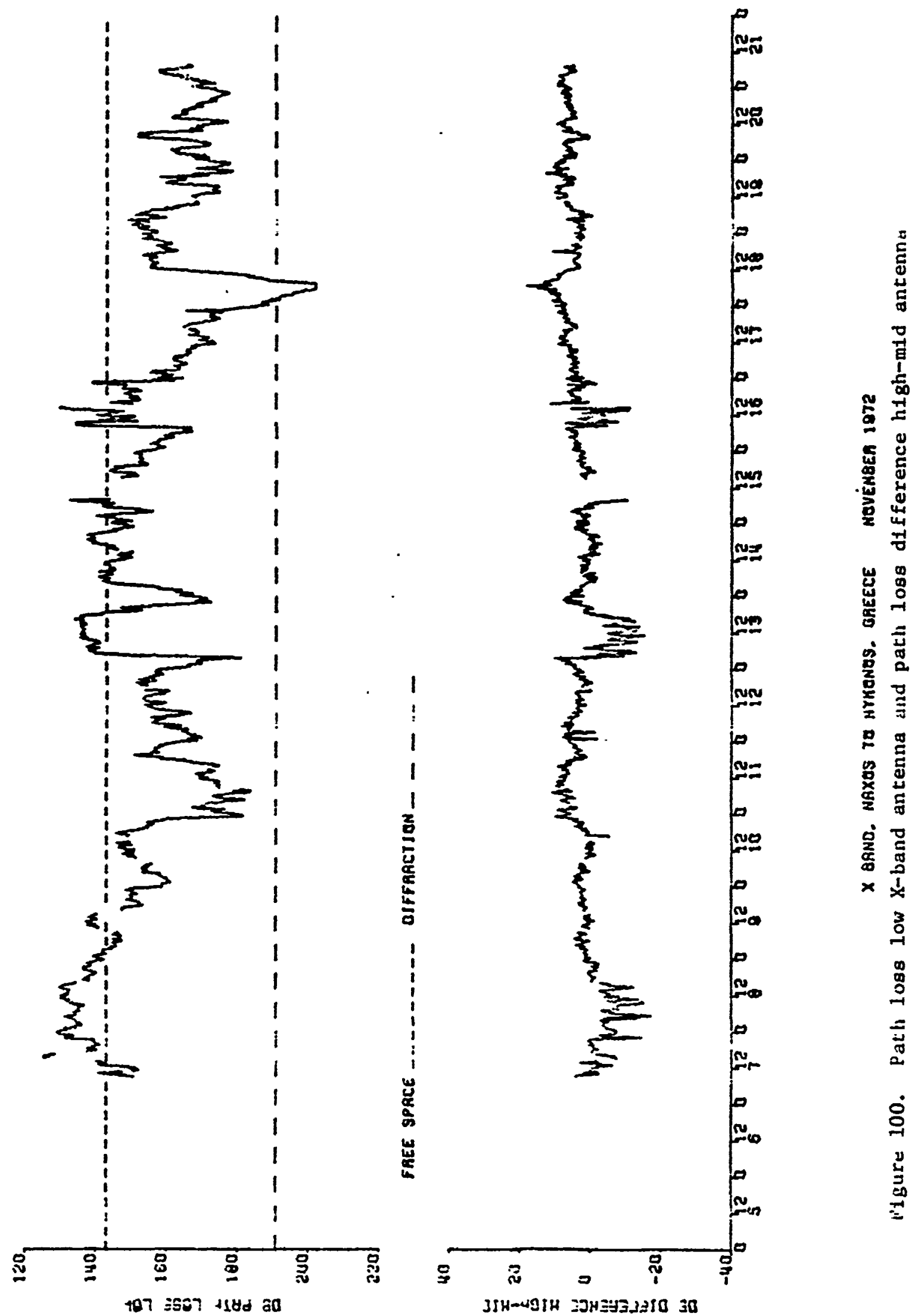

8

8

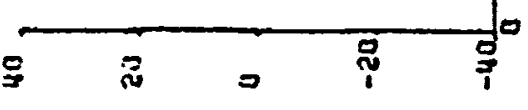

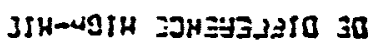

E. 

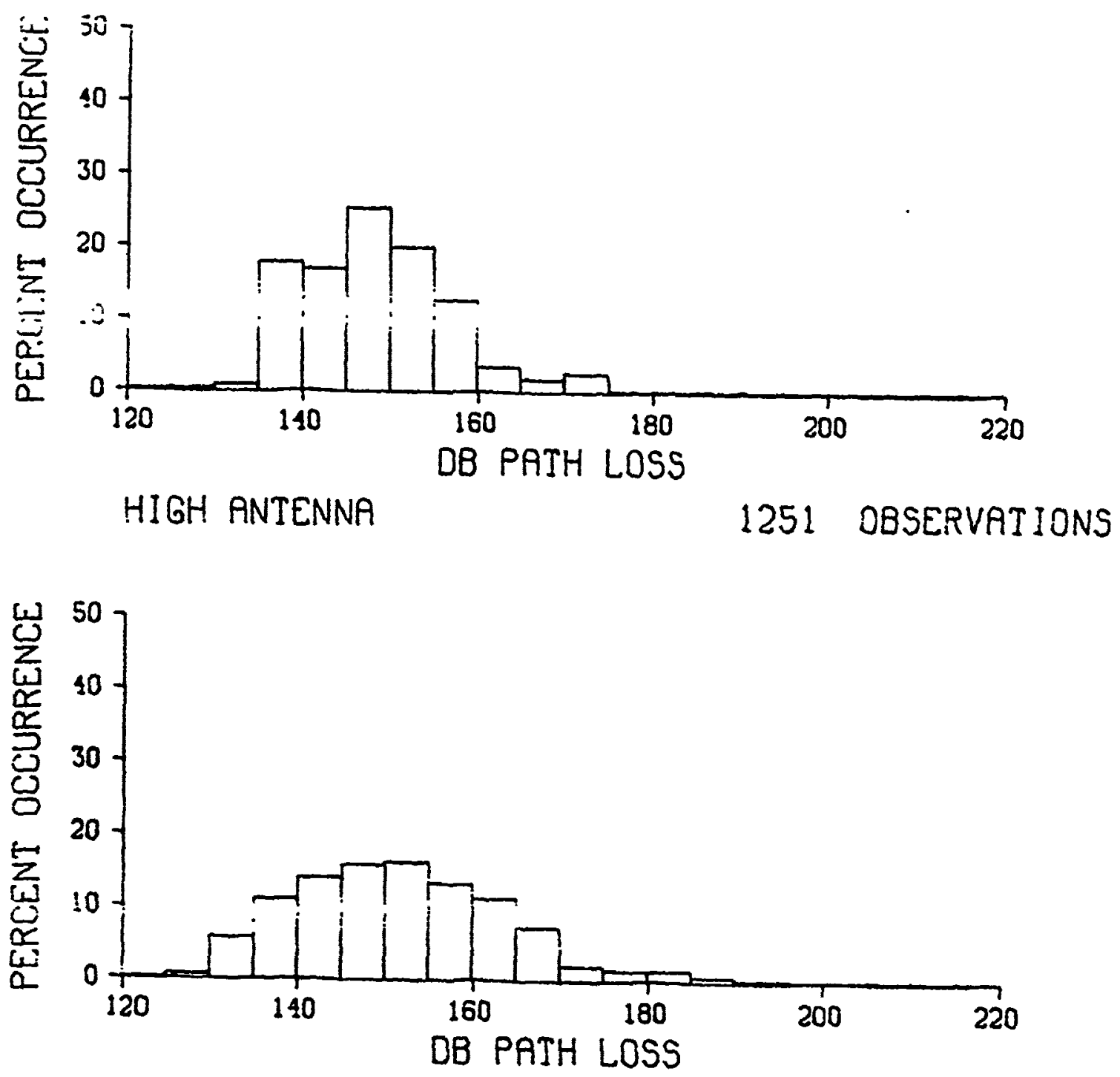

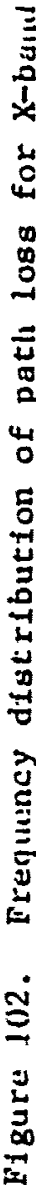
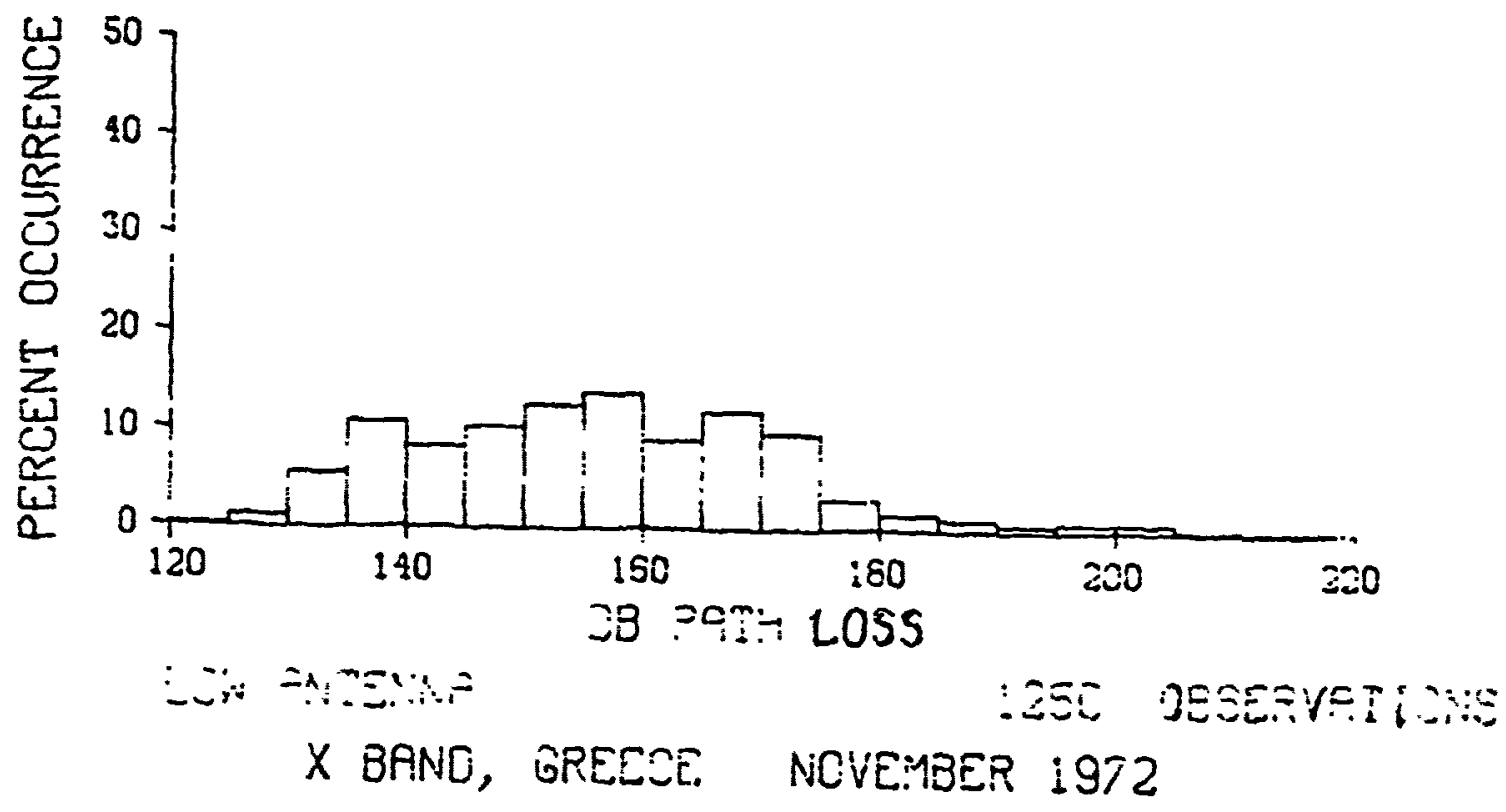

$E$ 


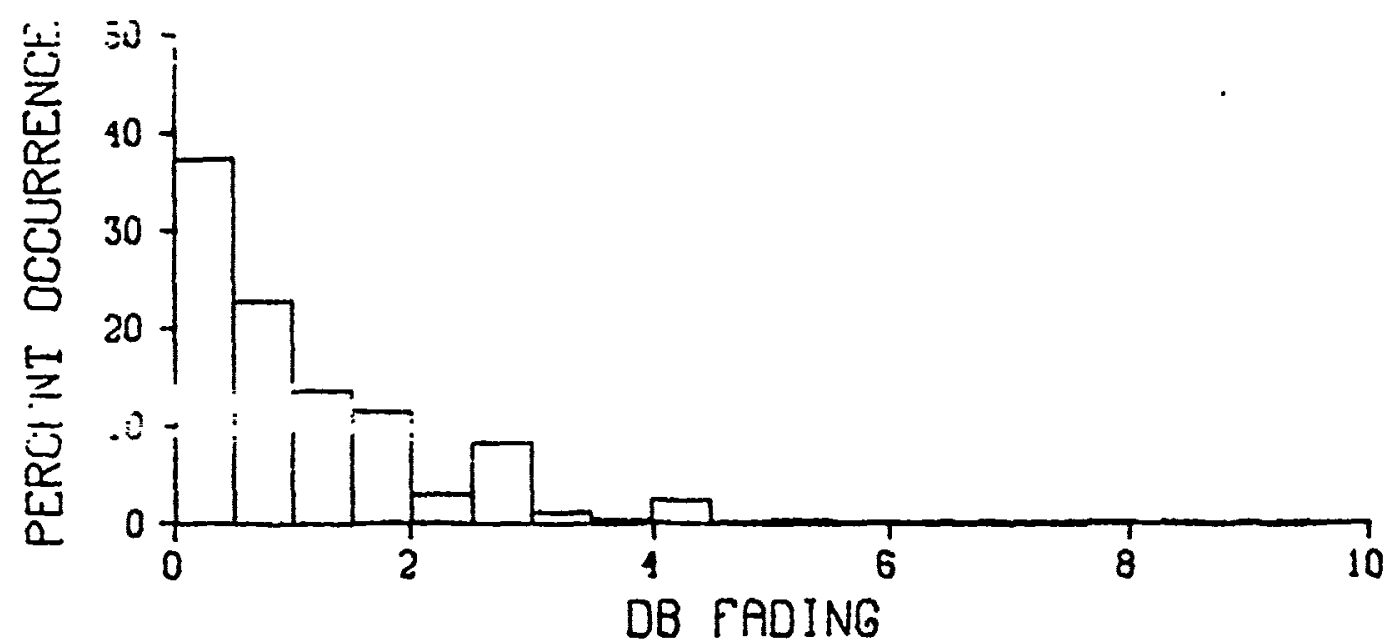

HIGH ANTENNA

1251 OBSERVATIONS
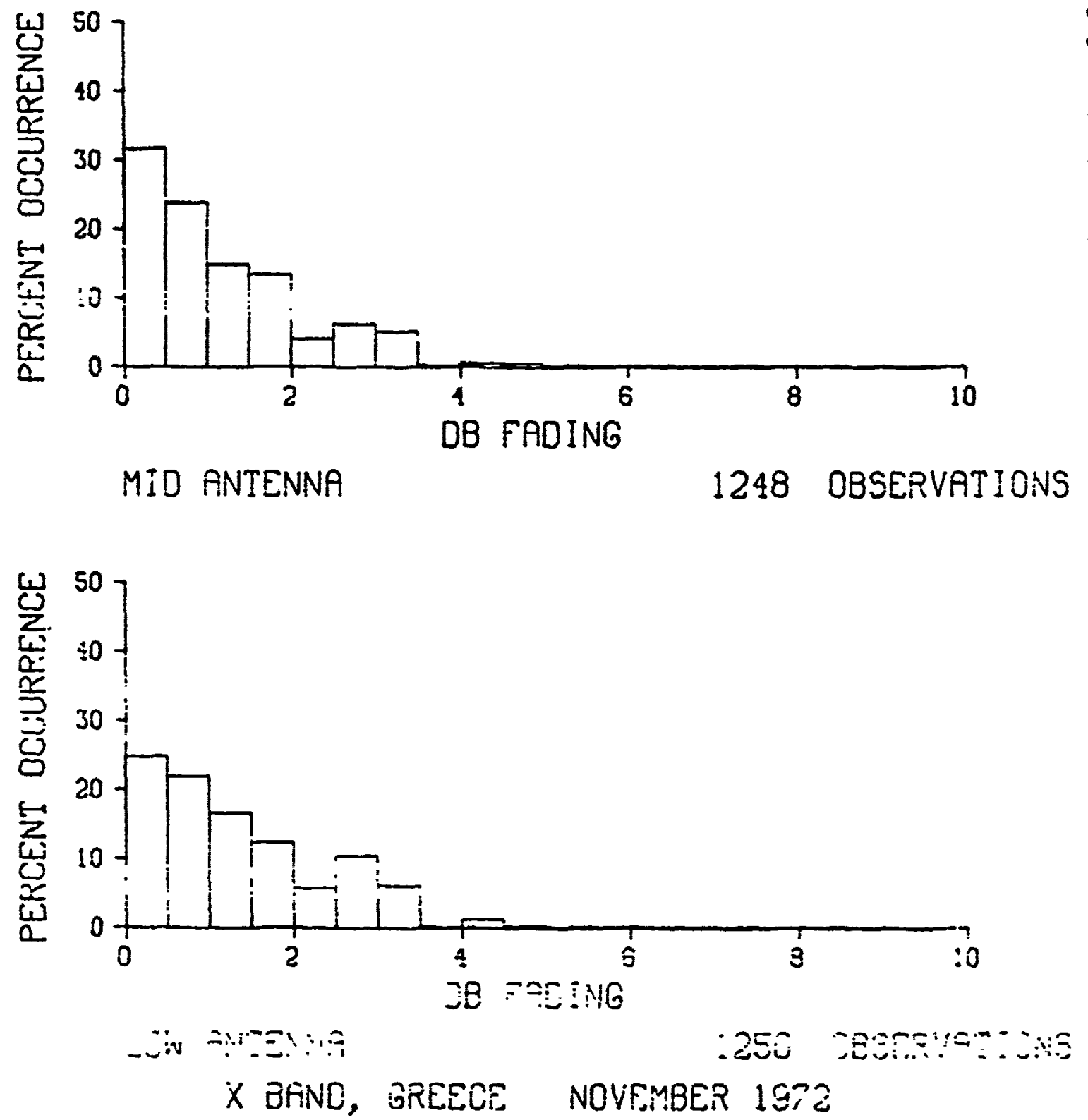


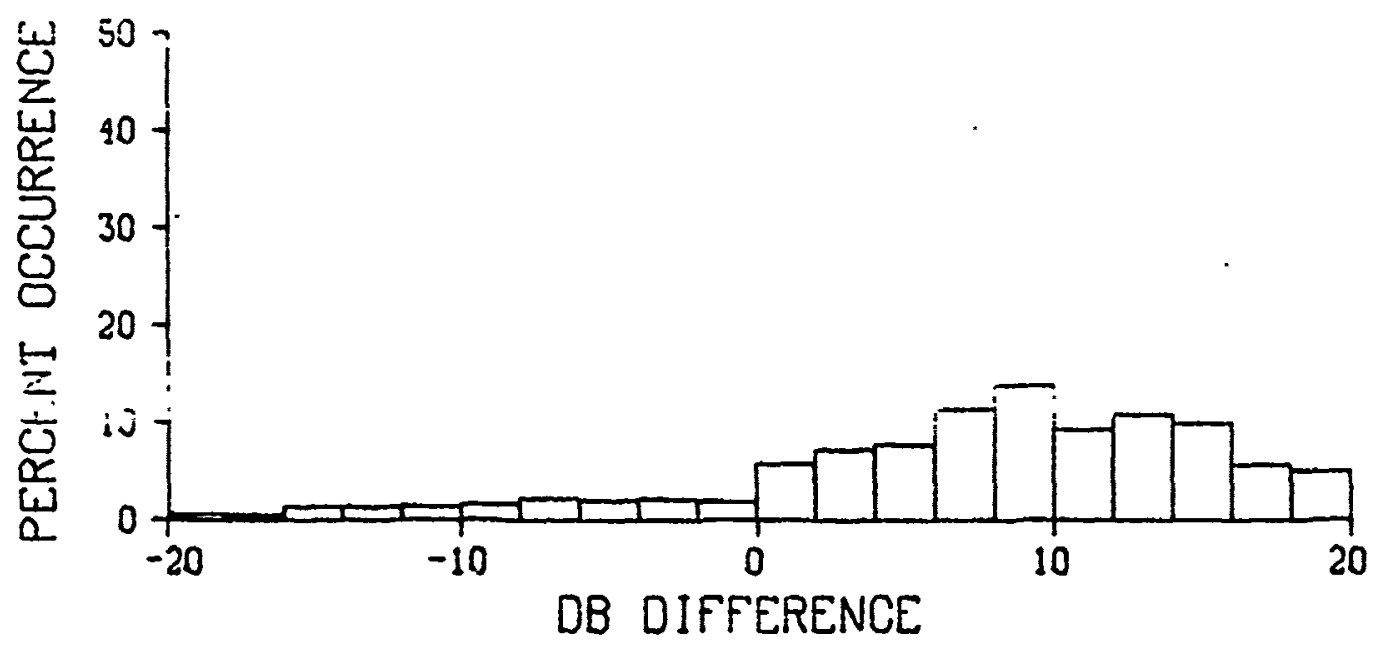

HIGH-LOW

1249 OBSERVATIONS

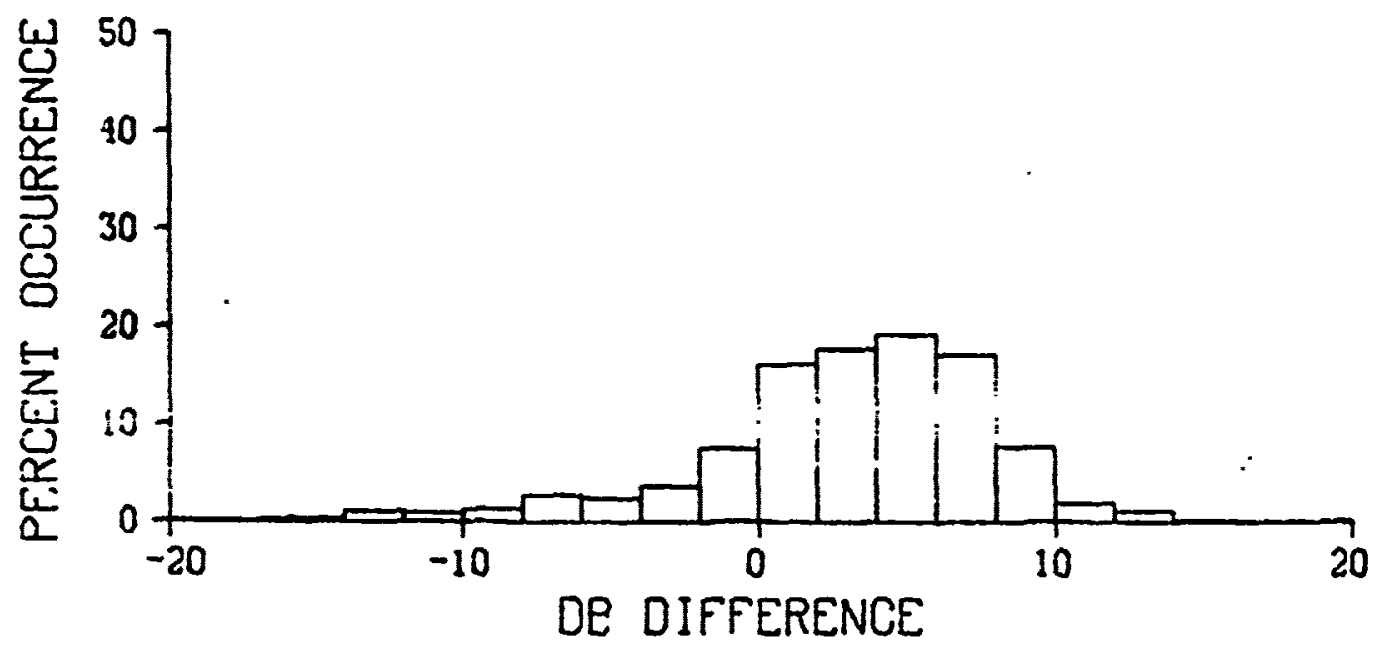

HIGH-MID

1247 OBSERVATIONS
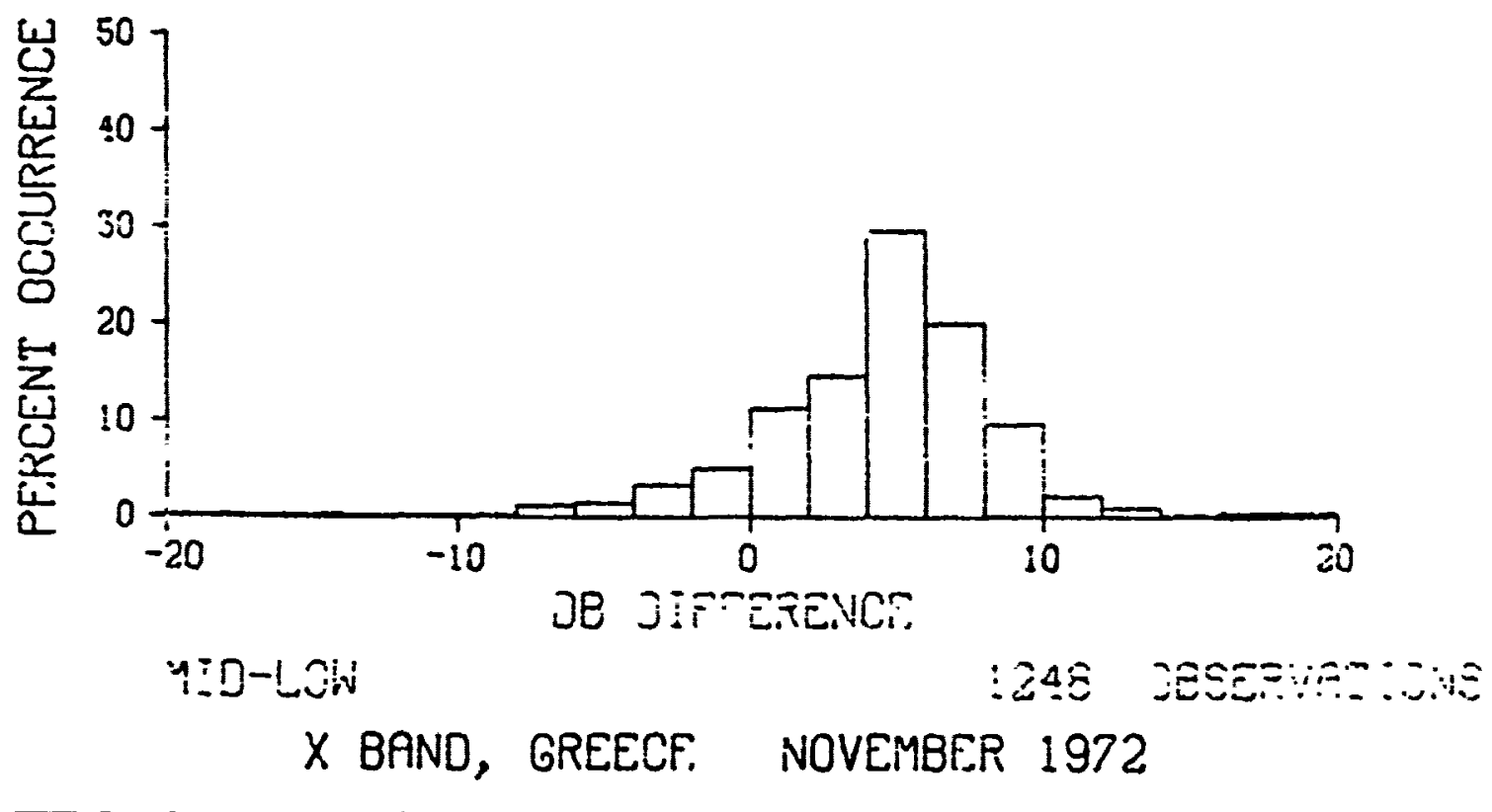


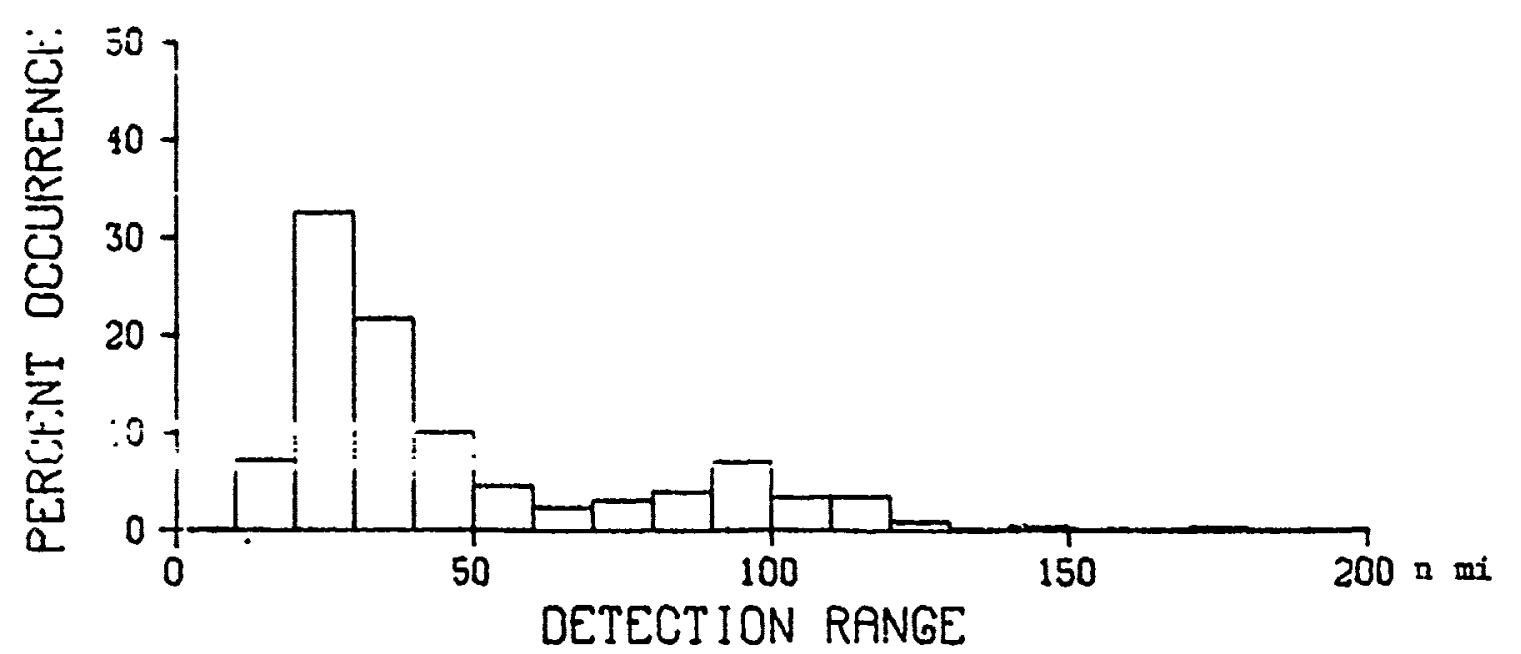

HIGH ANTENNA

1247 OBSERVATIONS

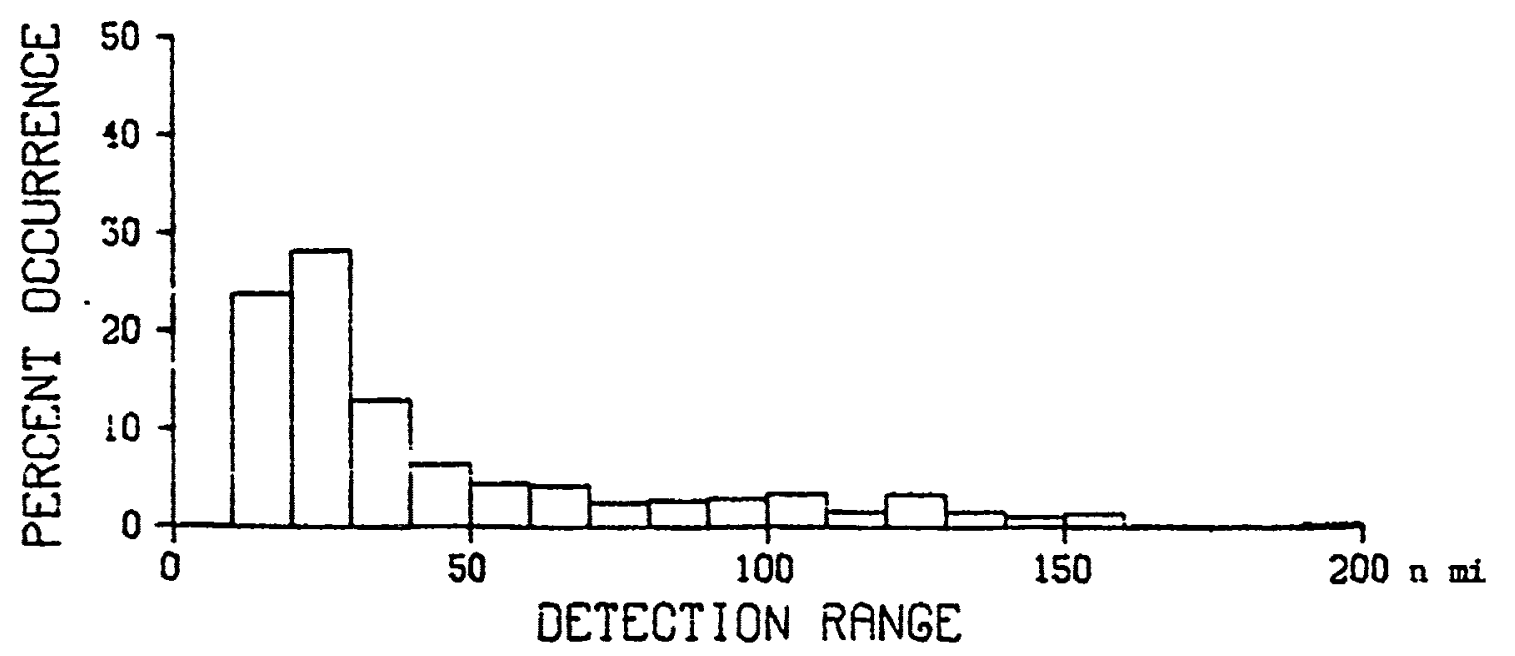

MID ANTENNA 1247 OBSERVATIONS

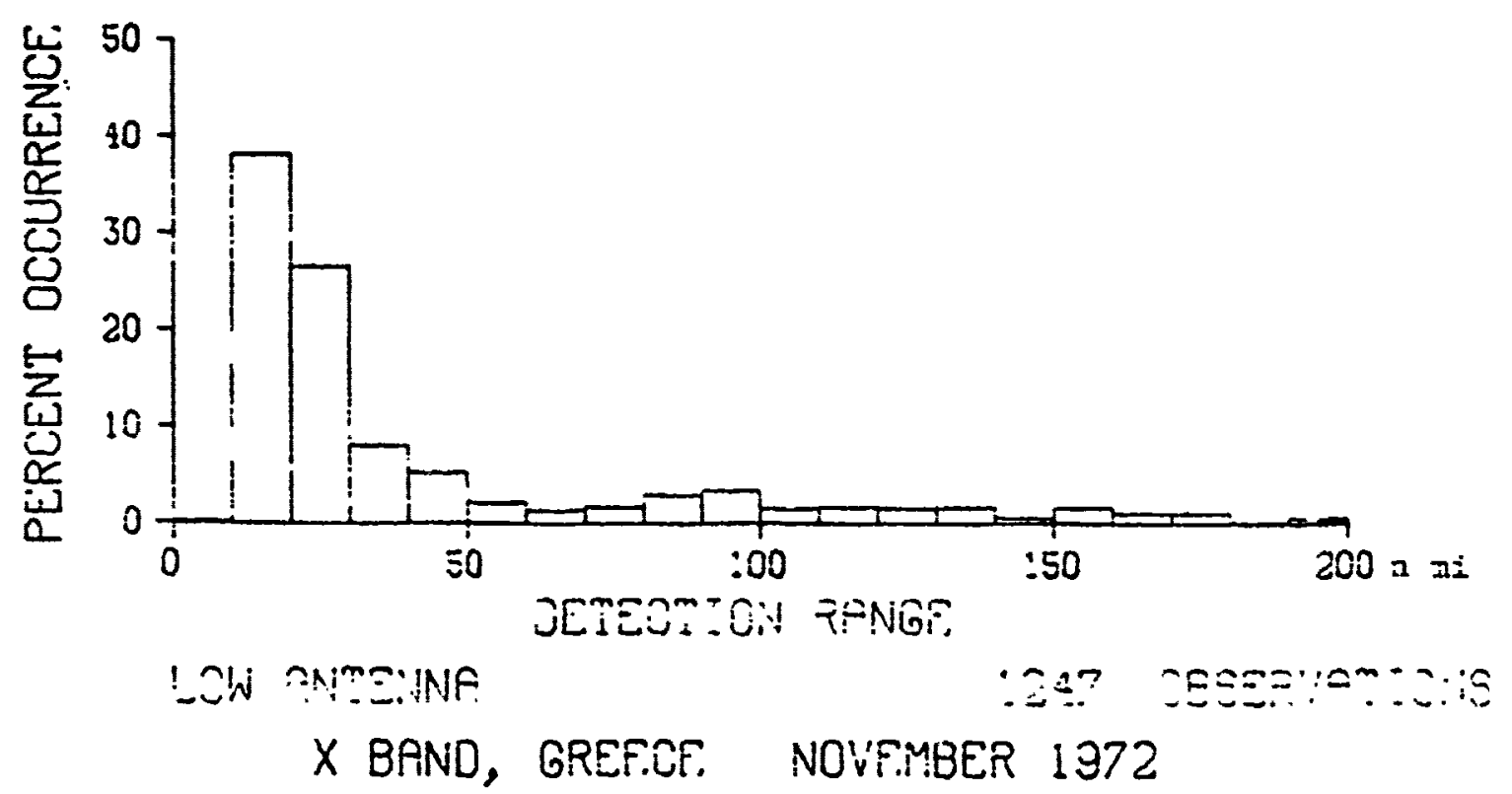

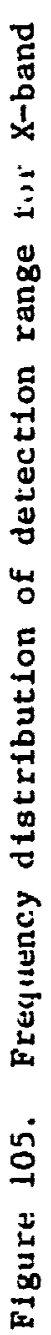

㷎

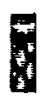

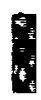

3
8
3

7
$=$ 

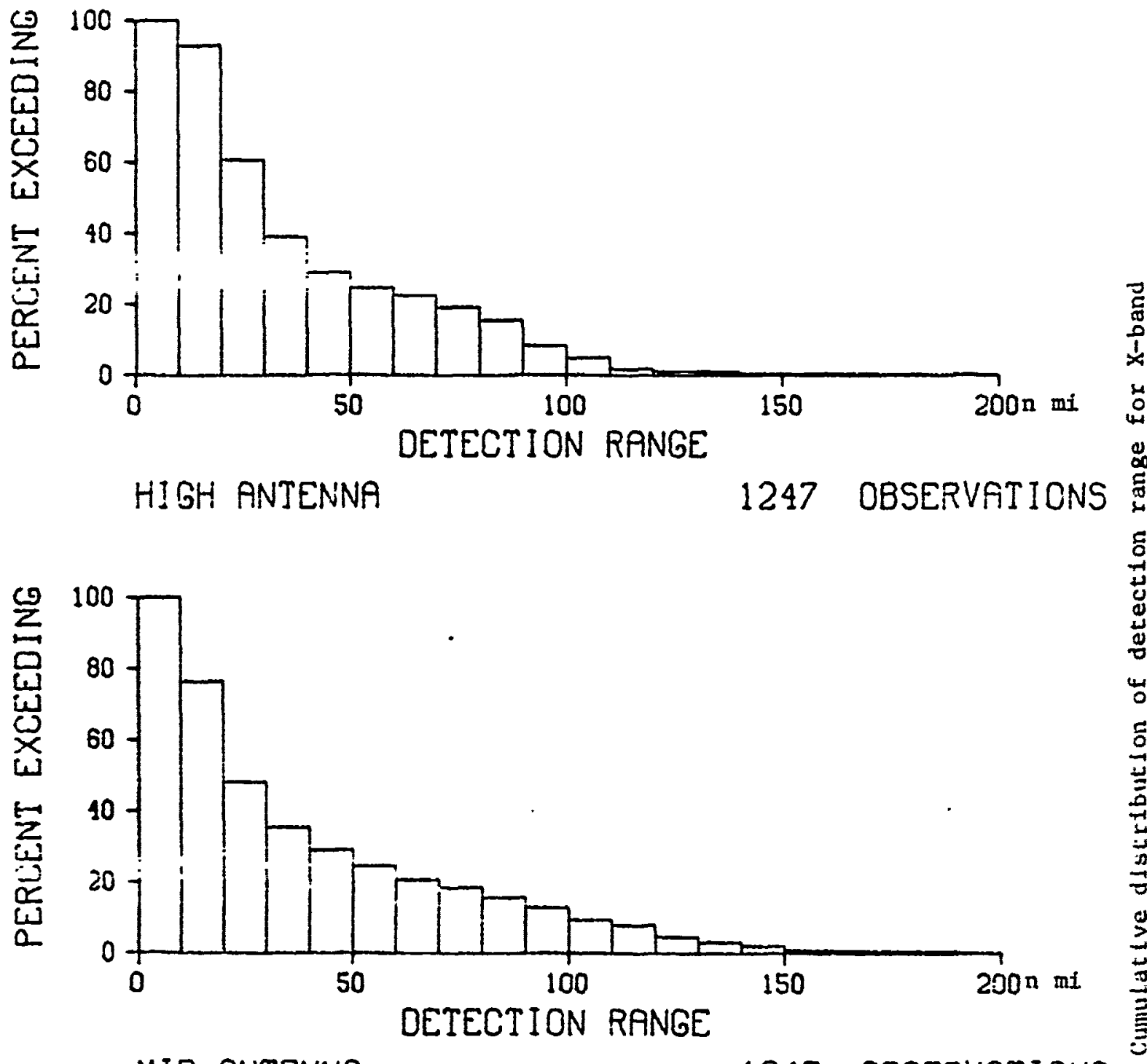

M H]GH ANTENNA 1247 OBSERVATIONS

8

8

MID ANTENNA

1247 OBSERVATIONS

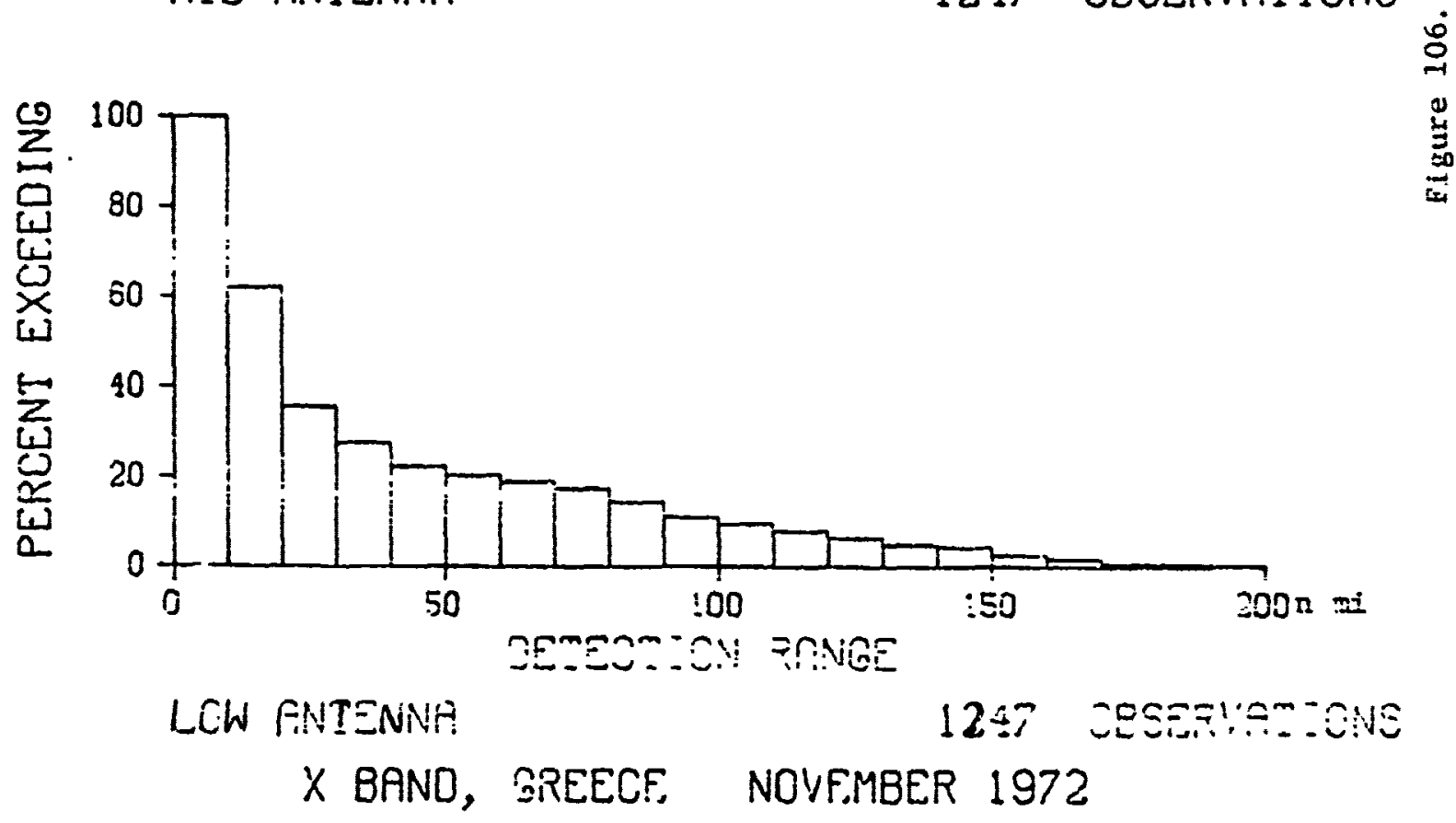

8

3

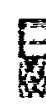

F

8

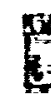




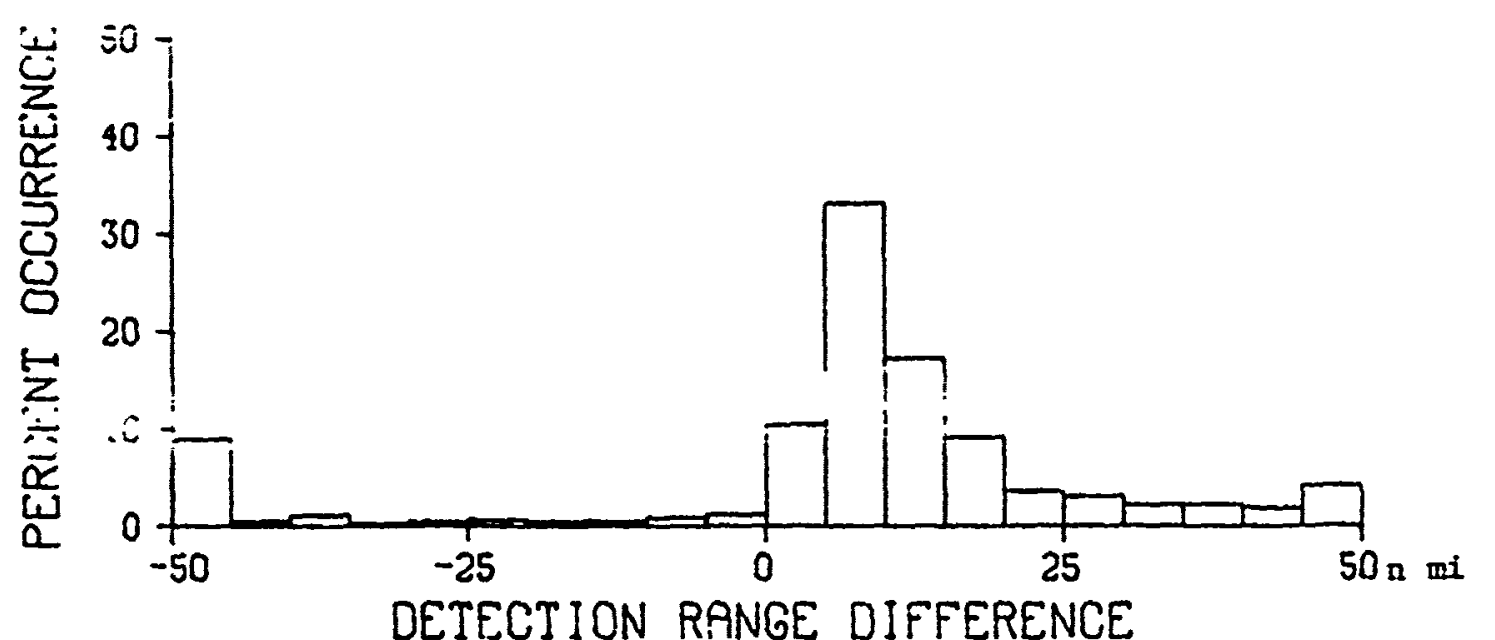
HIGH-LOW
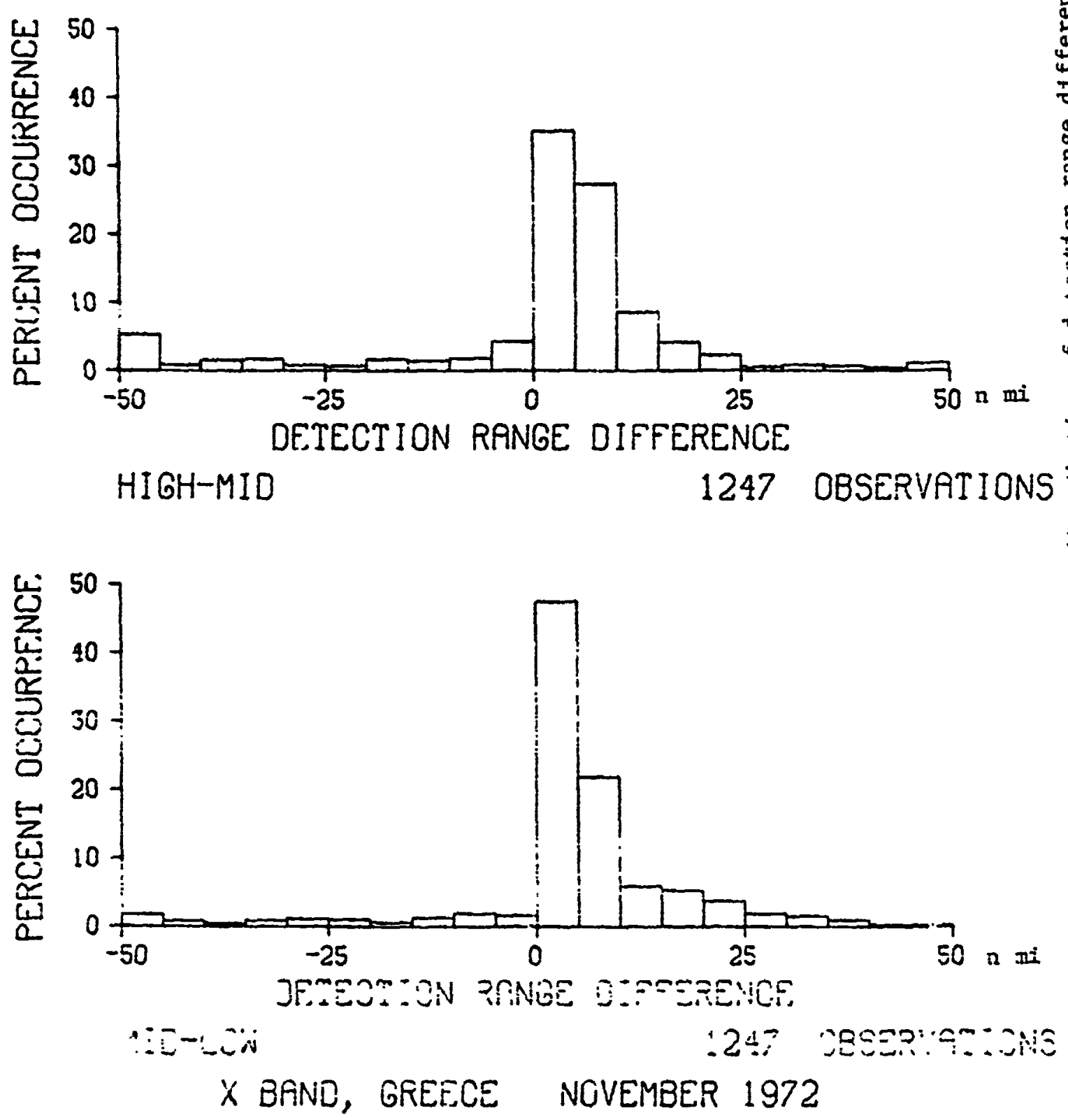

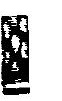

8 
W

8
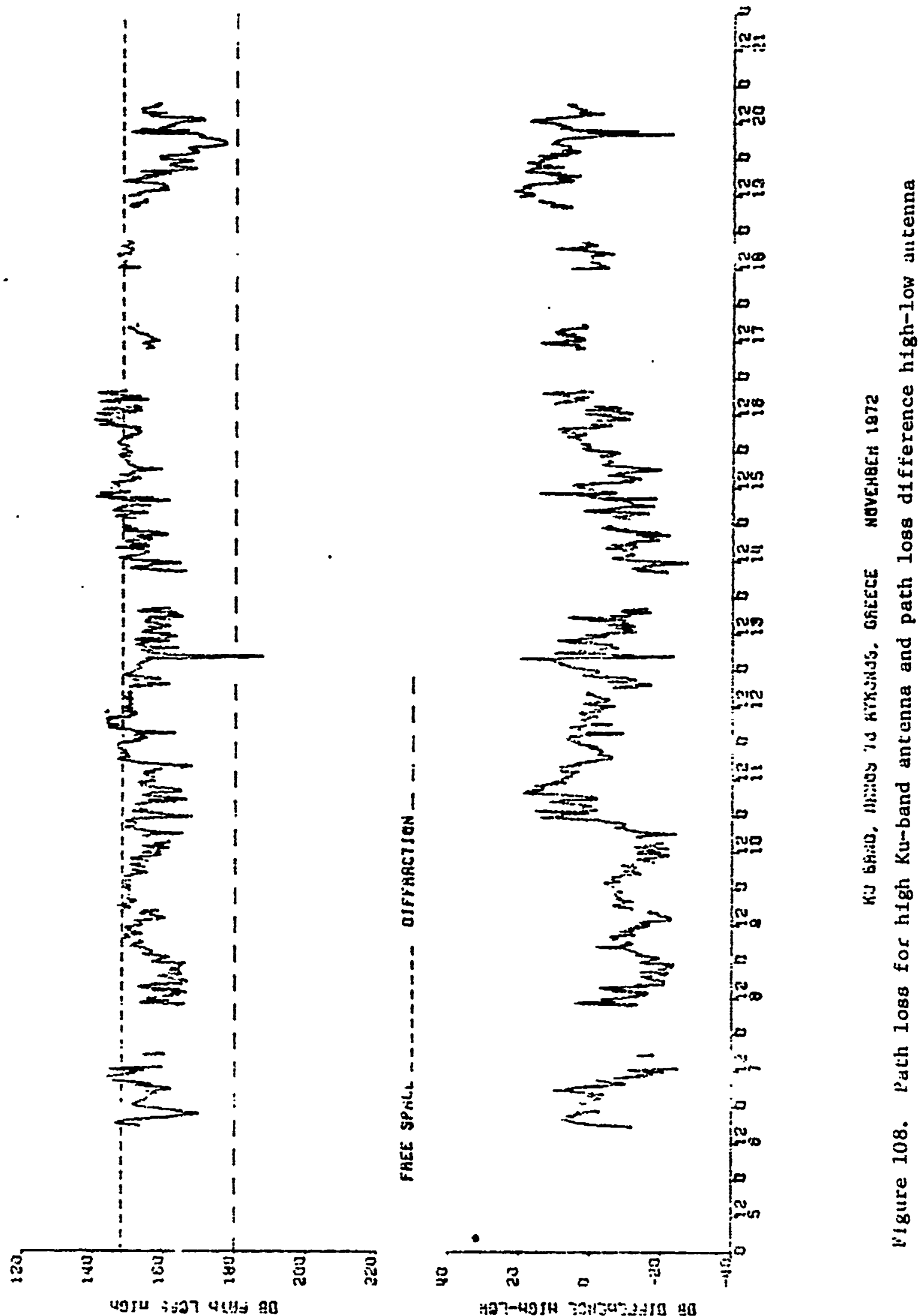

4

8

a

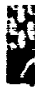

8

8

9

8

8

8

9

$d$

8

m

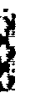

E 

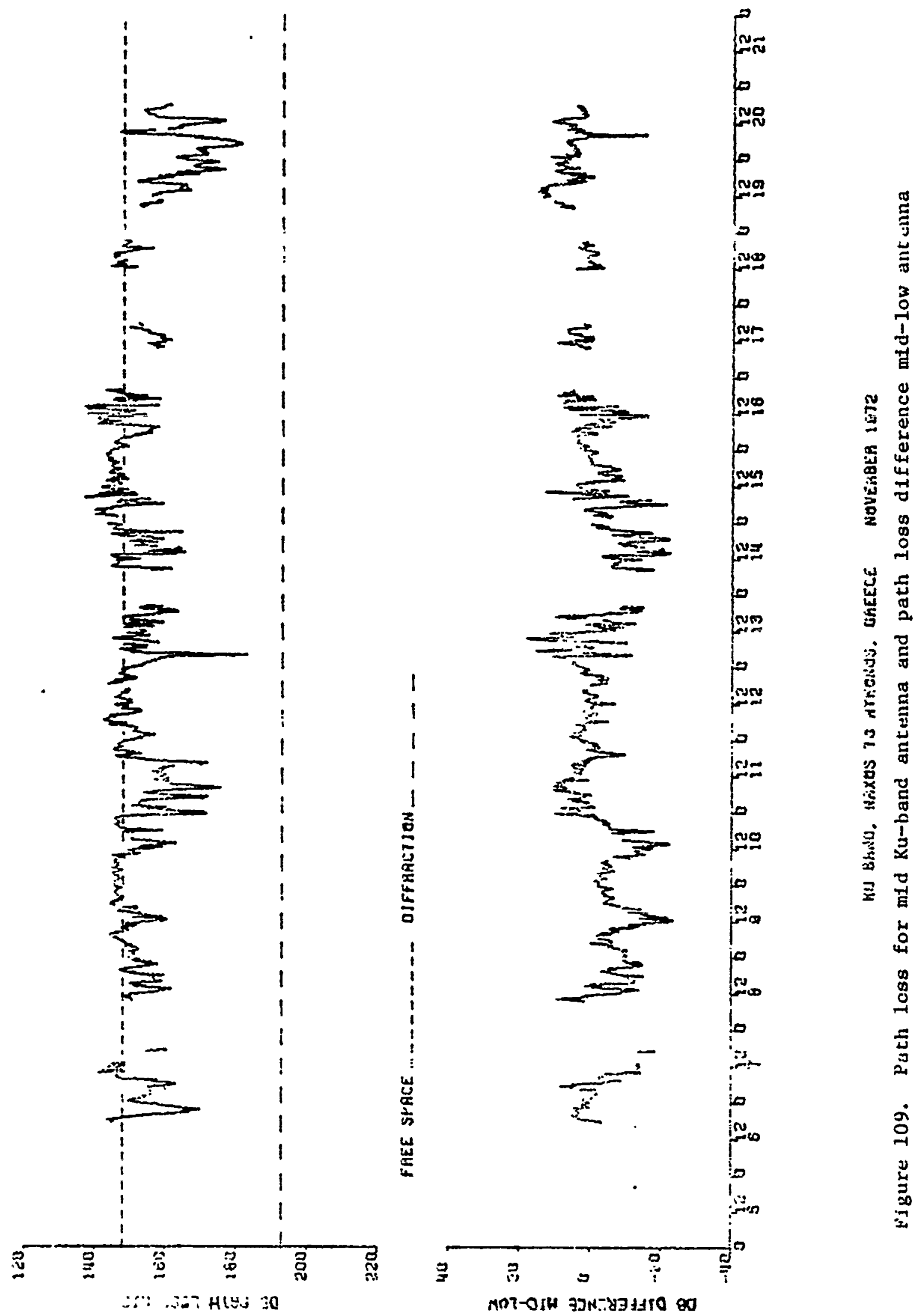

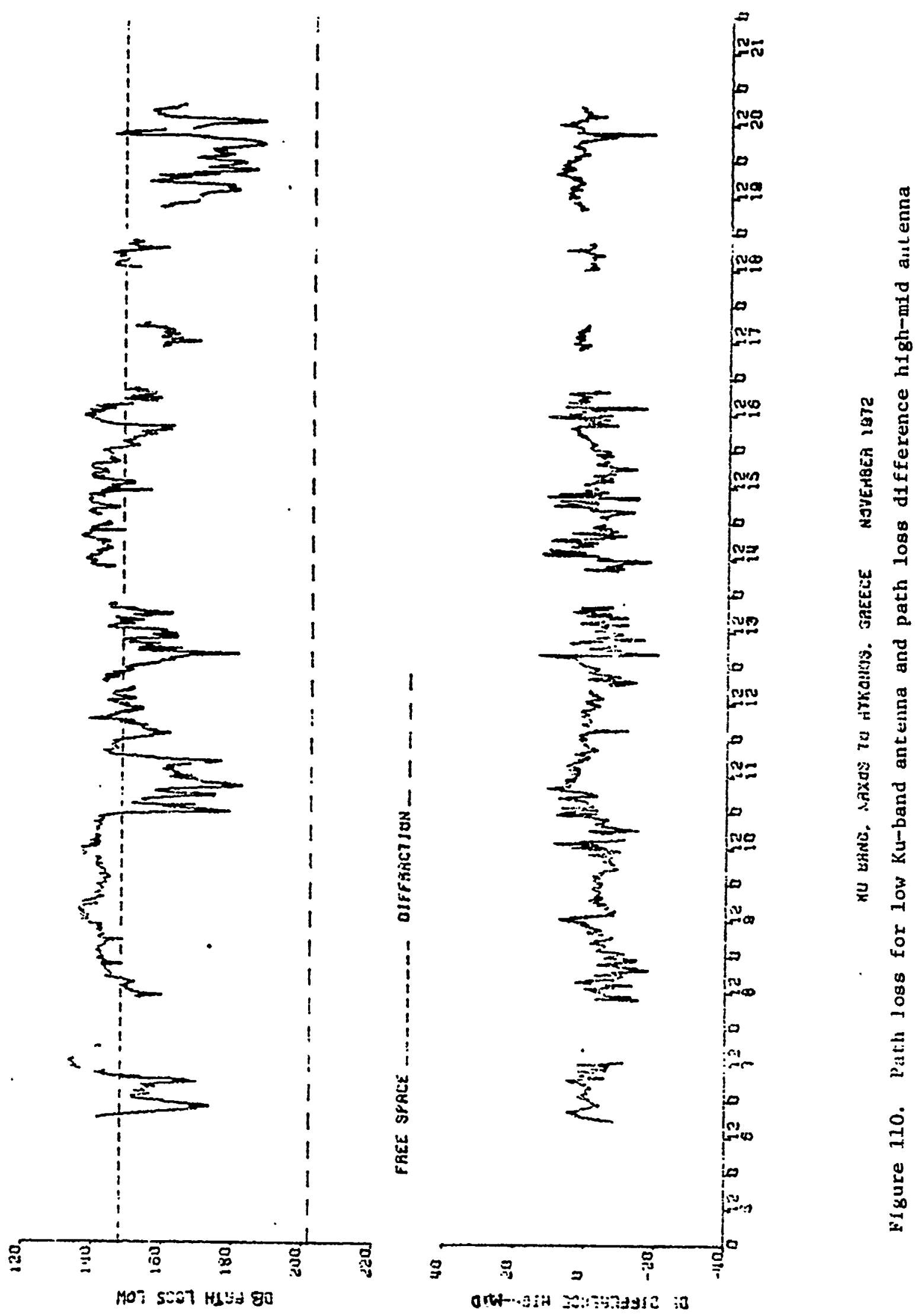


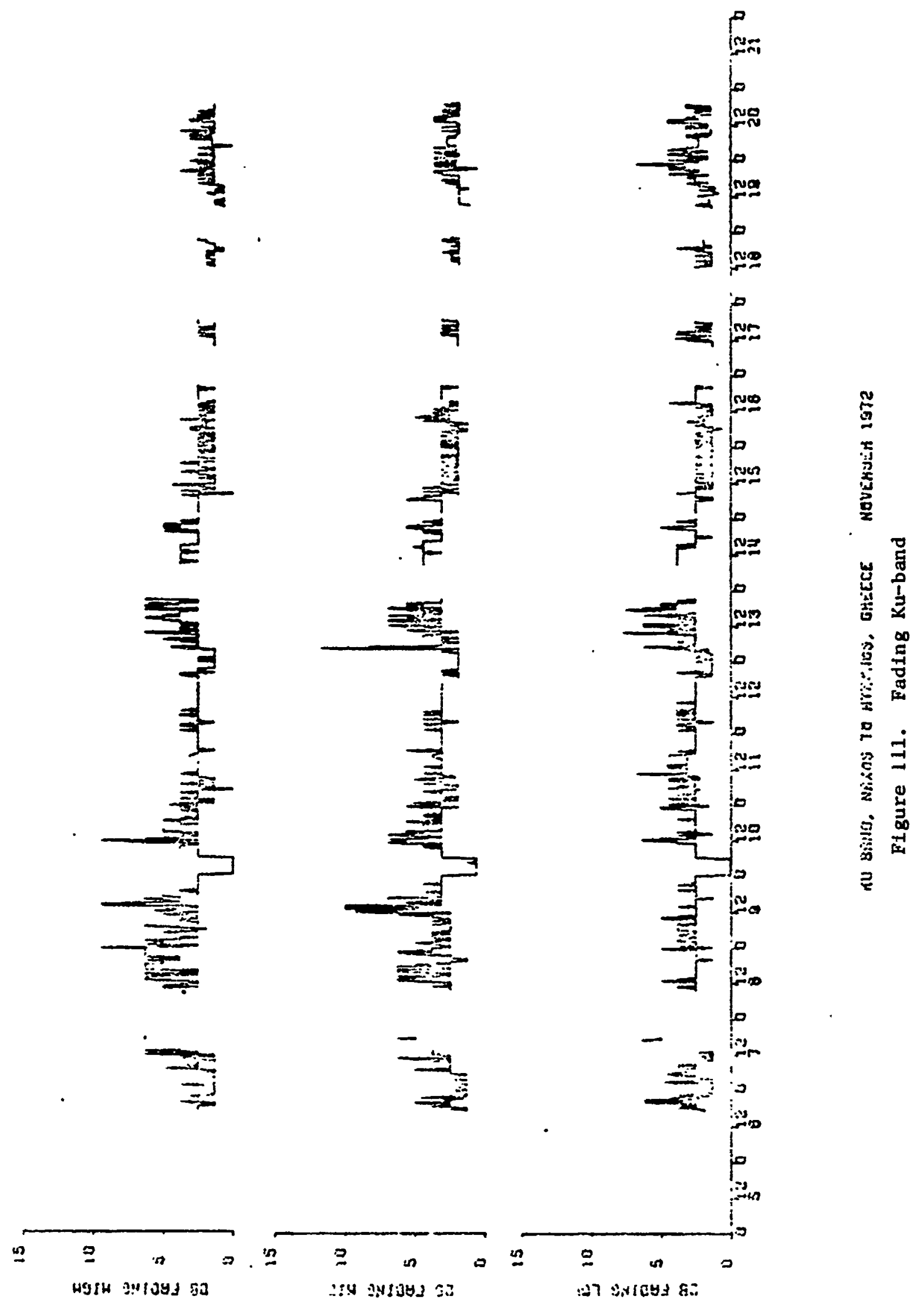




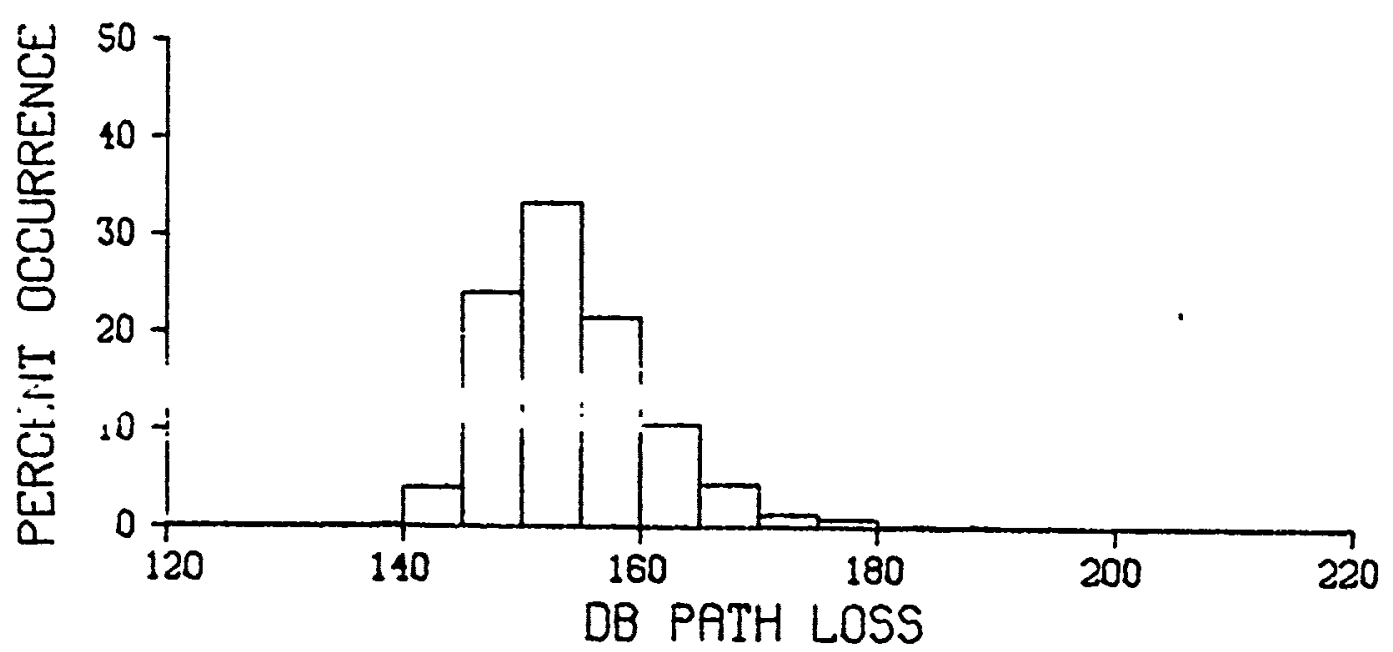

HIGH RNTENNA

1001 OBSERVATIONS

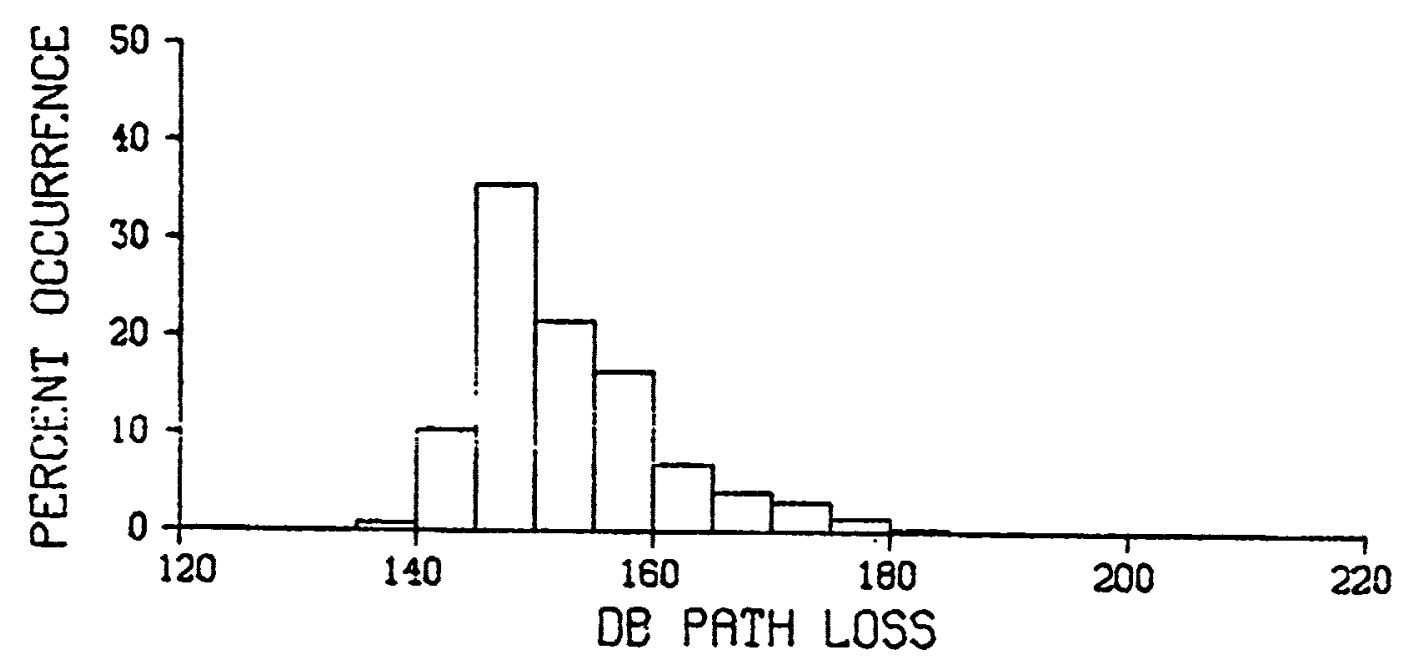

MID ANTENNA

998 OBSERVATIONS $\underset{\exists}{ت}$

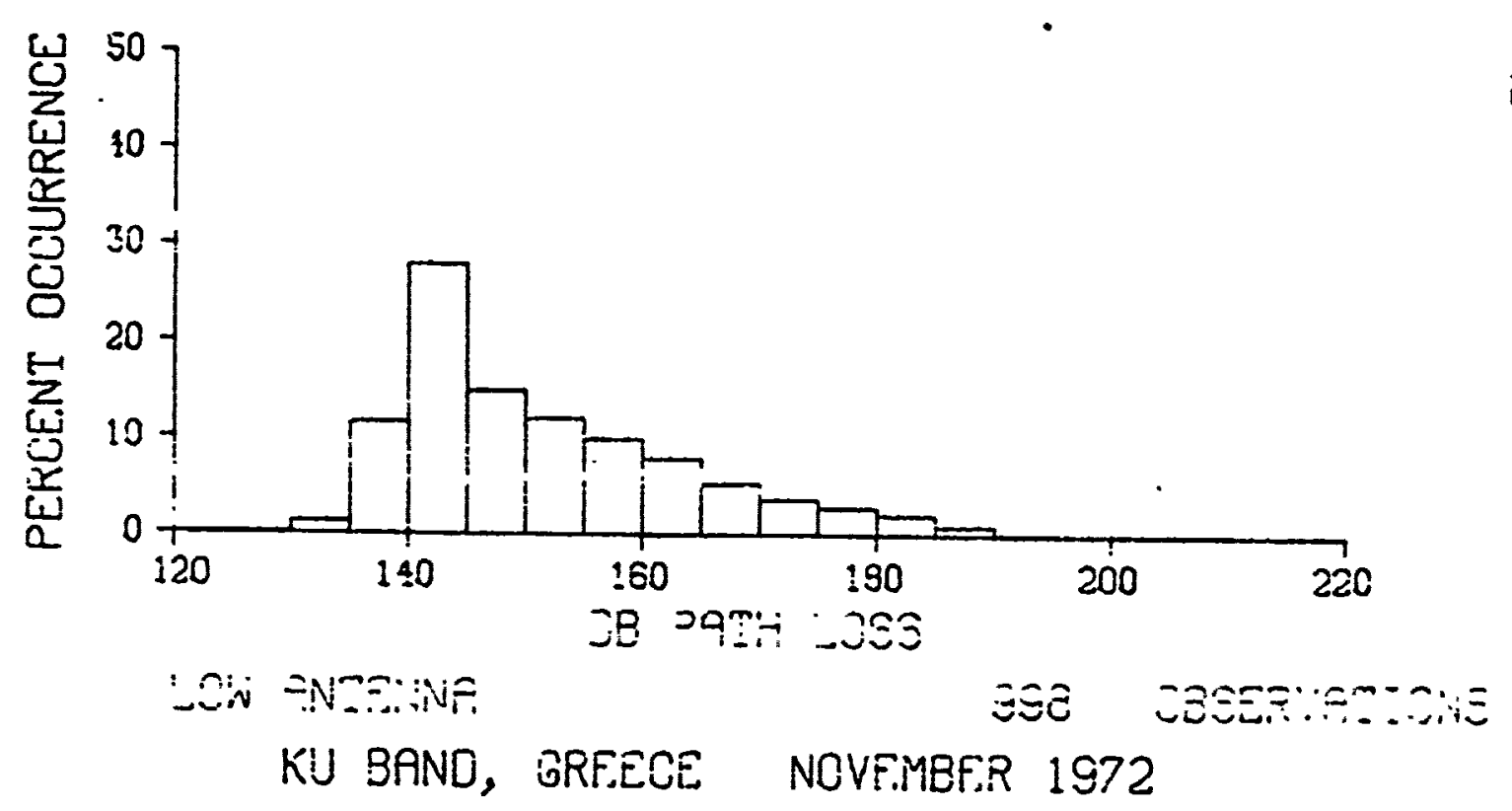



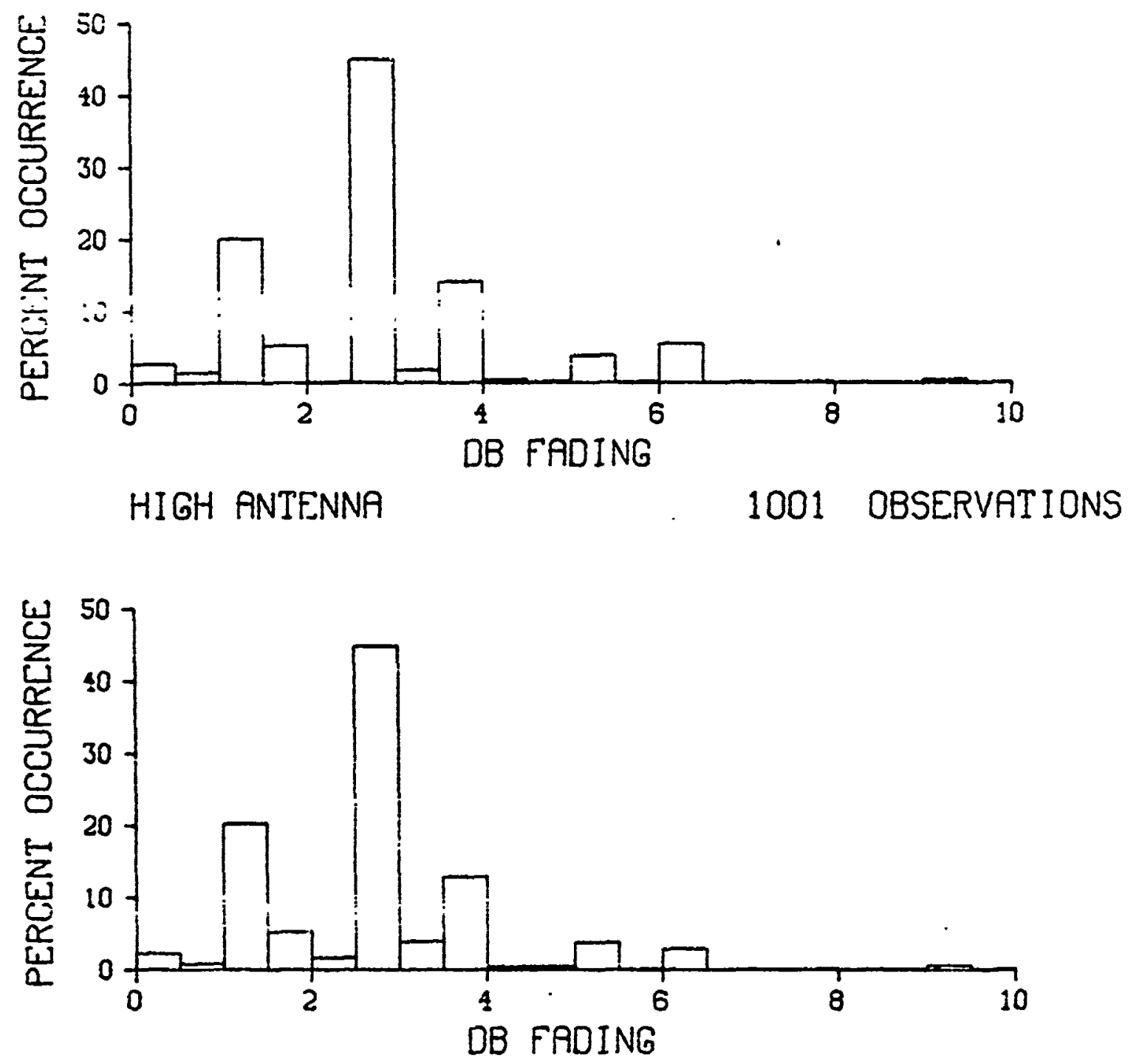

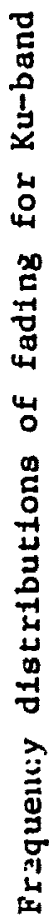
MID ANTENNA 998 OBSERVATIONS $\stackrel{\dot{m}}{=}$

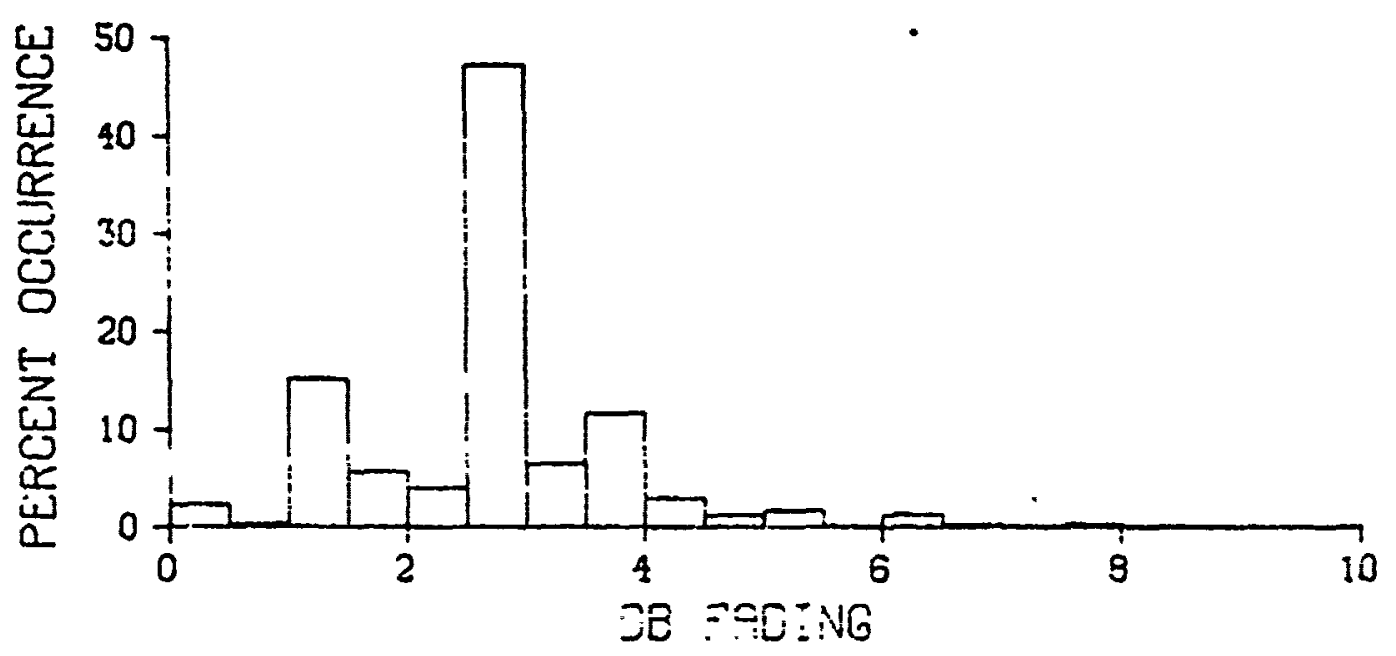

$$
\begin{aligned}
& \text { sén =neE:SHE }
\end{aligned}
$$

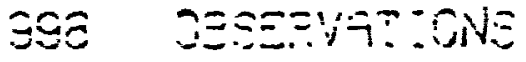

$$
\begin{aligned}
& \text { KU EAND, GREF.CF NOVEMBER } 1972
\end{aligned}
$$



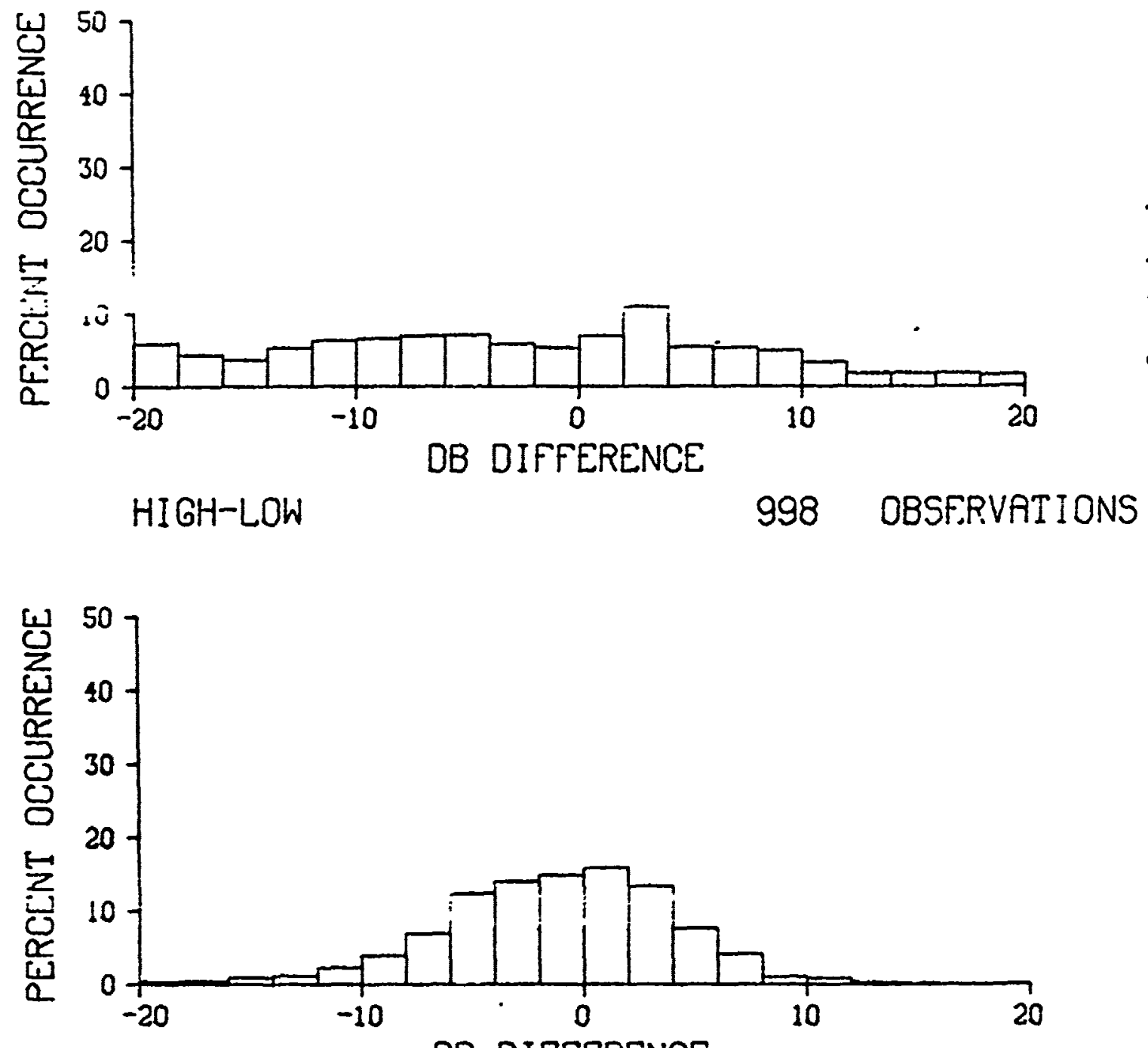

HIGH-MID

DB DIFFERENCE

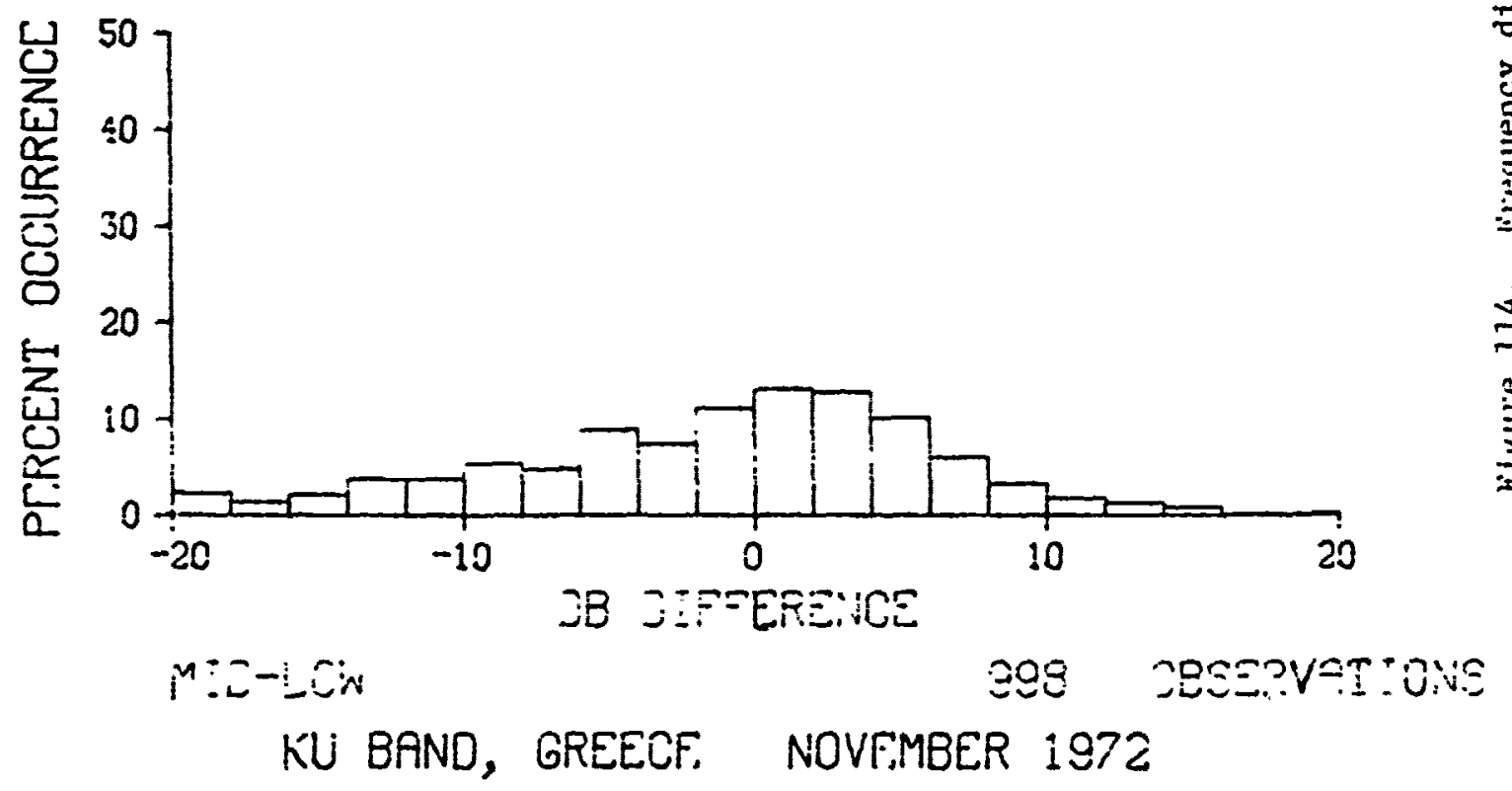



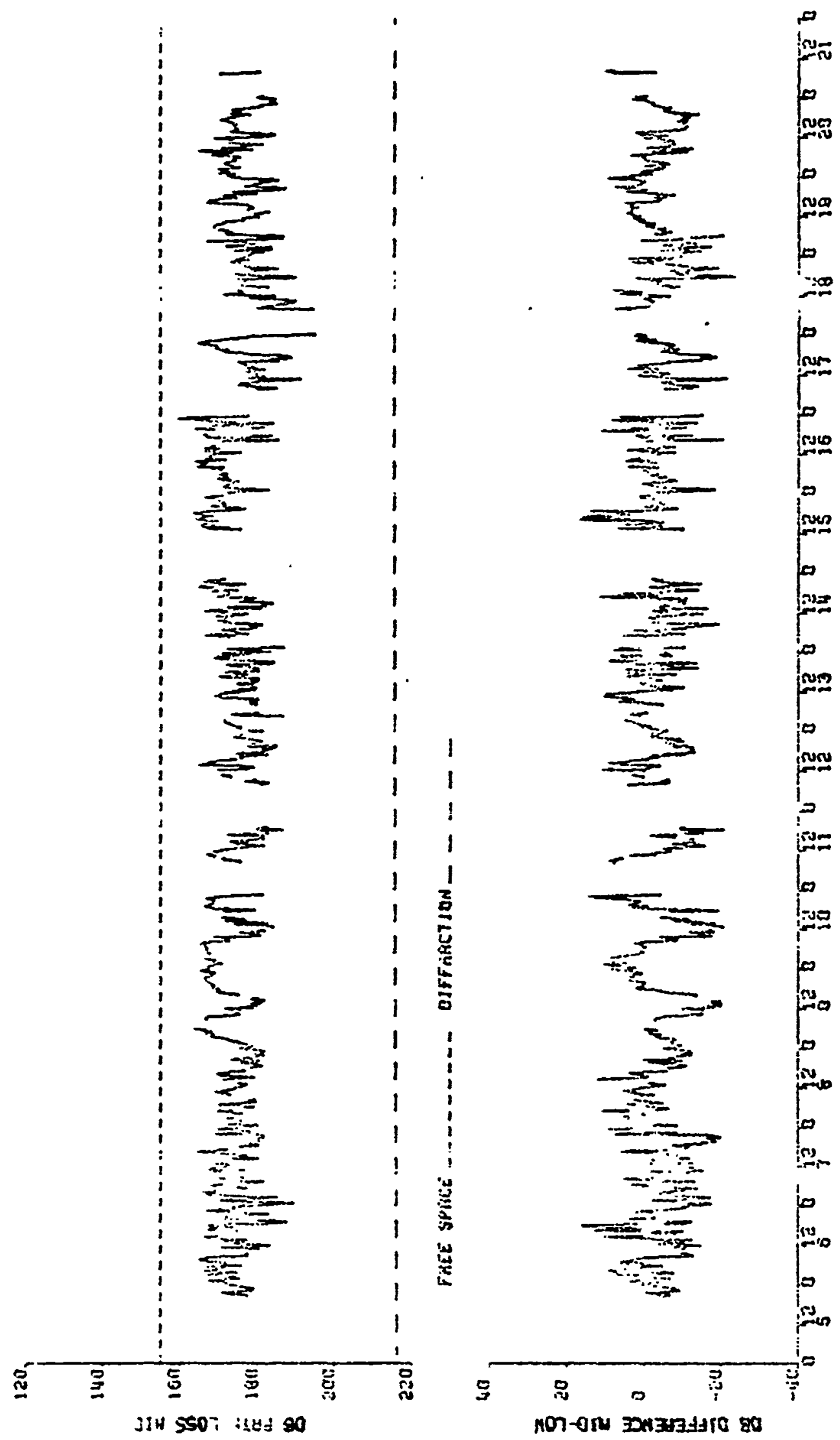


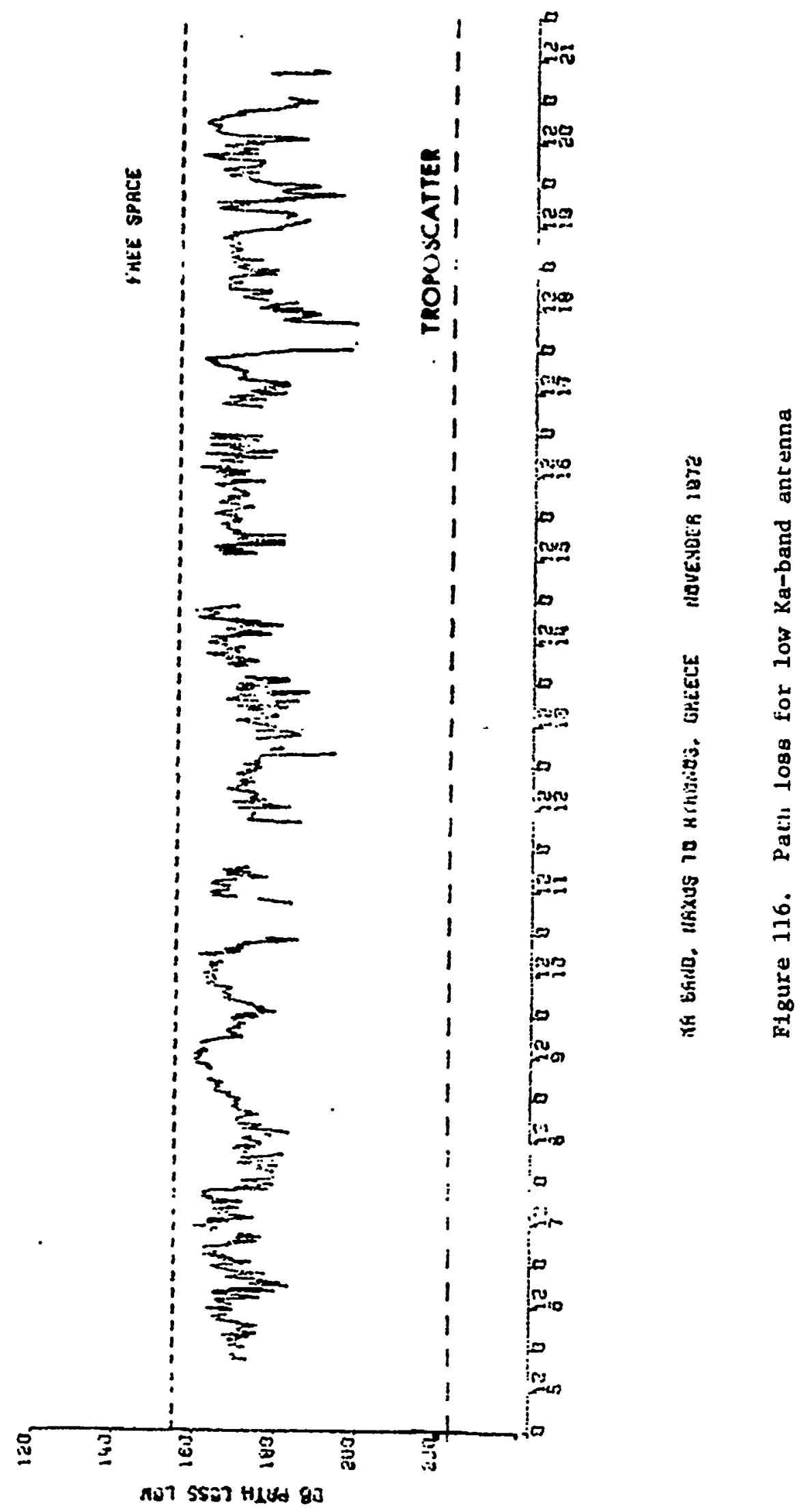




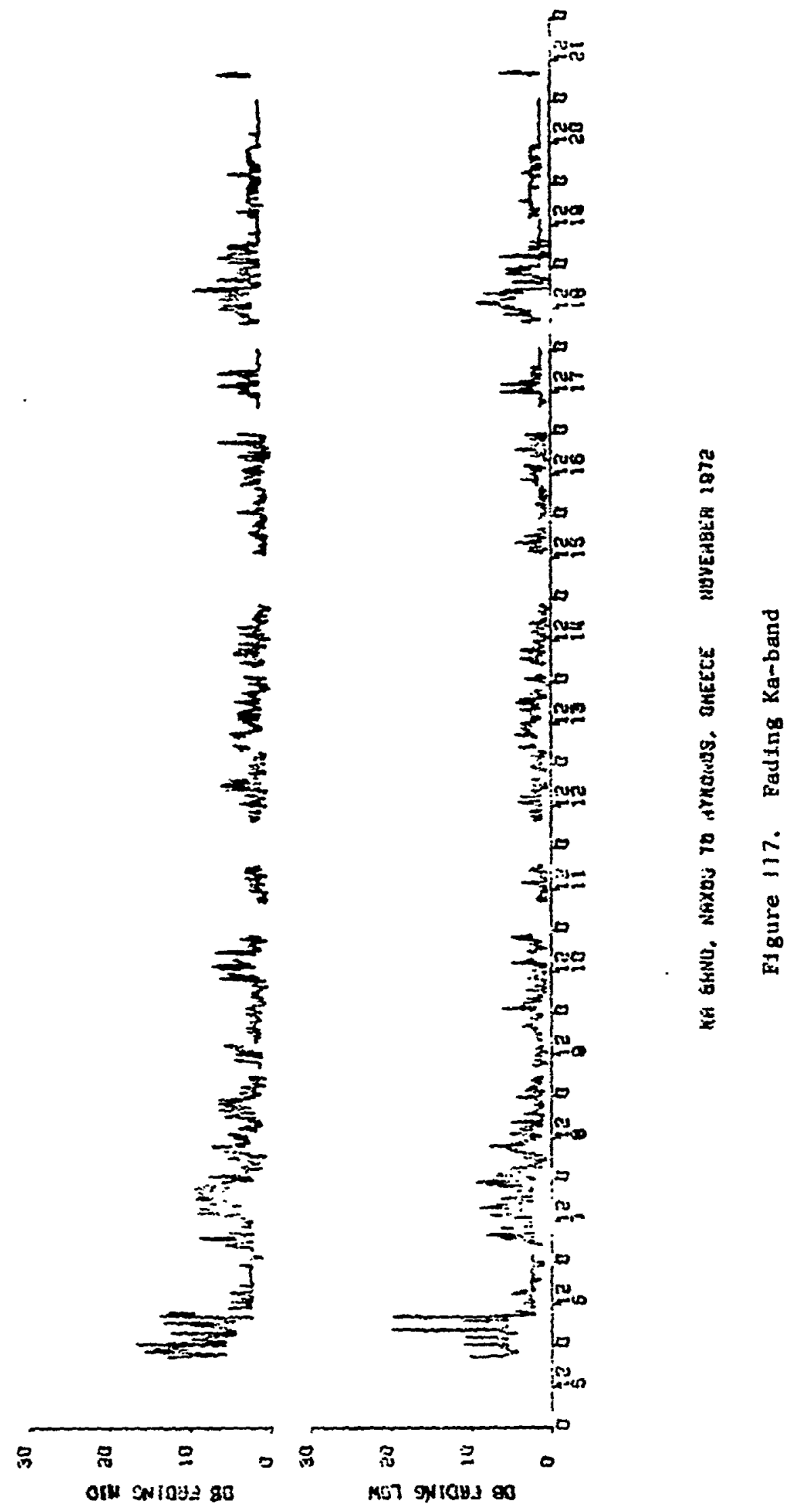




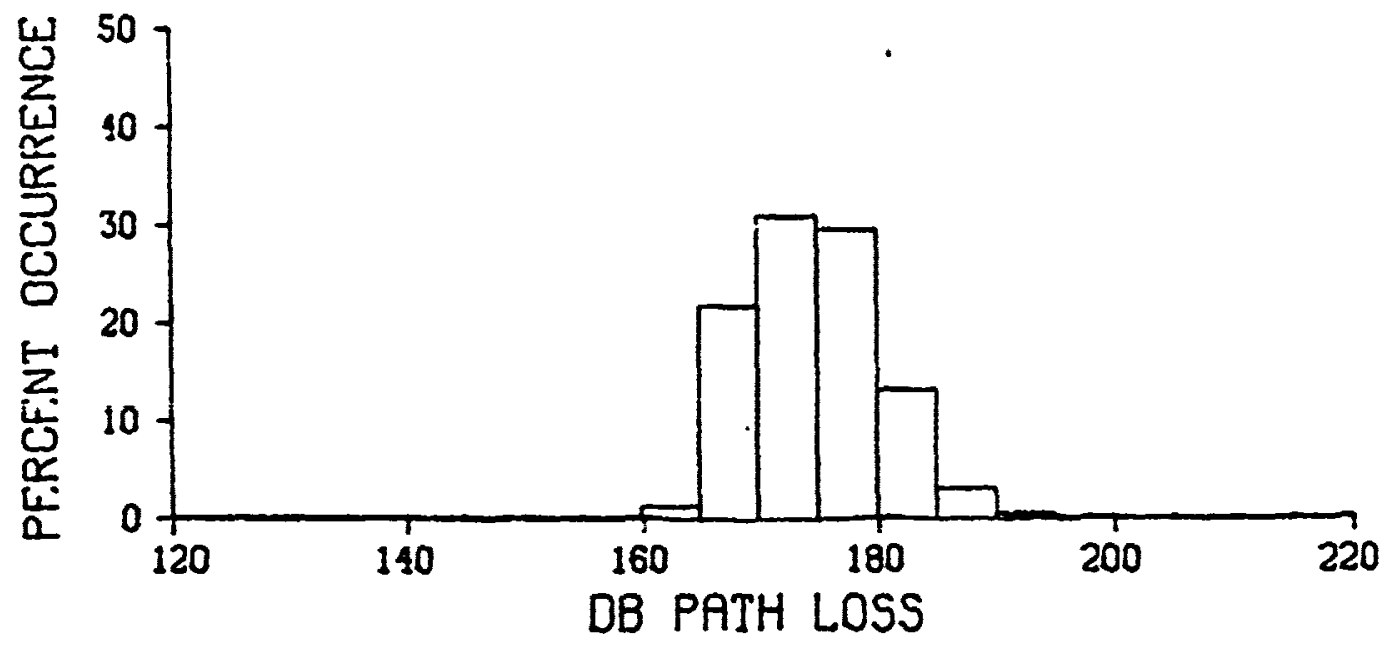

MID ANTENNA

1154 OESERVATIONS

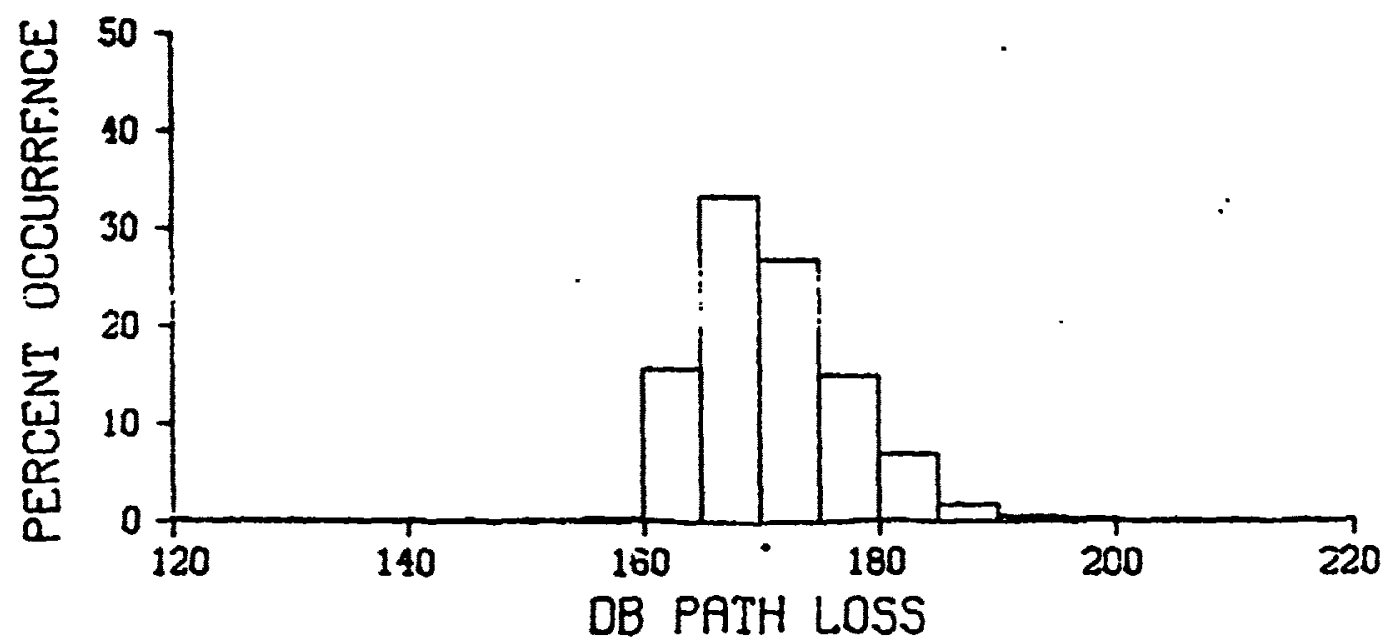

LOW ANTENNA

1155 OBSERVRTIONS

KA BAND. GREECE NCVE:1BF.R 1972

Figure 118. Frequency distribution of path loss for Ka-jand 


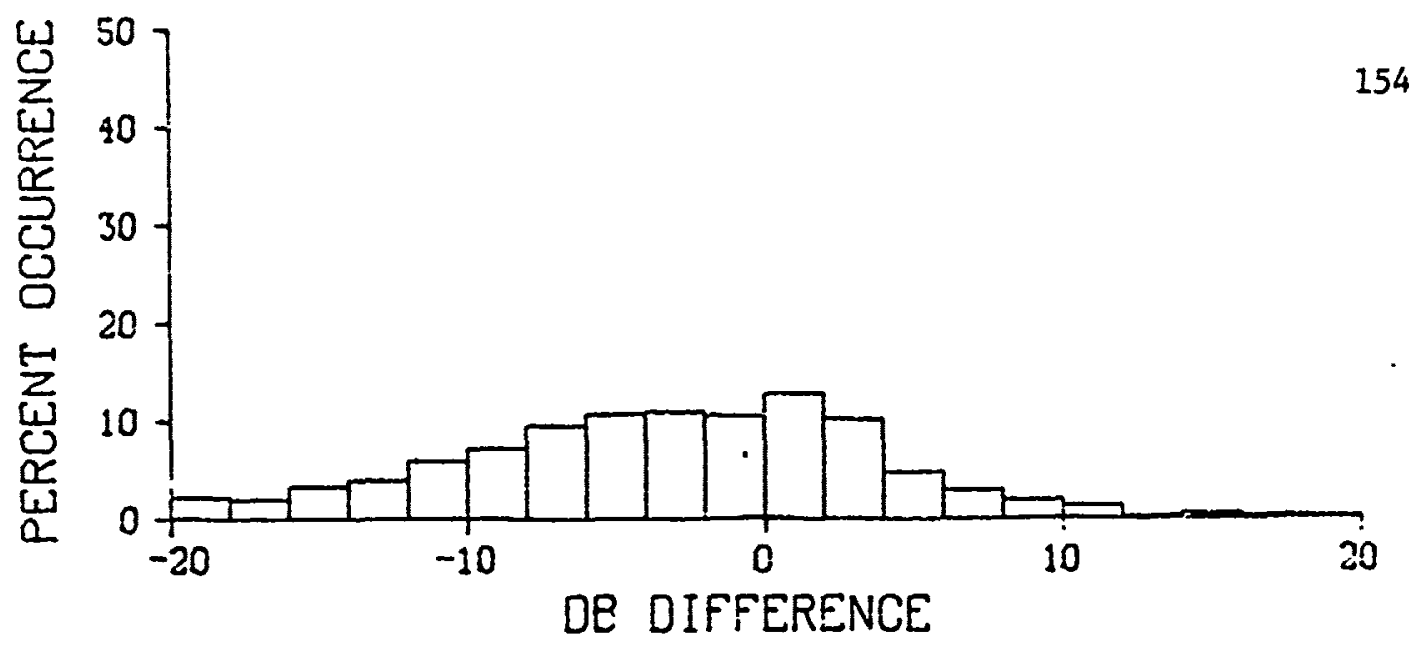

MID-LOW

1146 OESEP.VATIONS

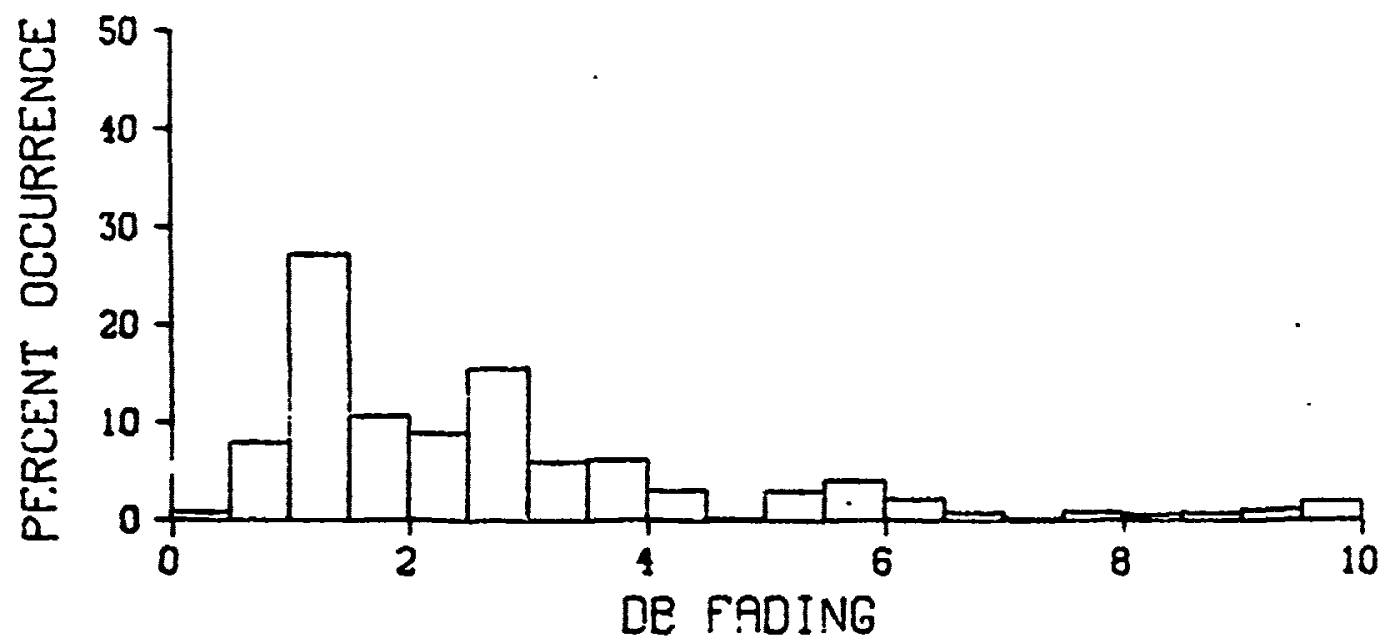

MID ANTENNR

1154 OBSERVATIONS

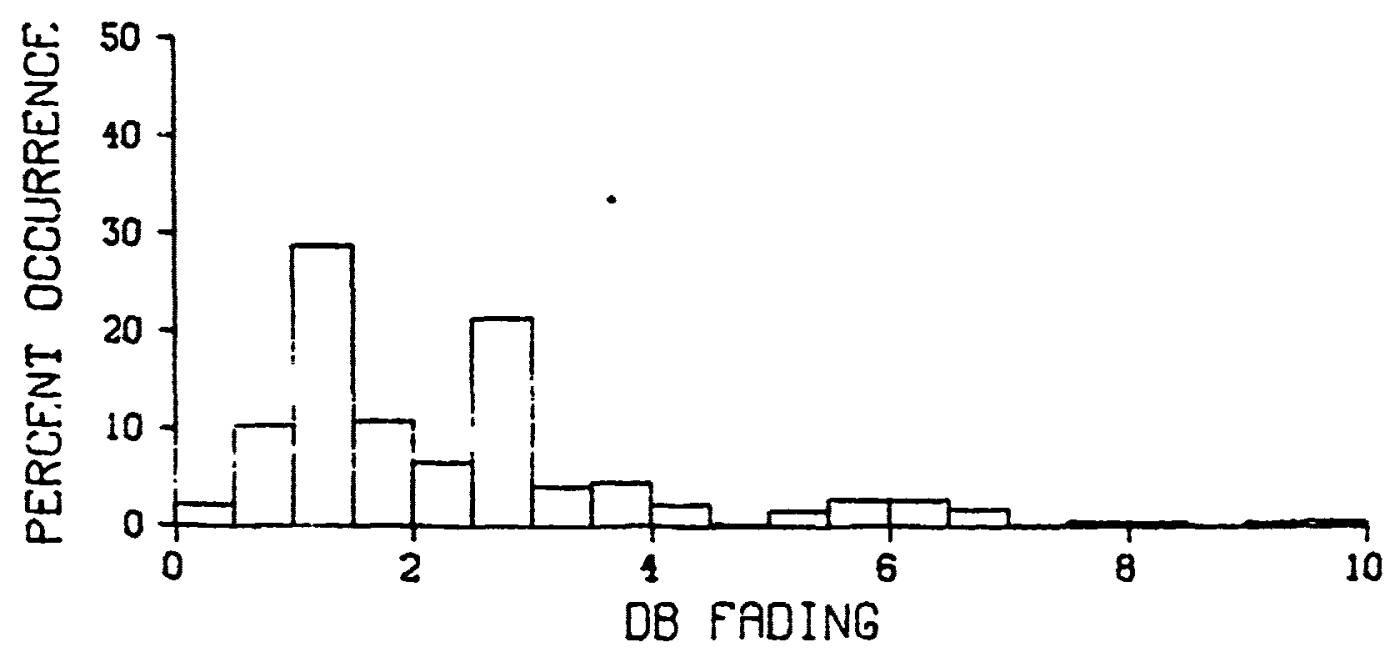

LOW ANTFNNA

1155 OBSERVATIONS KA BAND, GREECE NOVFMBEP. 197?

Figure 1ij. Frequency distrijurions uf fara loss iitierence between antennas ma jading is: la-iand 
8

$\sqrt{3}$
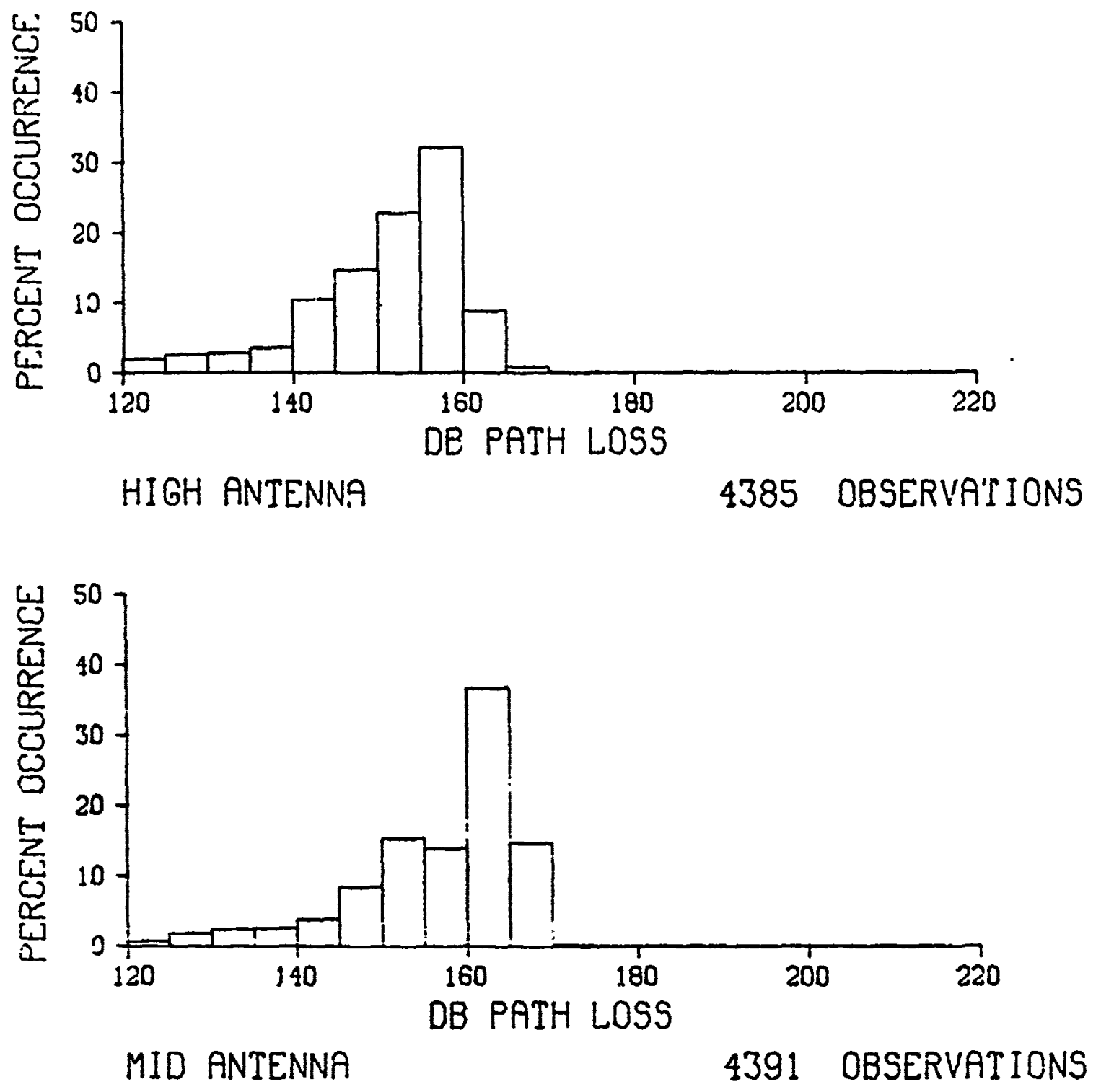

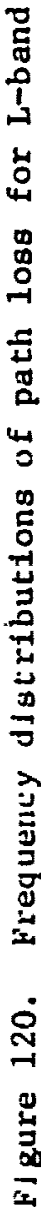

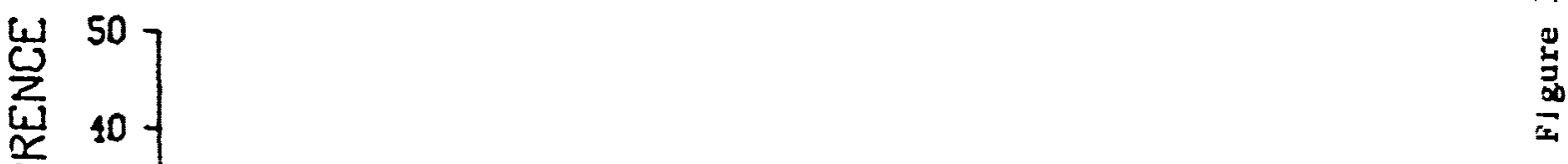

s

8

10

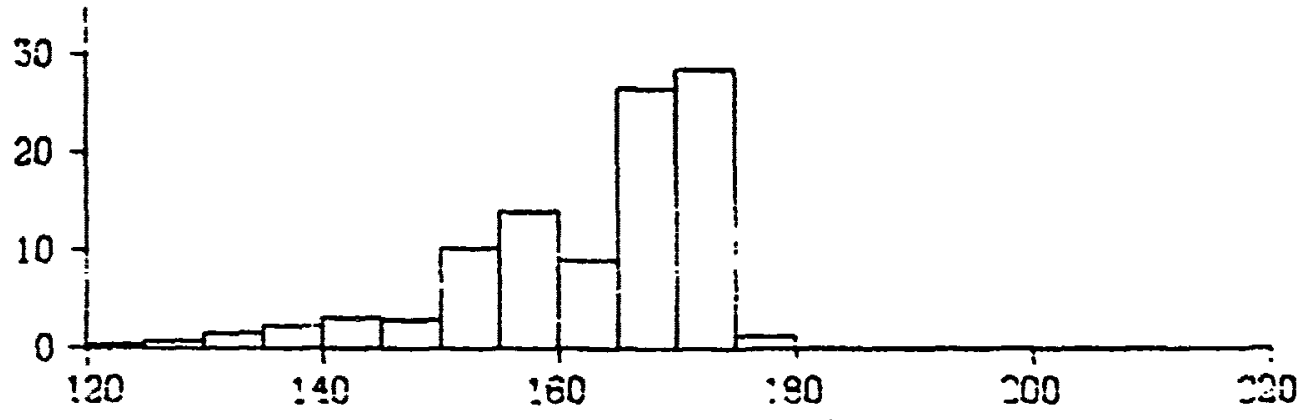

DS PATH LOSS

LOW FNTE:INT:

4387 OESERY $T$ TIONS

L BFND, ALL SEFSONS 1972 


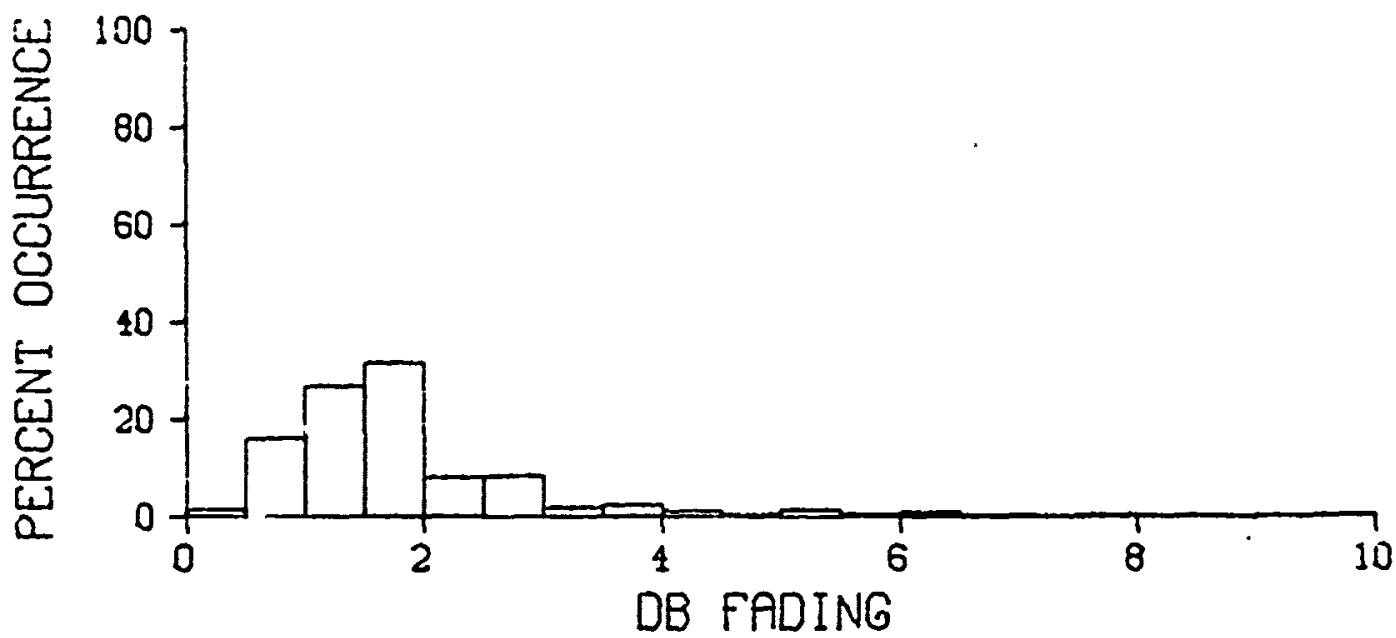

HIGH ANTENNA

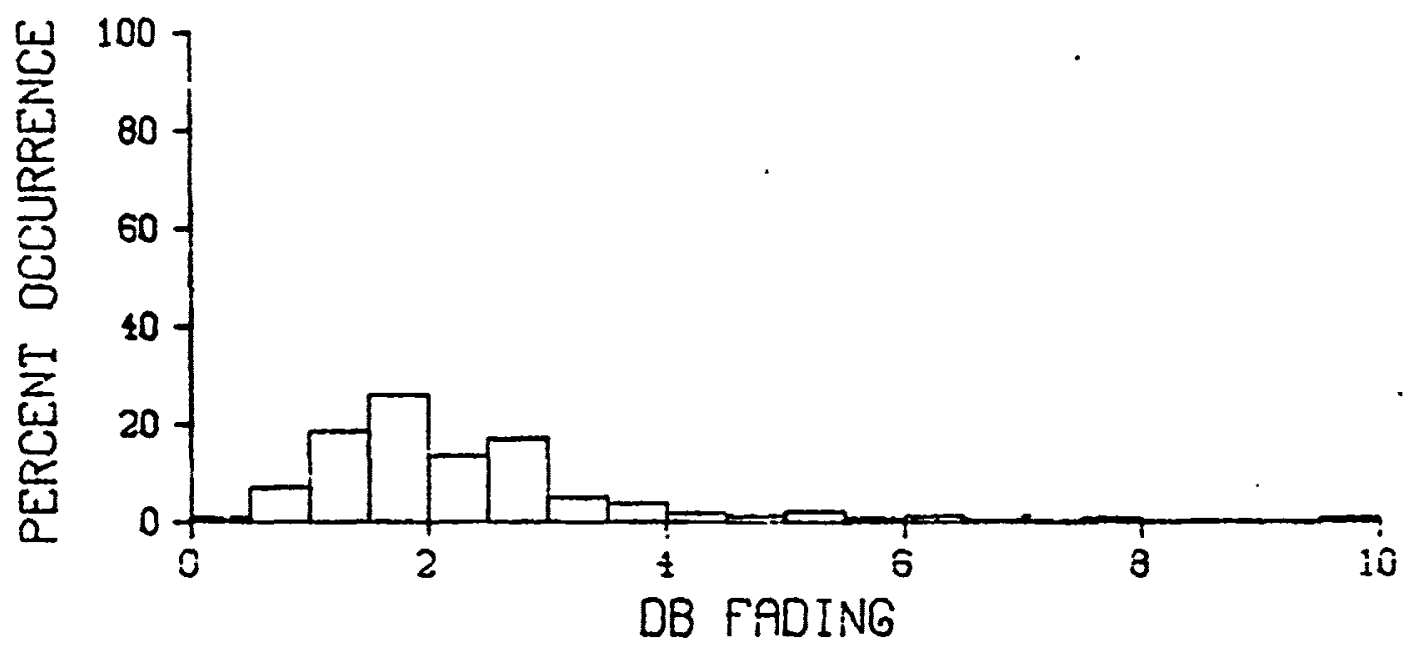

MID ANTENNA

4391 OBSERVATIONS

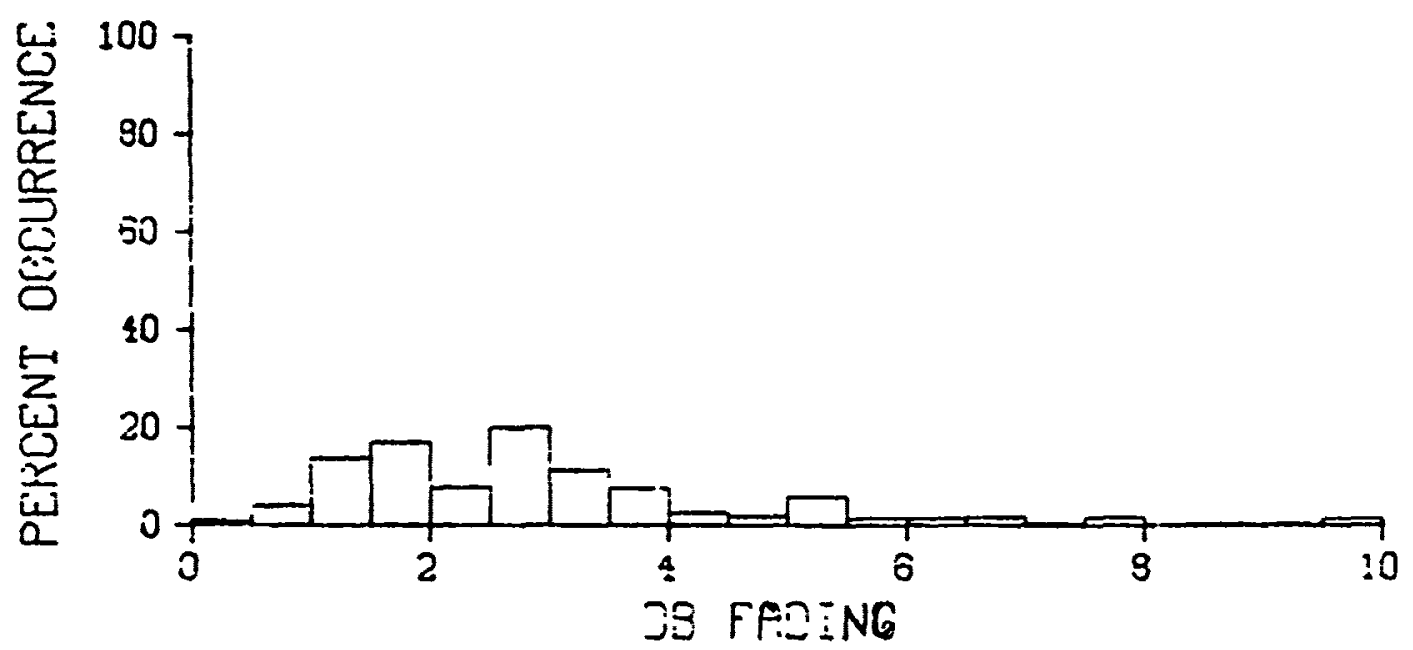

LCW RINTENNF:

4387 DESERVATIO::O

L BAND, FLL SEASEONS i972 

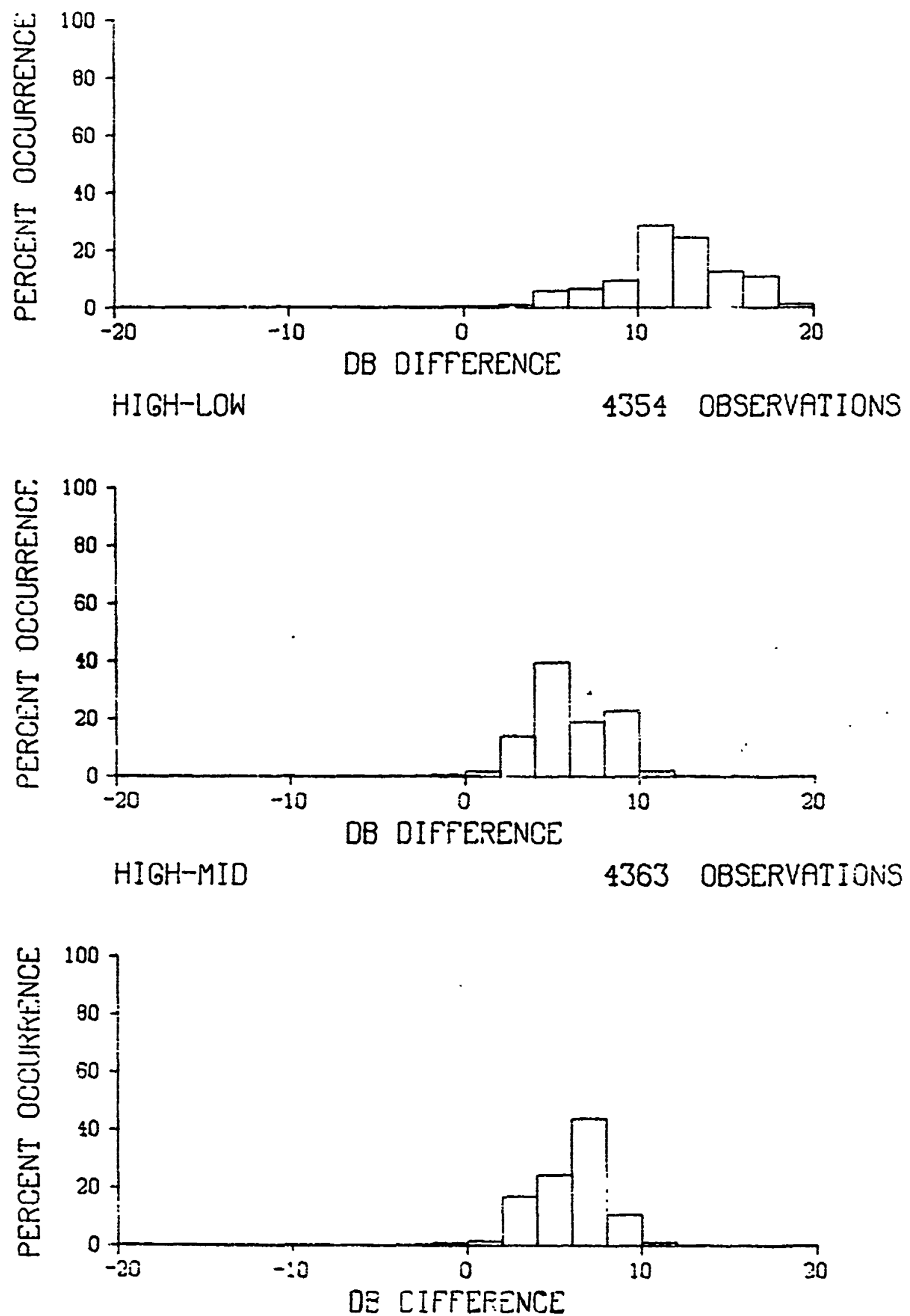
MED-LOH

L BAND, PILL SEASONS iG72 


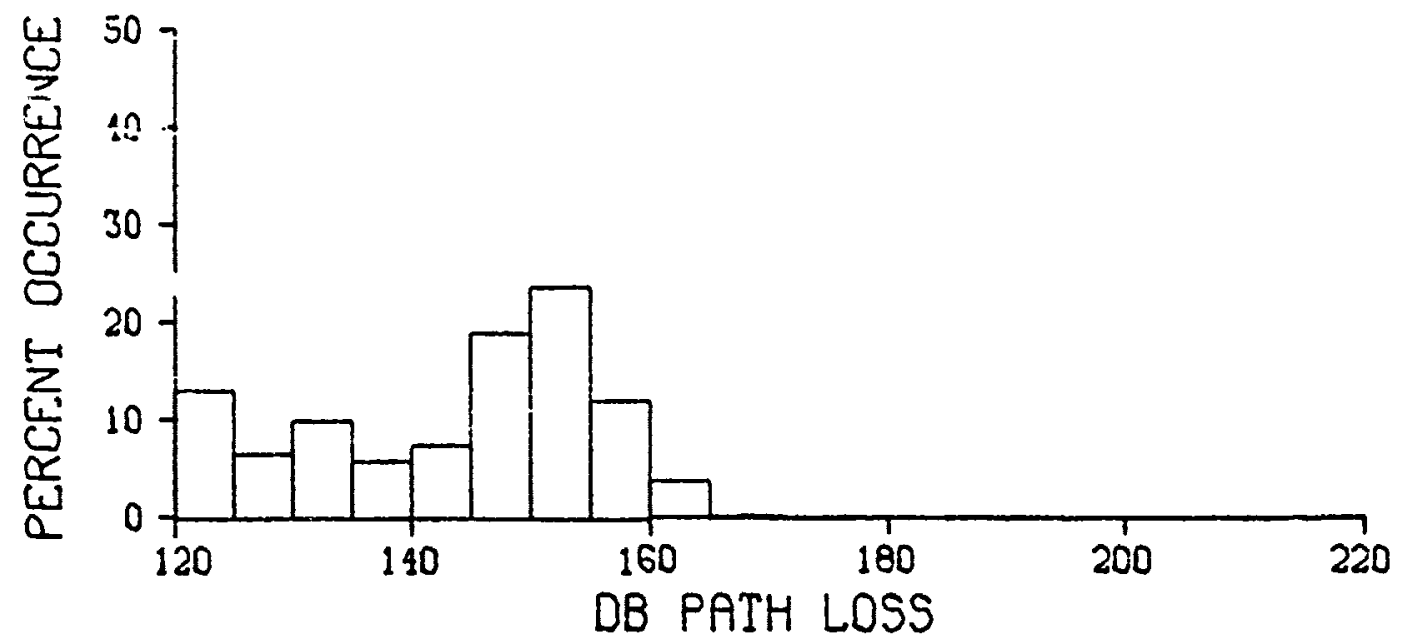

HIGH PINTENNA

4546 OBSERVATIONS
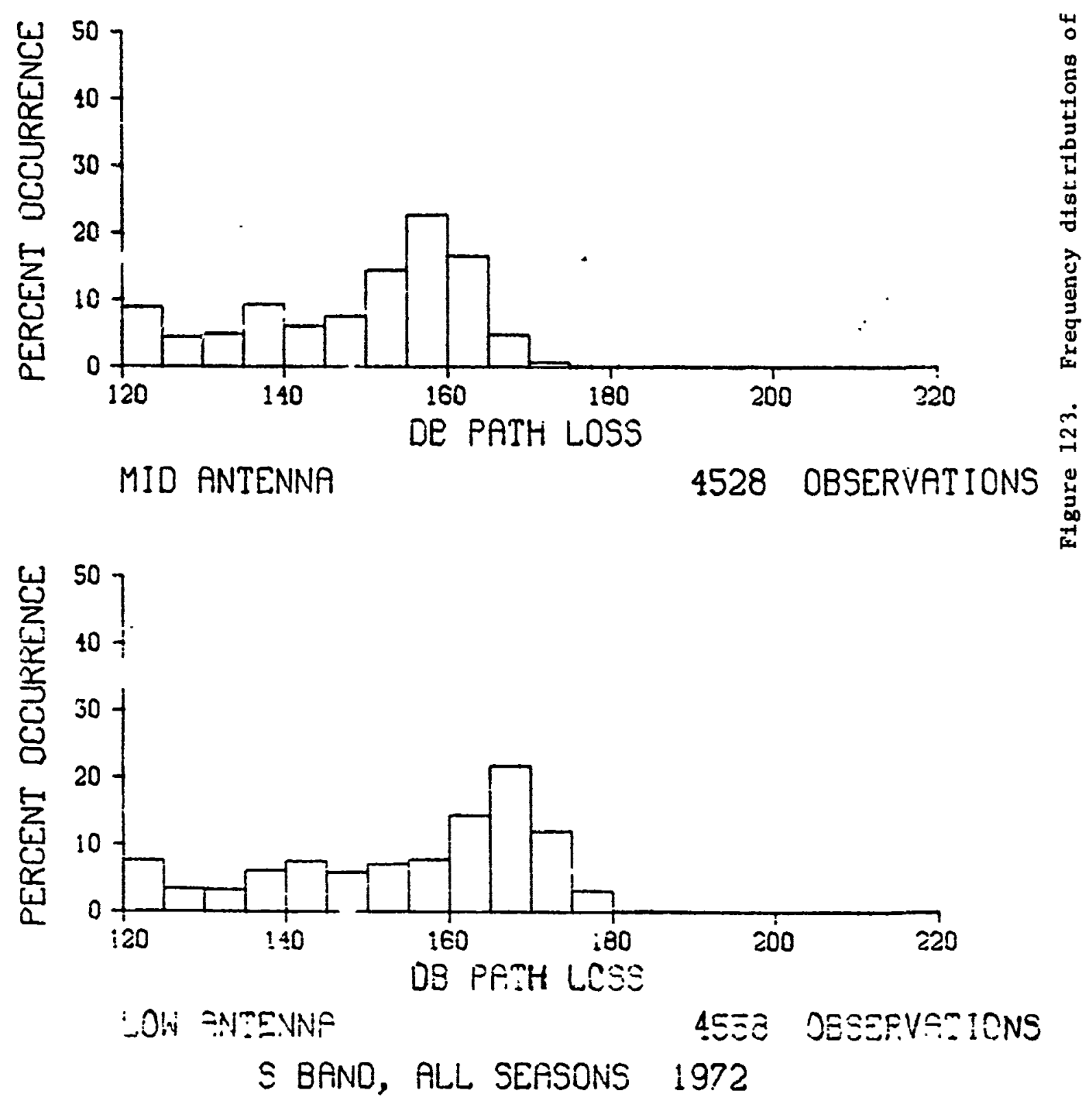
8

8

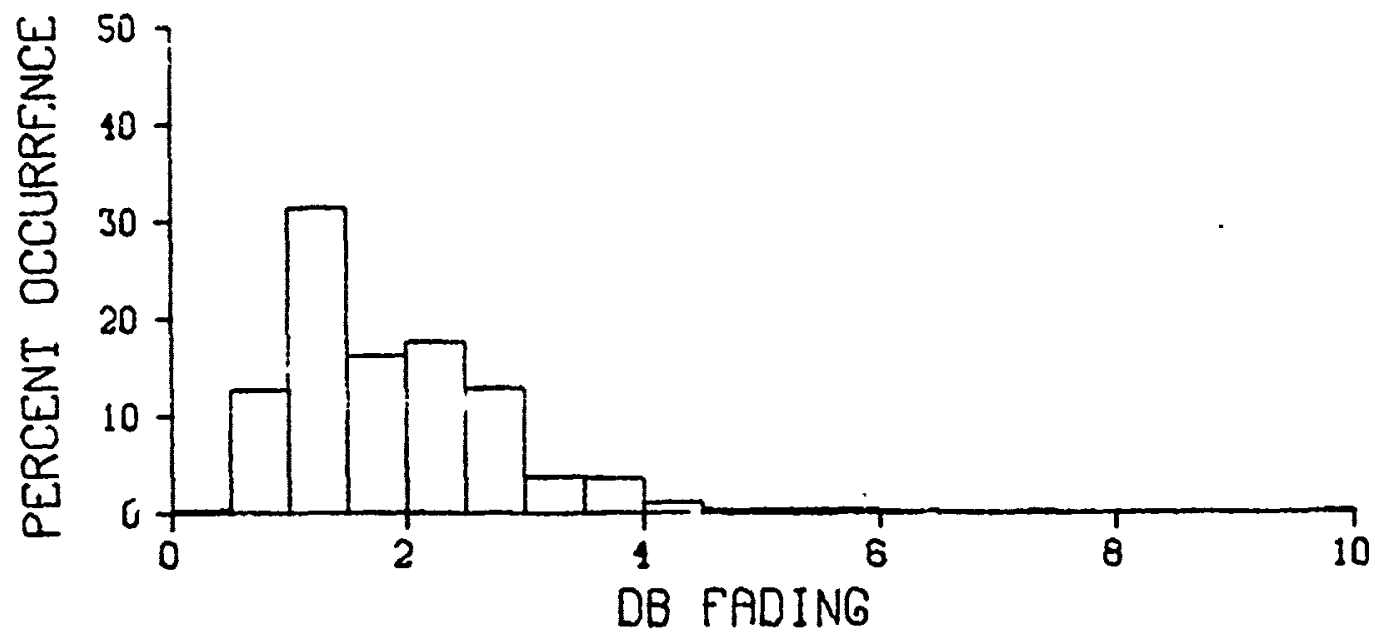

HIGH ANTENNA

4546 OBSERVATIONS

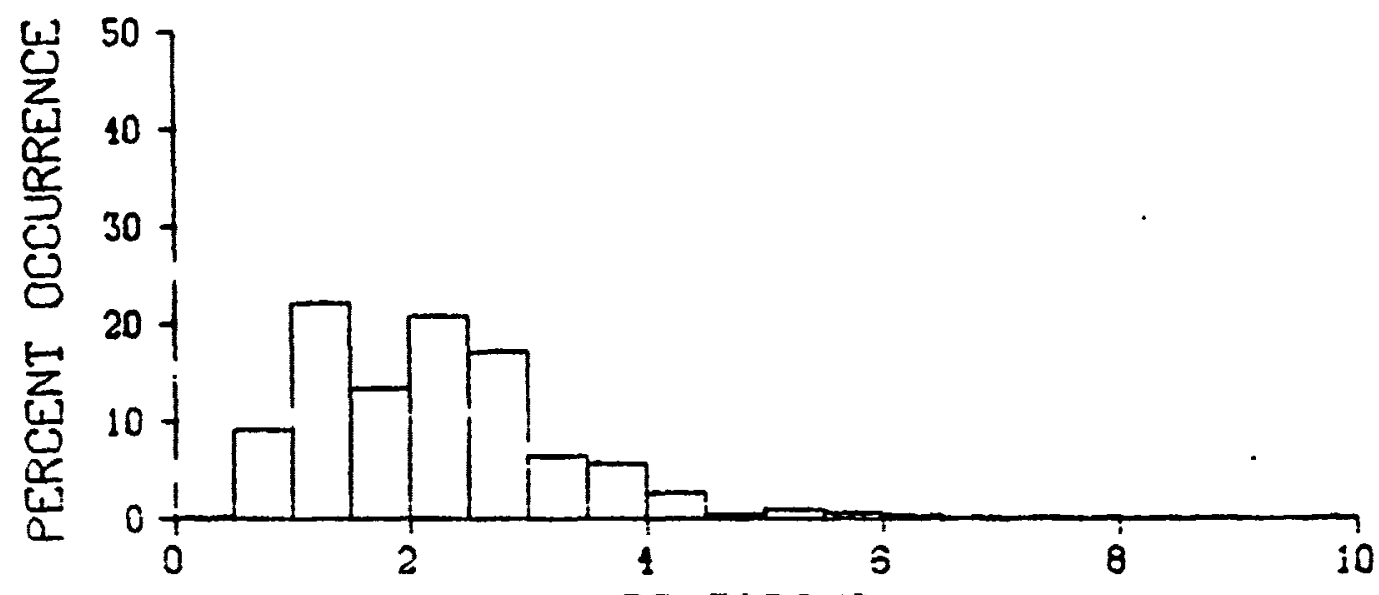

DB FADING

MID ANTENNA

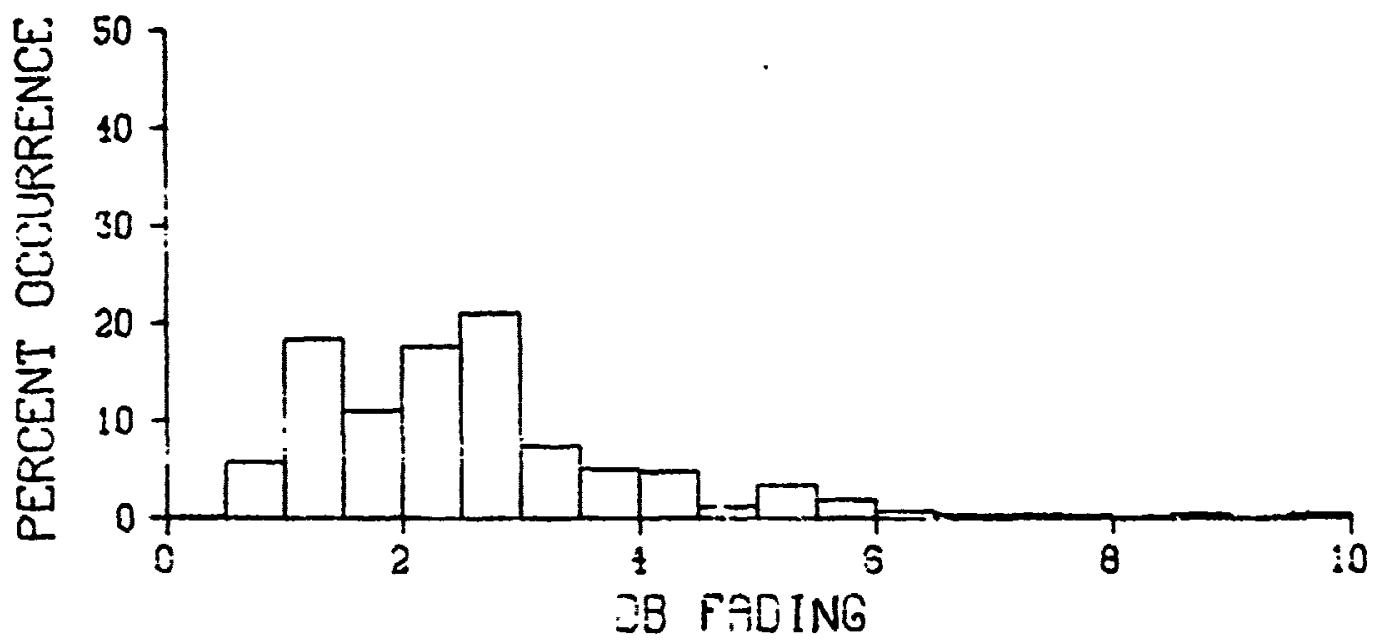

LIN GNTENNA

\5SC UESEFVF:- -US

$S$ BAND, ALL SEFISONS 1972 


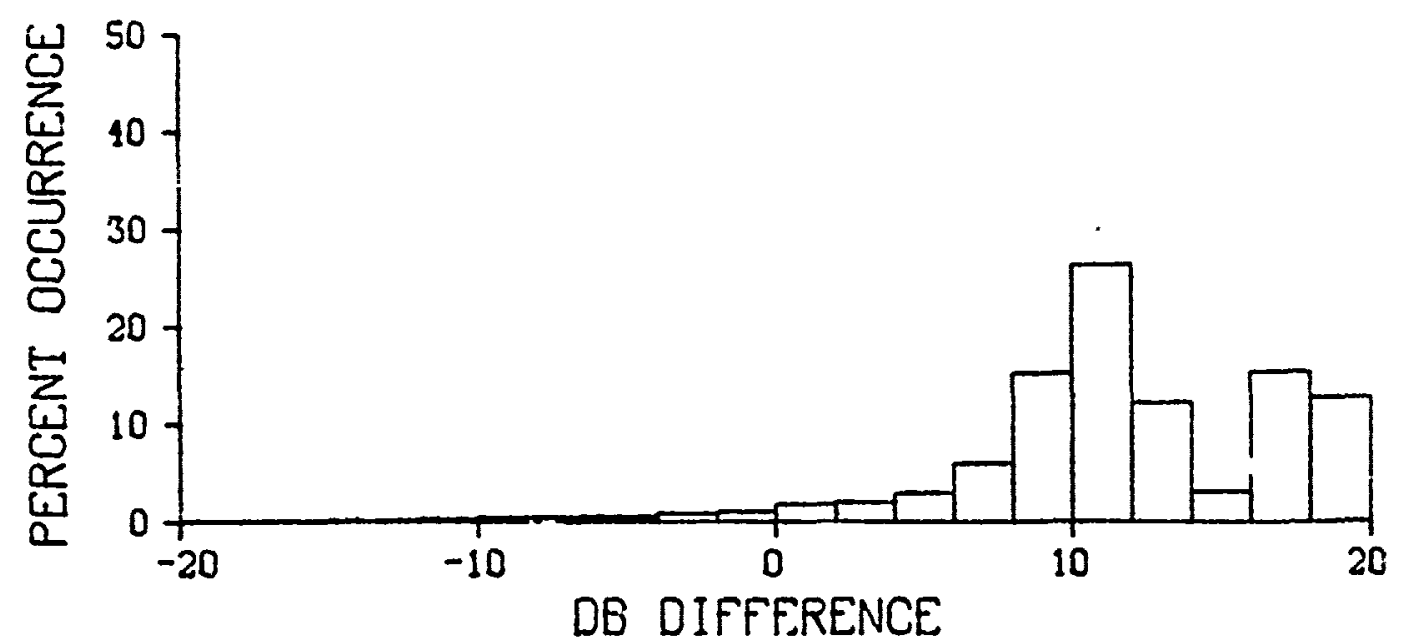

HIGH-LOW

4460 OBSERVATIONS

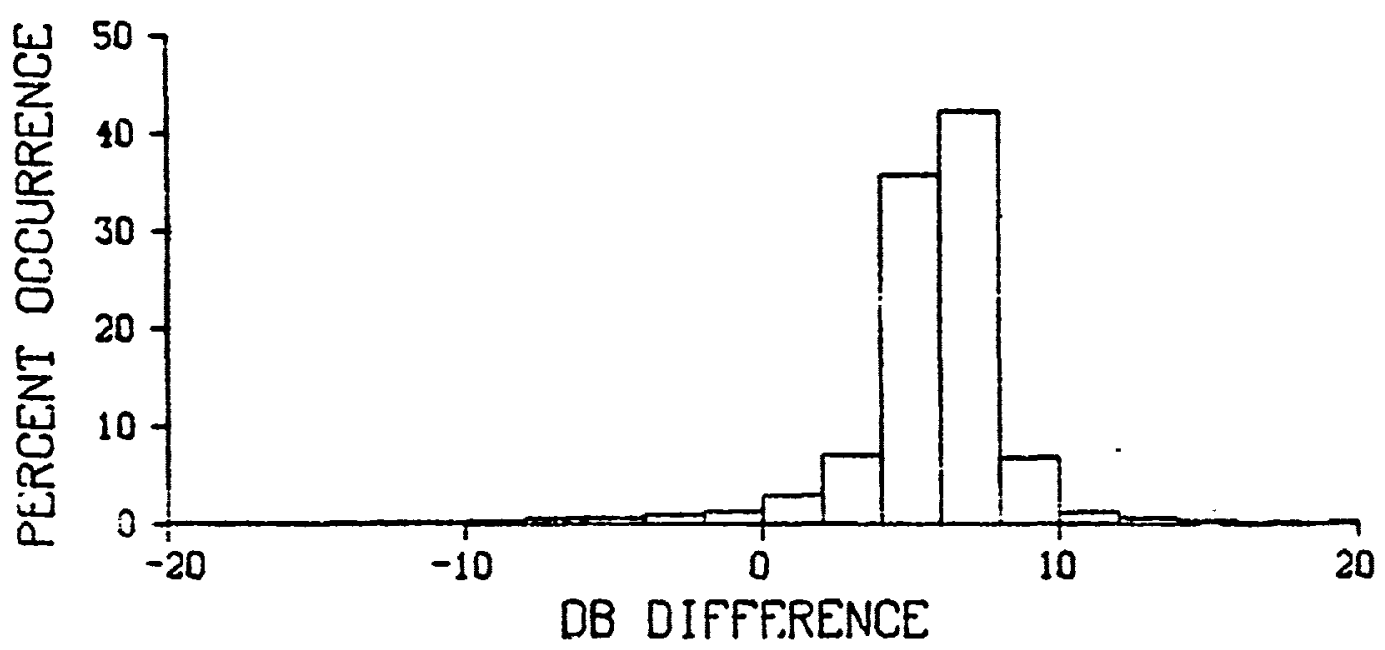

IIIGIT-MID

4440 OBSEPVATICNS

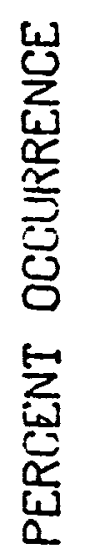
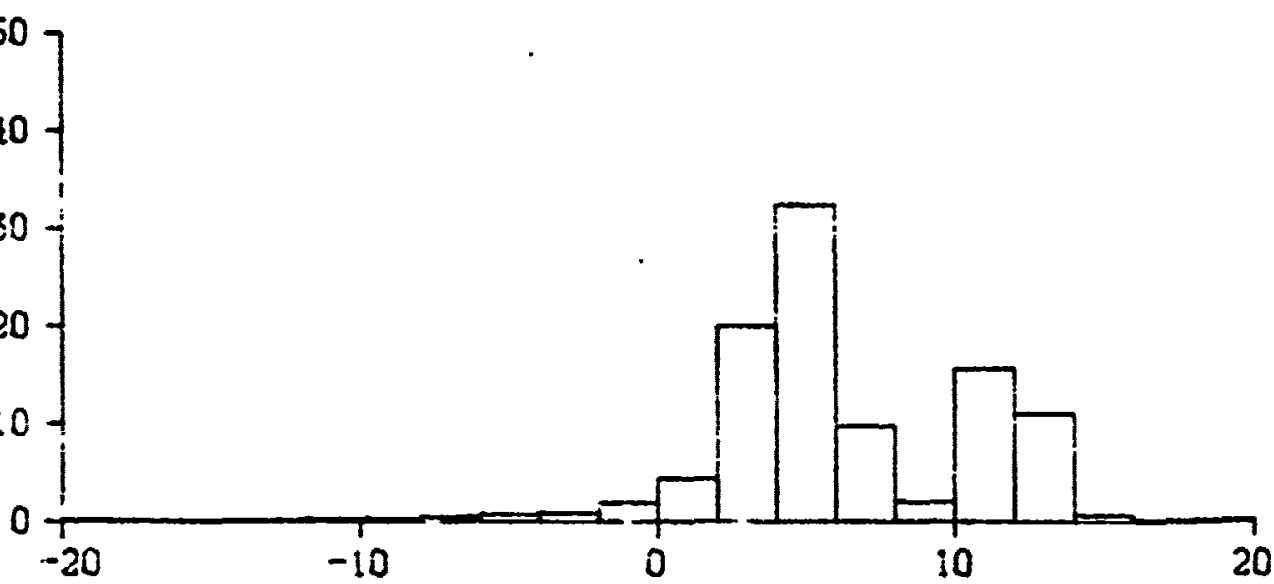

SB ZIFFEREICE

MID-LOW

4450 CBSERVATIONS

$S$ BFND, PLL SERSCNS $: 972$ 

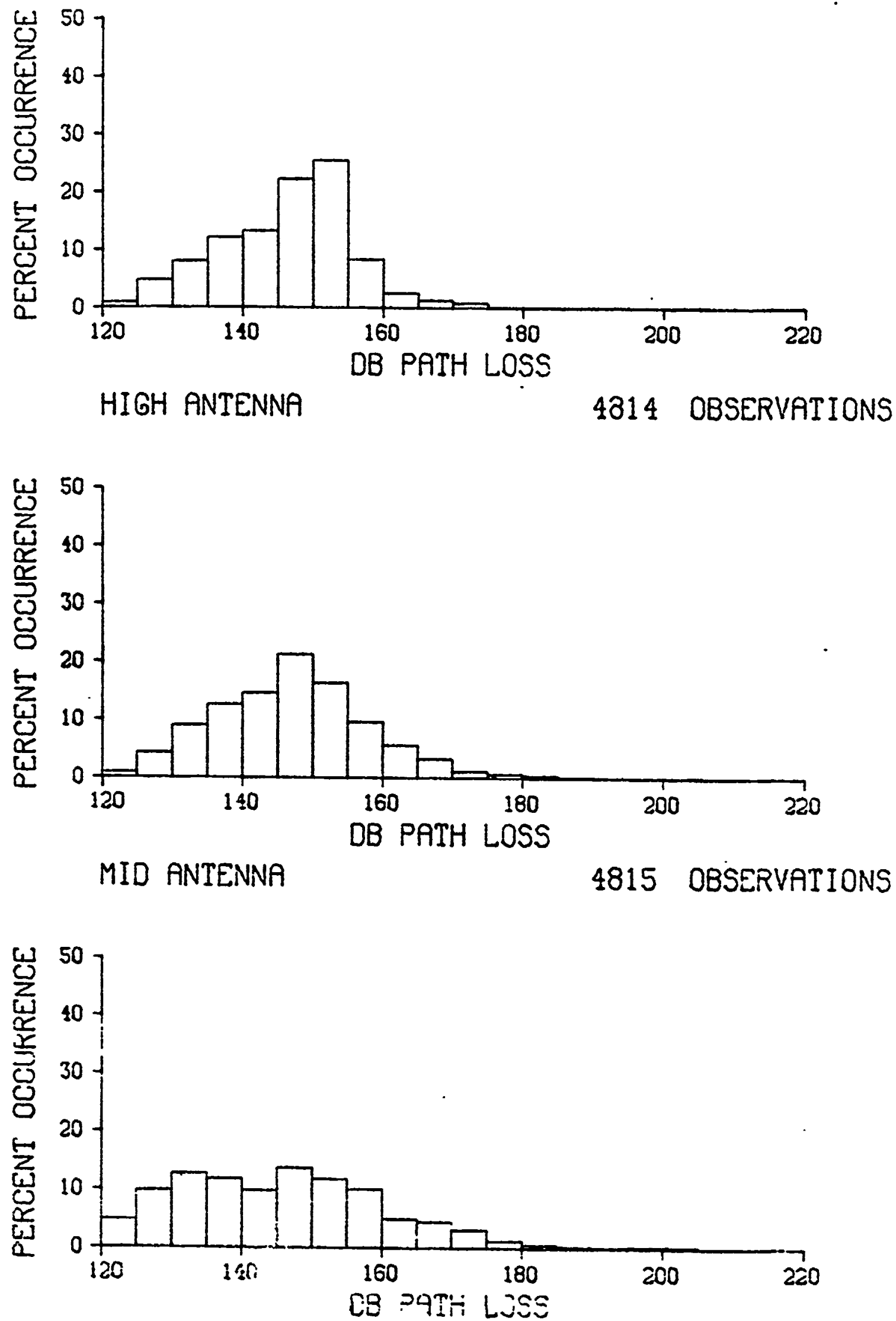

U.W INTENENA 


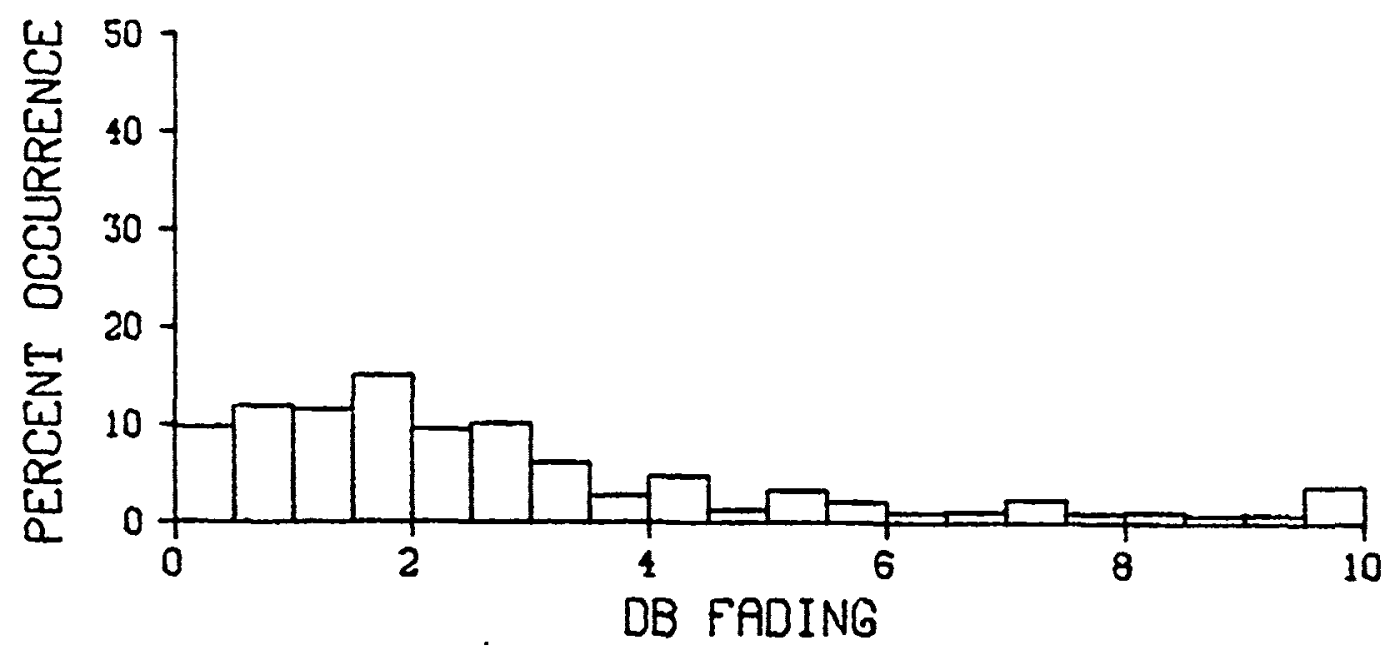

HIGH ANTENNA

4814 OBSERVATIONS

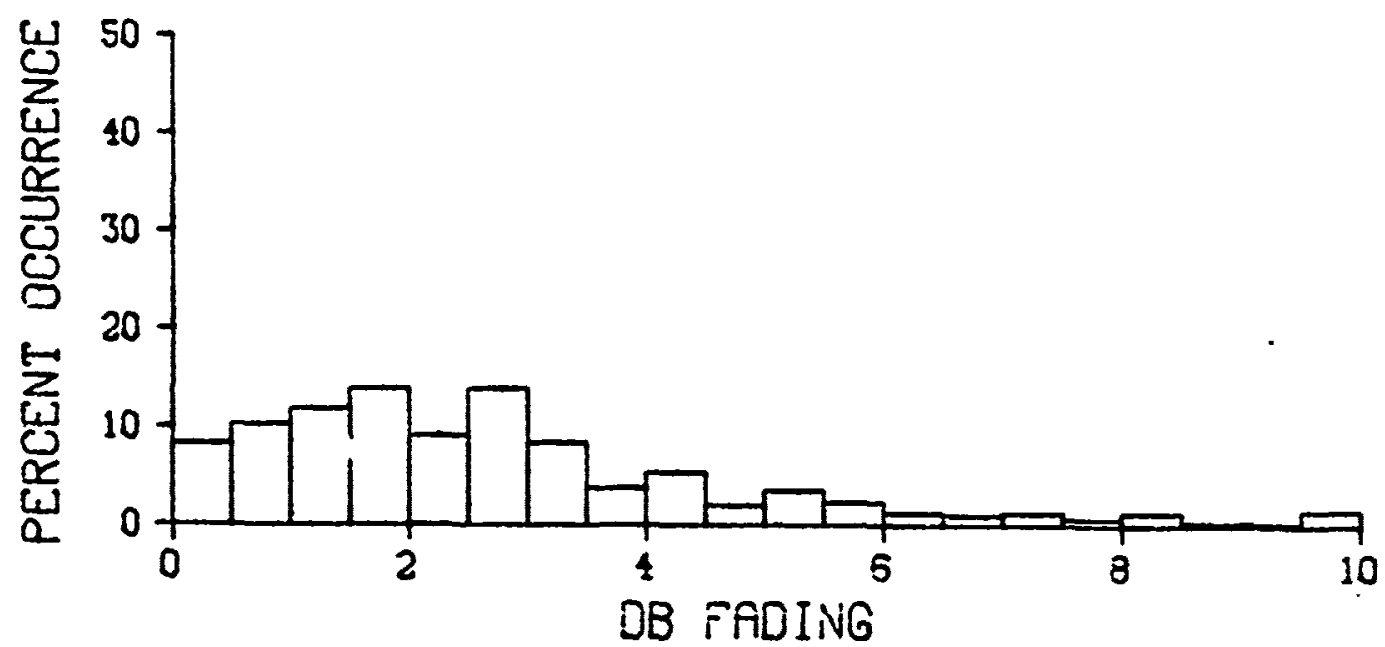

苟

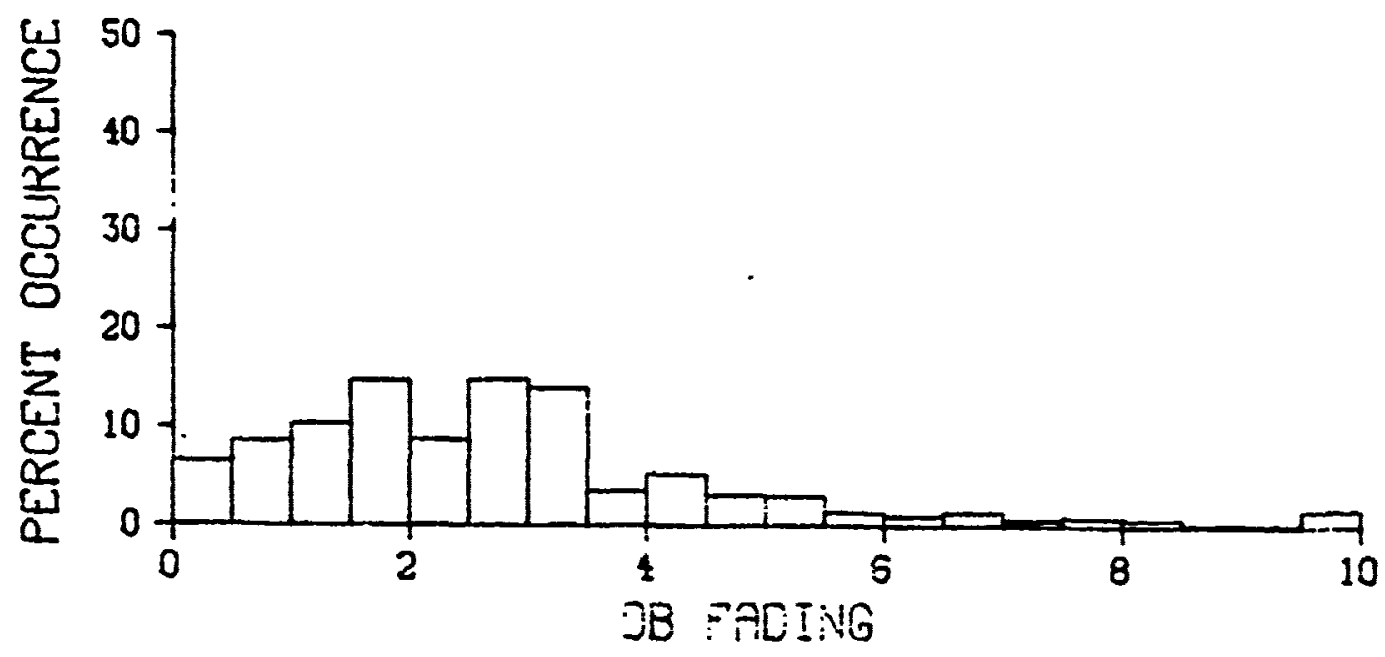

LOW ANTENNA

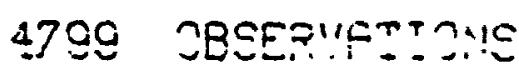

$X$ BAND, ALL SEASONS : 972 
s

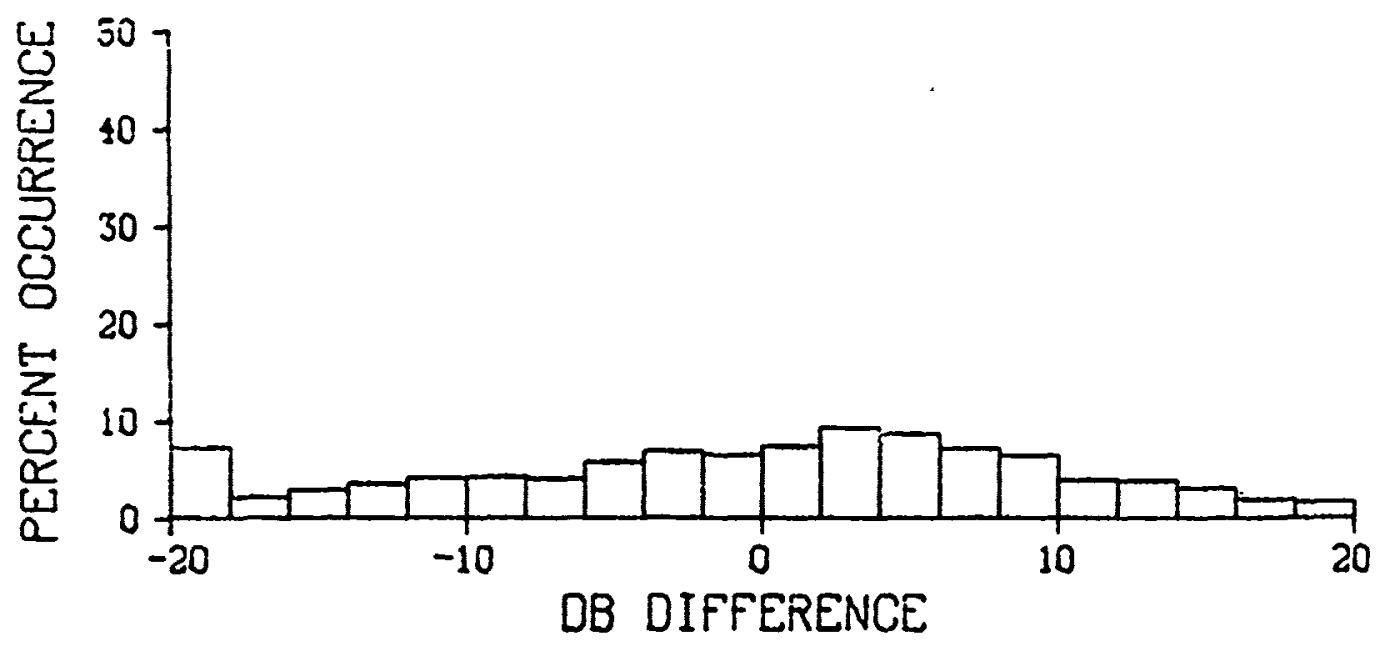

HIGH-LOW

4787 OBSERVATIONS

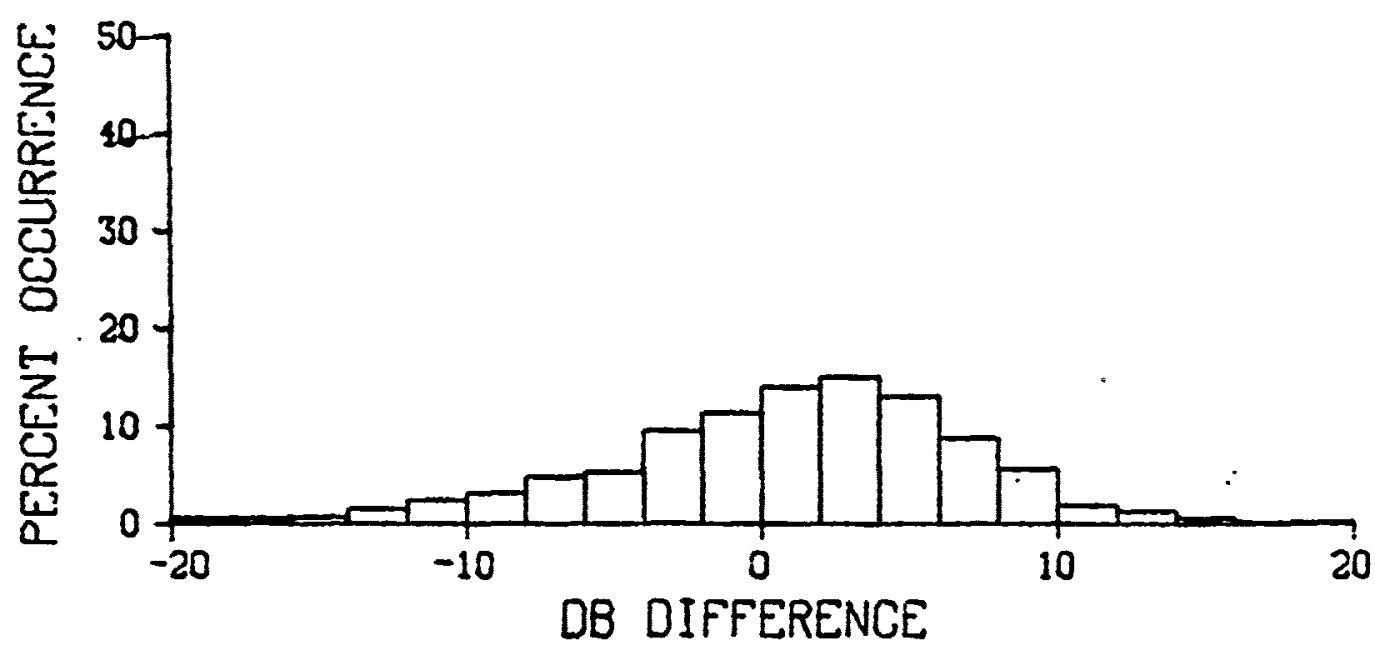

HIGH-MID

4803 OBSERVATIONS

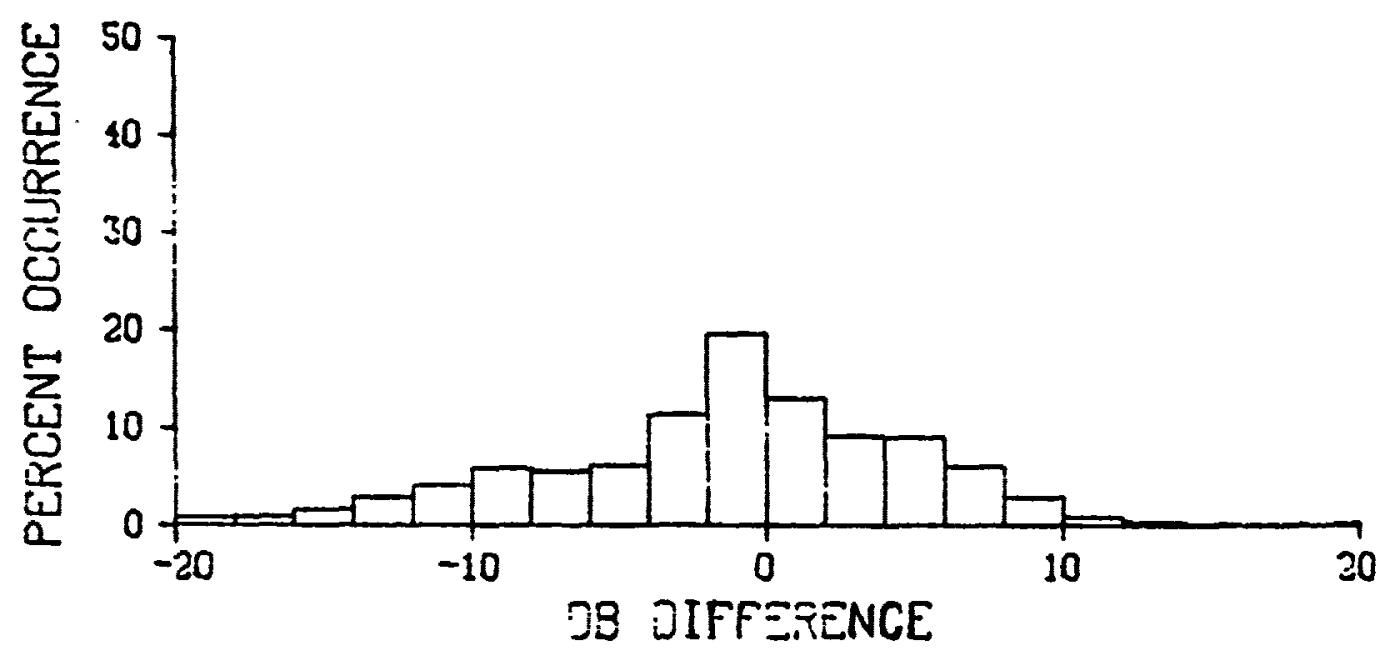

MiD-LOW

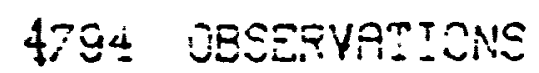
$X$ BAND, ALL GEÂSONS IGT2

s 


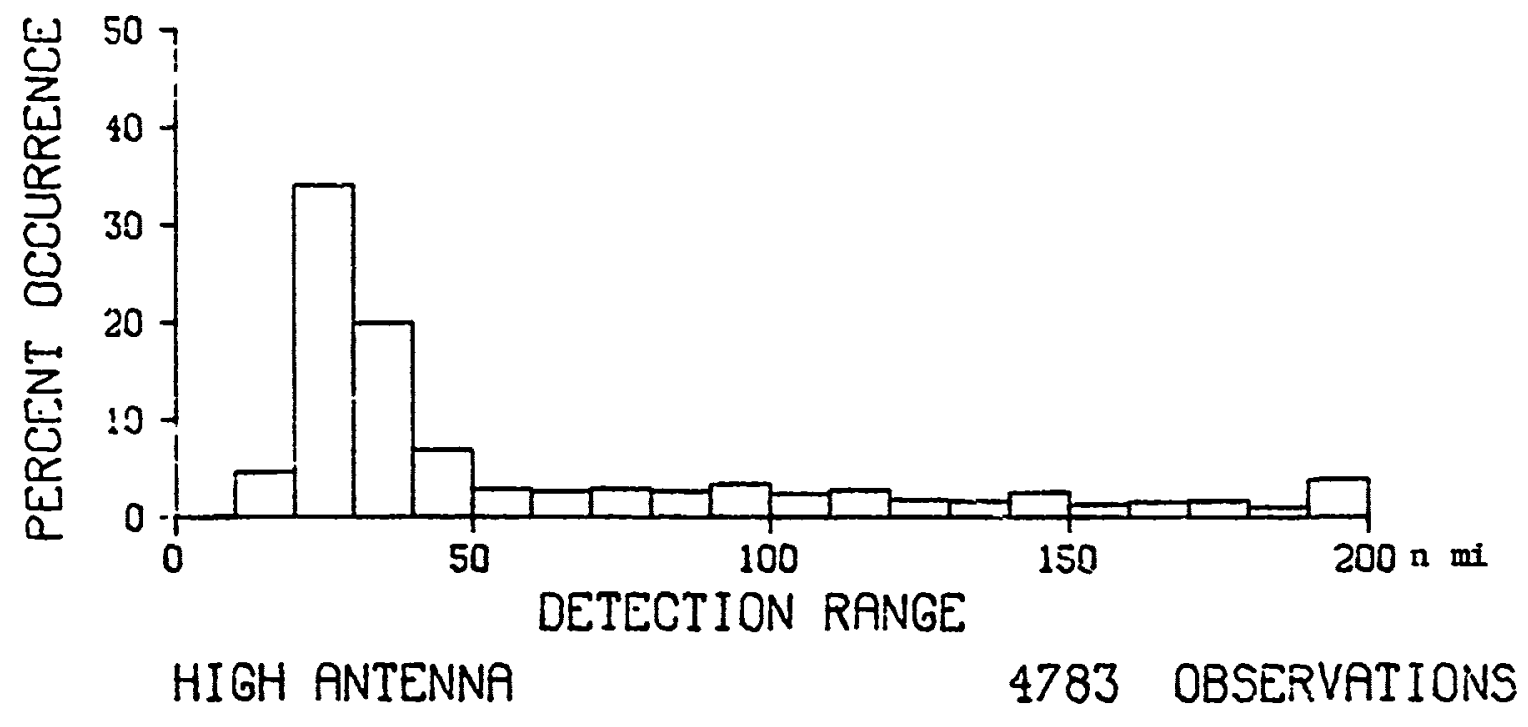

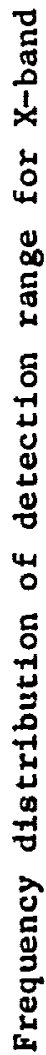
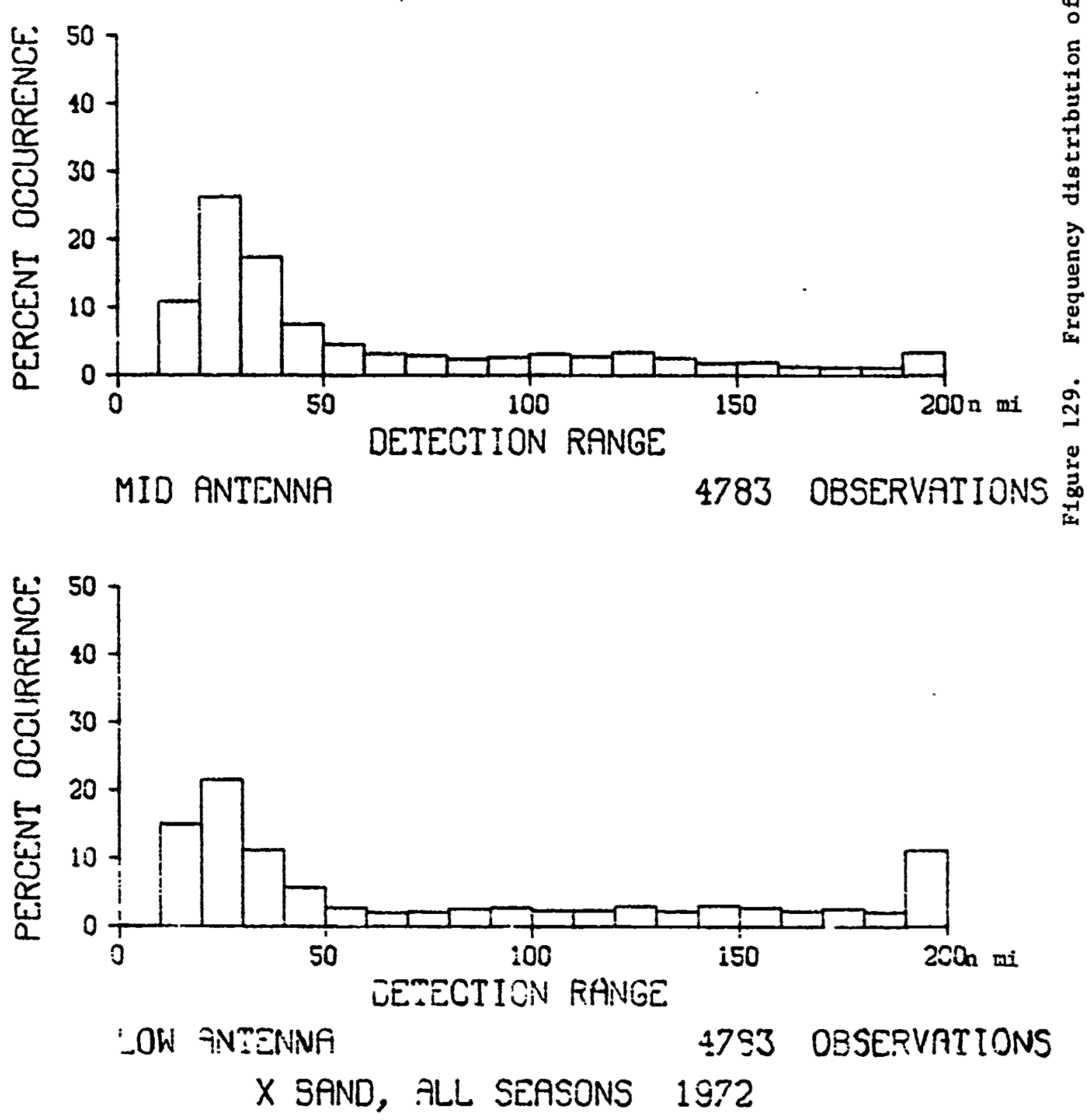
$\$$

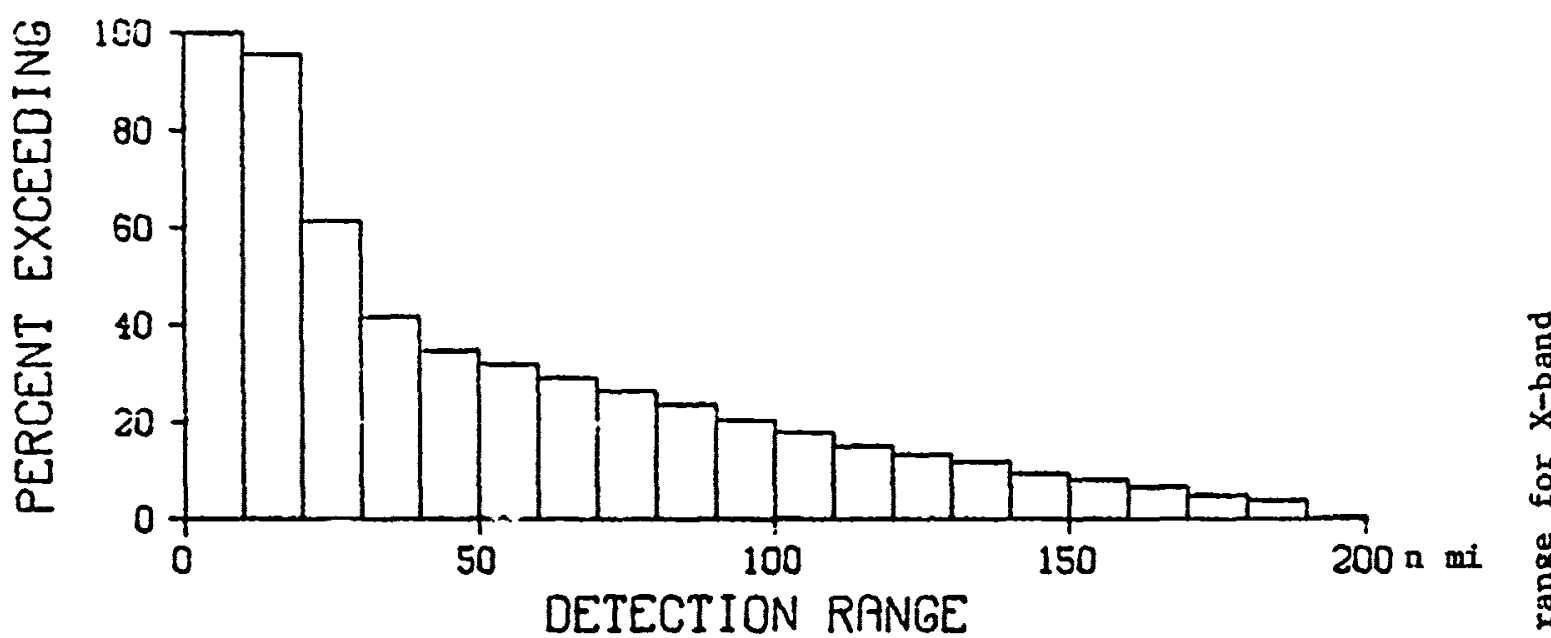

8 HIGH ANTENNA

4783 OBSERVATIONS
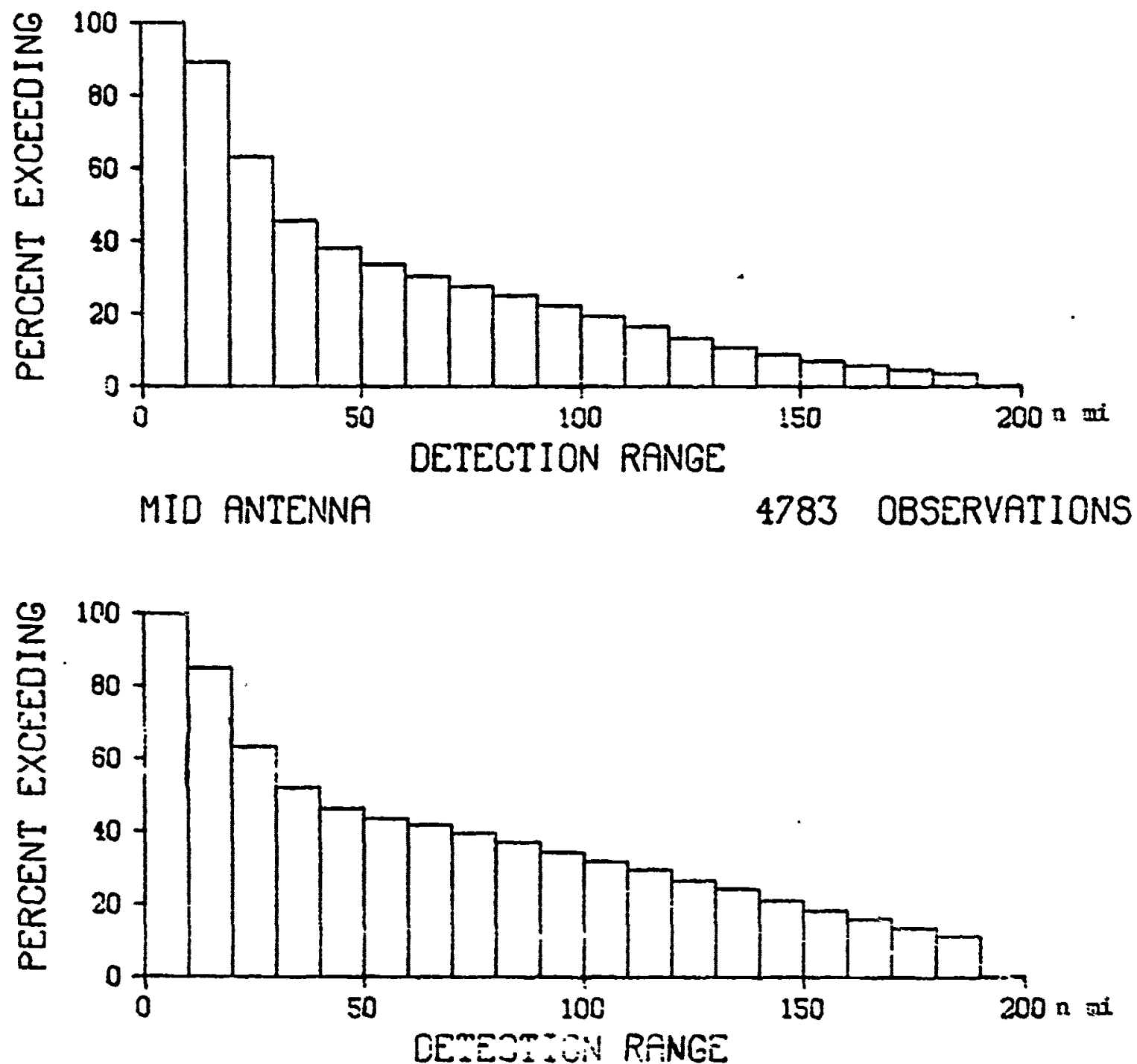

: IN ANTEINA

4.93 OREENVATIONS

$\because$ BAND, ALL SEASUNSS 1972 

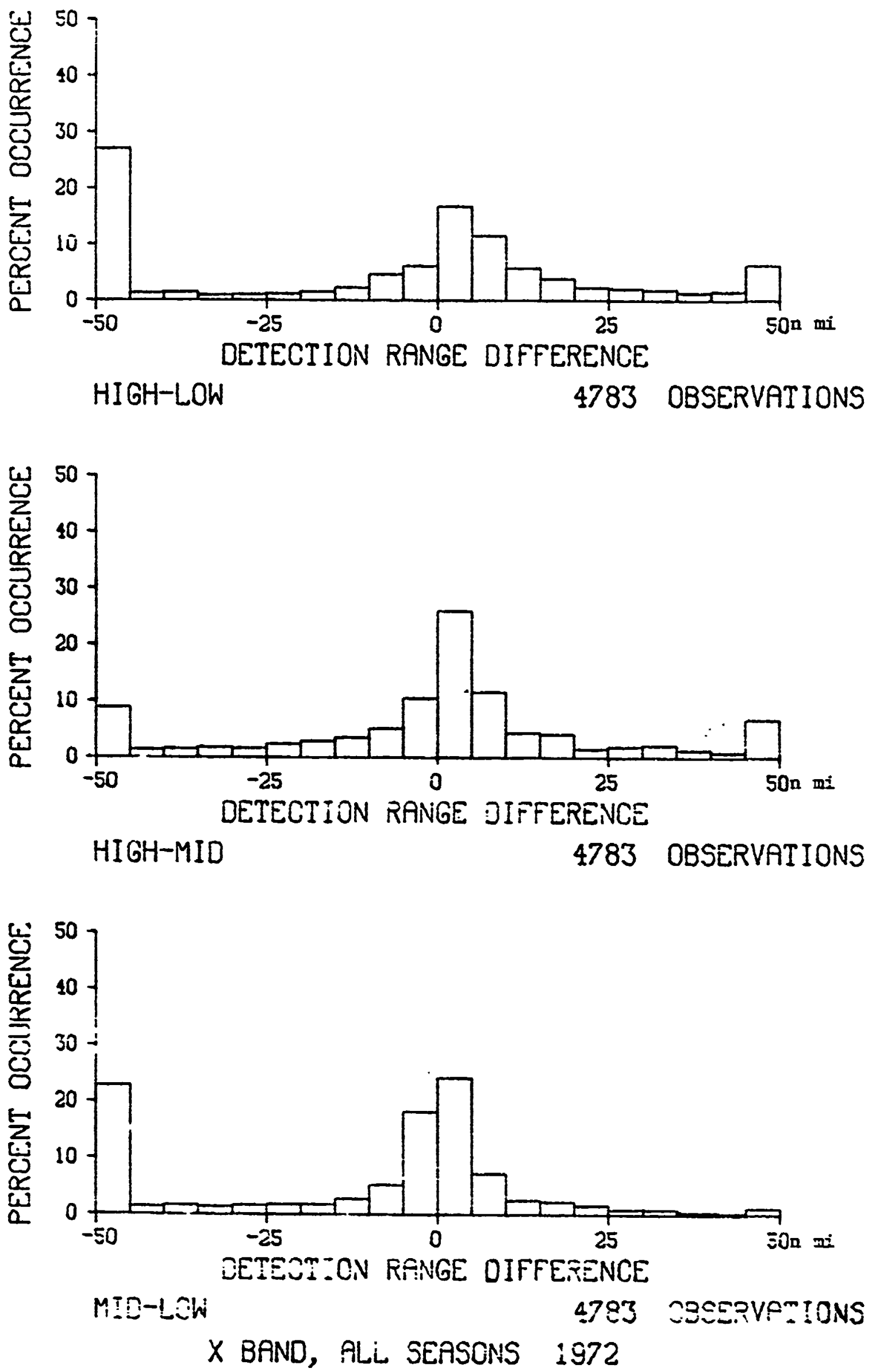


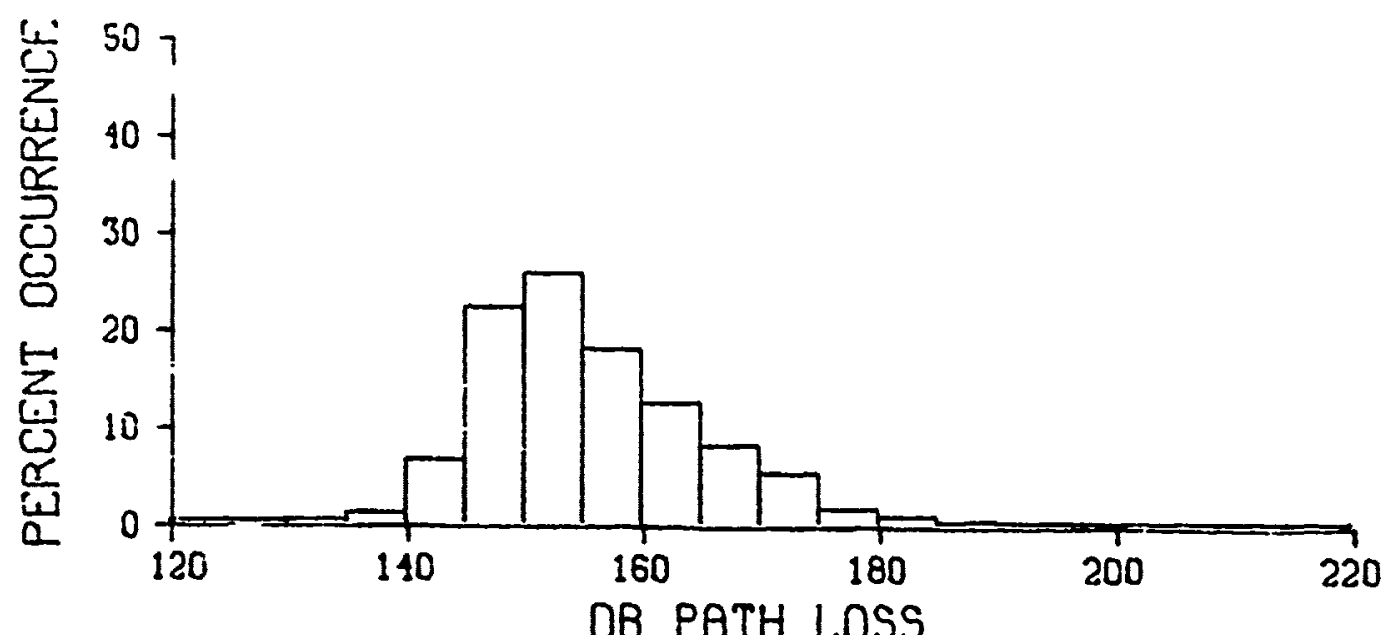

HIGH ANTENNA

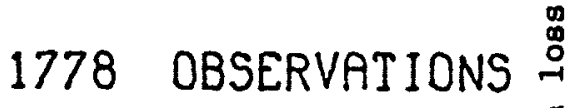

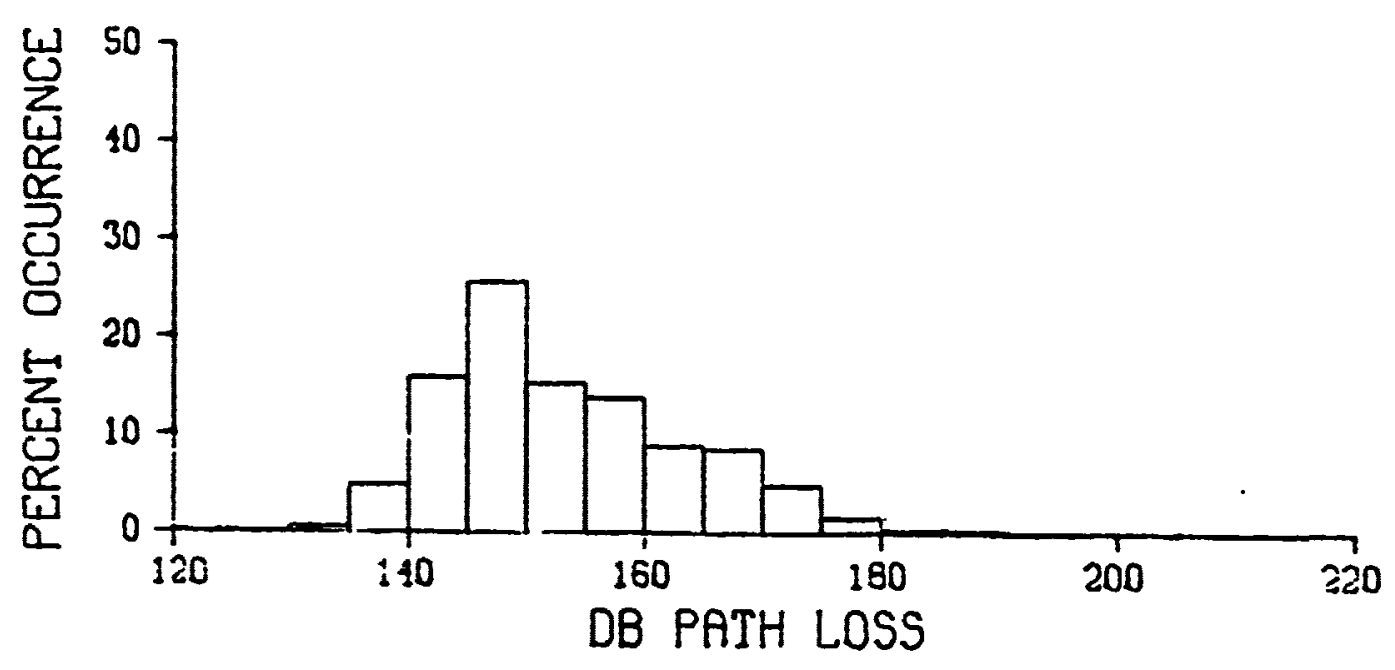

MID ANTENNA

1770 OBSERVATIONS

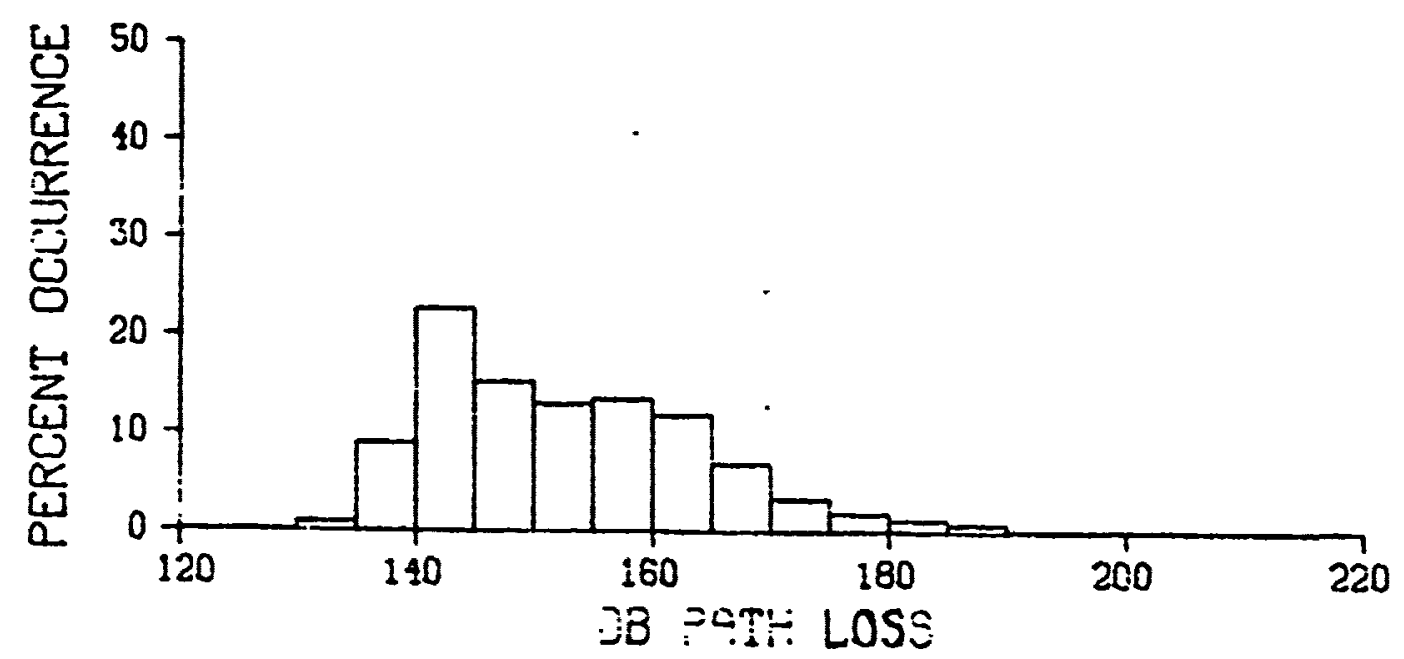

LÜ' PNTENNA

1770 CBSEFVATIOAS KU BANC, AUGUST - NOVEMBER 1972 


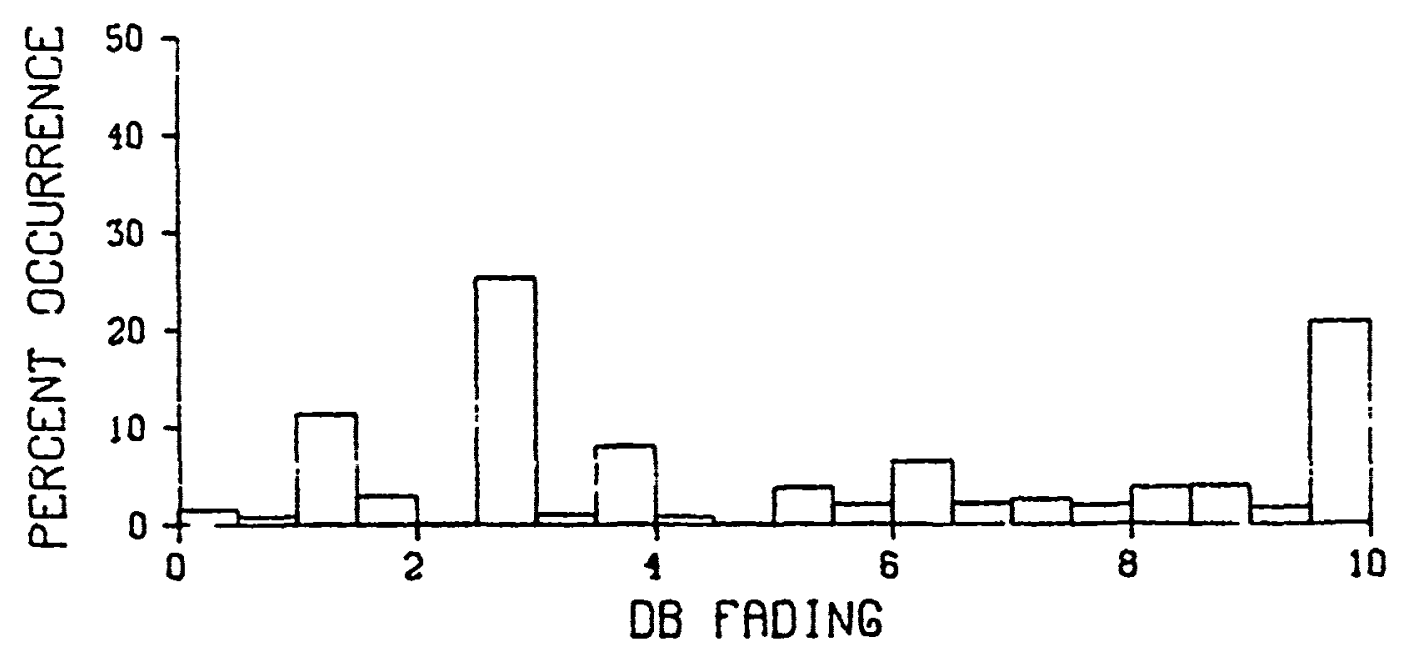

节

HIGH ANTENNA

1778 OBSERVATIONS
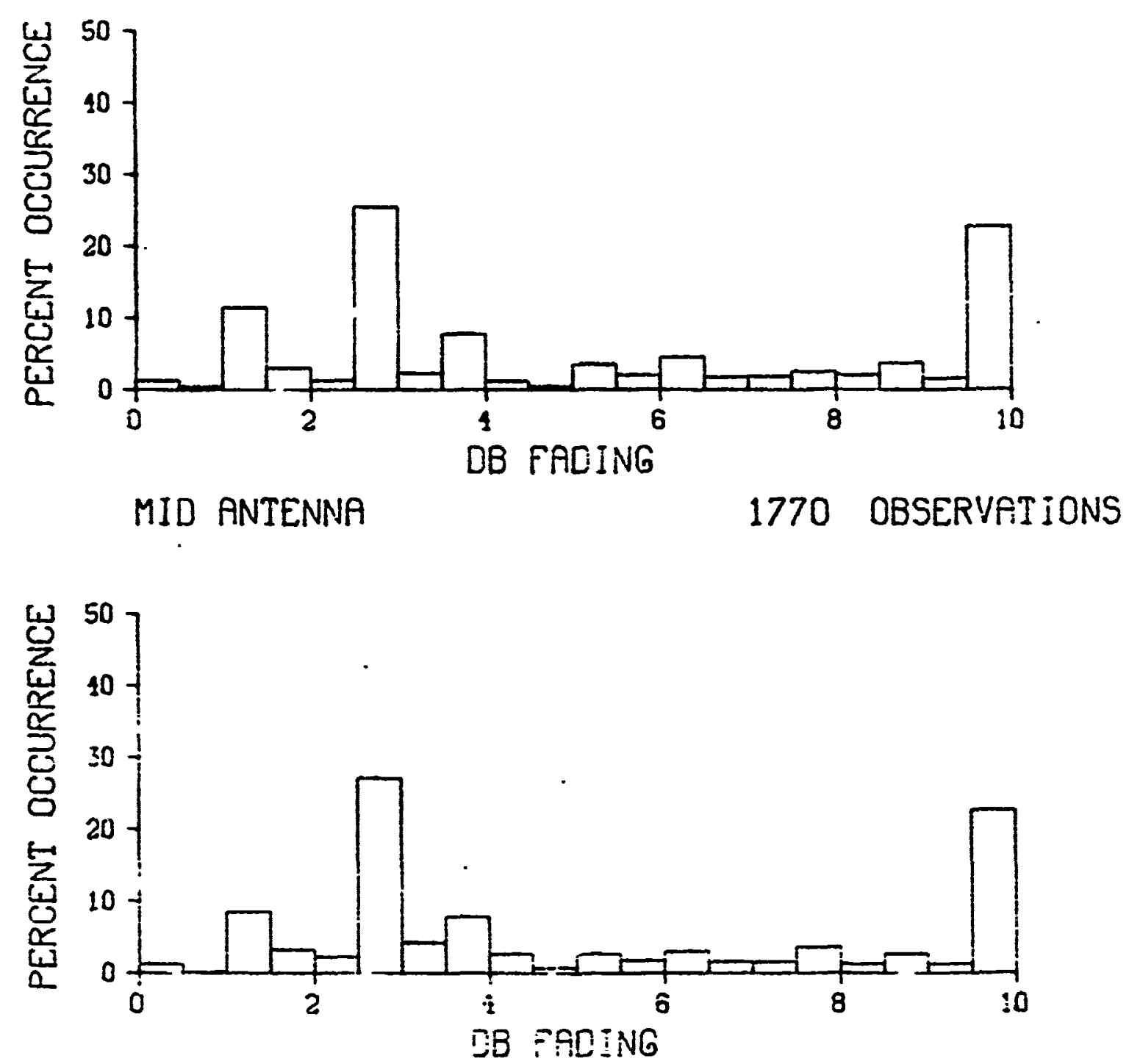

LOW ANTE, Wif.

1770 OBSETYFT:CNS KU BAND, HUGUST - NOVEMBER 1972 
8

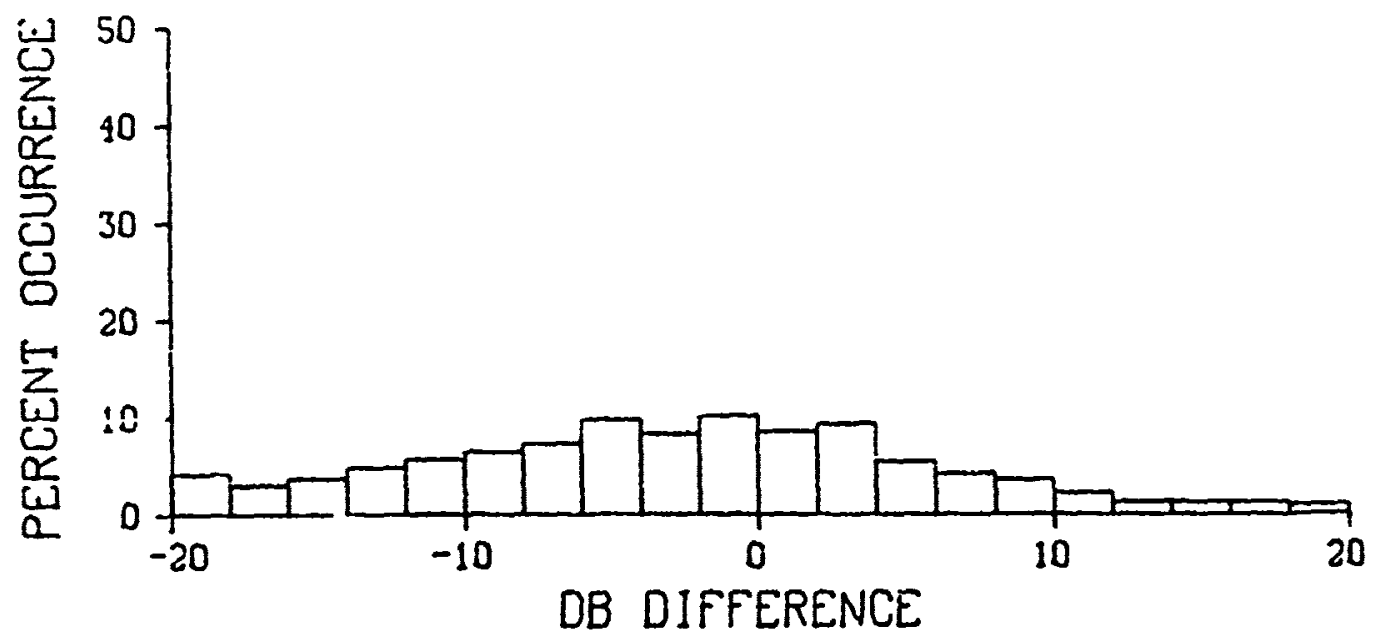

HIGH-LOW

1768 OBSERVATIONS

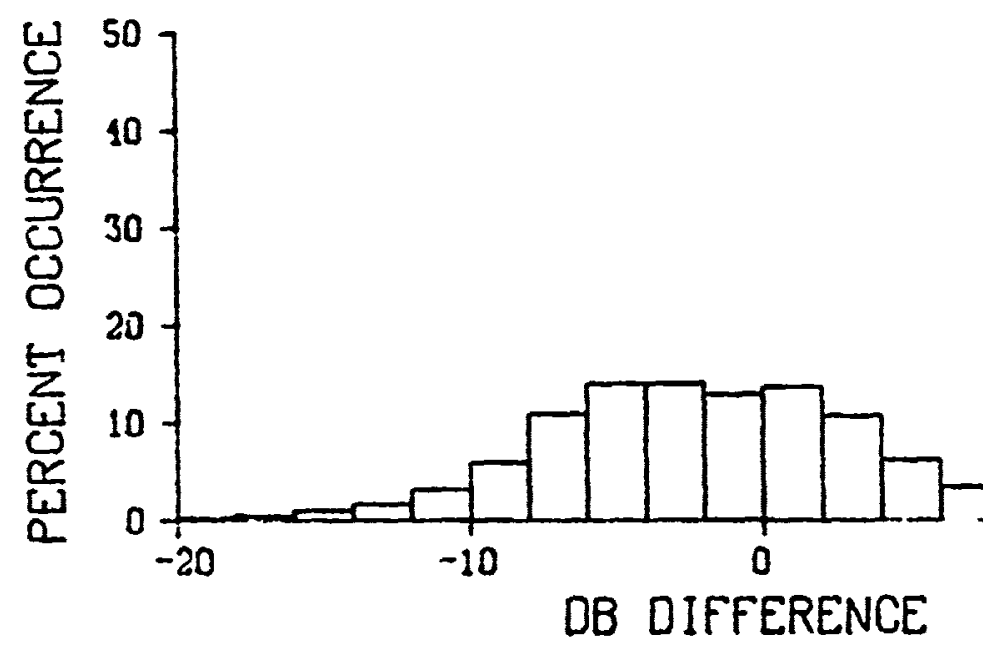

HIGH-MID

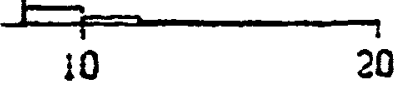

1769 OBSERVATIONS

粱

要

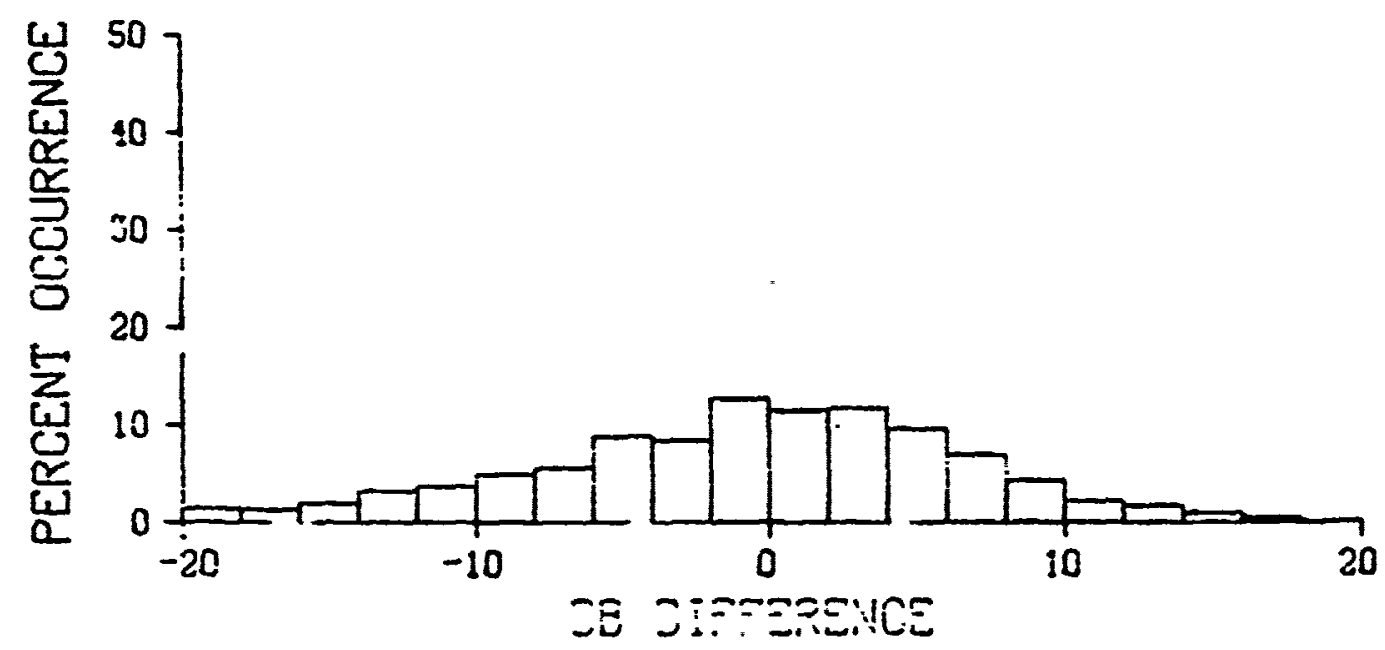

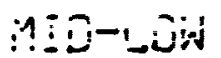

1755 OESERYA: :C:

KU BFND, RUGUST - NOVEMEER I972

tis 

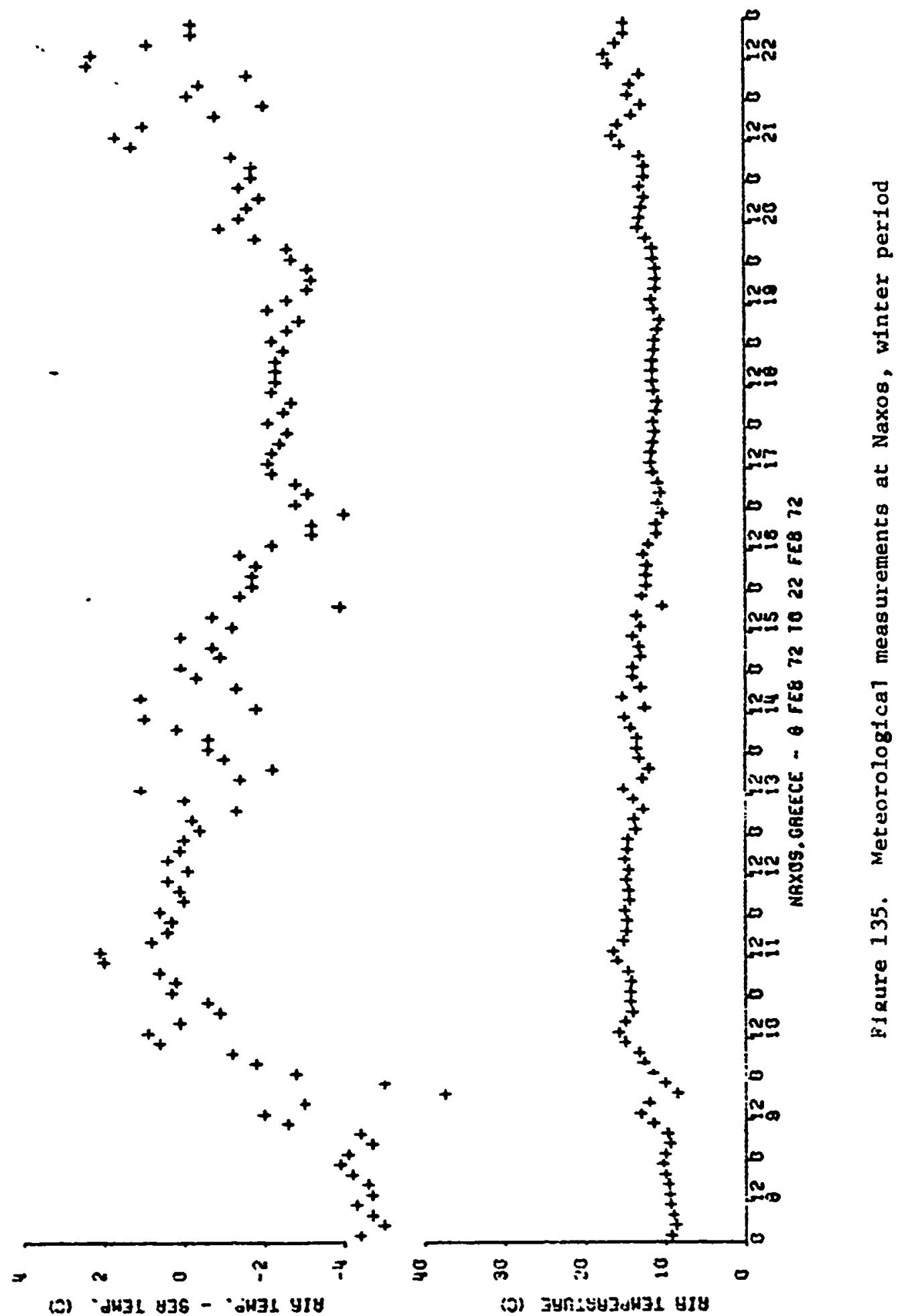


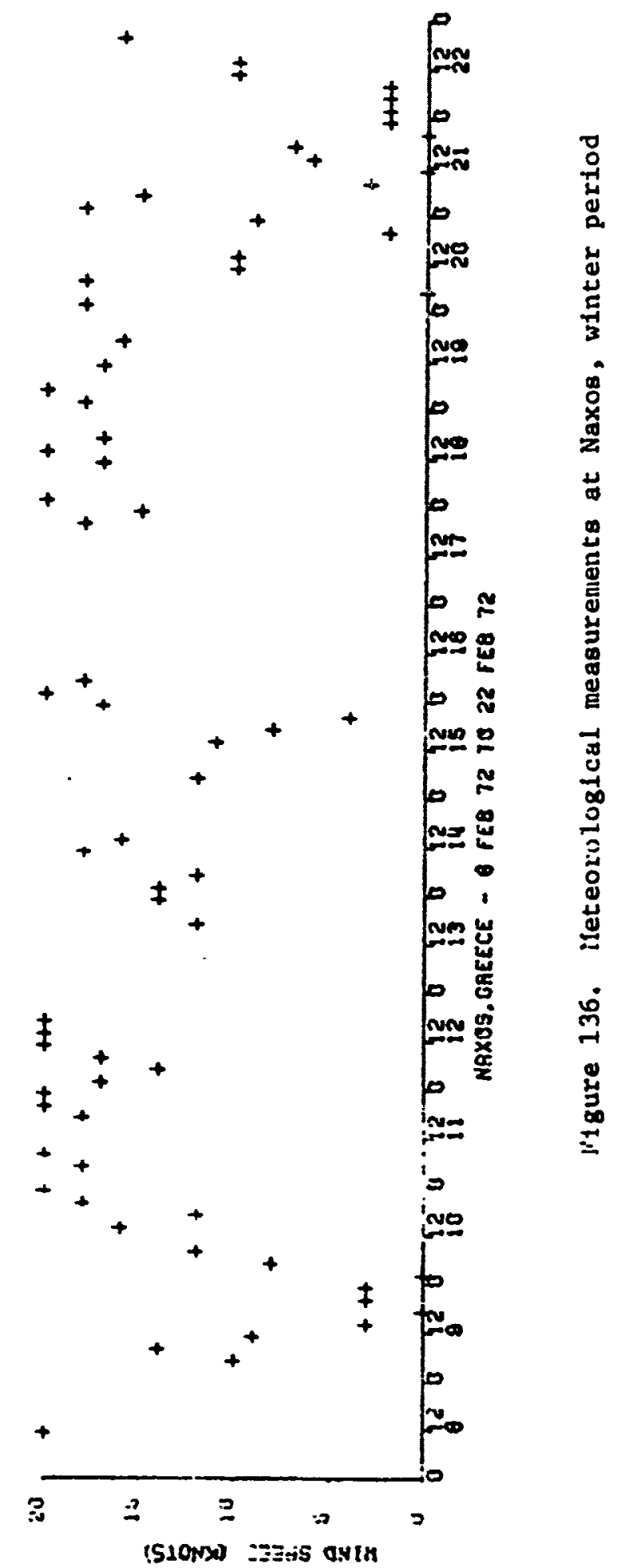

8

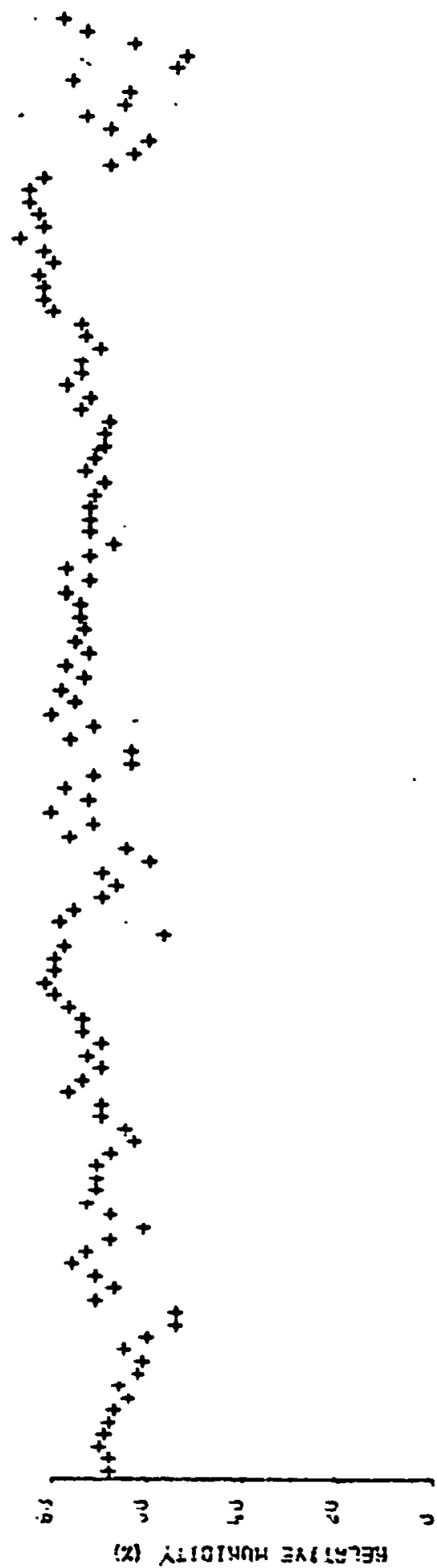

5 


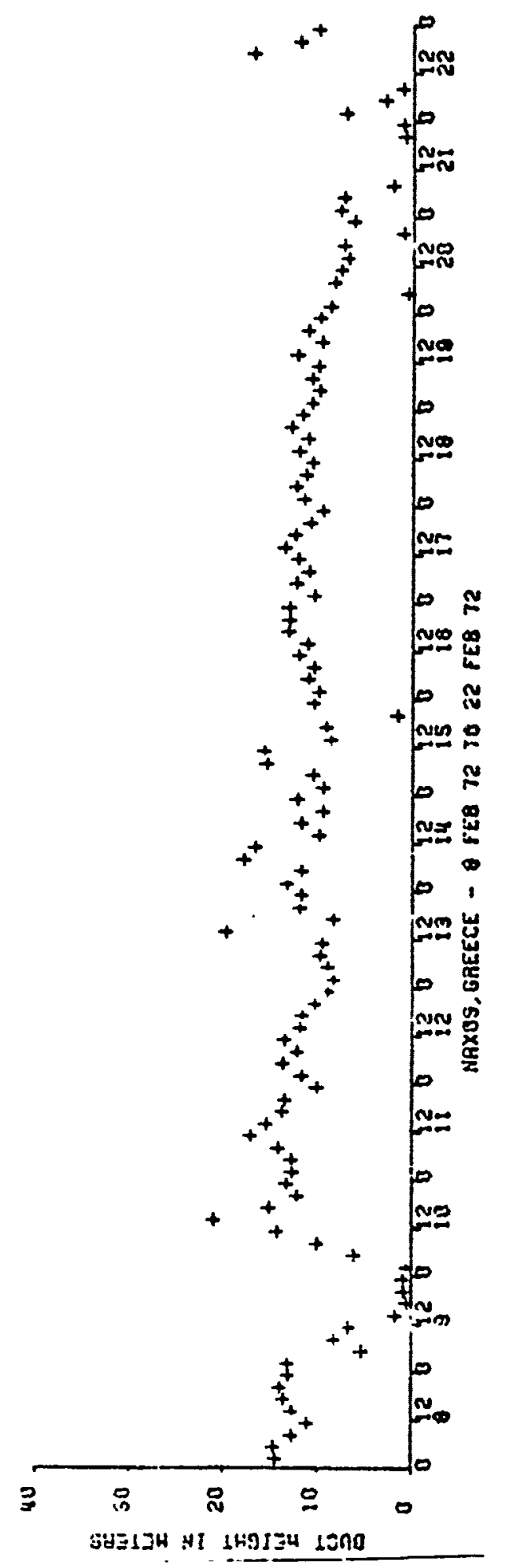

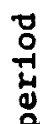

㟔

:

$z$

崩

号

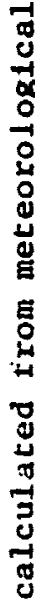

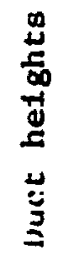

i

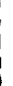


8

$\mathrm{N}$

的

$B$

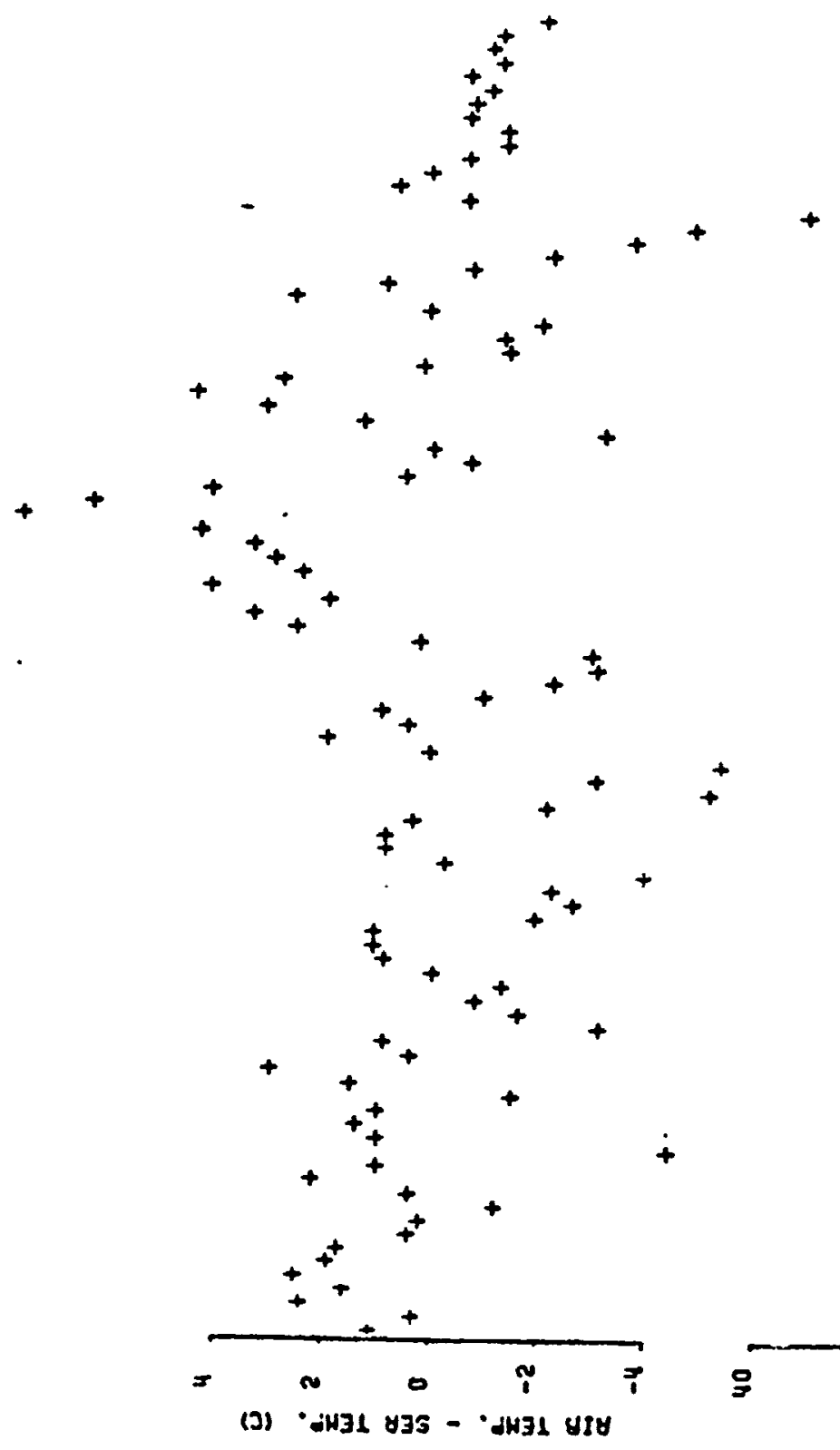

8

8

8

8

8

8

X

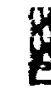

8

8 

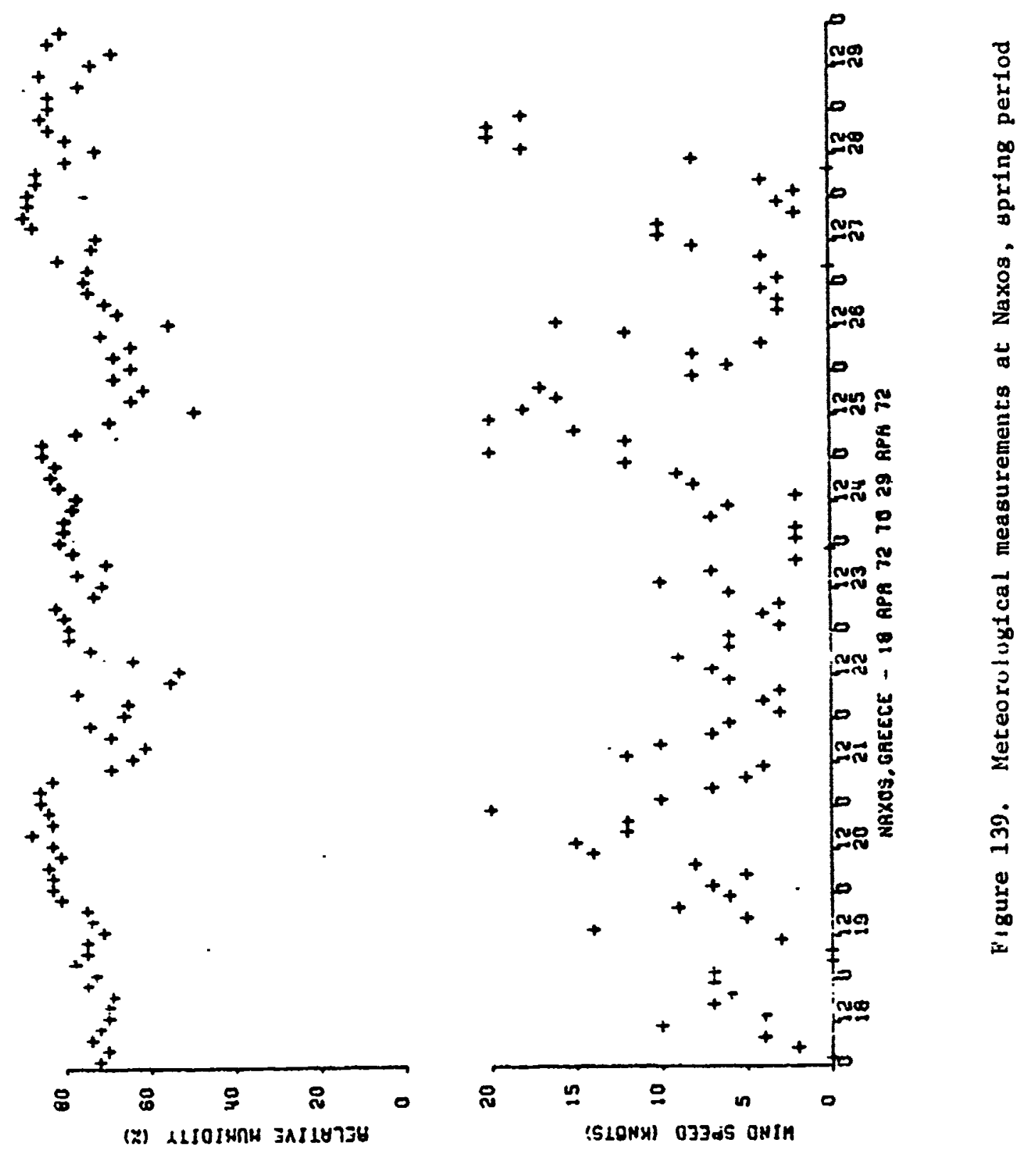


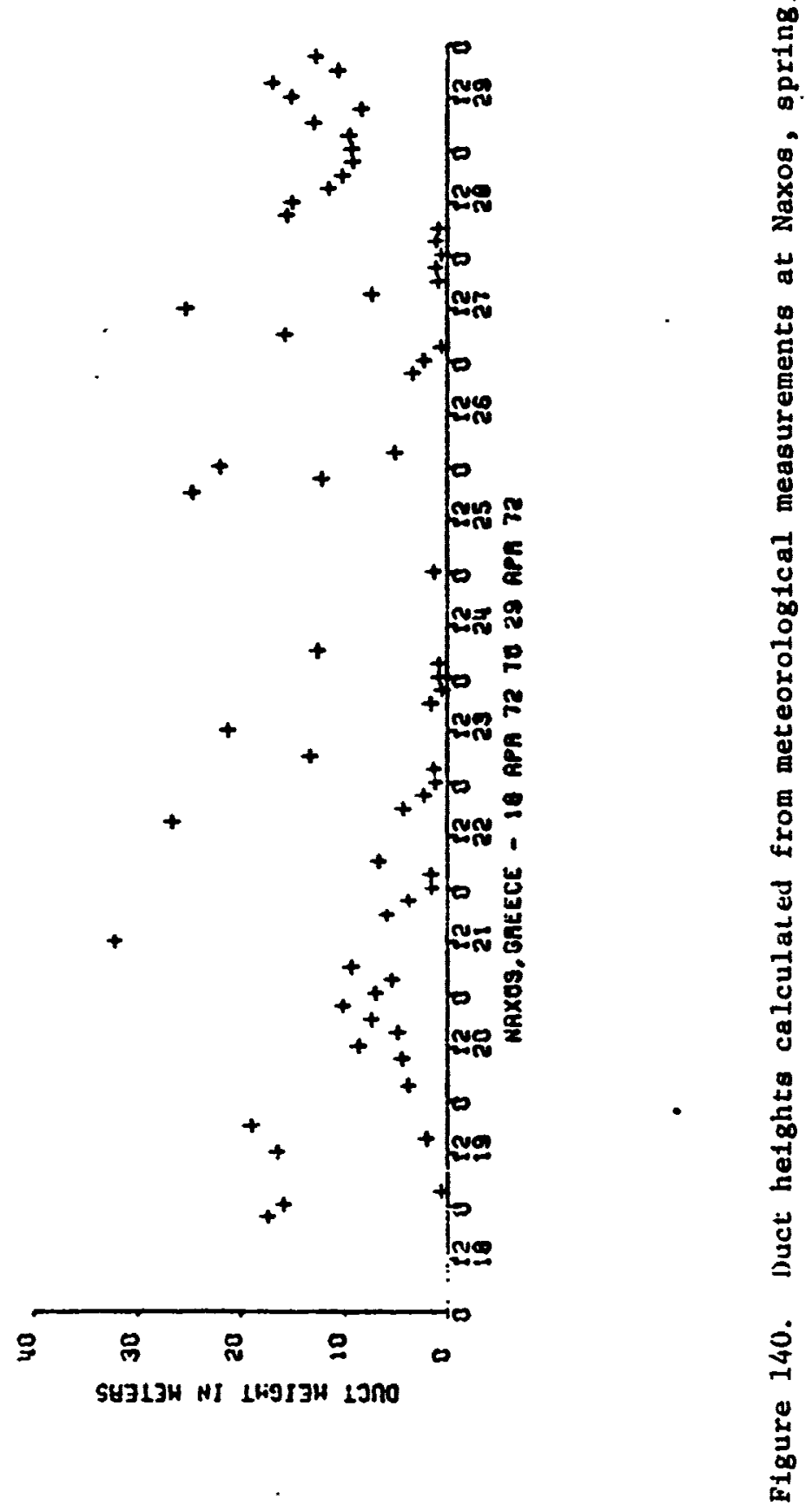




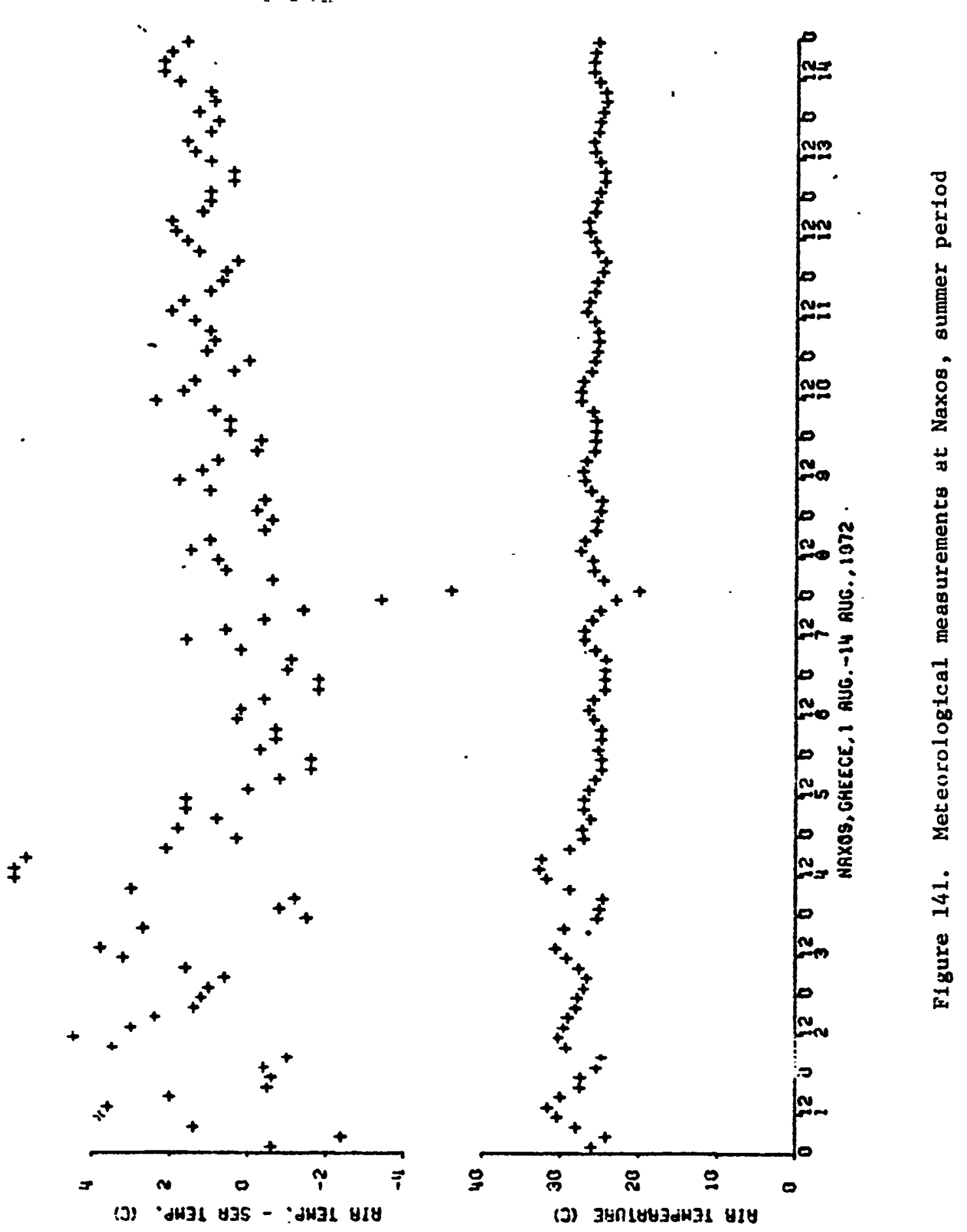


8

8

9

w

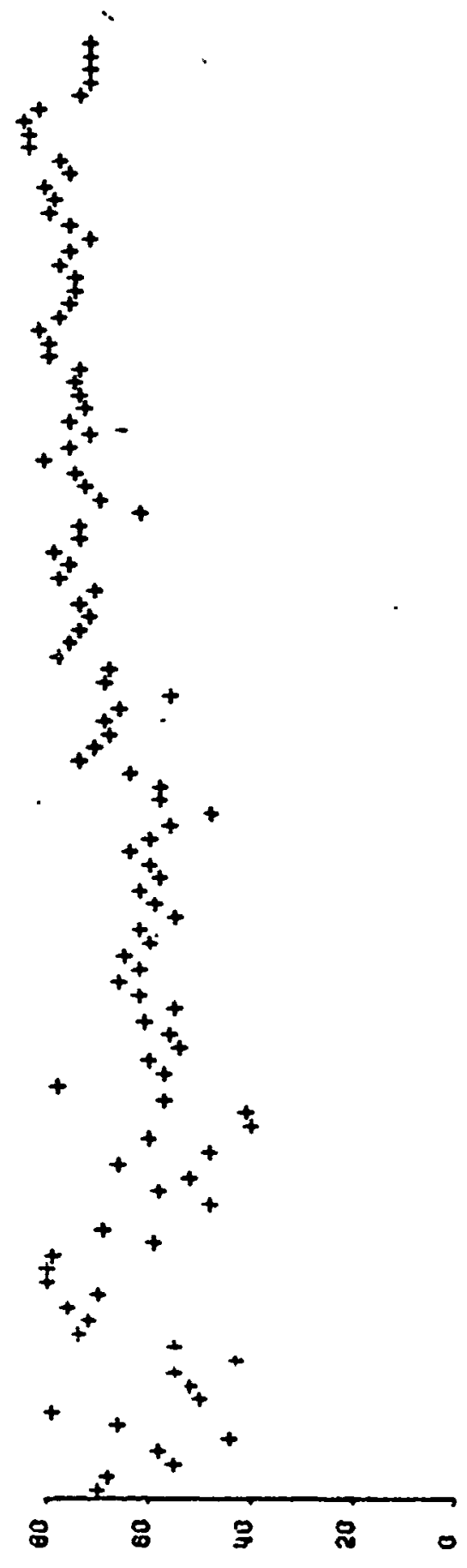

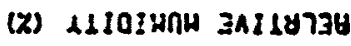

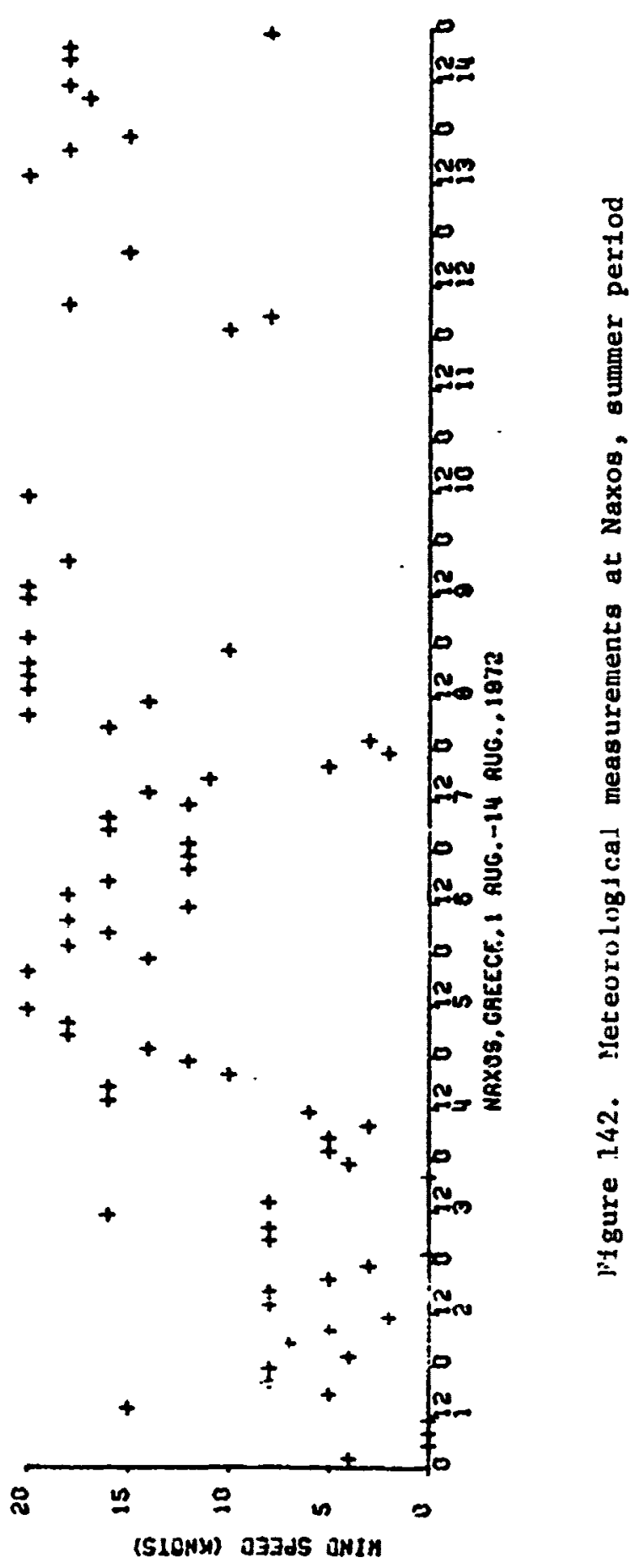

8 


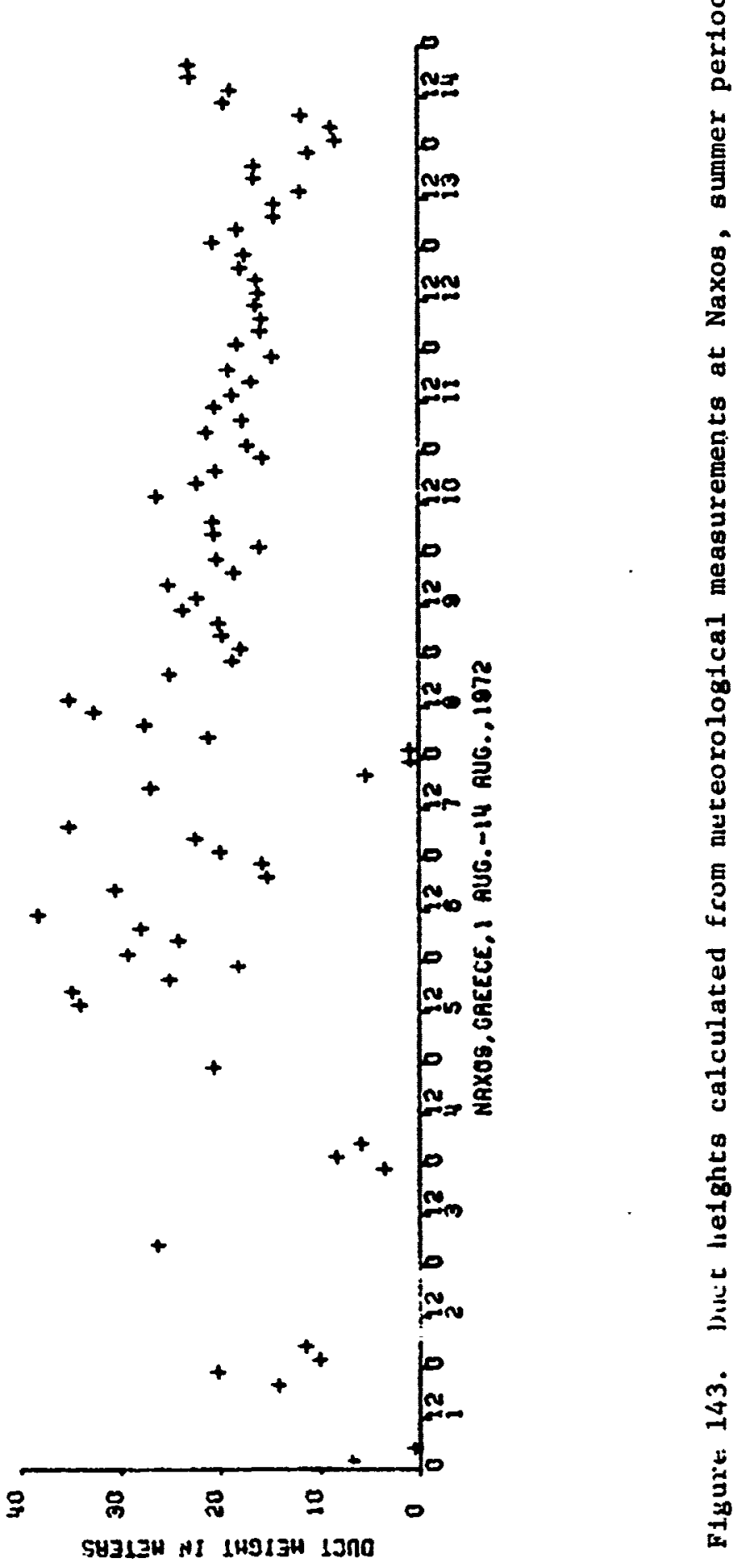

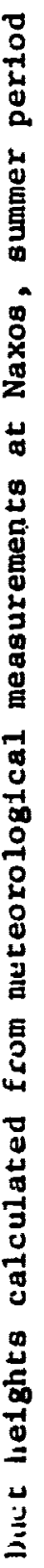

$\stackrel{9}{4}$

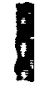

8 
8

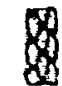

8

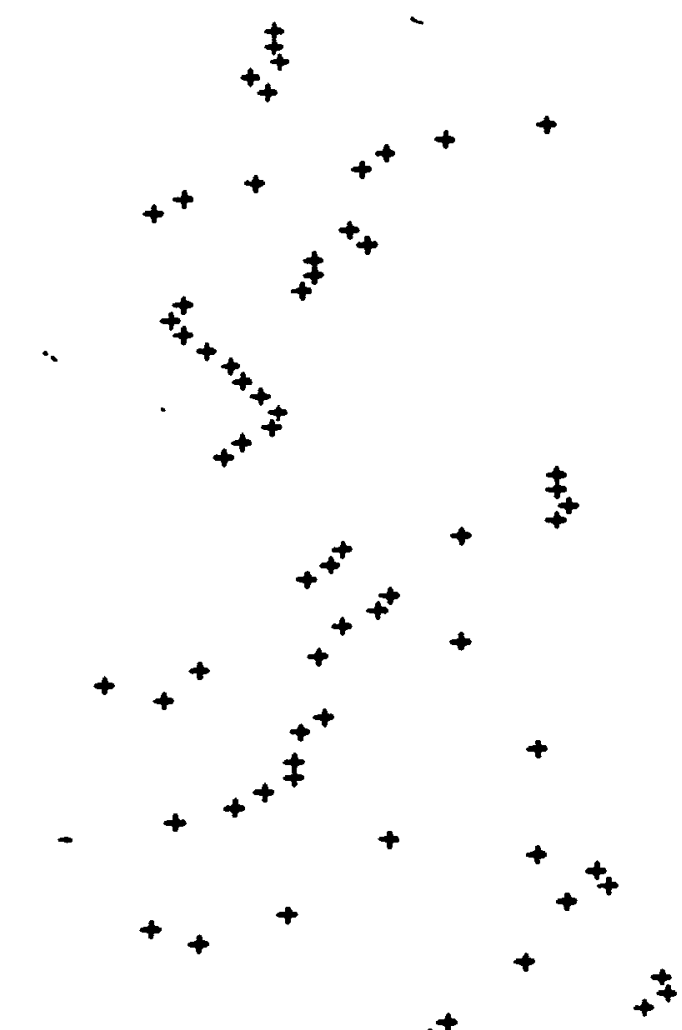

D

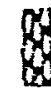

8

8

.

6

8

8

8

8

8

at

$x$

t)

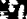

4

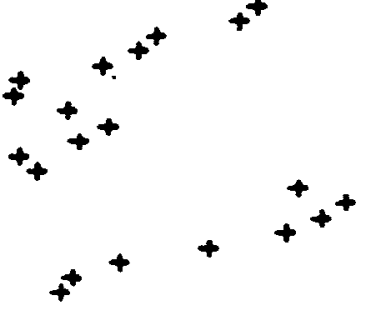

$+$

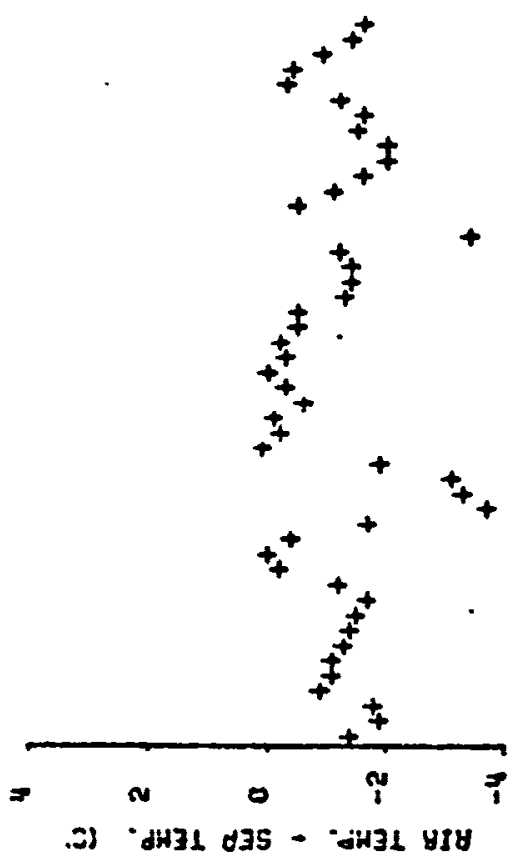

5 


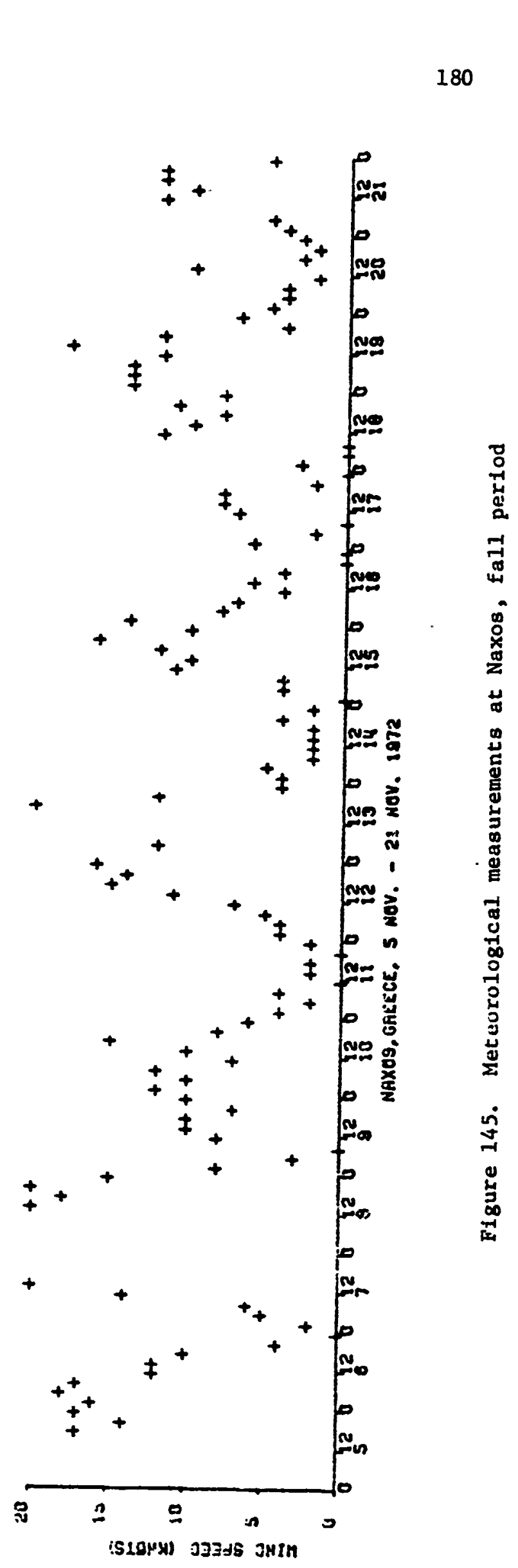

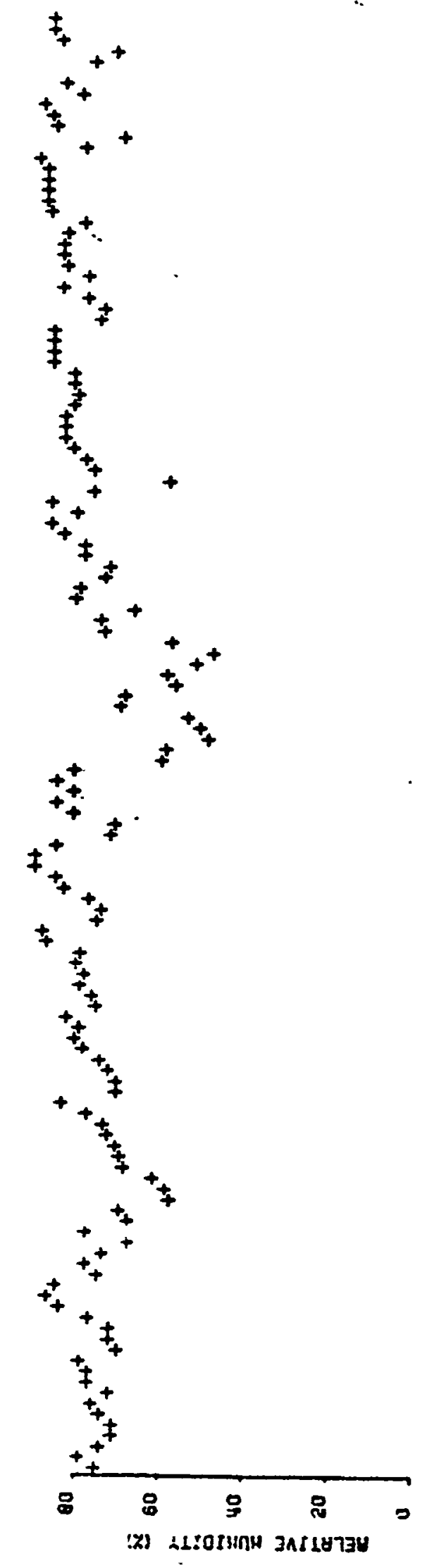

(1) 


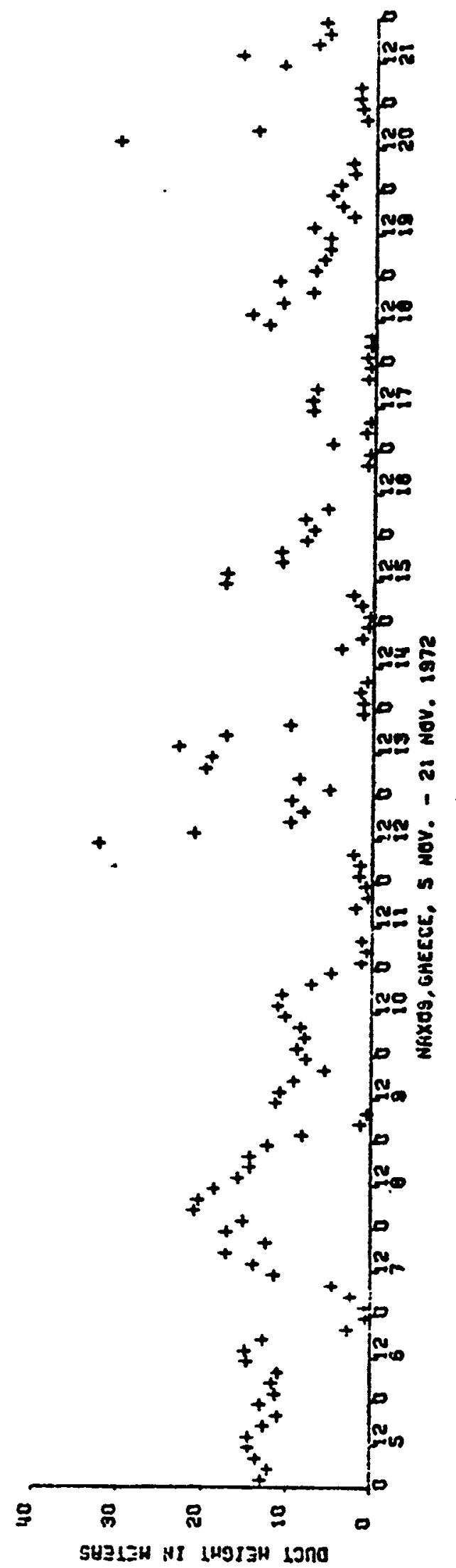

8

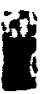

b

5

0

8

FE:

8

8

9

8

8

8 


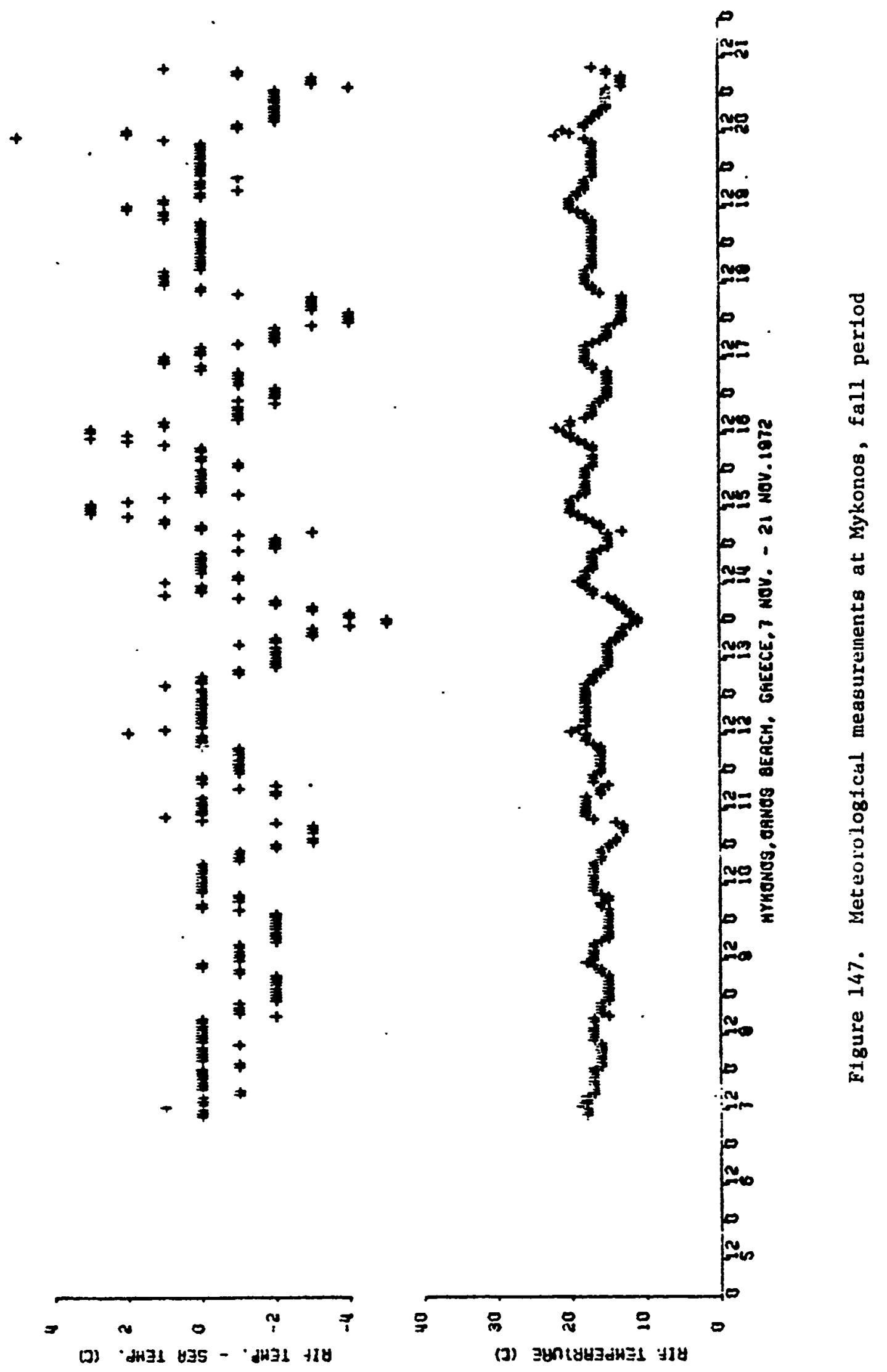



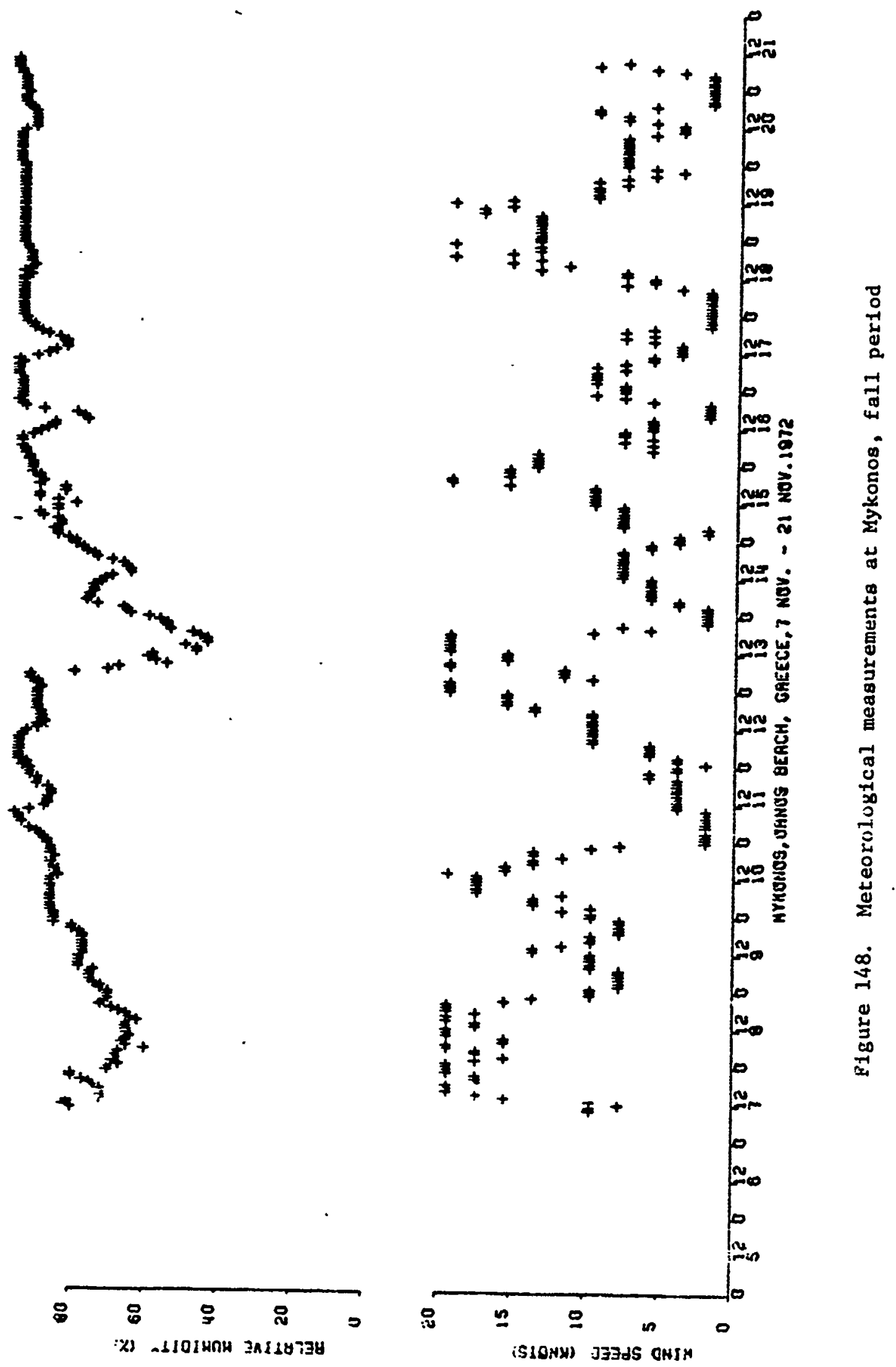

8

8

8

8

3

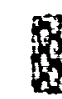

W

8

9

8

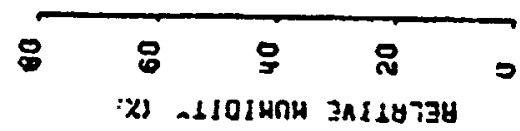

8 

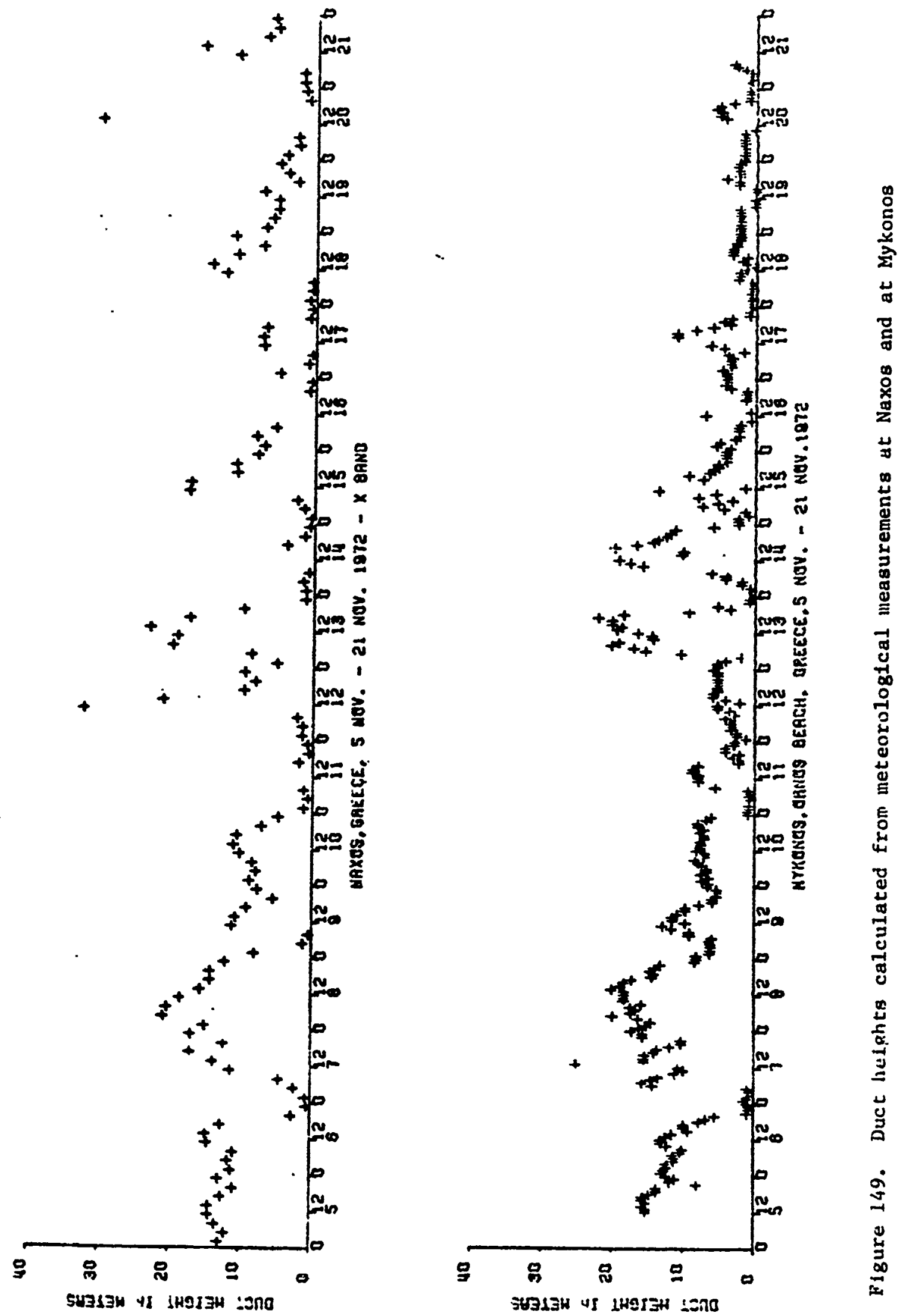

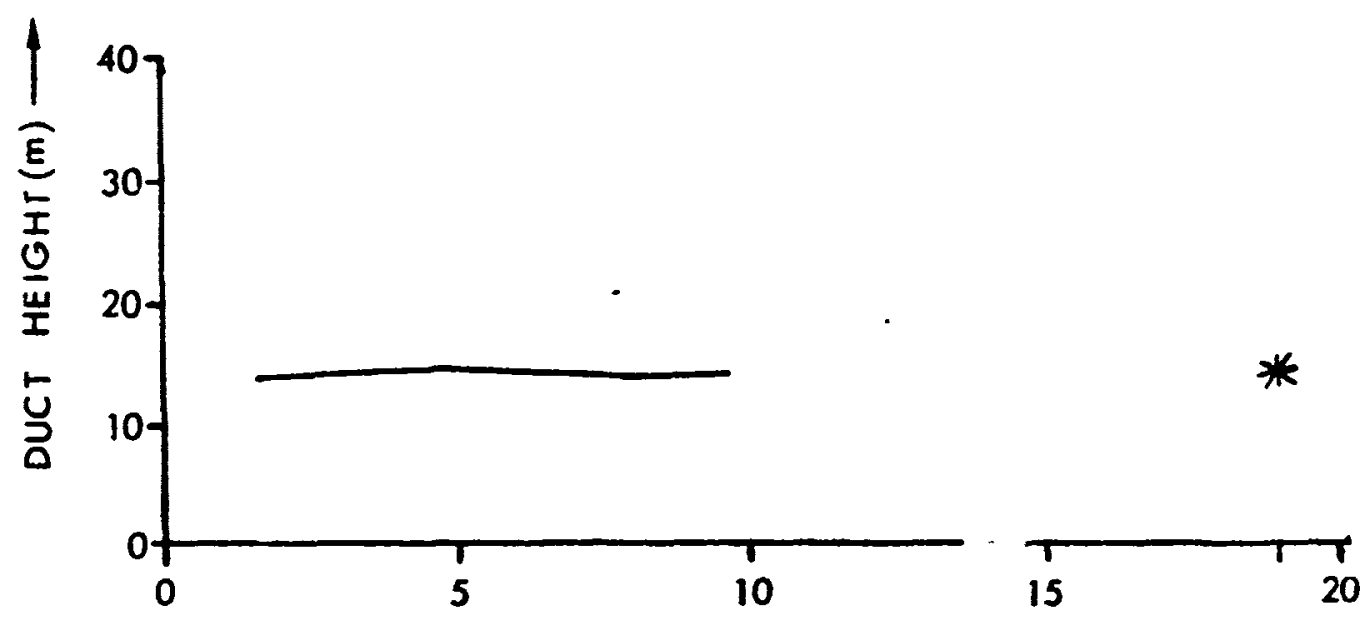

8



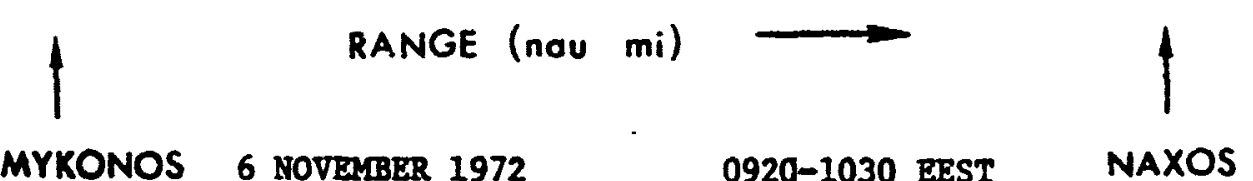

8

8.
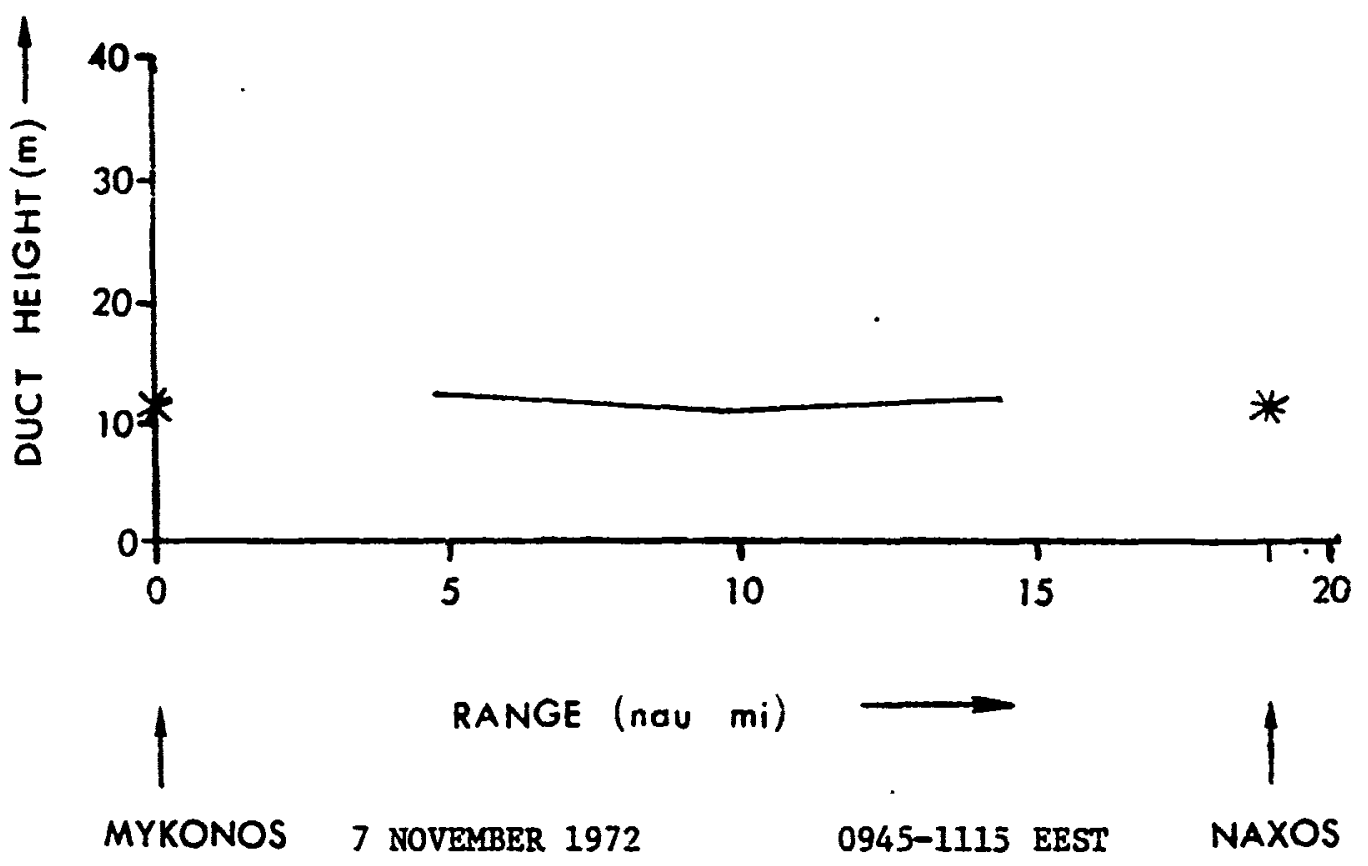

F1gure 150. Duct helght measurements along the propagation path 


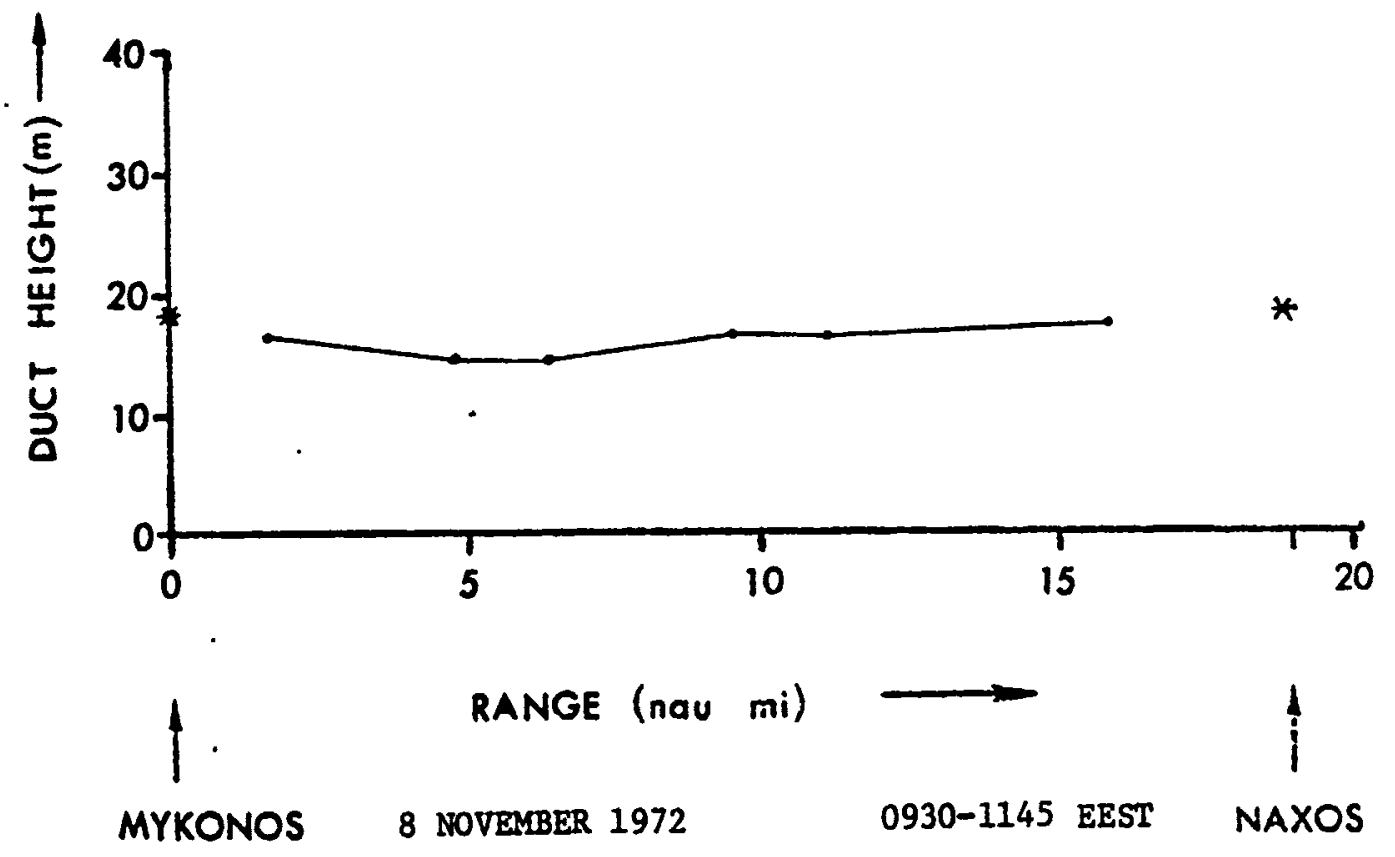

8

$\$$

d

8

B

3

13

M

$$
\begin{aligned}
& \frac{1}{E} \\
& \frac{1}{0} \\
& \frac{1}{I} \\
& \frac{U}{5} \\
& \frac{5}{0}
\end{aligned}
$$

407

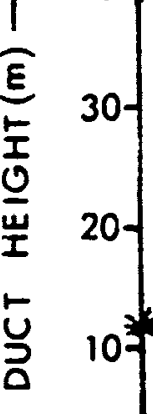

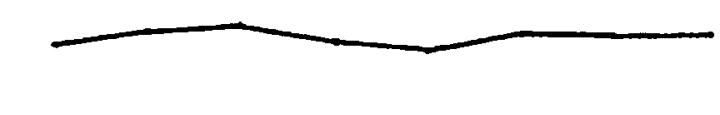

*

8

8

8

5

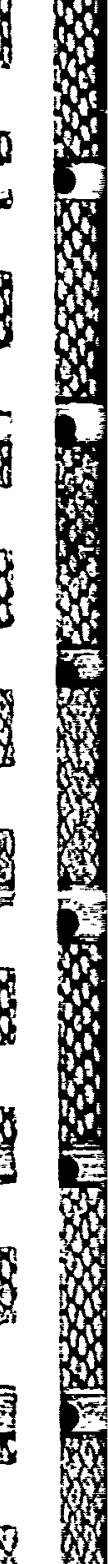

$\mathbb{W}$<smiles>[TeH][TeH]</smiles>

0

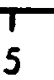

RANGE (nou mi)

1

MYKONOS 9 NOVEMBER 1972

1200-1400 EEST

NAXOS

Zigure 15i. Juct heizht measuzements alcng the propagation path 


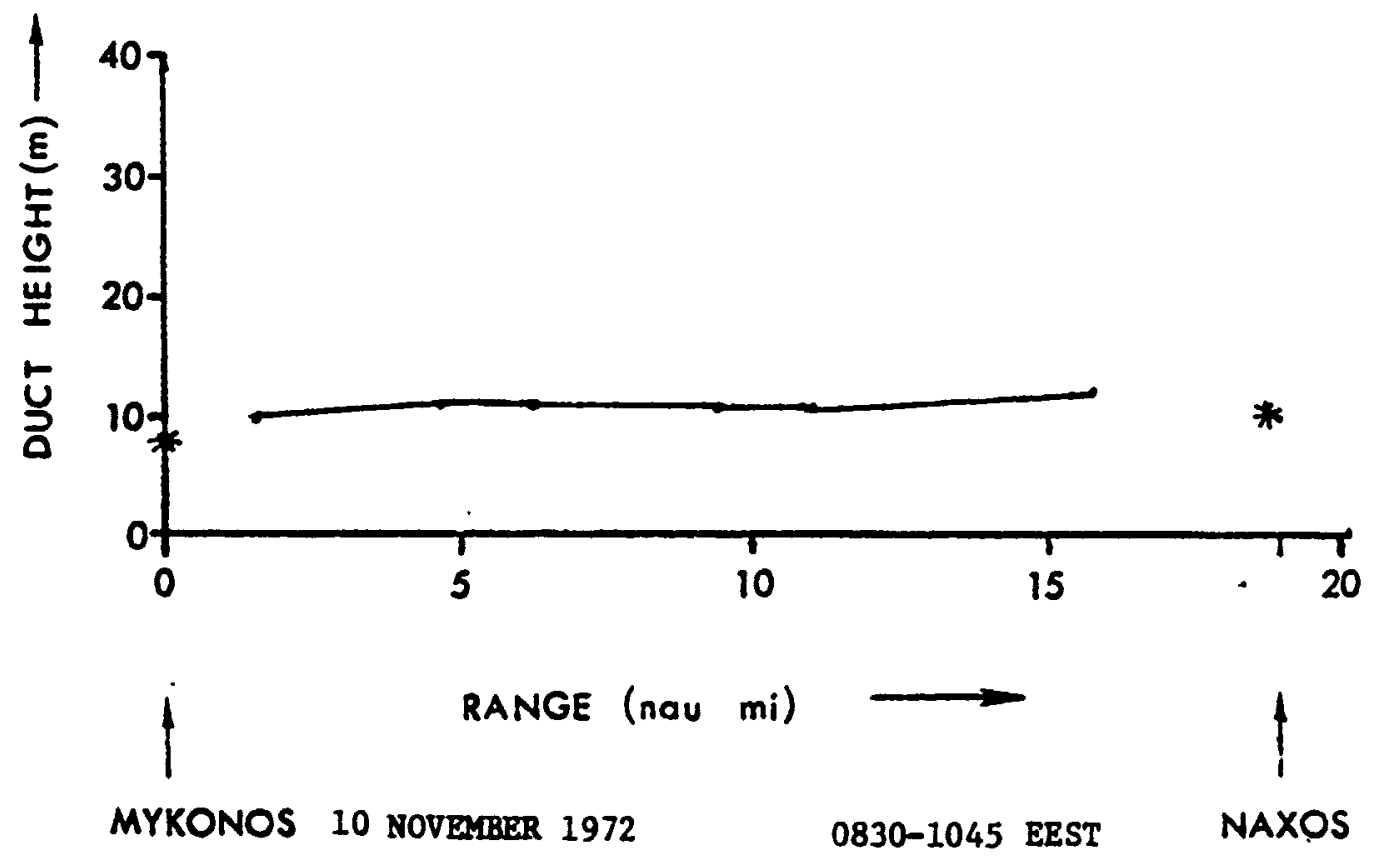

Figure 152. Duct height measurements along the propagation path 

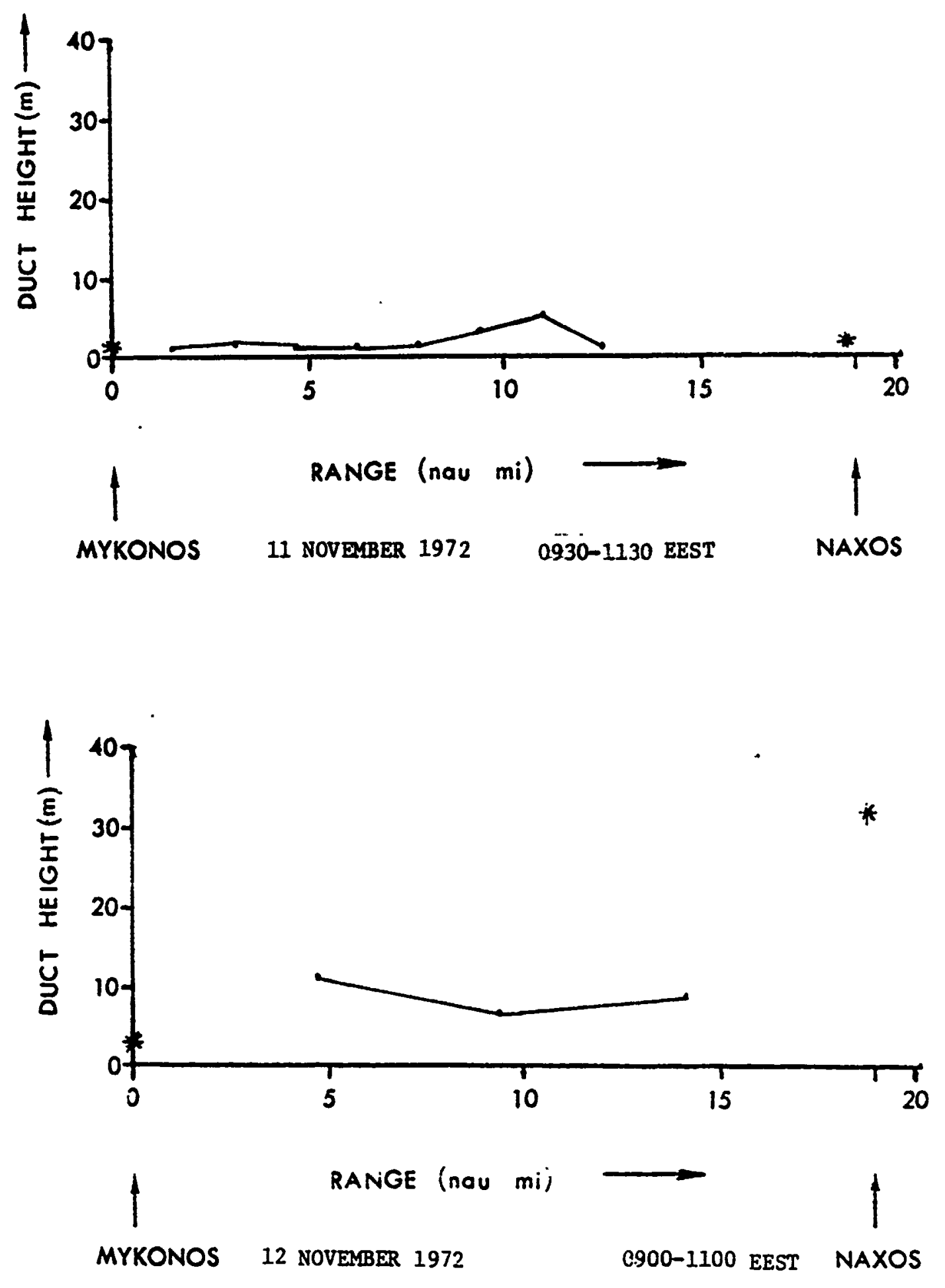

Figure 153. Duct height measurements along the propagation path 

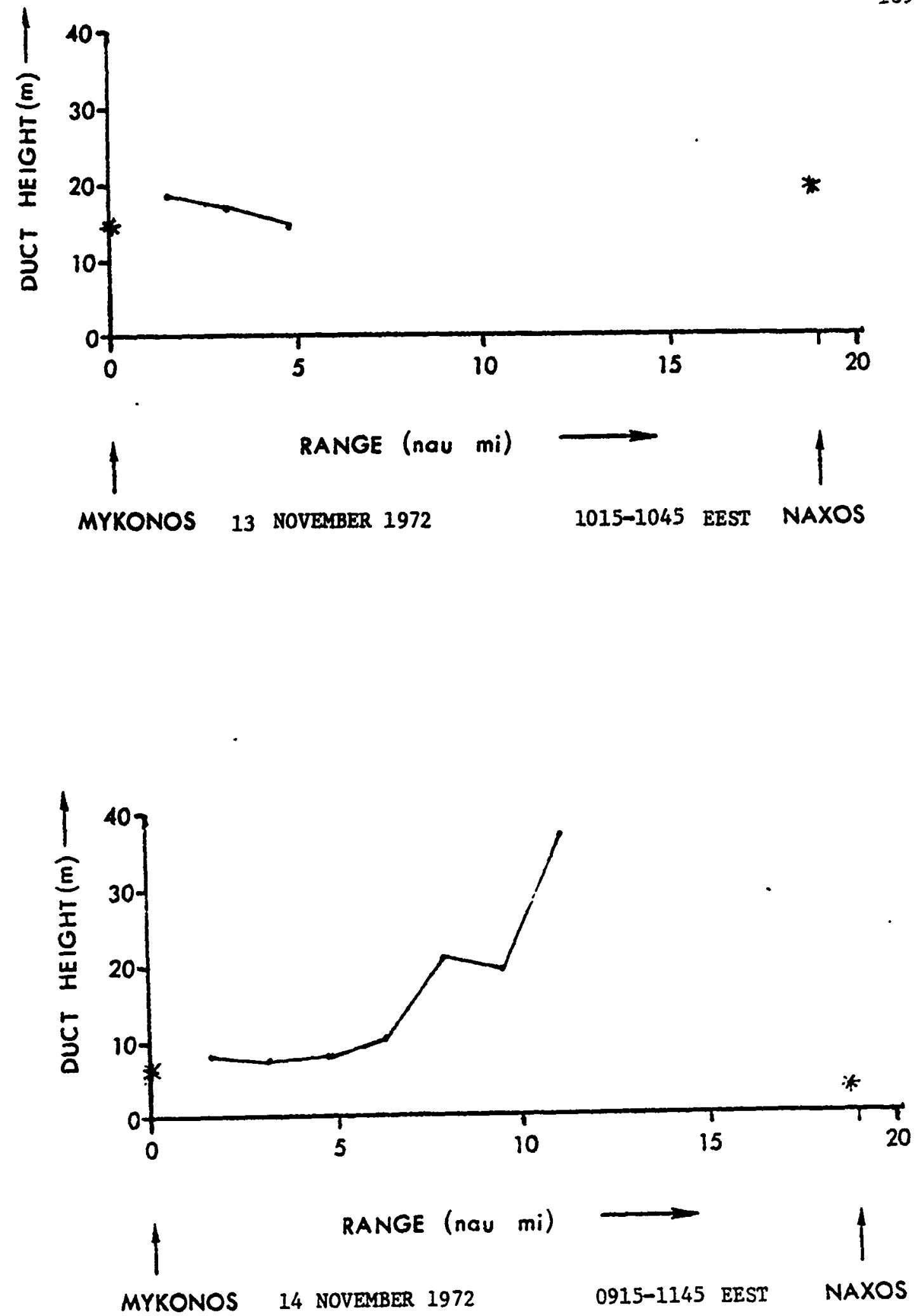

Figure 154. Duct height measurements along the propagation path 


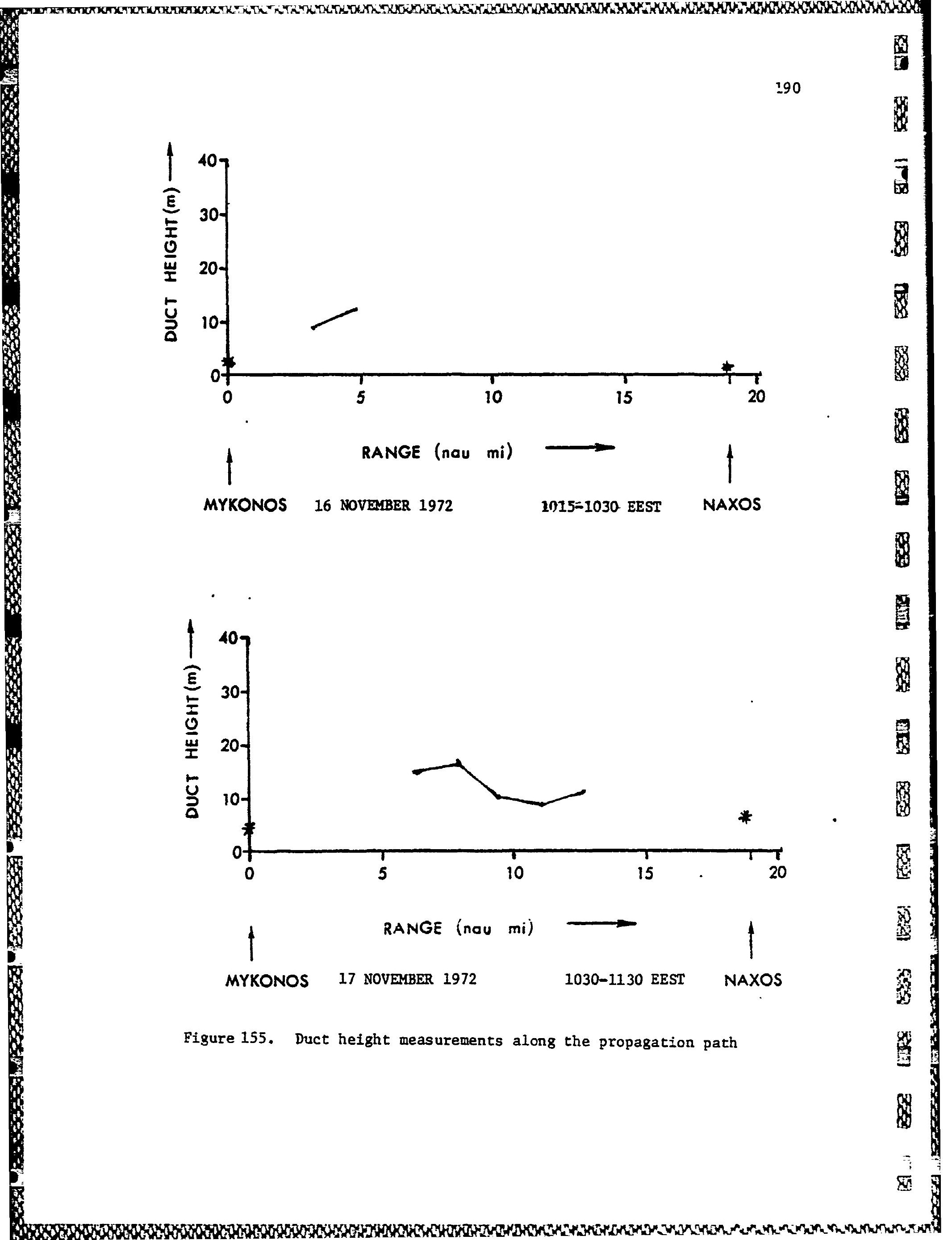



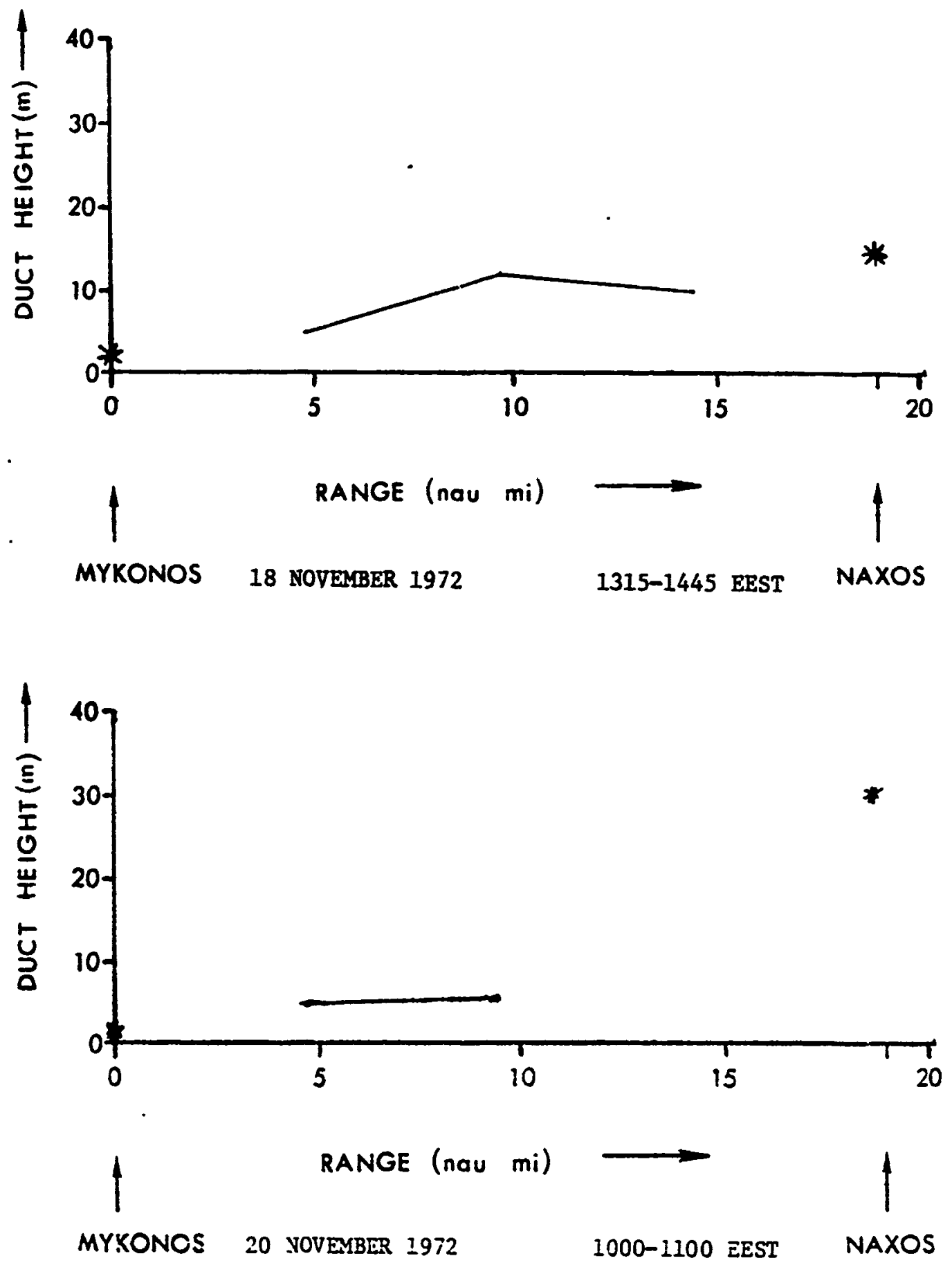

Ftgure 156. Cuct height seasurements Eiong :he propagation pach 


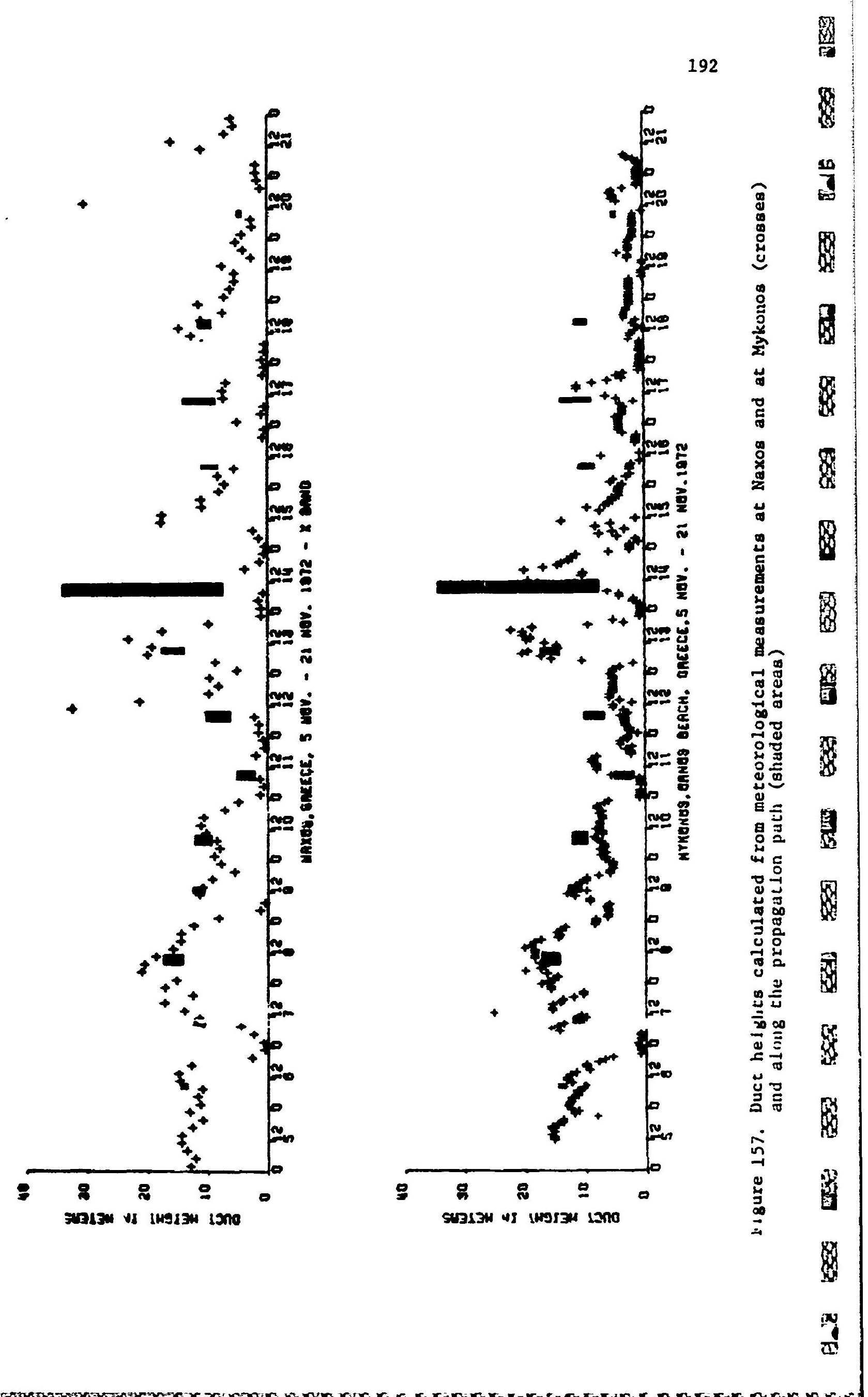




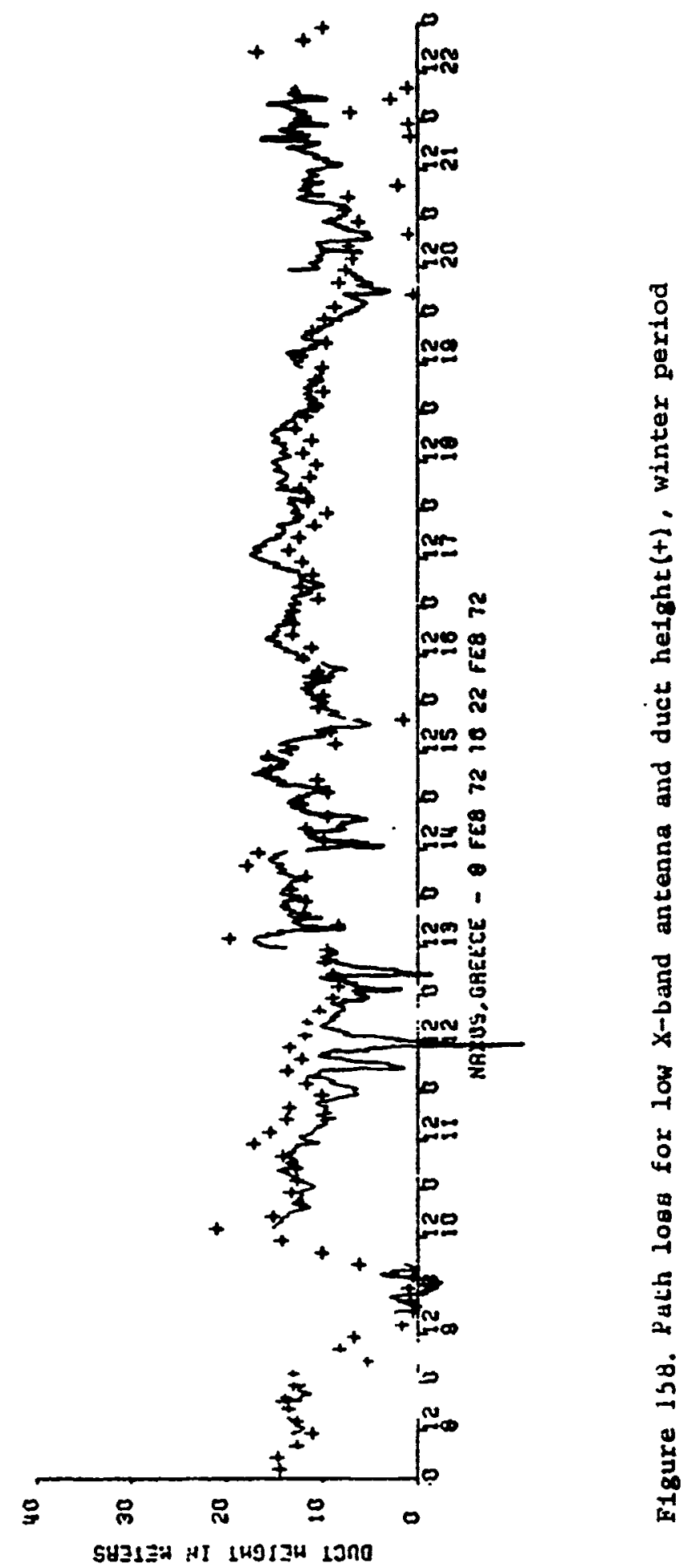

$$
\begin{aligned}
& \text { 일 } \\
& \text { ap UT SSOT ỵzo }
\end{aligned}
$$




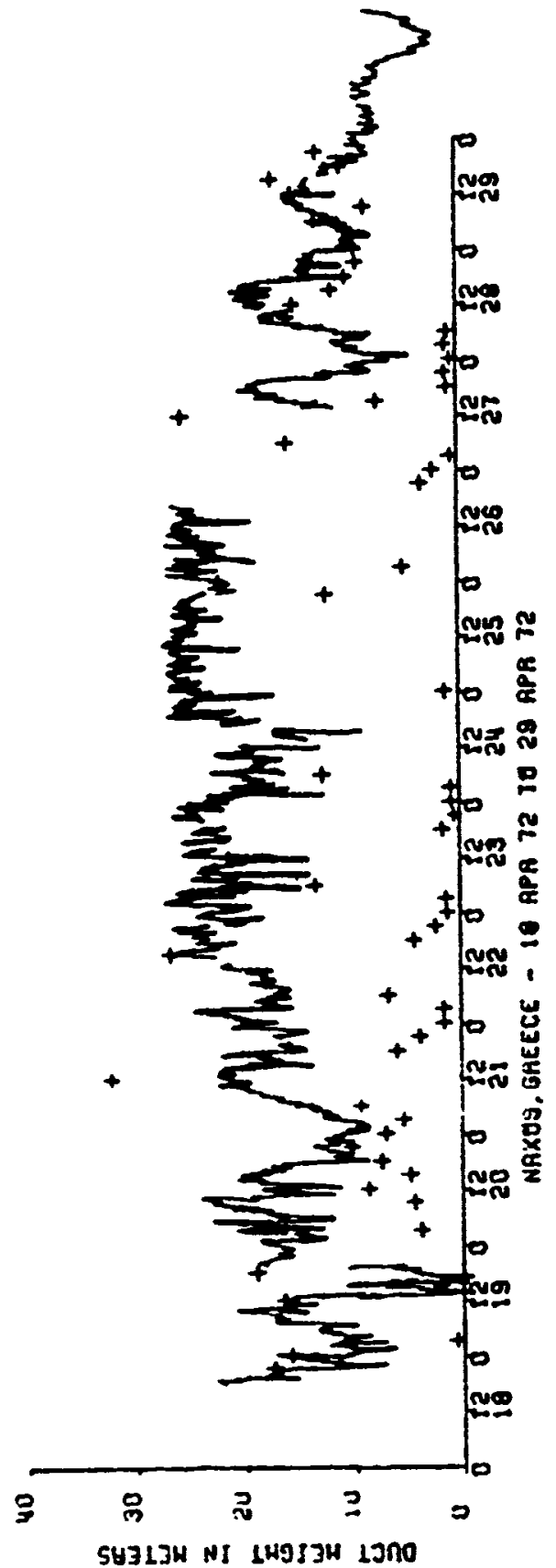



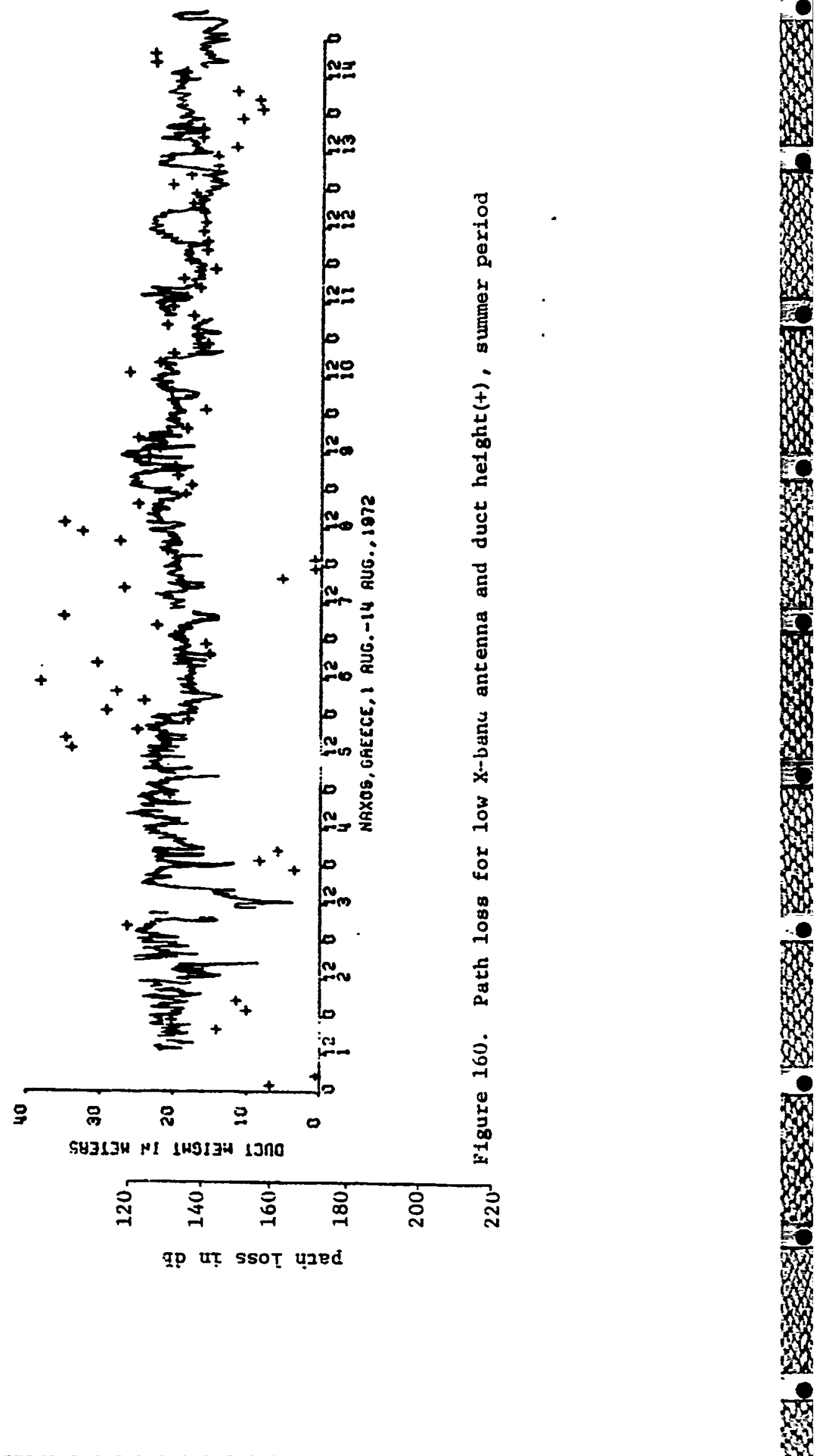

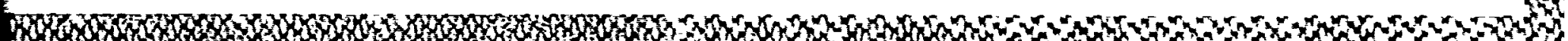

8

8

8

1

8

80

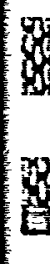

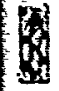

os

5 


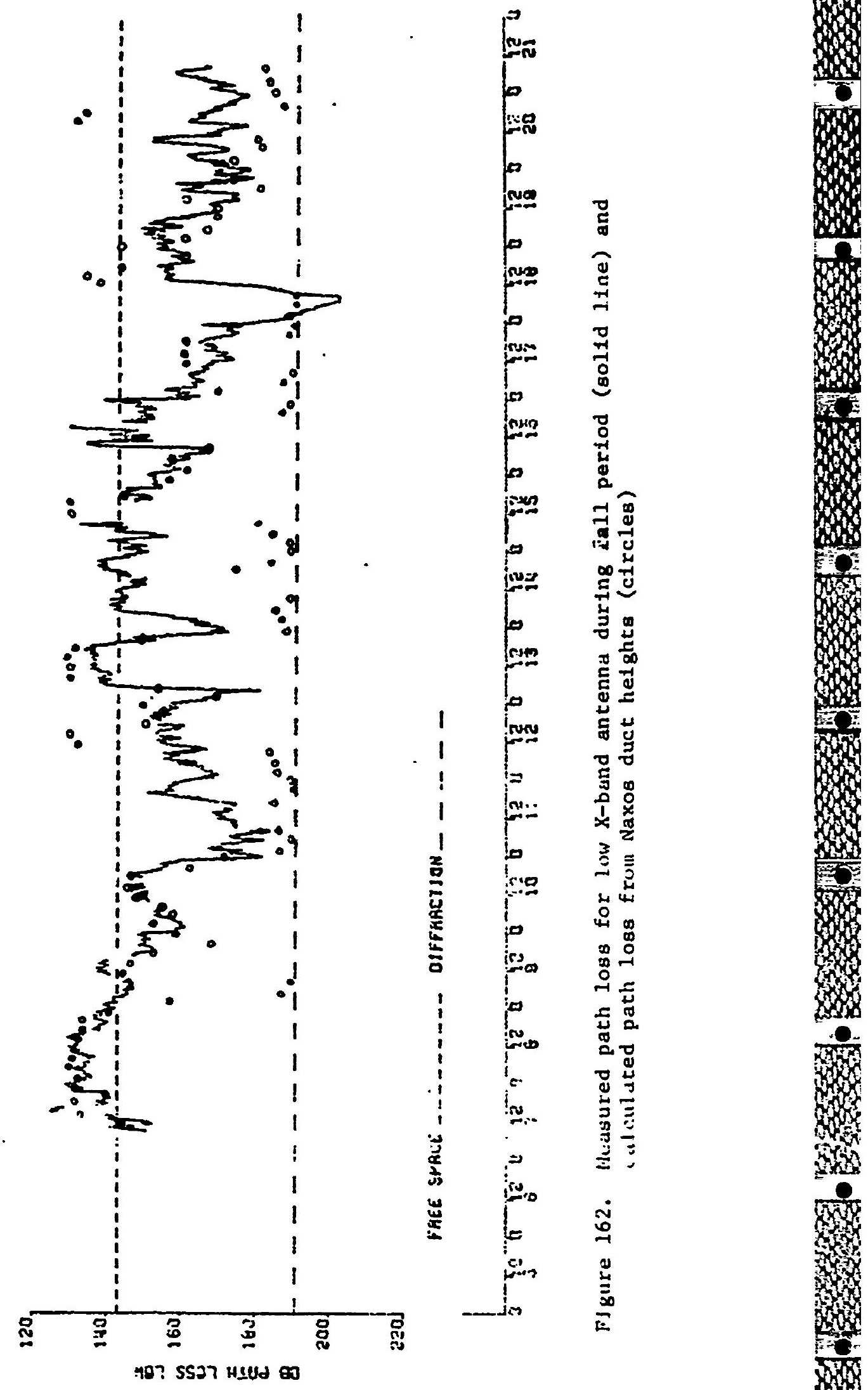


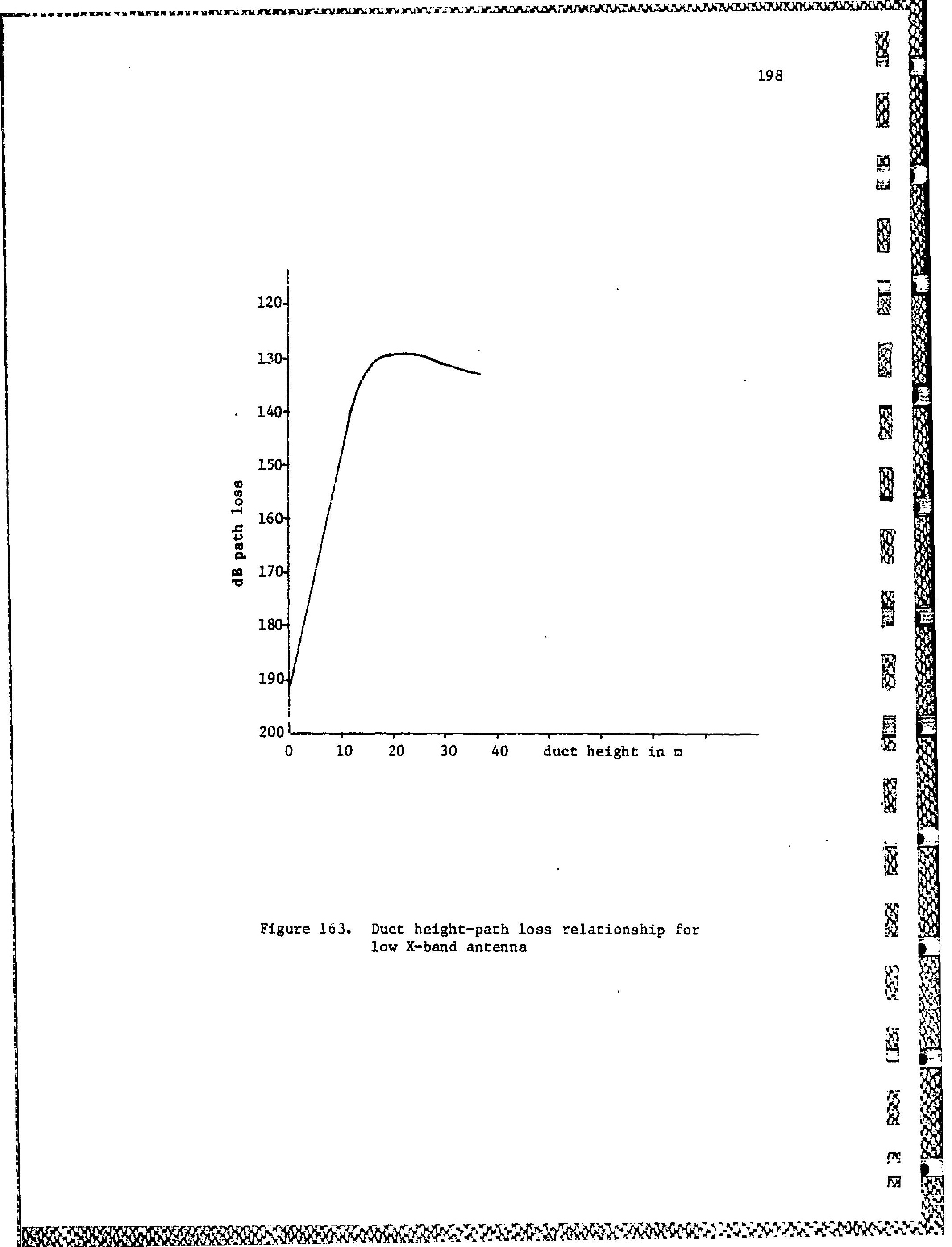



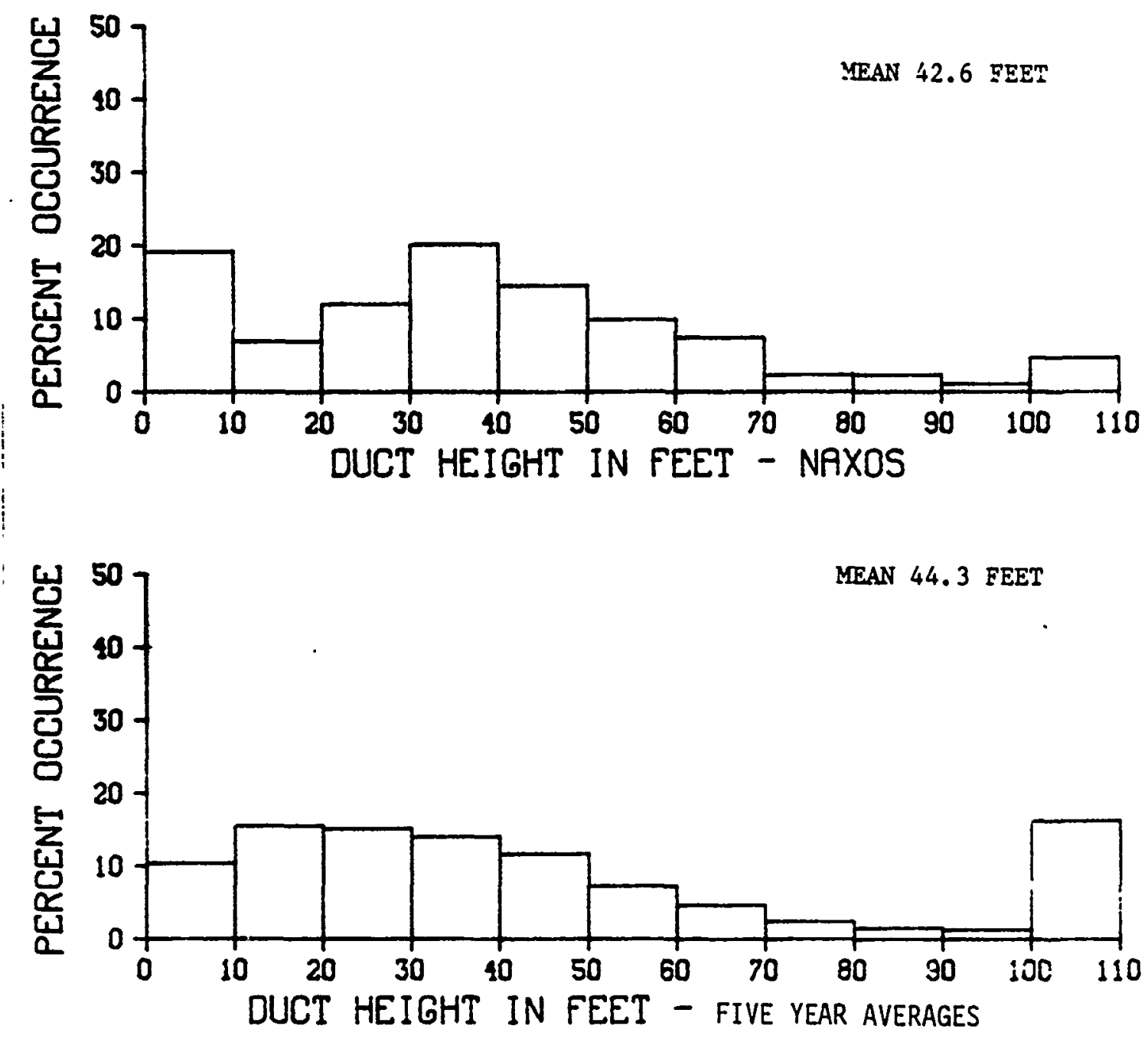

تigure 164. Duct height distribution from Naxos measurements and Erom five year meteorological averages for the area of the propagation path 
IX. TABLES

PAGE

1. Measurement periods and frequencles

2. Propagation link characteristics

WINTER PERIOD (8-22 FEBRUARY 1972)

3. Statistical presentation for L-band

4. Frequency distributiong of path loss and fading for L-band

5. Frequency distributions of path loss differences between antennas for $L$-band

6. Statistical presentation for s-band

209

7. Frequency distributions of path loss and fading for s-band

8. Frequency distributions of path loss differences between antennas for S-band

9. Statistical presentation for $X$-band

10. Frequency distributions of path loss and fading for $X$-band

11. Frequency distributions of path loss difference and detection range for $\mathrm{X}$-band

12. Cumulative distribution of detection range and frequency distribution of detection range differences for $X$-band

SPRING PERIOD (18-28 APRIL 1972)

13. Statistical presentation for L-band

14. Frequency distributions of path loss and fading for L-band

15. Frequency distributions of path loss differences between antennas For $L-b$ and 
i6. Statistical presentation for S-band

17. Frequency distributions of path $108 s$ and fading for S-band

18. Frequency distributions of path loss differences between antennas for S-band

19. Statistical presentation for $\mathrm{X}$-band

20. Frequency distributions of path $10 s$ and fading for $X$-band

21. Frequency distributions of path loss difference and detection range for $\mathrm{X}$-band

22. Cumulative distribution of detection range and frequency distribution of detection range differences for $\mathrm{X}$-band

SUMMER PERIOD (31 JULY - 14 AUGUST 1972)

23. Statistical presentation for L-band

24. Frequency distributions of path loss and fading for L-band

25. Frequency distributions of path loss differences between antennas for L-band

26. Statistical presentation Eor S-band

27. Frequency distributions of path loss and fading for S-band

28. Frequency distribuiions of path loss differences between antennas for S-band

29. Statistical presentation for X-band

30. Frequency fistributions of path loss and fading for $x$-band

31. Erequency jistributions of path loss difference and detection range for $\mathrm{X}$-band 
32. Cumulative distribution of detection range and frequency distribution of detection range differences for $x$-band

33. Statistical presentation Ku-band

34. Frequency distributions of path $10 s s$ and fading for Ku-band

35. Frequency distributions of path loss differences between antennas for Ru-band

WINTER PERIOD (7-21 NOVEMBER 1972)

36. Statistical presentation for L-band

37. Frequency distributions of path loss and fading for L-band

38. Frequency distributions of path inss differences betwern antennas for L-band

39. Statistical presentation for S-band

40. Frequency distributions of path loss and fading for S-band

41. Frequency distributions of path $108 \mathrm{~s}$ differences between antennas for S-band

42. Statistical presentation for X-band

43. Frequency distributions of path loss and fading for $\mathrm{X}$-band

44. Frequency distributions of path loss difference and detection range for $X$-band

45. Cumulative distribution of detection range and frequency distzibution of jetection range differences for $X$-band 
48. Frequency distributions of path loss differences between antennas for Ku-band

49. . Statistical presentation for Ka-band

50. Frequency listribution of path Icss for Ka-band

51. Frequency distributions of path loss difference and fading for Ka-band

\section{ALI SEASONS COMBINED}

52. Frequency distributions of path loss and fading for L-band

53. Frequency distributions of path loss differences between antennas for L-band

54. Frequency distributions of path loss and fading for S-band

55. Prequency distributions of path loss differences between ancennas for S-band

56. Percentage of time path ioss differences between high and Low $\mathrm{x}$-jand antennas exceed iertain $\mathrm{jB}$ values

57. Frequency distributions of path loss and fading for $X$-band

58. Frequency distributions of pach loss difference and detection range for $\mathrm{X}$-band

59. Cumulative distribution of detection range and frequency distribution of detection range differences for $\mathrm{x}$-band

50. Erequency dist=ibutions of path ioss and Eading Eor k̈u-band

61. Frequency distributions of path loss differences between antennas for Ku-band 


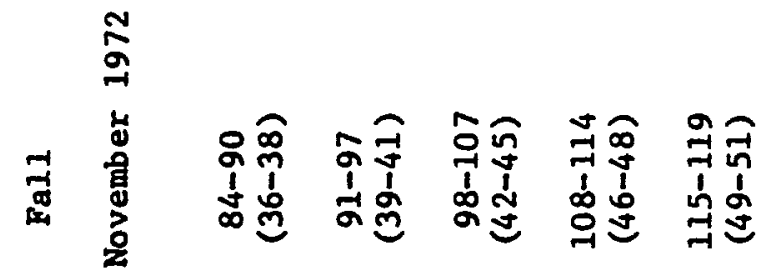

章

I

8

T

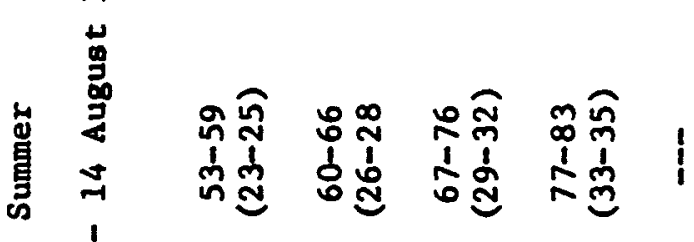

亭

范

3

$\Leftrightarrow$

d

8

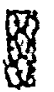

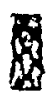

8

के

章

8

ฐิ

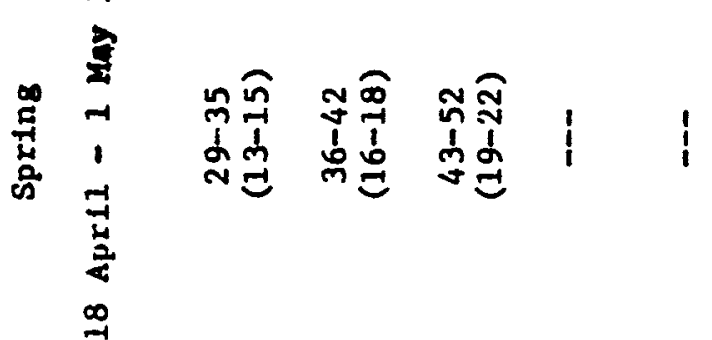

$\stackrel{N}{N}$

峁 总

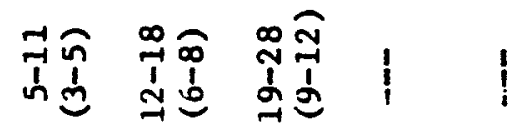

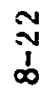

过

8

露

W

两

祭

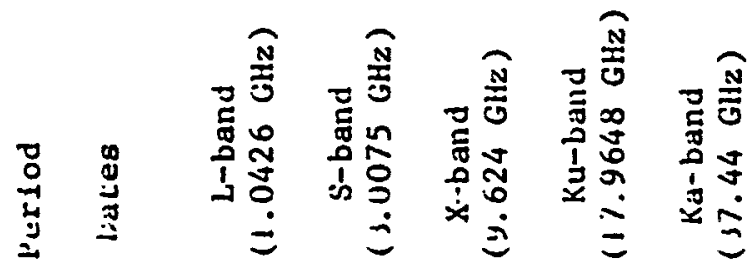

8 


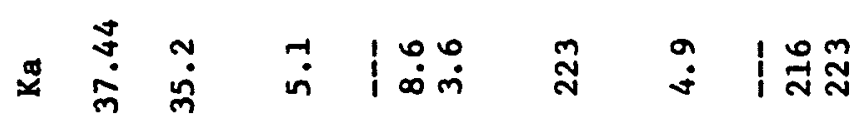

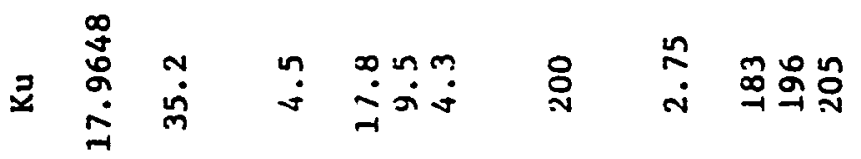

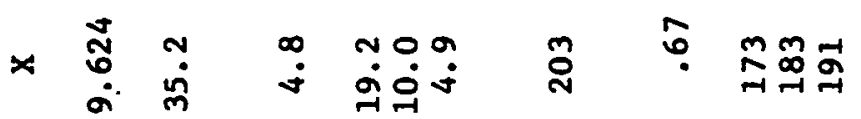

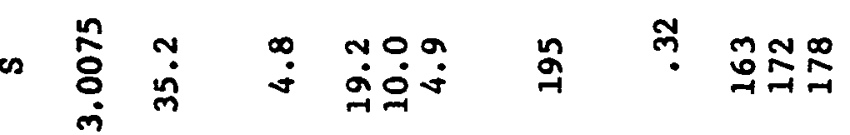

苟

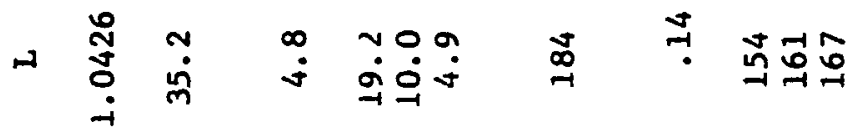

疍

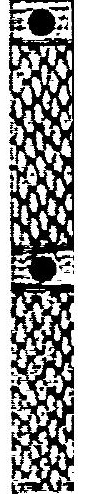

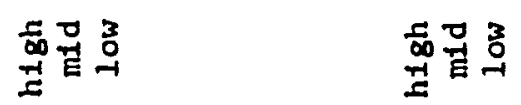

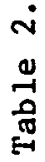

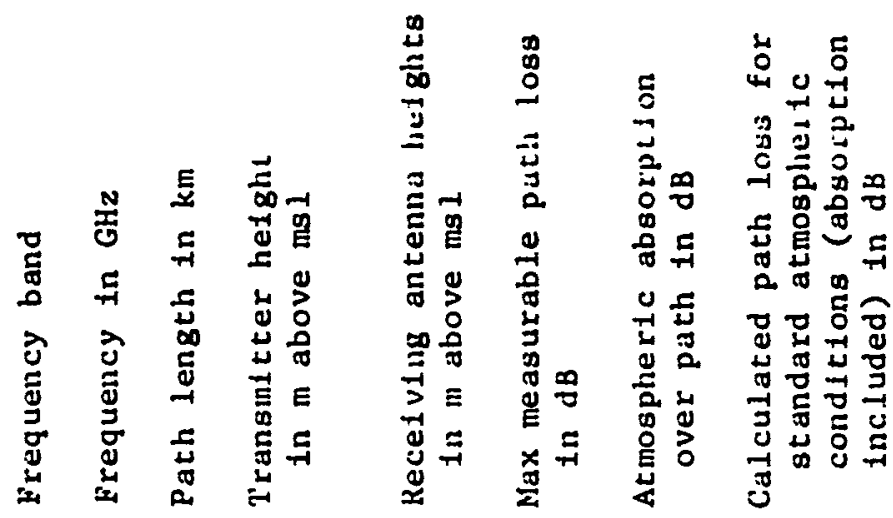

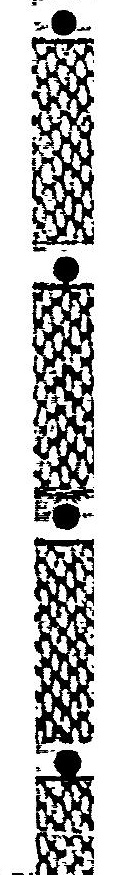




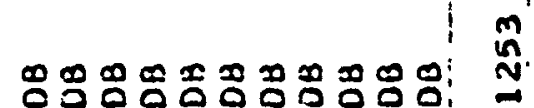

00000000000: "

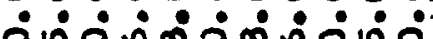

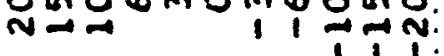

$\underset{w}{\sim}$

ヘヘヘヘヘヘヘヘヘヘヘ

a

2

I

เึ็

$\stackrel{n}{\simeq}$

ư

00000000000

H

뭉ํํ

윰ㅇำ

ヘヘヘヘヘヘヘヘヘヘヘ

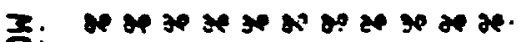

$000-0000000$

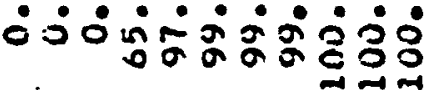

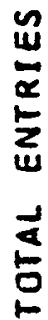

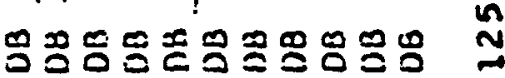

00000000000 "

:.

동ำ

i

$\wedge \wedge \wedge \wedge \wedge \wedge \wedge \wedge \wedge \wedge$

$\stackrel{\sim}{\sim}$

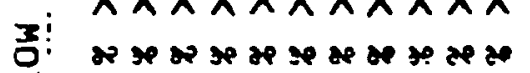

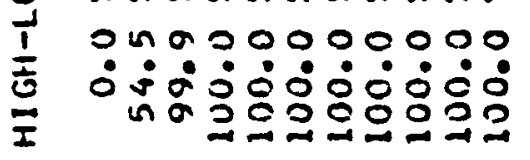

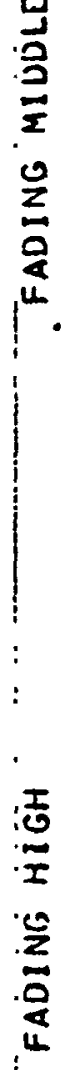

00 กิ

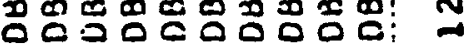

0000000000.1

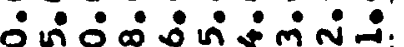

$\operatorname{Nan}$

us

ริ

ヘヘヘヘヘヘヘヘヘヘ

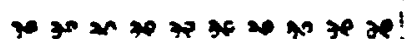

$\because$ is

0 o 00 in un n mm.

$\because 0.0 .0$

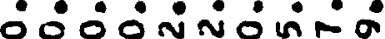

$\rightarrow m$ in

38

$\Delta$

5

8

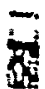

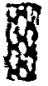

8if

i

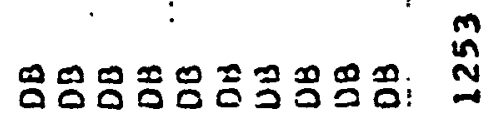

8 $0000000000 . " 1$

s.

in 000 in $5 \mathrm{mn}$ -

出

2

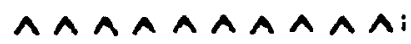

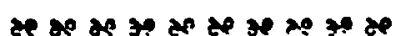

$0000=0 x n+n$

ن்

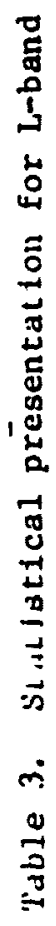

要

8

8

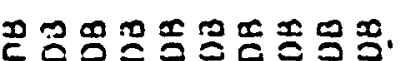

กิ

0000000000 .

$\dot{0} \dot{0} \dot{0} \dot{0} \dot{0} \dot{i} \dot{\sim}$

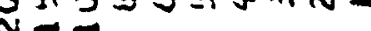

ヘヘヘヘヘヘヘヘヘヘ

x se se se se se se de

a

000000 TNO 000்000்

(1)

(x)

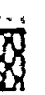

3

舀 


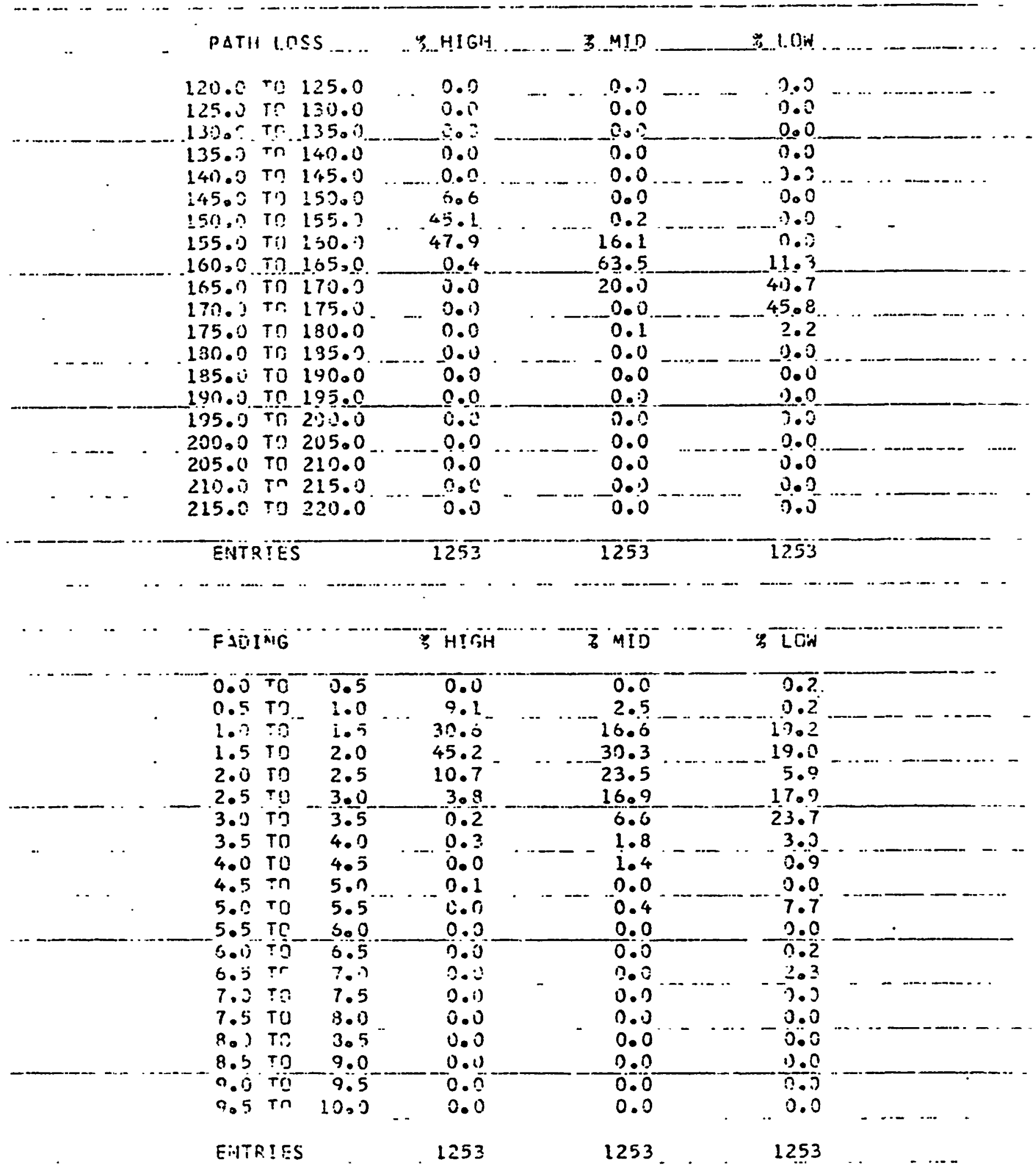

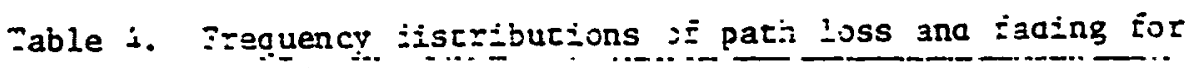
L-jand 
DIFFERENCE -

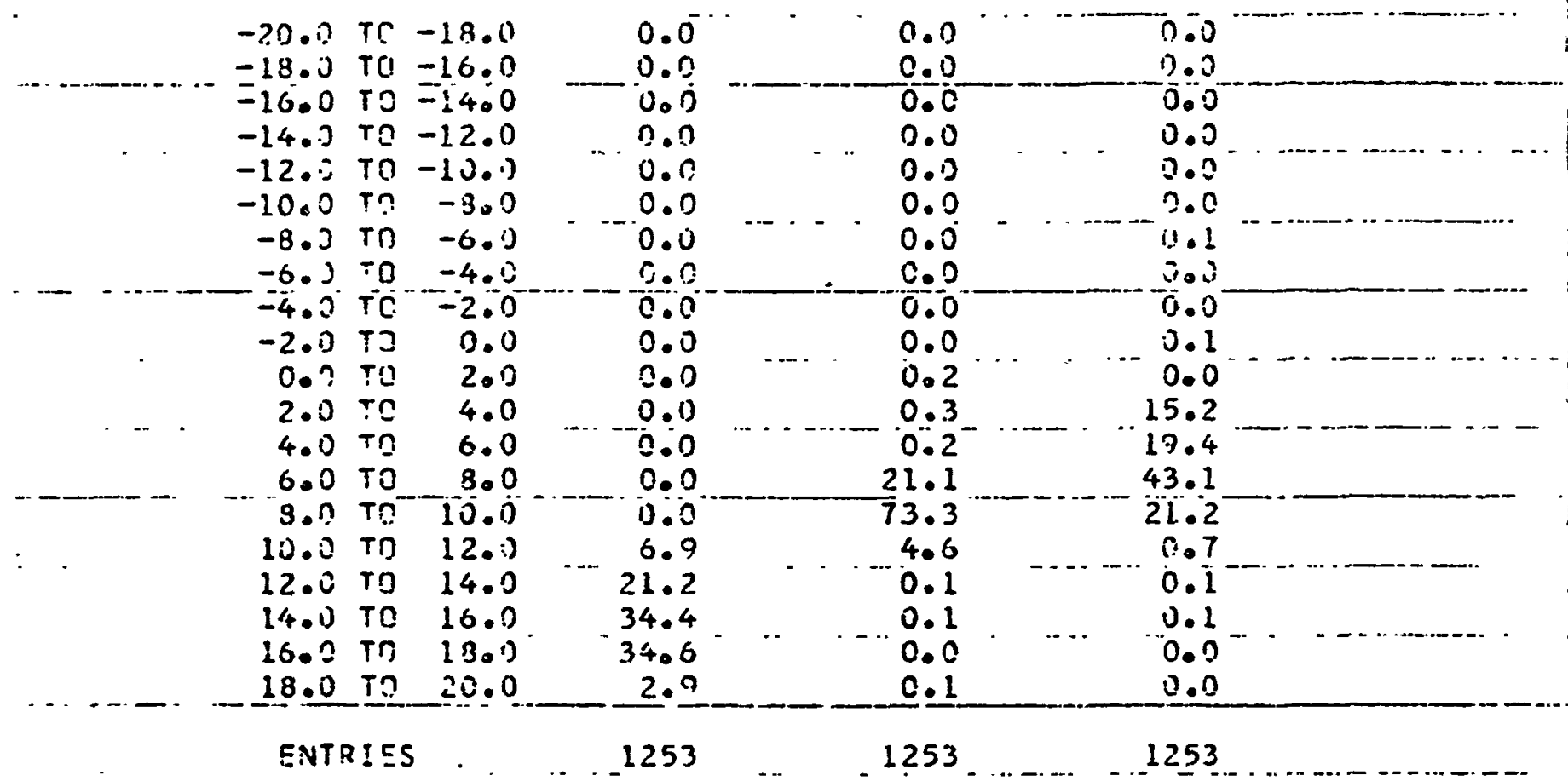

Table 5. Frequency distributions of path loss differences between ancemas for $L=t a n d$ 


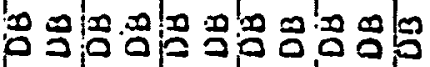

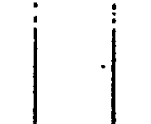

o:0 0,0000000 ல் மீ: NA. $11,1, N$

i 1 i

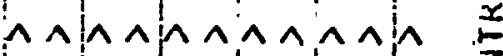

|

a

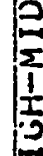

$\frac{1}{2}$

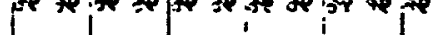

bolm m n m w

i.

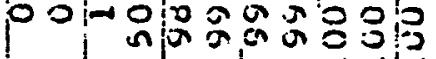
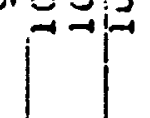

i

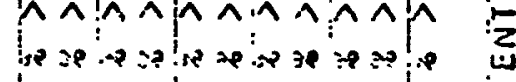

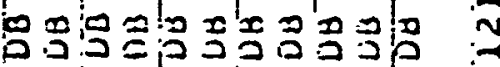

bo:00000000,0

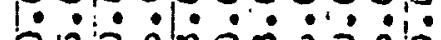

in om

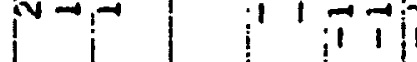

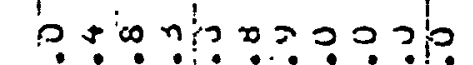

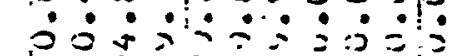

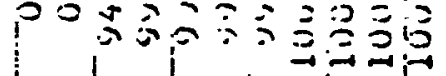

10

光

$\stackrel{0}{3}$

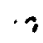

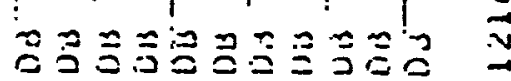

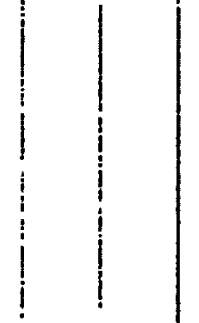

o $=0=2=202.001$

$\ddot{2} \dot{0} \dot{0} \dot{0} \dot{0} \dot{0} \dot{0}$

$\bar{N}=0$

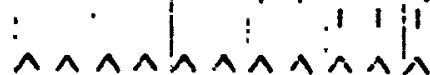

$=$

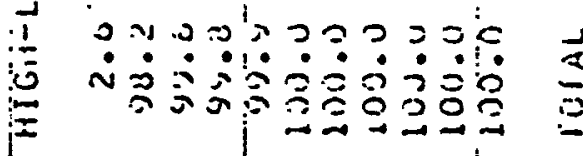

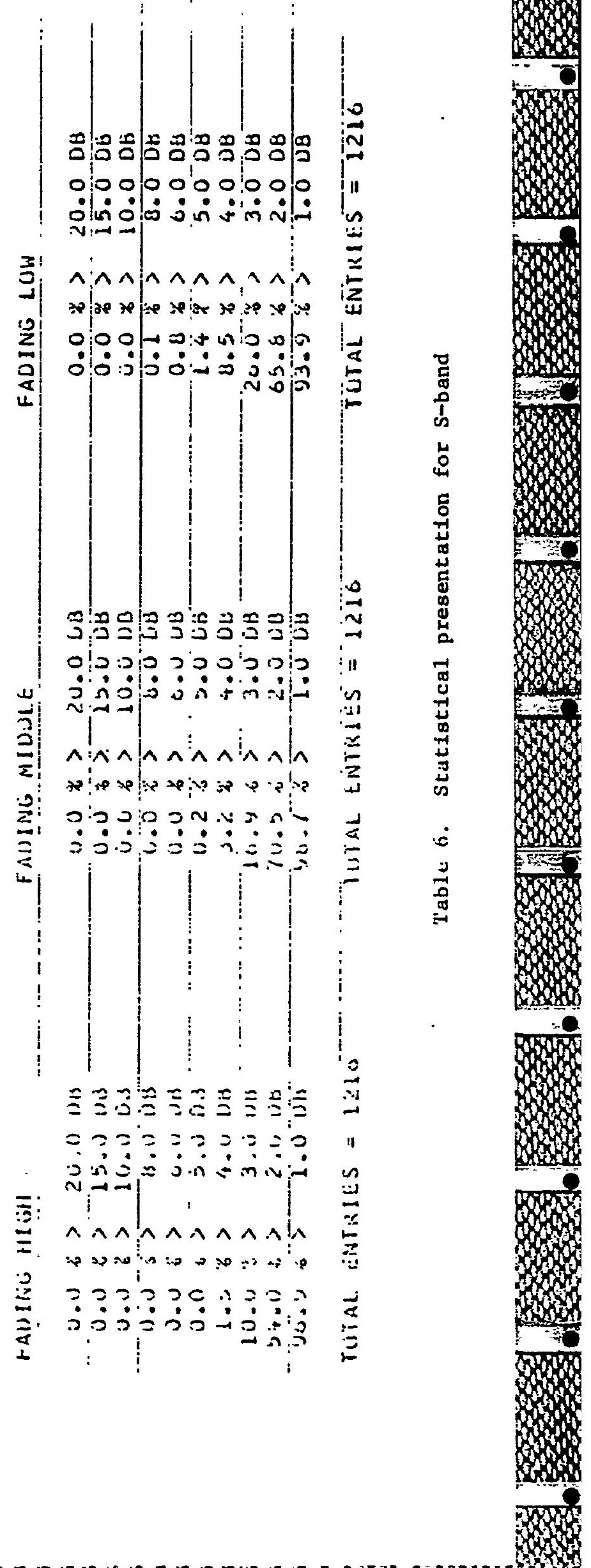


PATH LOSS \%. HIOH 6.910 $2 \ddot{10}$

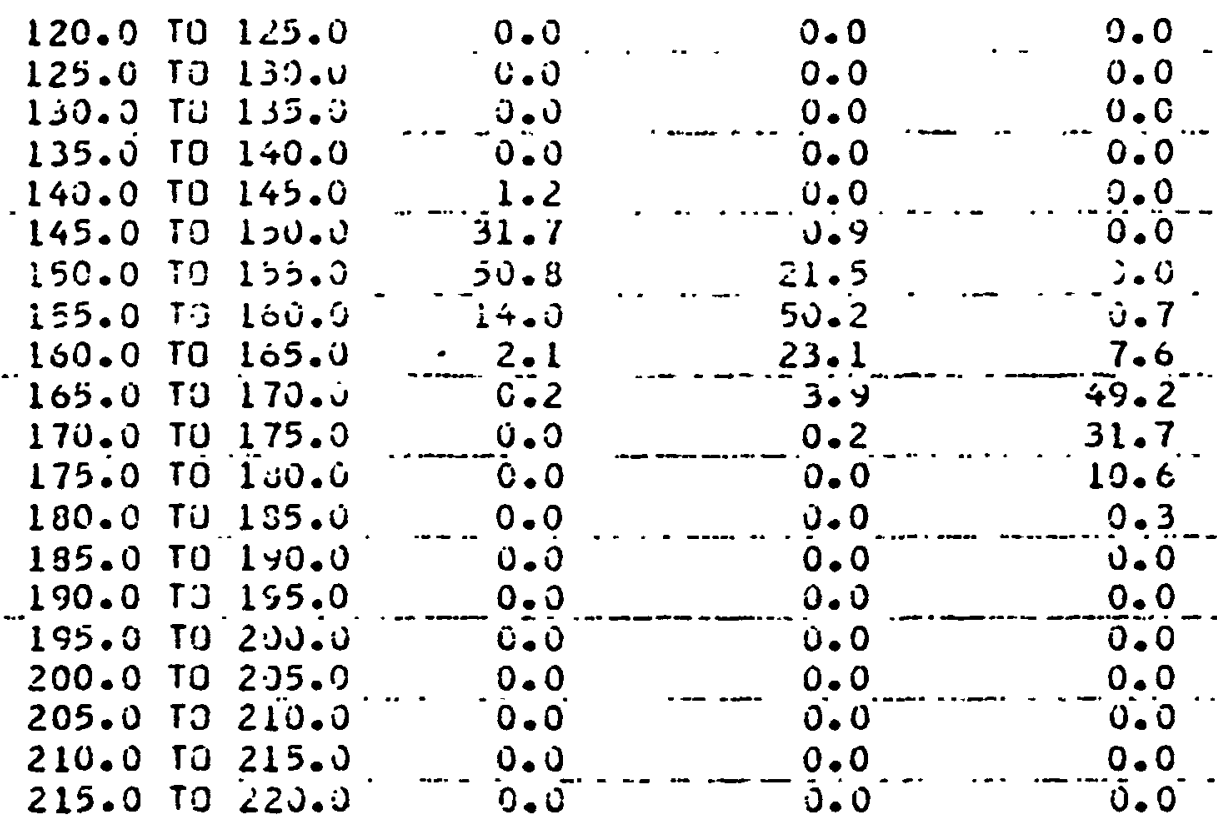

\section{ENTTKIES}

1216

$\sqrt{216}$

$121 i$

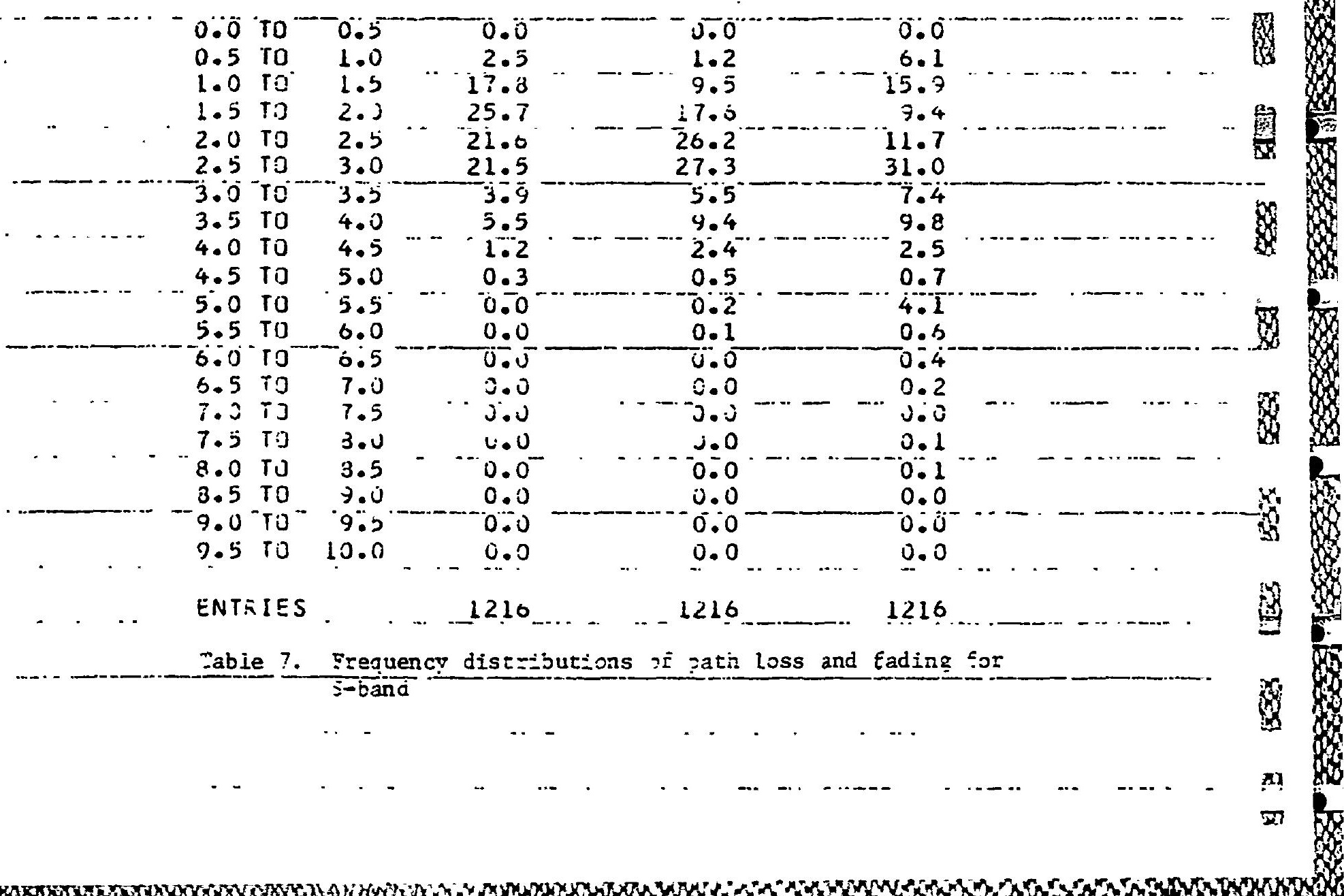

9 


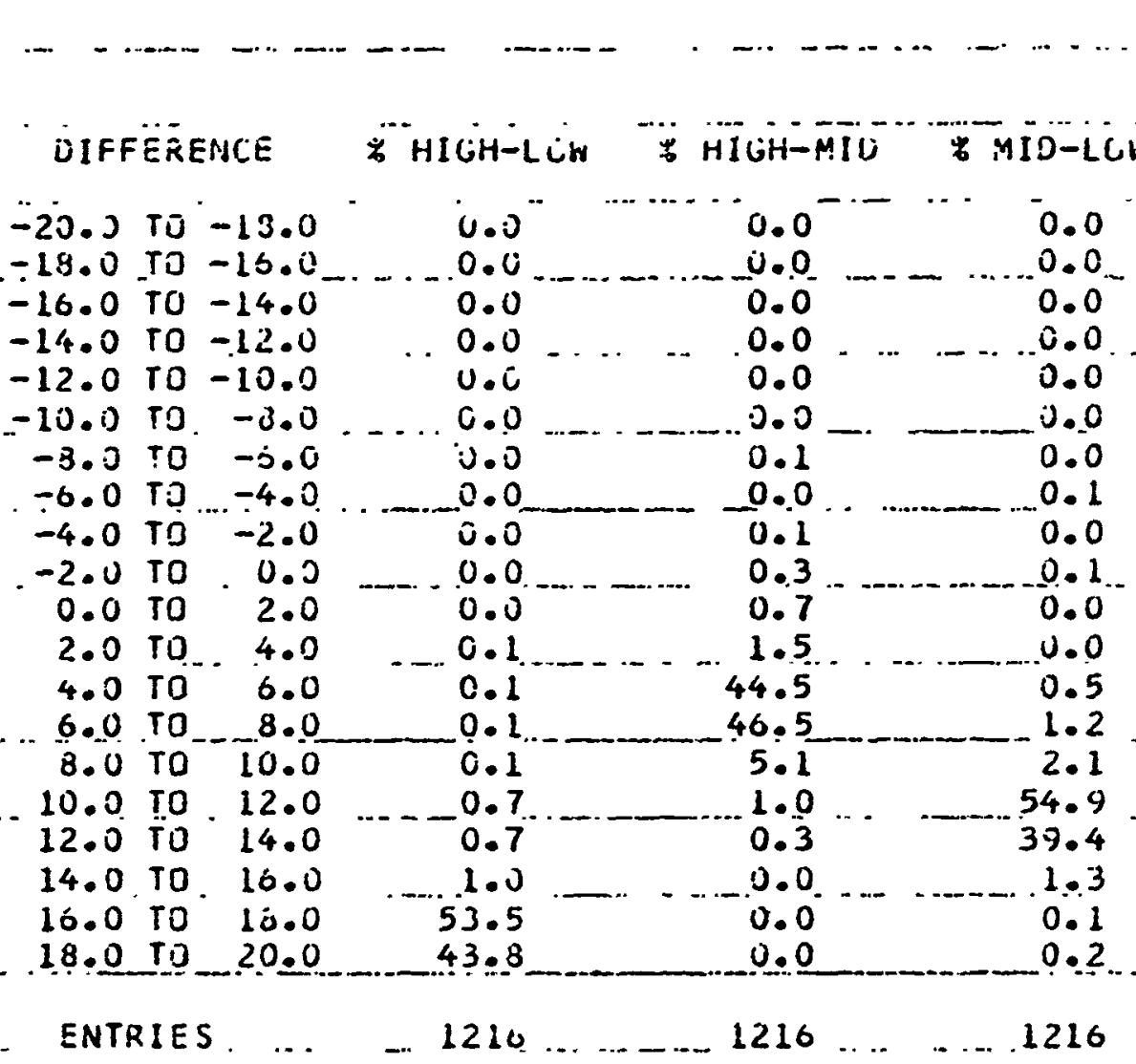

Table 8. Frequency distributions of path loss differences between

8
8
8
8
8
8
8
8 ànteñnas for $s=b a n d$ 


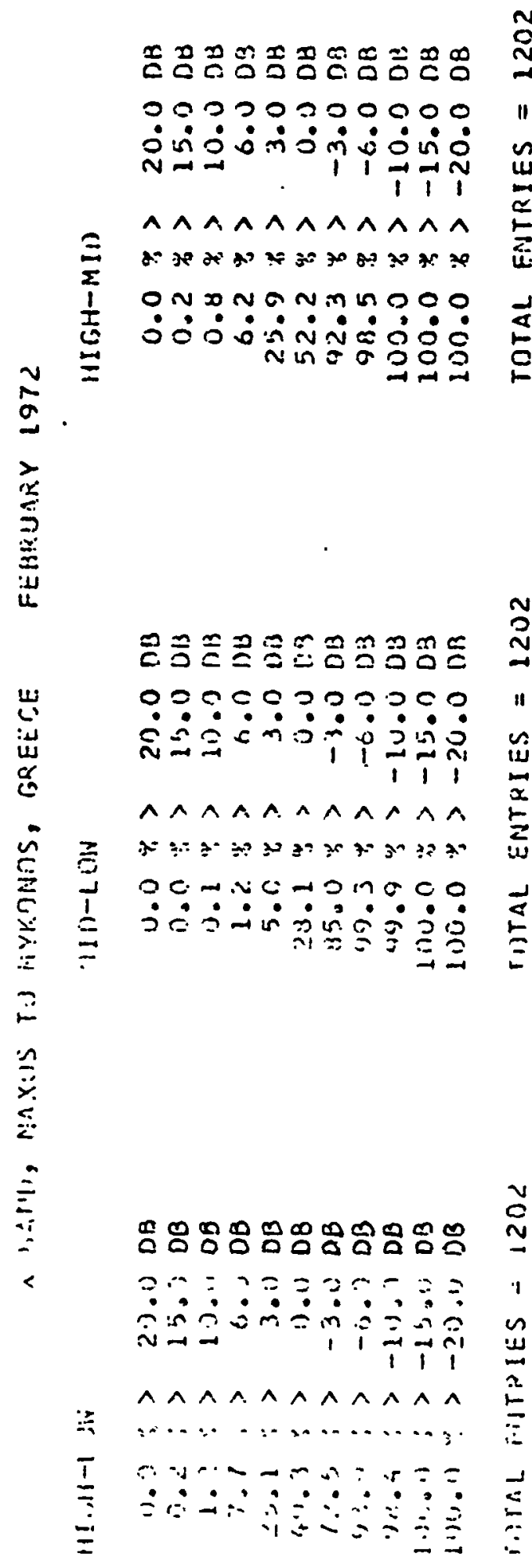

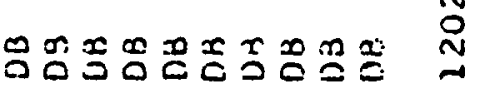

S 000000000011

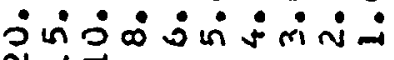

ヘヘヘヘヘヘヘヘヘヘ

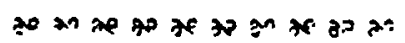

崩

OCONNOINNm

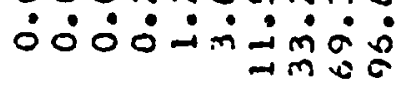

$\frac{1}{5}$
5
5

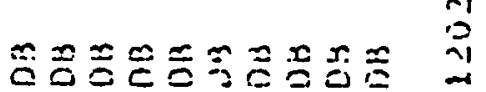

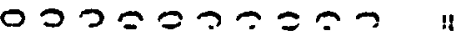

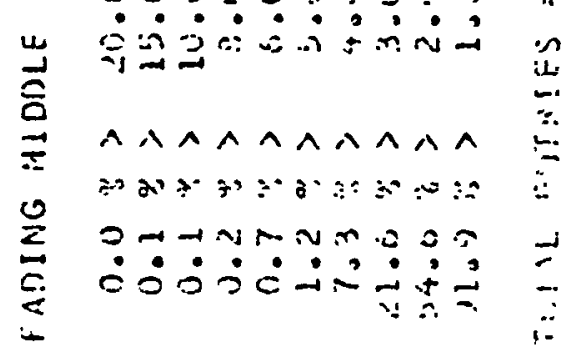

8

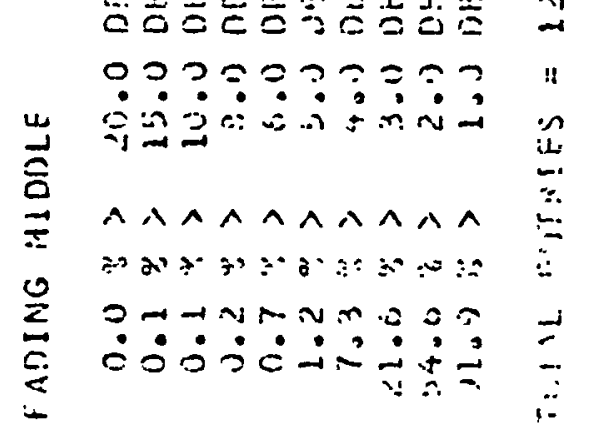


$X$ BAMU, GRELC:E EE3RUARY 1972

๑

PATH LUSS

$\because 4 ! 3 H$

$\Rightarrow 410$

\% L.OW

8

120.5 TC 123.')

i. is

125.0 TO 130.9

3.3

130.0 TO 135.3

?.1)

0.9

135.j TS $1 \% 2: 1$

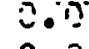

14C.0 T!! 145.0

0.8

145.0 T: 130.1

31.1

156.. T? 155.?

$4 ! .4$

155.0 Tก 1.20.1)

12.11

16u.0 TO 105.0

3.5

105.0 T. T" 170.0

2.3

170.0 TO 175.1 ?

0.5

175.J TO 13J.:

$\therefore .2$

100.0 TO 135.2

0.2

185.j TE $1 \geqslant 0.0$

$0.1)$

19..: is 195.

i. :

$145 . \approx$ Tu 203.0

$\therefore 0$

200.J Tn 253.0 .0

ง.c

2:5.j TT 2130.3

3. 3

210.0 TO 215.0

2.0

215.0 Tn 220.$)$

0.0

1202

0.0

0.0

6.0.

2.2

33.0

35.2

17.2

7.1

2.5

1.6

1.1

0.2

0.1

0.1

0.0

ن. 0

0.0

0.0

0.0

3.3

0.0

3.0

ENTRE:ST

1202

12.22

a

$\theta$

9

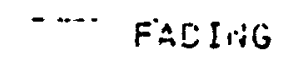

$\therefore \mathrm{HISH}$

? 410

* L. L :

8

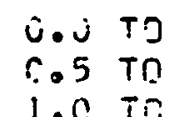

3.5

$\because 0.3$

1.5 TU

1.9

1.5

11.:

2. 0

$\div 8.0$

2. C TO

2.5

2.5 TI

3.0

3. 0 TO

3.5

18.4

25.0

$3.5 \mathrm{TO}$

$4.0 \mathrm{TO}$

4.0

12.3

4.5 TO

4.5

$7 . ?$

3.0

5.0 in

5.0

2.0

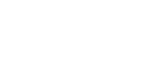

ㄱo.

3.0
2.9
3.4
3.9

$5.5 \mathrm{~T}: \mathrm{J}$

$5: 5$

0.7

(2.)

0.5

3.2

$\begin{array}{ll}6.0 T & T \\ 0.5 & T ?\end{array}$

7. 0 -

6.5

$\because 02$

7.5

$1 .:$

$7.5 \mathrm{TO}$

803

ง. 2

3.0 T3

3.5

0.1

9.5 TI

7.j

0.1

$2.0 \mathrm{TI}$

3.5

ن. 1

9.5 T?

1:).0

ล.1

3.1

6.7
17.4

14.1

22.4

17.5

EVTRI $\leqq S$

1202

$-9.5$

5.1

5.2
$-\quad 3.2$

2.9

$-0.6$

$0 .{ }^{4}+4$

u. 4

0.2

0.1

0.0

0.2

ن.

0.0

0.2

14.3

13.4

21.9

13.7

3.0

$\begin{array}{ll}\cdots & 6.0 \\ & 2.1\end{array}$

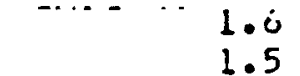

1.5

0.4

$\therefore=$

3.2

0.1

0.0

3.2

J. 1

$12 j 2$

1202

웅

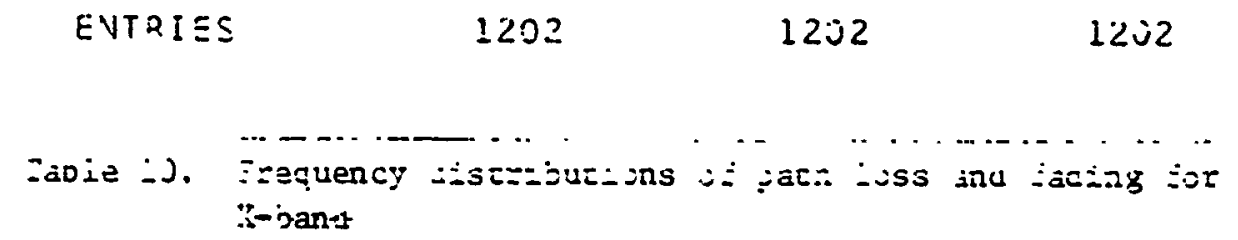

$\$$ $\therefore$-jants

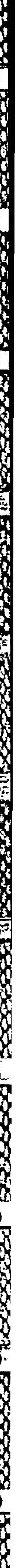


DIFFEREVCE ? HIGH-LDN \& HIGH-AID ; MID-LOH

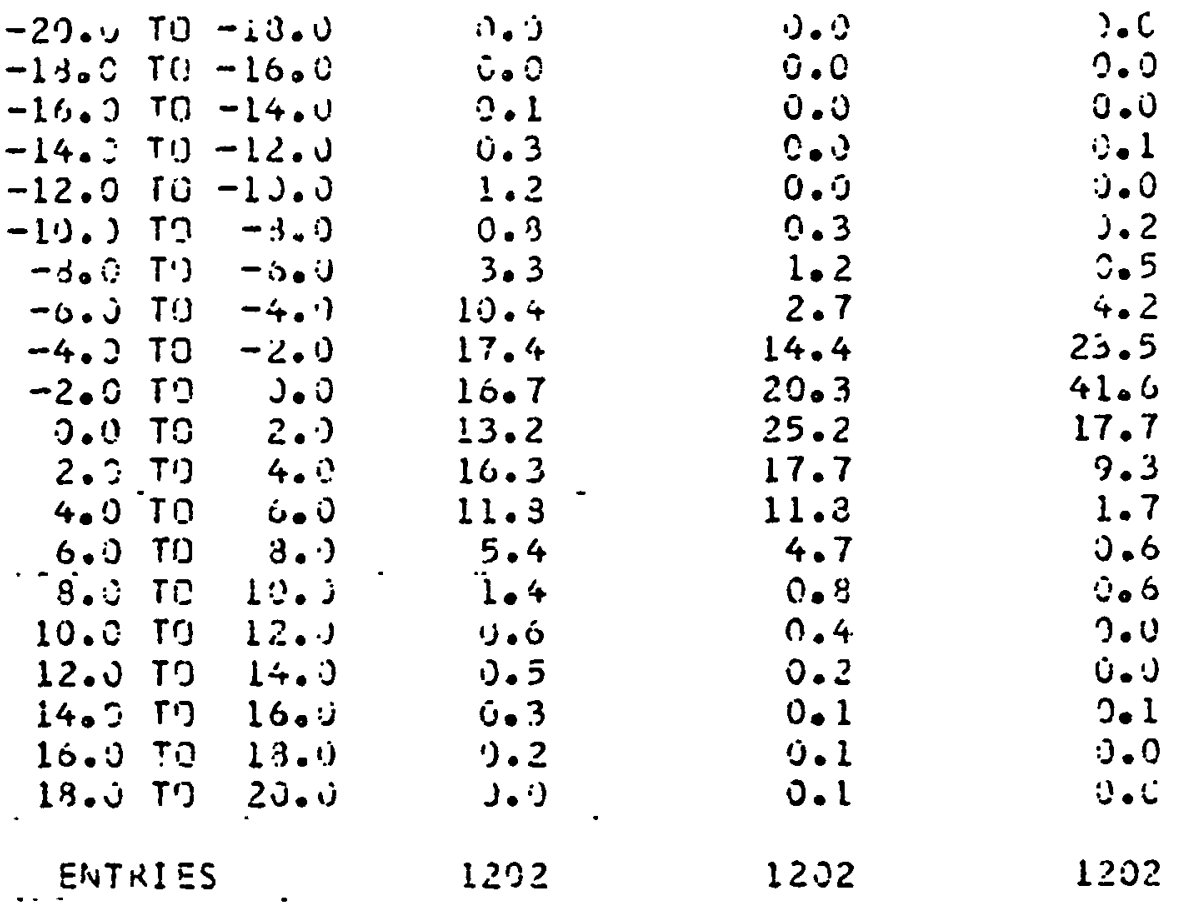

ENTRIES

1232

$\because$ iIO

31.34

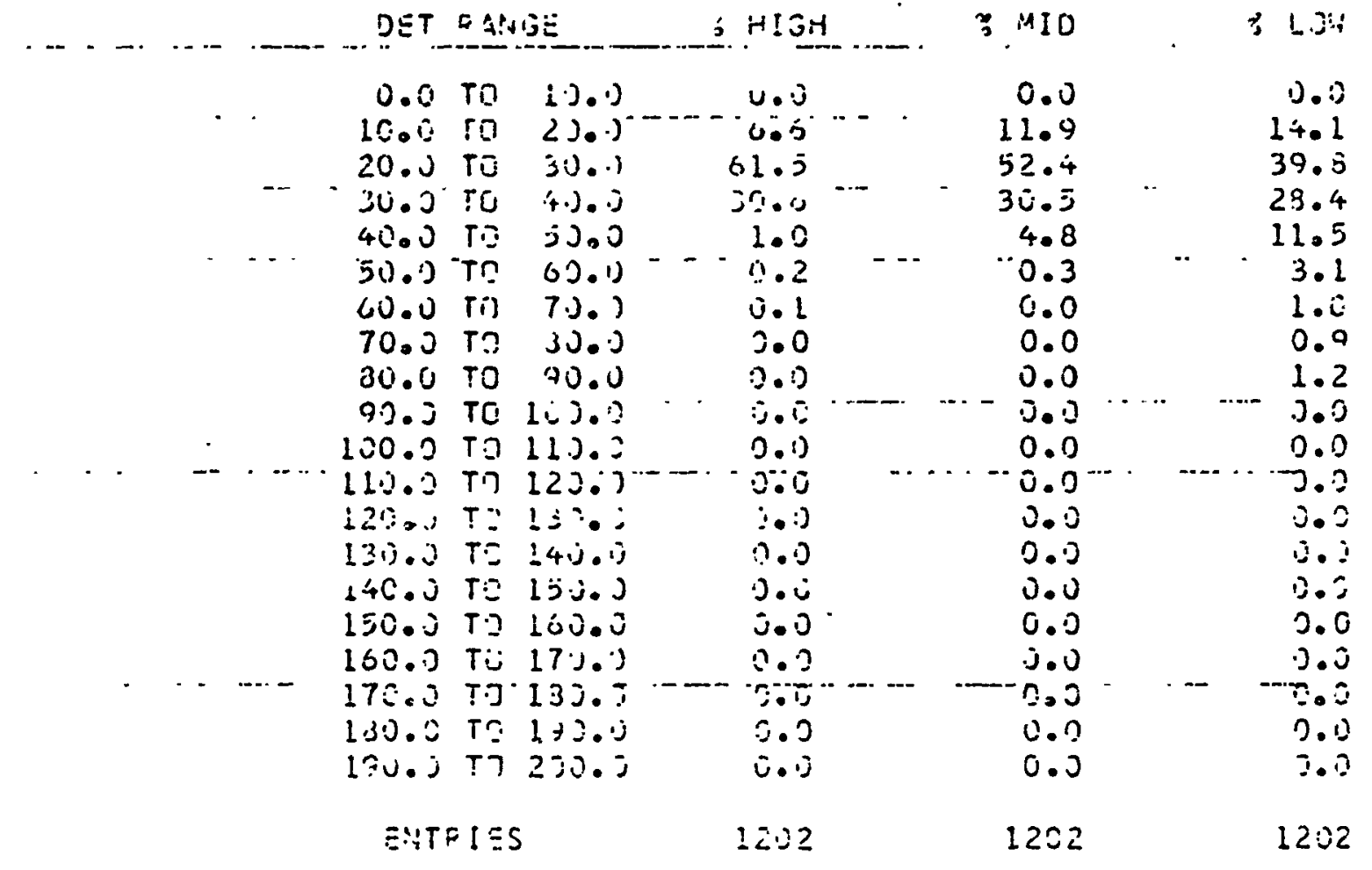

Tabie :i. Erequency istributions $2 \dot{f}$ pati loss $d i z$ ference and detection range Eor 3 -band 


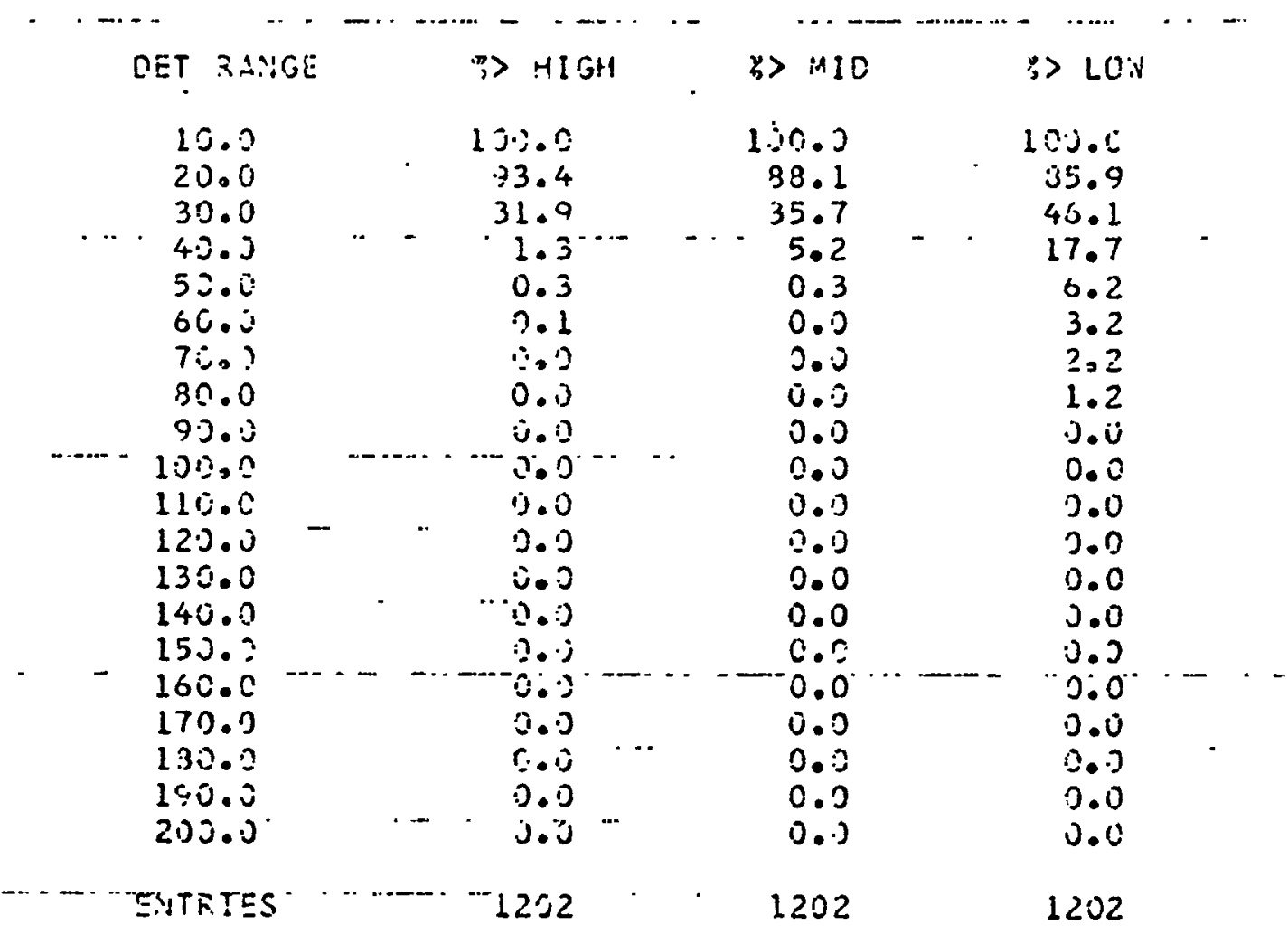

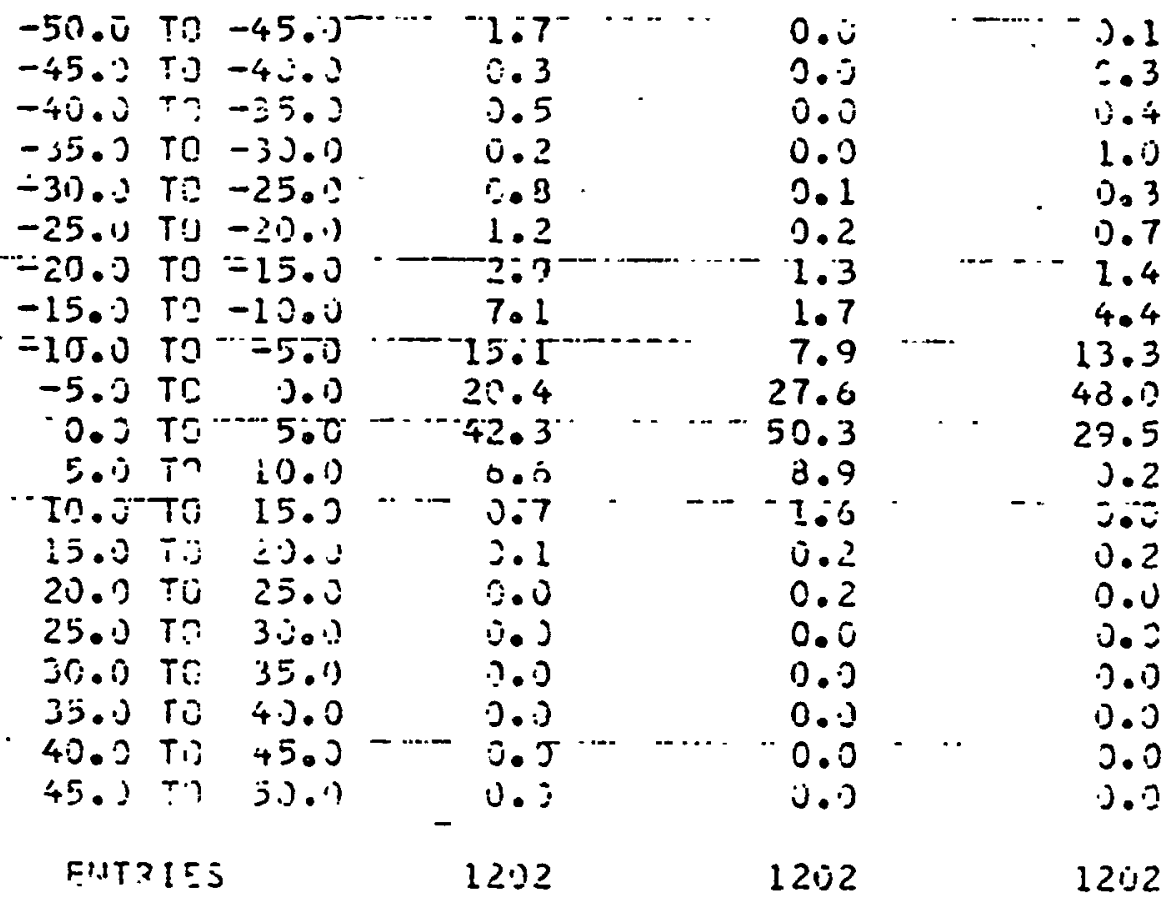

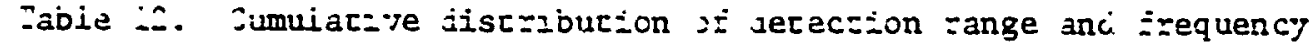

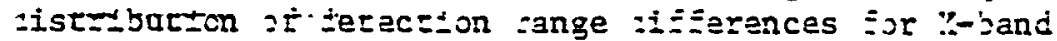

n: 


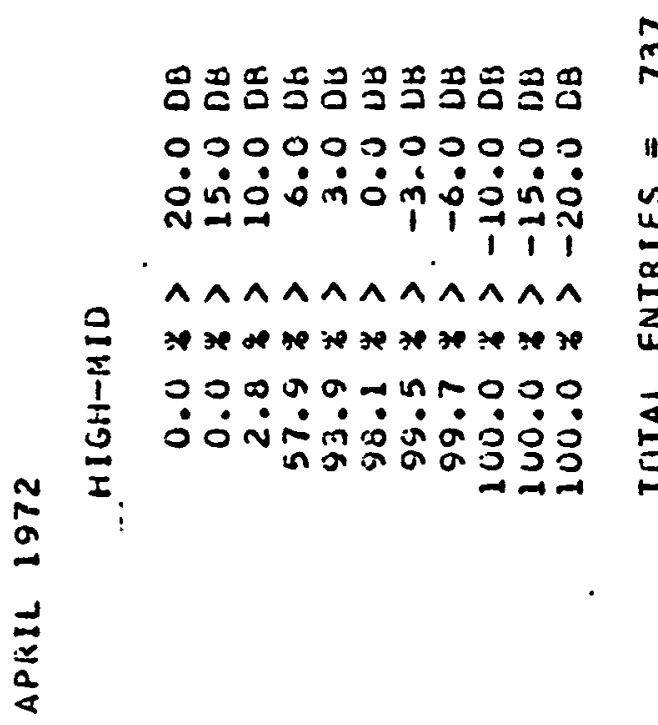

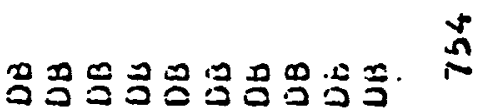

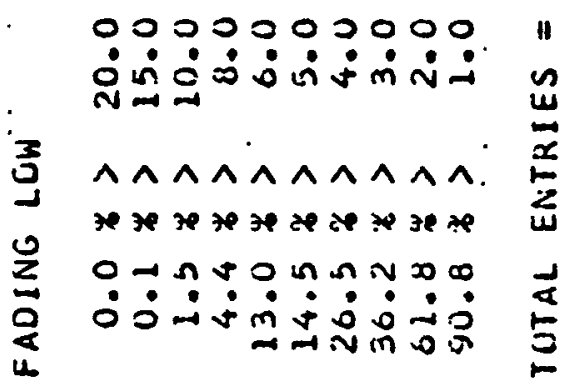

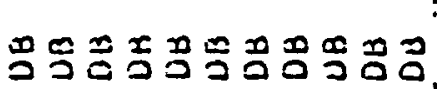

$\stackrel{a}{N}$

00037302000

: ํㅚㅁำ

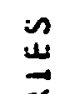

ヘヘヘヘヘヘヘヘヘヘヘ

$\ddot{n}$

.

$\underset{w}{z}$

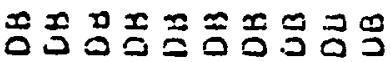

$00 \% 03.00000$

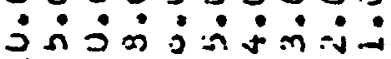
$!$

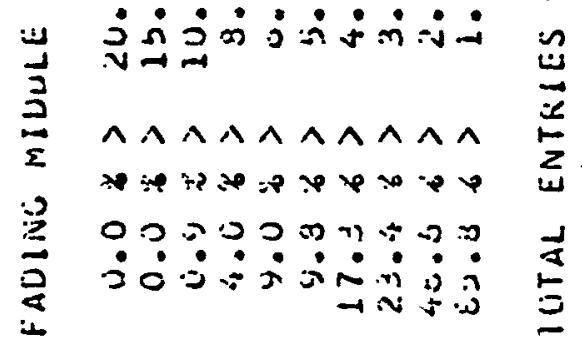

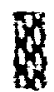

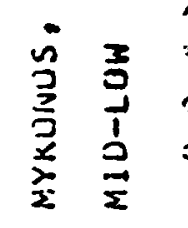

* >

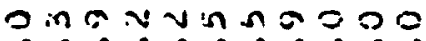

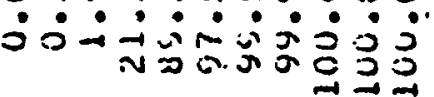

$\frac{1}{5}$

$\overrightarrow{2}$

$\rightarrow N=\infty$

g

a

高

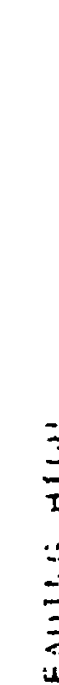

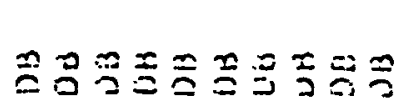

$\stackrel{n}{n}$

$00223000=0$ "

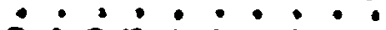

?:?0?0?

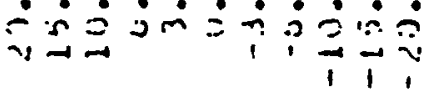

过

芯

$\wedge \wedge \wedge \wedge \wedge \wedge \wedge \wedge \wedge \wedge \wedge$

$\wedge \wedge \wedge \wedge \wedge \wedge \wedge \wedge \wedge \wedge$

:

PON $\because m \sim N=\because$

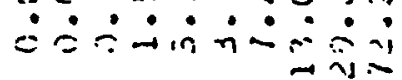

כ"ב 
PATH LCSS

120.0 TO 125.0
125.0 TO 130.0
130.0 TU 135.0
135.0 TO $1+0.0$
140.0 TO 145.0
141.0 TU 150.0
150.0 TO 155.0
155.0 TO 100.0
160.0 TO 105.0
165.0 TU 110.0
170.0 TO 175.0
175.0 TU 130.0
180.0 TO 135.0
185.0 TO 190.0
190.0 TO 195.0
195.0 TO. 200.0
200.0 TO 205.0
205.0 TU 210.0
210.0 TU 215.0
215.0 TO 220.0

6. HI ItI

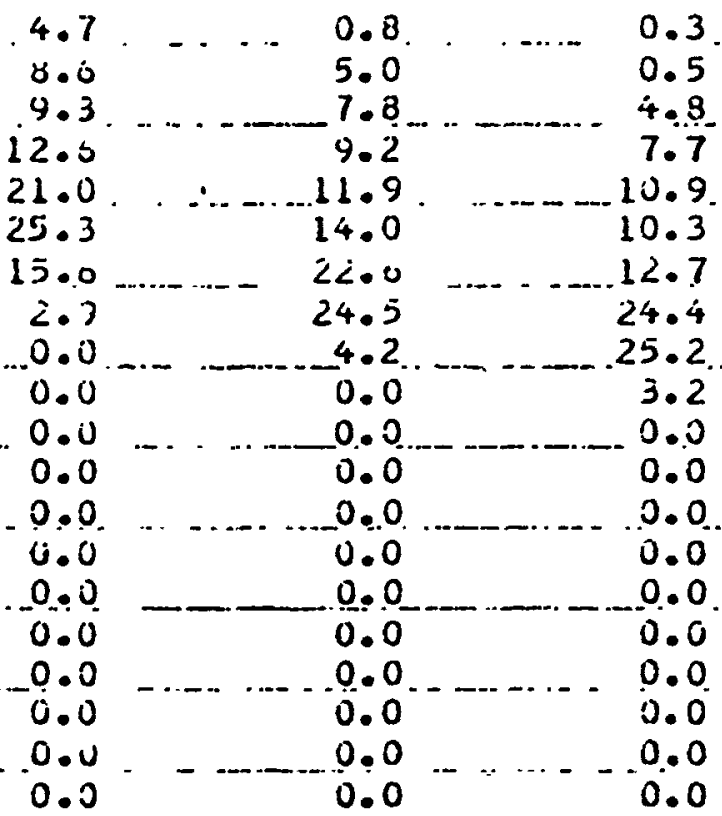

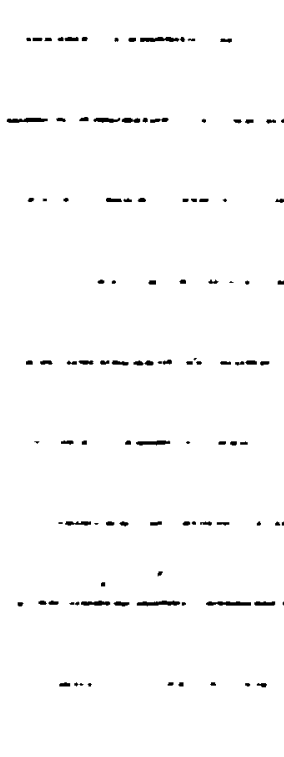

ENTRIES

752

758

754

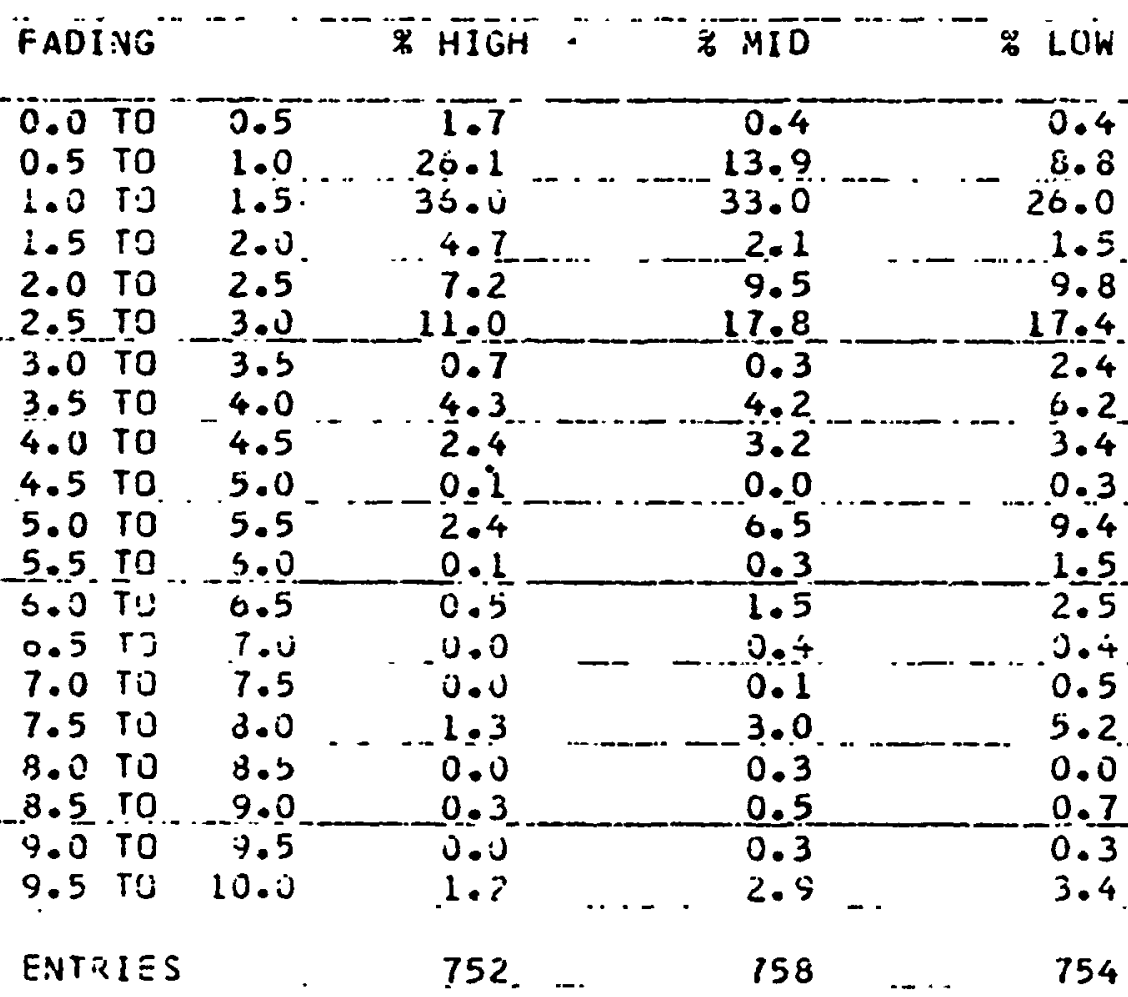

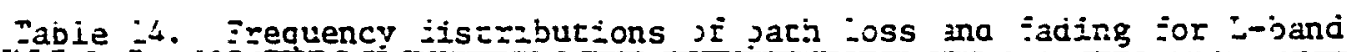




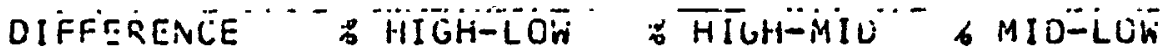

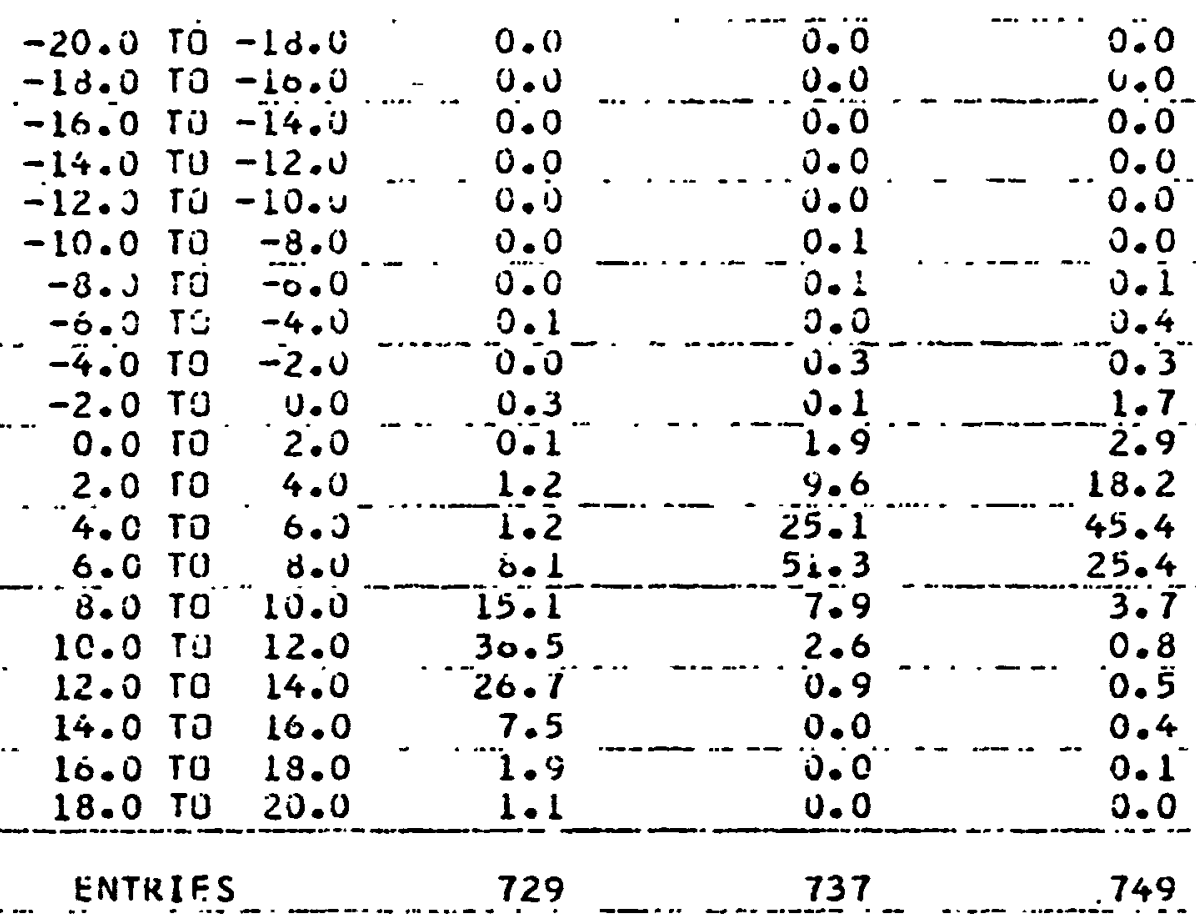

Table 15. Frequency distributions of path loss differences between antennas for $L-b a n d$ 


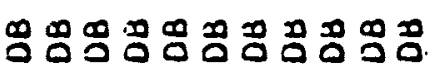

a 00000000000 욤

"

ヘヘヘヘヘヘヘヘヘヘヘ

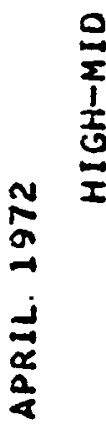

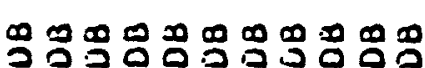

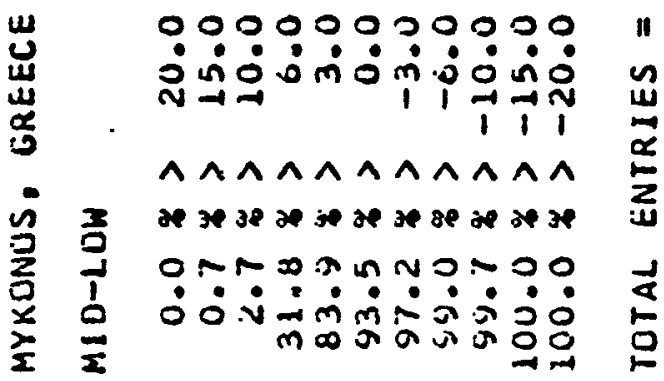
an$\dot{0} \dot{0} \dot{0} \dot{0} \dot{0} \dot{0} \dot{0} \dot{0}$ +

$\mathfrak{O}$

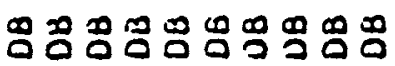

$\stackrel{2}{\sim}$ 0000000000.11

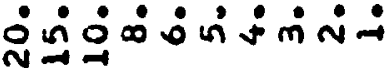

引ิヘヘヘヘヘヘヘヘヘヘ

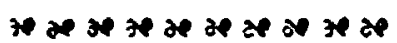

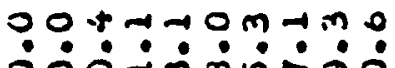
ல்ப்ச்ம் $\rightarrow$ ก

8

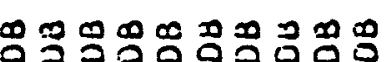
0000000000

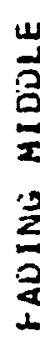

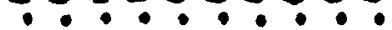

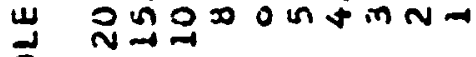
ヘヘヘヘヘハヘヘヘヘ

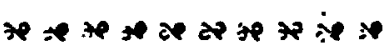

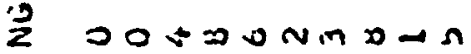
วั0்பட்

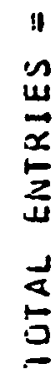

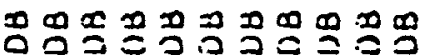

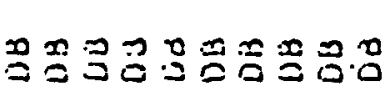
$\dot{0} \dot{0} \dot{0} \dot{0} \dot{0} \dot{0} \dot{0} \dot{0}$

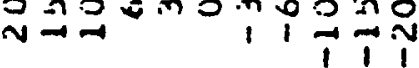
o $000=02=$ $\dot{\therefore} \dot{0} \dot{0} \dot{\Delta} \dot{\sim} \dot{\sim} \dot{\sim}$

$\sin$

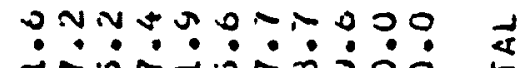

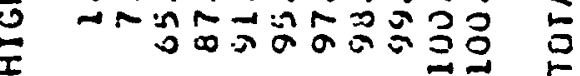

$\frac{9}{2}$

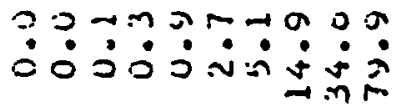


PATH LUSS - HIGH H LON

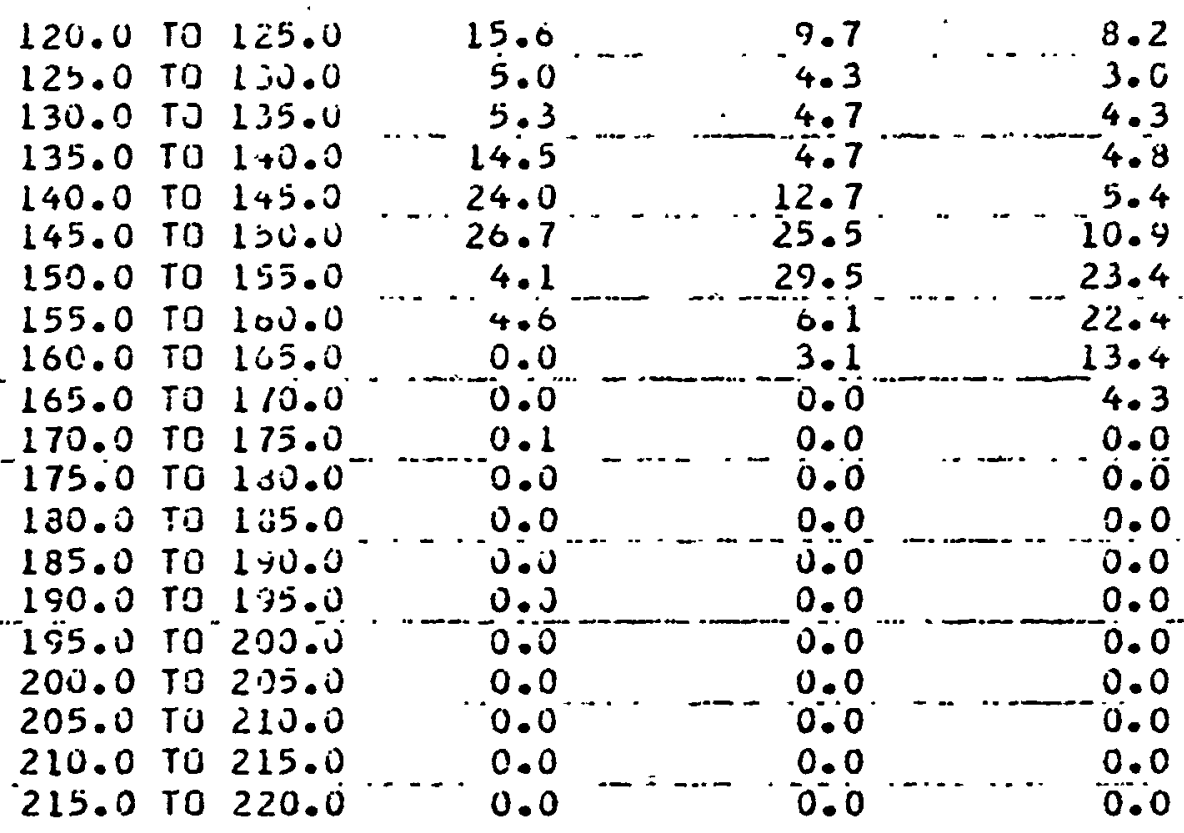

FAOTINE

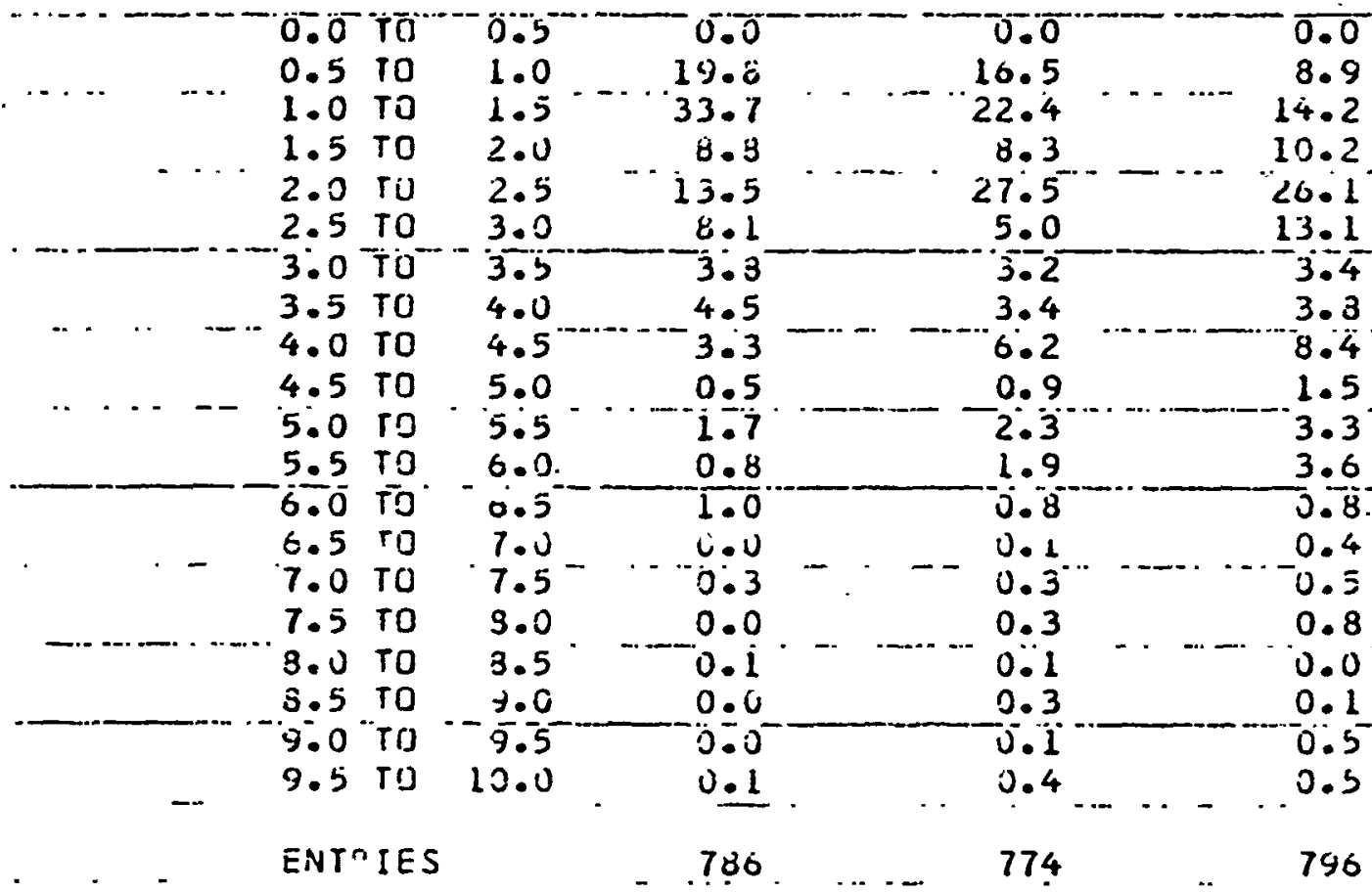

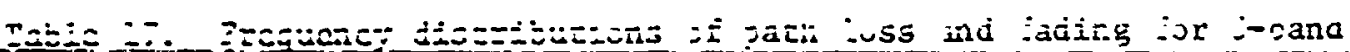


DIFFEREACE क HICH-LOW

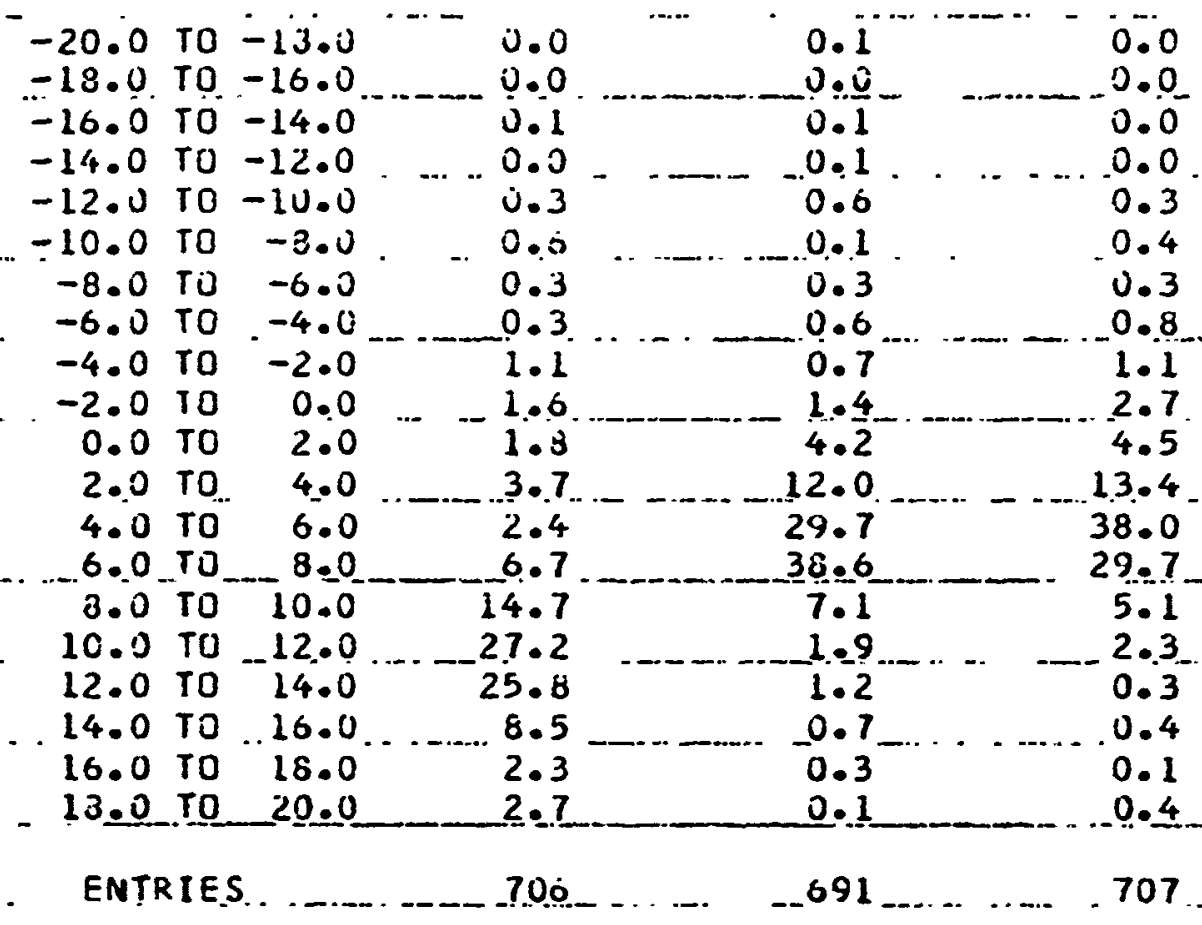

9

a..

10

Q

80

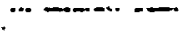

$-2.0$

0.3

1.1

1.6

0.0 TO

2.0

$1 \cdot 3$

07

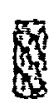

-. Täblë 18. "Frequency distributions of path loss differeñces between antennas for S-band

8

요

$\mathrm{B}$

8

$\mathrm{P}$

ing 

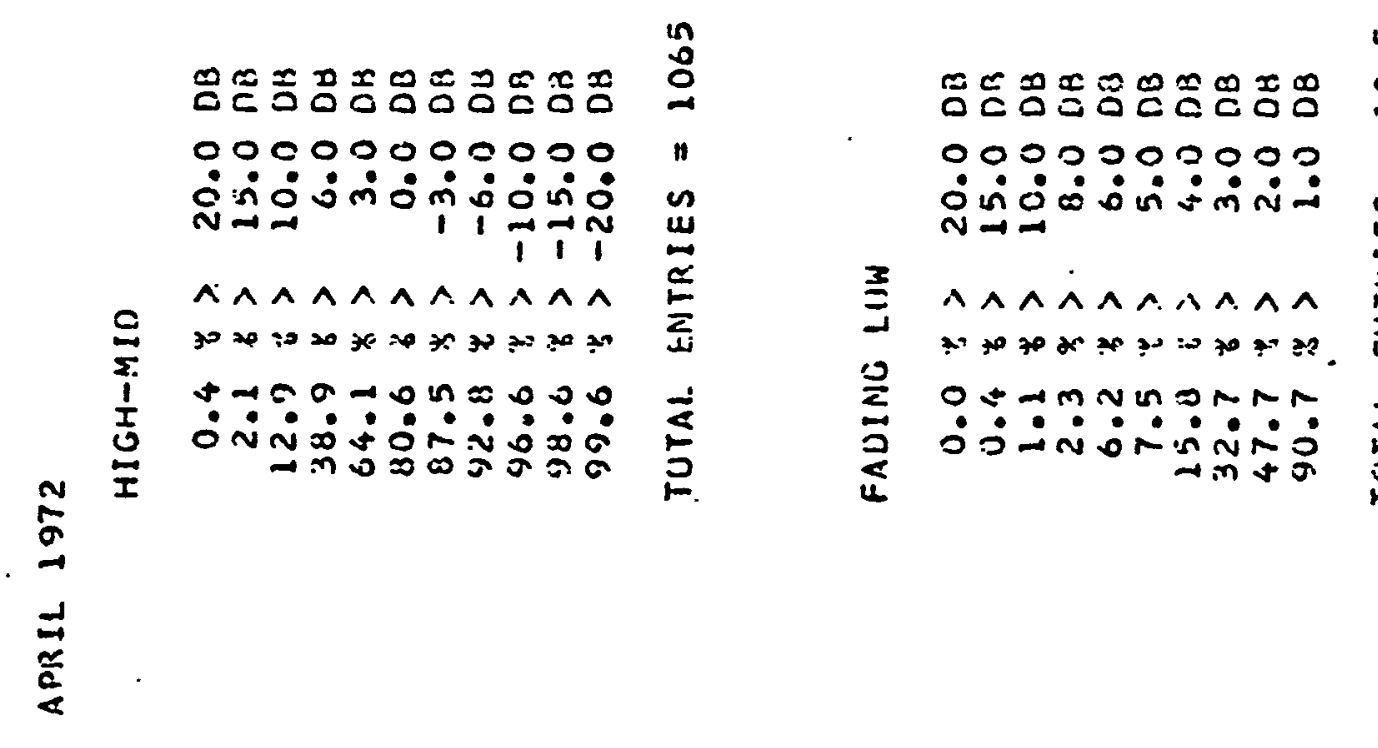

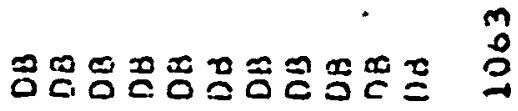

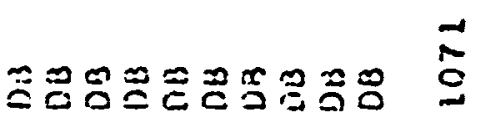

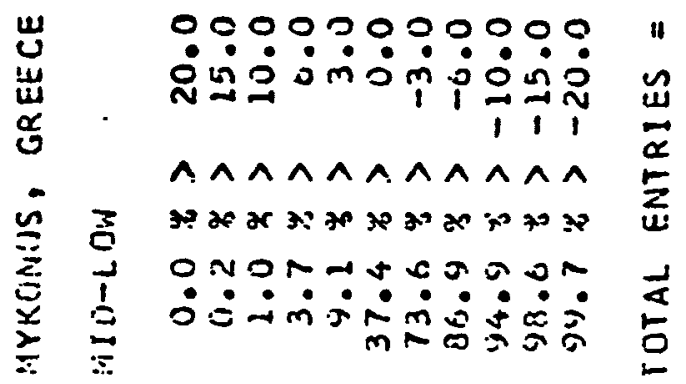
0000000000

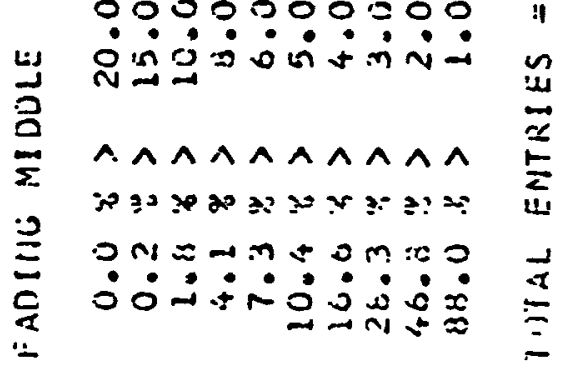




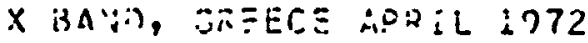

PATH LOSJ

12..1) TO $12 \% .0$

125.J T0 132.3

130.0 T.J 135.J

135.j TC 143.0

140.0 TO 145..)

$1+5.5 \div 2: 33.0$

1Ec.; ro ijs.j

155.0 T I

160.:) TC 155.3

165.j T. 17.J.J

17C.J TO 175.0

175.2 Tด 1 $1 \mathrm{~J} .1 \mathrm{~J}$

1CL. TC 135.?

105.0 TO $1+0.0$

190.0 TC 135.1

195.? T? 22\%.?

200.0 TO 20\%.0

2U5.D TO $21 \mathrm{~J} .0$

210.0 T.J 215.0

215.0 To 22.1 .13 $\because \mathrm{HIGH}$

3.9

18.7

23.7

17.1

16.5

3.9

7.5

3.2

2.1

¿. 3

3.0

ก.

נ.

0.0

0.0

9.5

0.0

0. 0

2. 0

0.0 $\div 910$

3.5

15.2

15.0

15.2

14.:

1.1

8.5

7. 3

4.4

3.6

1.0

0.2

ن. 1

0.)

1). 0

5.

0.0

0.0

0.0

0.0

1071
ᄂ?

14.4

11.5

11.7

13.1

$11 .+$

1.). 7

1.2 .0

10.4

2.3

3.1

0.6

ن. 3

.0 .5

0.0

. 0.1$]$

0.0

.00

2.0

2.0

2.0

$1=65$

ENTAIES

$1 \leq 59^{\circ-}$

$=$ MI

$\because 20$

Fãlivis

3) HIGH

0.2
10.7

0.2

0.0 TO 0.5

0.5

$1+.3$

13.6

$27 . ?$

7.3

11.7

9.5

2.1

2.7

1.1

2.7

2.9

0.7

j.?

1.1

0.0

1.5

0.0

0.5

2.1

9.1

:2.5

3J. 2

5.4

9.9

15.4

1.3

3.7

2.5

2.4

1.1

3.6

1.7

1.1

3.5

1.1

3.1

$\begin{array}{lll}8.5 & \text { TO } & 9.0 \\ 9.3 & \text { TI } & 9.5\end{array}$

9.5 TS 1.0.0

1.8

3.9

1.3

EHTRIES

1.069

1071

1365

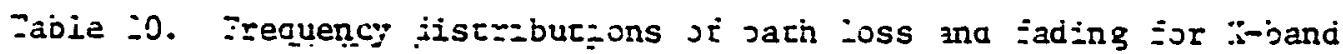




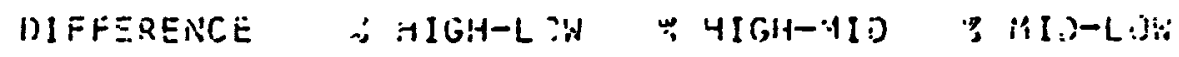

8

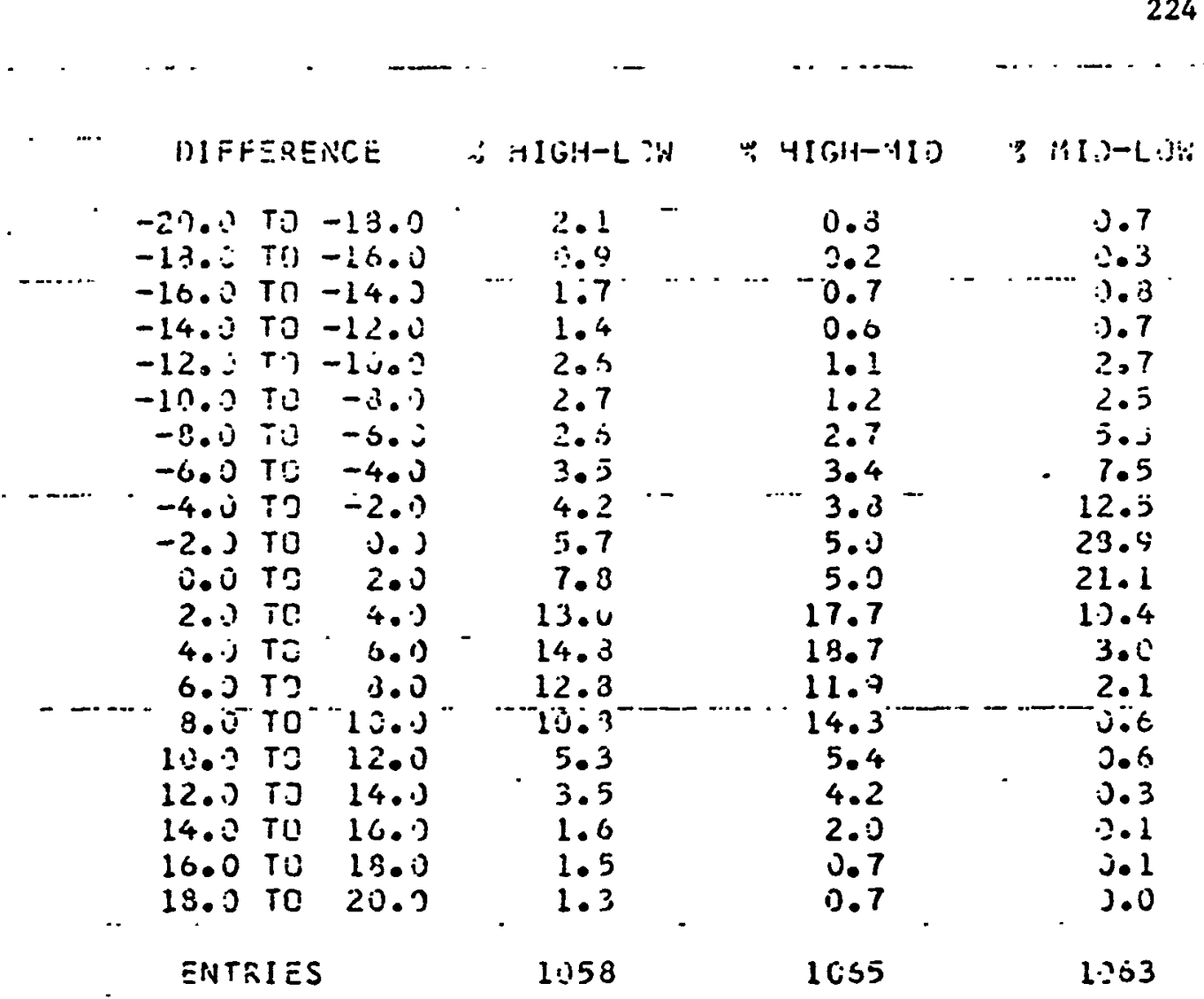

(8)

?

$\theta$

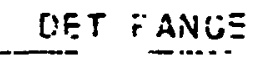

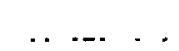

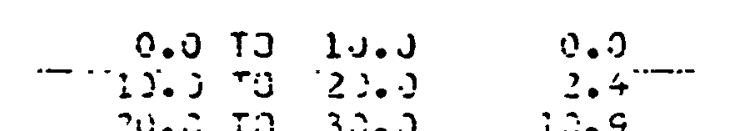

20.0 id 32,0

30.0 TC 41.0

$4: .0$ TO 5.9.:

50.0 TO 50.0

(20.0 T.

70.0 ? 39.0

30.0 TO 90.0

9C.D TO. 13J.1.

100.J TS 110.0

11..J T 12.0.J

12.3. J T? 13\%.J

iju.: ?? L+ง.J

$[\div 0.9$ io 150.J

155.0 TO 160.?

160.0 TO 17.).

17.j.) Tr 13.j.j

18.

170.0

5.0

7. $)$

2.7

4.2

4.7

2.1

2.0

$4 \cdot 3$

4.3

4.5

$+. j$

7.16

3.2

5.7

4.5

$+01$

$\therefore 6.0$

a 410

* LS:

EUTRISS

1.757

0.0
7.7
17.7
9.2
4.3

2.13

3.5

20.7

3.4

4.4

4.3

2.1

4.5

1.3

3.4

$3 . \div$

2.8

2.5

3.3

2.2

3.1

2.9

$-3.8$

2.6

4.3

3.2

2.2

4.2

3.0

3.7

4. 3

12.7

3.4

1.3

1.7

3.2

2.2

2.4

2.3

$22 \cdot 3$

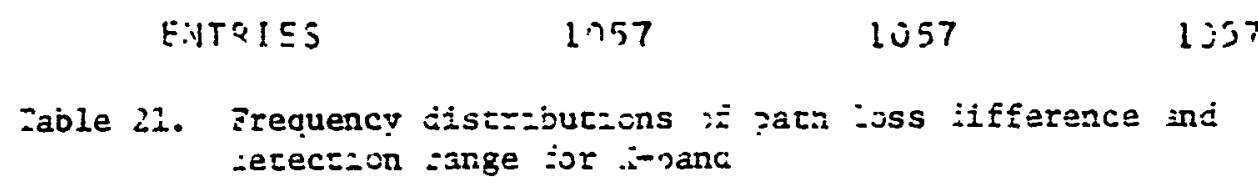

1357

粗

8

8

8

勿

9

奴

x

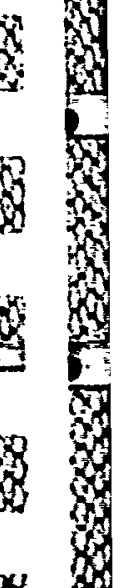




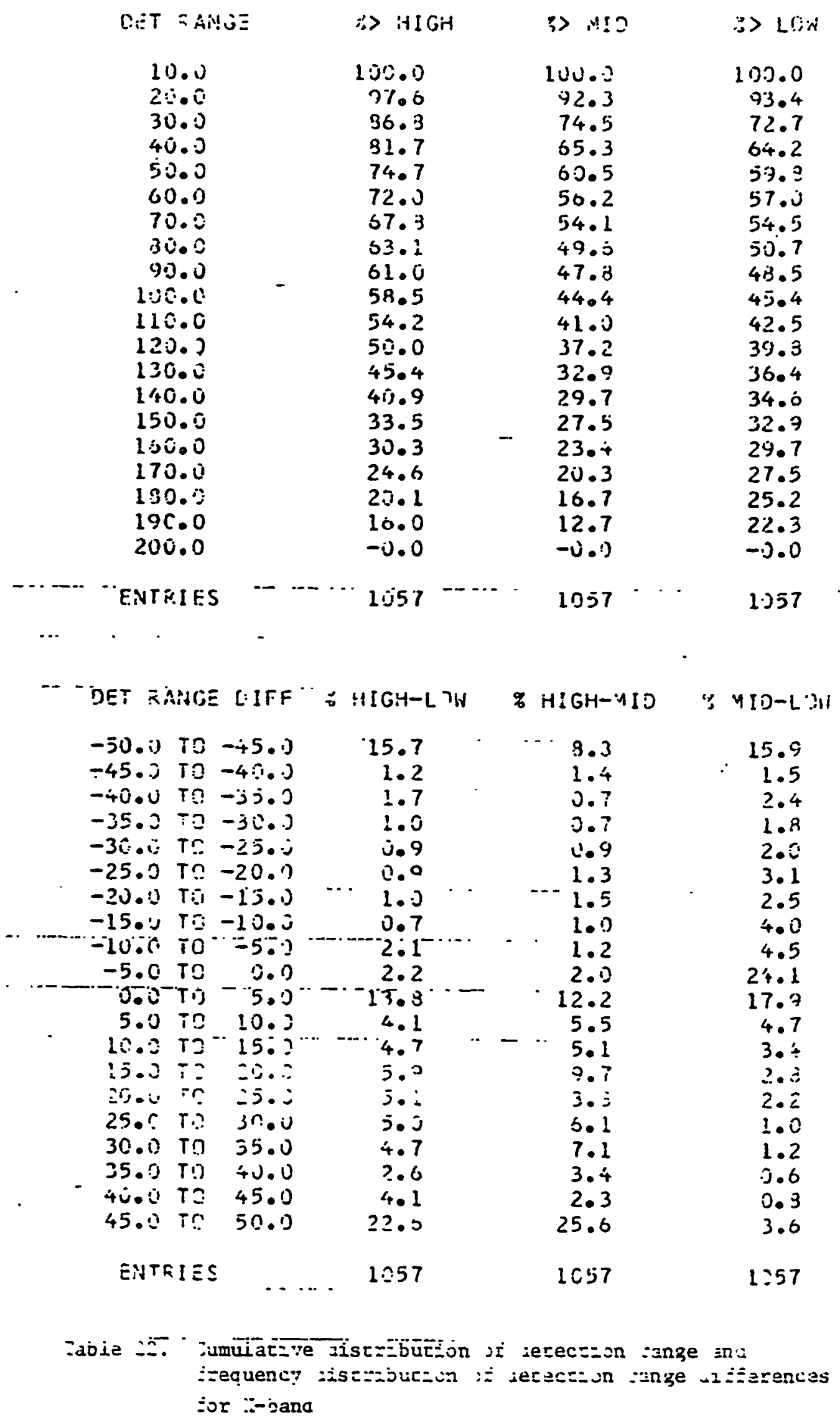


8

L BAND, GREECE AUGUST 1972

8

j

PATH LOSS

HIGH

$\therefore M 10$

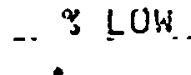

8

$120 . \therefore$ TO 125.J

125.0 TO 130.0

130.0 TO 135.0

135. 0 TO 145.0

140.0 TO 145.0

145.0 TO 150.0

150.0 TO 155.0

155.0 TO 160.0

160.0 TO 165.0

6

165.0 TO 170.0

170.0 to 175.0

173.0 TE 180.0

130.0 TO 135.0

185.0 TO 193.0

190.3 TU 195.0

195.0 TO 200.0

250.0 TO 235.0

205.0 TC 210.0

210.0 TO 215.0

215.0 TO 220.0

8

ENTRIES

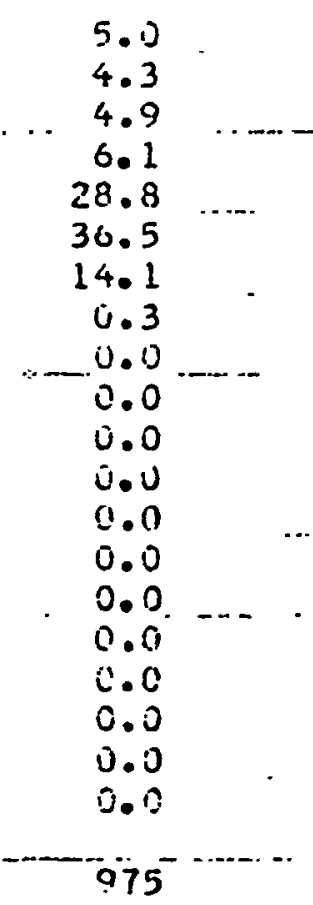

2.1

4.0

4.9

4.2

7.1
26.1

.49 .4 .

1.9

0.3

0.0

0.0

0.0

0.0

0.0

0.0

0.0

0.0

0.0

0.0

0.0

975

$2 \longdiv { 2 7 }$

$\$$

FAOING

$\because$ HIGH

6410

iojk

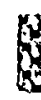

ט.j

0.5 TO 1.0

1.0 T: 1.5

1.5 ig 2.0

2.0 TO

2.5

2.5 TO

3. 0

3.0 TO

3.5

3.5 TC

4.0 TO

4.0

4.5

$4.5 \mathrm{TO}$

5.0

$5.0 \mathrm{TC}$

5.5

5.5 T.O

6.0 TO

6.3

$6.5 \mathrm{TI}$

$7 .:$ ro

7.5 TO

8.5 TO

3. 5 TS

5.5

T.

7.5

3.0

8.5

9.0 TD

9.0

9.5

\section{1}

15.0

18.2

28.6

6.1

10.1

5.5

4.9

1. 3

0.3

3.8

1.1

2.5

3.3

0.0

0.5

0.1

0.3

9.5 TC

1บ. 3

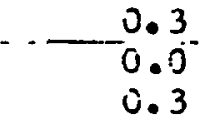

0.3

-

... $975_{-}$

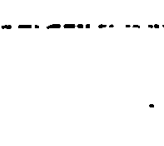

0.2

9.6

21.6

26.7

3.9

13.1

9.2

6.1

0.8

0.6

2.9

0.4

0.4

0.0

6. 3

0.8

0.8
2.3

2.3

4.4

ENTKIES

$+$

Tabie 24 .

Erequenc:

0.5

0.4

5.4

4.7

2.9

$\frac{1}{n} \cdot 0$

0.0

0.0

0.0

3.0

$0 . \mathrm{C}$

0.0

0.0

0.0

j.0

974 


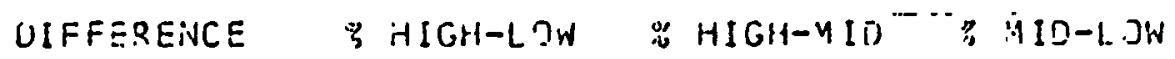

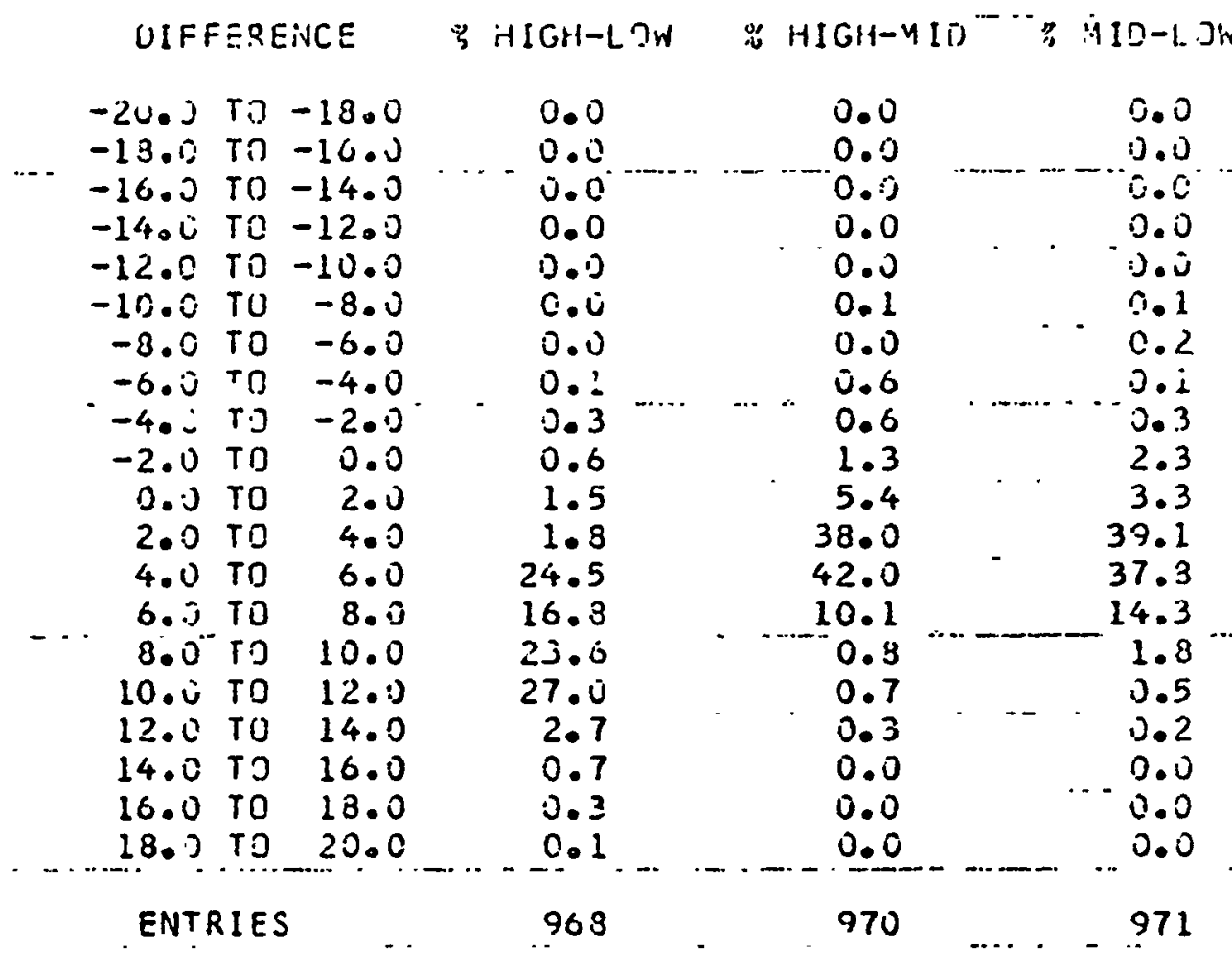

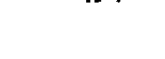

Table 25. Frequency distributions of path loss differences between antennas for L-band 
80

229

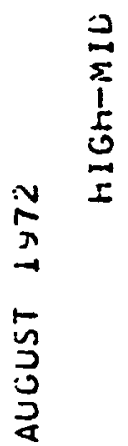

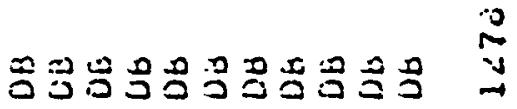

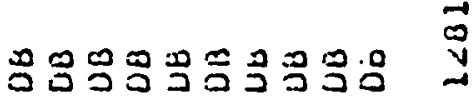
00000000000 "I ن் $\stackrel{4}{\sim}$

ヘヘヘヘヘヘヘヘヘヘヘ ż

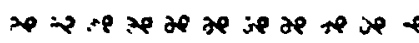
N.Jó $\dot{0} \dot{0} \dot{0} \dot{0} \dot{0} \dot{0}$

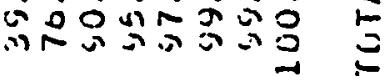
000ว 000000 $\dot{0} \dot{0} \dot{0} \dot{0} \dot{0} \dot{0} \dot{0}$

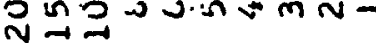

$\wedge \wedge \wedge \wedge \wedge \wedge \wedge \wedge \wedge \wedge$

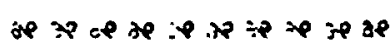
o

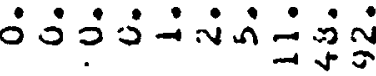
11

8 $e_{4}^{2}$
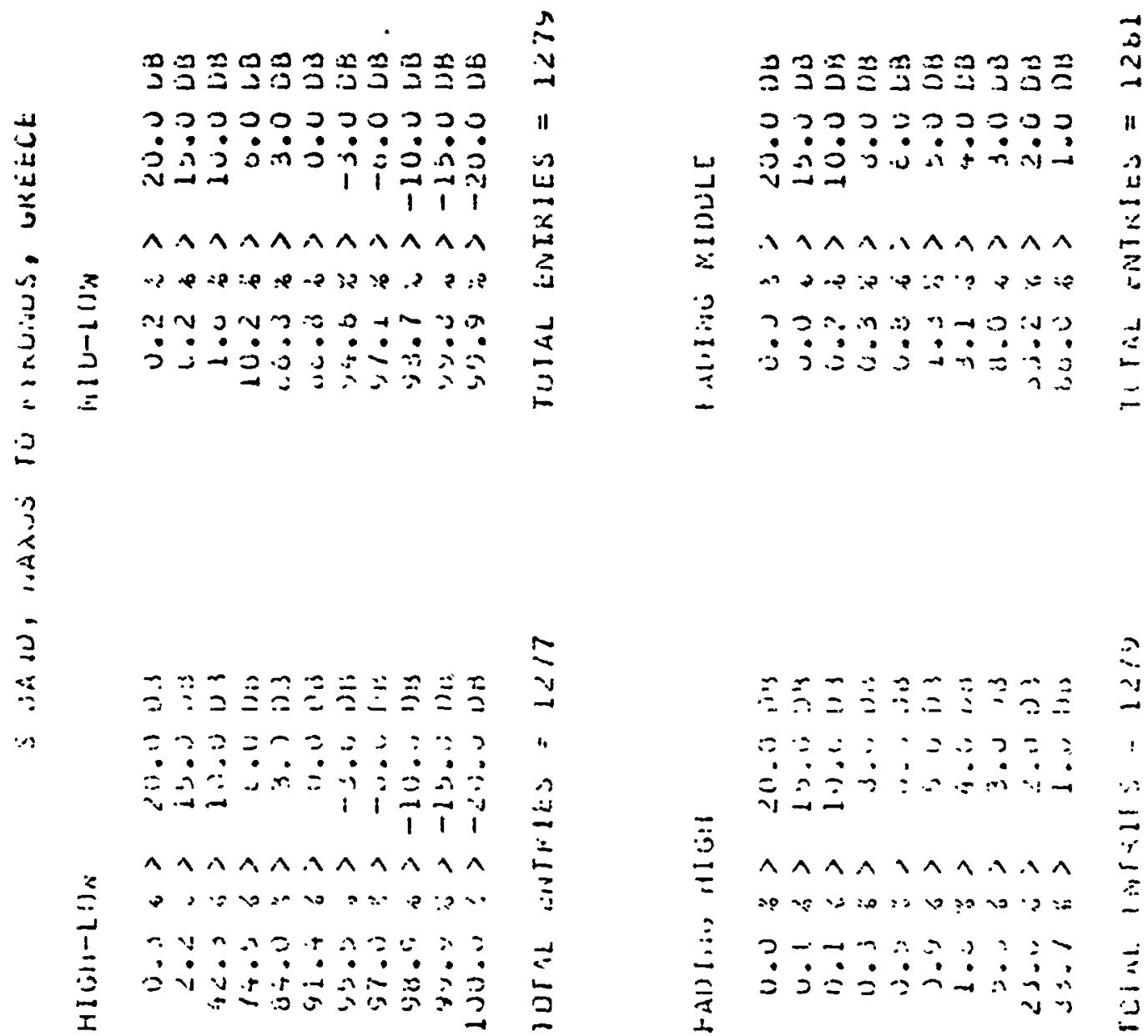

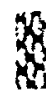

2

8

8

8

8

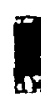

X

$\$$

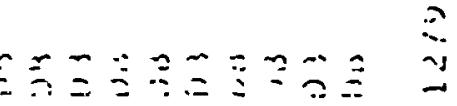

$\therefore=\div:=\therefore 2=: \quad$ : $\dot{0} \div \dot{0} \div \therefore \therefore \therefore$

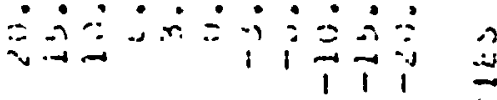

8

p

$\infty$ 


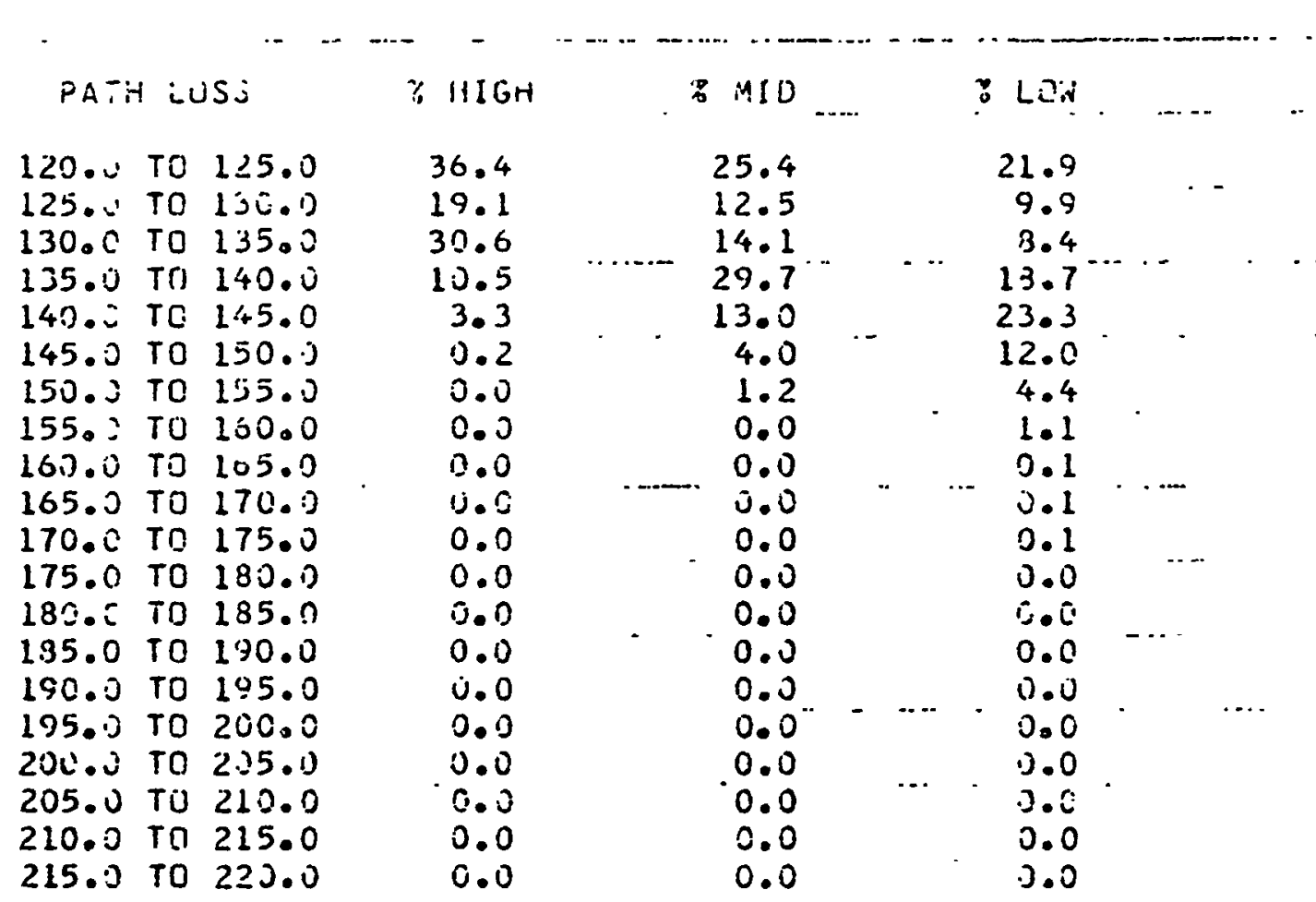


$S$ BAND, GREECE AUGUST 1972.

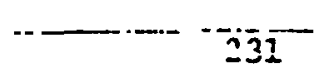

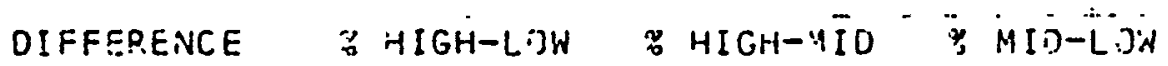
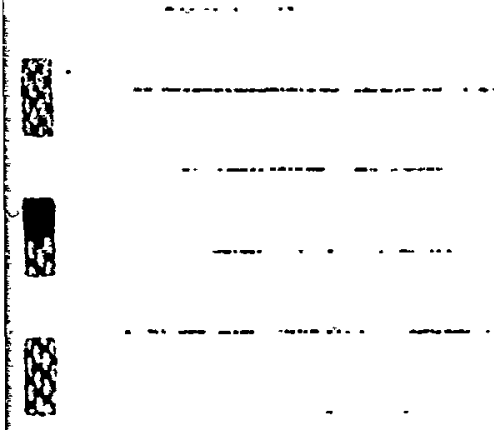

-20.0 TO -18.0

-18.3 TO -16.2

-16.0 TO -14.0

-14.0 TO -12.0

-12.0 TO -10.0

ง. 1

$-3.0$

0.13

-10.0 TO -8.0

-8.0 TO -6.5

-6.0 10 $=4.0$

-4.0 TO

$=2.0$

$-2.0 \div 0$

0.0

0.0 TO

2.0

4.0

2.0 TO

4.0 TO

6.0

6.0 .10

8.0

B. 0 TO 10.0

$-0.3$

0.5

-0.9 .

บ. 9

0.9.

1.8

2.5

4.9

4.2

7.7

11.6

10.0 TO 12.0

20.5

26.3

12.0 TO 14.0

11.7

3.6

16.0 TO

18.0

18.0 TO 20.0

0.6

0.7

0.2

3.2

1277

0.2

0.2

$0 . \mathrm{C}$

6

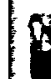

\section{ENTRIES}

19. 7

1.3

1.3

2.5

3.4

6.3

11.7

31.7

28.3

9.0

1.5

0.5

0.2

0.1

0.3

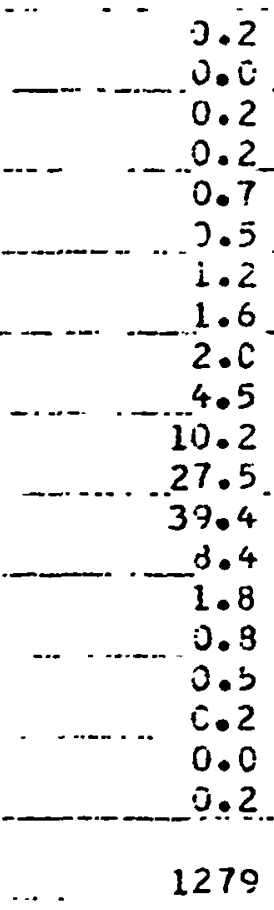

1278

1270

8

w

(5)

Table 28. "Frequency distributions of path loss differences between antennas for S-band
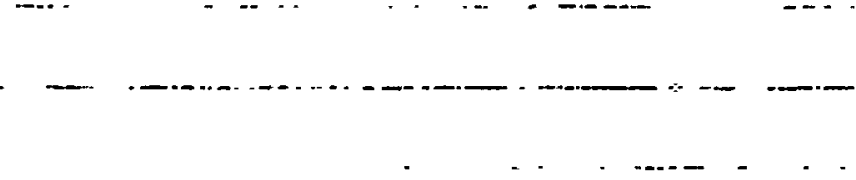


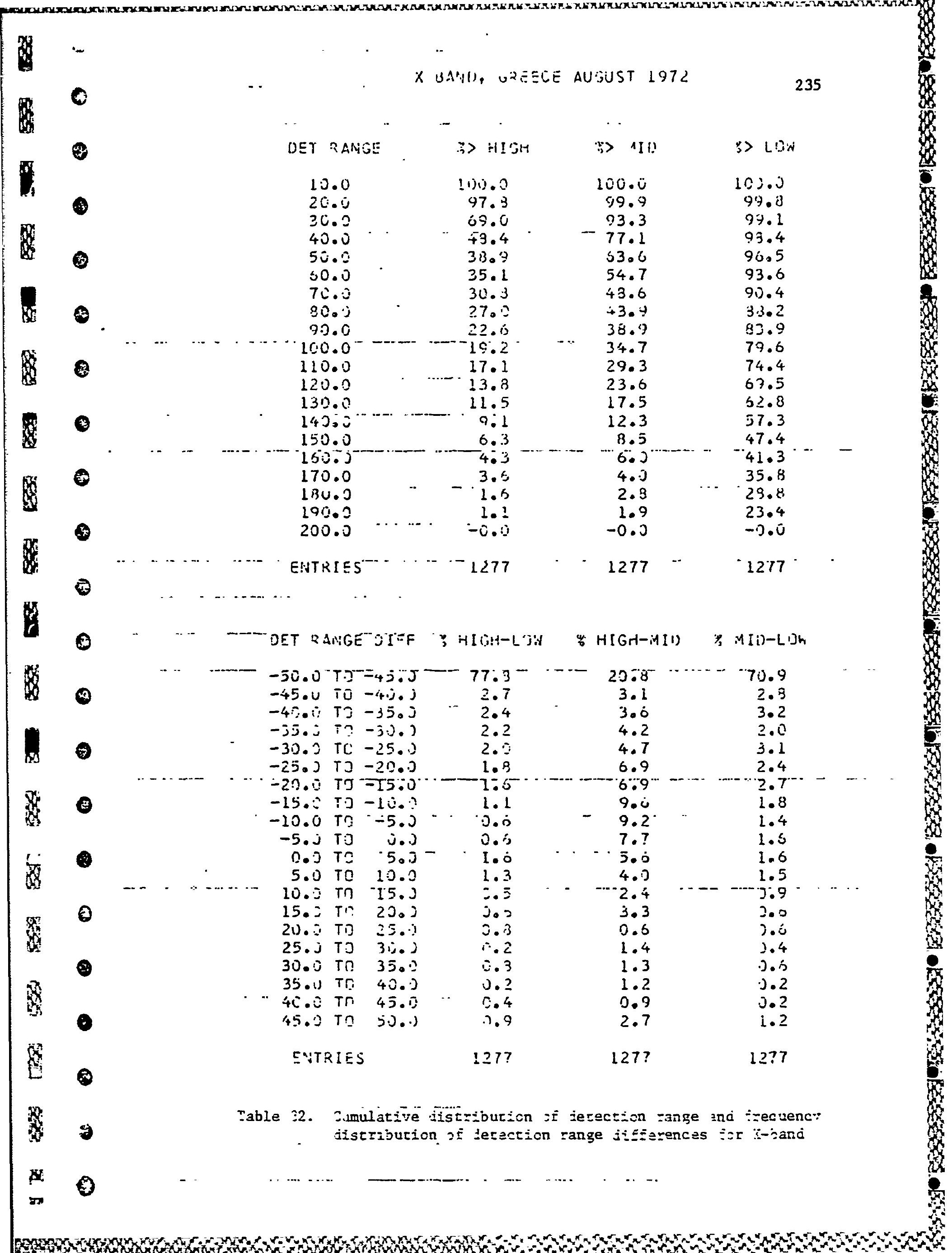



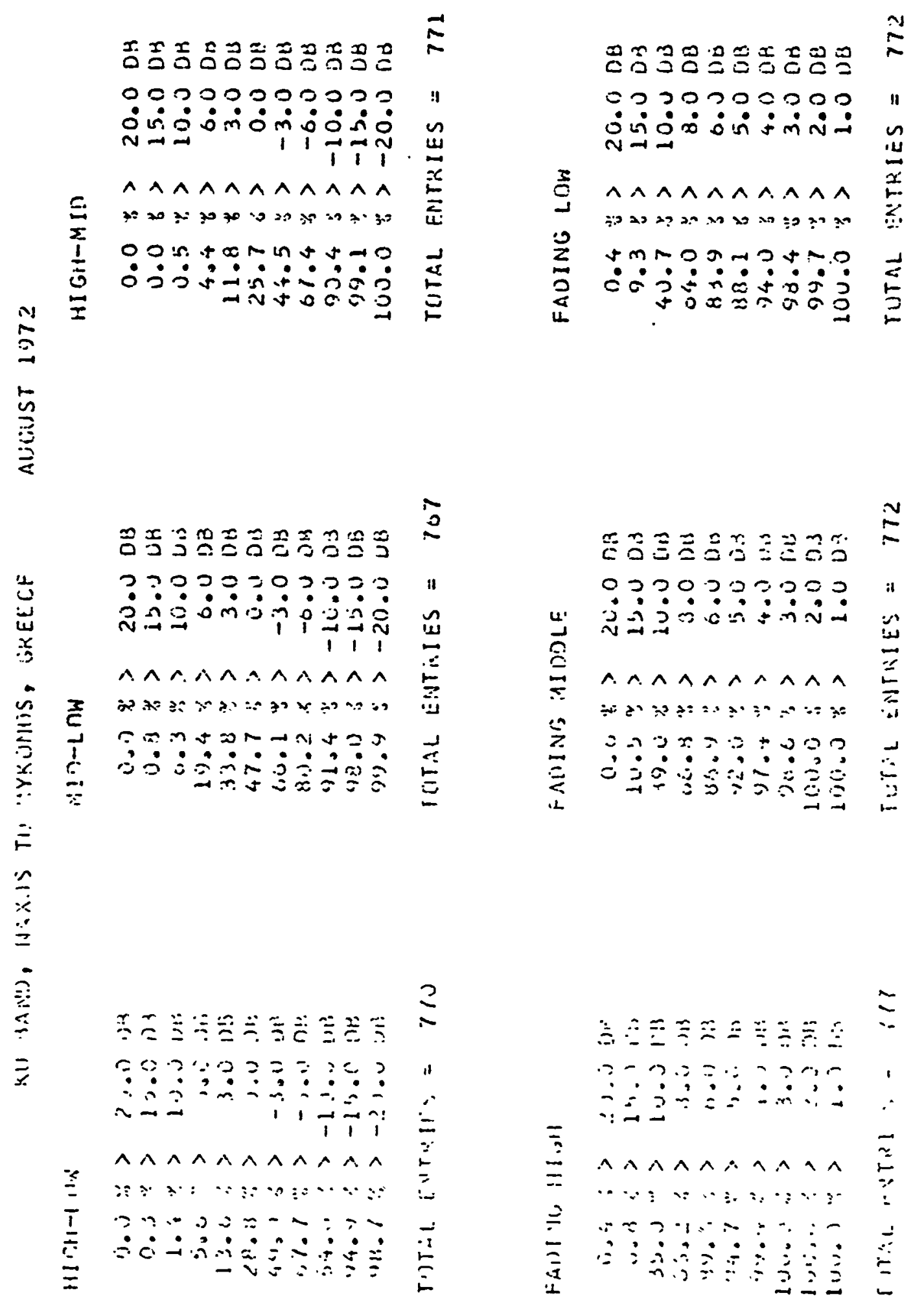
PATH LOSS

120.0 To 125.0

125.0 TO 130.0

130.0 TO 135.0

135.0 TO 140.0

140.0 TO 145.0

145.0 TO 150.0

150.0 TO 155.0

155.0 TO 100.0

160.0 TO 165.0

165.0 TO 170.0

170.0 TO 175.0

175.0 TO 180.0

180.0 TO 185.0

185.0 TO 190.0

190.0 TO 195.0

195.0 TO 200.0

200.0 TO 205.0

205.0 TO 210.0

210.0 TO 215.0

215.0 TO 220.0
$3 \mathrm{HIGH}$

0.0

0.0

0.4

1.8

9.5

19.6

15.6

12.9

14.5

12.4

9.8

2.1

1.3

0.1

0.1

0.0

0.0

0.0

0.0

0.0
* MID

0.0

0.0

1.2

10.1

22.8

13.0

7.1

10.4

11.5

14.4

7.1

2.7

0.3

0.5

0.0

0.0

0.0

0.0

0.0

0.0
: LON

0.0

0.0

0.5

5.7

16.1

15.8

14.2

13.3

17.1

9.9

2.8

0.4

0.0

0.1

0.0

0.0

0.0

0.0

0.0

0.0

ENTRIES

777

772

772

FADING

\% HIGH

$\%$ M10

* LOW

0.0 TO

0.5 TO

0.5

$1.0 \mathrm{TI}$

1.0

1.5

1. To

2.0

$2.0 \div 0$

2.5 TO

3.0 TO

3.5 TO

4.0 TO

2.5

3.0

3.5

4.0

4.5 TO

5.0 TO

5.5 TO

6.1) TO

5.5 TO

7.0 TO

7.5 TO

8.0 TO

8.5 TO

4.5

5.0

5.5

6.0

6.5

7.0

7.5

8.0

8.5

9.0

9.5

0.0

0.0

0.0

0.0

0.0

0.0

0.0

0.4

1.2

0.0

3.7

4.5

9.0 TO

10.0

$-7.7$

4.5

5.3

4.1

8.6

9.0

3.3

47.1

0.0

0.0

0.0

0.0

0.9

0.5

0.1

1.0

2.2

0.3

3.1

4.4

6.6

3.6

4.0

5.6

4.7

8.4

2.7

51.8

777

772

0.0

0.0

0.3

0.0

1.2

1.3

3.0

2.2

0.0

4.0

4.0

5.2

3.2

3.5

8.0

2.8

6.1

3.0

52.2

ENTRIES

772

Table 34.

Frequency listributions lf sath loss int EAdino cor ku-jand -. 


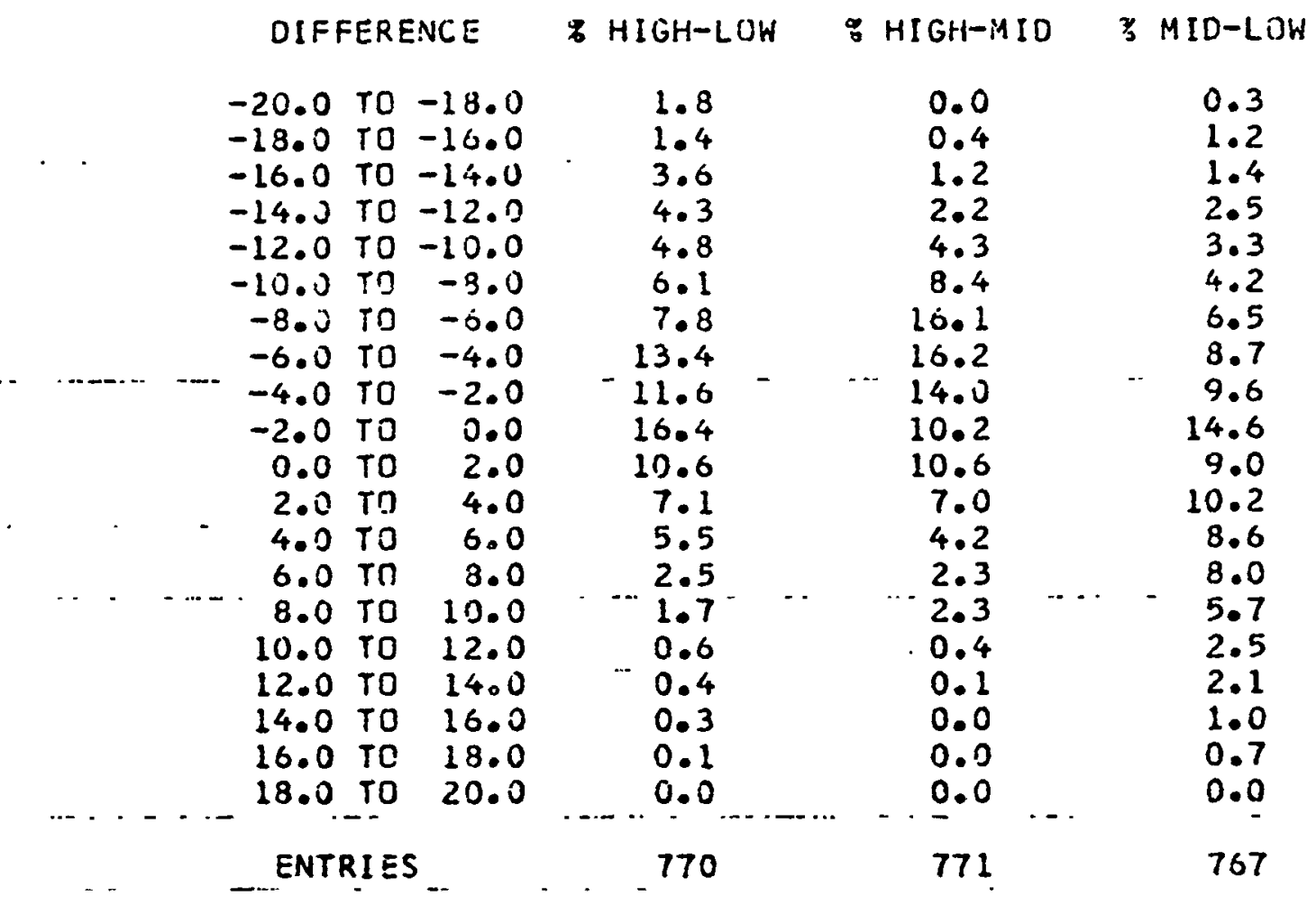

Table 35. Frequency distributions of path loss differences between antennas for Ku-band 


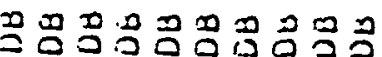

$\stackrel{2}{5}$ 00000000000 "I

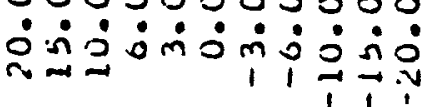
$\tilde{u}$ ヘヘヘヘヘヘヘヘヘヘヘ

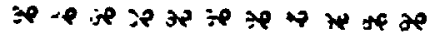
o.s.

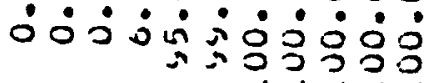
곡ำ

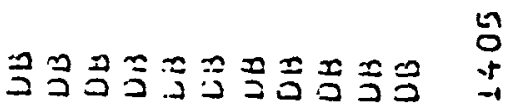
$000.000: 000$ $\dot{\vec{i}} \dot{\mathrm{i}} \dot{\mathbf{j}} \dot{\mathrm{i}} \dot{\mathbf{r}} \dot{\mathrm{j}} \dot{\mathrm{j}}$ 3
0
3
3
4
4 $\therefore \rightarrow=$

B

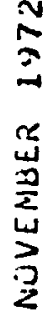

8

ヘヘヘヘヘヘヘヘヘヘ ק.

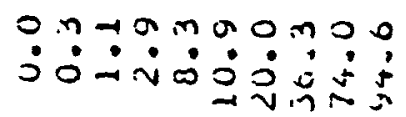
"

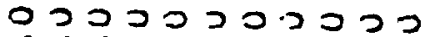

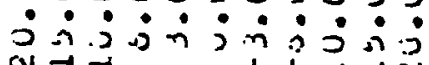
un $\rightarrow 10 \frac{1}{1}$ $\wedge \wedge \wedge \wedge \wedge \wedge \wedge \wedge \wedge \wedge \wedge$

出

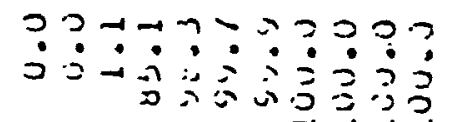

$\underset{5}{2}$

3

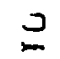

ב⿱⺈

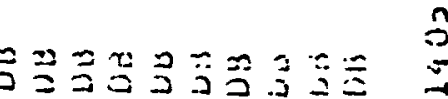

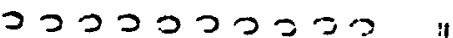
$\therefore i j i j i: \dot{0}:$

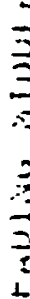

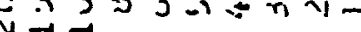

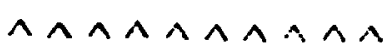
$\therefore ?$ ?

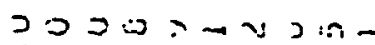

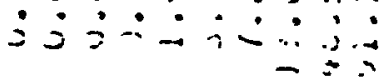
ji $: i a n=0$

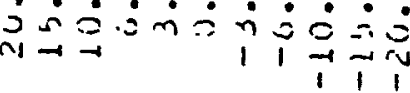

$\because$
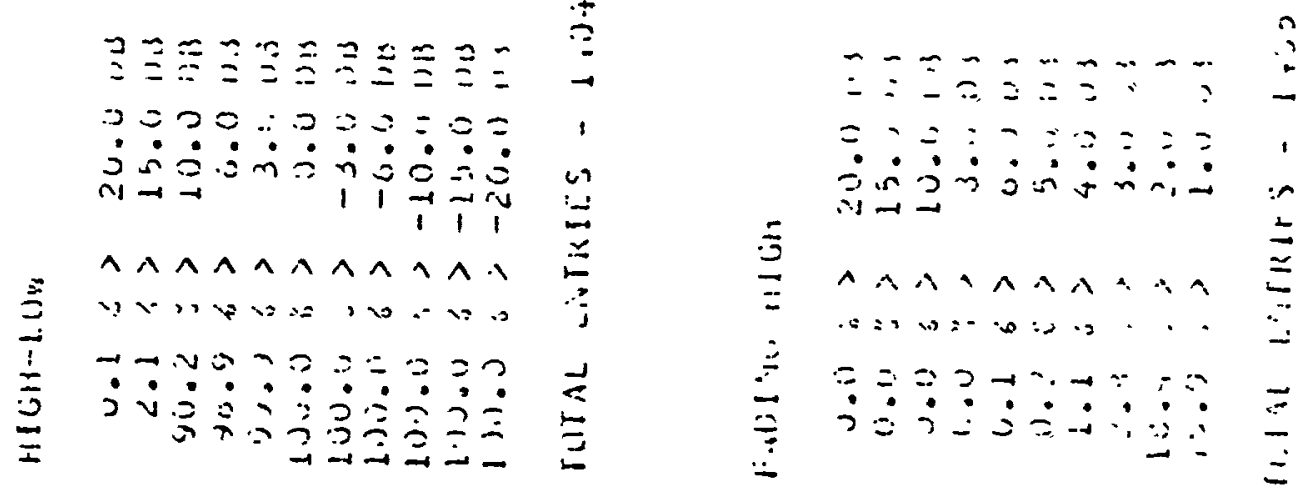


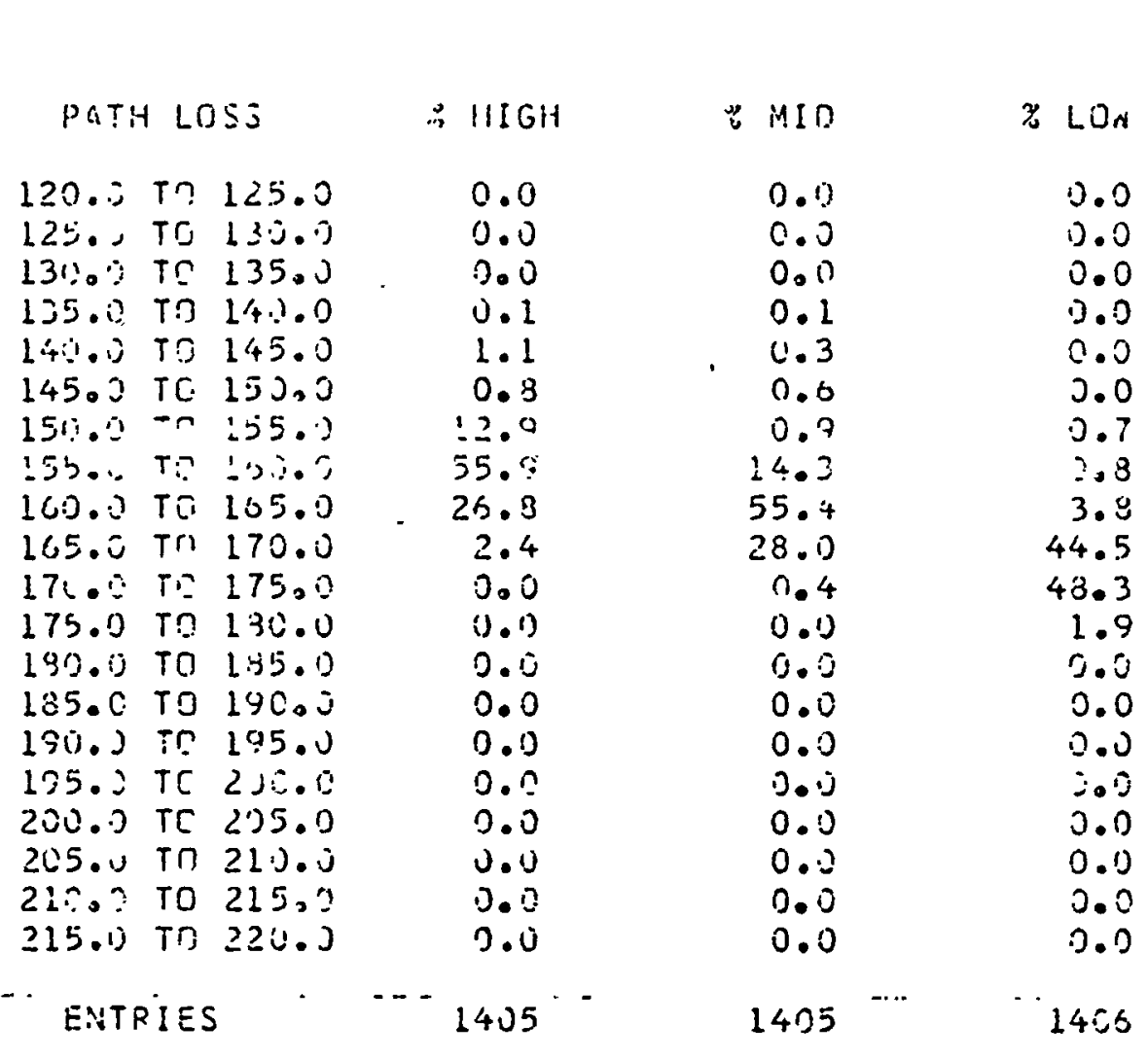

\begin{tabular}{|c|c|c|c|c|c|c|c|c|c|c|}
\hline$=A E$ & ט & & 6 & $\mathrm{HISH}$ & & : & MID & & & $\%$ LUin \\
\hline 0.0 & חד & 0.5 & & $2.2^{\circ}$ & & & 2.2 & & & i. 9 \\
\hline 0.5 & TS & (1.1) & & $1 ?$ & & & 5.7 & & & $\because$ \\
\hline 1. 3 & $T \Gamma$ & 1.5 & & 24 & & & 10.2 & & & \\
\hline $1 . \equiv$ & is & 2.1 & & 35. & & & 34. & & & 17 \\
\hline 2.3 & TO & 2.5 & & 7.1 & & & 13 & & & \\
\hline 2.5 & $T S$ & 3.0 & $\cdots$ & 9.5 & $\ldots-$ & & 19 & & & \\
\hline $3 . ?$ & TO & 3.5 & & 1. & & & 3.6 & & & \\
\hline .5 & TO & 4.5 & & 0.9 & & & 3. & & & 11 \\
\hline .3 & TC & 4. & & 0.5 & & & 2. & & & \\
\hline 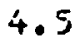 & TO & 5. & & $0 . \dot{0}$ & - & & & & & \\
\hline .0 & $T, 7$ & 5. & & S. 2 & & & 0 & & & \\
\hline 5.5 & Tว. & 6.0 &.. & $0 . !$ & $\cdots$ & .. & 1 & & & 2 \\
\hline 0.0 & TO & 6. & & 0.0 & & & & & & 1. \\
\hline 5.5 & $\because$ & 7. & & ;. ; & & & 3. & & & 8 \\
\hline .6 & $T r$ & 7. & & 3. & & & 3 & & & \\
\hline 7.5 & $T r$ & 8. & & 0. & & & 0. & & & \\
\hline$B .5$ & $T C$ & 3. & & 0.1 & & & 0.0 & & & 0 \\
\hline 8.5 & TO & 3.0 & & 0.0 & & & 0.4 & & & 0.4 \\
\hline o. & $T r_{-}$ & 9. & $\cdots$ & 2. & & & 0.1 & - & - & 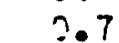 \\
\hline 9.5 & Tq & 10.0 & & 0.0 & & & 0.3 & & & \\
\hline FNT: & RIES & & & 14135 & & & 14.25 & & & $1+06$ \\
\hline
\end{tabular}

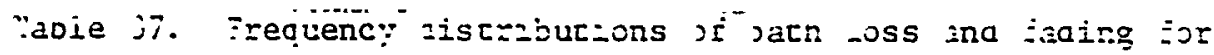
isiand 


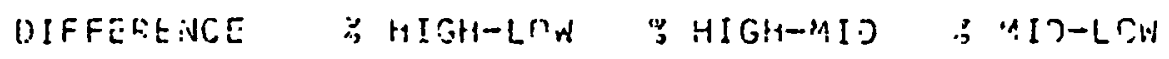

8.

$-26 . . T C-13.3$

$-10 . \therefore$ TS $-16 .: 5$

-16.5 TC -14.0

$-14 .$.$) TO -12 . \mathrm{J}$

$-12 . \therefore$ tr $-1.20:$

-10.0 TR -3.?

$-3.6 T L-0.0$

$-5.0 \mathrm{Tr}-4.0$

-4.2 TC -2.3

-2.0 TI 0.0

$0.0 \mathrm{TS}$

2.) TO

2.0

4.0

0.0

3.0

2. 0

0.0

2.3

3.2

3.

0.0

0.0

2.0

0.0

4. T TO

o.. J

0.3

i). 5

S.

8.u $\overline{10}$

10.0

4.3

10.3 T. 12.0

12.0 TR

14.0

4.5

44.9

40.7

3.0

14.0 TO 16.0

16.? Tr 13.0

1.0

13.6 TO 20.0

0.1

ENTFIES

1404

0.0

0.0

0.0

0.0

0.3

0.0

0.3

0.0

0.1

0.3

0.6

12.2

30.1

$5 . ?$

0.6

0.1

0.1

0.0

0.0

0.0

.0 .0

2.3

ก.

2.0

0.0

3.0

3.5

0.0

0.1

0.1

0.6

2.4

3.5

74.9

11.7

1.4

0.1

0.1

0.0

$14 \mathrm{ij}$

1405

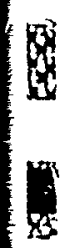

Table 38. Frequency distributions of path loss differences between antennas for L-band 

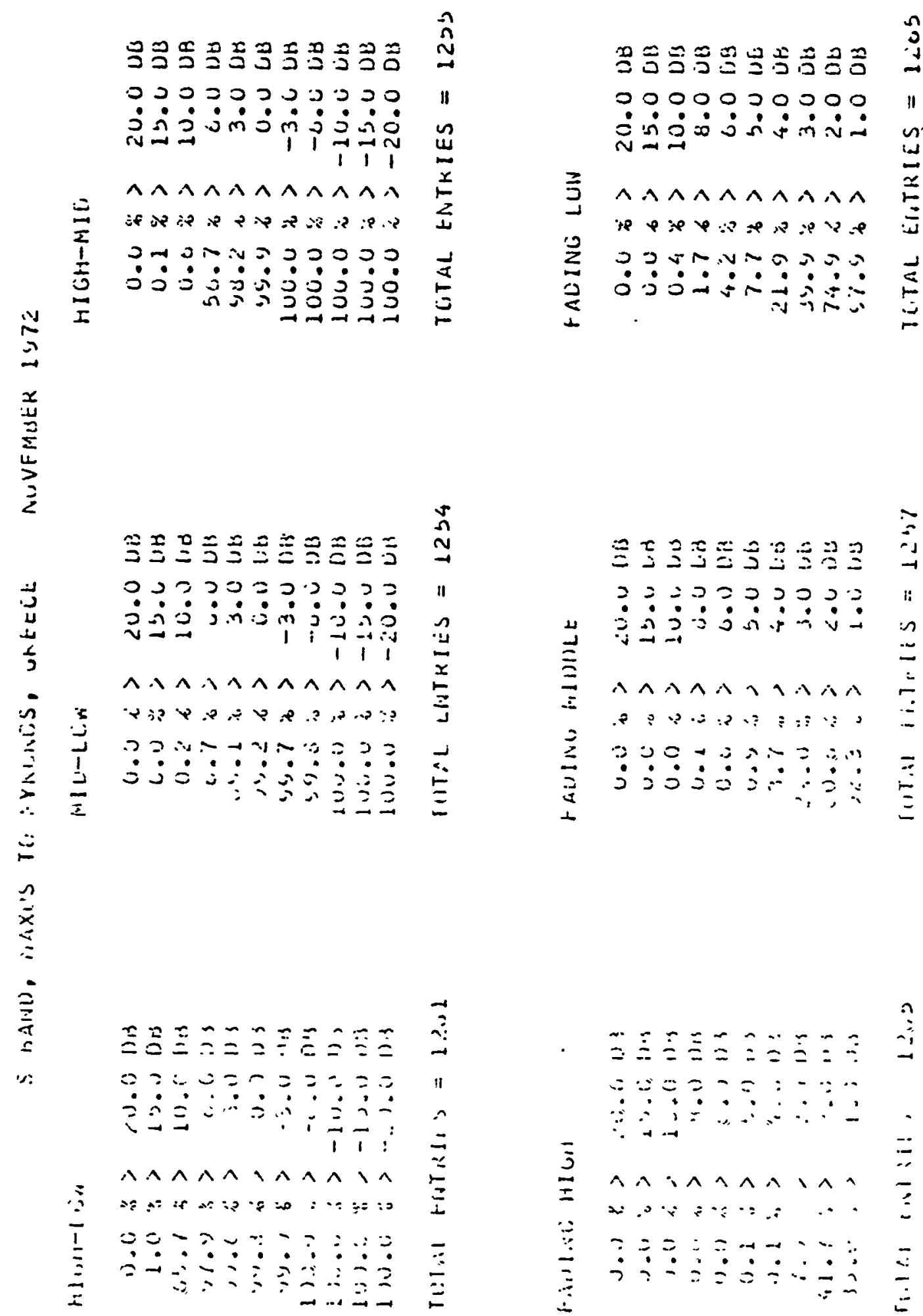

xix

$-$

i

A

8

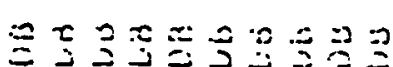

$\because$
$\because$
$=1$
$\because$
$\because$
$\therefore$
$\because$
$\because$
$\Xi$

in

8

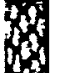

\begin{tabular}{l}
2 \\
$\Xi$ \\
$\Xi$ \\
$\vdots$ \\
$\vdots$ \\
$\vdots$ \\
$\vdots$ \\
\hline
\end{tabular}

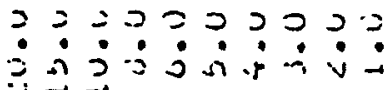

$x$

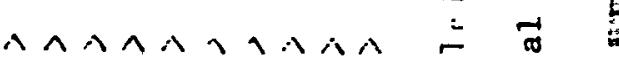

$\because \div 0 \div \because \div=\because \cdots$

2ं $\dot{2} \dot{2} \dot{2} \therefore \dot{2}$

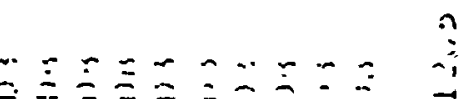

采

$\because \because \because \approx-\div:-\cdot \cdot$

$\because$

$\vdots$
$\vdots$
$\vdots$
$\vdots$

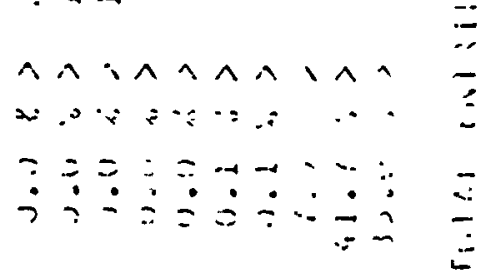

8

3 


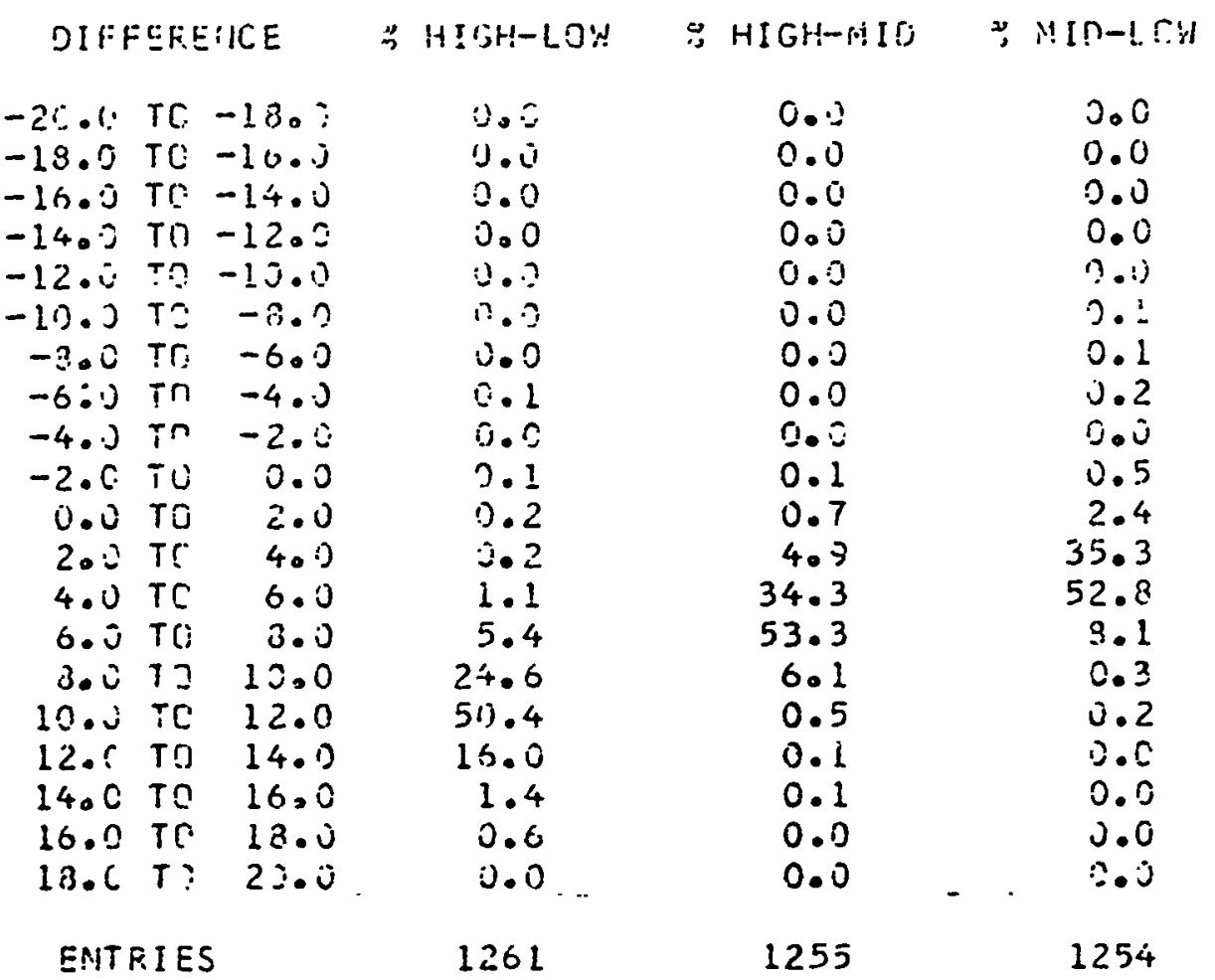

Table 41. Frequency distributions of path loss differences between antennas for S-band 


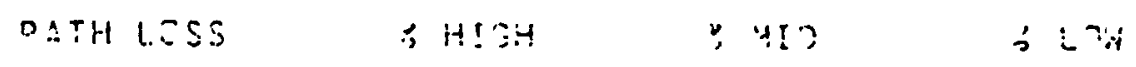

0.9

$125.5 \div C$ 13jo.

130.0 TO 133.J

135.J Tก L4J.J

140.0 TO 14500

145.0 TO 15.3 .0

15:.0 TI 155.

155.3 T 100.3

160.1 io 105.)

165.2 TO 17:. J

170.0 TO 175.0

175.0 TS 10.5 .0

$180 . \cong$ is 135.2

18.5 .0 TC 19.J.1)

19.0.: TO 195.:

195.0 TO $200 . \Omega$

200.? TC 205.0

205.3 TO 21.0 .1

210.0 TO 215.S

225.1 TO 220.0

\section{ENTRIES}

- . .

i

FADING

$\because \mathrm{H} \square \mathrm{GH}$

अ MID

บ. ว

1). 6

17.6

10. 3

25.2

10.7

12.5

3.4

1. 5

2.5

1. 0

ग. $C$

$0 . ?$

0.0

0.0

0.0

2.0

0.0

3.0

1251

0.5

5.5

11.1

14.0

15.7

16.2

13.2

11.2

7,2

1.9

1.4

1.4

0.6

0.1

0.3

0.0

0.0

0.0

1]. 0

1248

1250

0.0

1. 0

5.4

10.8

3.2

10.2

12.6

13.3

11.9

9.7

3.0

1.4

1.0

0.5

0.9

0.7

10.0

1). 0

3.0

3 LOW

0.0 TC

$$
0.5^{-} 37.3^{\cdots}
$$

0.5 TO

1.0 T?

1.)

22.6

$1.5 \mathrm{TO}$

1.5

13.3

2.3

2.3

2. 5

11.2
$-\quad 2.2$

$2.5 \mathrm{~T}$

$$
\text { .. } 3.0
$$

ล. 2

3.i) TO

3.5

3. $5 \mathrm{~T}$

4.2

1.0

0.2

4.5 T

4.5

2.3

0.2

5.0 TO

$5 . ?$

0.2

$5.5 \mathrm{TO}$

6.1

6.J TC

5.5

0.1

6.5 T.?

-.

7.5

‥?

2.1

(i.)

7.5 TC

3. 2

$\because .2$

8.0 T?

2. 5

0.1

0.0

0.1

.30

10.19

1251

ENTRIFS

1251

-

Tadie $4:$. Fecuency tistributions $2 f$ path loss and fading for X-hand
1243

\section{6}

$23.3^{\circ}$

14.7

13.4

$4 . .2$

6.2

5.0

0.2

0.6

0.3

0.1

0.0

0.2

0.0

0.1

0.3

0.0

0.0

1.0

0.0

1250

24.3

21.9

16.5

12.4

5.3

10.3

6.0

$0 . c$

1.2

0.2

0.1

0.2

2.2

3.2

0.1

2.2

3.2

10.0

3.0
(3)

W

12.

5

8

0

\%

d

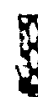

㽞

3

형

果

X

8

is

$B$

3

D

is

$\square$ 
$\Leftrightarrow$

우

DIFFERENCE" : HIGH-1.?:I \% HIGH-UID ? :ID-L:IH:

8

C.

(ิ)

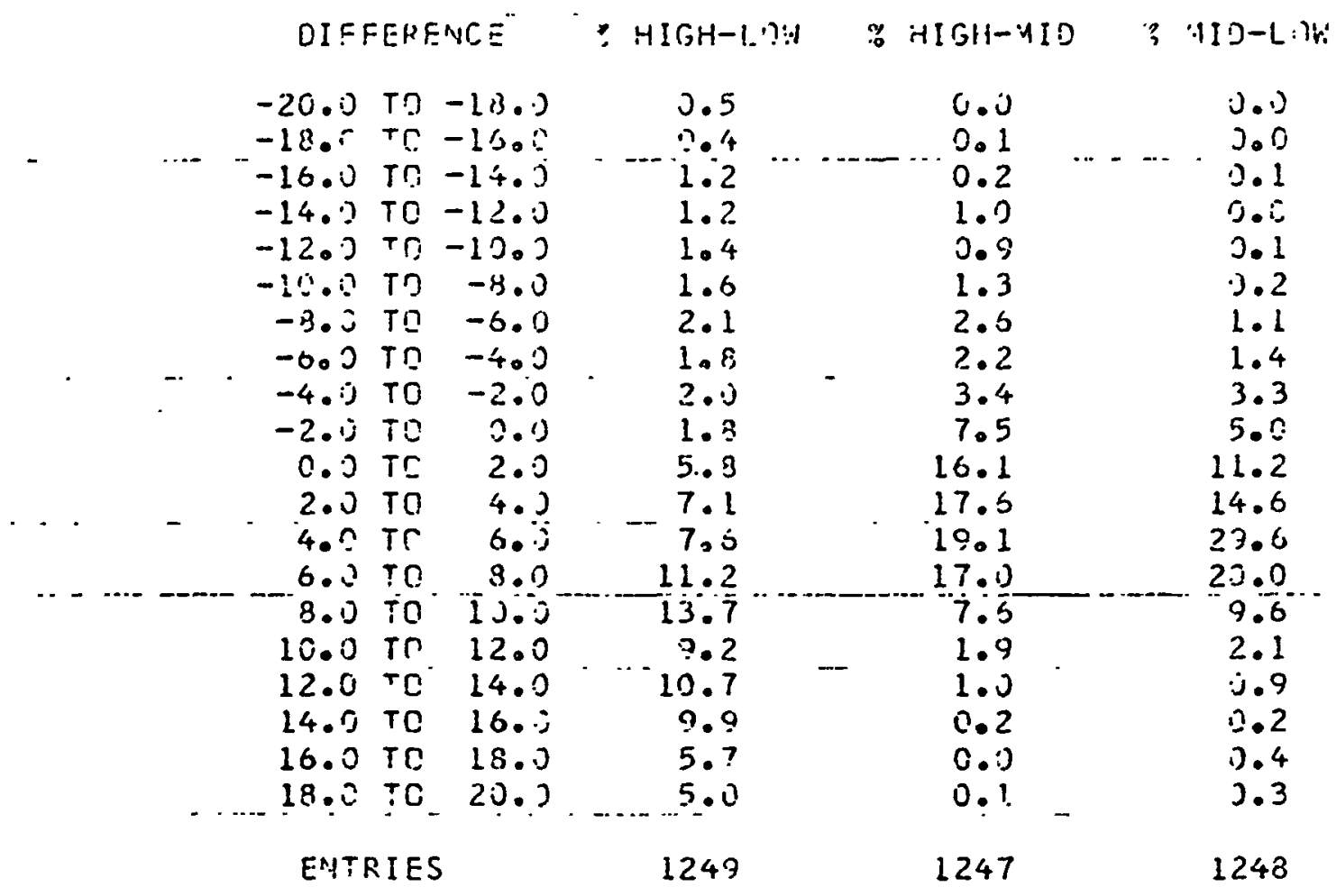

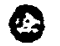

.

3

3

?

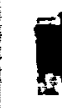

$B$

8

8

9

5

3 UET RANGE $+1 ! 9: 1$ 2. $4 ! D$

$$
\begin{aligned}
& \text {... } 0.0 \text { TC } 10.0 \quad \ldots .09 \\
& 10.3 \text { TC 20.? 7\%1 } \\
& 20.3 \text { in 30.) } \quad 32.5 \\
& 30.0 \text { Tत } 40.0 \quad-21.6 \\
& \text { 40.? Tก 50.0 9.? } \\
& 50.0 \text { in } 60.0)^{-1.5} \\
& 60.0 \text { TO } 73.0 \quad 2.2 \\
& 70.0 \text { TO } 30.0 \quad 3.0 \\
& 90.0 \text { T. } 90.0 \quad 3.3 \\
& 9 r_{0.3} \text { TS } 1.2 .0 .3 \quad \cdots \quad 7.1 \\
& 100.0 \text { r } 110.0 \\
& 110.2 \text { Tก 12J.J } \\
& \text { 12:. j it :3:30.j }
\end{aligned}
$$

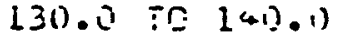

$$
\begin{aligned}
& 140.0 \text { Tr } 15 \mathrm{~J} .9 \\
& 150.0 \text { TO 160.0 } \\
& 160.9 \text { TR 172.? } \\
& -\ldots-3.4 \\
& \begin{array}{r}
3.4 \\
.07
\end{array} \\
& 0 . ? \\
& 3.4 \\
& 0.1 \\
& \text { ก. : } \\
& 170.0 \text { T0. 13.). } 0^{-\cdots} \text { 2.? } \\
& 130.0 \text { t? } 172.3 \\
& \therefore \text { : } \\
& \text { [3(.) T? EuJ.) } \\
& \text { j.: }
\end{aligned}
$$

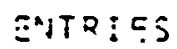

$$
\begin{aligned}
& : 247
\end{aligned}
$$

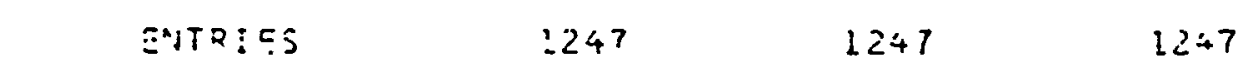

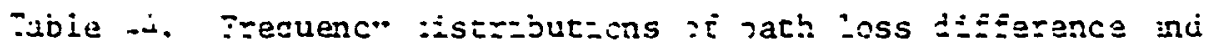
detection :ange Sor :"-band

p

is

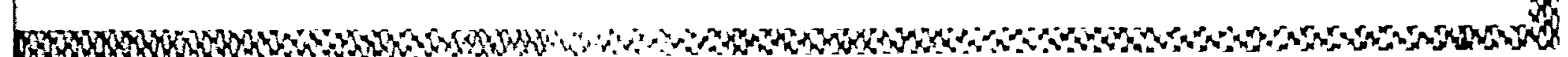




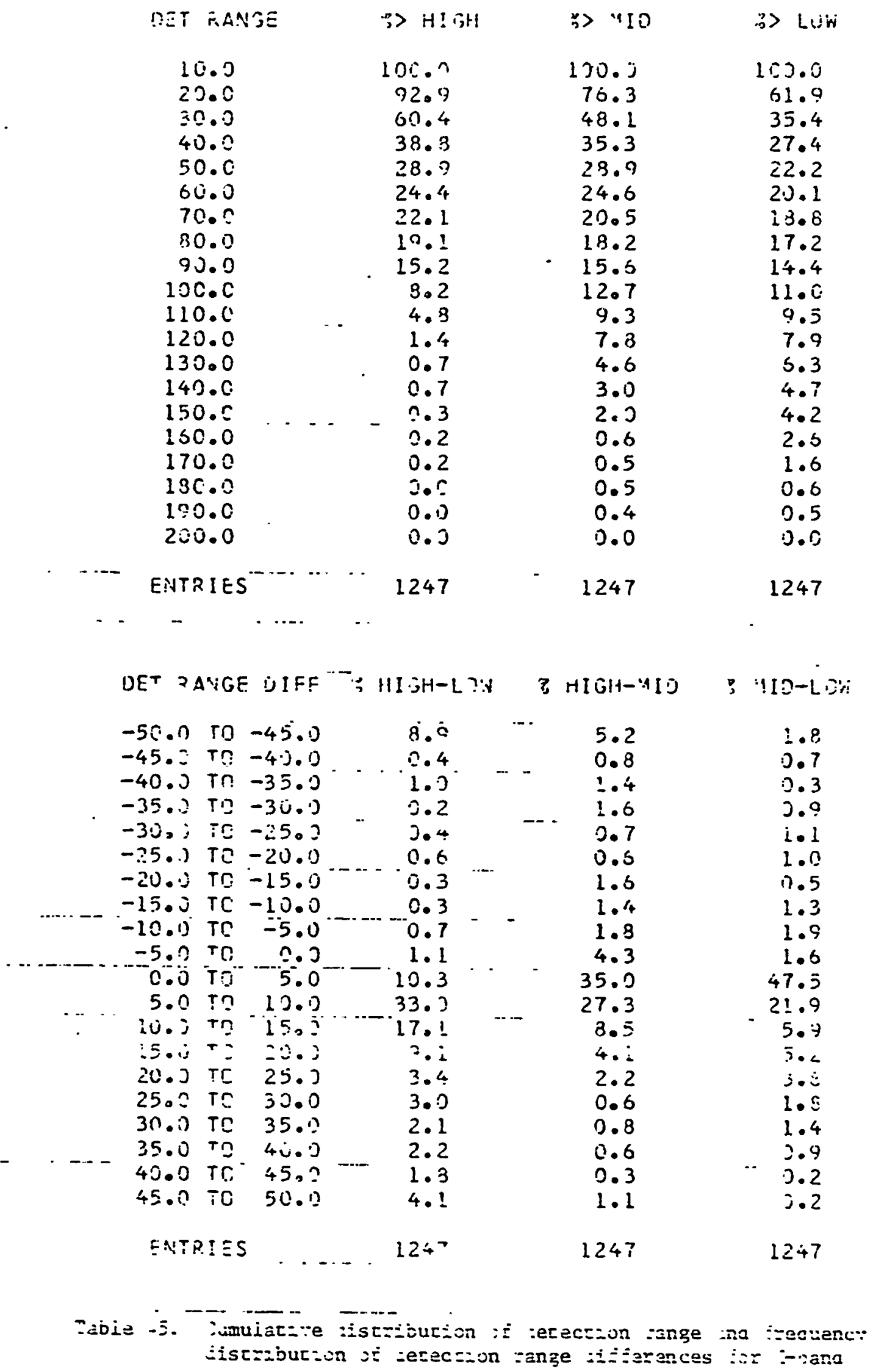


(x)

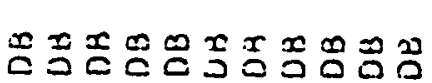

$\stackrel{2}{\circ}$ 03000003000 " பெ $\dot{0} \dot{0} \dot{0} \dot{0} \dot{0} \dot{0}$

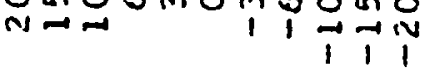

$\tilde{w}$

$\wedge \wedge \wedge \wedge \wedge \wedge \wedge \wedge \wedge \wedge \wedge$

密

m

I

$00 \pi 00 \rightarrow n \rightarrow-00$

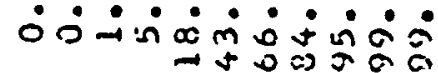

$\sqrt{I}$

is

E

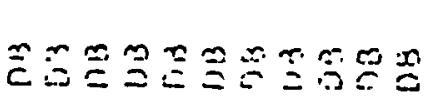

$\stackrel{\infty}{\sigma}$

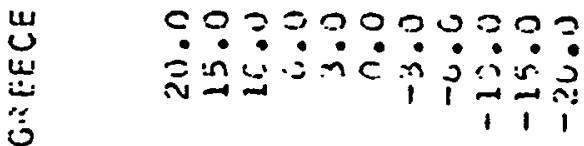

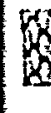
$\wedge \wedge \wedge \wedge \wedge \wedge \wedge \wedge \wedge \wedge \wedge$

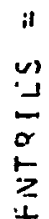

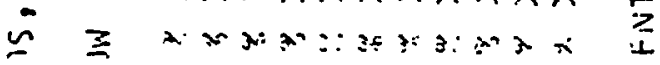

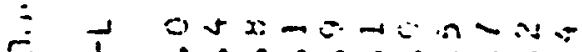

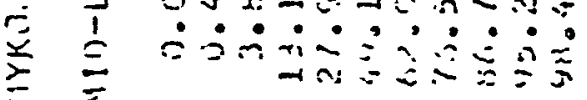

$\stackrel{\vec{E}}{E}$

$\leftleftarrows$

8

$\stackrel{n}{\ddot{x}}$

$\stackrel{2}{2}$

ํㅡㄴ

$\stackrel{5}{\sigma}$ $c=000000=011$

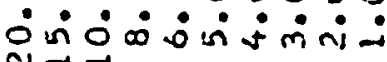

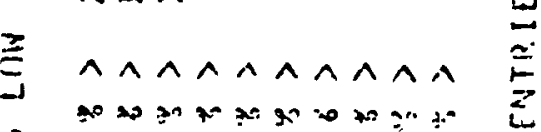

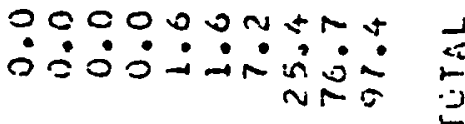

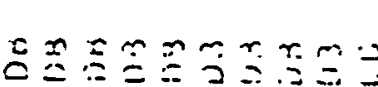
2\%00.00ว= $=0$

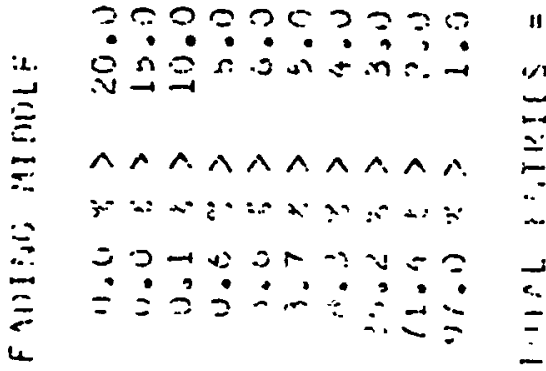

1

1

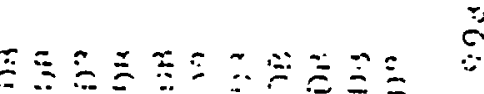

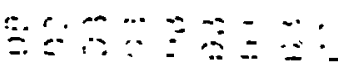

$\overrightarrow{=}$

$\because \because \because \because \because \because ? \div \because$

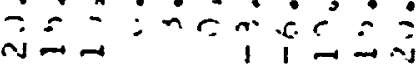

$\because \because \because \vdots \vdots . \vdots$

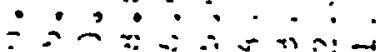

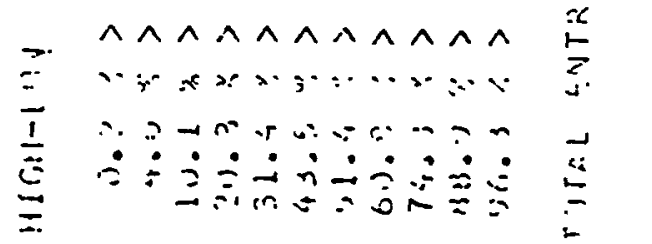

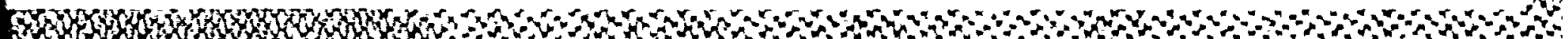




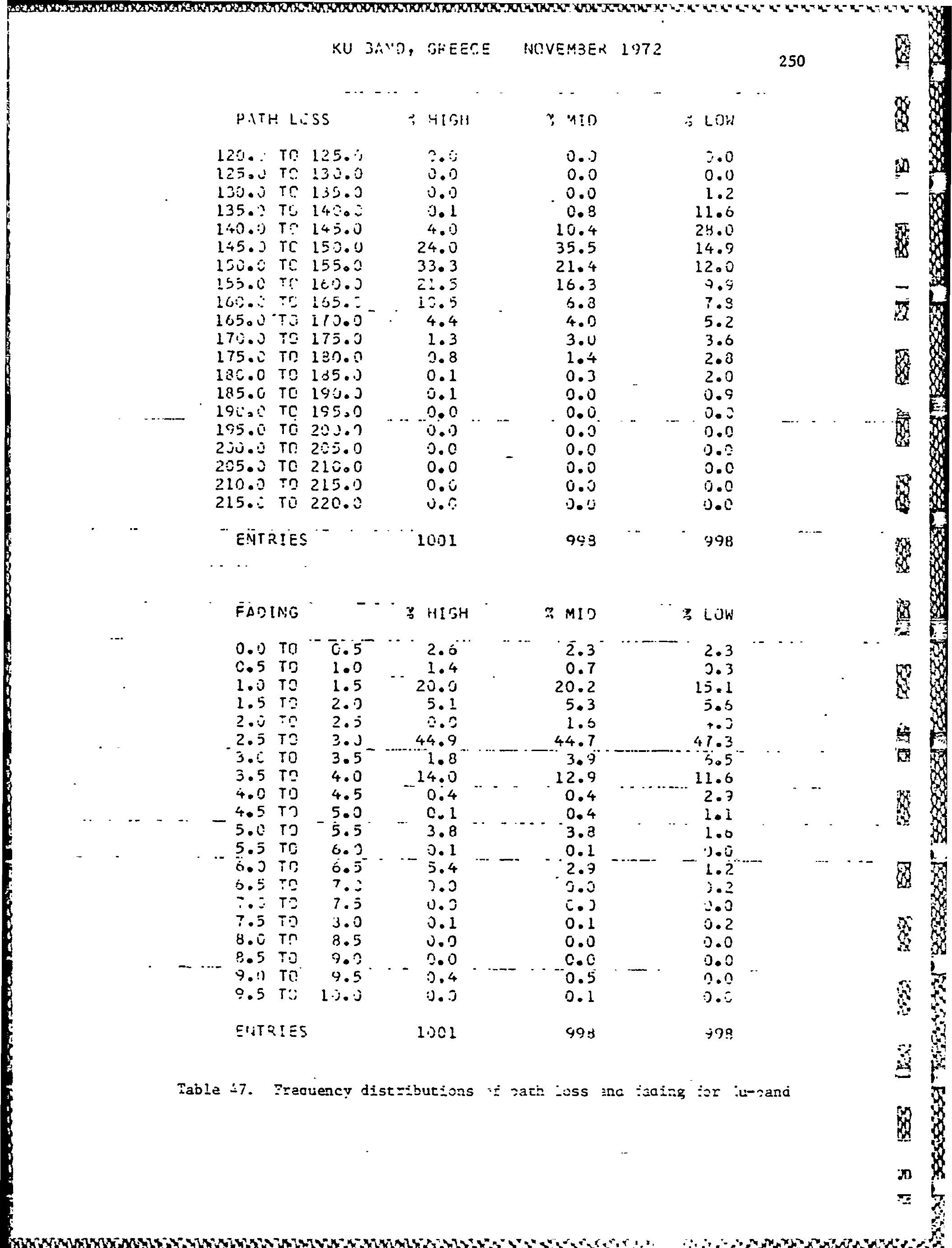




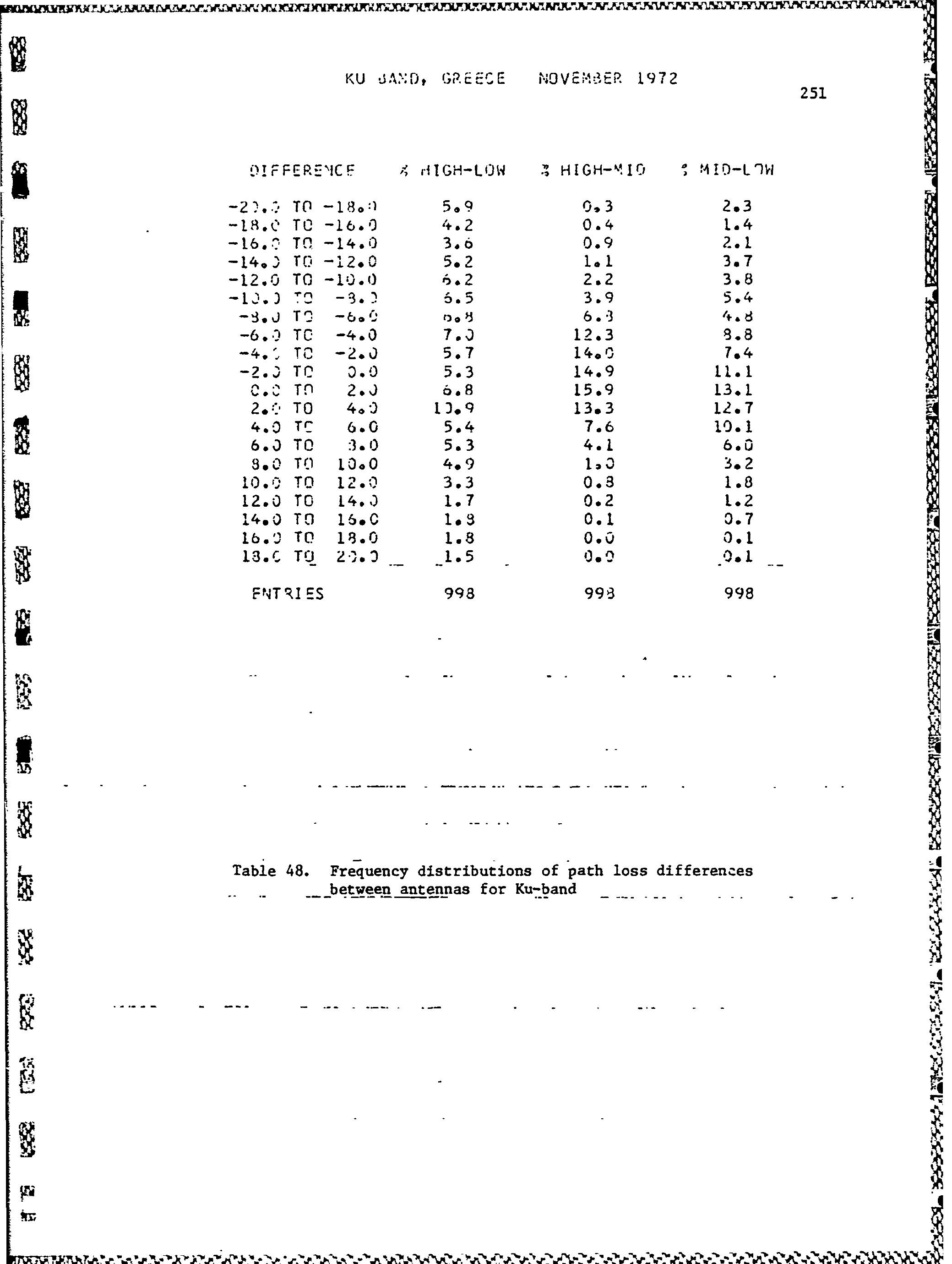

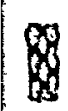

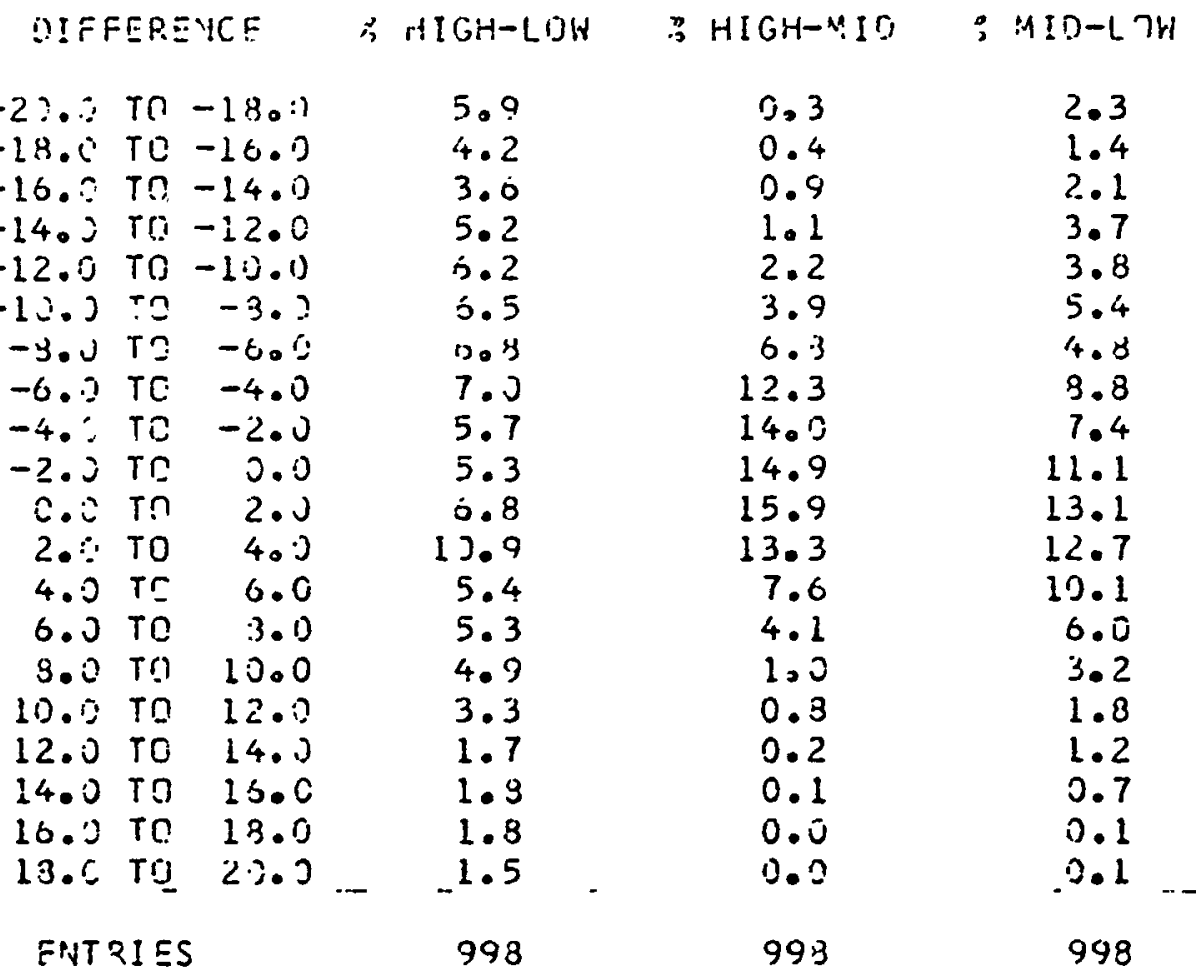




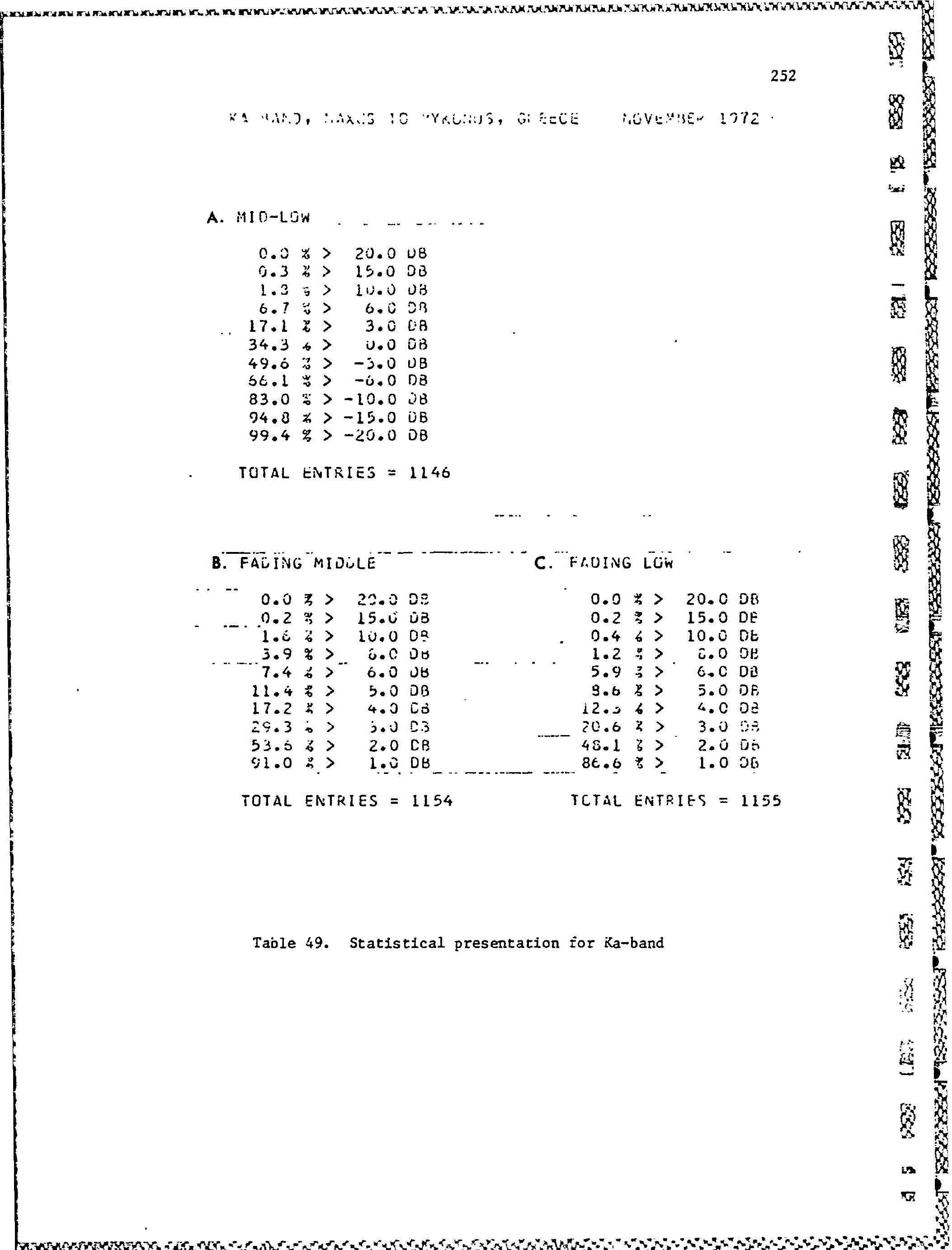




\section{KA BAND, GREECE NULEMAER 1972}

PATH LCSS

120.0 TO 125.0

125.0 TO 130.0

130.0 TO 135.0

$13 \mathrm{b.0}$ Tü 140.0

140.0 TO 145.0

145.0 TJ 150.0

150.0 TU 125.3

155.0 Ti! 100.0

160.0 Tu 105.0

165.0 TU $1 \% .0$

170.0 Tú 175.0

175.0 TJ $1 \doteq 0.0$

ijo.j TU 1 T 130.0

$133.0 \mathrm{TH} 1>0.0$

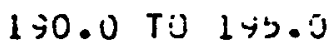

195.0 TJ 20.3

200.0 TO $\angle \mathrm{O} 2 . \mathrm{U}$

205.0 T:J 210.0

210.0 io $21 \% .0$

$215.0 \mathrm{~T} 3220.0$

ENTRIES
$6 M 1 D$

0.0

0.0

0.0

C. 0

0.0

0.0

0.0

0.1

1.2

$\angle 1.0$

30.8

24.5

$1 j \cdot 1$

3.0

0.3

). 1

0.0

i. 0

0.0

0.0

1154
6 Lin

0.0

0.0

0.0

0.0

ง. 0

0.0

0.0

0.3

15.7

33.2

26.8

14.5

6.0

1.6

0.4

0.3

0.0

0.0

C. 0

0.0

1155

Table 50. Frequency distribution of path loss for Ka-band

$\infty$ 


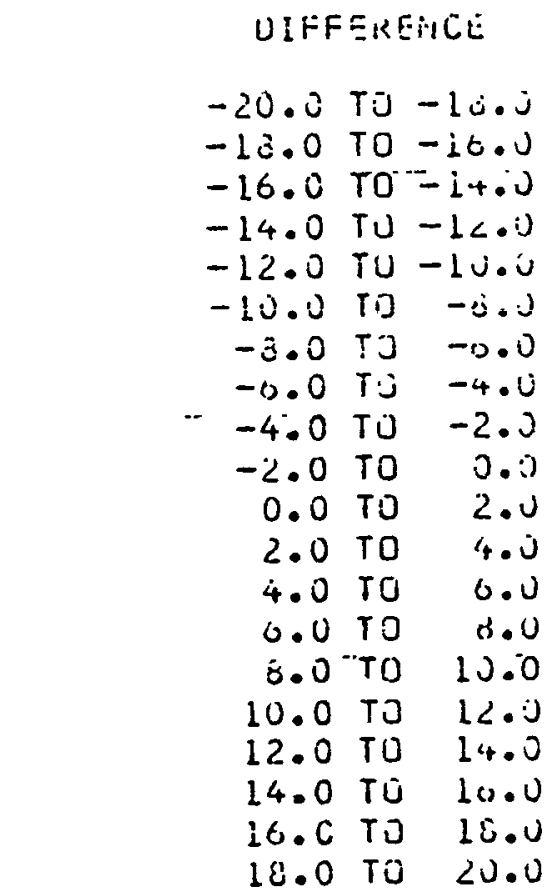

ENTRIES $\ddot{6} 1$ IU-LU.

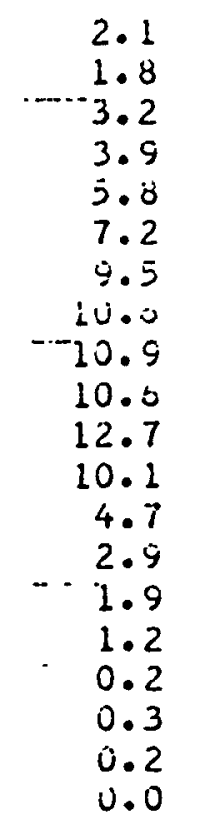

1140

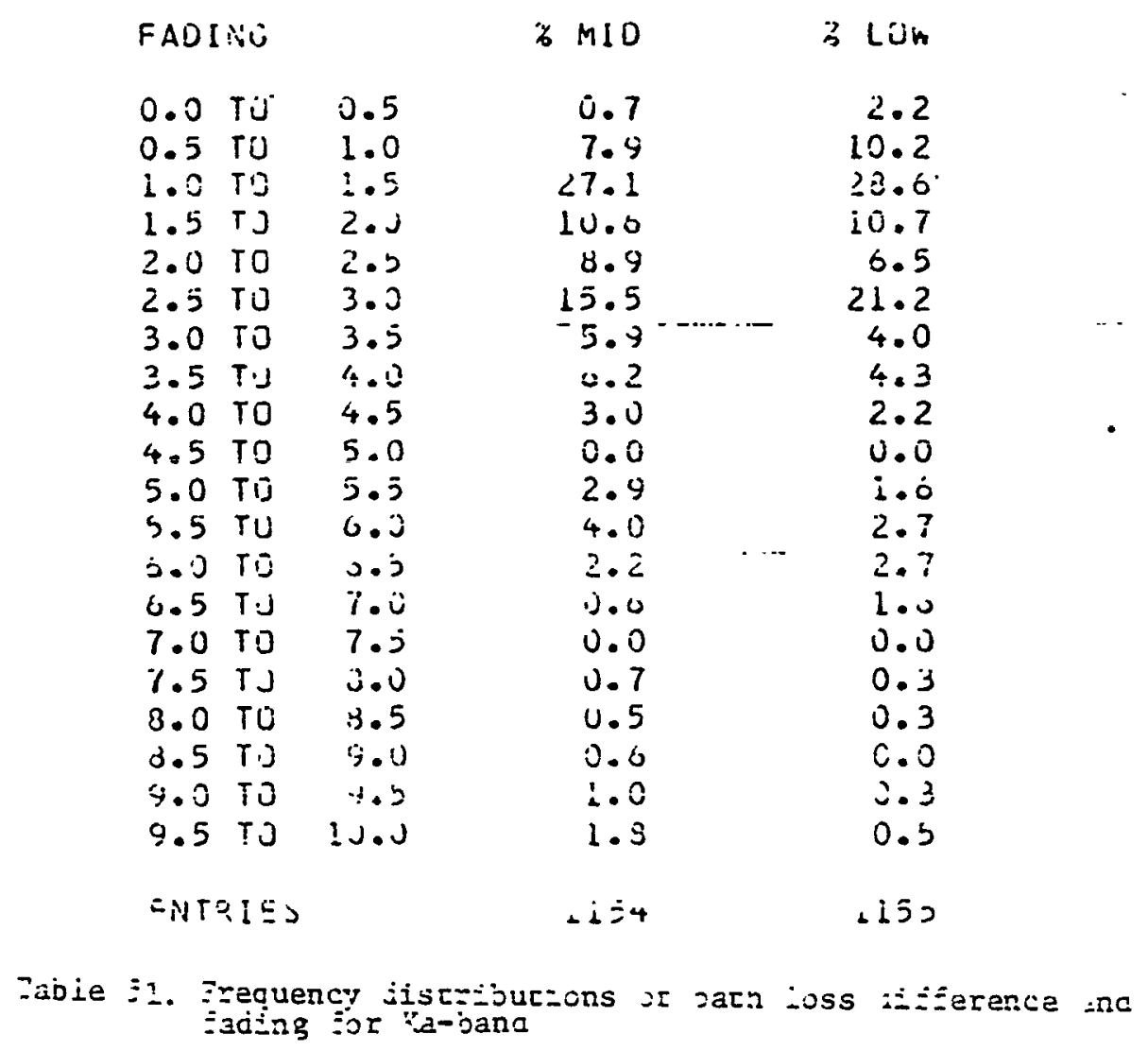




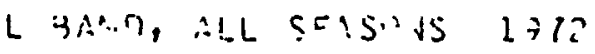

PATH LCSS

123.073125 .0

125.0 in 1.30.0

130.: TE $135 . ?$

135.0 TC 14.0.0

$140.0 \mathrm{Tin} i+5.0$

145. TO 150.1

15n.? TR 155.0

155.0 T. 360.0

1ن.

$165.0 \mathrm{T.1} 172.0$

170.0 TS 175.1

$175 . \therefore T$ T. 132.

$100 . ? 19125.0$

185.3 TI 110.0

10. 2 TO 105.9

$195 . .5 T R ?, 0.0$

200.0 TU 2:5.0

205.0 TO 210.0

210.0 TC 215.0

215.0 T: 223.0
$" H ! ; H$

1.5

\section{4}

2.7

3.0

10.4

14.6

$2 ? .3$

$32 . ?$

3. 7

$0 . ?$

(1.)

is 9

0.9

1). !?

1). 0

0.0

0.0

0.2

B.

$\therefore 1.8$

4395

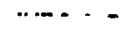

ENTRIES

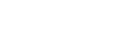

$\because$ 的同

$\Rightarrow 6$

2.2

0.6

1.5

2. 3

3.1

2.3

10.2

24.0

9.3

20.4

23.6

0.1

$0.1)$

3.

0.3

0.10

0.1

$0.1)$

0.0

0.0

1.2 .

0.0

0.0

0.0

0.0

3.2

0.0

0.0

3.5

4337

S

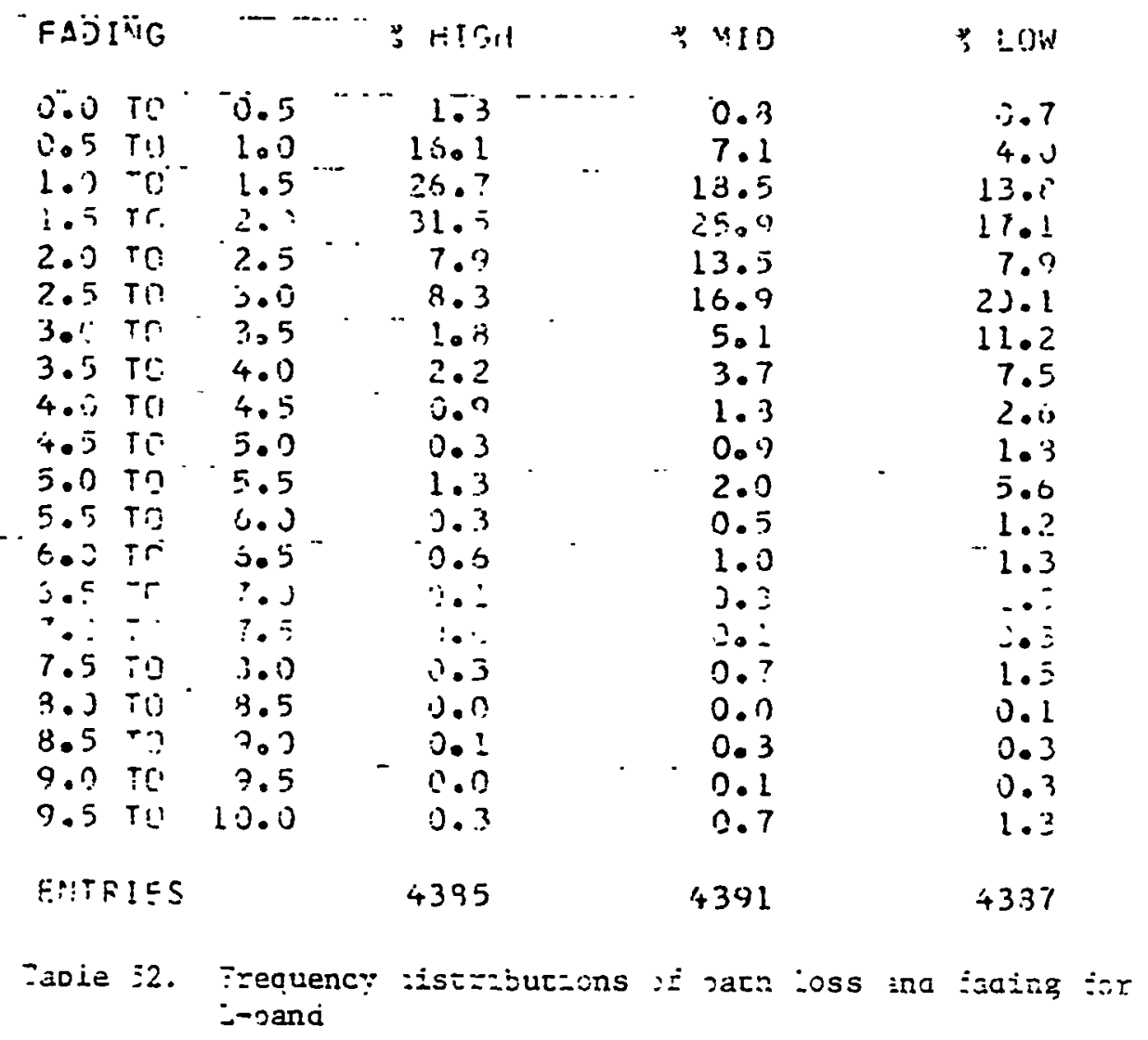

$x$

8

8

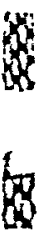

3



4

8

4371
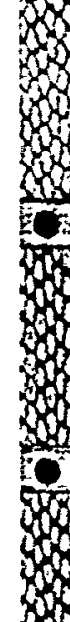
DSEFEDESG

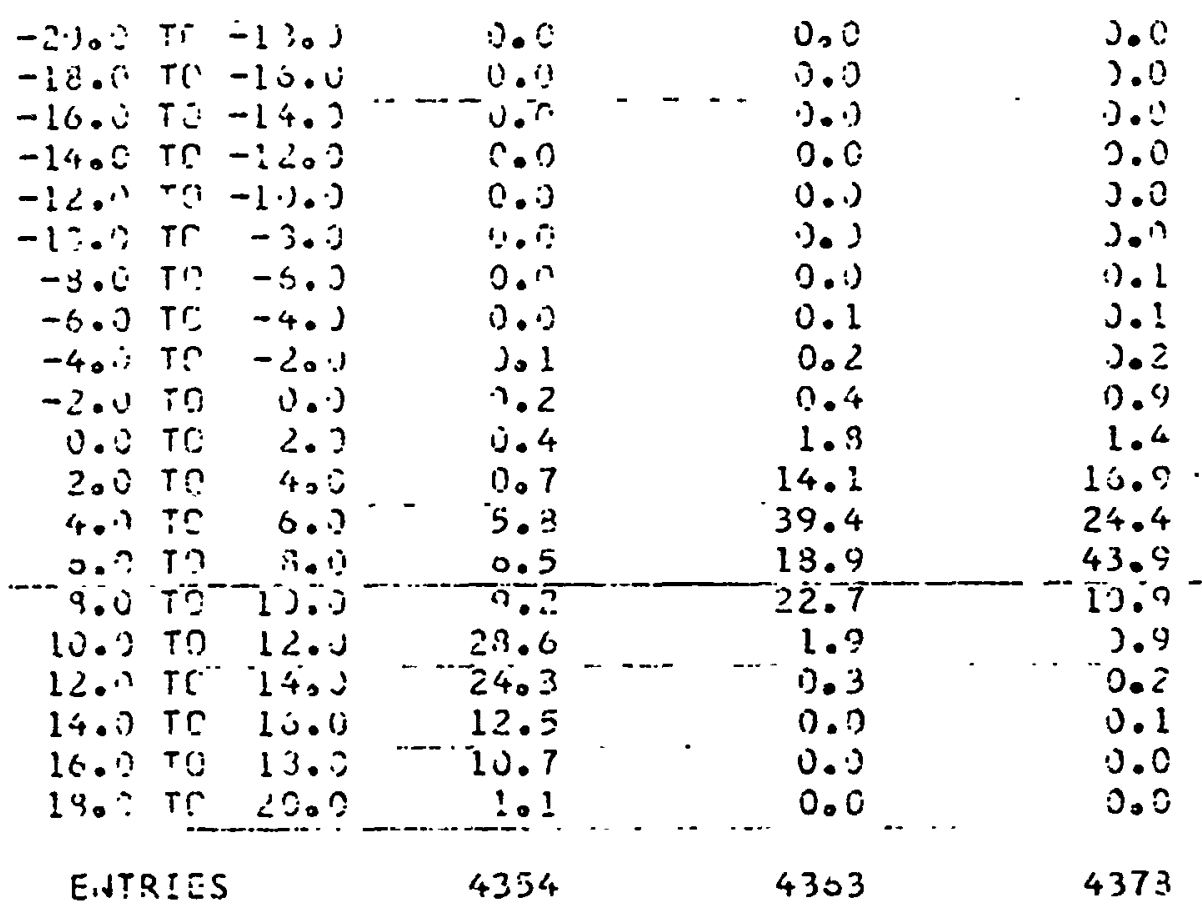

-2.100 Ti $-130 j$

2.0

0,0

3

- $1 \mathrm{cos} T(\mathrm{~T}-10.0$

-16.0 T5 -14.3

).

3.0

-1400 Tr -1200

0.0

2.0

-12.0 ra

0.1

3.0

$0.1)$

2.19

). 1

3.2

$-40 . ;$ TR -20.1

-2.0 in 0.0

0.0 TC 2.2

200 TC 400

0.7

5.3

$\cdots$

$0 . ?$ T?

9.0 T. 13.0

10.2 TO 12.0

$12.1 T T C^{-1} 190$

14.0) TR IU.j

16.0 TO 13.0

R!ES

4303

4373

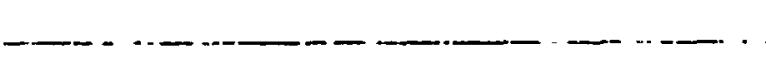

\section{9}

0.2

0.1

0.0

Table 53. Frequency distributions of Dath loss differences between antennas $=0$ I $i$-band 
DIFFERENCE \& HIGH-LOW : HIGH-YID : YID-LOW

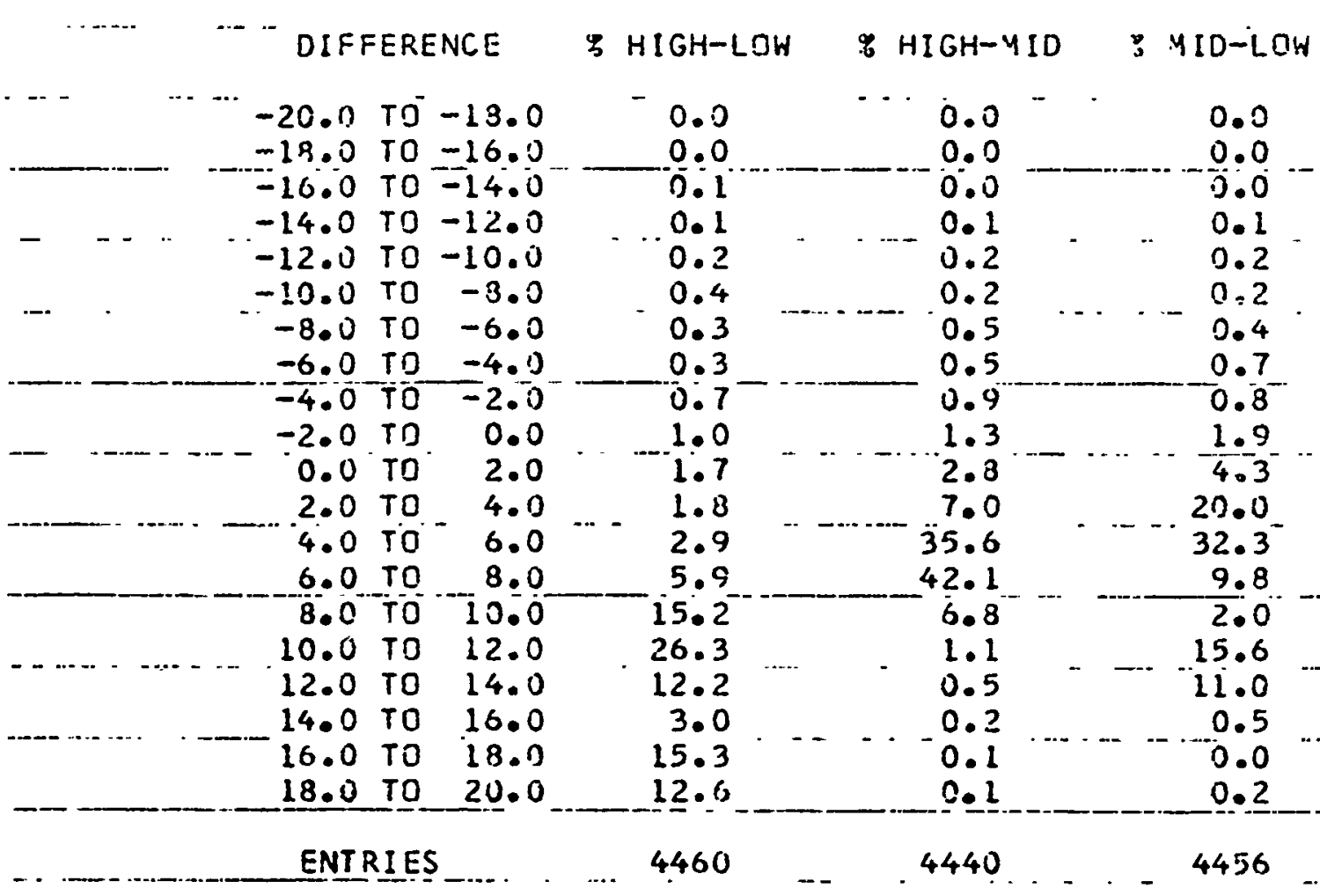

Table 55. Frequency distributions ó path loss differencés between antennas for S-band 


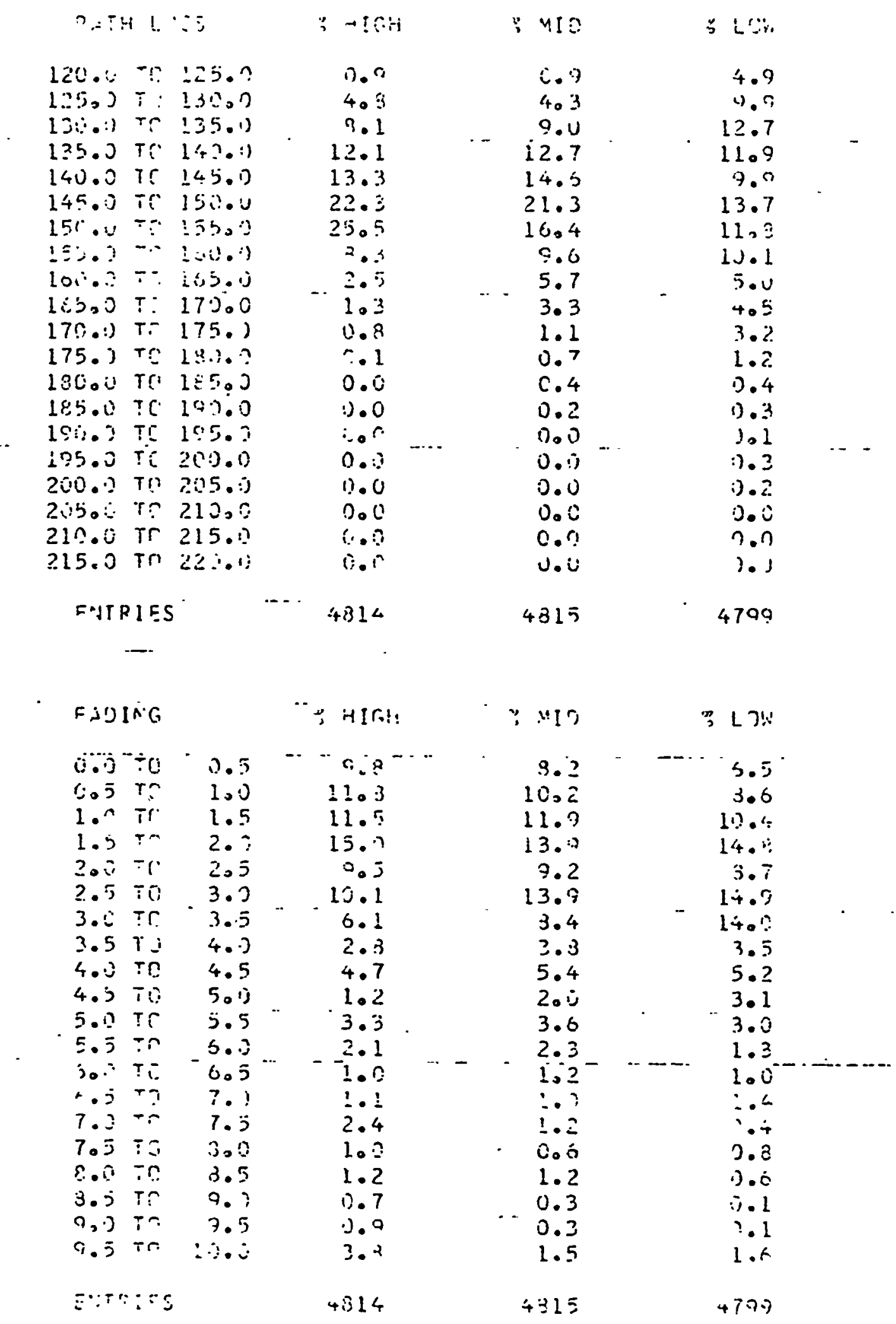

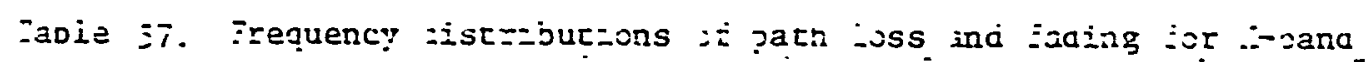

$0.0 \div \div \cdot 0.5$

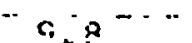

10,2

11.9

0

$1.5 T$

9.2

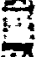

3.0

3.5

13.7

3.3

5.4

$2 . i 5$

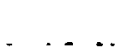




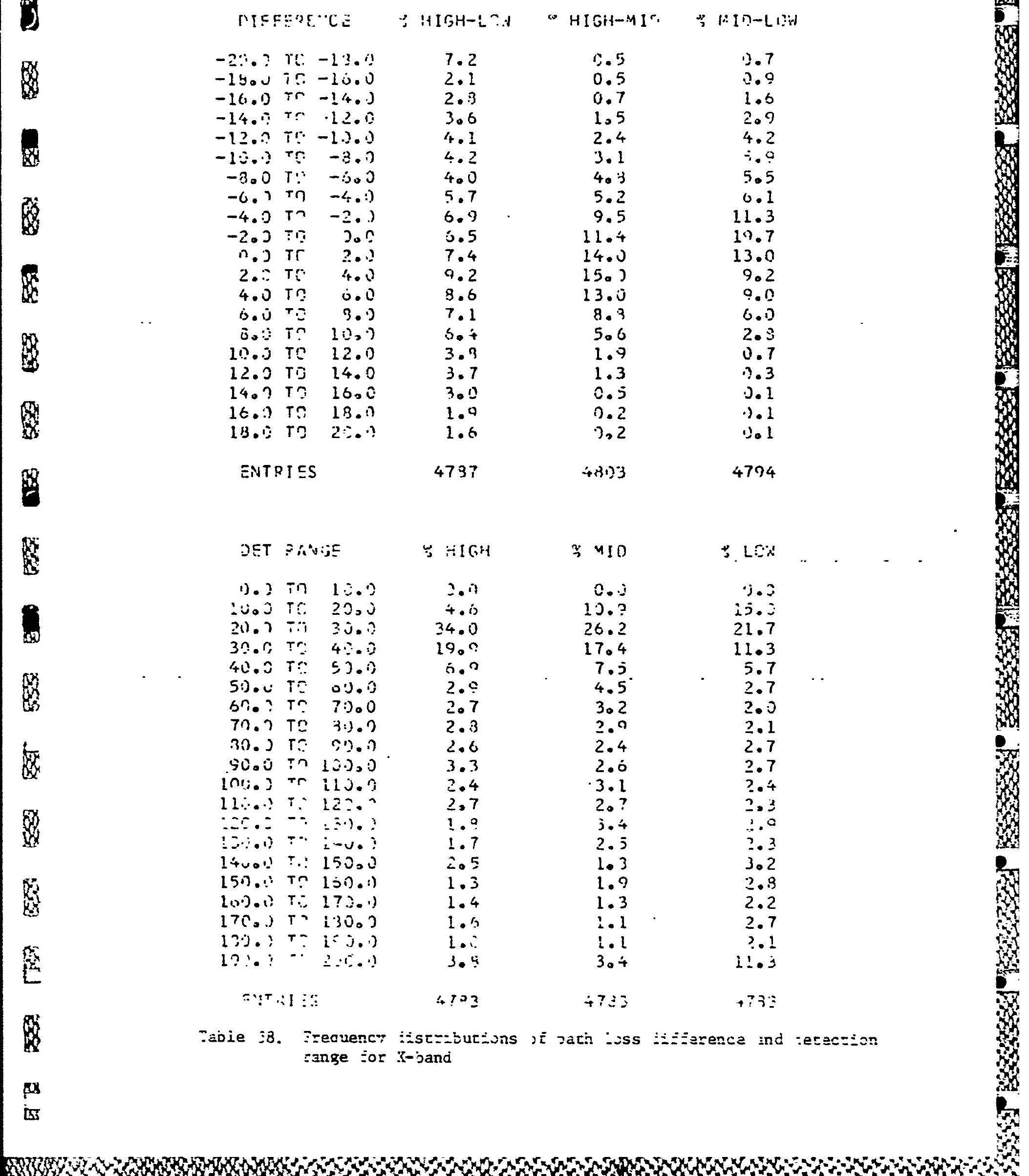




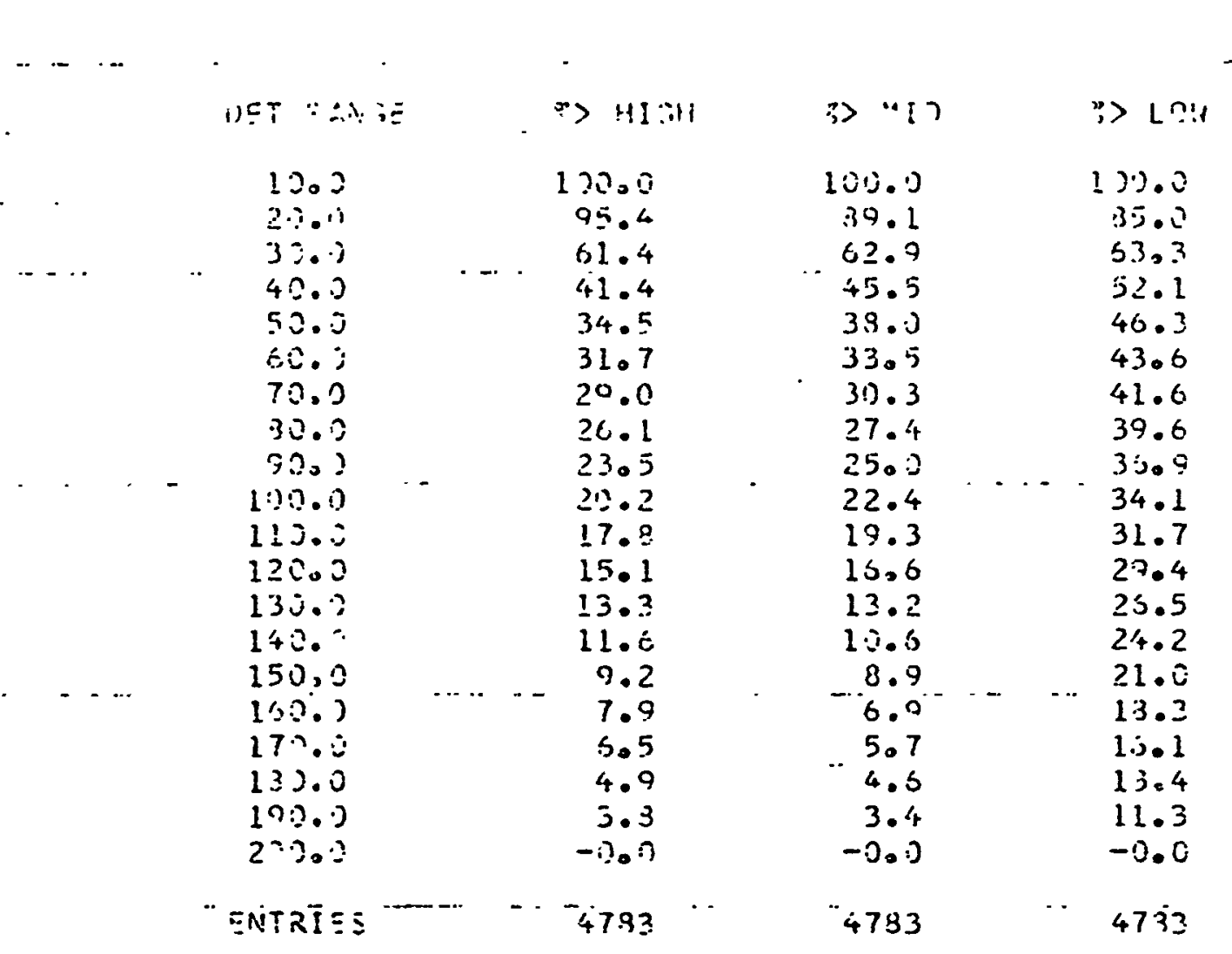

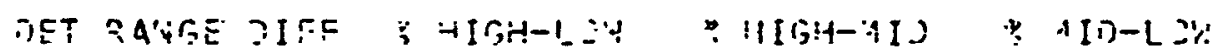

$-5 . \therefore$ r.

$-45.212-40.0$

27.9

$8.9^{\circ}$

22.9

$-4: 1 . ?-35.2$

1.2

1.3

1.5

1.7

$.9 ?$

i. 7

$-30 . \div \div-23.0$

$1 . .7$

-25.0 is:-2..

1.1

2.4

-15.7 r $20-13.11$

2,9

3.0

5.2

-160.9 T: -5.3

2.3

4.6.

10.5
20.0

3.3 i. 5.0

o. 1

10.3

110 is

11.5

4.4

$3 .$.

2.2

$2300 \div \quad 3500$

$25.2 \div \because \quad \therefore .7$

1.9

3.0. ? $35 . ?$

- 35,2 4040.0

40.7 tr 45.3

1.8

$\frac{1.2}{1.5}$

$1 \cdot 5$

1.5

i. 9

2.1

$-1 \cdot 2$

.5 .7

1.4
1.6
1.4

1.4
1.7

$1 . \bar{c}$

1.7

2. 2

5.2

13.3

24.2

(1)

$=\cup \div: 1: 5$

$5 \quad 478: \quad 4733 \quad 4733$

$5 \quad 47834732473$

7.2

?. 5

2.2

1.5

3.5

3.9

0.4

1). 3

1.2

5

Tabie 59. Cumulative distribution os derection range and freouency

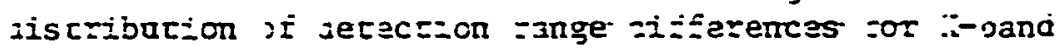

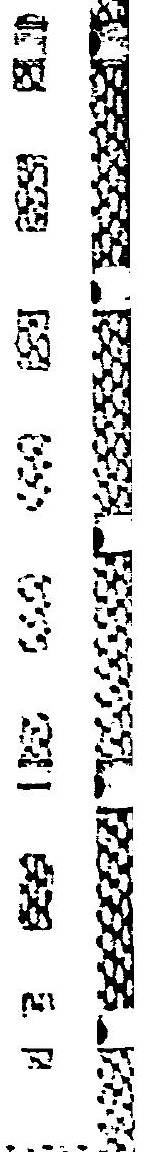

10010 
KU BAND, NUGUST - NULVEMBSE 1972.

PATH LUSZ

120.0 TO 125.0

125.0 TU 130.0

130.0 TO 135.0

135.0 TO 140.0

140.0 TJ 145.0

145.0 TO 150.0

i50.0 Tu i 25.0

$155 . j$ TJ 160.0

160.0 TO 105.0

165.0 T0 110.0

170.0 TÓ 175.0

175.0 TO 130.0

130.0 ru 185.0

185.0 TJ 130.0

190.0 T 135.0

$193.0^{-}$TO 230.0

200.0 To 205.0

205.0 TO 210.0

210.0 To 215.0

215.0 TO 220.0
: HIGH

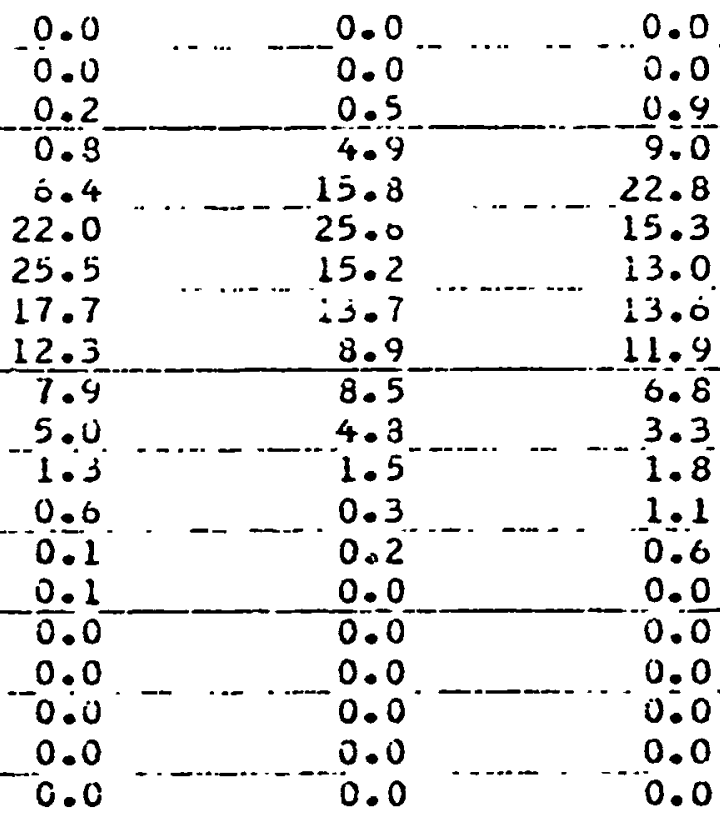

$6+9$ క L L

1770
1770
$17 \overline{7}$

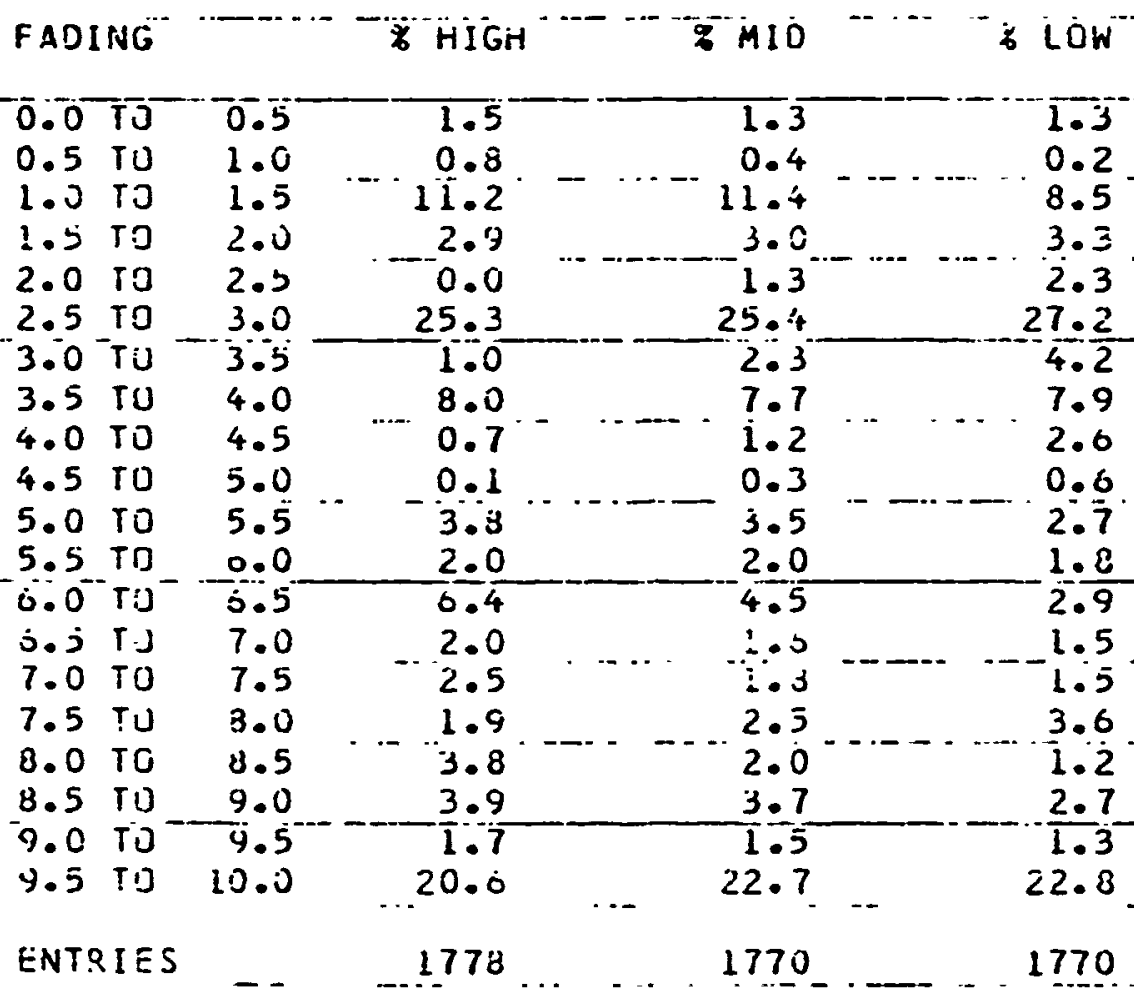

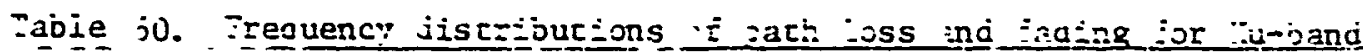

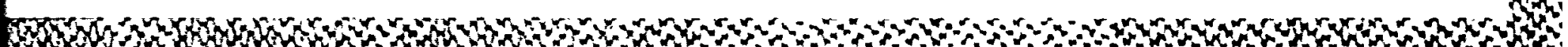




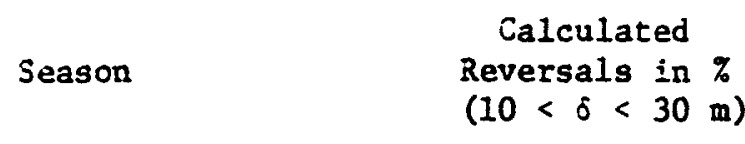

Winter

Spring

Summer

Fall

Year Average
41

36

33

48

39
Measured

Reversals in \%

51

28

93

14

47

Table 62. Calculated and measured antenna reversals 
X. APPENDICES 


\section{A. Detection Range Calculations}

An important consideration in interpreting path loss results is the implied maximum range of detection for some assumed radar and taiget. A technique has been developed that directly relates the measured path loss at 19 nautical miles for all three antenna helghts to predicted decacion range. The radar that is assumed is one which can just detect the target at 200 naurical miles in free space. All detection range results presented here are for this same radar and target combination. Figure A 1 shows the theoretical dependence of path loss on range for all three antenna heights at $9.6 \mathrm{GHz}$ for a variety of duct heights. These calculations are the results of a full wave computer solution using realistic log-linear distributions of refractive index in the boundary layer. The free space path loss at 200 nautical miles at $9.6 \mathrm{GEz}$ is $163.5 \mathrm{~dB}$ and is indicated in Figure $A 1$ as a threshold. Any case for which the path loss is less than this threshold is defined as being detectable and any case for which the path loss is greater than this threshold is defined as being undetectable. Thus the maximum range of detection Eor any of the sample duct heigints shown in Figure $A 1$ is the range at which the path loss first exceeds the 200 nautical mfle free space threshold. From the curves in Figure A 1 it is therefore possible to relate certain discrete path loss values at 19 nautical miles to detection range. Figure $A 2$ shows a tabulation and a plot of these path loss values versus the corresponding detection range for each duct height and antenna height of Figured $:$. The solid curye in Figure $A 2$ is an empirical fit given by the relation

$$
\begin{array}{ll}
\mathbf{R}=6-338 /(136-\mathrm{L}) & \mathrm{L} \geq 142 \\
\mathbf{R}=139 i-9.4 \mathrm{~L} & \mathrm{~L}<142
\end{array}
$$




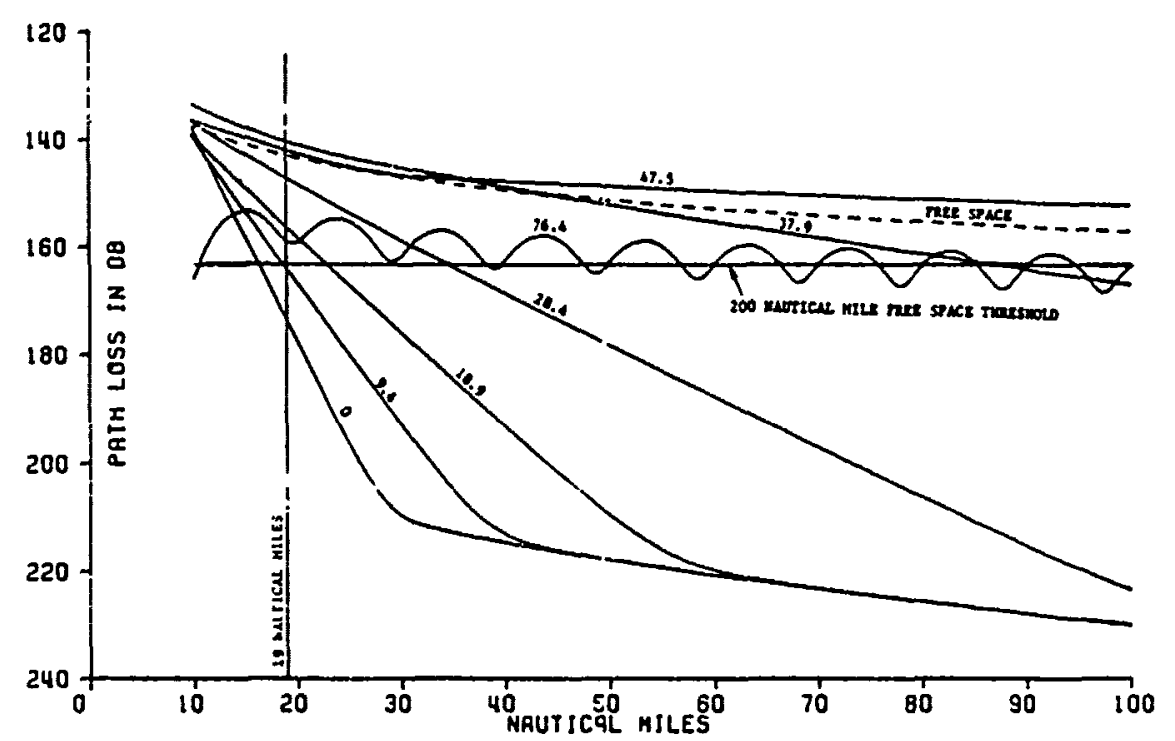

(a) High antenna

Frequency: $9.6 \mathrm{GHz}$

Transmitter antenna:

Receiver antenna: 64 Duct heights in feet : indicated

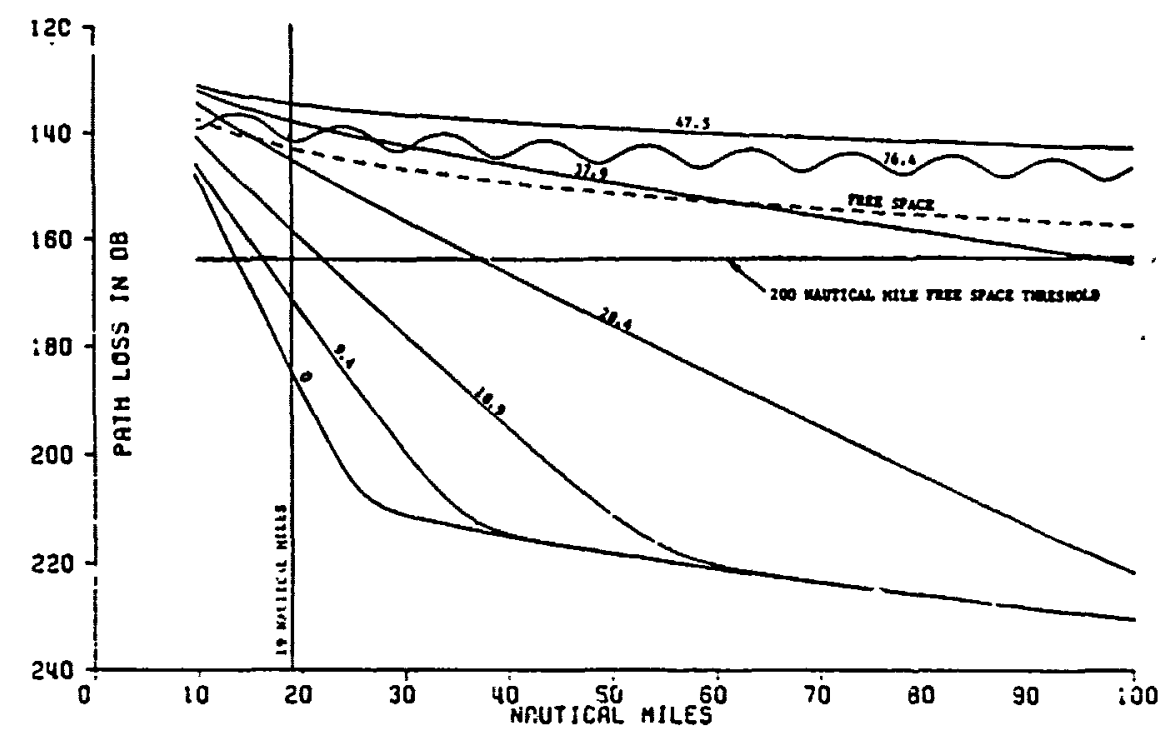

(b) Mid antenna

Receiver antenna: 32

Frequency: $9.6 \mathrm{Grz}$

Transmitter antenna: :

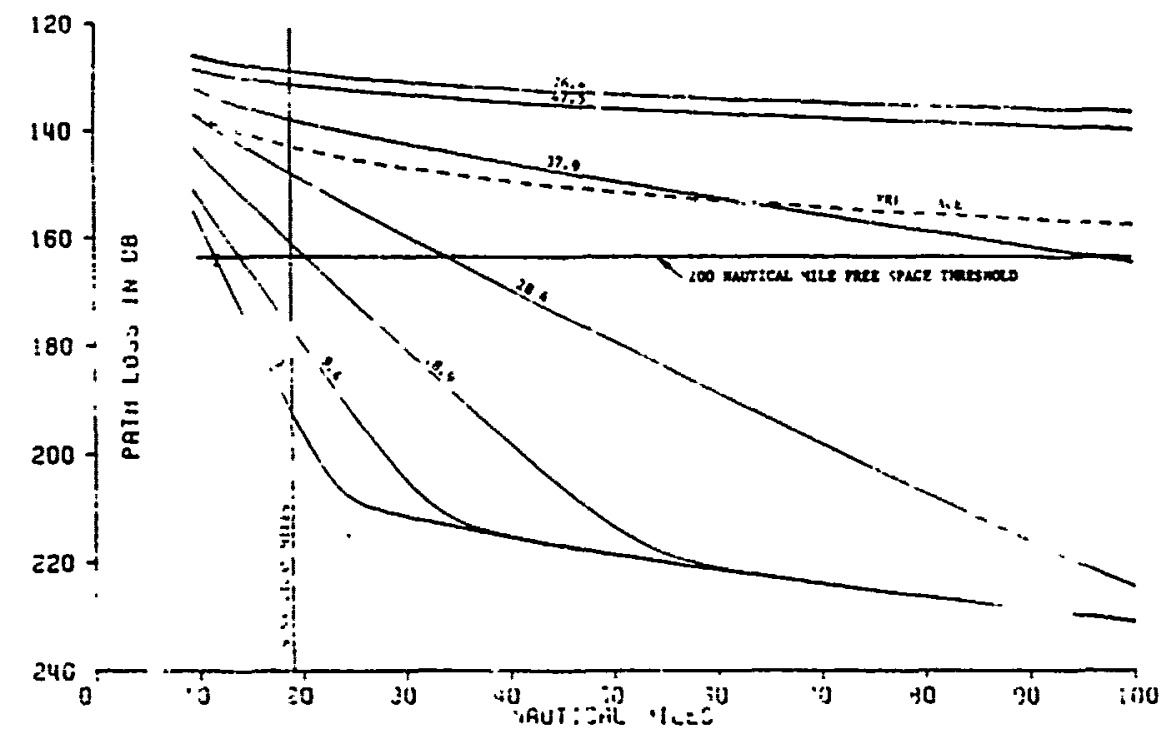

(c) Low antenna

Receiver antenna: 16 Frequency: $9.6 \mathrm{GHz}$ Transmitter antenna: :

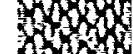

Figure A 1. Salcuiaced pacn loss "er"us range for the three ancanna hezghrs 



\section{B. Radiosonde Profiles}

Radiosonde profiles were obtained using $403 \mathrm{MHz}$ transmitters and Beukers receivers. They were optically tracked for wind information. The Individual profiles with the launch time in GMT are listed in the following presentation wich is self explanatory. Some of the profiles for the : November 1972, $0751 \mathrm{Z}$ launch are shown twice. In the second presentation the original chart recorder trace was read in very small increments and with maximum resolution. This was done in order to show that small fluctuations lgnored in the first presentation did not represent significant refractive changes. 


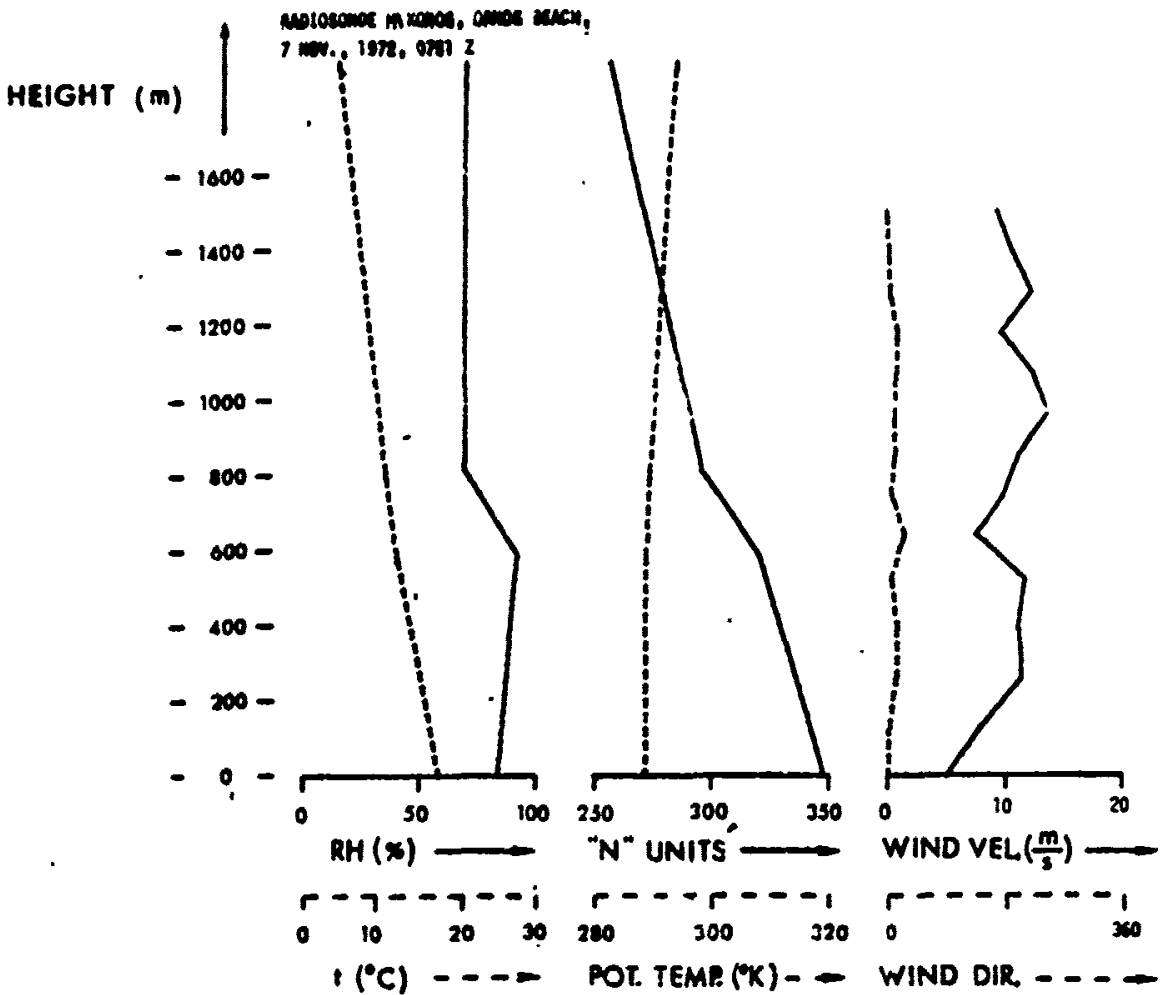

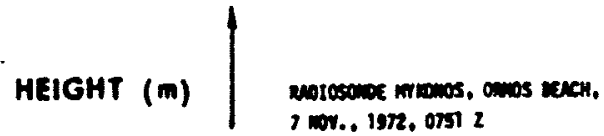

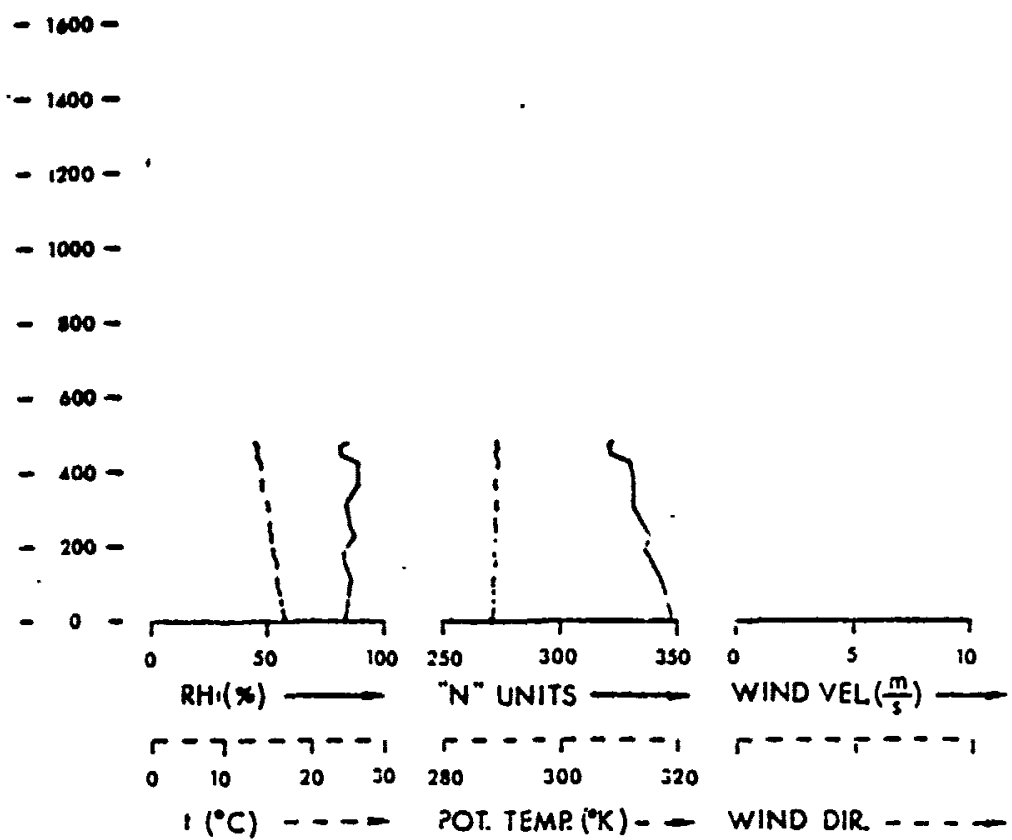


8
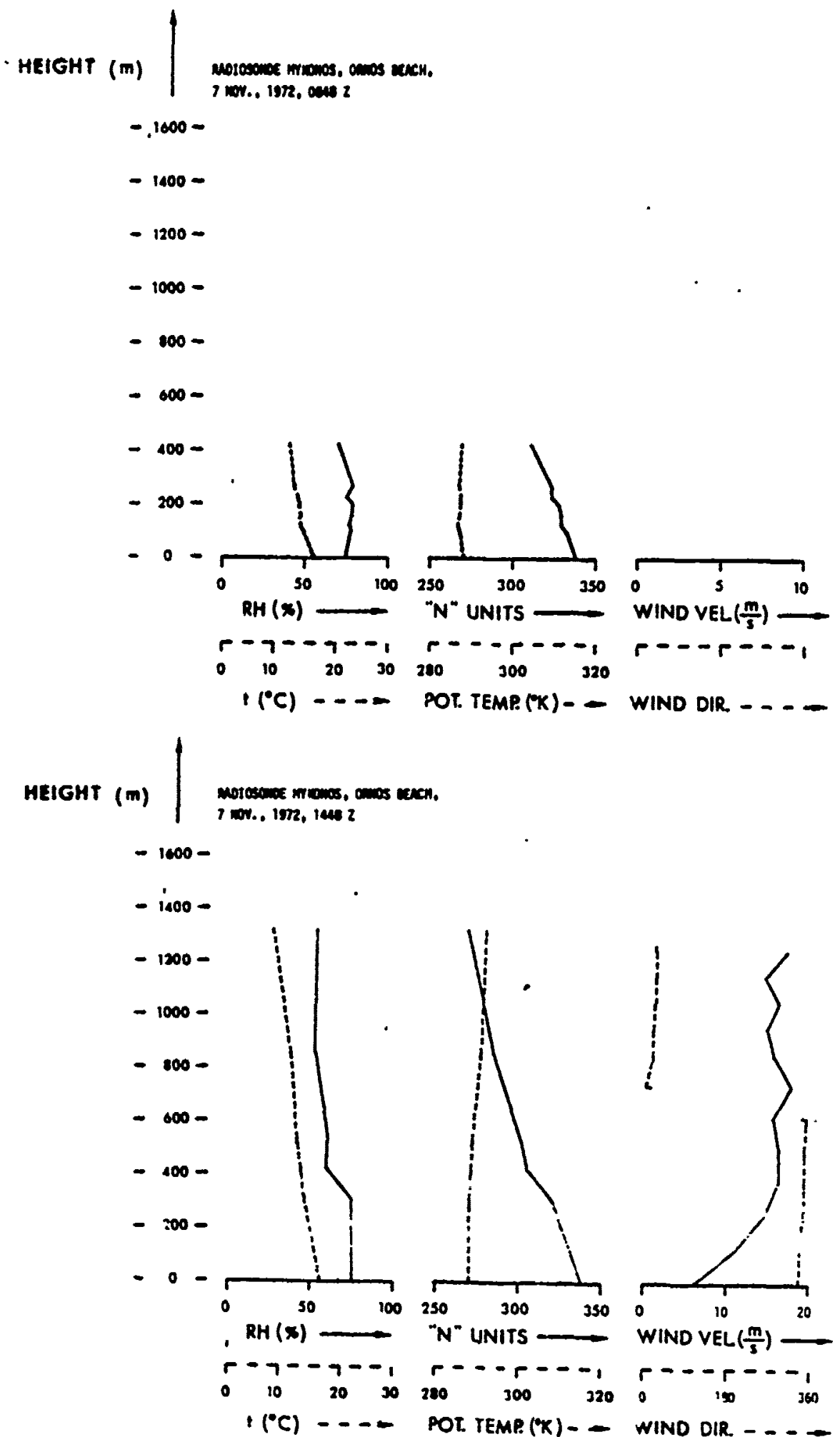

8

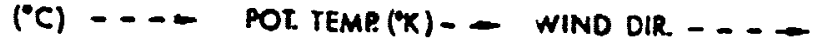




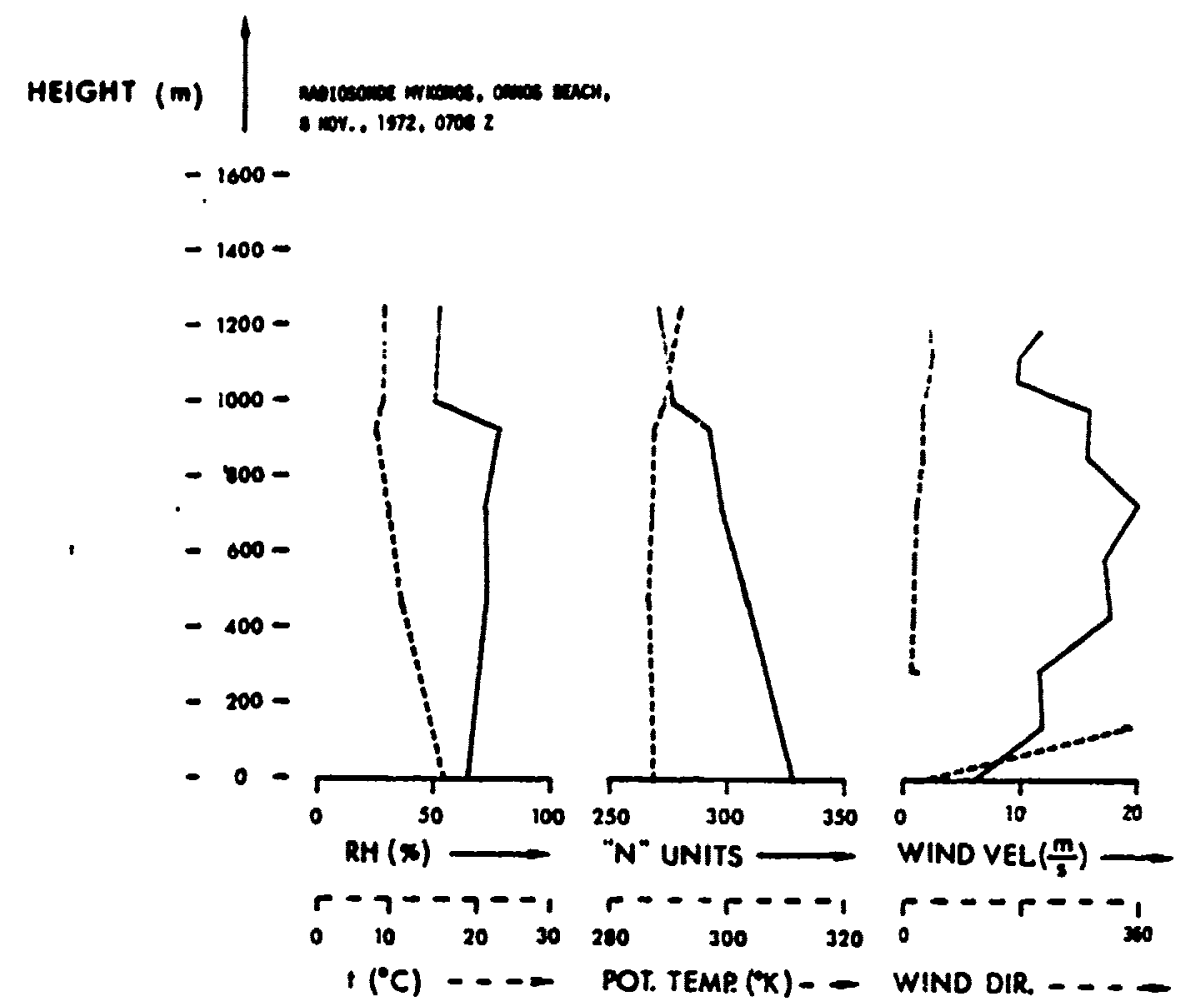

W

0

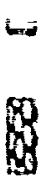

in

$-800-$

$-\infty 00-$

$-400-$

$-200-$

(C).--

POT. TEMP (KX) - - WIND OIR - . -

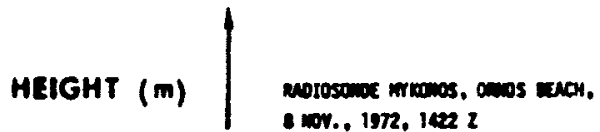

$-1600-$

$-1400-$

$-1200-$
$-1000-$
$-100-$
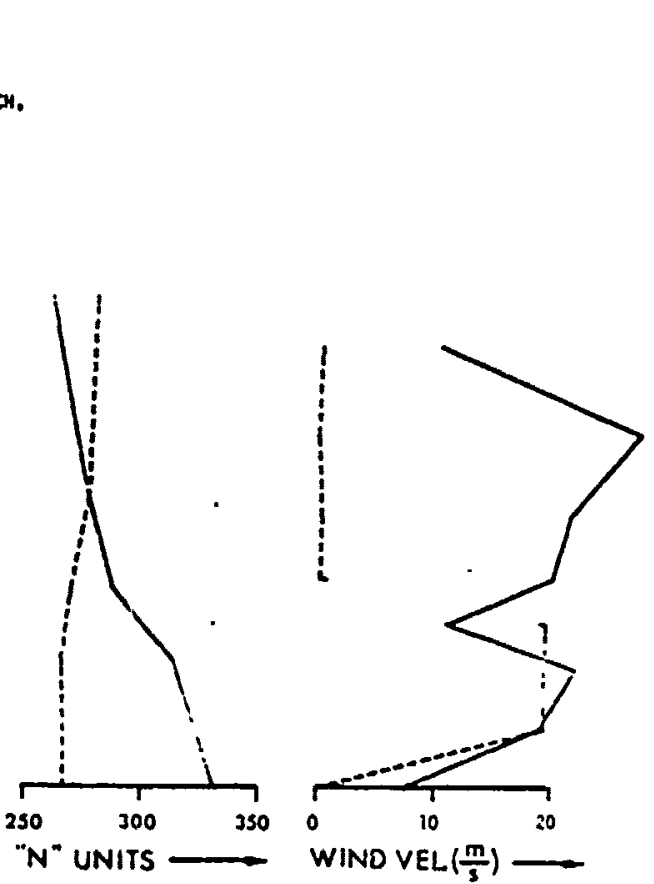

(x)

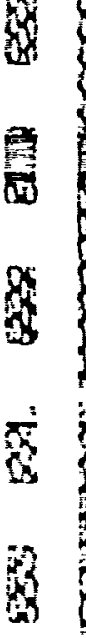

a

$\beta$

3

8

3

$\Delta$

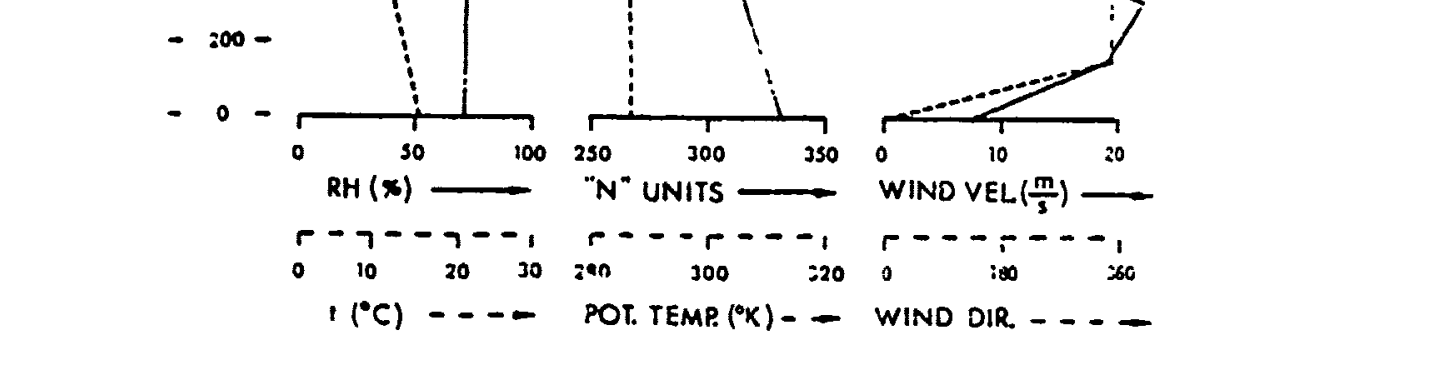

The 
Q
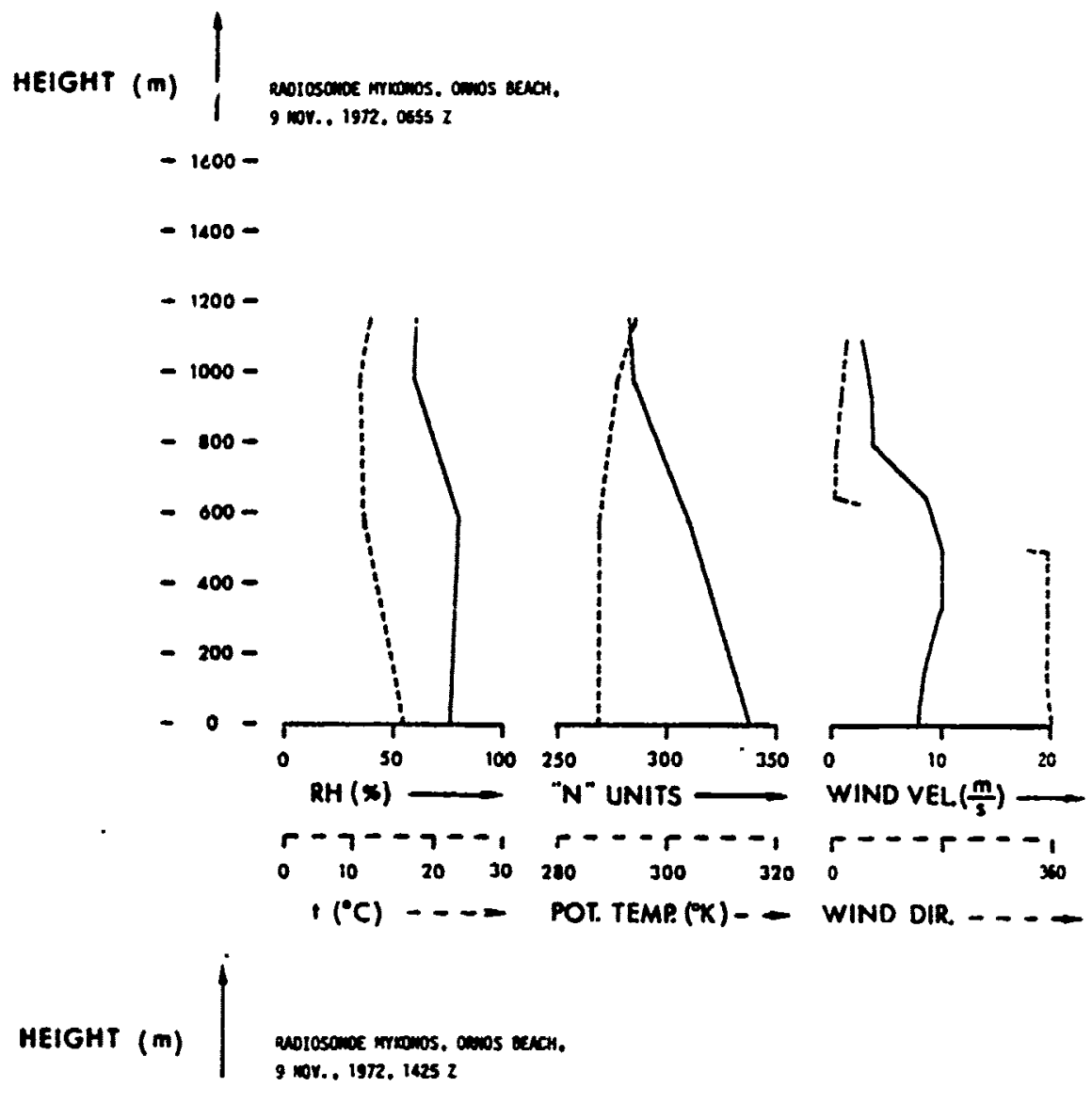

$-1800=$

- 11000-

$-1200-$

$-1000-$

$-800-$

$-600-$

$-400-$

$-200-$

$-.0$
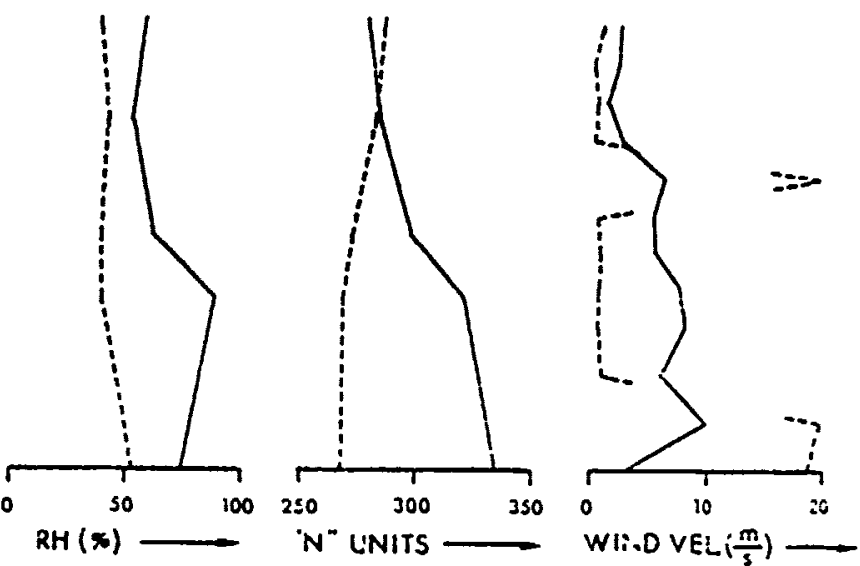

r- - - - t -
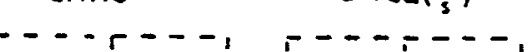

0 in

( $\left.{ }^{\circ} \mathrm{C}\right)=-$.

300

3200

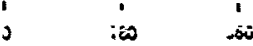

M

POT. TEMP $\left({ }^{\circ} \mathrm{K}\right)$ - - WINO DIR. - - -

to 

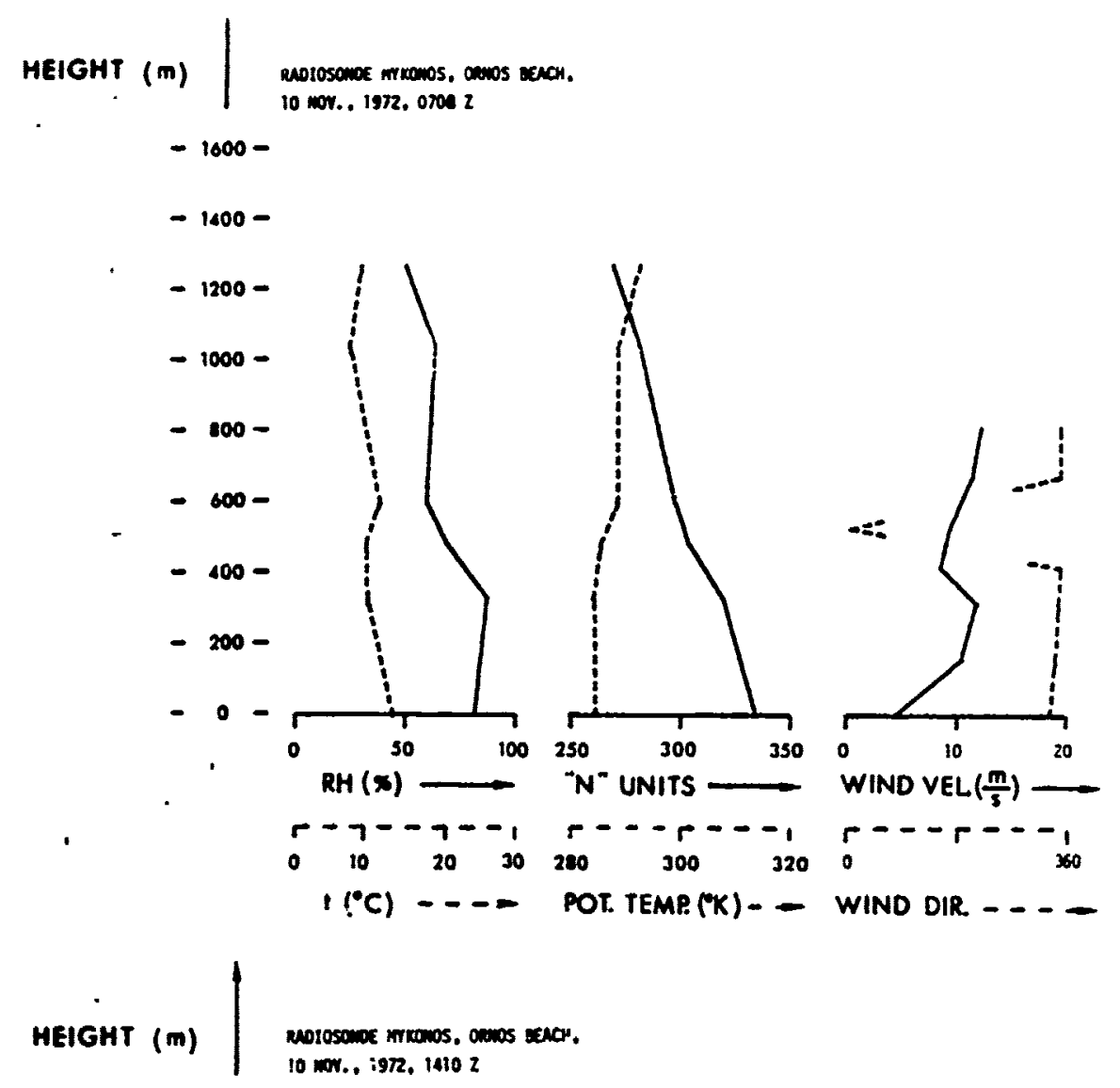

- $1600-$

$-1400-$

- $1200-$

- 1000 -

- 800 -

- $.00-$

$-400-$

- 200 -

- $200-$

- 0 $10 \mathrm{mor} . \mathrm{.}$ : $972.1410 \mathrm{Z}$
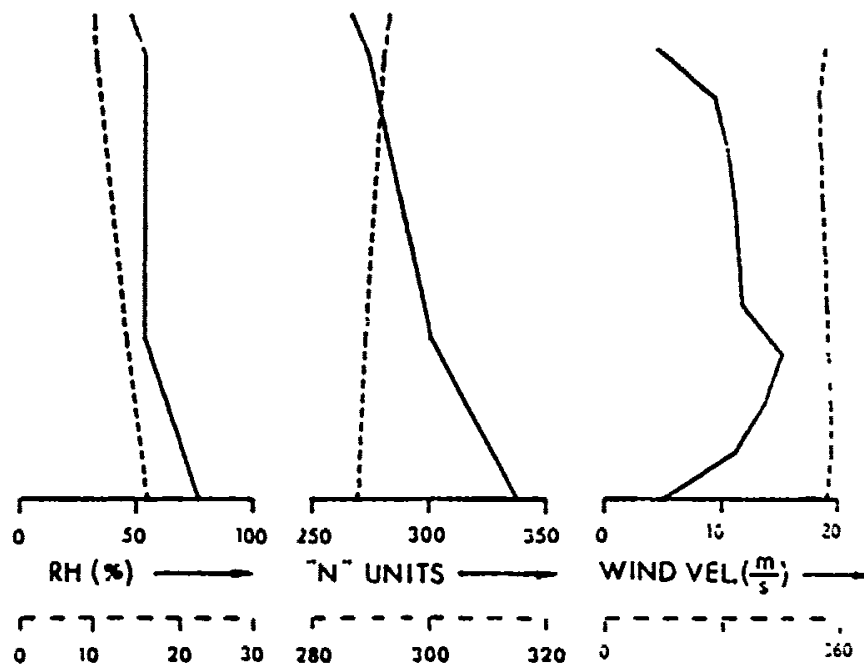

( $\left.{ }^{\circ} \mathrm{C}\right)$ - - POT. TEMP $\left({ }^{\circ} \mathrm{K}\right)$ - - WIND DIR. - . - 


8



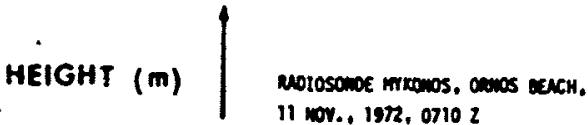

- $1000-$

$-1400-$

$-1200-$

$-1000-$

$-100-$

$-800-$

$-400-$

- $200-$

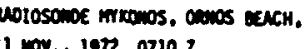

$-0-$
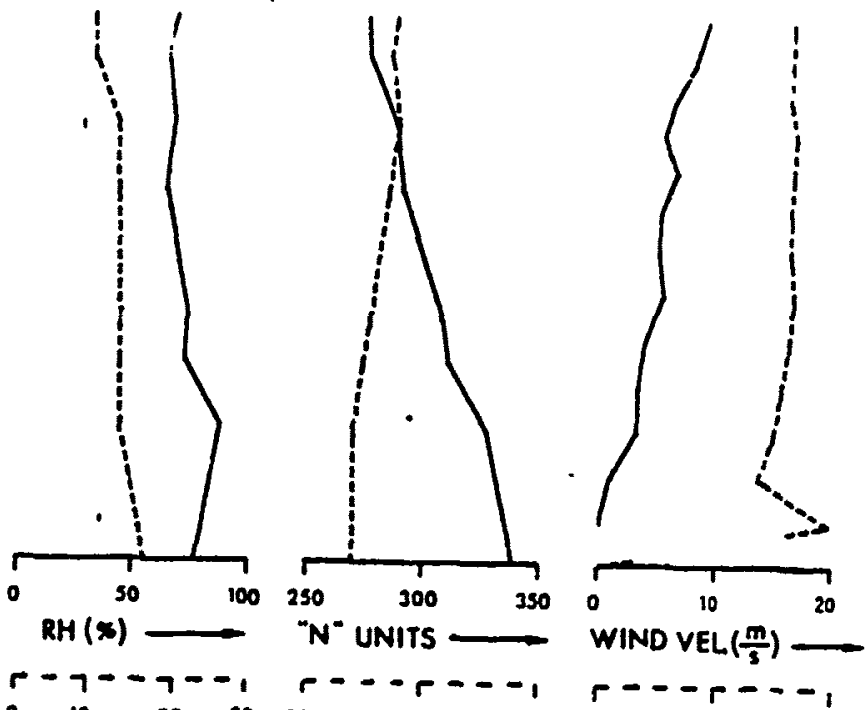

200

( ("C) $^{\circ} \mathrm{-}-\mathrm{-}$

r

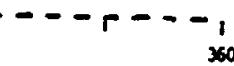

HEICHT (m)

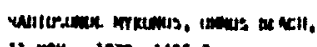

11 mov. . 1972. 1405 2

$-1600-$

$-1000-$

$-1200-$

$-1000-$

$-100-$

- 600 -

$-.100-$

- $200-$

$-0$
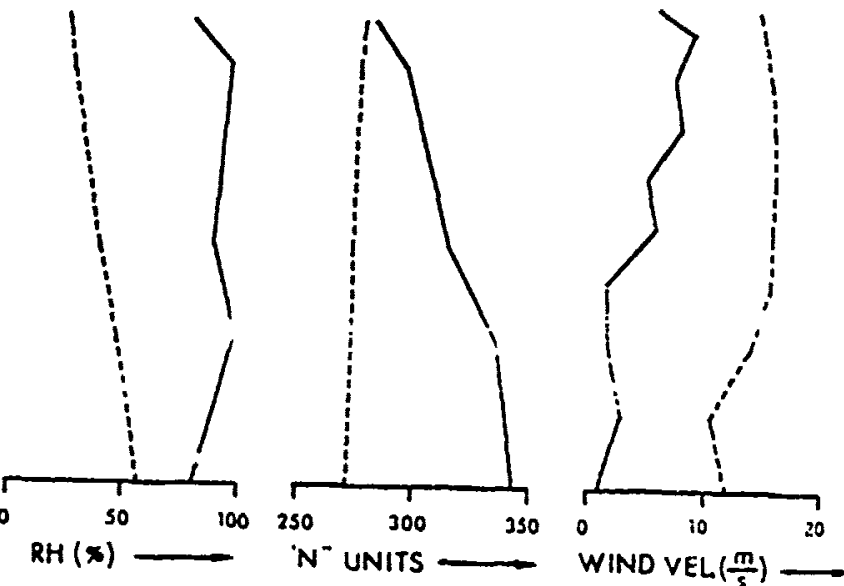

r-ר-・--

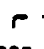

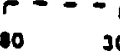

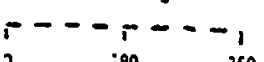

$\left({ }^{\circ} \mathrm{C}\right)$

POT. TEMP ( $\mathrm{X}$ ) - -

WIND DIR.

$-$

8

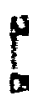




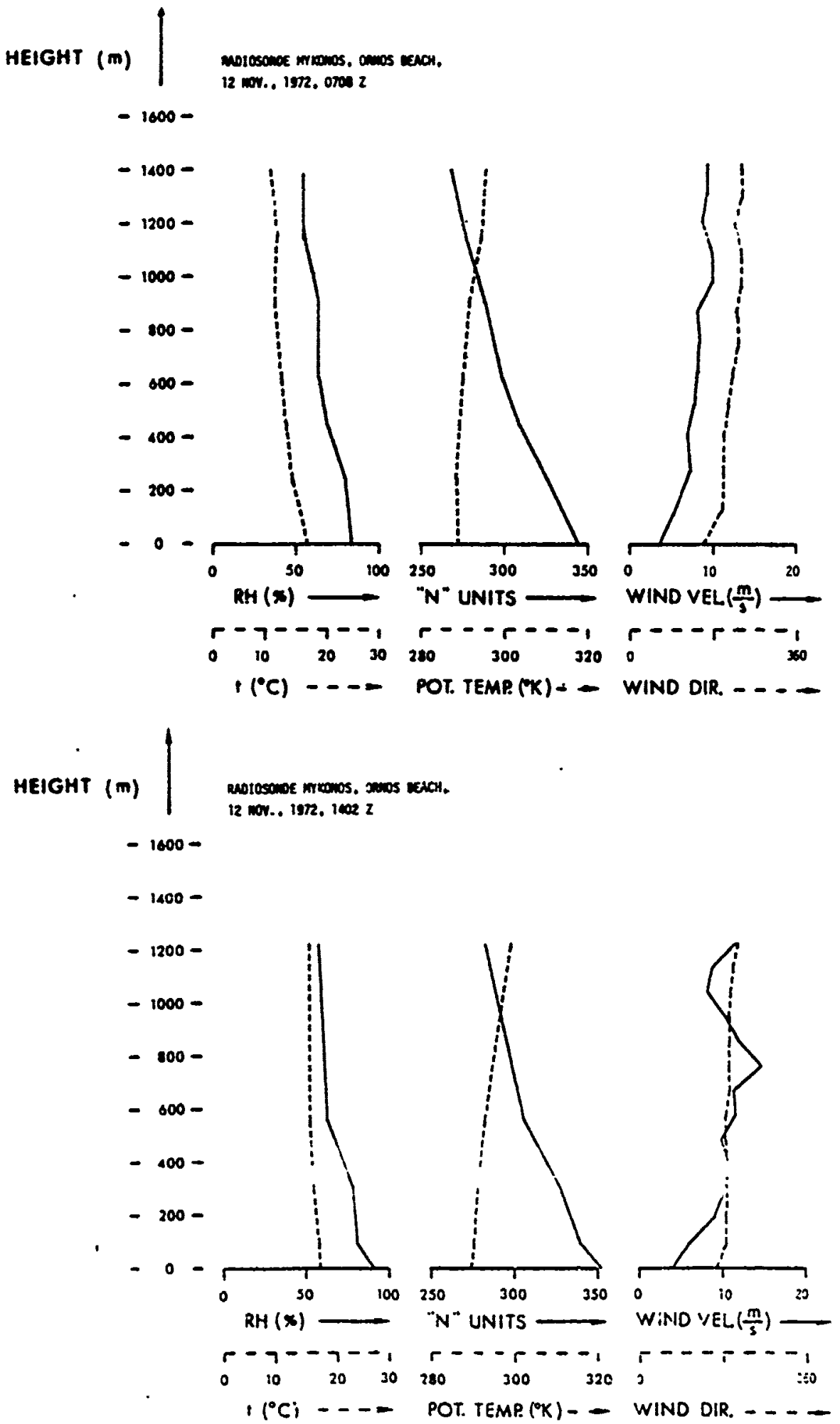



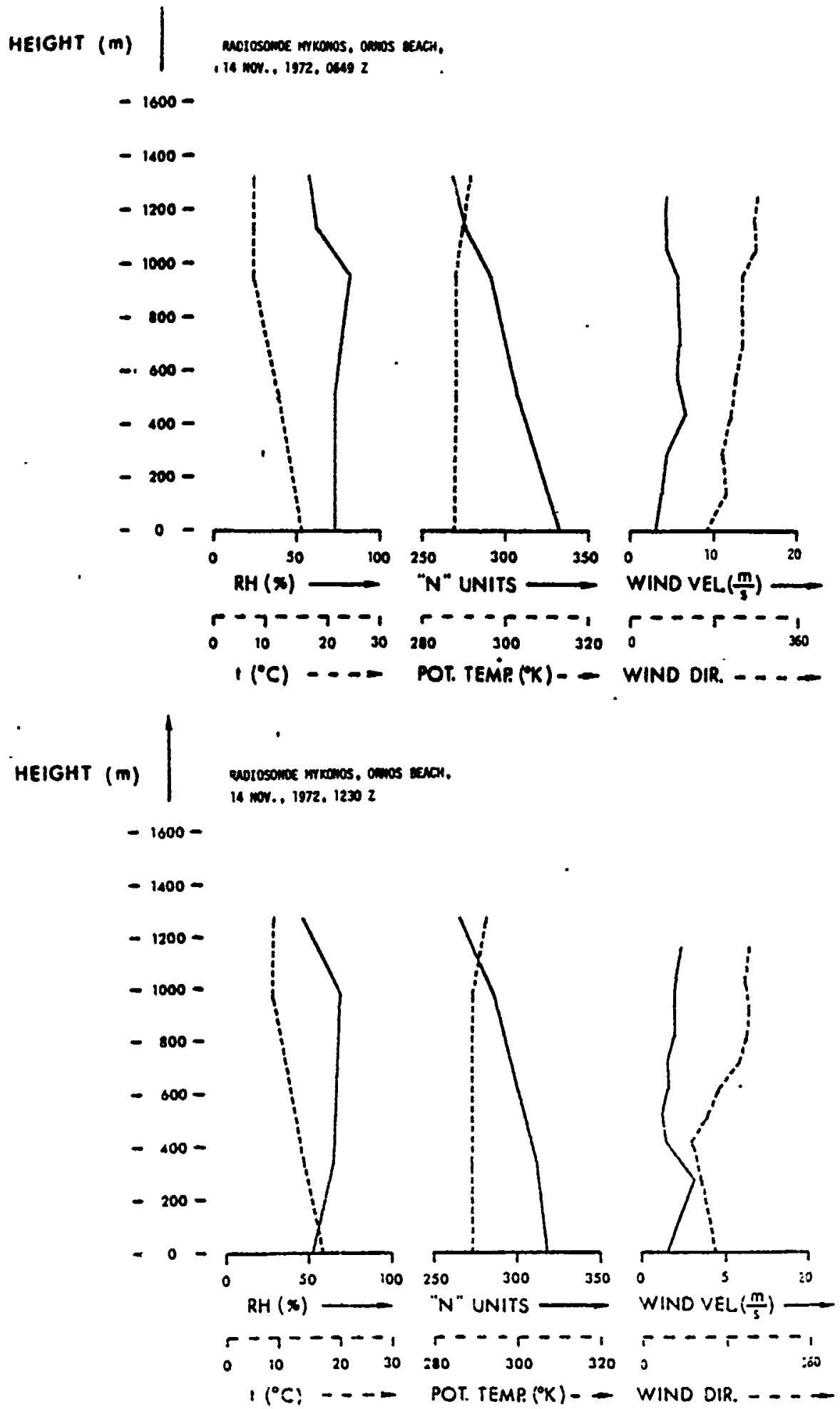


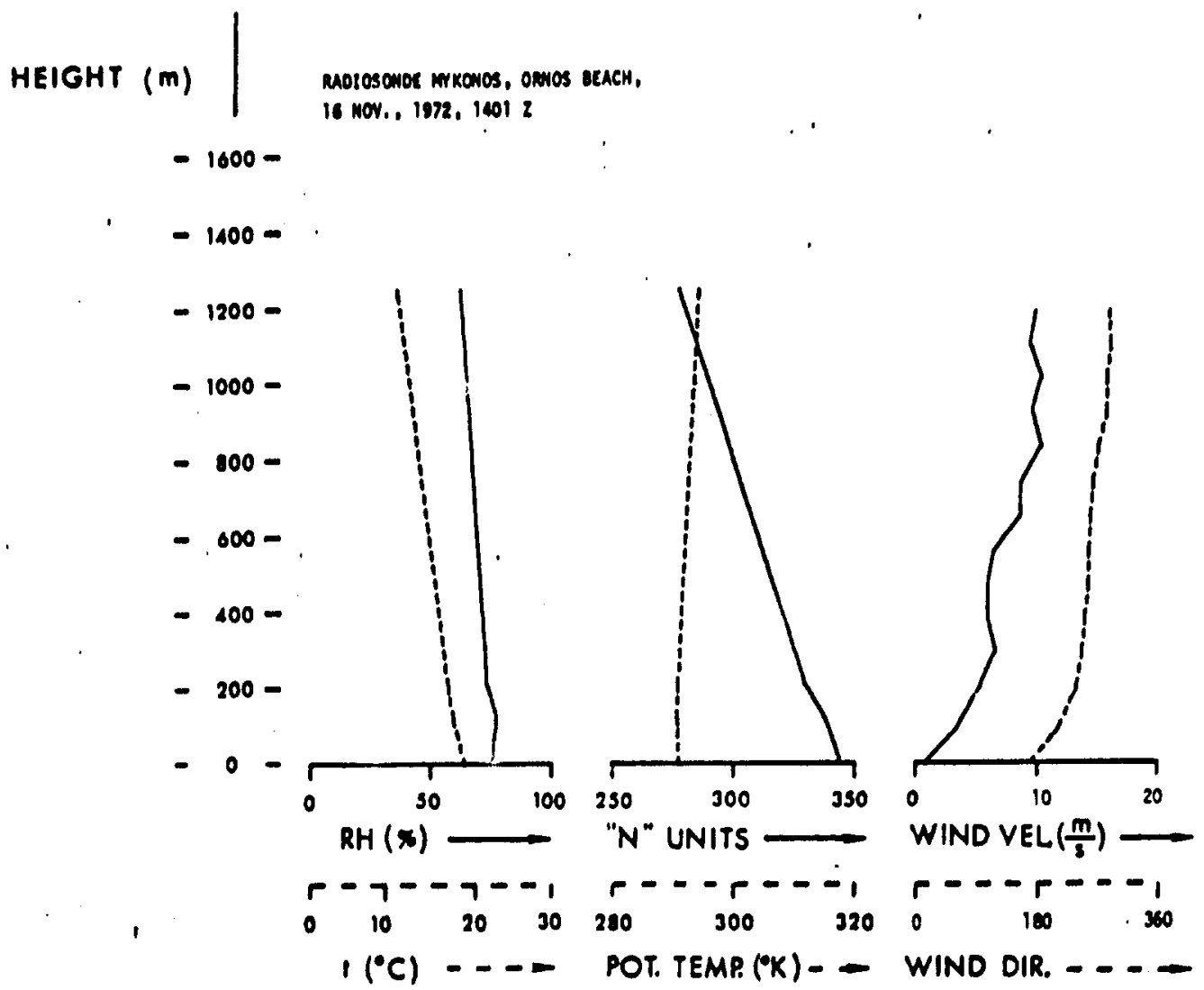




\section{ACKNOWLEDGEMENT}

The measurement program and the data analysis in this report involved many people. The conscientious effort and enthusiasm of the following people were essential for the successful outcome of the project: K. D. Anderson, L. J. Goodson, W. K. Horner, Dr. D. R. Jensen, M. L. Phares, and J. F. Theisen.

The measurements were conducted with the assistance of University of Athens personnel. We thank Professor Anastassiadis for his support and C. Dragatsis, D. Mavrakis, and S. Prionas for their help during the program.

Also gratefully acknowledged is the assistance and support of the Naval Communication Station in Greece. Captain Brabant took a personal Interest in our program and gave us assistance through the entire measurement period. Final thanks go to the $7206 \mathrm{th}$ Support Group USAFE in Athens

- who provided disbursing support and use of their facilities. 